NISTIR 8209

\title{
Models for Viscosity, Thermal Conductivity, and Surface Tension of Selected Pure Fluids as Implemented in REFPROP v10.0
}

\author{
Marcia L. Huber
}

This publication is available free of charge from: https://doi.org/10.6028/NIST.IR.8209 
NISTIR 8209

\section{Models for Viscosity, Thermal Conductivity, and Surface Tension of Selected Pure Fluids as Implemented in REFPROP v10.0}

Marcia L. Huber Applied Chemicals and Materials Division Material Measurement Laboratory

This publication is available free of charge from:

https://doi.org/10.6028/NIST.IR.8209

August 2018

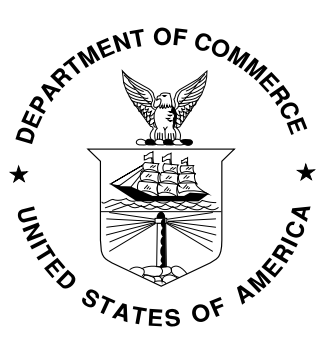

U.S. Department of Commerce Wilbur L. Ross, Jr., Secretary

National Institute of Standards and Technology Walter Copan, NIST Director and Under Secretary of Commerce for Standards and Technology 


\section{Disclaimer}

Certain commercial entities, equipment, or materials may be identified in this document in order to describe an experimental procedure or concept adequately. Such identification is not intended to imply recommendation or endorsement by the National Institute of Standards and Technology, nor is it intended to imply that the entities, materials, or equipment are necessarily the best available for the purpose. 


\begin{abstract}
We describe models for the viscosity, thermal conductivity, and surface tension for selected fluids implemented in version 10.0 of the NIST computer program, NIST Standard Reference Database 23, also known as NIST Reference Fluid Thermodynamic and Transport Properties Database (REFPROP). These fluids do not presently have published reference fluid quality models in the open literature, so we provide preliminary models based on available data as an interim measure to allow calculations of these properties. Comparisons with available experimental data are given.
\end{abstract}

\title{
Keywords
}

natural gas; refrigerants; REFPROP; surface tension; thermal conductivity; viscosity. 


\section{Table of Contents}

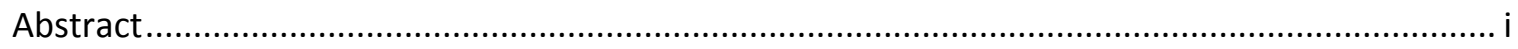

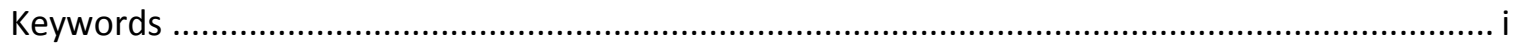

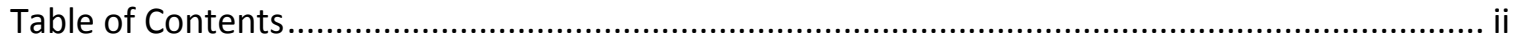

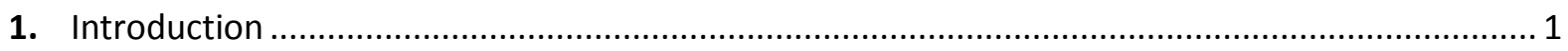

2. Pure-Fluid Extended Corresponding States Viscosity Model ..................................................... 1

3. Pure-Fluid Extended Corresponding States Thermal Conductivity Model .................................... 3

4. Pure-Fluid Dedicated Polynomial Thermal Conductivity Model .................................................... 4

5. Pure-Fluid Surface Tension Model ............................................................................................. 5

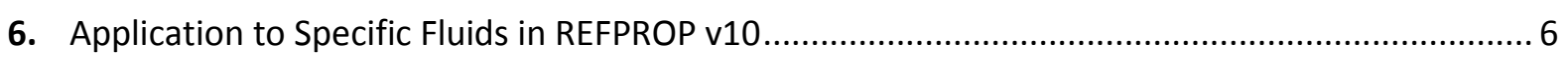

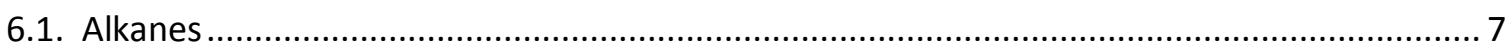

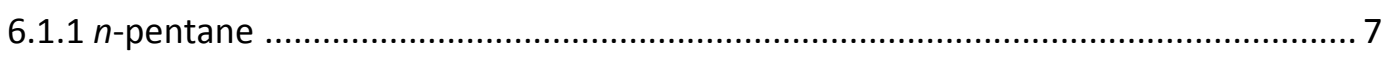

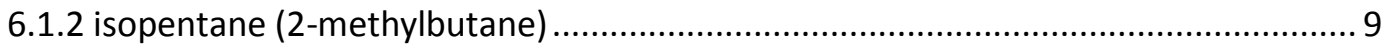

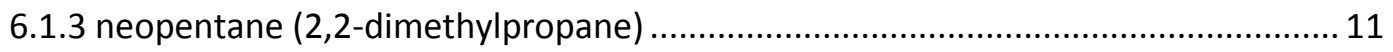

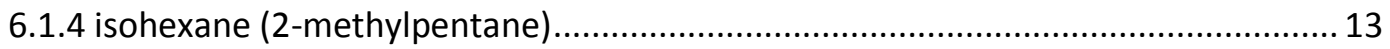

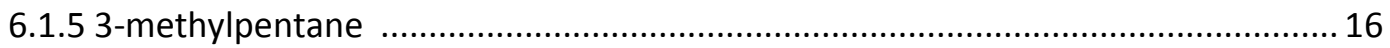

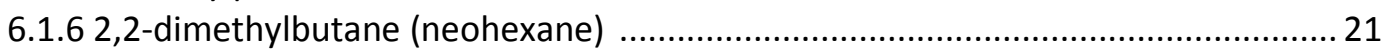

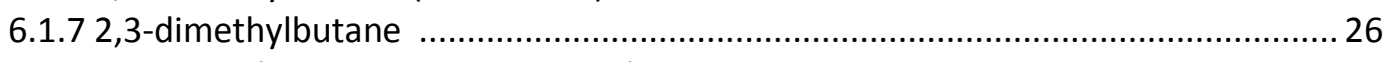

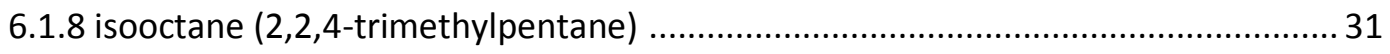

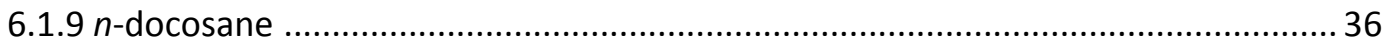

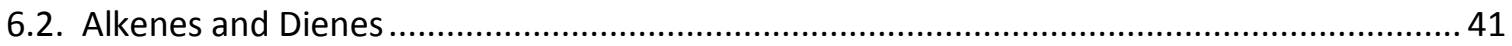

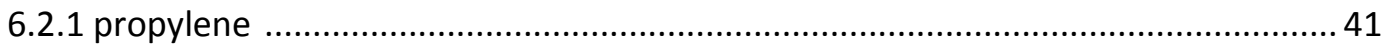

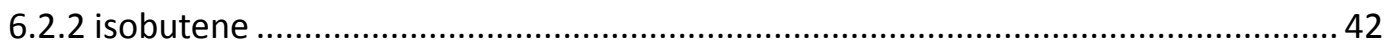

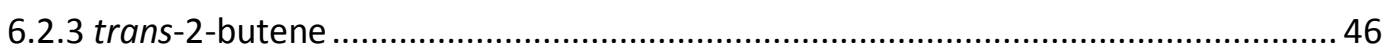

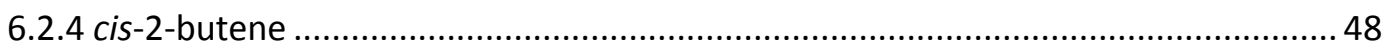

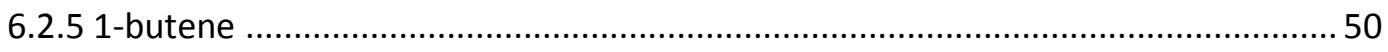

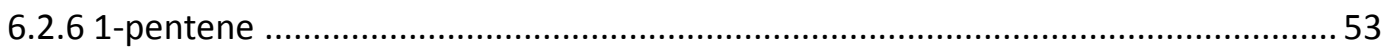

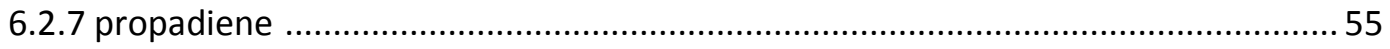

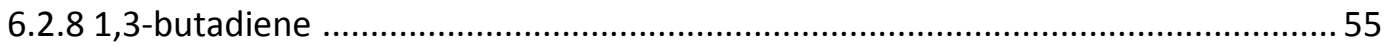

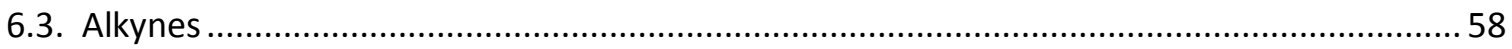

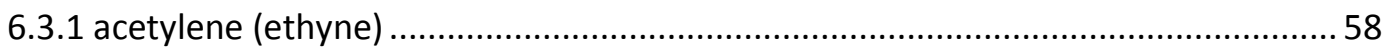

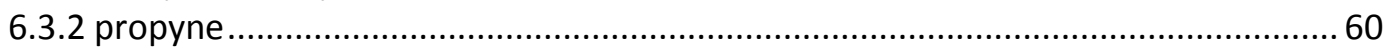

6.3.3 1-butyne

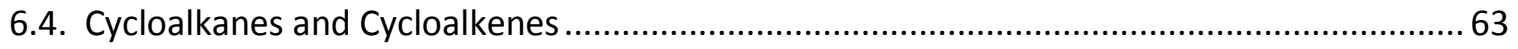

6.4 .1 cyclopropane

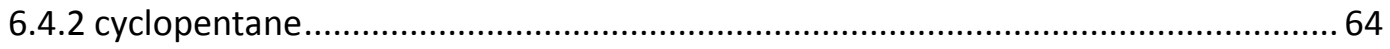

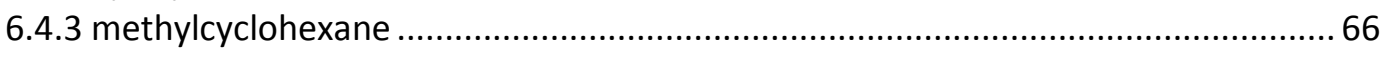

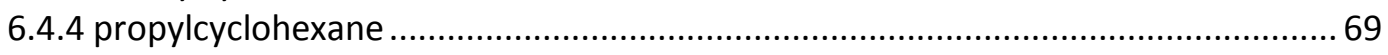

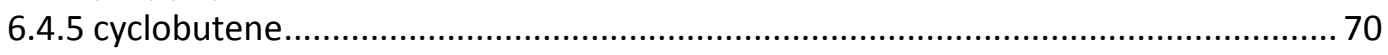




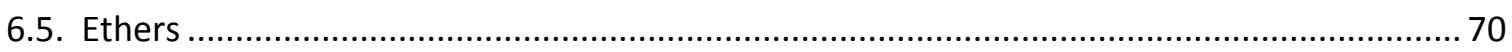

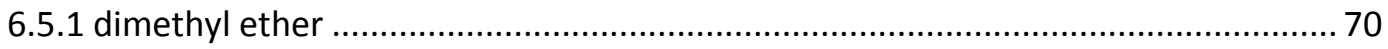

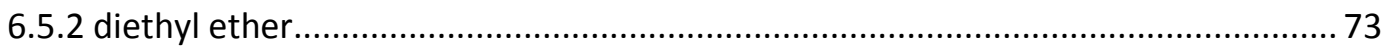

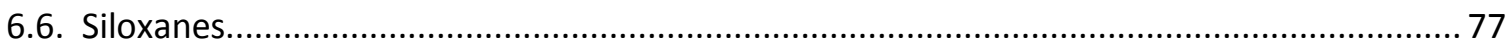

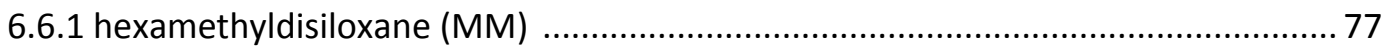

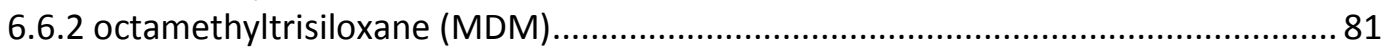

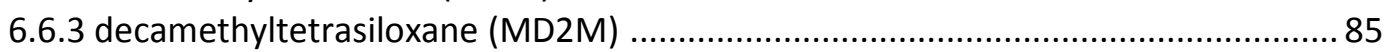

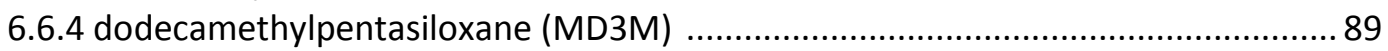

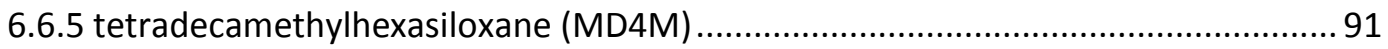

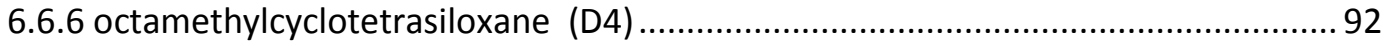

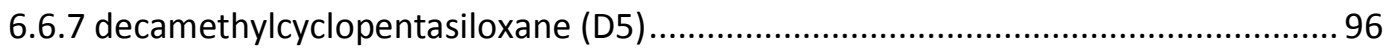

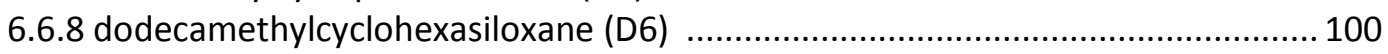

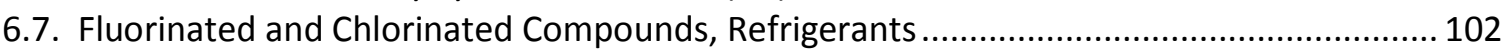

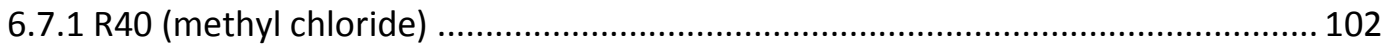

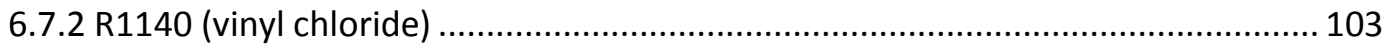

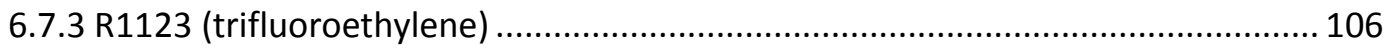

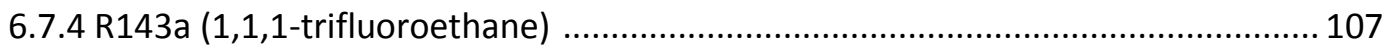

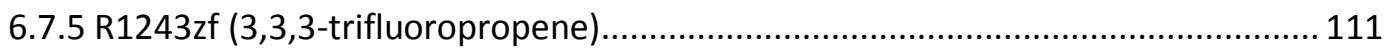

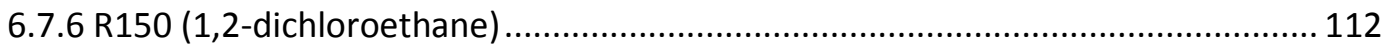

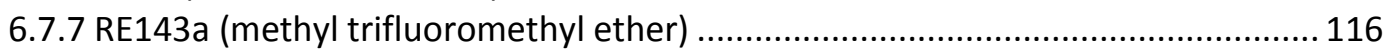

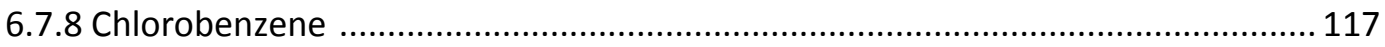

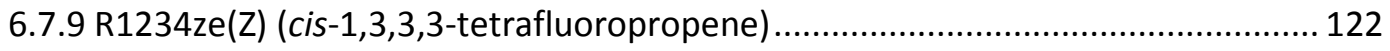

6.7.10 R1233zd(E) (trans-1-chloro-3,3,3-trifluoro-1-propene).................................... 124

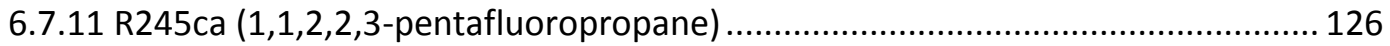

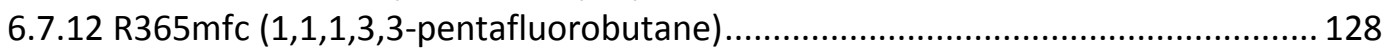

6.7.13 R1224yd(Z) ((Z)-1-chloro-2,3,3,3-tetrafluoropropene).................................... 130

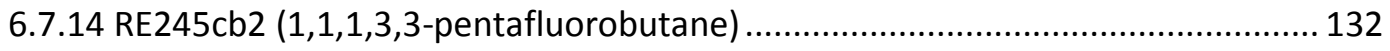

6.7.15 RE245fa2 (2,2,2-trifluoroethyl-difluoromethyl ether) .................................... 136

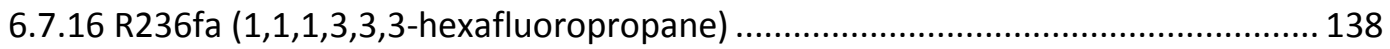

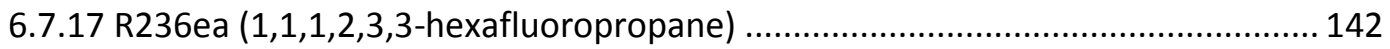

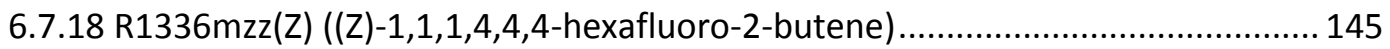

6.7.19 R114 (1,2-dichloro-1,1,2,2-tetrafluoroethane)................................................ 148

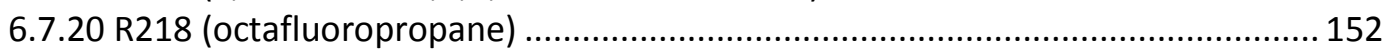

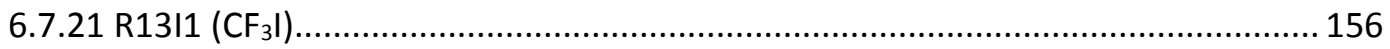

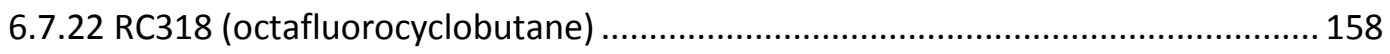

6.7.23 RE347mcc (1,1,1,2,2,3,3-heptafluoro-3-methoxypropane) ............................... 162

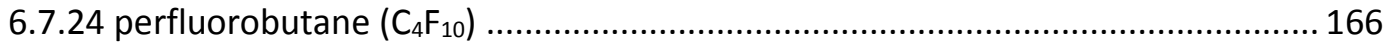

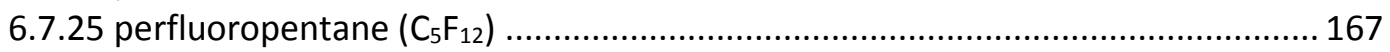

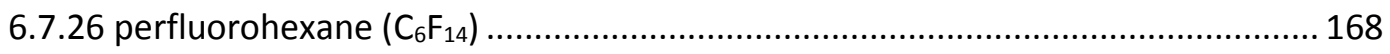

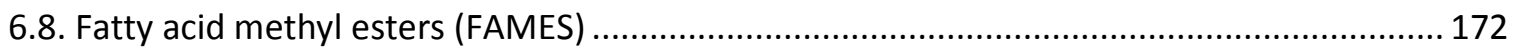

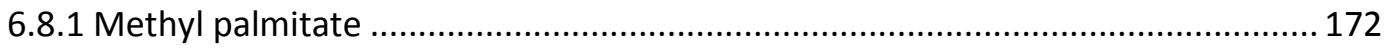

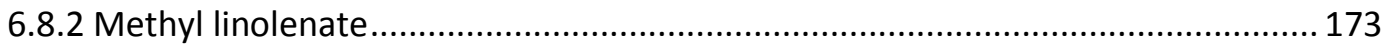

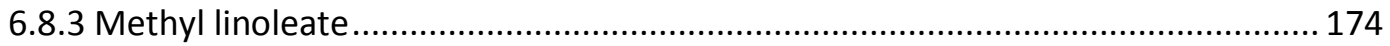

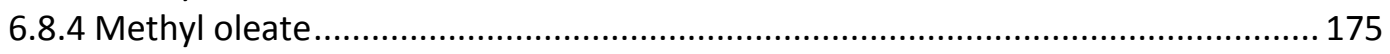

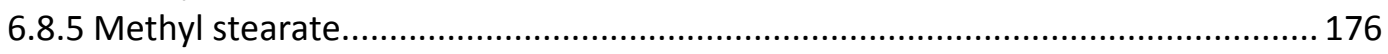




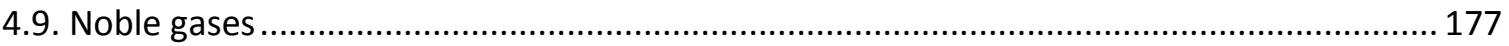

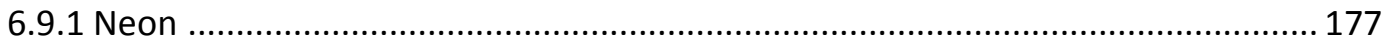

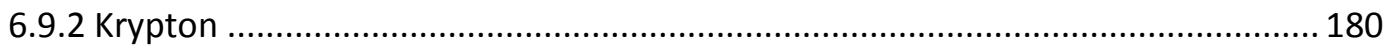

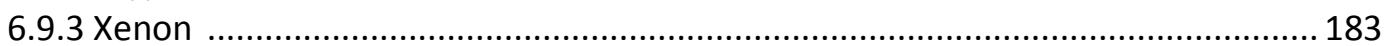

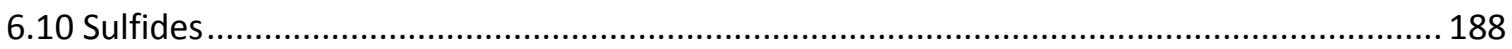

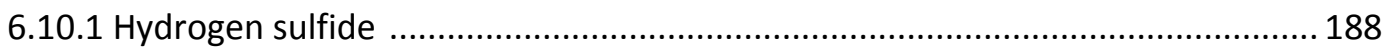

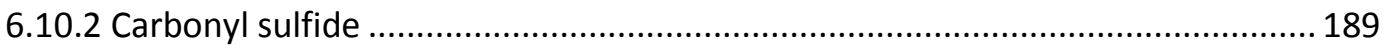

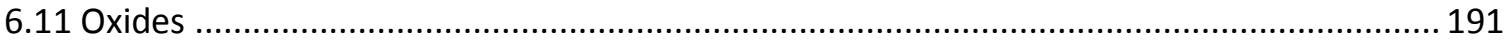

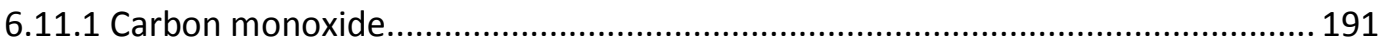

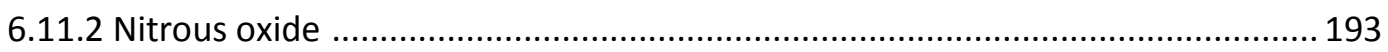

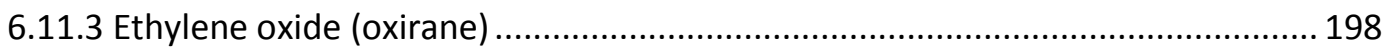

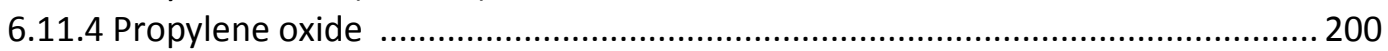

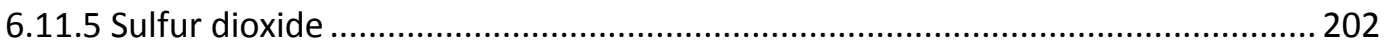

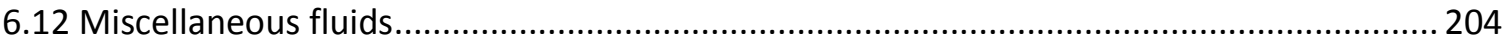

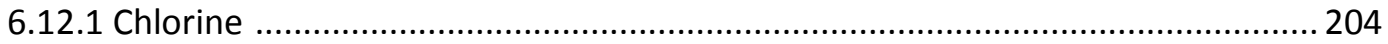

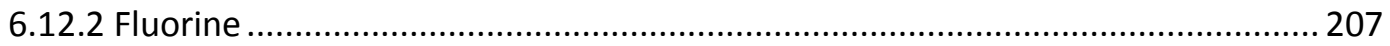

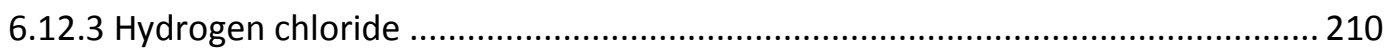

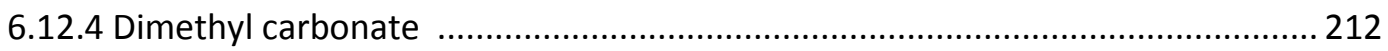

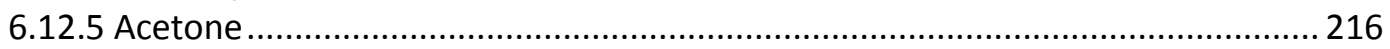

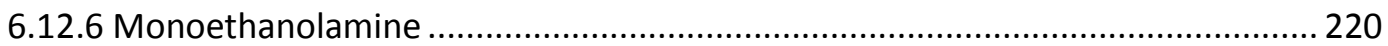

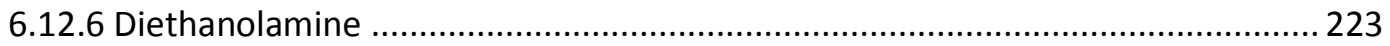

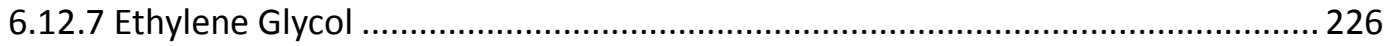

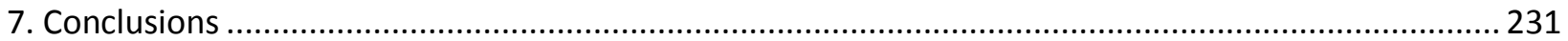

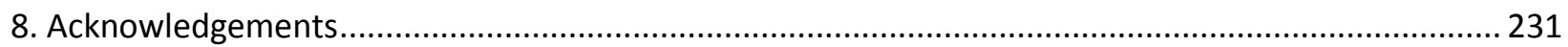

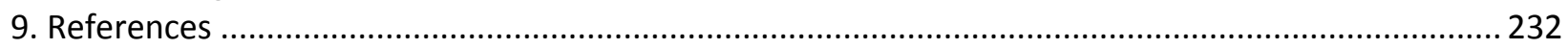

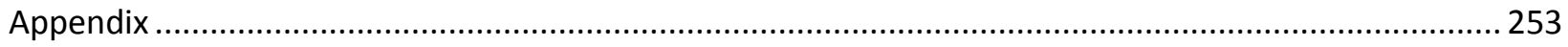

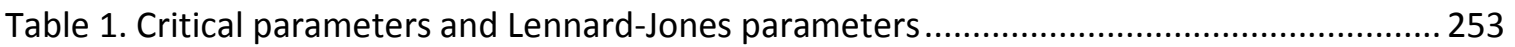

Table 2. Coefficients for the residual viscosity, Eq. (11), and $F_{c}$ from Eq. (8)............................ 256

Table 3. Coefficients for the dilute gas and residual thermal conductivity, Eq. (16), Eq. (21). ... 259

Table 4. Coefficients for the critical enhancement of thermal conductivity ................................262

Table 5. Coefficients for the surface tension, Eq. (25)............................................................ 264

Table 6. Coefficients for the dedicated polynomial correlations of thermal conductivity,

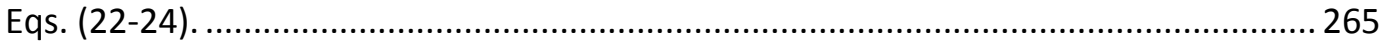

Table 7. Sample points for checking computer calculations for the viscosity,

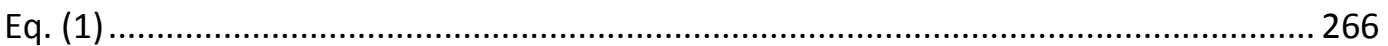

Table 8. Sample points for checking computer calculations for the thermal conductivity,

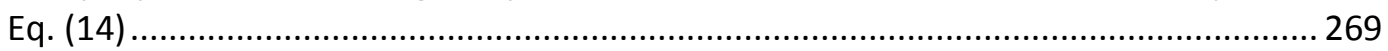

Table 9. Sample points for checking computer calculations for the thermal conductivity,

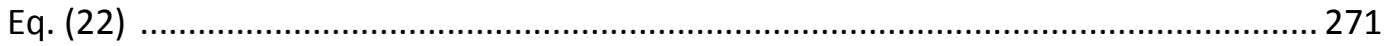

Table 10. Sample points for checking computer calculations for the surface tension, Eq. (25). 


\section{Introduction}

A NIST computer program, NIST Standard Reference Database 23, also known as NIST Reference Fluid Thermodynamic and Transport Properties Database (REFPROP) (E. W. Lemmon, Bell, Huber, \& McLinden, 2018), provides models for the thermophysical properties of commonly used industrial fluids, including refrigerants, natural gas fluids, cryogens, alcohols, and other fluids. The goal is to provide high-accuracy models that can represent the thermophysical properties of the fluids to within their experimental uncertainty. For some fluids, lack of experimental data prevents this goal from being achieved. As a result, we provide here some models (considered preliminary) that can be used to compute thermophysical properties in REFPROP until more data and better models become available. A previous publication (Chichester \& Huber, 2008) described the extended corresponding states model for viscosity and thermal conductivity implemented in REFPROP, which is based on the earlier publications by Ely and Hanley (J.F. Ely \& Hanley, 1981, 1983). This method is also discussed in additional publications (Huber \& Ely, 1992a, 1992b; Huber, Laesecke, \& Perkins, 2003; Klein, McLinden, \& Laesecke, 1997; M.O. McLinden, Klein, \& Perkins, 2000); here we will present the coefficients and comparisons with data; the reader is referred to earlier publications for details on the methodology. A worked example of the use of extended corresponding states to calculate the viscosity and thermal conductivity of pure fluids can be found in the work of Bell et al. (Bell, Wronski, Quoilin, \& Lemort, 2014).

\section{Pure-Fluid Extended Corresponding States Viscosity Model}

The viscosity of a pure fluid is represented as a sum of a dilute-gas and a residual contribution. Only the residual contribution is treated via corresponding states:

$$
\eta(T, \rho)=\eta^{*}(T)+\Delta \eta(T, \rho)=\eta^{*}(T)+\Delta \eta_{0}\left(T_{0}, \rho_{0}\right) F_{\eta}(T, \rho)
$$

where the superscript $*$ denotes a dilute gas value, and the subscript 0 denotes a reference fluid value. The viscosity of the reference fluid is evaluated at a conformal temperature $T_{0}$ and molar density $\rho_{0}$ given by

$$
T_{0}=T / f(T, \rho)
$$

and

$$
\rho_{0}=\rho h(T, \rho)
$$

The quantities $f$ and $h$ are called equivalent substance reducing ratios, and relate the reference fluid to the fluid of interest using a ratio of critical parameters (denoted by the subscript $\mathrm{c}$ ) and functions of temperature and density known as shape functions $\theta$ and $\phi$, 


$$
f=\frac{T_{\mathrm{c}}}{T_{\mathrm{c} 0}} \theta(T, \rho)
$$

and

$$
h=\frac{\rho_{\mathrm{c} 0}}{\rho_{\mathrm{c}}} \phi(T, \rho),
$$

where all densities are molar densities. The shape factors can be considered functions of both temperature and density. In this work, we generally have available accurate formulations for the thermodynamic properties of the fluids, either in terms of a Helmholtz energy equation or a PVT equation of state (EOS), and we use a form of the "exact" shape factor method (J. F. Ely \& Magee, 1989). It is a requirement in this method to first determine the thermodynamic shape factors.

The dilute-gas viscosity in Eq. (1) is found by Chapman-Enskog theory (Hirschfelder, Curtiss, \& Bird, 1964)

$$
\eta^{*}(T)=\frac{5 \sqrt{m \pi k_{\mathrm{B}} T}}{16 \pi \sigma^{2} \Omega^{(2,2)}},
$$

where the dilute-gas viscosity is $\eta^{*}$ in $\mathrm{Pa} \cdot \mathrm{s}, \mathrm{m}$ is the molecular mass in $\mathrm{kg}, k_{\mathrm{B}}$ is the Boltzmann constant $\left(1.38064852 \times 10^{-23} \mathrm{~m}^{2} \cdot \mathrm{kg} \cdot \mathrm{s}^{-2} \cdot \mathrm{K}^{-1}\right.$ ) (Mohr, Newell, \& Taylor, 2016), $\sigma$ is a collision diameter in $\mathrm{m}$, and $T$ is the absolute temperature in $\mathrm{K}$. We will further assume that a Lennard-Jones 12-6 potential applies, and use the Lennard-Jones collision diameter for $\sigma$. Neufeld et al. (Neufeld, Janzen, \& Aziz, 1972) gave the following empirical correlation (neglecting the sinusoidal term) for the calculation of the collision integral $\Omega^{(2,2)}$

$$
\Omega^{(2,2)}=1.16145\left(T^{*}\right)^{-0.14874}+0.52487 \mathrm{e}^{-0.77320 T^{*}}+2.16178 \mathrm{e}^{-2.43787 T^{*}},
$$

with the dimensionless temperature $T^{*}=k_{\mathrm{B}} T / \varepsilon$, and $\varepsilon$ the minimum of the Lennard-Jones pairpotential energy. The range of validity of this empirical correlation is $0.3<T^{*}<100$.

To account for polyatomic gases, Chung et al. (Chung, Ajlan, Lee, \& Starling, 1988) introduced a correction factor $F_{\mathrm{c}}$ defined by $F_{\mathrm{c}}=1-0.2756 \omega+0.059035 \mu_{\mathrm{r}}^{4}$ where $\omega$ is the acentric factor and $\mu_{\mathrm{r}}$ is a reduced dipole moment defined by $\mu_{\mathrm{r}}=131.3 \mu /\left(V_{\mathrm{c}} T_{\mathrm{c}}\right)^{0.5}$ such that Eq. (6) may be written (Chung et al., 1988)

$$
\eta^{*}(T)=4.0785 \frac{(M T)^{0.5}}{V_{\mathrm{c}}^{2 / 3} \Omega^{(2,2)}} F_{\mathrm{c}},
$$

where the units on critical volume $V_{\mathrm{c}}$ are $\mathrm{cm}^{3} \cdot \mathrm{mol}^{-1}$ and $T$ is in $\mathrm{K}$, and $M$ is the molar mass of the fluid in $\mathrm{g} \cdot \mathrm{mol}^{-1}$, and the viscosity is in $\mu \mathrm{Pa} \cdot \mathrm{s}$.

The function $F_{\eta}$ in Eq. (1) is found using the expression 


$$
F_{\eta}(T, \rho)=f^{1 / 2} h^{-2 / 3}\left[\frac{M}{M_{0}}\right]^{1 / 2},
$$

where $M$ is the molar mass of the fluid and $M_{0}$ is the molar mass of the reference fluid. The model as developed to this point is predictive, and does not use any information on the viscosity of the fluid (except for the dilute-gas piece that requires Lennard-Jones $\varepsilon$ and $\sigma$ ). The nondimensional functions $f$ and $h$ are found from thermodynamic data as described above. In order to improve the representation of the viscosity, an empirical correction factor may be used if there are experimental viscosity data available. We then evaluate Eq. (1) at $\rho_{0, v}$ instead of $\rho_{0}$, where (Huber et al., 2003)

$$
\rho_{0, v}(T, \rho)=\rho_{0}(T, \rho) \psi\left(\rho_{\mathrm{r}}\right),
$$

and $\psi$ is a polynomial in reduced density $\rho_{\mathrm{r}}=\rho / \rho_{\mathrm{c}}$ of the form

$$
\psi\left(\rho_{r}\right)=\sum_{k=0}^{n} c_{k} \rho_{\mathrm{r}}^{k},
$$

where the coefficients $c_{k}$ are constants found from fitting the experimental viscosity data. As indicated in Eq. (1), in order to evaluate the viscosity of a particular fluid, the value of the residual viscosity of a reference fluid is required. It is not necessary to use the same reference fluid for all fluids; however, when using the model in a predictive mode, it is best to select the reference fluid that is most similar in chemical nature to the fluid of interest. The reference fluid should also have a very accurate equation of state and viscosity surface. When using pure-fluid experimental viscosity to essentially "correct" the viscosity, the choice of reference fluid is not as important, since an empirical correction factor determined from data is applied as in Eqs. (10-11).

\section{Pure-Fluid Extended Corresponding States Thermal Conductivity Model}

We start with the procedure of Ely and Hanley (J.F. Ely \& Hanley, 1983) and represent the thermal conductivity of a fluid as the sum of translational (from collisions between molecules) and internal (due to internal motions of the molecule) modes of energy transfer,

$$
\lambda(T, \rho)=\lambda^{\text {int }}(T)+\lambda^{\text {trans }}(T, \rho) .
$$

The translational contribution may be further divided into a dilute-gas contribution (denoted here by a superscript $*$ ) that is a function only of temperature, a residual contribution, and a critical enhancement,

$$
\lambda^{\text {trans }}(T, \rho)=\lambda^{*}(T)+\lambda^{\mathrm{r}}(T, \rho)+\lambda^{\text {crit }}(T, \rho),
$$

leading to the following expression for the thermal conductivity

$$
\begin{aligned}
\lambda(T, \rho) & =\lambda^{\mathrm{int}}(T)+\lambda^{*}(T)+\lambda^{\mathrm{r}}(T, \rho)+\lambda^{\mathrm{crit}}(T, \rho) . \\
& =\lambda^{0}(T)+\lambda^{\mathrm{r}}(T, \rho)+\lambda^{\mathrm{crit}}(T, \rho)
\end{aligned}
$$


We use an Eucken correlation for the internal contribution

$$
\lambda^{\text {int }}(T)=\frac{f_{\text {int }} \eta^{*}}{M}\left[C_{p}^{*}-\frac{5}{2} R\right],
$$

where $C_{p} *$ is the ideal-gas heat capacity (in $\mathrm{J} \cdot \mathrm{mol}^{-1} \cdot \mathrm{K}^{-1}$ ), $R$ is the molar gas constant (Mohr et al., 2016) $\left(8.314472 \mathrm{~J} \cdot \mathrm{mol}^{-1} \cdot \mathrm{K}^{-1}\right), \eta^{*}$ is the dilute-gas viscosity $(\mu \mathrm{Pa} \cdot \mathrm{s})$ as given in $\mathrm{Eq} .(8), f_{\text {int }}$ is set to $1.32 \times 10^{-3}$, and $\lambda$ is in $\mathrm{W} \cdot \mathrm{m}^{-1} \cdot \mathrm{K}^{-1}$. If sufficient dilute-gas thermal conductivity data are available, $f_{\text {int }}$ is fit to a polynomial in temperature,

$$
f_{\mathrm{int}}=\sum_{i=0}^{n} a_{i} T^{i}
$$

where the number of terms in the summation depends upon the fluid. For the dilute-gas translational contribution (in $\mathrm{W} \cdot \mathrm{m}^{-1} \cdot \mathrm{K}^{-1}$ ), we use

$$
\lambda^{*}(T)=\frac{15 \times 10^{-3} R \eta^{*}}{4 M},
$$

where the dilute-gas viscosity, $\eta^{*}$, is from Eq. (8) (in $\mu \mathrm{Pa} \cdot \mathrm{s}$ ). The residual contribution is found using extended corresponding states:

$$
\lambda^{\mathrm{r}}(T, \rho)=\lambda_{0}^{\mathrm{r}}\left(T_{0}, \rho_{0}\right) F_{\lambda},
$$

with

$$
F_{\lambda}(T, \rho)=f^{1 / 2} h^{-2 / 3}\left[\frac{M_{0}}{M}\right]^{1 / 2} .
$$

In order to improve the representation of the thermal conductivity, an empirical correction factor may be used if there are experimental thermal conductivity data available. We then evaluate Eq. (28) at $\rho_{0, k}$ instead of $\rho_{0}$, where (M.O. McLinden et al., 2000)

$$
\rho_{0, k}(T, \rho)=\rho_{0}(T, \rho) \chi\left(\rho_{\mathrm{r}}\right)
$$

and $\chi$ is a polynomial in reduced density $\rho_{\mathrm{r}}=\rho / \rho_{\mathrm{c}}$ of the form

$$
\chi\left(\rho_{\mathrm{r}}\right)=\sum_{k=0}^{n} b_{k} \rho_{\mathrm{r}}^{k},
$$

where the coefficients $b_{k}$ are found from fitting the experimental thermal conductivity data.

The critical contribution is computed using a simplified crossover model developed by Olchowy and Sengers (Olchowy \& Sengers, 1989), and later generalized (R. A. Perkins, Sengers, Abdulagatov, \& Huber, 2013) so that it may be used knowing only $T_{\mathrm{c}}, \rho_{\mathrm{c}}, p_{\mathrm{c}}$, the acentric factor $\omega$ and the molecular weight of the component. Unless specified otherwise, all critical enhancement 
parameters in this document are obtained from the method of Perkins et al. (R. A. Perkins et al., 2013) with critical parameters and an acentric factor obtained from the specific equation of state used for thermodynamic properties for each fluid.

\section{Pure-Fluid Dedicated Polynomial Thermal Conductivity Model}

When there are sufficient high-quality data available that span the entire liquid, gas, and fluid regions, one can make a dedicated fluid correlation that does not require the use of corresponding states. These correlations are much simpler to implement, faster to calculate, and generally are preferred when sufficient data are present. Typically, the thermal conductivity is expressed in terms of a dilute gas, residual, and critical enhancement term:

$$
\lambda(T, \rho)=\lambda^{0}(T)+\Delta \lambda^{\text {res }}(T, \rho)+\lambda^{\text {cit }}(T, \rho),
$$

where the dilute gas contribution $\lambda^{0}$ is a function of $T$ only. Note that the dilute-gas contribution here includes both the translational and rotational contributions mentioned earlier in Eq. (14). More complicated forms, including ones that are more theoretically based, have been used in other works (Assael et al., 2013; Assael, Koutian, Huber, \& Perkins, 2016; Assael, Mylona, Huber, \& Perkins, 2012), but here we will use a simple polynomial in temperature,

$$
\lambda^{0}(T)=\sum_{k=0}^{n} \alpha_{k} T^{k}
$$

The residual contribution $\lambda^{\text {res }}$ is written as a polynomial in terms of temperature and density,

$\Delta \lambda^{\mathrm{res}}(T, \rho)=\sum_{l=0}^{m}\left(\beta_{1, l}+\beta_{2, l}\left(T / T_{\mathrm{c}}\right)\right)\left(\rho / \rho_{\mathrm{c}}\right)^{l}$,

and the critical enhancement contribution is computed as discussed earlier using a simplified crossover model developed by Olchowy and Sengers (Olchowy \& Sengers, 1989), and later generalized (R. A. Perkins et al., 2013). When data in the critical region are present, the parameter $q_{\mathrm{d}}{ }^{-1}$ (the cutoff wavelength, see (R. A. Perkins et al., 2013)) may be found by fitting instead of using the generalized method in (R. A. Perkins et al., 2013).

\section{Pure-Fluid Surface Tension Model}

We fit surface tension data to a commonly used equation that has been used successfully for other fluids in the REFPROP program (Mulero \& Cachadiña, 2014):

$$
\sigma(T)=\sum_{i=0}^{k} \sigma_{i}\left(1-\frac{T}{T_{\mathrm{c}}}\right)^{n_{i}}
$$


where $\sigma_{\mathrm{i}}$ and $n_{\mathrm{i}}$ are coefficients obtained from fitting data and $T_{\mathrm{c}}$ is the critical temperature in $\mathrm{K}$, and the surface tension is in $\mathrm{N} \cdot \mathrm{m}^{-1}$. Since this function is zero at the critical point, uncertainty estimates expressed in the text only apply to temperatures not close to critical. 


\section{Application to Specific Fluids in REFPROP v10}

The following section gives details on the data used to develop correlations for the viscosity, thermal conductivity, and surface tension for selected pure fluids in REFPROP v10. Extensive use of the NIST ThermoDataEngine database (Diky et al., 2017) was made to locate data sources. A reference fluid is necessary to implement the corresponding states method; the same reference fluid is not used for all fluids. The specific reference fluid will be noted for each application, selected from these fluids: propane, $n$-octane, $n$-dodecane, $\mathrm{R} 134 \mathrm{a}$, and nitrogen. The associated equations of state and transport correlations are those adopted as the default choices in REFPROP v10, that can be found in Ref.(Beckmueller, Thol, Lemmon, \& Span, 2018; Huber et al., 2003; Huber, Laesecke, \& Perkins, 2004; Huber, Laesecke, \& Xiang, 2004; Huber \& Perkins, 2005; E.W. Lemmon \& Huber, 2004; E.W. Lemmon \& Jacobsen, 2004; E. W. Lemmon, McLinden, \& Wagner, 2009; Marsh, Perkins, \& Ramires, 2002; R. A. Perkins, Laesecke, A., Howley, J., Ramires, M.L.V., Gurova, A.N. and Cusco, L., 2000; Span, Lemmon, Jacobsen, Wagner, \& Yokozeki, 2000; Tilner-Roth \& Baehr, 1994; E. Vogel \& Herrmann, 2016). The only exception to this is that for propane, the older viscosity model of Vogel (E. Vogel, Küchenmeister, Bich, \& Laesecke, 1998) is used instead of the very recent Vogel publication (E. Vogel \& Herrmann, 2016). This is helpful for backwards compatibility, since the newer model is not available in older versions of REFPROP. When uncertainty is discussed in this document, it is the expanded uncertainty with a coverage factor of two, that is approximately a $95 \%$ confidence interval. Tables for checking computer programming of the equations can be found in the Appendix. The data point for the calculations corresponds to a temperature of $0.9 T_{\mathrm{c}}$ and a pressure of $0.9 p_{\mathrm{c}}$, rounded to the number of digits shown in the table, ensuring that there will be a contribution for the critical enhancement of the thermal conductivity. 


\subsection{Alkanes}

\subsection{1 $n$-Pentane}

The equation of state of Thol et al. (Thol, Uhde, Lemmon, \& Span, 2018) was used to provide the density and the critical parameters. Lennard-Jones parameters were calculated by fitting the zero-density values for pentane found in Vogel and Holdt (E. Vogel \& Holdt, 1991). These values are presented in Table 1 (located in the Appendix). Numerous data sets for liquidphase viscosity were found and we selected several wide-ranging sets (Audonnet \& Padua, 2001; Estrada-Baltazar, 1998; Giller \& Drickamer, 1949; P. S. Ma, Zhou, Yang, \& Xia, 2004; Oliveira \& Wakeham, 1992; Tohidi, 2001) for regression to obtain the coefficients in Table 2 (located in the Appendix). Figure 1 shows the viscosity deviations as a function of temperature and Figure 2 gives the deviations as a function of pressure. The experimental data exceed $100 \mathrm{MPa}$ in some cases, but we restrict the usage of the correlation to $100 \mathrm{MPa}$ (the limit of the EOS). Based primarily on comparisons with the data of Vogel, we estimate the uncertainty for the gas phase is $1 \%$. There is considerable scatter among the liquid data sets, and we estimate the uncertainty for the liquid phase is $5 \%$ for pressures up to $100 \mathrm{MPa}$. We do not cover the thermal conductivity or surface tension here as there are existing correlations for these properties for $n$-pentane in literature publications (Mulero, Cachadiña, \& Parra, 2012; C.-M. Vassiliou, M.J. Assael, M. L. Huber, \& R. A. Perkins, 2015). 


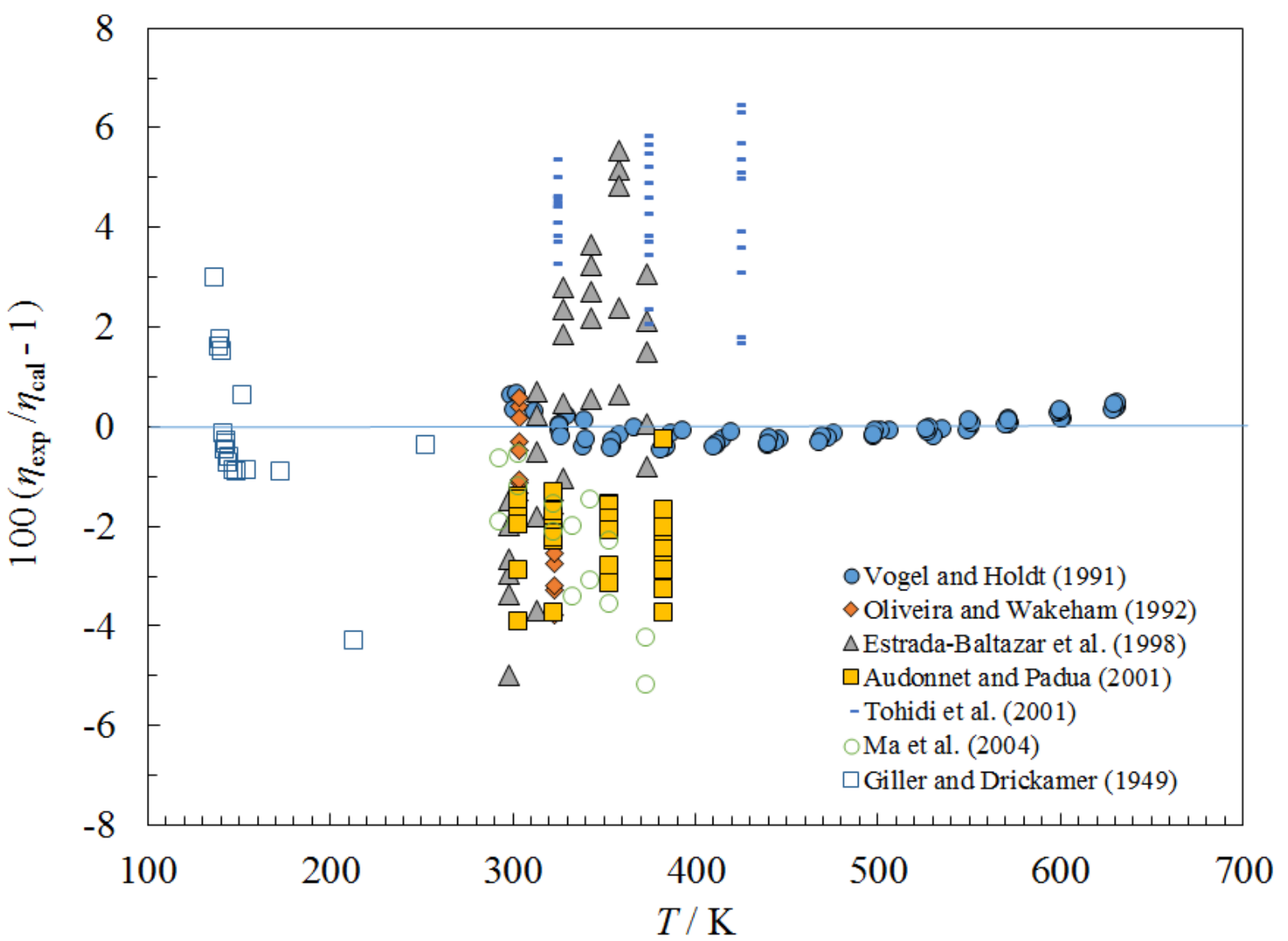

Figure 1. Percentage deviations between the model and the experimental viscosity data for $n$-pentane as a function of temperature. 


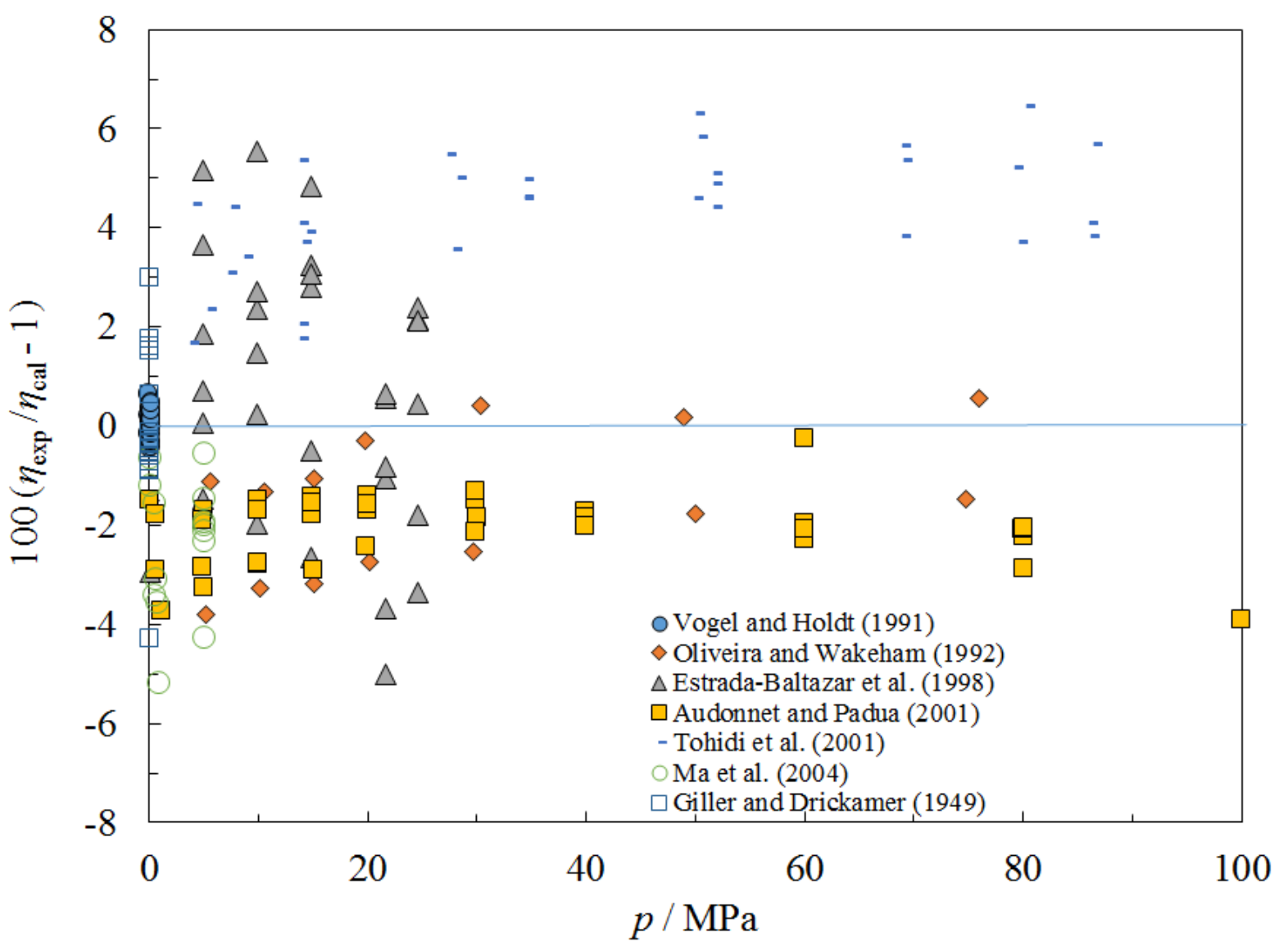

Figure 2. Percentage deviations between the model and the experimental viscosity data for $n$-pentane as a function of pressure.

\subsubsection{Isopentane (2-methylbutane)}

The equation of state of Lemmon and Span (E.W. Lemmon \& Span, 2006) was used to provide the density and the critical parameters. Lennard-Jones parameters were estimated with the method of Chung et al. (Chung et al., 1988) with parameters as given in Table 1 . The value of $F_{\mathrm{c}}$ in Table 2 was adjusted to provide better agreement with the data of Lambert et al. (Lambert et al., 1955) and with McCoubrey and Singh (J.C. McCoubrey \& Singh, 1963). Very limited liquid-phase viscosity data were available, with no data above saturation pressure. The data of Lesche et al. (Lesche, Klemp, \& Nickel, 1984), Batchinski (Batschinski, 1913), and Ma et al. (R. F. Ma, Shi, Duan, Han, \& Liu, 2003) were used to obtain the coefficients in Table 2, and deviations from viscosity data are given in Figure 3. Based on comparisons of the gas-phase data of Lambert et al. (Lambert et al., 1955) and with McCoubrey and Singh (J.C. McCoubrey \& Singh, 1963), the estimated uncertainty for the gas-phase viscosity is $3 \%$. For the liquid phase we estimate the uncertainty is $10 \%$ along saturation at temperatures above $120 \mathrm{~K}$. At lower temperatures and at higher pressures, the uncertainty is larger. We do not cover the thermal conductivity or surface tension here as there are existing correlations for these properties for isopentane in the literature (Mulero et al., 2012; Vassiliou et al., 2015). 


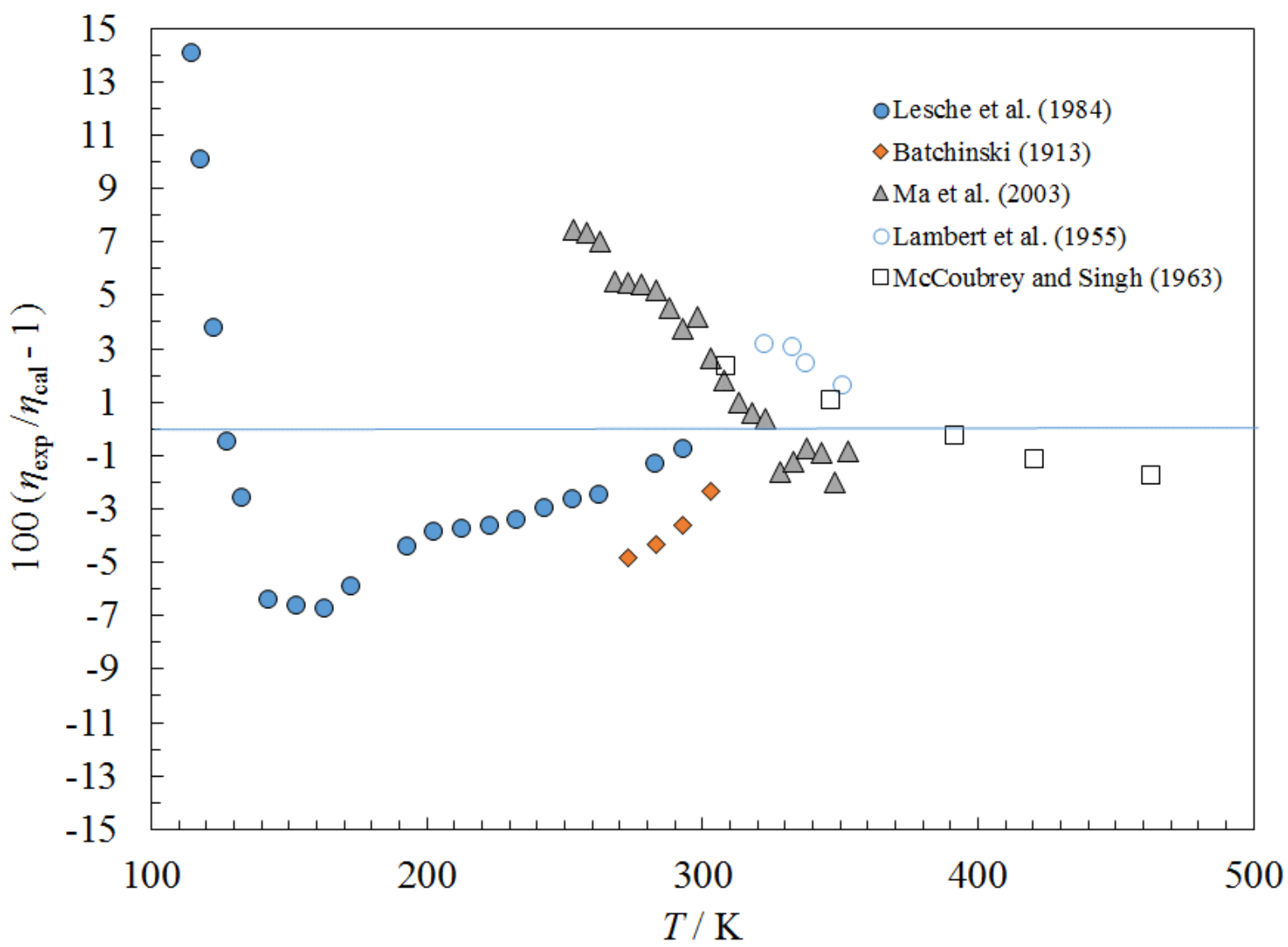

Figure 3. Percentage deviations between the model and the experimental viscosity data for isopentane as a function of temperature. 


\subsubsection{Neopentane (2,2-dimethylpropane)}

For density, we use the equation of state of Lemmon and Span (E.W. Lemmon \& Span, 2006) to provide density and values for the critical point. The dilute-gas viscosity data of Vogel et al. (E. Vogel, Holdt, \& Strehlow, 1988) were fit to provide the LJ parameters in Table 1, and the liquid-phase data of Gonzalez and Lee (Gonzalez \& Lee, 1968) and Van Wijk et al. (Van Wijk, Van der Veen, Brinkman, \& Seeder, 1940) (for pressures less than $100 \mathrm{MPa}$ ) were fit to give the coefficients in Table 2. Based on comparisons with the liquid data, we estimate the uncertainty to be $10 \%$ for pressures up to $100 \mathrm{MPa}$. Comparisons with the Vogel data show agreement to within $1.5 \%$ for the low density points spanning the temperature range from 299 to $633 \mathrm{~K}$ at densities up to $0.069 \mathrm{~mol} \cdot \mathrm{dm}^{-3}$; Figure 4 shows the viscosity deviations from experimental data. Only two data sources for thermal conductivity were found: Lambert et al. and Parkinson et al. (Lambert et al., 1955; Parkinson \& Gray, 1972). Both of these are in the dilute-gas region; we were unable to locate any experimental data for the thermal conductivity of liquid neopentane. The very limited data were fit to give the dilute-gas parameters in Table 3 (located in the Appendix). Parameters for the critical enhancement are presented in Table 4 (located in the Appendix). The AAD with this extremely limited data is $1 \%$, and we estimate the uncertainty in the dilute gas as $5 \%$. Deviations are shown in Figure 5. Since there were no liquid-phase data available, to estimate the liquid-phase thermal conductivity of neopentane, we selected a value of $b_{0}$ in Eq. (21) such that when propane is used as a reference fluid, the saturated liquid value of thermal conductivity of $n$-pentane at a reduced temperature of 0.7 matches its value from a published reference correlation (C.-M. Vassiliou, M.J. Assael, M.L. Huber, \& R.A. Perkins, 2015). With this procedure we estimate the uncertainty in the liquid phase is on the order of $10 \%$. Surface tension is discussed in a separate publication (Mulero \& Cachadiña, 2014). 


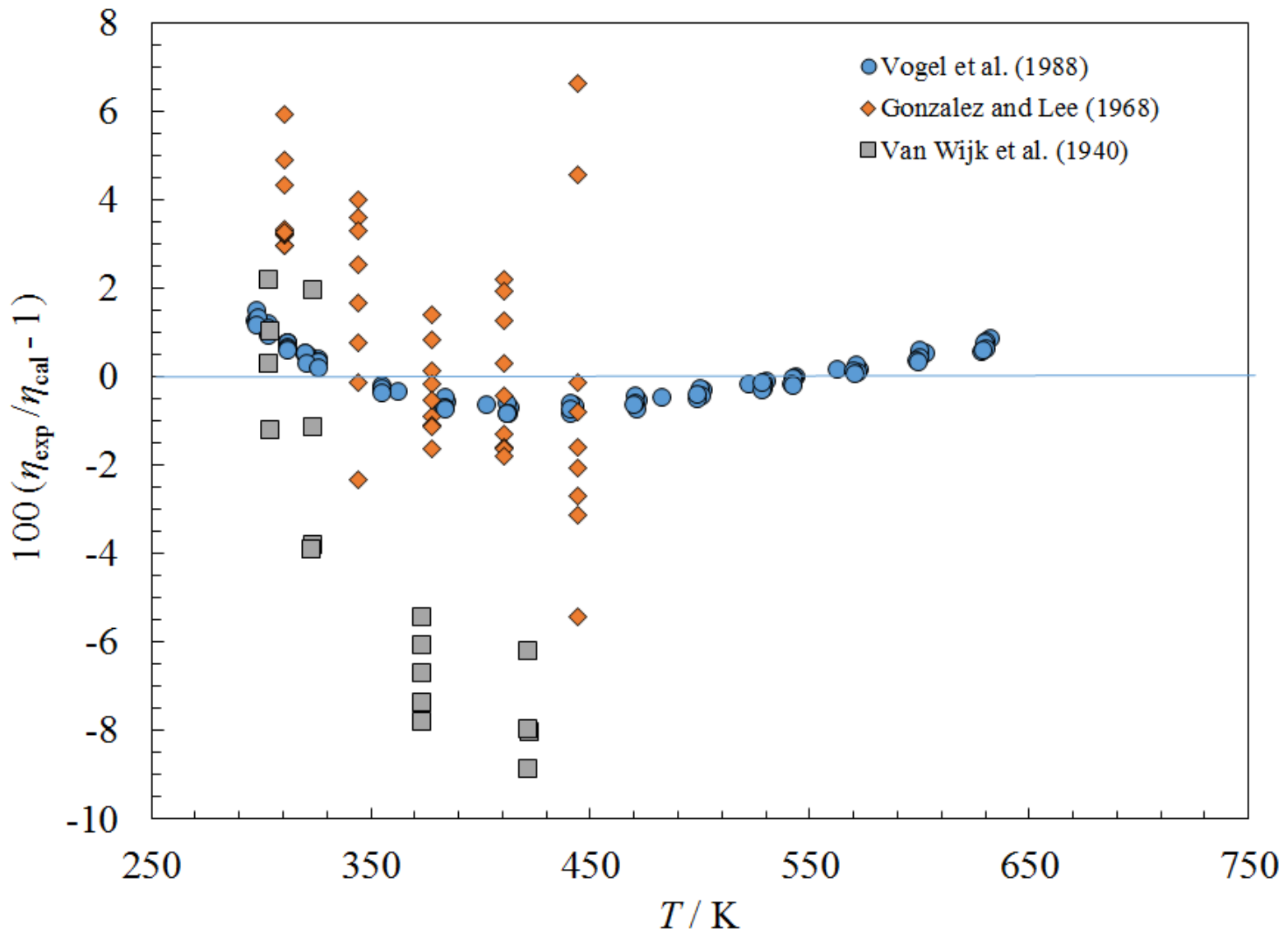

Figure 4. Percentage deviations between the model and the experimental viscosity data for neopentane. 


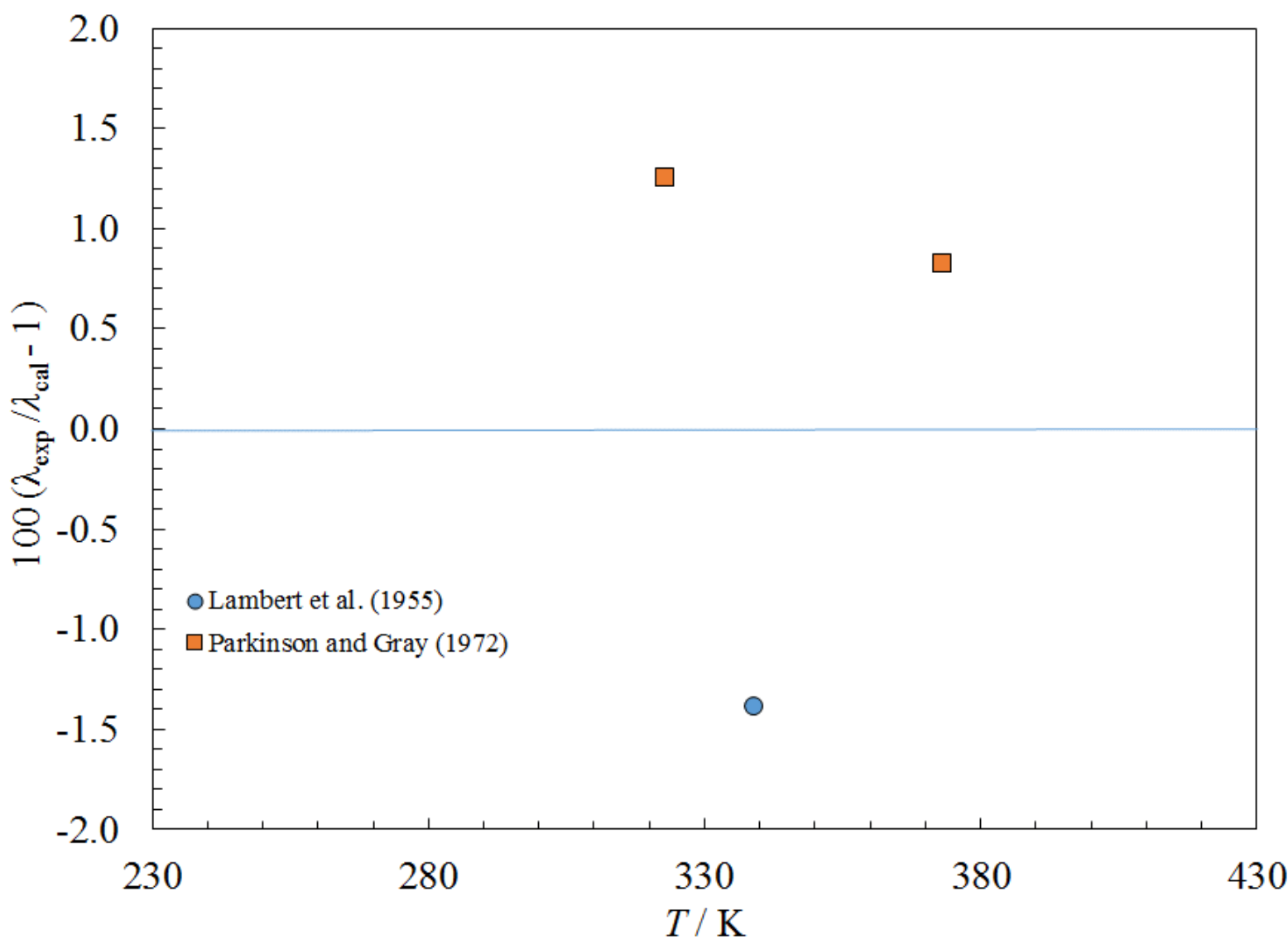

Figure 5. Percentage deviations between the model and the experimental thermal conductivity data for neopentane.

\subsubsection{Isohexane (2-methylpentane)}

The equation of state of Lemmon and Span (E.W. Lemmon \& Span, 2006) was used to provide the density and the critical parameters. Lennard-Jones parameters were computed using the method of Chung et al. (Chung et al., 1988). No data sources for vapor-phase viscosity were found. The liquid-phase data of Wen et al. (Wen, Meng, Wei, \& Wu, 2017) were used to obtain the coefficients in Table 2. Deviations with viscosity data as a function of temperature are shown in Figure 6, and with pressure in Figure 7. Data of Thorpe and Rodger (Thorpe \& Rodger, 1894), Batschinski (Batschinski, 1913), and Chavanne and Van Risseghem (Chavanne, 1922) are shown only for comparison purposes, as they were not used in fitting since the more recent data of Wen et al. (Wen et al., 2017) were considered superior. The estimated uncertainty for the liquid phase from $303 \mathrm{~K}$ to $343 \mathrm{~K}$ at pressures to $30 \mathrm{MPa}$ is $2 \%$. Estimated uncertainty in the gas phase is 10 $\%$. Gas-phase thermal conductivity data were not available. As an estimate, we adopted the same dilute-gas coefficient as for isooctane. The liquid-phase thermal conductivity data of Watanabe (Watanabe, 2003) and Filipov and Laushkina (Filipov, 1984) were fit to obtain the coefficients in Table 3. Parameters for the critical enhancement are presented in Table 4. Sakiadis and Coates (Sakiadis \& Coates, 1955) data are shown for comparison purposes only. All experimental data are at atmospheric pressure. Figure 8 shows deviations between the data and the model as a 
function of temperature. The estimated uncertainty of the thermal conductivity of the liquid phase is $3 \%$ for the saturated liquid, $10 \%$ for pressures to $100 \mathrm{MPa}, 10 \%$ for the gas phase, and larger in the critical region. Surface tension is discussed in a separate publication (Mulero et al., 2012).

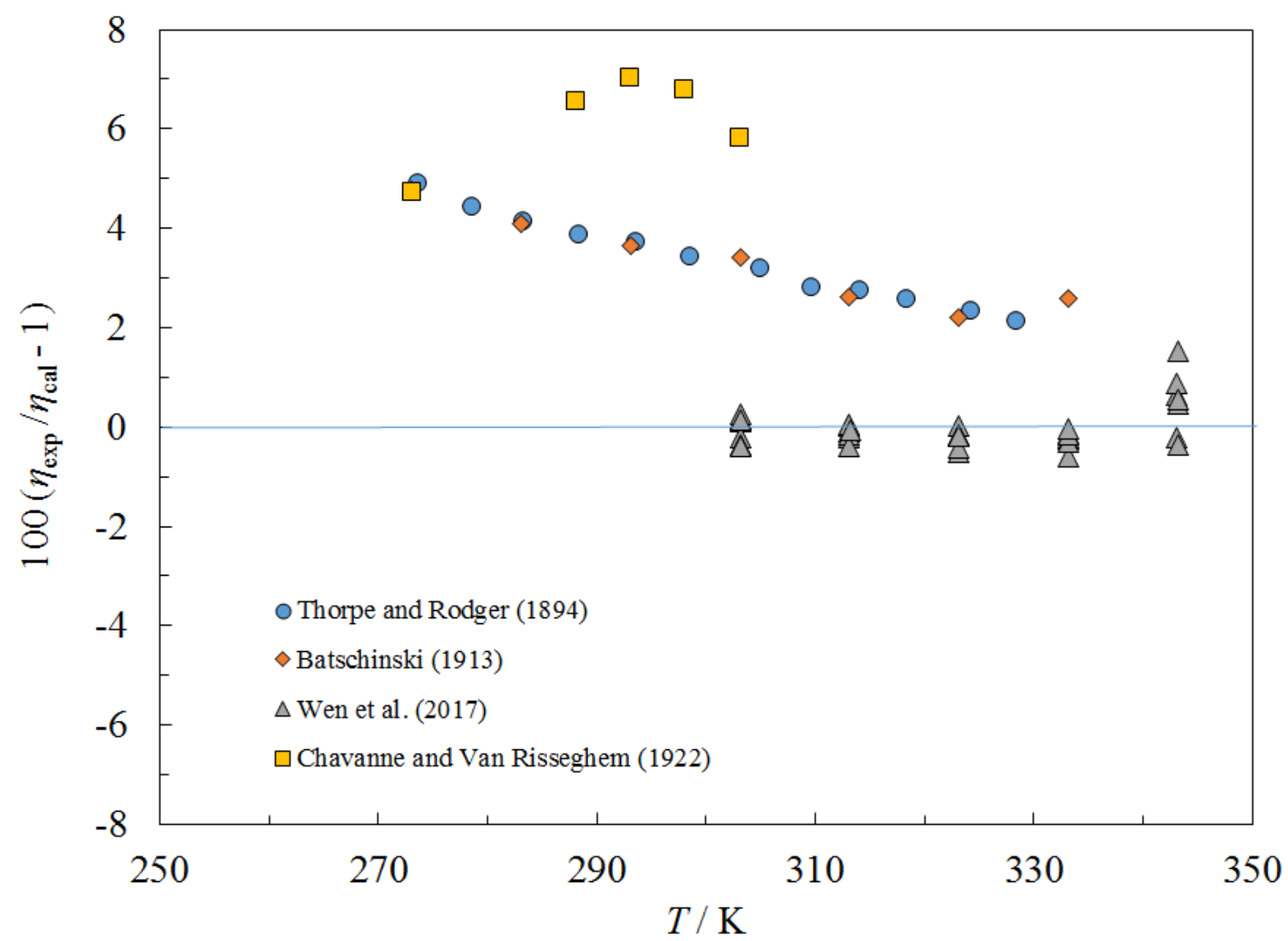

Figure 6. Percentage deviations between the model and the experimental viscosity data for isohexane as a function of temperature. 


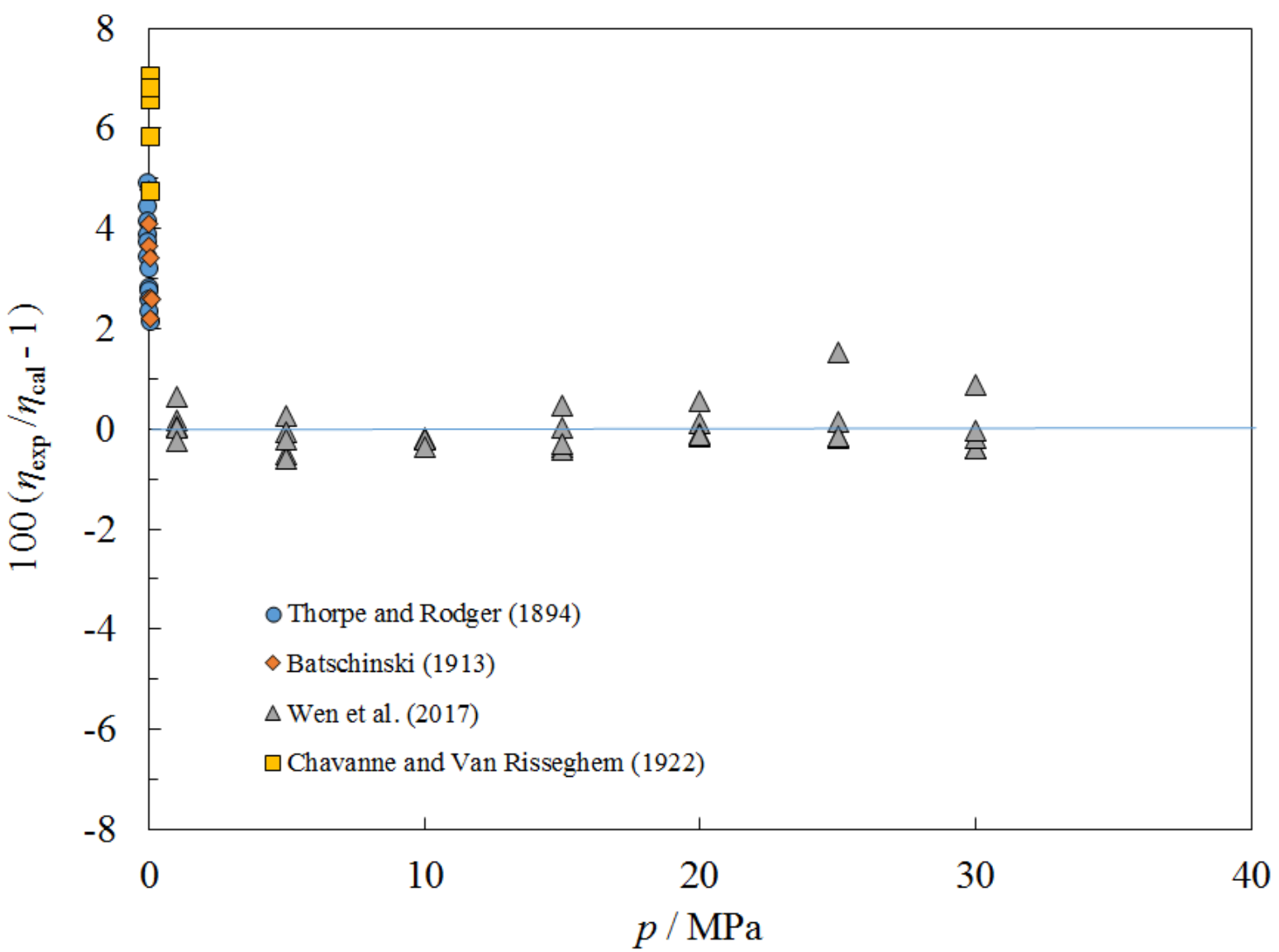

Figure 7. Percentage deviations between the model and the experimental viscosity data for isohexane as a function of pressure. 


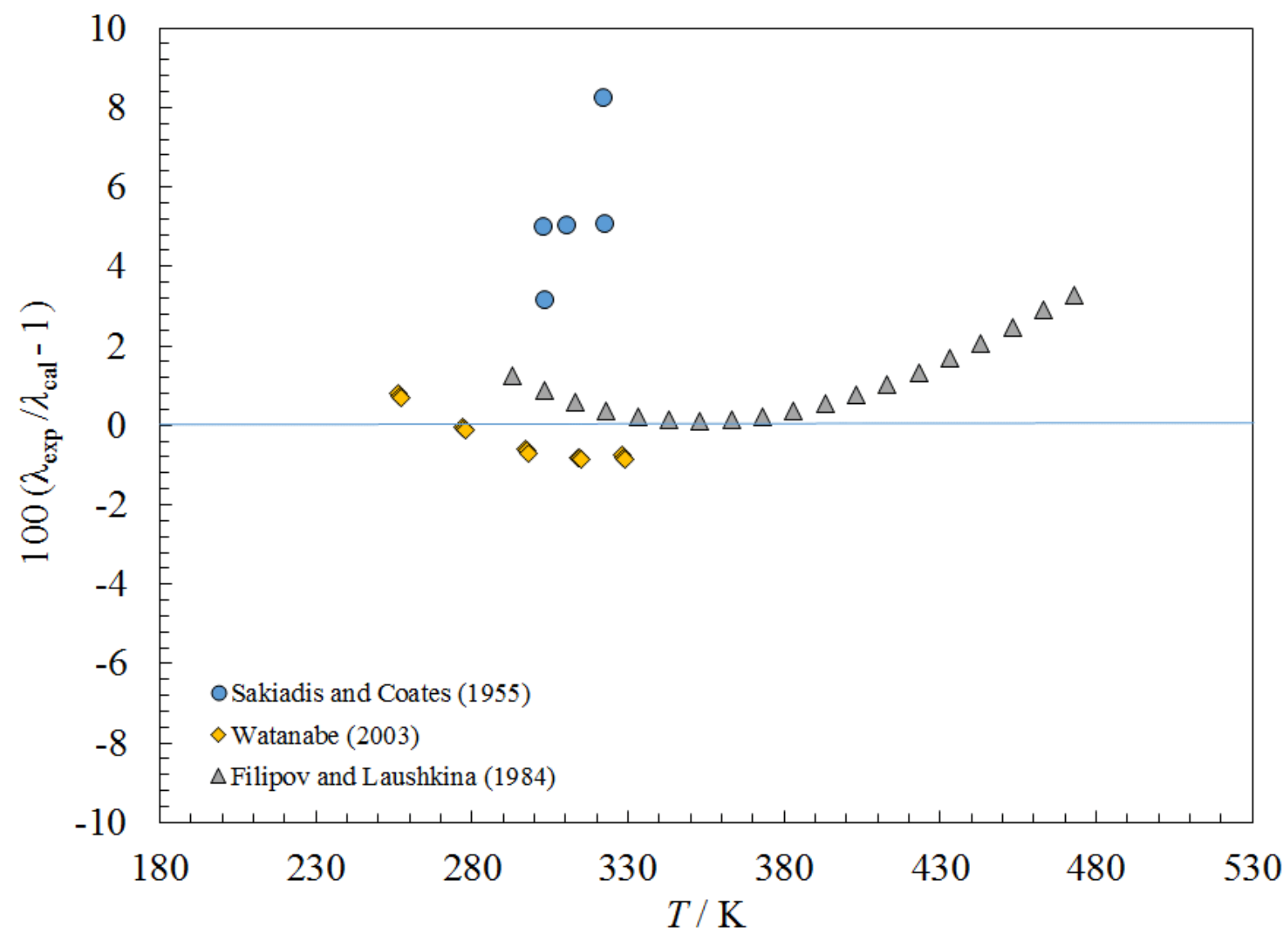

Figure 8. Percentage deviations between the model and the experimental thermal conductivity data for isohexane as a function of temperature.

\subsubsection{3-Methylpentane}

The equation of state of Gao et al. (K. Gao, J. Wu, \& E. W. Lemmon, 2017g) was used to provide the density and the critical parameters. Lennard-Jones parameters were calculated by the method of Chung et al. (Chung et al., 1988), values are presented in Table 1. Liquid-phase data from Wen et al. (Wen et al., 2017) were fit to obtain the coefficients in Table 2. Deviations with viscosity data as a function of temperature are shown in Figure 9, and as a function of pressure in Figure 10. The estimated uncertainty for the liquid phase at pressures to $40 \mathrm{MPa}$ is $2 \%$, the uncertainty level of Wen's data. At higher pressures the uncertainty is larger. There are no data in the gas phase for viscosity for comparison; the estimated uncertainty in the gas phase is $10 \%$. The equation of state is valid up to $1000 \mathrm{MPa}$, but we do not recommend the use of the correlation above $100 \mathrm{MPa}$. Liquid-phase thermal conductivity data of Watanabe (Watanabe, 2003) were fit to obtain the coefficients in Table 3. Parameters for the critical enhancement are presented in Table 4. Data at pressures above saturation were not available. Gas-phase thermal conductivity data were also unavailable. A value for $a_{0}$ in Table 3 was selected that gave a reasonable fit for a similar fluid, isopentane, for which gas-phase data were available. Figure 11 shows deviations between the data and the model as a function of temperature. The data of Sakiadis and Coates (Sakiadis \& Coates, 1955) are shown only for comparison. They were not used in the fitting process as they were considered not as reliable as Watanabe (Watanabe, 2003). The estimated uncertainty of the 
liquid phase is $2 \%$ along saturation over the temperature range $256 \mathrm{~K}$ to $330 \mathrm{~K}, 10 \%$ for the gas phase, and larger in the critical region and at pressures above saturation. Extremely limited surface tension data from two sources (Quayle, 1944; Wibaut, 1939) were fit to obtain the coefficients in Table 5 (located in the Appendix). The estimated uncertainty is $1 \%$ for $290 \mathrm{~K}$ to $315 \mathrm{~K}$. Figure 12 shows the deviations of the model from the surface tension data.

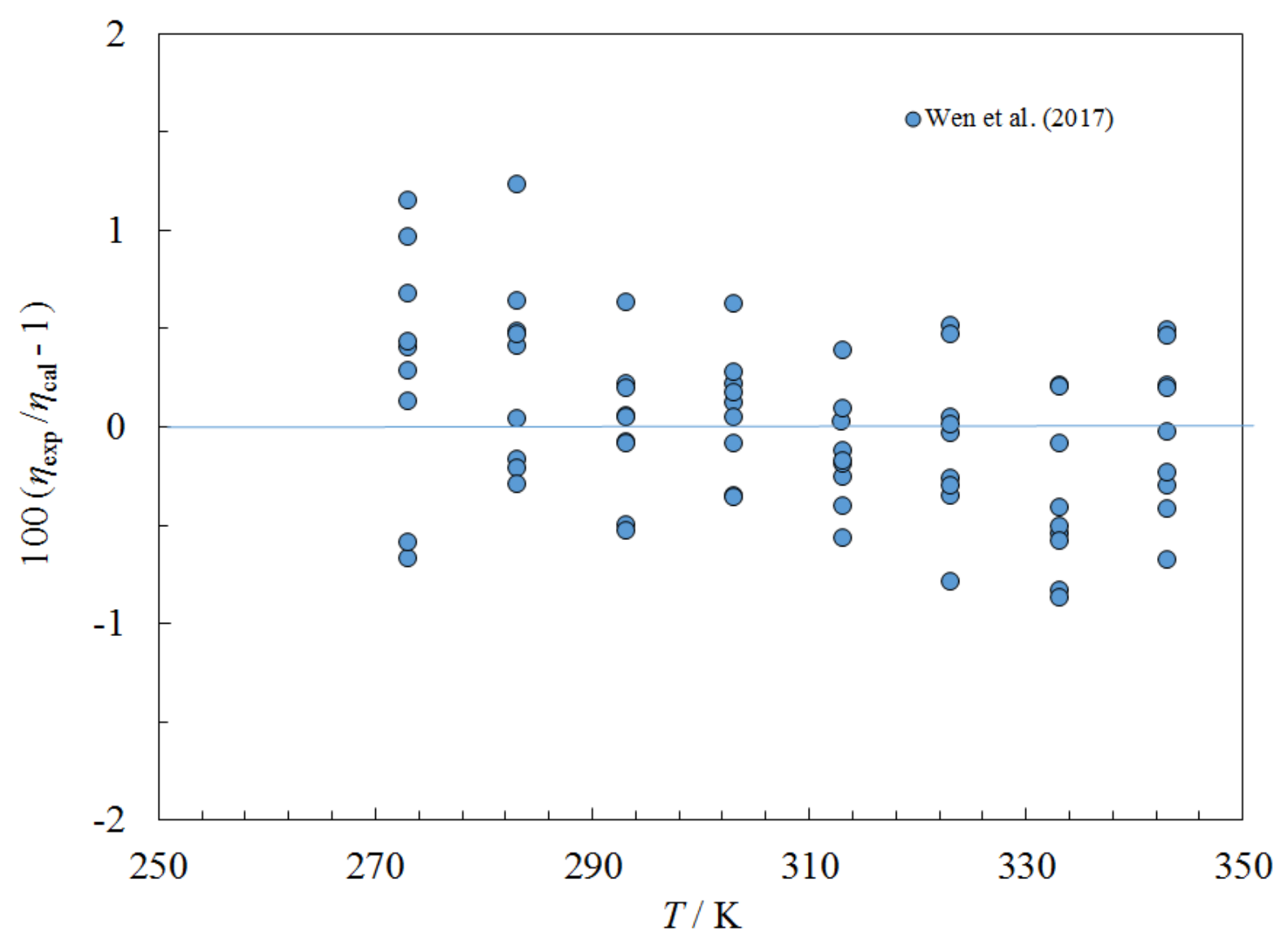

Figure 9. Percentage deviations between the model and the experimental viscosity data for 3-methylpentane as a function of temperature. 


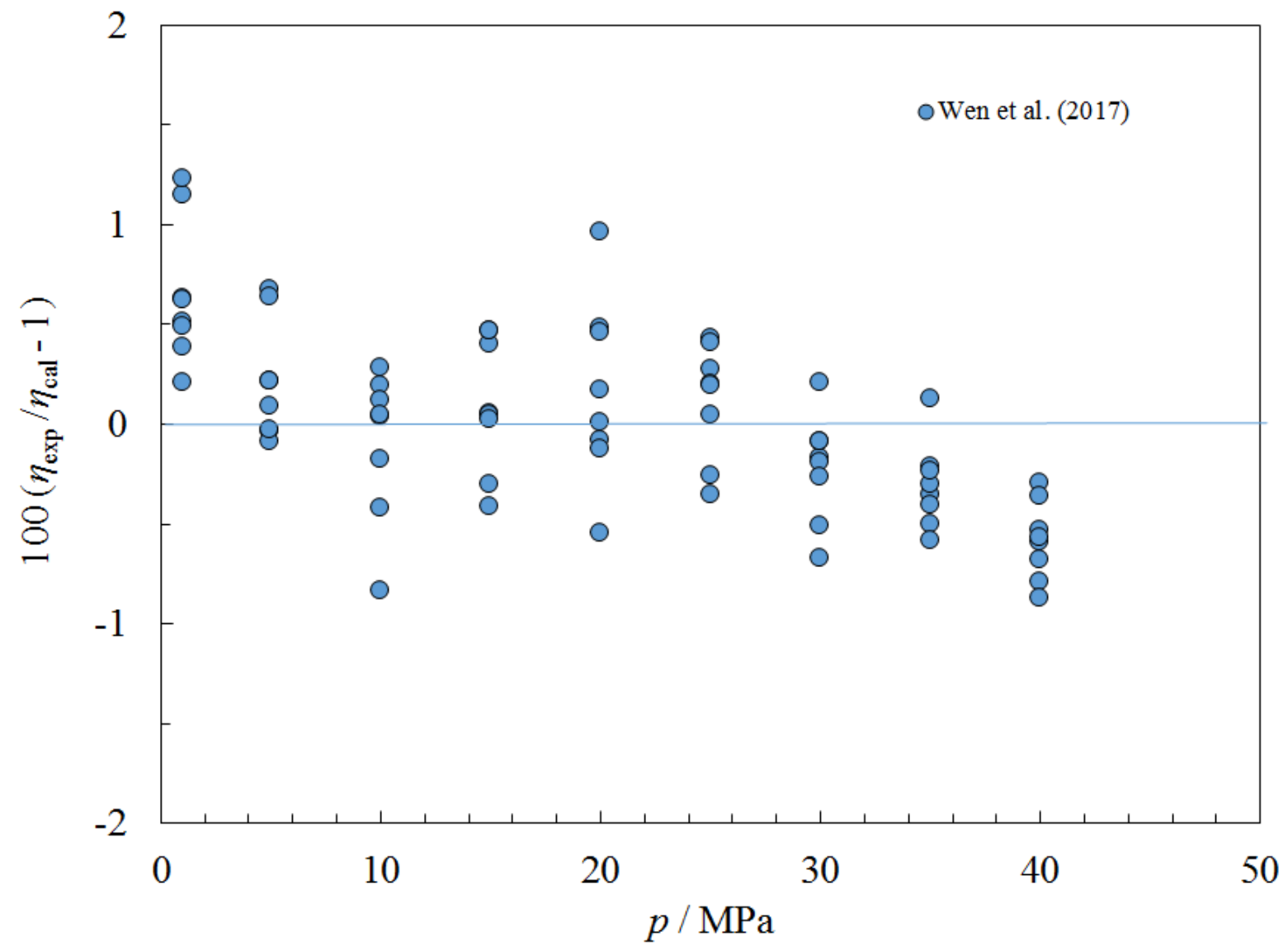

Figure 10. Percentage deviations between the model and the experimental viscosity data for 3-methylpentane as a function of pressure. 


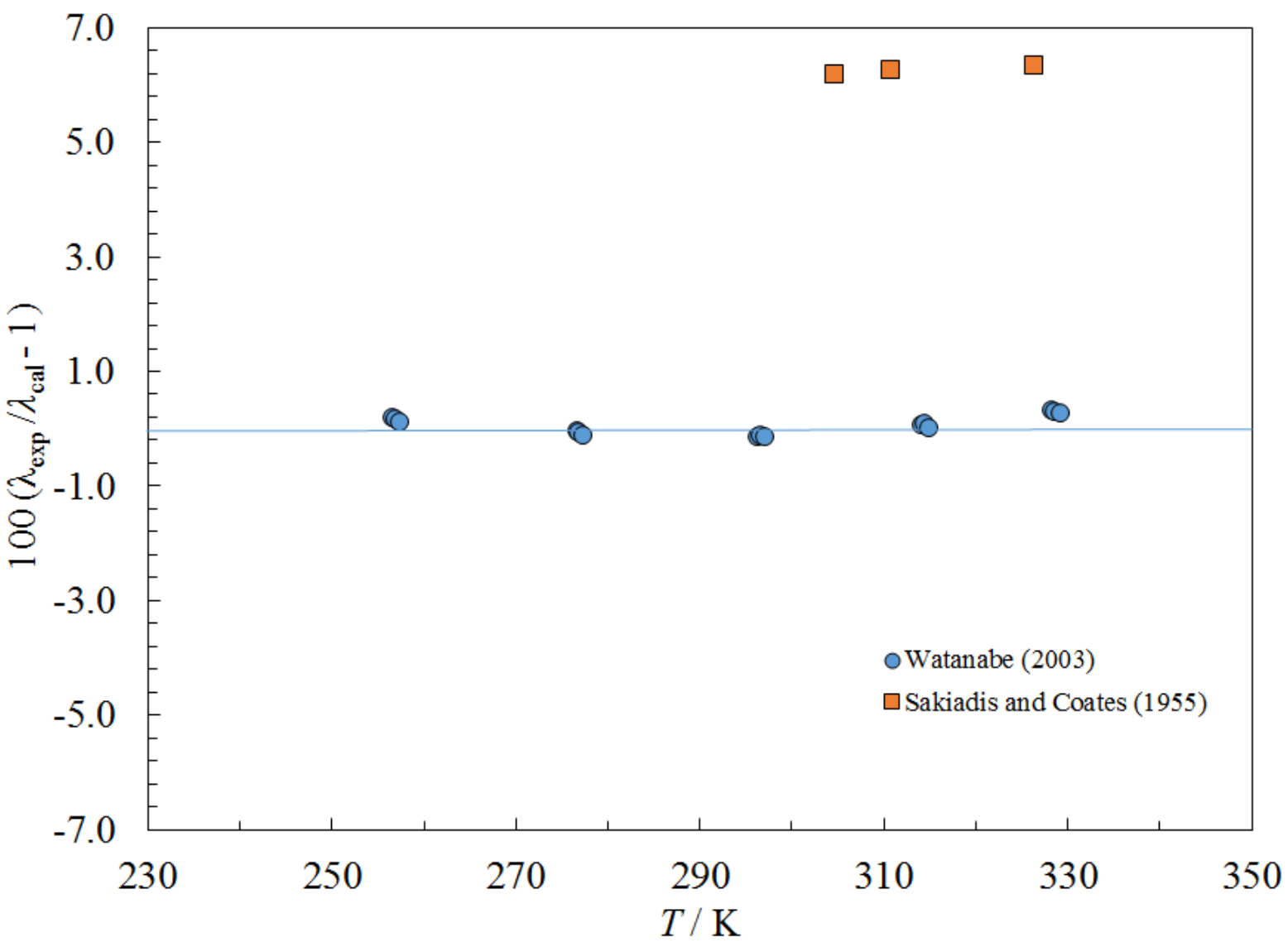

Figure 11. Percentage deviations between the model and the experimental thermal conductivity data for 3-methylpentane as a function of temperature. 


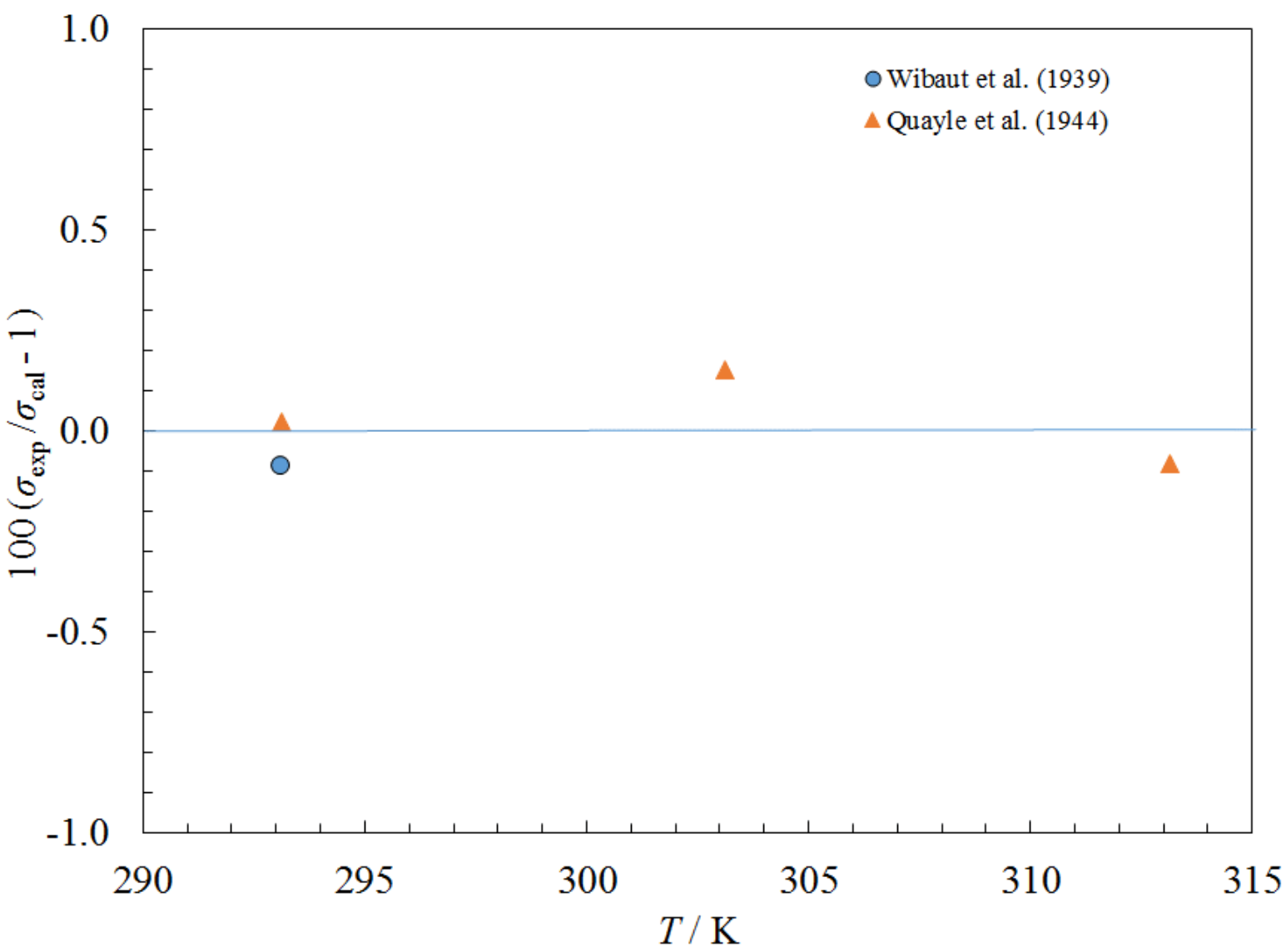

Figure 12. Percentage deviations between the model and the experimental surface tension data for 3-methylpentane as a function of temperature. 


\subsubsection{2,2-Dimethylbutane (neohexane)}

The equation of state of Gao et al. (K. Gao, J. Wu, \& E. W. Lemmon, 2017e) was used to provide the density and the critical parameters. Lennard-Jones parameters were calculated by the method of Chung et al. (Chung et al., 1988); values are presented in Table 1. Liquid-phase data from Chavanne and Van Risseghem (Chavanne, 1922), Brazier and Freeman (Brazier, 1969), and Lambert et al. (Lambert et al., 1955) were fit to obtain the coefficients in Table 2. Lambert et al. (Lambert et al., 1955) give data in the gas phase. Deviations with viscosity data as a function of temperature are shown in Figure 13, and as a function of pressure in Figure 14. The data of Brazier and Freeman (Brazier, 1969) extend to $400 \mathrm{MPa}$, but we limit the use of the correlation to 100 $\mathrm{MPa}$. The estimated uncertainty for the liquid phase at pressures to $100 \mathrm{MPa}$ is $5 \%$. The estimated uncertainty in the gas phase is also $5 \%$. Liquid-phase thermal conductivity data of Watanabe (Watanabe, 2003) and gas-phase data of Lambert et al. (Lambert et al., 1955) were fit to obtain the coefficients in Table 3. Parameters for the critical enhancement are presented in Table 4. Data at pressures above saturation were not available. Figure 15 shows deviations between the data and the model as a function of temperature. The data of Sakiadis and Coates (Sakiadis \& Coates, 1955) are shown only for comparison. They were not used in the fitting process as they were considered not as reliable as Watanabe (Watanabe, 2003). The estimated uncertainty of the liquid phase is $2 \%$ along saturation over the temperature range $257 \mathrm{~K}$ to $321 \mathrm{~K}, 5 \%$ for the gas phase, and larger in the critical region and at pressures above saturation. Surface tension data from Wibaut et al. (Wibaut, 1939) and Gao et al. (W. Z. Gao, X.; Liu, Z., 2009) were fit to obtain the coefficients in Table 5 . The estimated uncertainty is $2.5 \%$ over the temperature range $230 \mathrm{~K}$ to $380 \mathrm{~K}$, due to the stated uncertainty of $2.5 \%$ for the highest temperatures for the data of Gao et al. (W. Z. Gao, X.; Liu, Z., 2009) Figure 16 shows the deviations of the model from the surface tension data. 


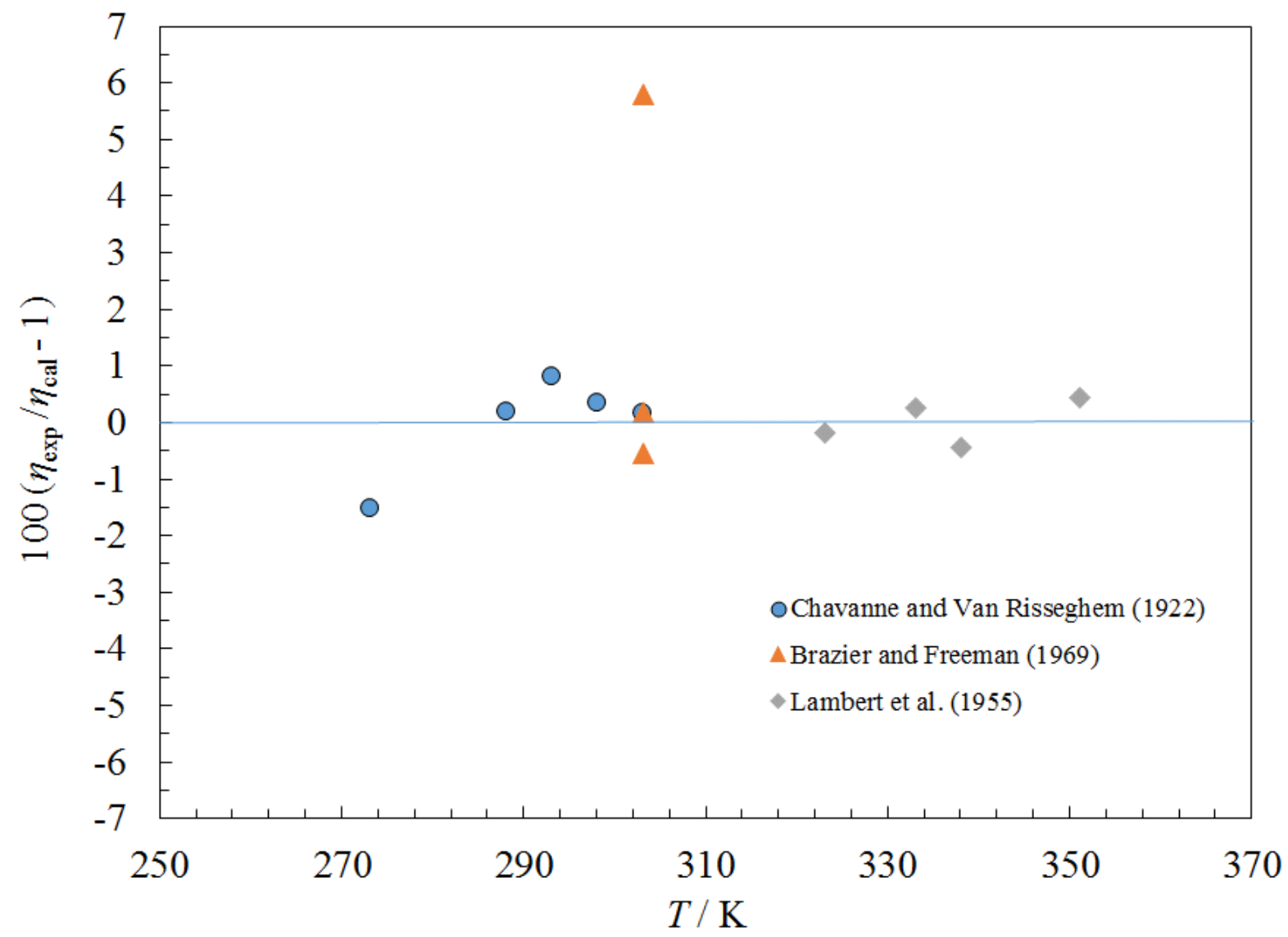

Figure 13. Percentage deviations between the model and the experimental viscosity data for 2,2-dimethylbutane as a function of temperature. 


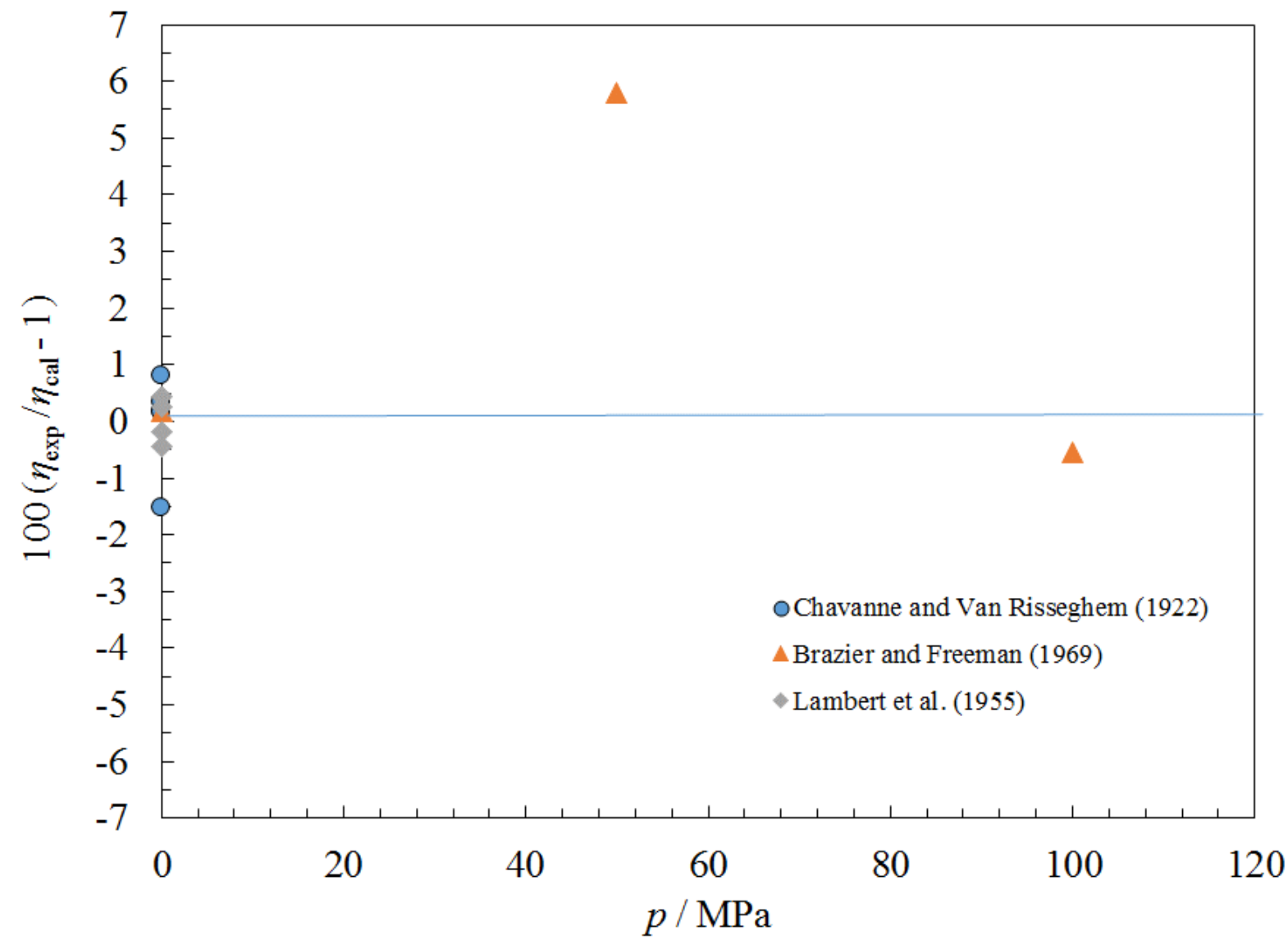

Figure 14. Percentage deviations between the model and the experimental viscosity data for 2,2-dimethylbutane as a function of pressure. 


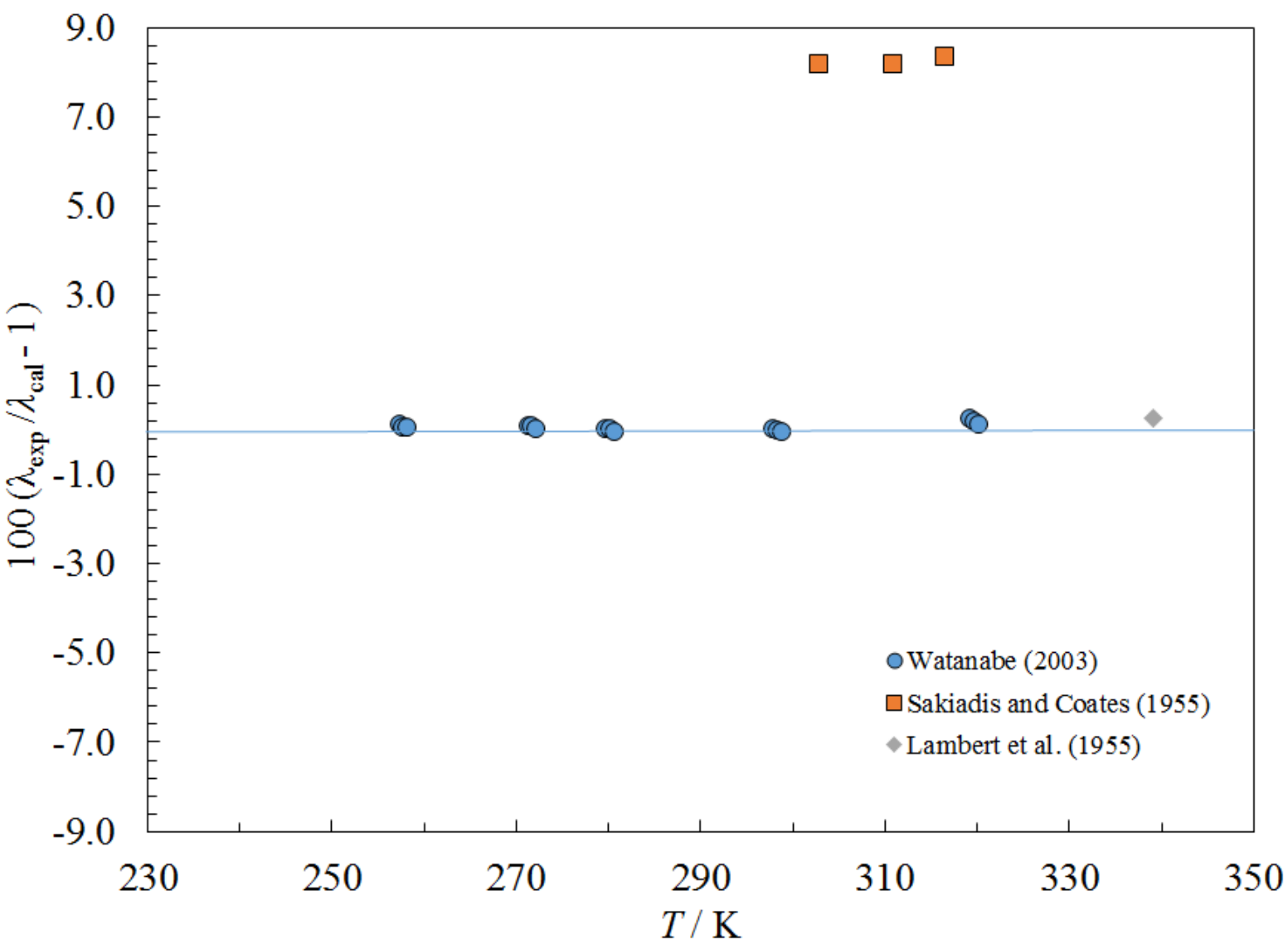

Figure 15. Percentage deviations between the model and the experimental thermal conductivity data for 2,2-dimethylbutane as a function of temperature. 


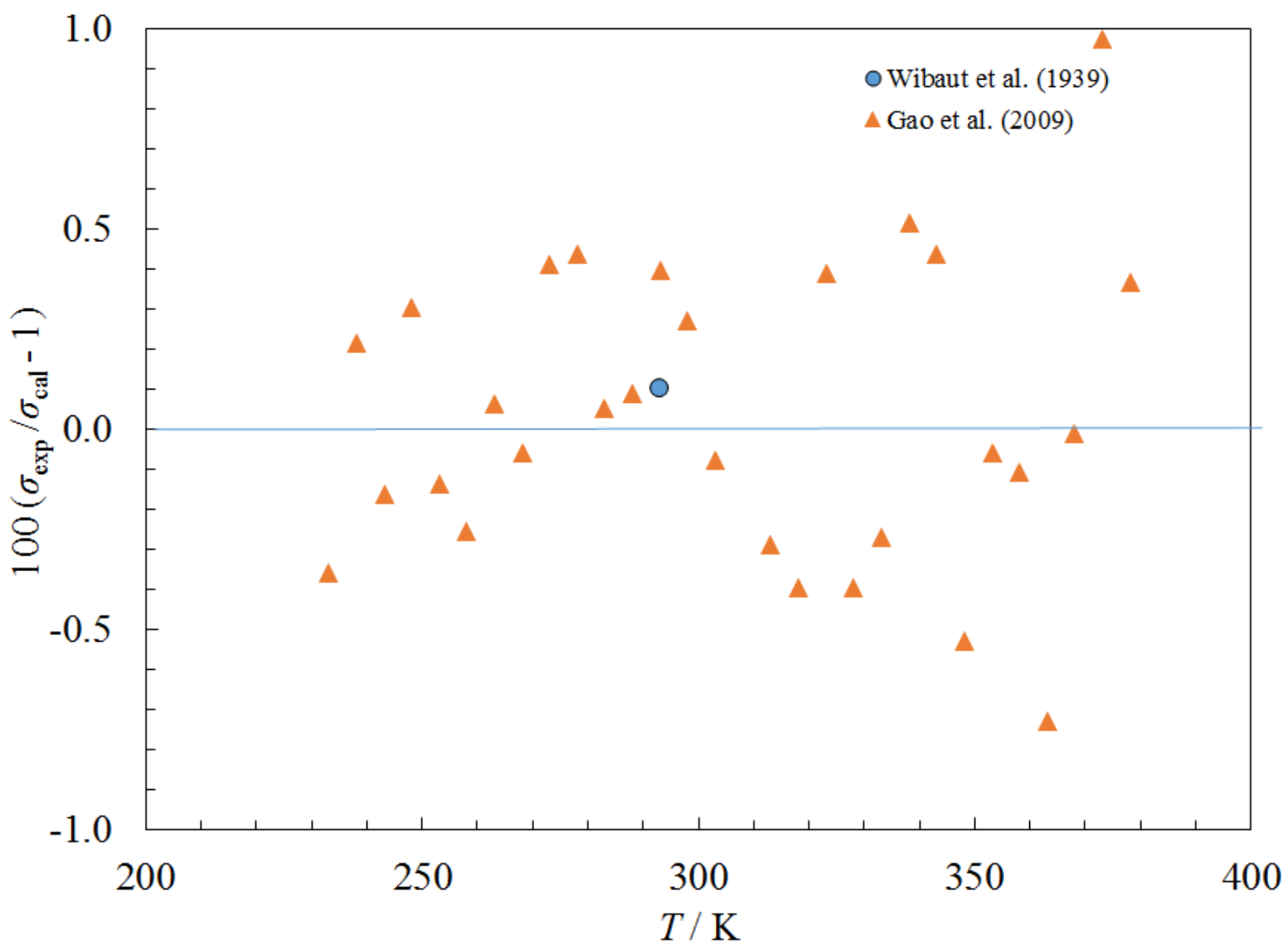

Figure 16. Percentage deviations between the model and the experimental surface tension data for 2,2-dimethylbutane as a function of temperature. 


\subsubsection{2,3-Dimethylbutane}

The equation of state of Gao et al. (K. Gao, J. Wu, \& E. W. Lemmon, 2017f) was used to provide the density and the critical parameters. Lennard-Jones parameters were calculated by the method of Chung et al. (Chung et al., 1988), values are presented in Table 1. Liquid-phase data from Wen et al. (Wen et al., 2017) were used to obtain the coefficients in Table 2. Chavanne and Van Risseghem (Chavanne, 1922) also measured a few points but they deviate significantly from Wen et al. (Wen et al., 2017) and only Wen et al. (Wen et al., 2017) were used in fitting. No gasphase viscosity data were found. Deviations with viscosity data as a function of temperature are shown in Figure 17, and as a function of pressure in Figure 18. The estimated uncertainty for the liquid phase at pressures to $40 \mathrm{MPa}$ for $273 \mathrm{~K}$ to $343 \mathrm{~K}$ is $2 \%$, rising to $5 \%$ at pressures to 100 $\mathrm{MPa}$. The estimated uncertainty in the gas phase is $10 \%$. Liquid-phase thermal conductivity data of Watanabe (Watanabe, 2003) and also Filipov and Laushkina (Filipov, 1984) were fit to obtain the coefficients in Table 3. Parameters for the critical enhancement are presented in Table 4. Data at pressures above saturation were not available. Figure 19 shows deviations between the data and the model as a function of temperature. The data of Sakiadis and Coates (Sakiadis \& Coates, 1955) are shown only for comparison. They were not used in the fitting process as they were considered not as reliable as the other data. The estimated uncertainty of the liquid phase is $2 \%$ along saturation over the temperature range $257 \mathrm{~K}$ to $321 \mathrm{~K}, 5 \%$ for the gas phase, and larger in the critical region and at pressures above saturation. Only one data point for surface tension from Wibaut et al. (Wibaut, 1939) was available, so we use the same value for $n_{0}$ in Eq. (25) as for a similar fluid (2,2-dimethylbutane) and adjusted the value of $\sigma_{0}$ to fit the single data point of Wibaut et al. (Wibaut, 1939). The estimated uncertainty is estimated to be $3 \%$, slightly larger than for 2,2dimethylbutane. Figure 20 shows the deviations of the model from the surface tension data. 


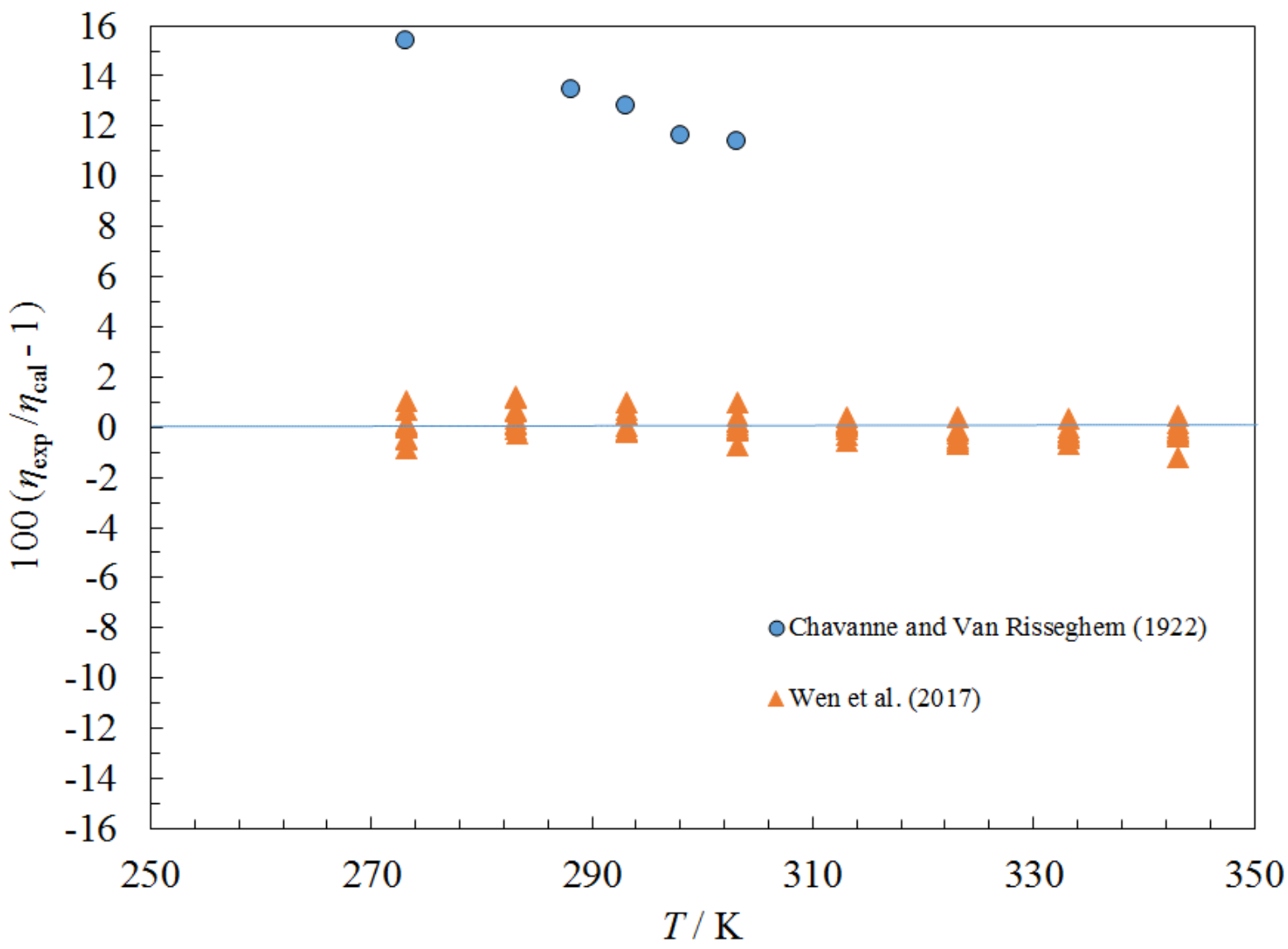

Figure 17. Percentage deviations between the model and the experimental viscosity data for 2,3-dimethylbutane as a function of temperature. 


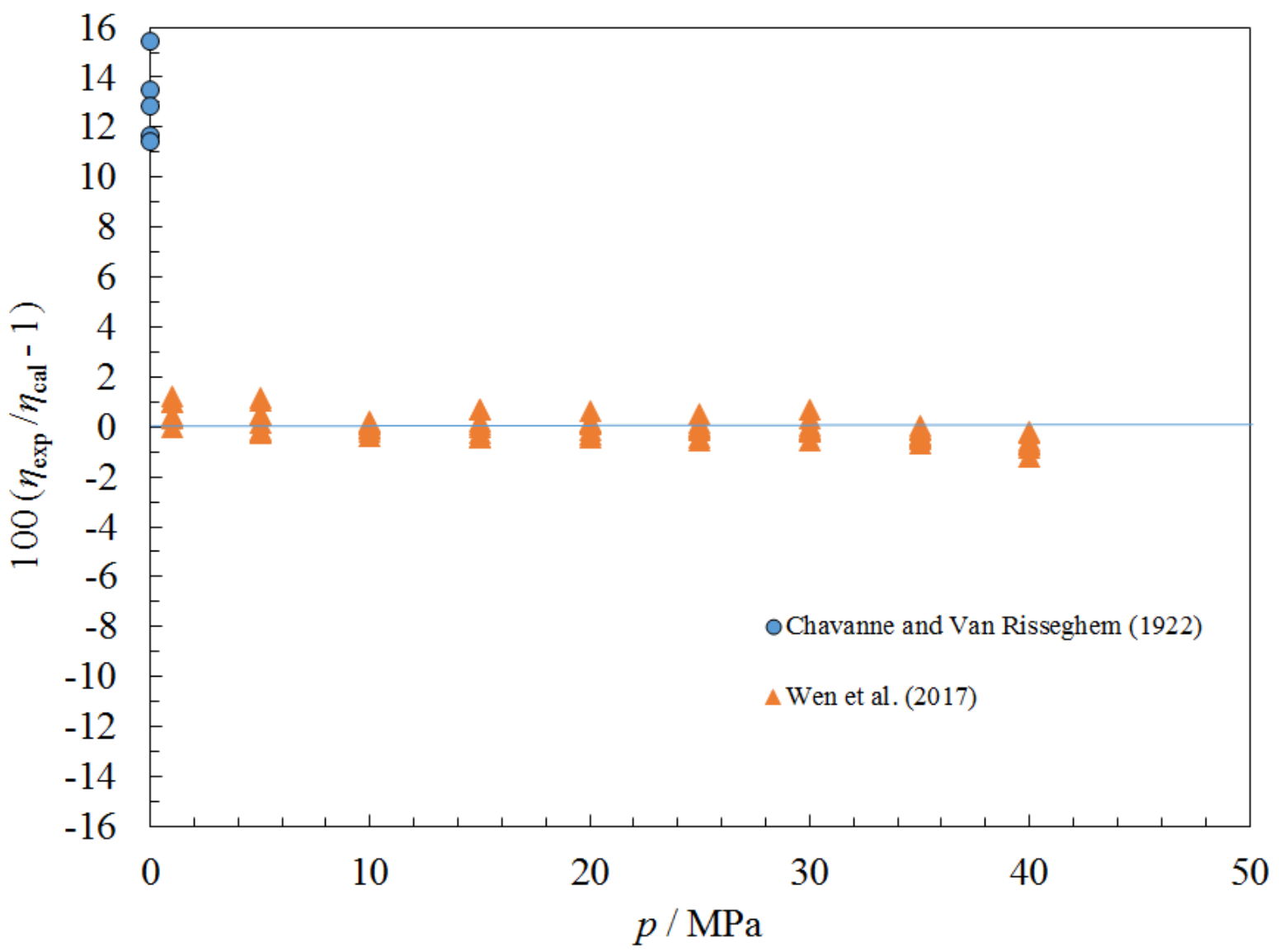

Figure 18. Percentage deviations between the model and the experimental viscosity data for 2,3-dimethylbutane as a function of pressure. 


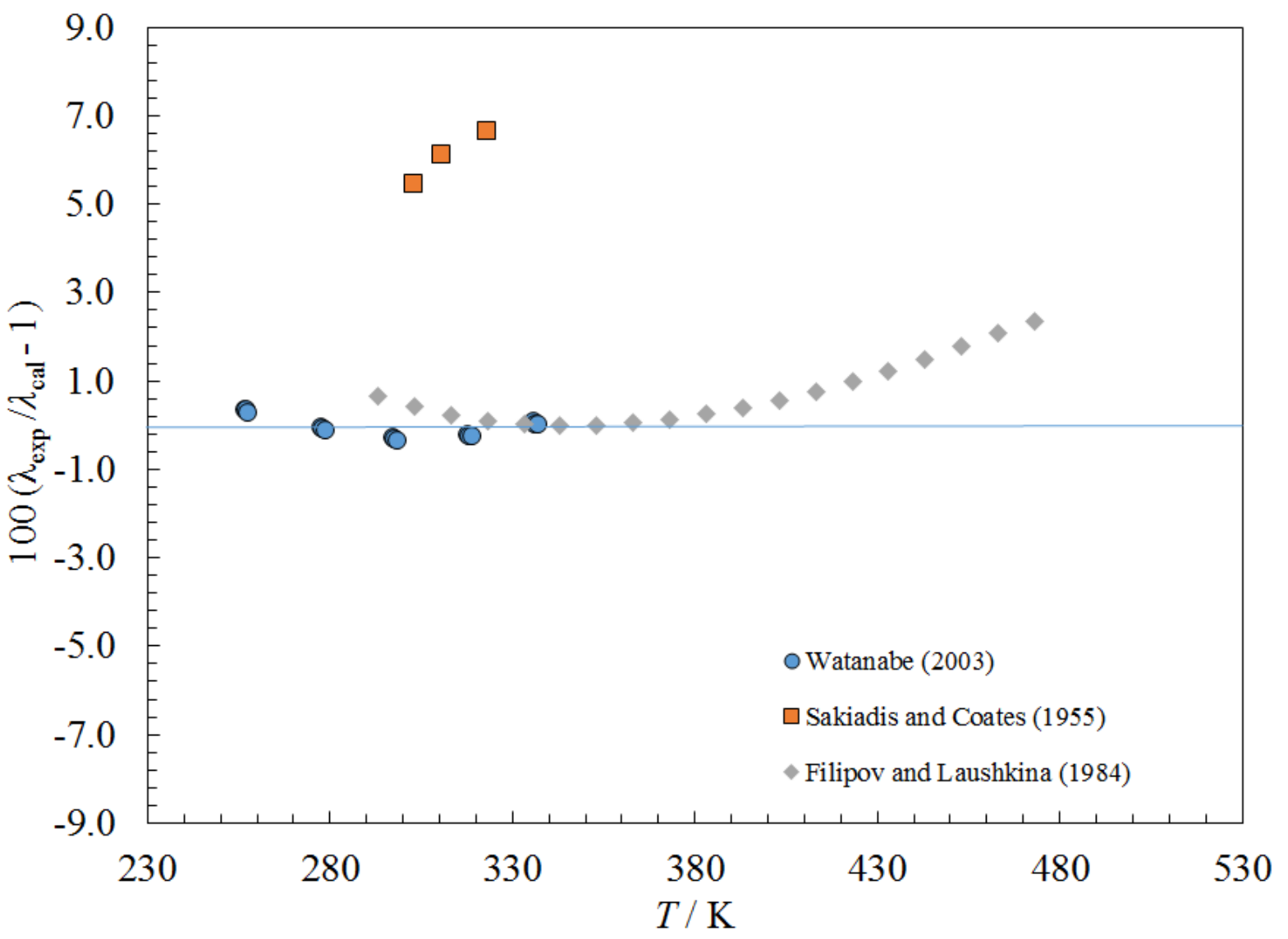

Figure 19. Percentage deviations between the model and the experimental thermal conductivity data for 2,3-dimethylbutane as a function of temperature. 


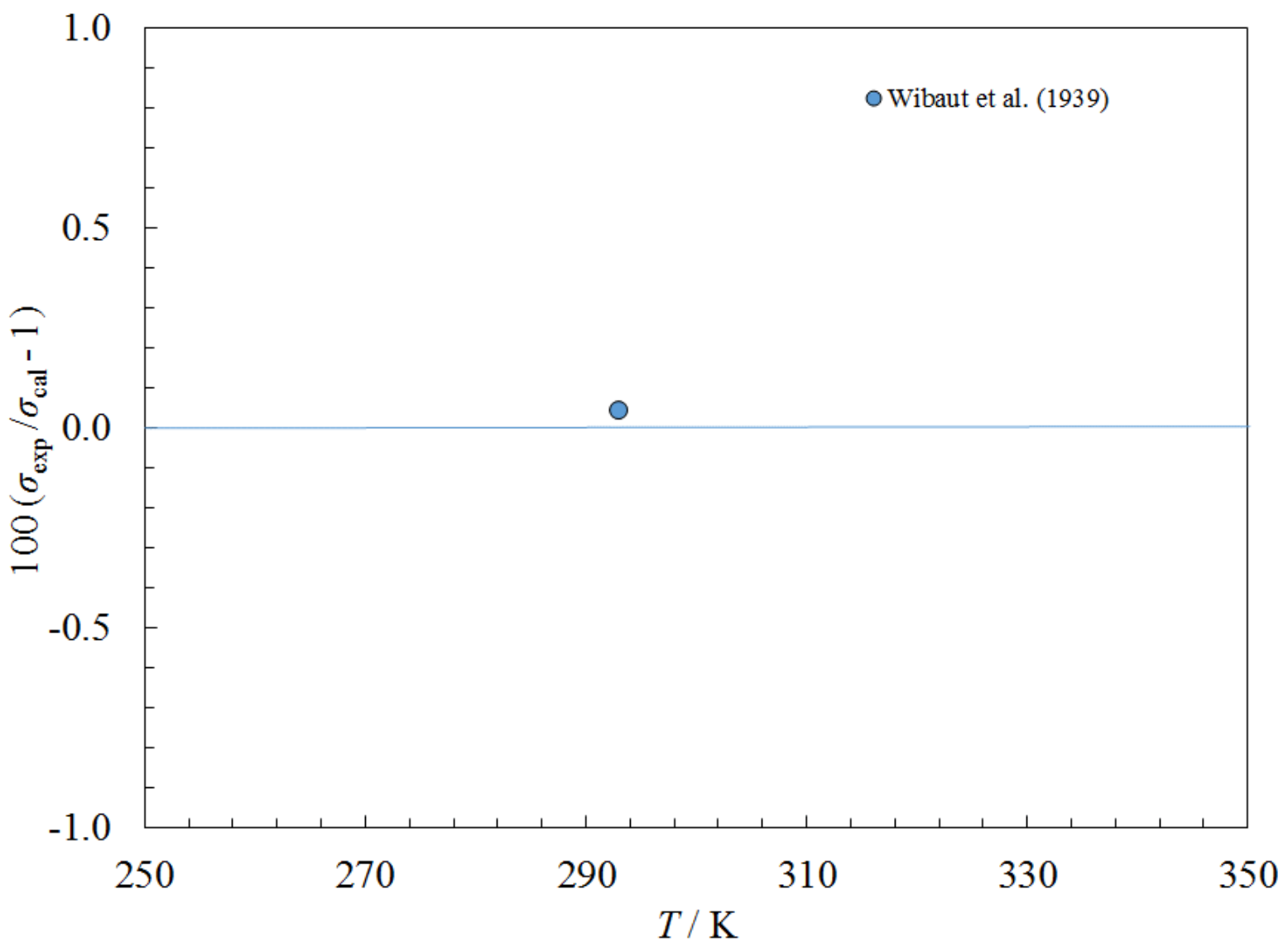

Figure 20. Percentage deviations between the model and the experimental surface tension data for 2,3-dimethylbutane as a function of temperature. 


\subsubsection{Isooctane (2,2,4-trimethylpentane)}

The equation of state of Blackham et al. (Blackham, Lemmon, \& Lemmon, 2018) was used to provide the density and the critical parameters. The Lennard-Jones parameters were obtained from fitting the viscosity data of (Diaz Pena, Cabello, \& Cheda, 1975; Lusternik \& Zdanov, 1973; J. C. McCoubrey, McCrea, \& Ubbelohde, 1951) and are presented in Table 1. Liquid-phase data from Padua et al. (Padua, Fareleira, Calado, \& Wakeham, 1996), Zambrano et al. (Zambrano et al., 2016), Ma et al. (P. S. Ma et al., 2004) and Dymond et al. (Dymond, Glen, \& Isdale, 1985) were fit to obtain the coefficients in Table 2. Deviations with viscosity data as a function of temperature are shown in Figure 21, and as a function of pressure in Figure 22. Figure 21 only shows points up to $200 \mathrm{MPa}$. Several data points by Dymond et al. (Dymond et al., 1985) that extend to $500 \mathrm{MPa}$ have larger deviations (up to $25 \%$ at $500 \mathrm{MPa}$ ) and are not shown in this plot. Figure 22 indicates that the representation of the data by the model begins to show substantial deviations above $150 \mathrm{MPa}$. The estimated uncertainty for the liquid phase at pressures to $100 \mathrm{MPa}$ is $5 \%$, rising to $\sim 25 \%$ at pressures to $500 \mathrm{MPa}$. The estimated uncertainty in the gas phase is 10 $\%$. The equation of state is valid up to $1000 \mathrm{MPa}$, but we do not recommend the use of the correlation above $100 \mathrm{MPa}$. Gas-phase thermal conductivity data of Naziev and Aliev (Y. M. Naziev \& Aliev, 1973), and the liquid-phase data of Naziev and Aliev (Y. M. Naziev \& Aliev, 1973) and Watanabe (Watanabe, 2003) were fit to obtain the coefficients in Table 3. Parameters for the critical enhancement are presented in Table 4 . Figure 23 shows deviations between the data and the model as a function of temperature, and Figure 24 shows the deviations as a function of pressure. The estimated uncertainty of the liquid phase is $7 \%$ for pressures to $100 \mathrm{MPa}, 10 \%$ for the gas phase, and larger in the critical region. Surface tension is discussed in a separate publication (Mulero \& Cachadiña, 2014). 


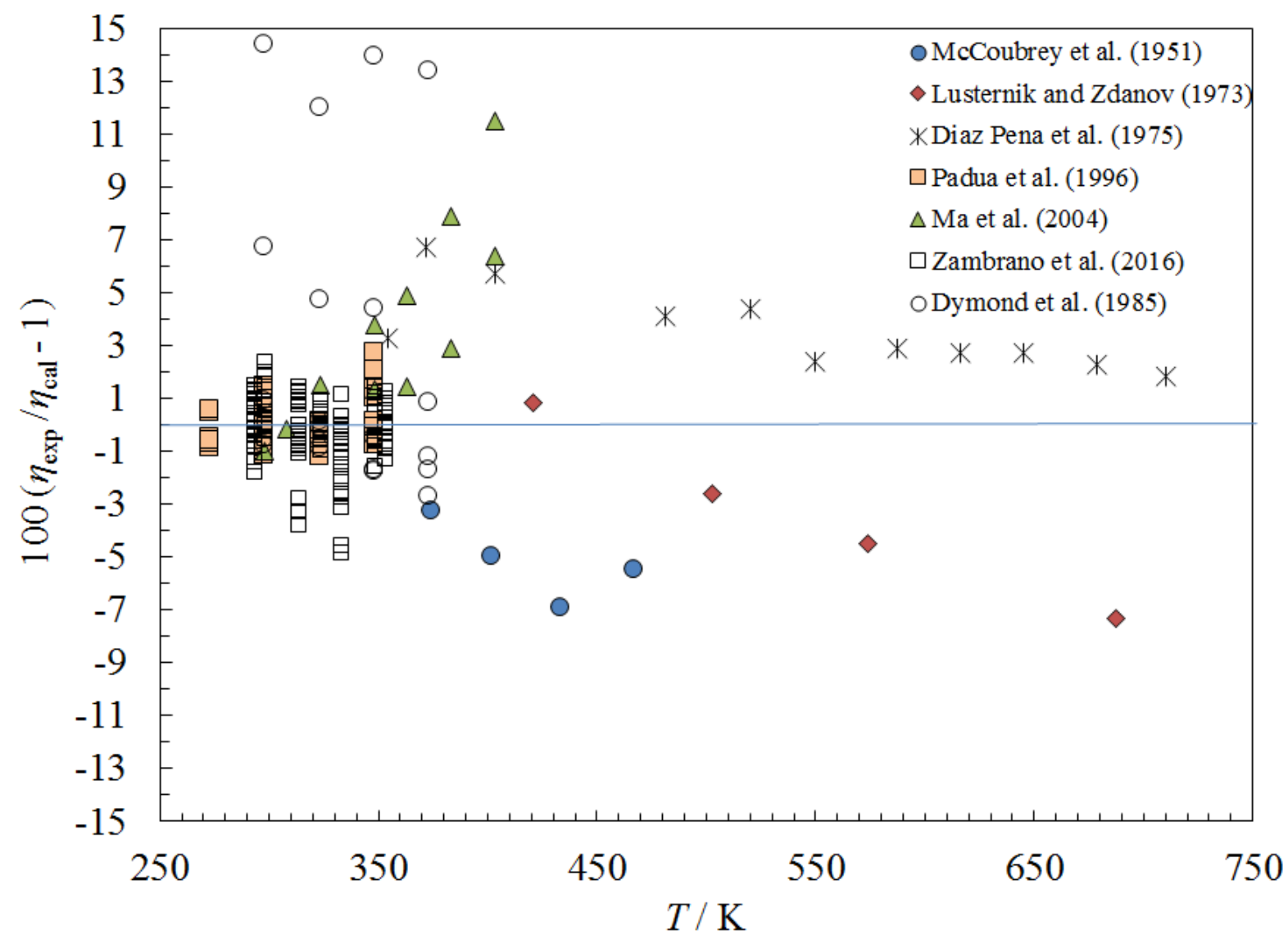

Figure 21. Percentage deviations between the model and the experimental viscosity data for isooctane as a function of temperature. 


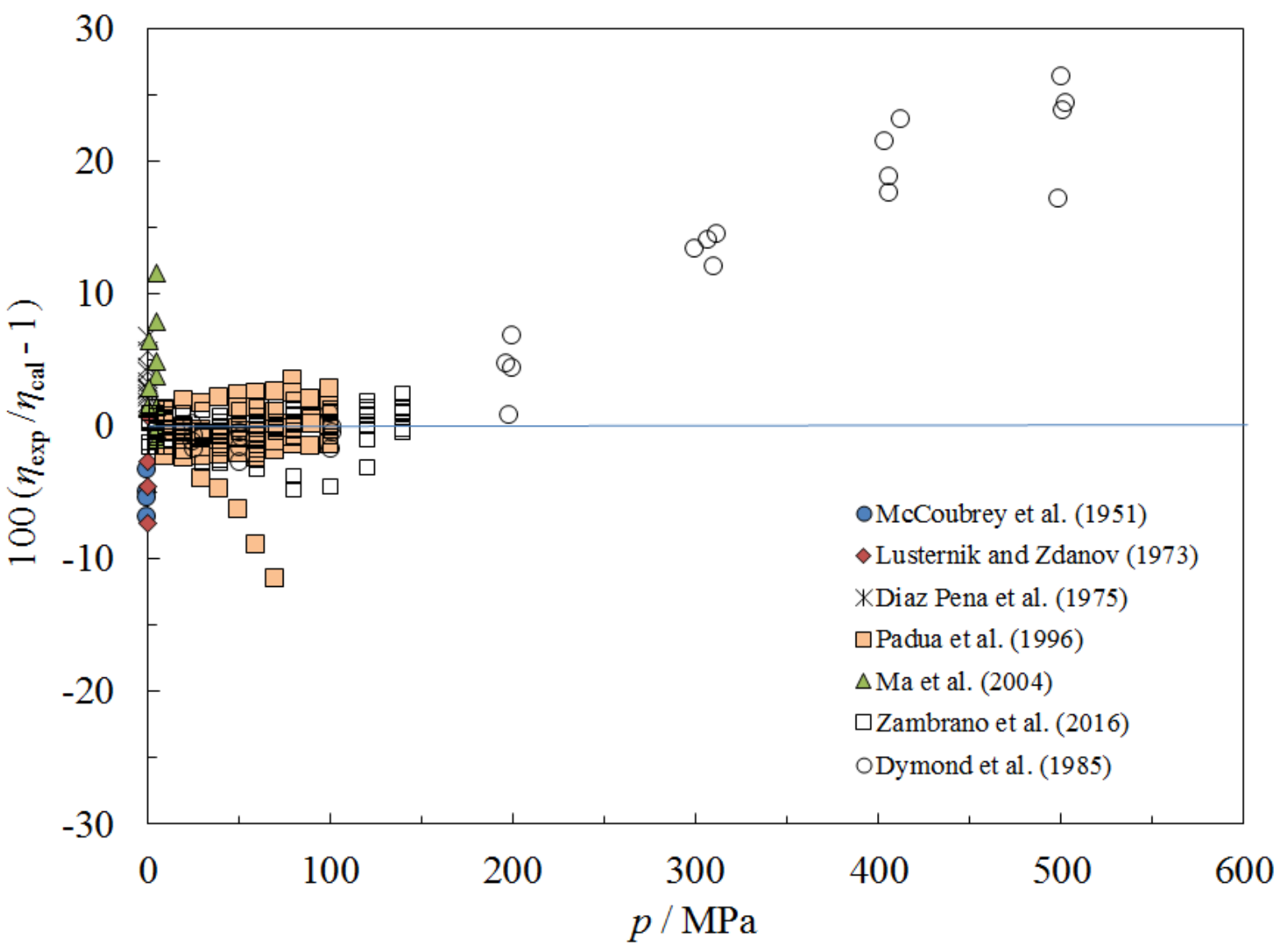

Figure 22. Percentage deviations between the model and the experimental viscosity data for isooctane as a function of pressure. 


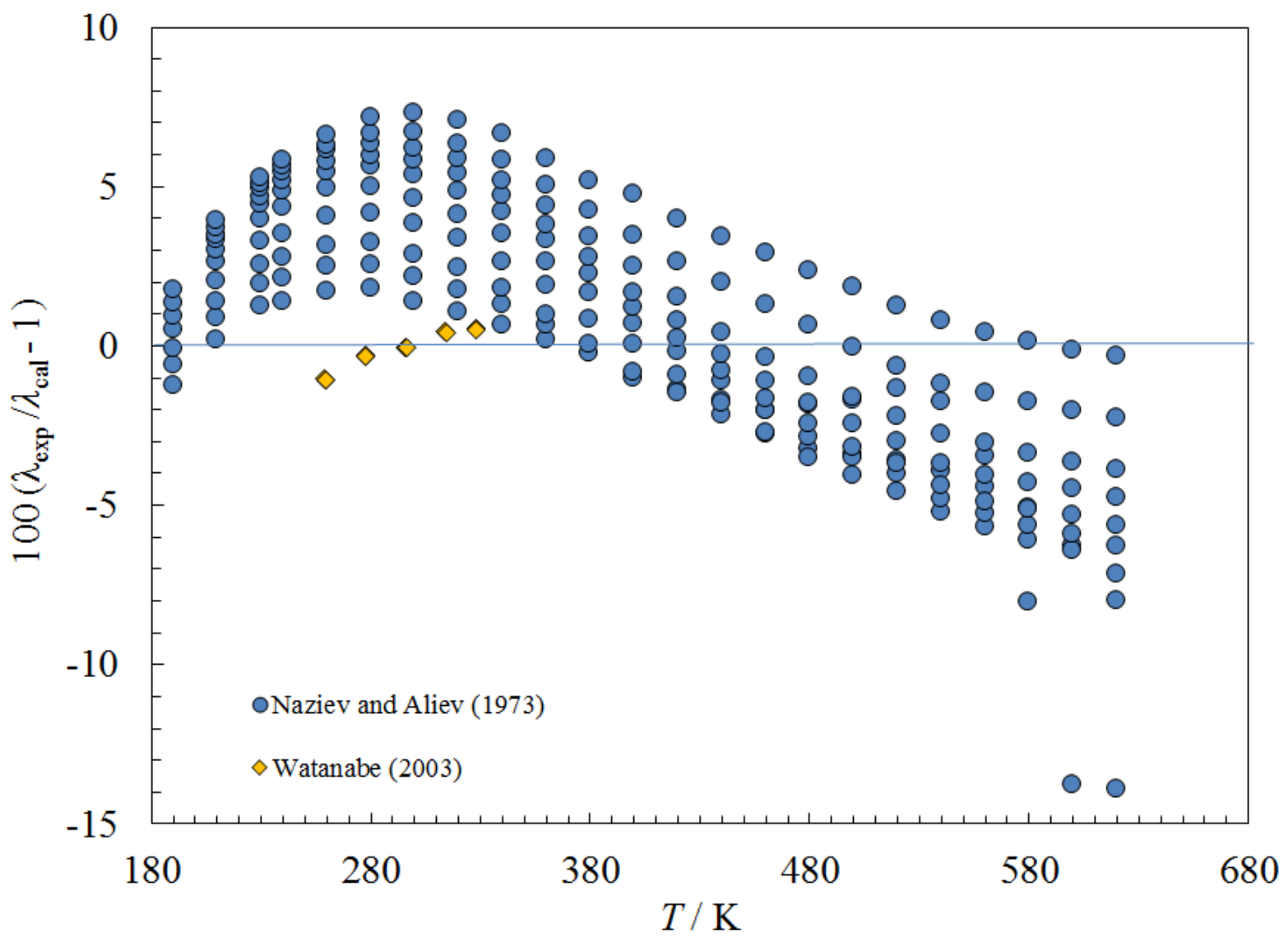

Figure 23. Percentage deviations between the model and the experimental thermal conductivity data for isooctane as a function of temperature. 


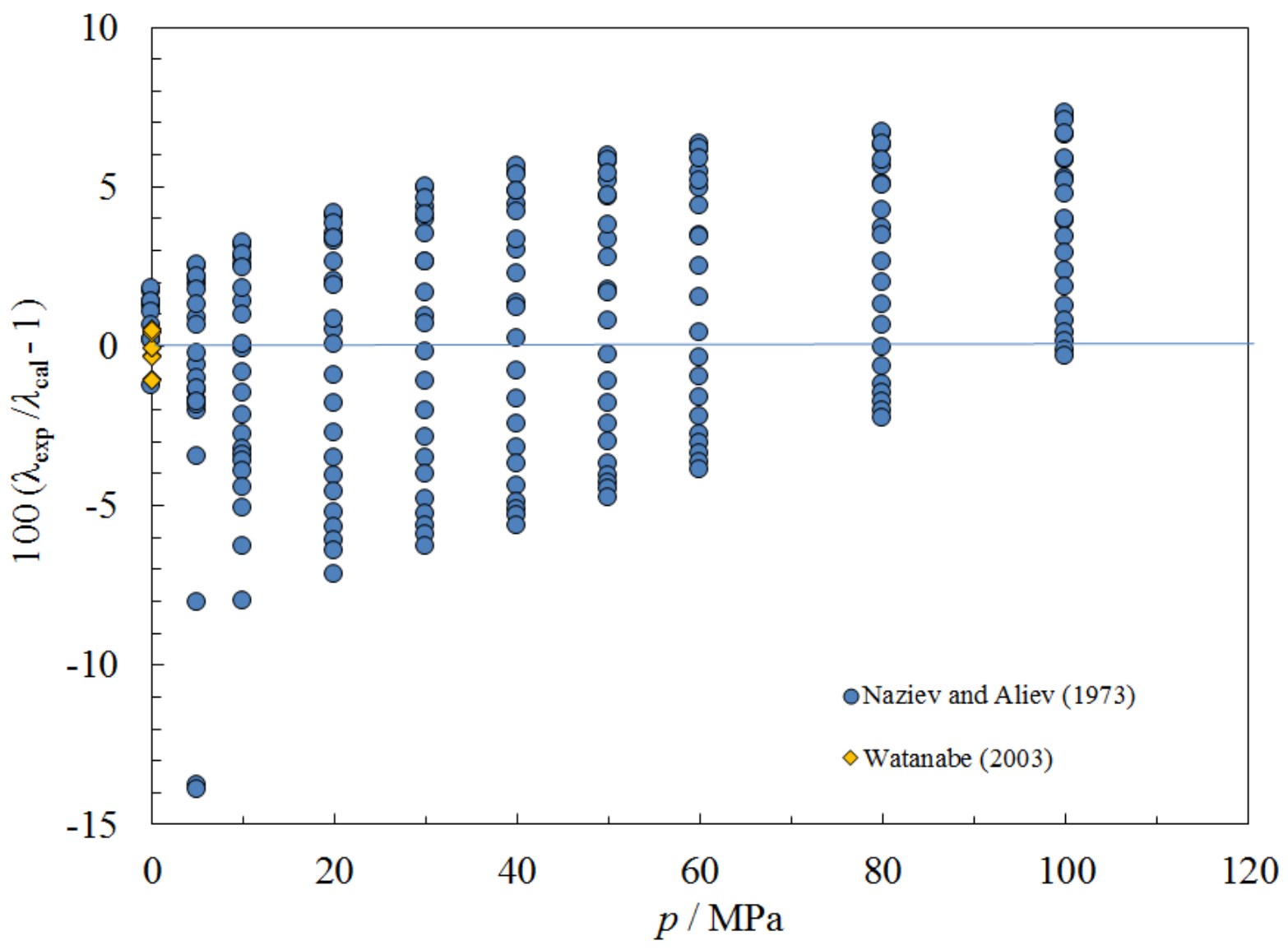

Figure 24. Percentage deviations between the model and the experimental thermal conductivity data for isooctane as a function of pressure. 


\subsection{9 n-Docosane}

The equation of state of Romeo and Lemmon (Romeo \& Lemmon, 2017) was used to provide the density and the critical parameters. The procedure described in Riesco and Vesovic (Riesco \& Vesovic, 2016) was used to estimate the Lennard-Jones parameters. This method is tuned for long-chain alkanes. We were unable to locate gas-phase viscosity data, and liquid-phase data are limited to values at atmospheric pressure (Briggs \& Comings, 1943; Karapet'yants \& Kuosen, 1963; Queimada, Quiñones-Cisneros, Marrucho, Coutinho, \& Stenby, 2005). In addition, the values from Karpet'yants and Kuo-sen (Karapet'yants \& Kuo-sen, 1963) are predictions based on family behavior, and we use them to guide the higher temperature behavior, as the experimental data only cover the range $318 \mathrm{~K}$ to $358 \mathrm{~K}$. These data were fit to obtain the coefficients presented in Table 2. A deviation plot for the viscosity is shown in Figure 25. The uncertainty in the liquid phase at saturation is estimated to be $5 \%$, rising to $10 \%$ at pressures to $50 \mathrm{MPa}$. The equation behaves in a physically reasonable manner at higher pressures and may be extrapolated to $50 \mathrm{MPa}$, but uncertainties are larger; we estimate $10 \%$. The estimated uncertainty in the gas phase is $10 \%$. We do not recommend the use of the correlation above $50 \mathrm{MPa}$. Two data sources of liquid-phase thermal conductivity were found (Mukhamedzyanov \& Usmanov, 1967; Yu.L. Rastorguev, Bogatov, \& Grigov'ev, 1974). No gas-phase data were located. Rastorguev et al.(Yu.L. Rastorguev et al., 1974) covers a wide range of temperatures, $333 \mathrm{~K}$ to $473 \mathrm{~K}$, at pressures up to $50 \mathrm{MPa}$. We fit only the data of Rastorguev et al. (Yu.L. Rastorguev et al., 1974) to obtain the parameters in Table 3, and comparisons with all experimental data are shown in Figures 26 and 27 as a function of temperature and pressure respectively. Parameters for the critical enhancement are presented in Table 4. The estimated uncertainty for the liquid at pressures to $50 \mathrm{MPa}$ is $3 \%$; uncertainty is larger at higher pressures and in the critical region. The estimated uncertainty in the gas phase is $25 \%$. Three sets of surface tension data were found (Nemzer, 1985; Queimada, Caco, Marrucho, \& Coutinho, 2005; Queimada, Silva, Caco, Marrucho, \& Coutinho, 2003) and fit to obtain the coefficients in Table 5. Figure 28 shows percentage deviations between the model and experimental data. The estimated uncertainty for surface tension at temperatures below $573 \mathrm{~K}$ is $2 \%$. 


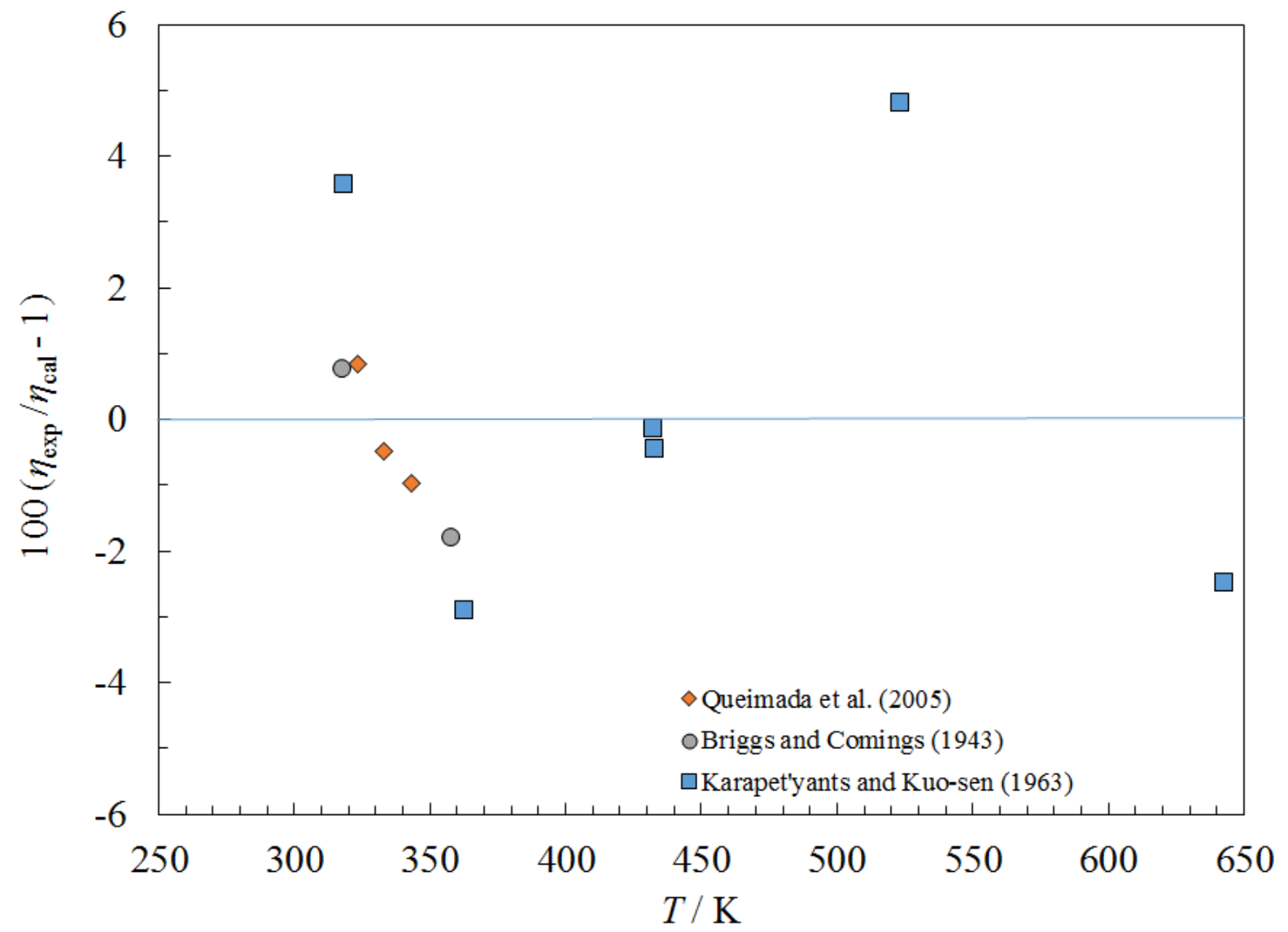

Figure 25. Percentage deviations between the model and the experimental viscosity data for $n$-docosane. 


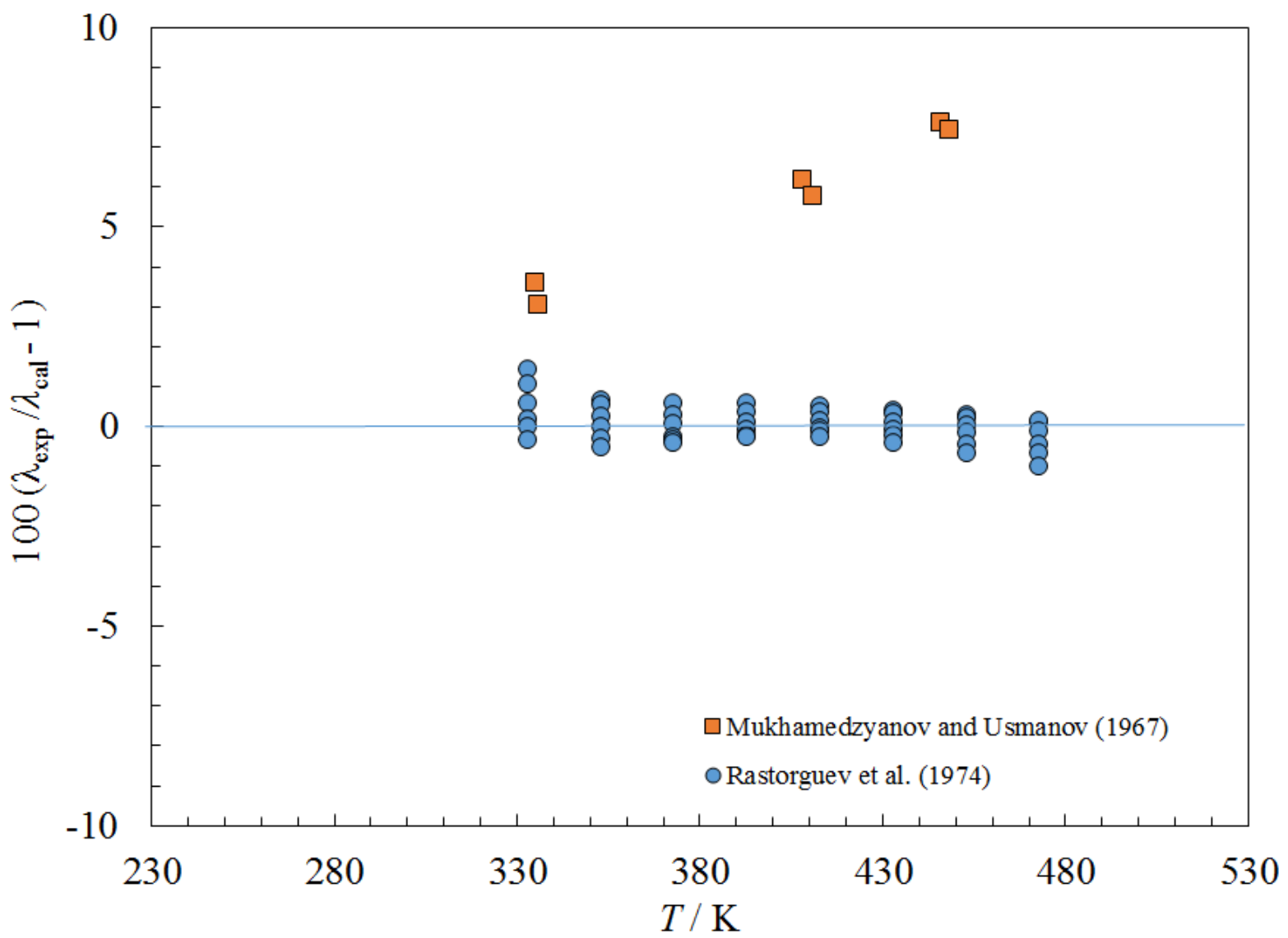

Figure 26. Percentage deviations between the model and the experimental thermal conductivity data for $n$-docosane as a function of temperature. 


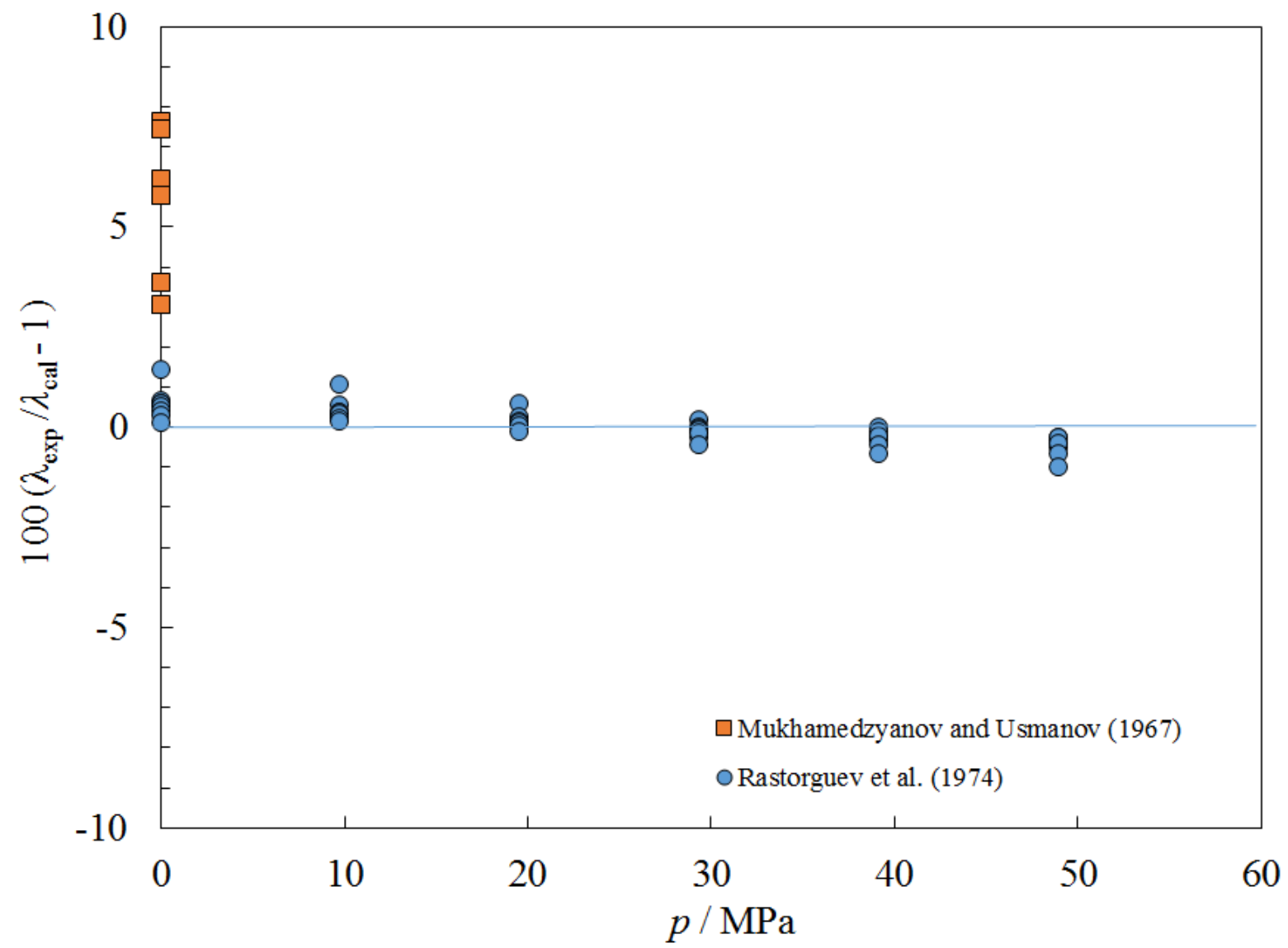

Figure 27. Percentage deviations between the model and the experimental thermal conductivity data for $n$-docosane as a function of pressure. 


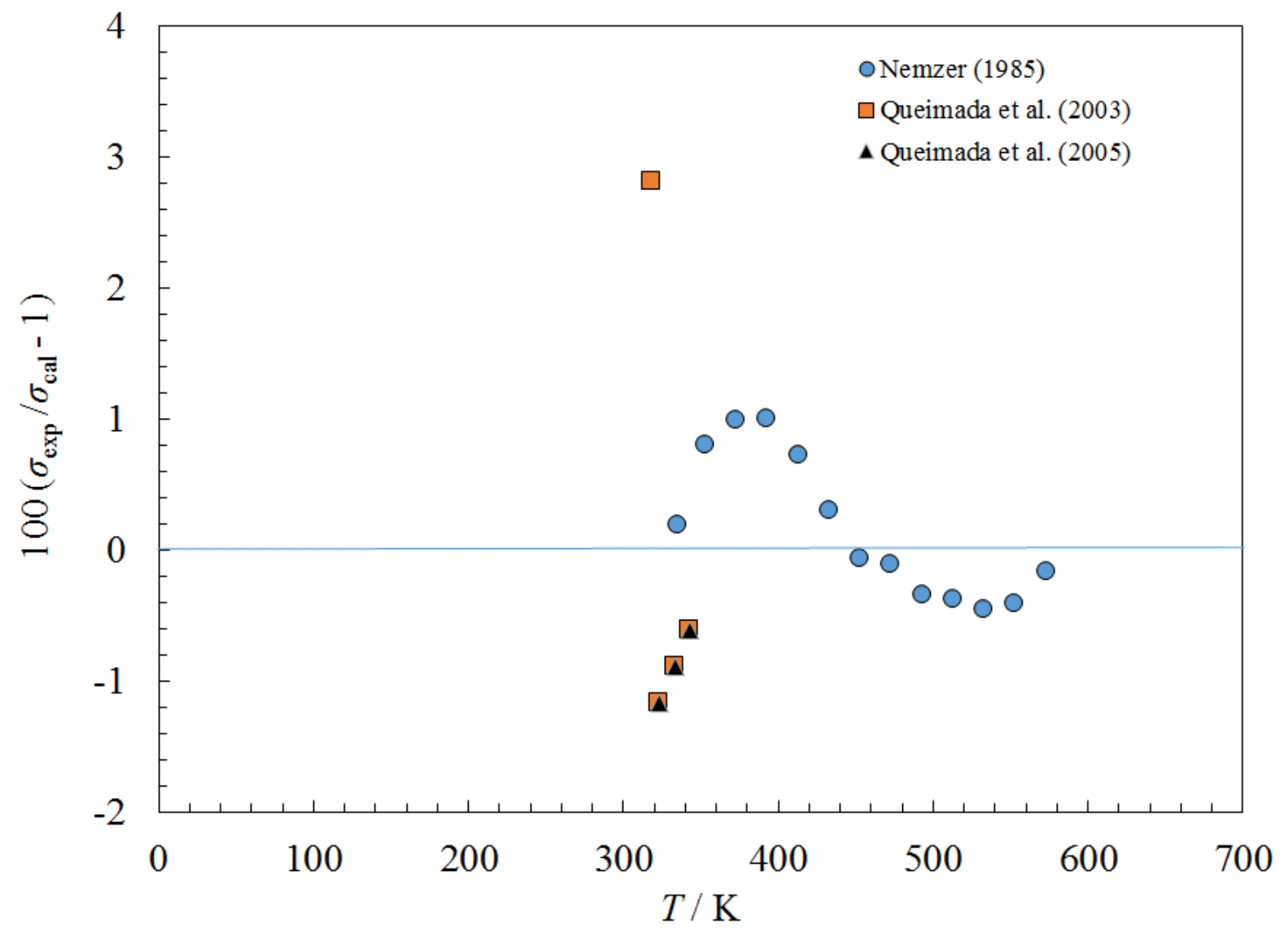

Figure 28. Percentage deviations between the model and the experimental surface tension data for $n$-docosane. 


\subsection{Alkenes and Dienes}

\subsubsection{Propylene (propene)}

The equation of state of Lemmon et al. (E.W. Lemmon, McLinden, Overhoff, \& Wagner, 2017) was used to provide the density and the critical parameters. Lennard-Jones parameters were taken from Reid et al. (Reid, 1987) and are given in Table 1. Several data sources for viscosity including both gas and liquid phases (Adzumi, 1937; Galkov \& Gerf, 1941; Gerf \& Galkov, 1940; Golubev, 1959; Haepp, 1976; Lambert et al., 1955; Neduzij \& Khmara, 1968; Titani, 1930) were used to obtain the coefficients in Table 2, and deviation plots for viscosity as a function of temperature and of pressure are given in Figures 29 and 30. The estimated uncertainty in the gas phase is $5 \%, 10 \%$ in the liquid above $100 \mathrm{~K}$ to pressures of $80 \mathrm{MPa}$, and higher elsewhere. The data of Neduzij and Khmara (Neduzij \& Khmara, 1968) differ from the data of Golubev (Golubev, 1959) by 5-10\% in the region of overlap, and it is not clear what set is superior so the regression used both sets. Future measurements are recommended to clarify this situation. Thermal conductivity (Assael et al., 2016) and surface tension (Mulero et al., 2012) are treated in separate papers and are not discussed here.

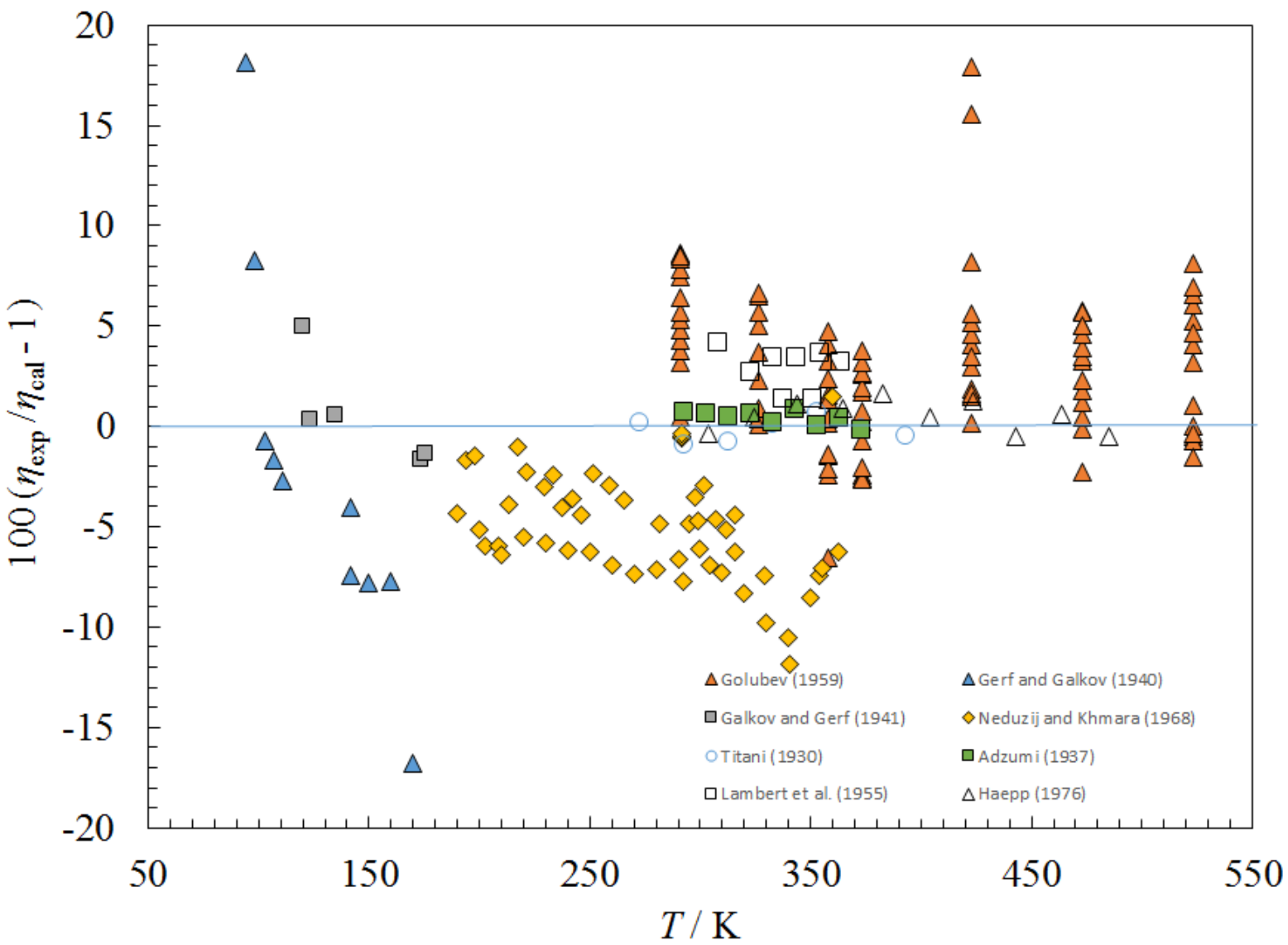

Figure 29. Percentage deviations between the model and the experimental viscosity data for propylene as a function of temperature. 


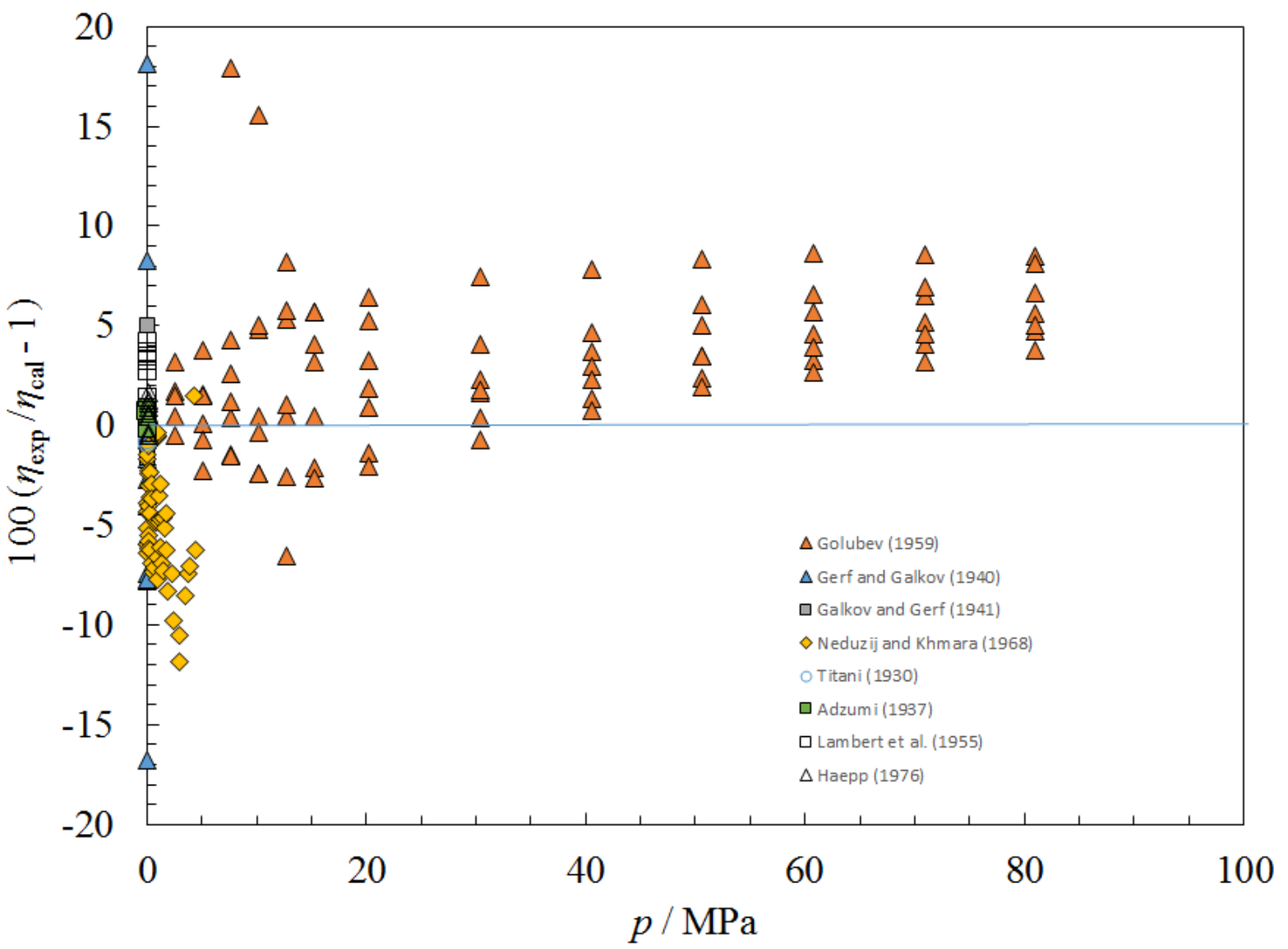

Figure 30. Percentage deviations between the model and the experimental viscosity data for propylene as a function of pressure.

\subsubsection{Isobutene (isobutylene)}

The equation of state of Lemmon and Ihmels (E.W. Lemmon \& Ihmels, 2005) was used to provide the density and the critical parameters. Lennard-Jones parameters were obtained from Hirschfelder et al. (Hirschfelder et al., 1964) and are given in Table 1. In the liquid phase we fit the data of Neduzij and Khmara (Neduzij \& Khmara, 1968) to obtain the coefficients in Table 2. Figures 31 and 32 show deviations from the experimental viscosity data. The liquid-phase data are limited to the liquid saturation boundary. Due to the extremely limited data, we estimate the uncertainty of the viscosity correlation in the liquid phase at saturation is $5 \%$, rising to $10 \%$ at pressures to $50 \mathrm{MPa}$. In the gas phase, based on comparisons with the gas-phase viscosity data of Titani (Titani, 1930), the estimated uncertainty is $3 \%$. For thermal conductivity, the gas-phase data of Vilim (Vilim, 1960), Senftleben (Senftleben, 1964), and Ryabtsev and Kazaryan (Ryabtsev \& Kazaryan, 1970) were fit to obtain the coefficients in Table 3. Parameters for the critical enhancement are presented in Table 4. For the liquid phase and supercritical phases, we fit the data of Ryabtsev and Kazaryan (Ryabtsev \& Kazaryan, 1970) that extend to $50 \mathrm{MPa}$. Deviations are shown as a function of temperature in Figure 33, and as a function of pressure in Figure 34. We estimate that the uncertainty for thermal conductivity is $5 \%$ for pressures to $50 \mathrm{MPa}$, except in the critical region where it is larger. Surface tension is discussed in a separate publication (Mulero et al., 2012). 


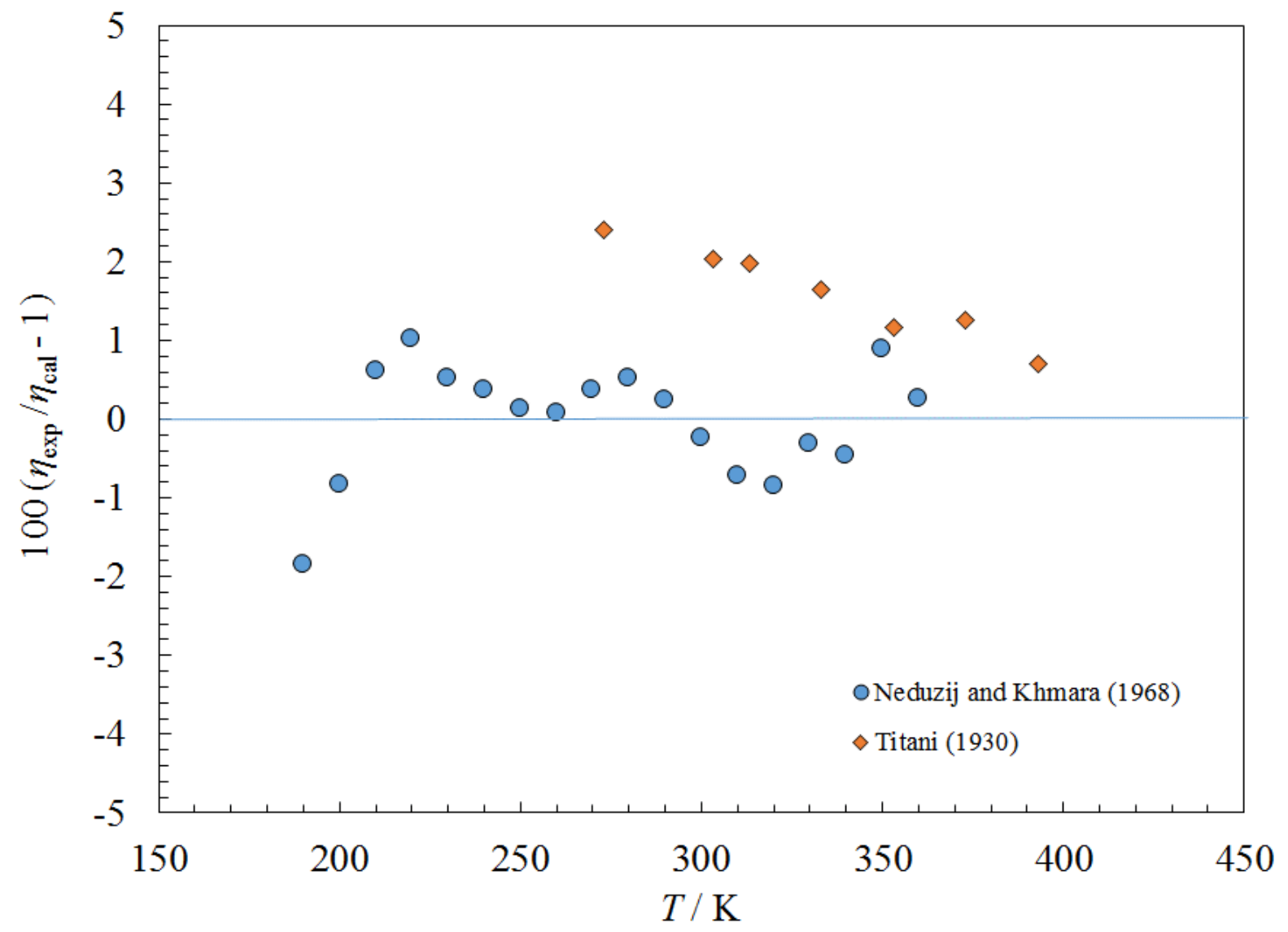

Figure 31. Percentage deviations between the model and the experimental viscosity data for isobutene as a function of temperature. 


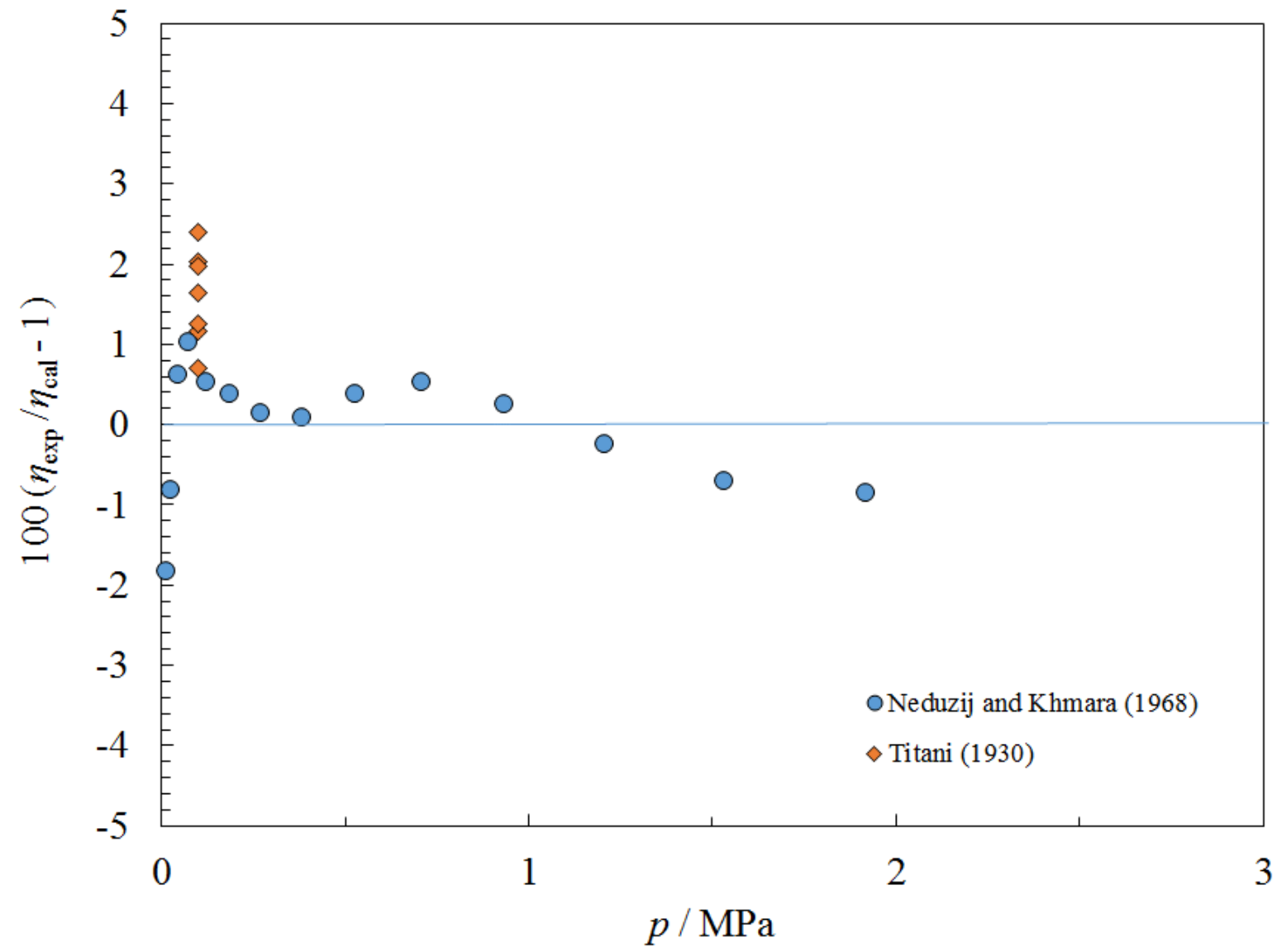

Figure 32. Percentage deviations between the model and the experimental viscosity data for isobutene as a function of pressure. 


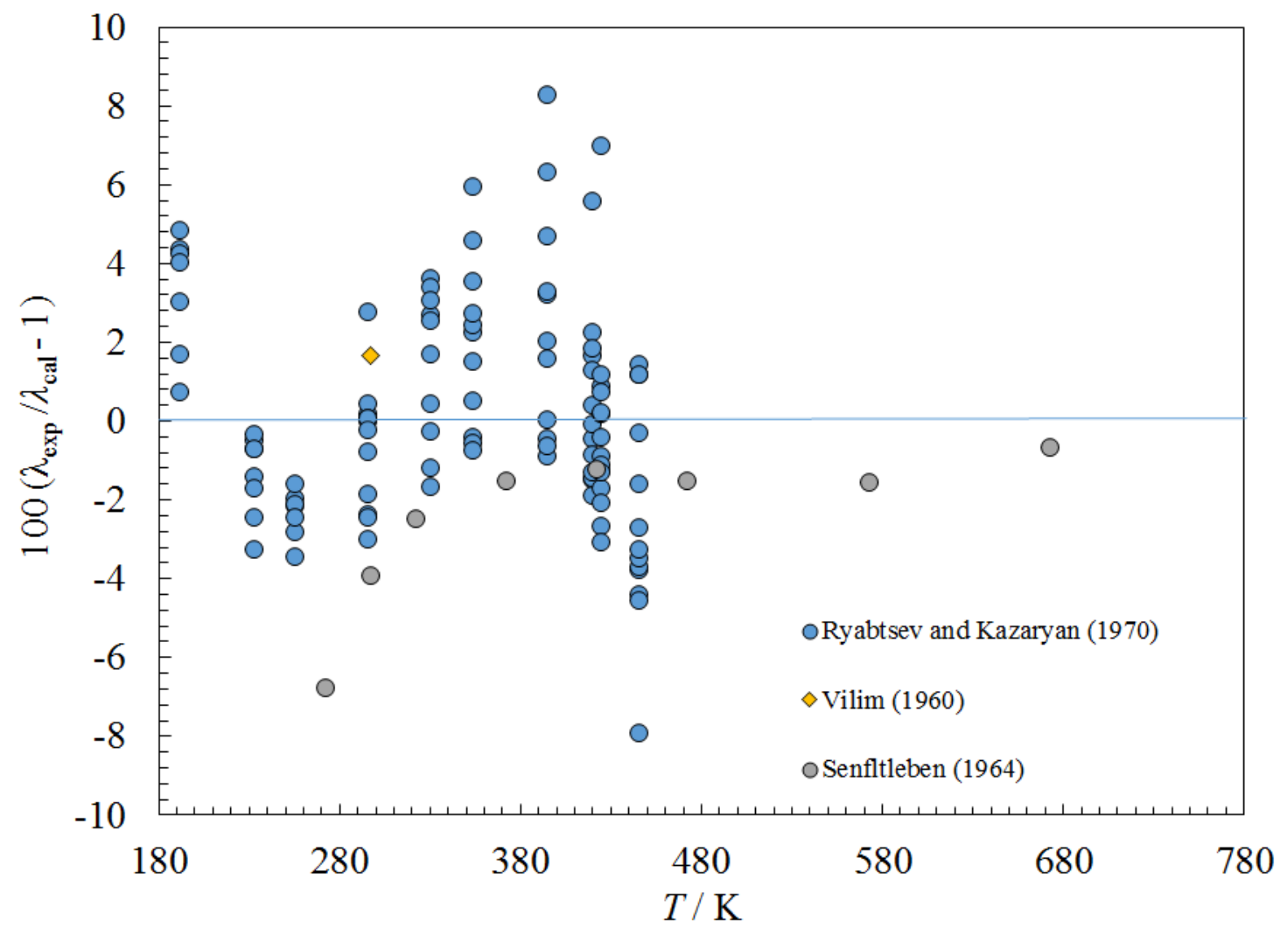

Figure 33. Percentage deviations between the model and the experimental thermal conductivity data for isobutene as a function of temperature. 


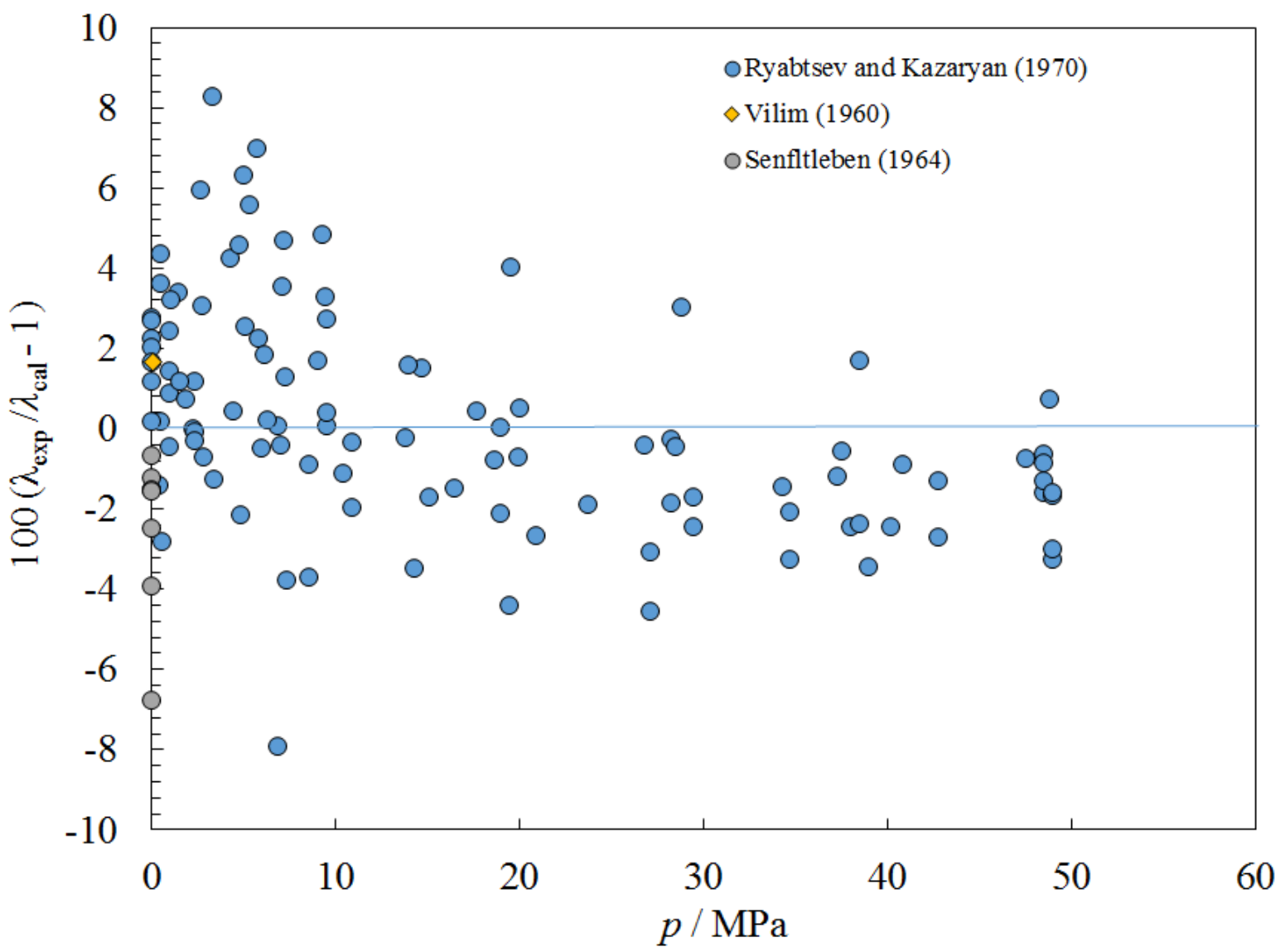

Figure 34. Percentage deviations between the model and the experimental thermal conductivity data for isobutene as a function of pressure.

\subsubsection{Trans-2-butene}

The equation of state of Lemmon and Ihmels (E.W. Lemmon \& Ihmels, 2005) was used to provide the density and the critical parameters. Lennard-Jones parameters were obtained from Hirschfelder et al. (Hirschfelder et al., 1964) and are reported in Table 1. There were no viscosity data found in the liquid phase; we use the same coefficients as isobutene for an estimate, that are given in Table 2. Figure 35 shows deviations with available gas-phase data (Lambert et al., 1955; Titani, 1930). We estimate the uncertainty of the viscosity correlation in the gas phase is $5 \%$, and as high as $20 \%$ in the liquid. For thermal conductivity, we again used the same coefficients as were used for isobutene, in Table 3, since no liquid-phase thermal conductivity data were found. Parameters for the critical enhancement are presented in Table 4. Figure 36 shows deviations with available gas-phase data (Lambert et al., 1955; Parkinson, Mukhopadhyay, \& Gray, 1972). Due to the extremely limited data, we estimate that the uncertainty for thermal conductivity is $5 \%$ in the gas phase, and $20 \%$ elsewhere. Surface tension is discussed in a separate publication (Mulero \& Cachadiña, 2014). 


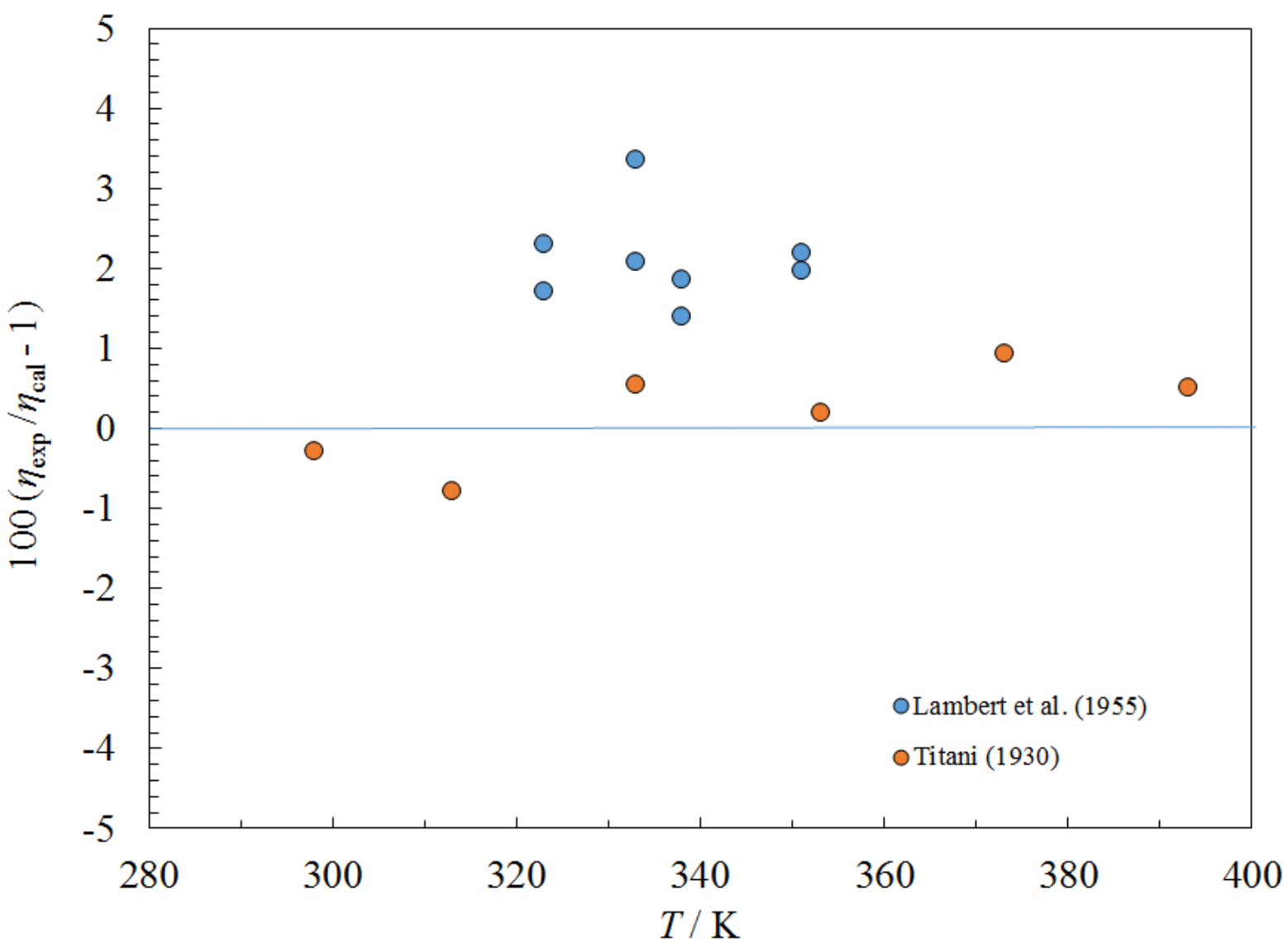

Figure 52. Percentage deviations between the model and the experimental viscosity data for trans-2-butene as a function of temperature. 


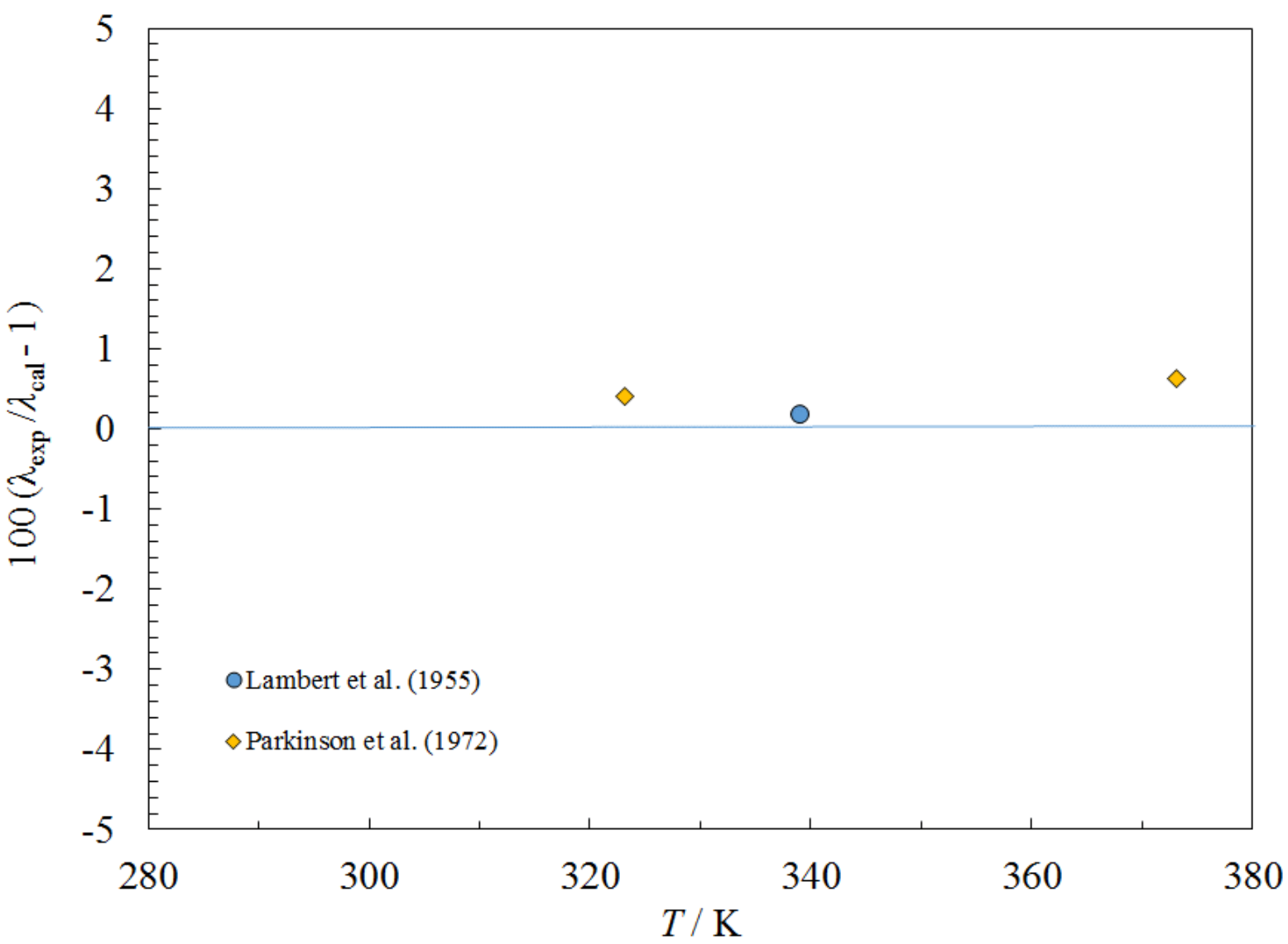

Figure 53. Percentage deviations between the model and the experimental thermal conductivity data for trans-2-butene as a function of temperature.

\subsubsection{Cis-2-butene}

The equation of state of Lemmon and Ihmels (E.W. Lemmon \& Ihmels, 2005) was used to provide the density and the critical parameters. Lennard-Jones parameters were obtained from Hirschfelder et al. (Hirschfelder et al., 1964). Similar to the situation for trans-2-butene, there were no data found in the liquid phase, we use the same coefficients as isobutene as an estimate, given in Table 2. Figure 54 shows deviations with available gas-phase data (Lambert et al., 1955; Titani, 1930; Trautz \& Husseini, 1934). We estimate the uncertainty of the viscosity correlation in the gas phase is $5 \%$, and as high as $20 \%$ in the liquid. For thermal conductivity, we again used the same coefficients as were used for isobutene, shown in Table 3, since no liquid-phase thermal conductivity data were found. Parameters for the critical enhancement are presented in Table 4. Figure 55 shows deviations with available gas-phase data (Lambert et al., 1955; Parkinson et al., 1972). We estimate that the uncertainty for thermal conductivity is $5 \%$ in the gas phase, and $20 \%$ elsewhere. Surface tension is discussed in a separate publication (Mulero \& Cachadiña, 2014). 


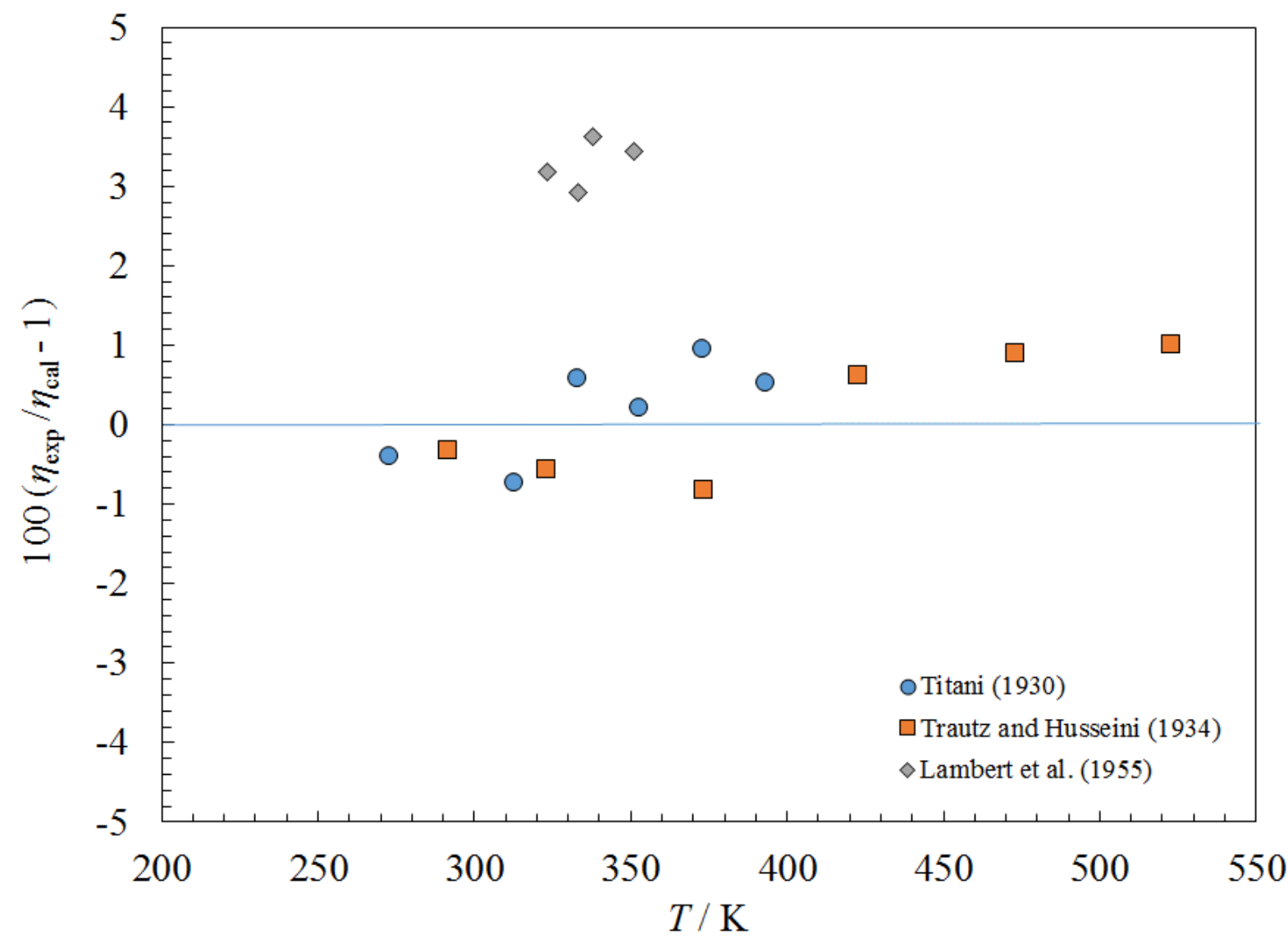

Figure 54. Percentage deviations between the model and the experimental viscosity data for cis-2-butene as a function of temperature. 


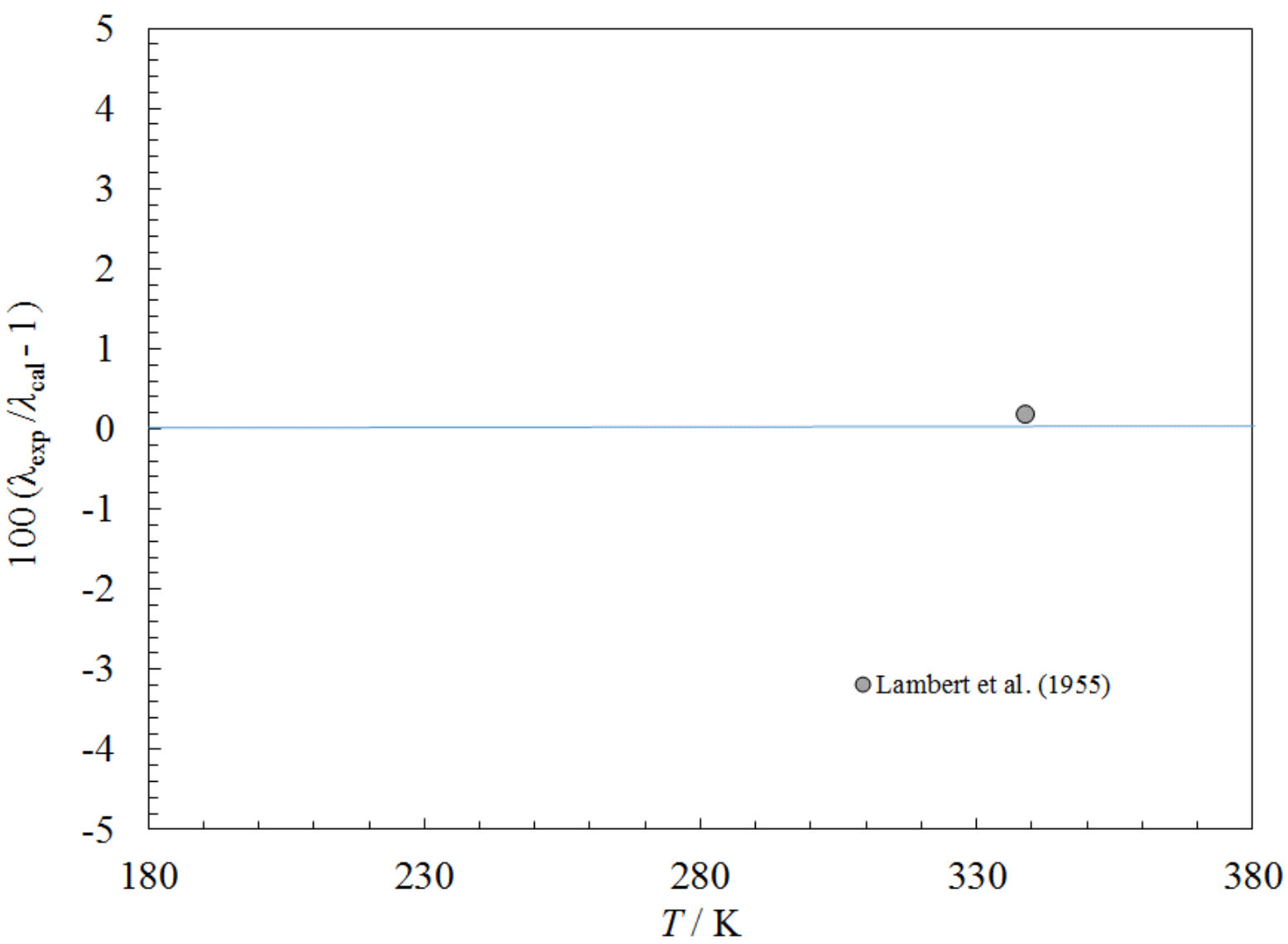

Figure 55. Percentage deviations between the model and the experimental thermal conductivity data for cis-2-butene as a function of temperature.

\subsubsection{1-Butene}

The equation of state of Lemmon and Ihmels (E.W. Lemmon \& Ihmels, 2005) was used to provide the density and the critical parameters. Lennard-Jones parameters were obtained from Hirschfelder et al. (Hirschfelder et al., 1964) and are shown in Table 1. There were no data found in the liquid phase, we use the same coefficients as isobutene as an estimate, given in Table 2. Figure 56 shows deviations with available gas-phase data (Titani, 1930). We estimate the uncertainty of the viscosity correlation in the gas phase is $5 \%$, and as high as $20 \%$ in the liquid. For thermal conductivity, we again used the same coefficients as were used for isobutene for the liquid phase, presented in Table 3, since no liquid-phase thermal conductivity data were found. Parameters for the critical enhancement are presented in Table 4. The data of Senftleben (Senftleben, 1964) were fit to obtain the gas-phase coefficients in Table 3. Figure 57 shows deviations with available gas-phase data (Parkinson et al., 1972; Senftleben, 1964; Vilim, 1960) We estimate that the uncertainty for thermal conductivity is $5 \%$ in the gas phase, and $20 \%$ elsewhere. Surface tension is discussed in a separate publication (Mulero et al., 2012). 


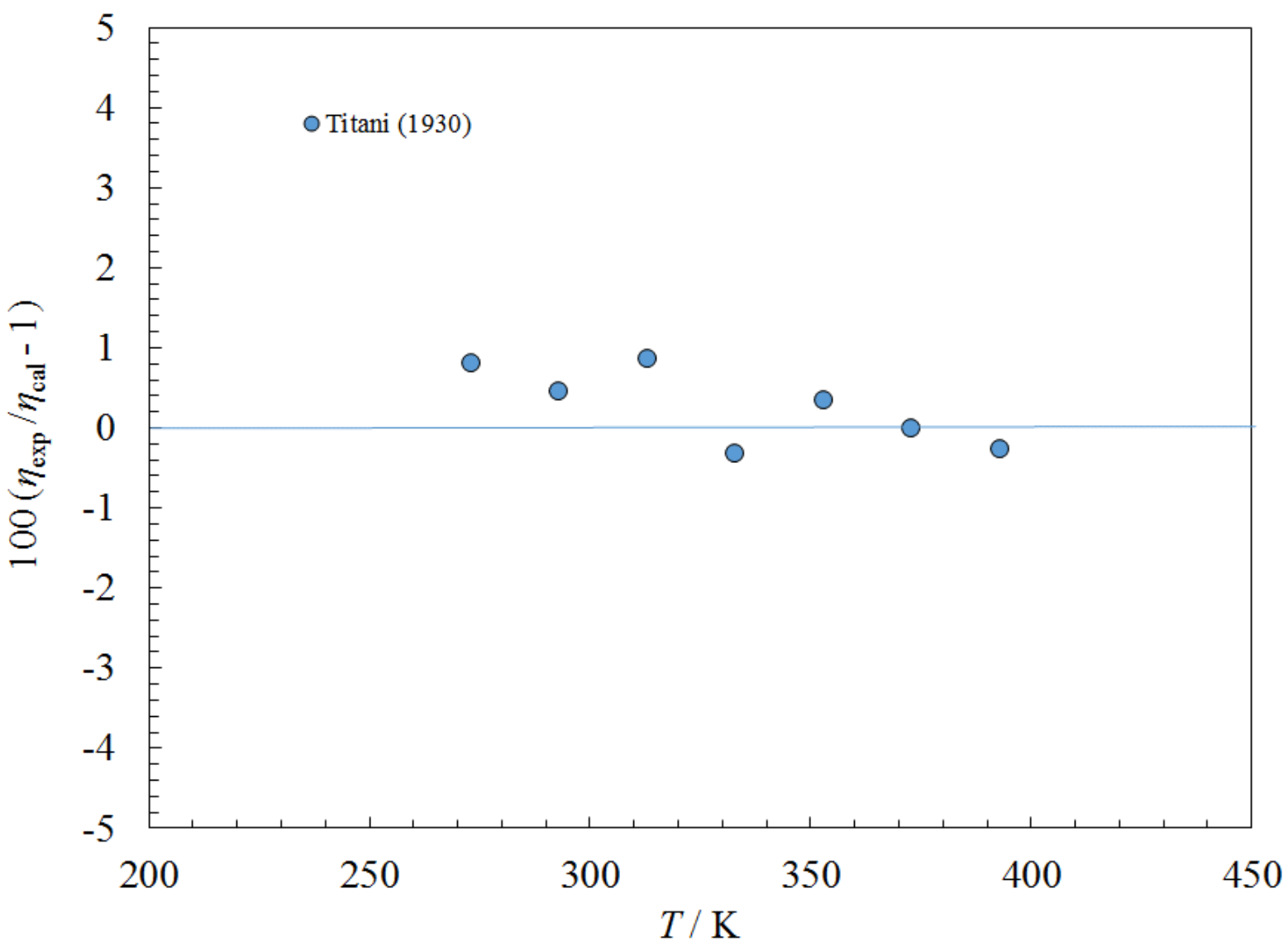

Figure 56. Percentage deviations between the model and the experimental viscosity data for 1-butene as a function of temperature. 


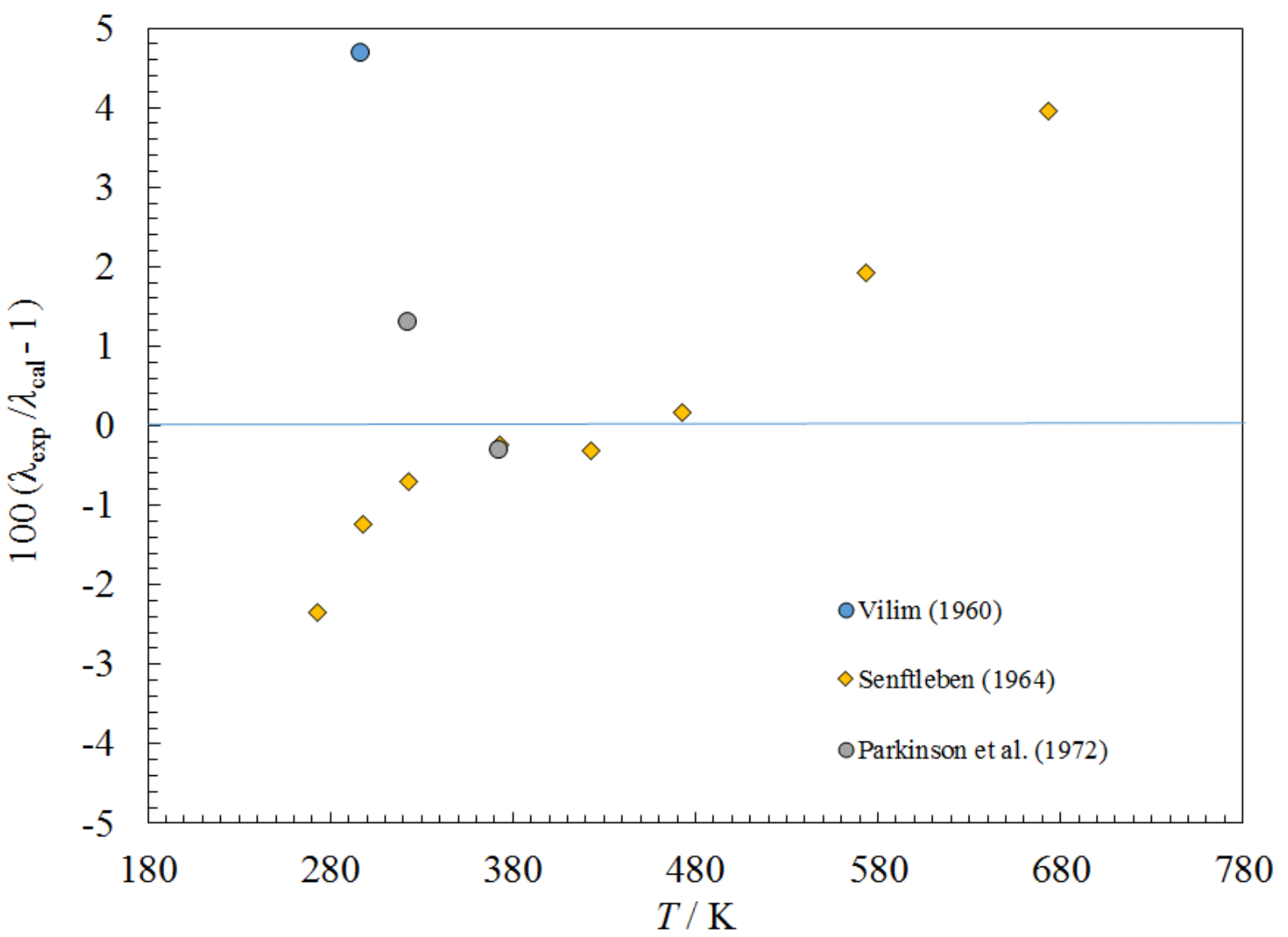

Figure 57. Percentage deviations between the model and the experimental thermal conductivity data for 1-butene as a function of temperature. 


\subsubsection{1-Pentene}

The equation of state of Gao et al. (K. Gao, J. Wu, \& E. W. Lemmon, 2017c) was used to provide the density and the critical parameters. Lennard-Jones parameters were calculated by the method of Chung et al. (Chung et al., 1988), values are presented in Table 1. The experimental data for this fluid are extremely limited. Experimental viscosity data were unavailable. The values given in Table 2 are totally predictive and based on family behavior. Estimated uncertainty for viscosity is $20 \%$. Vapor-phase thermal conductivity data were not found, but Naziev and Abasov (Y.M. Naziev \& Abasov, 1968) presented data for 1-hexene and 1-heptene and gave a correlation for 1-pentene that was used to estimate the values in Table 3. Parameters for the critical enhancement are presented in Table 4 . The estimated uncertainty in the gas phase is $10 \%$. Limited liquid-phase thermal conductivity data at atmospheric pressure from Watanabe and Kato (Watanabe \& Kato, 2004) were used to obtain the coefficient in Table 3 for the liquid phase. Figure 58 shows the deviations with the experimental thermal conductivity data. The estimated uncertainty for thermal conductivity at atmospheric pressure is $1 \%$ over the temperature range of the Watanabe and Kato data $(257 \mathrm{~K}$ to $302 \mathrm{~K})$ and higher at increased pressures and in the critical region. Recommended values for surface tension from Jasper (Jasper, 1972) were used to obtain the coefficients in Table 6, and a deviation plot is shown in Figure 59. The estimated uncertainty is $5 \%$.

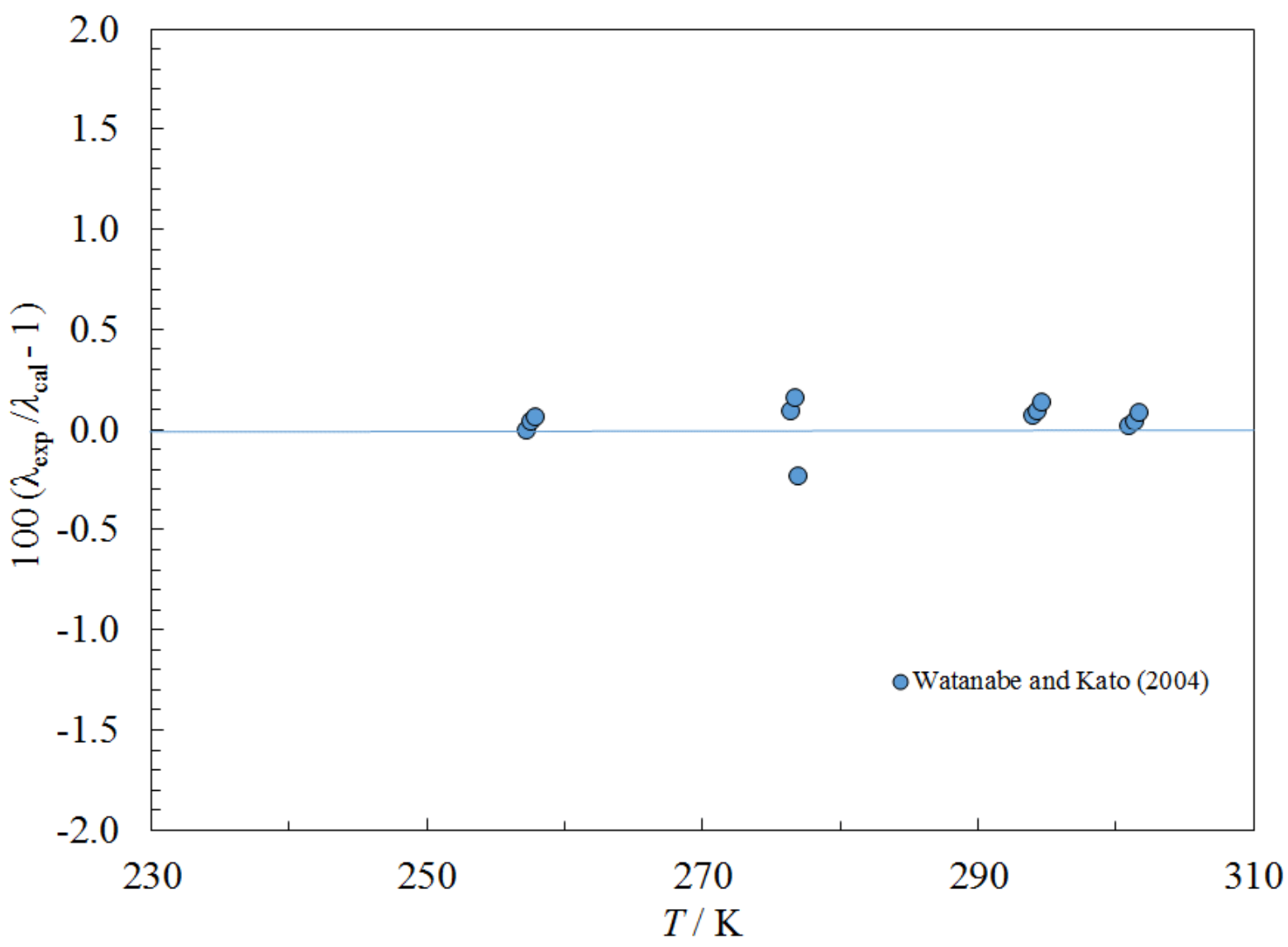

Figure 58. Percentage deviations between the model and the experimental thermal conductivity data for 1-pentene as a function of temperature. 


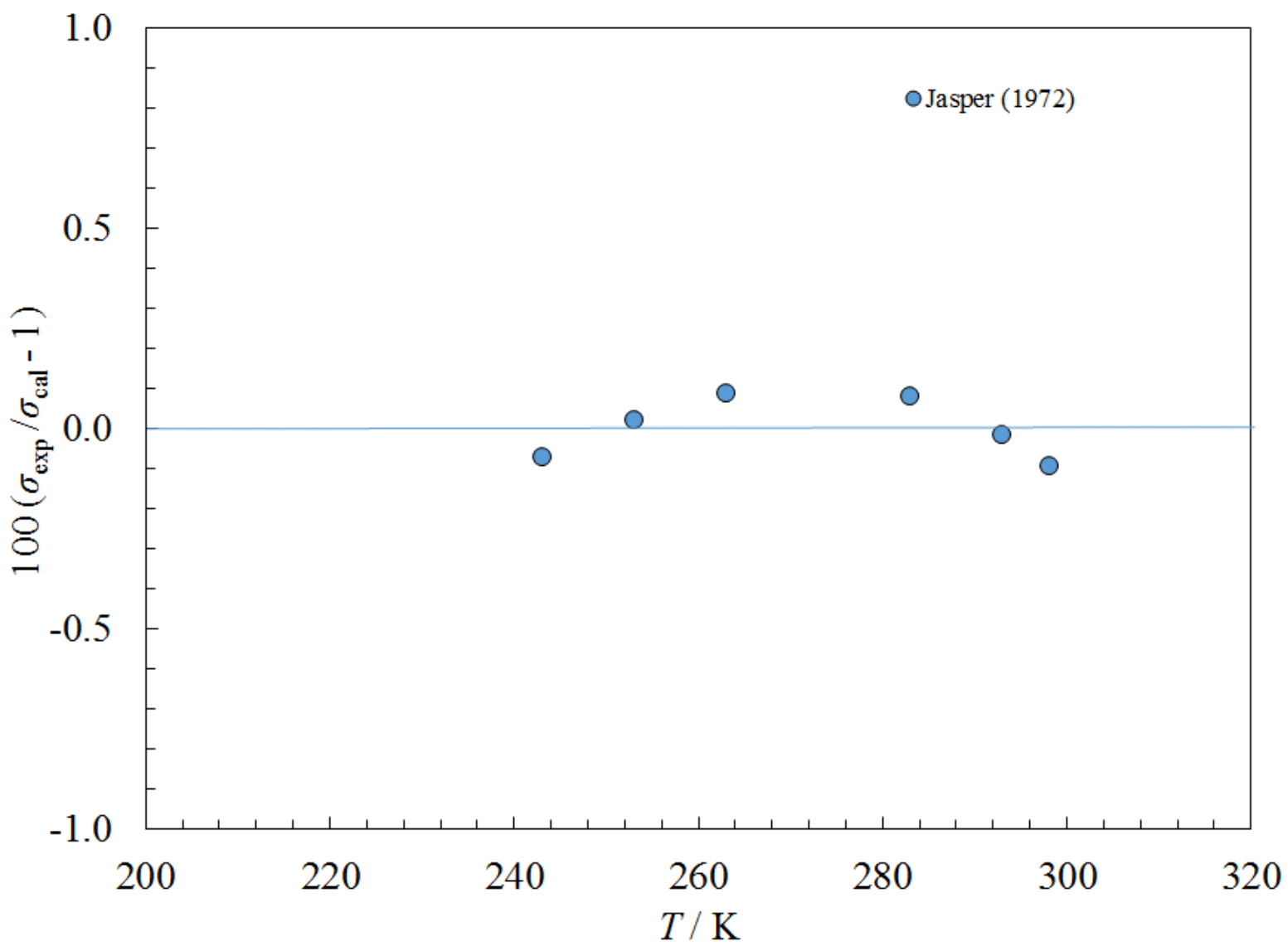

Figure 59. Percentage deviations between the model and the experimental surface tension data for 1-pentene as a function of temperature. 


\subsubsection{Propadiene}

The equation of state of Gao et al. (K. Gao, J. Wu, \& E. W. Lemmon, 20171) was used to provide the density and the critical parameters. Lennard-Jones parameters were calculated by the method of Chung et al. (Chung et al., 1988); values are presented in Table 1. Experimental data for viscosity, thermal conductivity, and surface tension were not found, so the model is totally predictive and should be considered extremely preliminary. Coefficients are given in Tables 2-4. For surface tension, the value of $n_{0}$ for propyne from Mulero et al. (Mulero et al., 2012) was used, and $\sigma_{0}$ was adjusted so that the surface tension of propadiene fell in between propylene and propyne as suggested by the simulations of Werth et al. (Werth et al., 2015). Coefficients are given in Table 5 for surface tension. The estimated uncertainty for viscosity and thermal conductivity is $20 \%, 10 \%$ for surface tension. Measurements should be made to enable improvements in the models for this fluid.

\subsubsection{1,3-Butadiene}

The equation of state of Gao et al. (K. Gao, J. Wu, \& E. W. Lemmon, 2017d) was used to provide the density and the critical parameters. Lennard-Jones parameters were calculated by the method of Chung et al. (Chung et al., 1988), values are presented in Table 1. Gas-phase data of Dunlop (Dunlop, 1994) and Lambert et al. (Lambert et al., 1955) and liquid-phase data of Golubev (Golubev, 1959), and Neduzij and Khmara (Neduzij \& Khmara, 1968) were used to obtain the coefficients in Table 2. Deviations with viscosity data as a function of temperature are shown in Figure 60. No liquid viscosity data were found off the saturation boundary. The estimated uncertainty for the liquid phase at saturation is $5 \%$; the estimated uncertainty in the gas phase is also $5 \%$. Very limited gas-phase thermal conductivity data from Lambert et al. (Lambert et al., 1955) and from Vilim (Vilim, 1960) were used to obtain the coefficients in Table 3. Parameters for the critical enhancement are presented in Table 4. Liquid-phase data were not found. Figure 61 shows the deviations of the thermal conductivity model from the gas-phase data. The estimated uncertainty in the liquid phase is $20 \%, 10 \%$ for the gas phase. We were unable to locate surface tension data. Yaws (Yaws, 2014) presents a correlation for surface tension, but it incorporates a slightly different critical temperature $(425.37 \mathrm{~K})$ than what the equation of state of Gao et al. (K. Gao et al., 2017d) uses, so we adjusted the coefficients (given in Table 5) slightly. The estimated uncertainty is $10 \%$. 


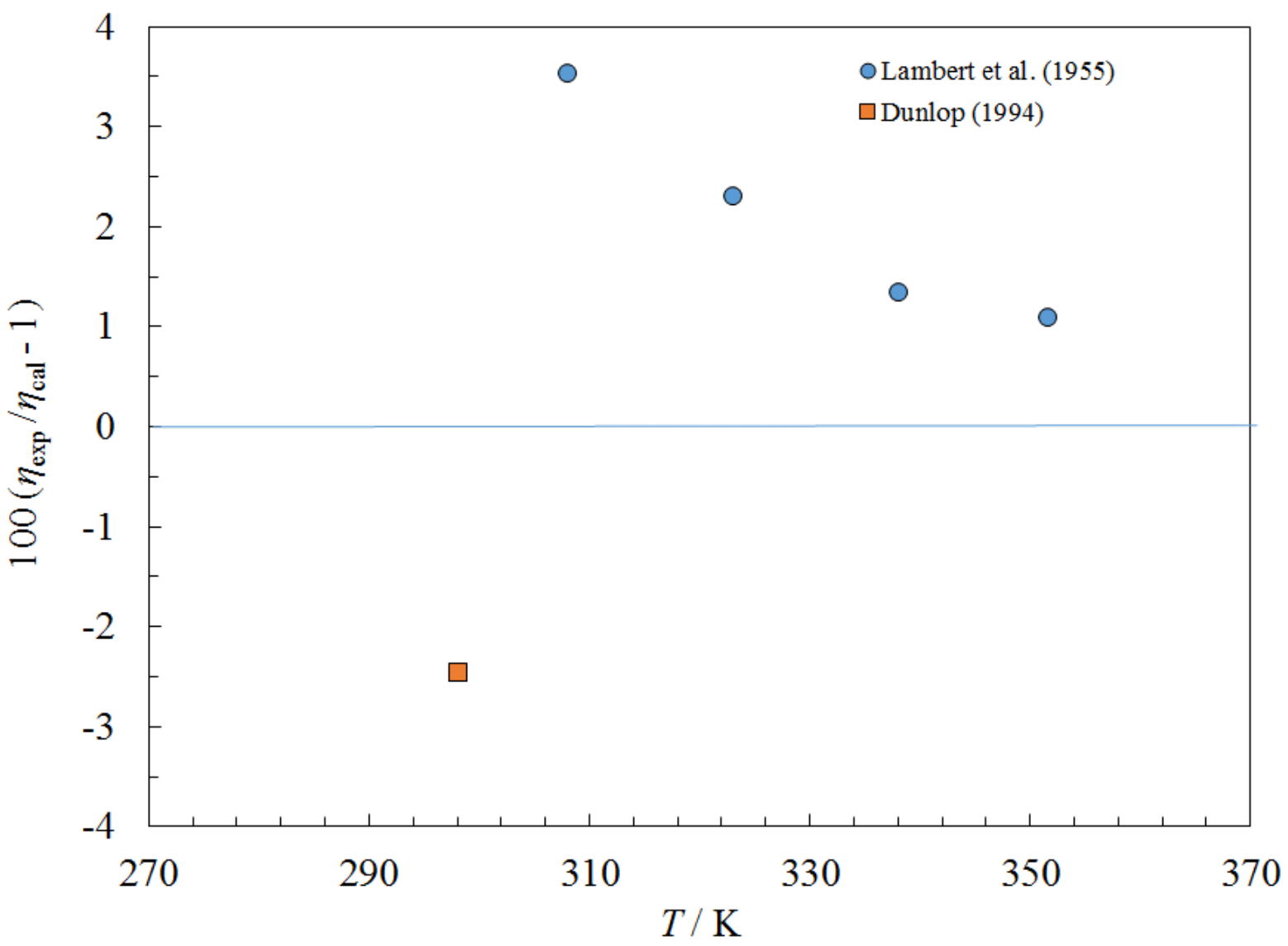

Figure 60. Percentage deviations between the model and the experimental viscosity data for 1,3-butadiene as a function of temperature. 


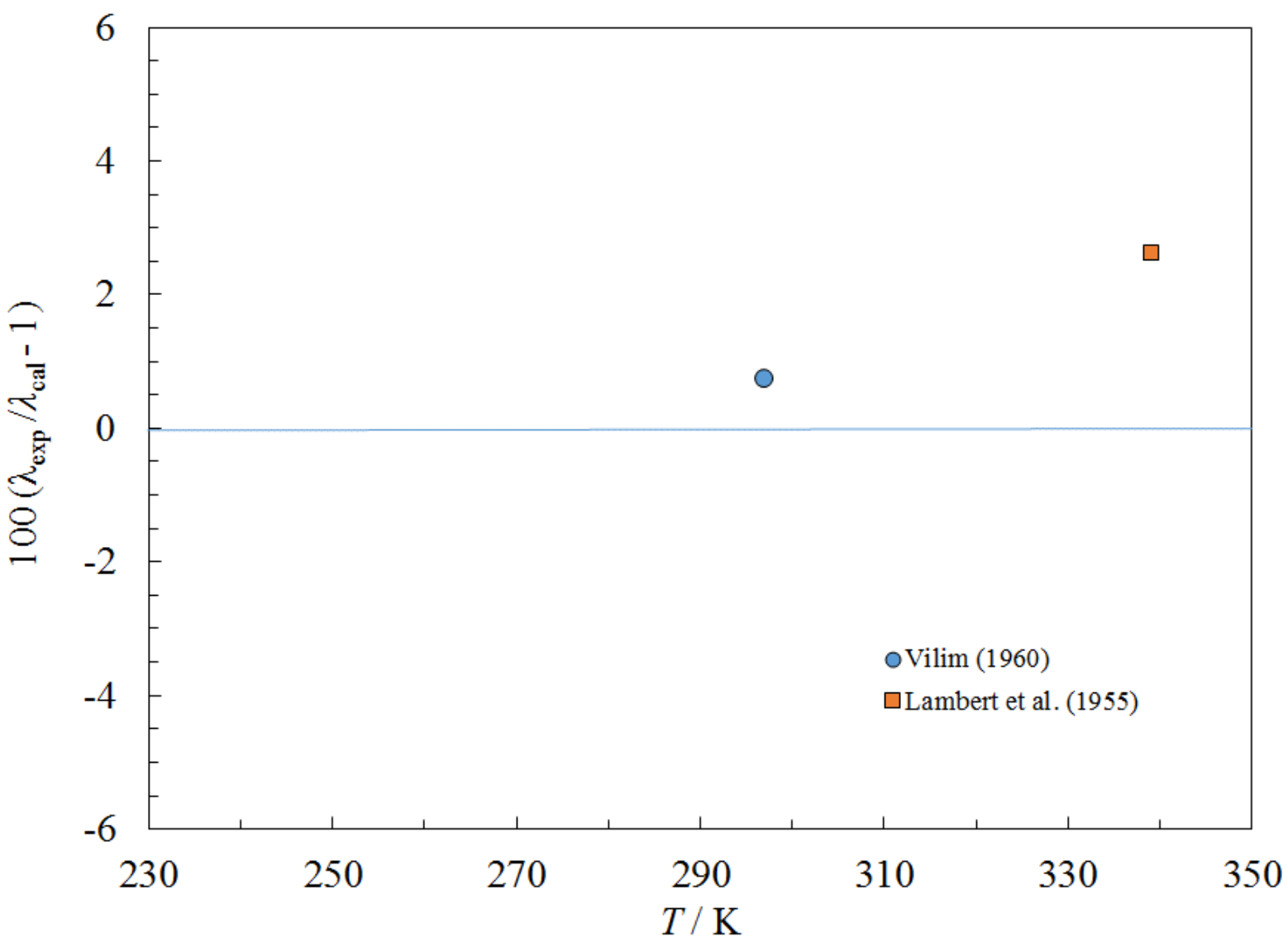

Figure 61. Percentage deviations between the model and the experimental thermal conductivity data for 1,3-butadiene as a function of temperature. 


\subsection{Alkynes}

\subsubsection{Acetylene (ethyne)}

The equation of state of Gao et al. (K. Gao, J. Wu, \& E. W. Lemmon, 2017h) was used to provide the density and the critical parameters. Lennard-Jones parameters were calculated by the method of Chung et al. (Chung et al., 1988); values are presented in Table 1. We were unable to locate any experimental data for liquid-phase viscosity or thermal conductivity. Gas-phase viscosity data of Titani (Titani, 1930), Adzumi (Adzumi, 1937), and Wobser and Muller (Wobser $\&$ Muller, 1941) were used to obtain the coefficient $F_{\mathrm{c}}$ in Table 2. Deviations are shown in Figure 62. The EOS is limited to $310 \mathrm{~K}$; values above that temperature are extrapolations. The estimated uncertainty for the gas-phase viscosity is $3 \%$. Similarly, the gas-phase data of Senftleben (Senftleben, 1964) were used to obtain coefficients in Table 3, and deviations are shown in Figure 63. Parameters for the critical enhancement are presented in Table 4. The Senftleben data extend to $673 \mathrm{~K}$, but the EOS is limited to $310 \mathrm{~K}$. The estimated uncertainty of the thermal conductivity in the gas phase is $10 \%$. The estimated uncertainty for liquid-phase viscosity and thermal conductivity is $20 \%$, although it is difficult to determine due to the lack of experimental data. One set of experimental data for surface tension (Maass \& Wright, 1921) was used to determine the coefficients in Table 5. Figure 64 shows the deviations from the experimental surface tension data. The estimated uncertainty is $2 \%$ over the temperature range $242 \mathrm{~K}$ to $282 \mathrm{~K}$.

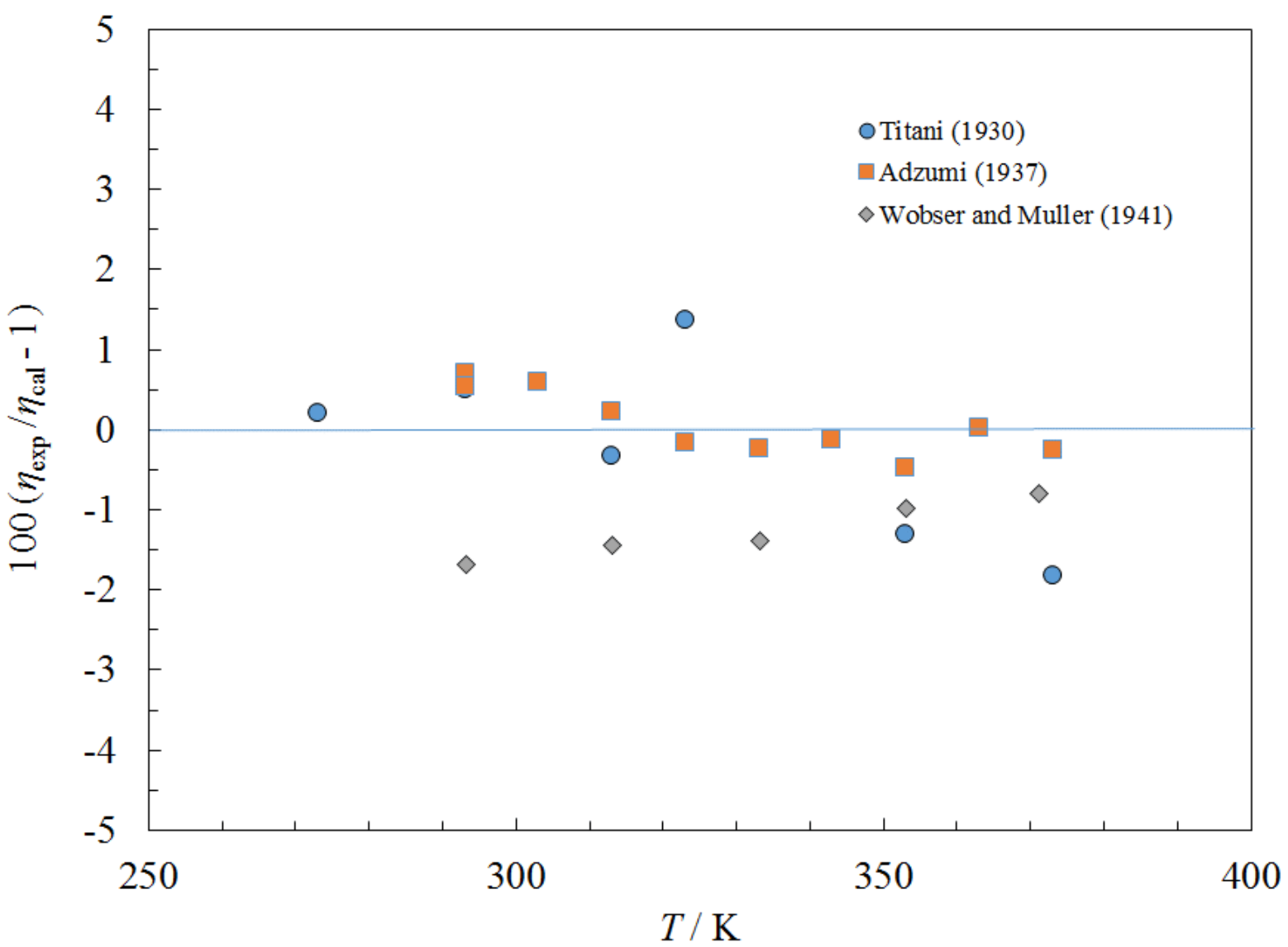

Figure 62. Percentage deviations between the model and the experimental viscosity data for acetylene as a function of temperature. 


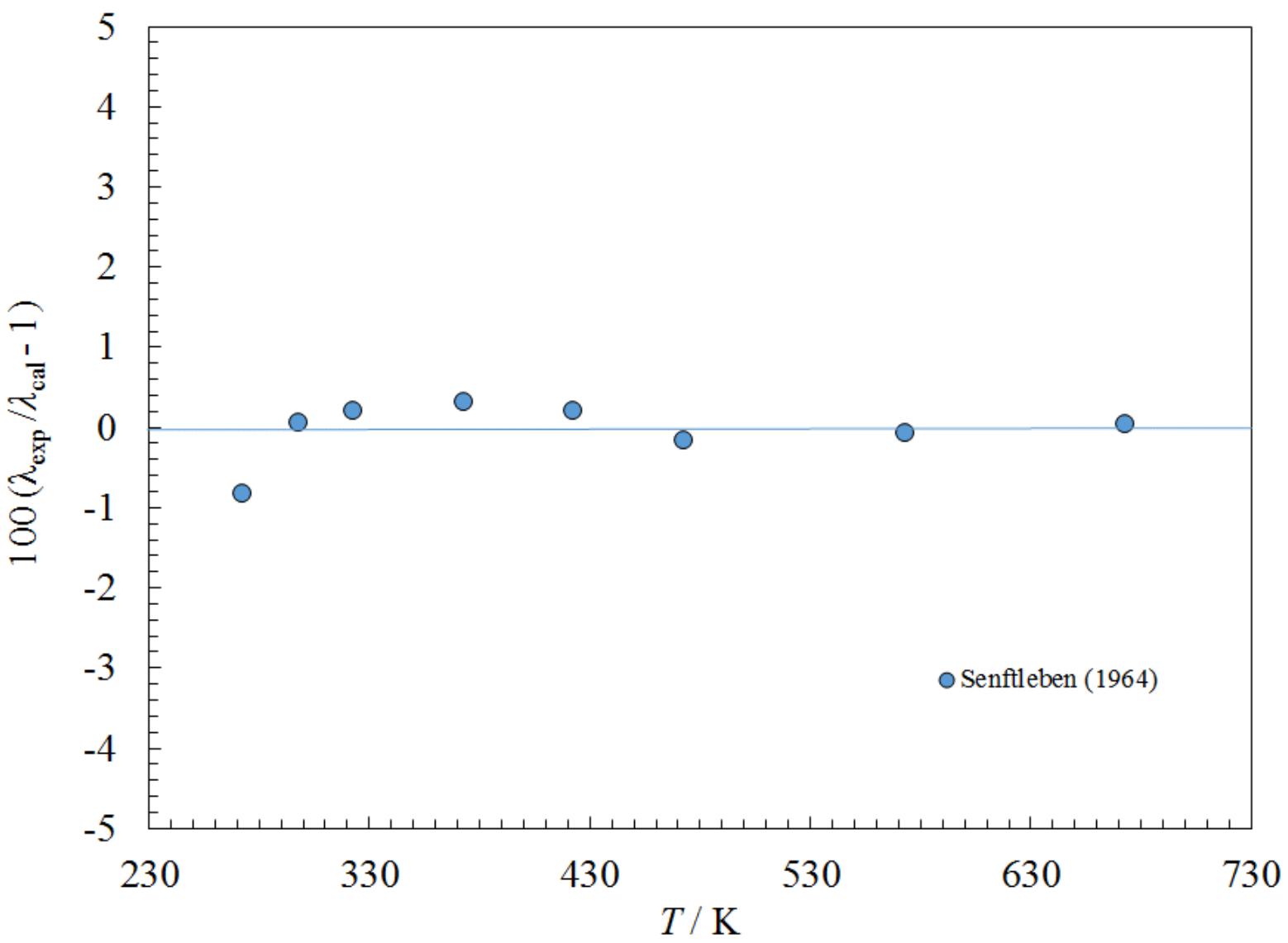

Figure 63. Percentage deviations between the model and the experimental thermal conductivity data for acetylene as a function of temperature. 


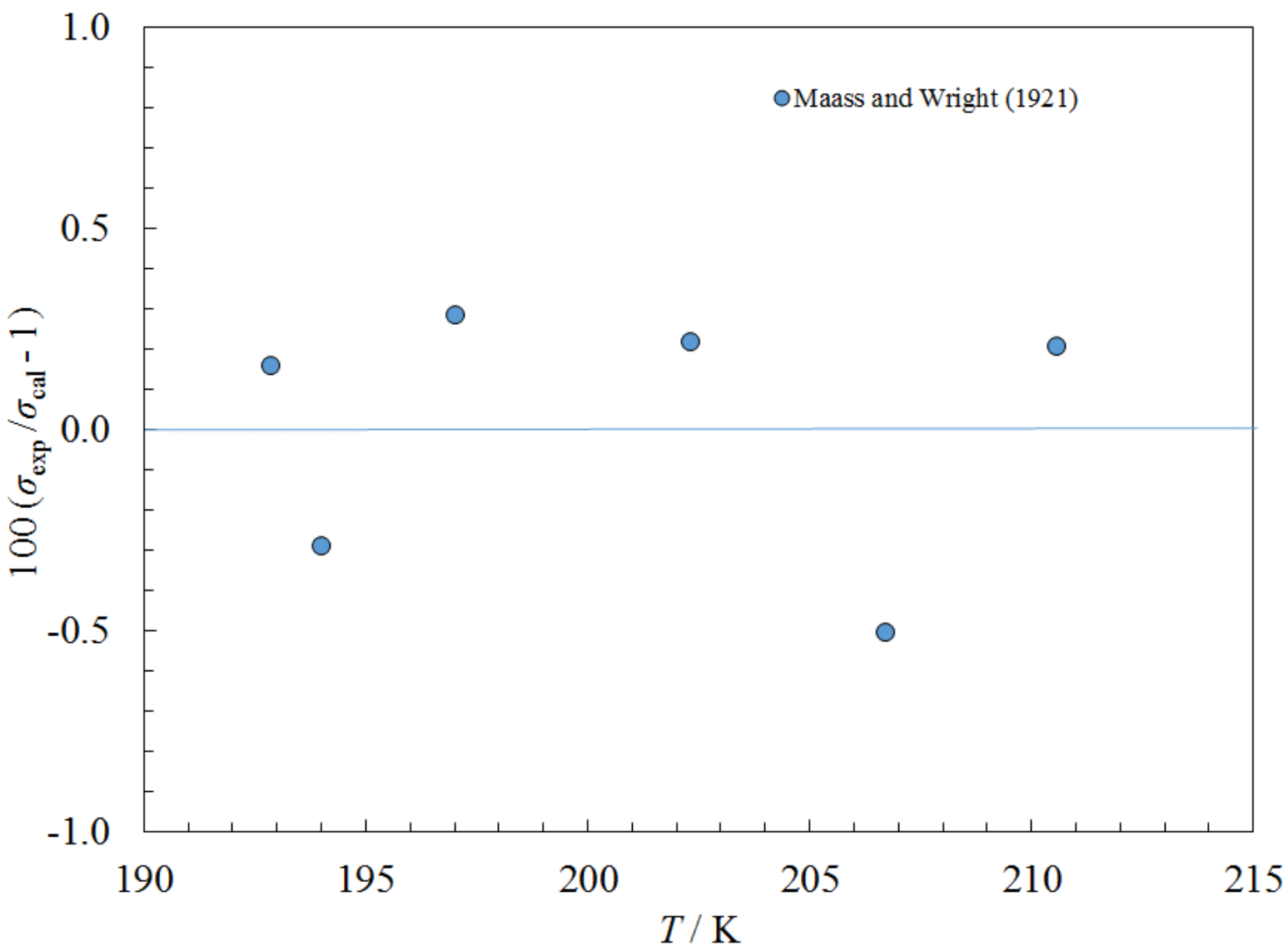

Figure 64. Percentage deviations between the model and the experimental surface tension data for acetylene as a function of temperature.

\subsubsection{Propyne}

The equation of state of Polt et al. (Polt, Platzer, \& Maurer, 1992) was used to provide the density and the critical parameters. Lennard-Jones parameters were obtained by fitting the viscosity data of Titani (Titani, 1930) and are presented in Table 1. A deviation plot with the viscosity data is shown in Figure 65. The estimated uncertainty in the gas phase is $3 \%$. We were unable to locate any liquid-phase viscosity data, or any thermal conductivity data at all. The values given in Tables 2-4 are predictive and based on comparisons with propane and propylene. The estimated uncertainty for the liquid viscosity and thermal conductivity is $20 \%$, and the estimated uncertainty for the thermal conductivity of the gas phase is $10 \%$. Surface tension is discussed in a separate publication (Mulero et al., 2012). 


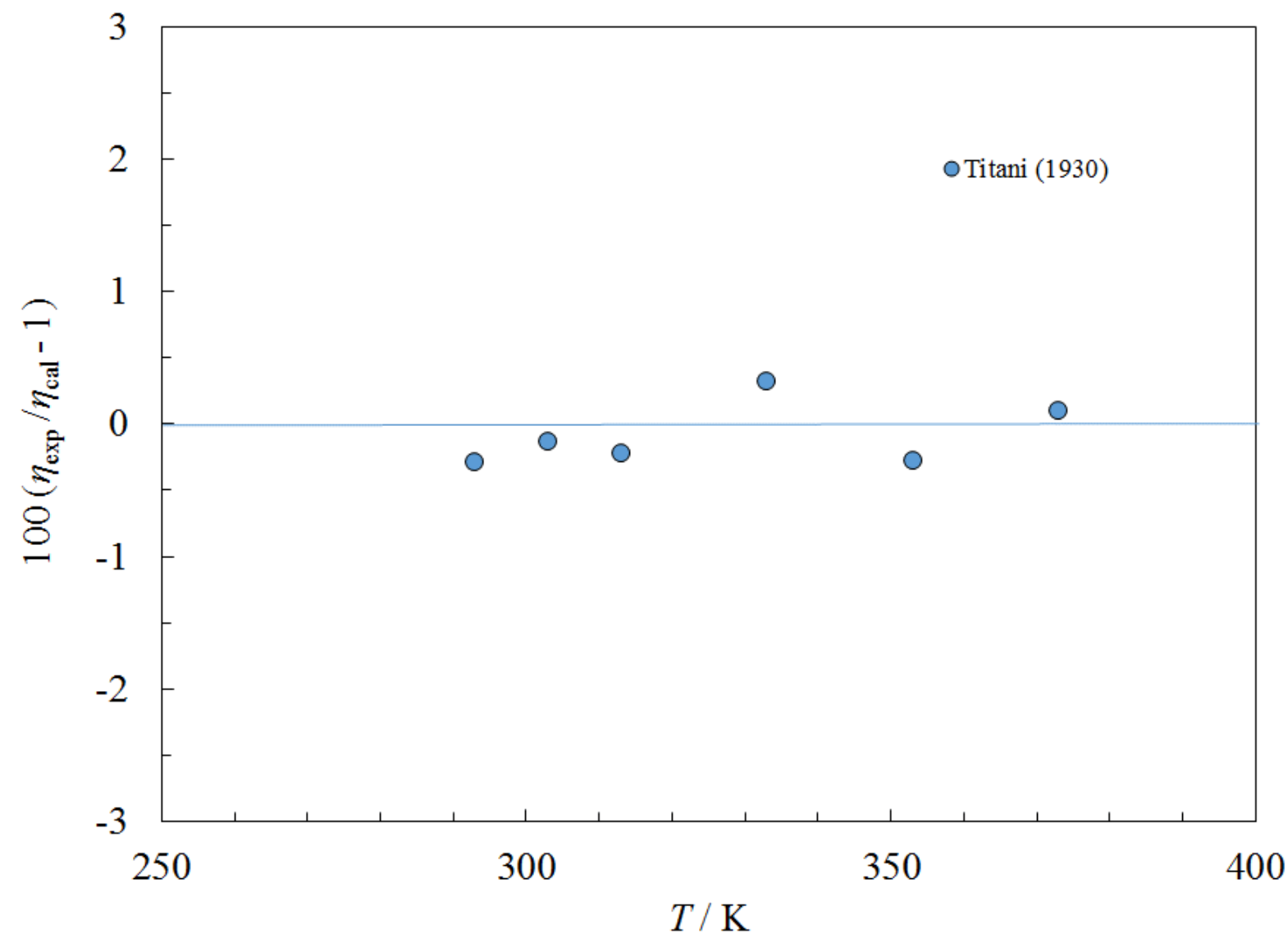

Figure 65. Percentage deviations between the model and the experimental viscosity data for propyne as a function of temperature. 


\subsubsection{1-Butyne (ethylacetylene)}

The equation of state of Gao et al. (K. Gao, J. Wu, \& E. W. Lemmon, 2017b) was used to provide the density and the critical parameters. Lennard-Jones parameters were calculated by the method of Chung et al. (Chung et al., 1988); values are presented in Table 1. We were unable to locate any experimental data for viscosity or thermal conductivity; the model is completely predictive. Coefficients are given in Tables 2-4, and are based on expected behavior with respect to propane. The estimated uncertainty for viscosity is $20 \%$ and the estimated uncertainty for thermal conductivity is also $20 \%$. One set of experimental data for surface tension (Morehouse \& Maass, 1931) was used to determine the coefficients in Table 5. Figure 66 shows the deviations from the experimental surface tension data. The estimated uncertainty is $3 \%$ over the temperature range $242 \mathrm{~K}$ to $282 \mathrm{~K}$.

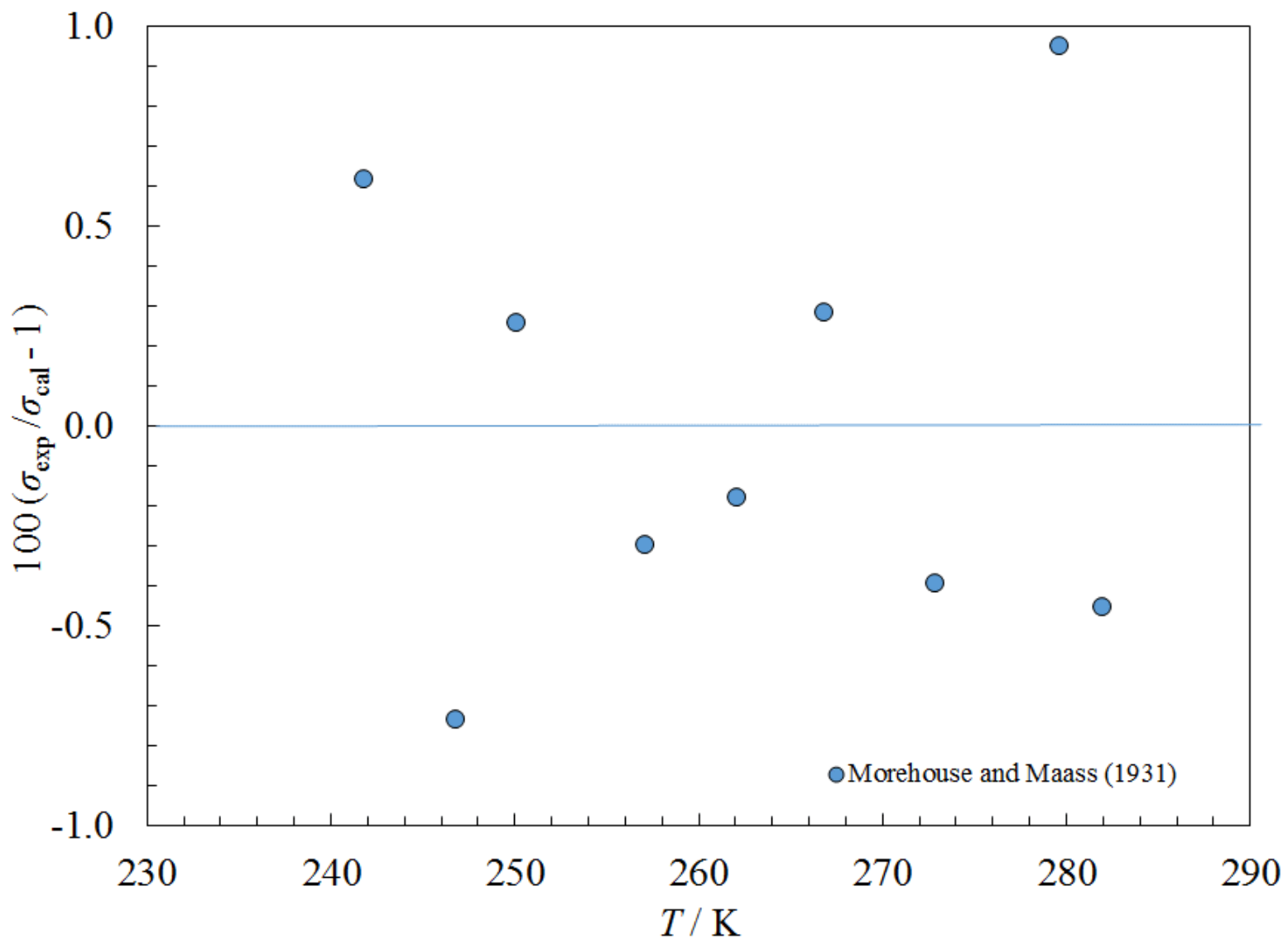

Figure 66. Percentage deviations between the model and the experimental surface tension data for 1-butyne as a function of temperature. 


\subsection{Cycloalkanes and Cycloakenes}

\subsubsection{Cyclopropane}

The equation of state of Polt et al. (Polt et al., 1992) was used to provide the density and the critical parameters. Lennard-Jones parameters were obtained from the method of Chung et al. (Chung et al., 1988) and are given in Table 1. Comparisons with the gas-phase data of Lambert et al. (Lambert et al., 1955) are shown in Figure 67. The estimated uncertainty in the gas phase is $5 \%$. There were no data available in the liquid phase for viscosity; propane was used as a reference fluid, and a completely predictive model was used. Coefficients are given in Table 2. It is difficult to assign uncertainty due to lack of experimental data; however, we estimated the uncertainty for the liquid phase as $30 \%$. Cycloalkane viscosity is difficult to model with corresponding states models, so we have adjusted the uncertainty accordingly. Figure 68 shows deviations between the model and the gas-phase thermal conductivity data of Lambert et al. (Lambert et al., 1955), Vines and Bennett (Vines \& Bennett, 1954), and Parkinson et al. (Parkinson et al., 1972). The estimated uncertainty in the gas phase is $5 \%$. No data at all were located for the thermal conductivity for the liquid phase. Coefficients are given in Table 3 and 4. The estimated uncertainty is $20 \%$ in the liquid phase. Surface tension is discussed in a separate publication (Mulero \& Cachadiña, 2014).

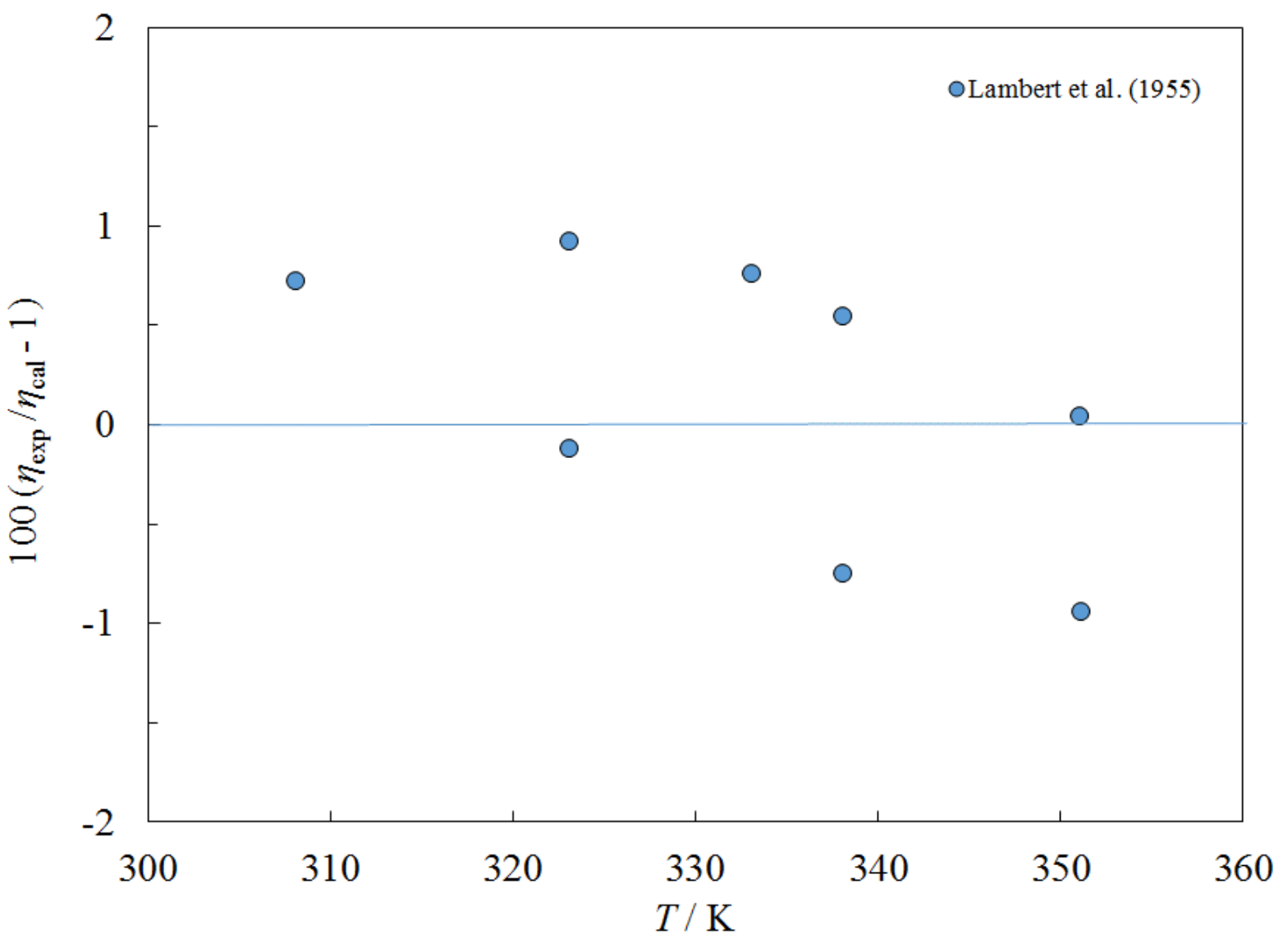


Figure 67. Percentage deviations between the model and the experimental viscosity data for cyclopropane as a function of temperature.

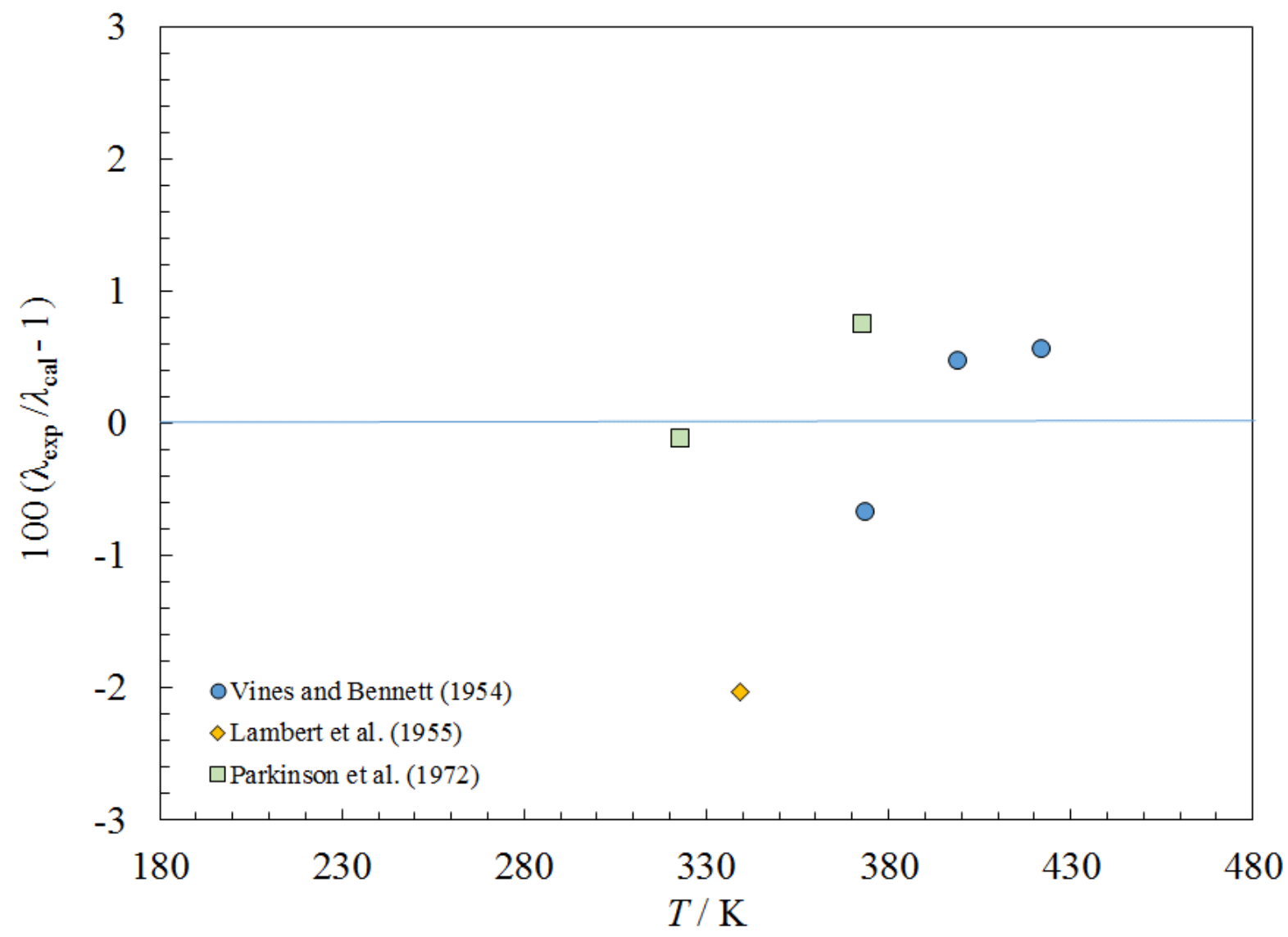

Figure 68. Percentage deviations between the model and the experimental thermal conductivity data for cyclopropane as a function of temperature.

\subsubsection{Cyclopentane}

The equation of state of Gedanitz et al. (Gedanitz, Dávila, \& Lemmon, 2015) was used to provide the density and the critical parameters. Lennard-Jones parameters were obtained from the method of Chung et al. (Chung et al., 1988) Viscosity data from several sources (Assael \& Dalaouti, 2000; K. R. Harris, Newitt, \& Woolf, 2004; Kurihara, Kandil, Marsh, \& Goodwin, 2007; Lambert et al., 1955; R. F. Ma et al., 2003) were used to obtain the coefficients in Table 2, and Figures 69 and 70 give deviation plots as a function of temperature and pressure respectively. The estimated uncertainty in the gas phase is $3 \%$, and in the liquid at temperatures above $223 \mathrm{~K}$ and pressures up to $400 \mathrm{MPa}$ is $2 \%$. Thermal conductivity (Vassiliou et al., 2015) and surface tension (Mulero \& Cachadiña, 2014) are dealt with in separate manuscripts and are not covered here. 


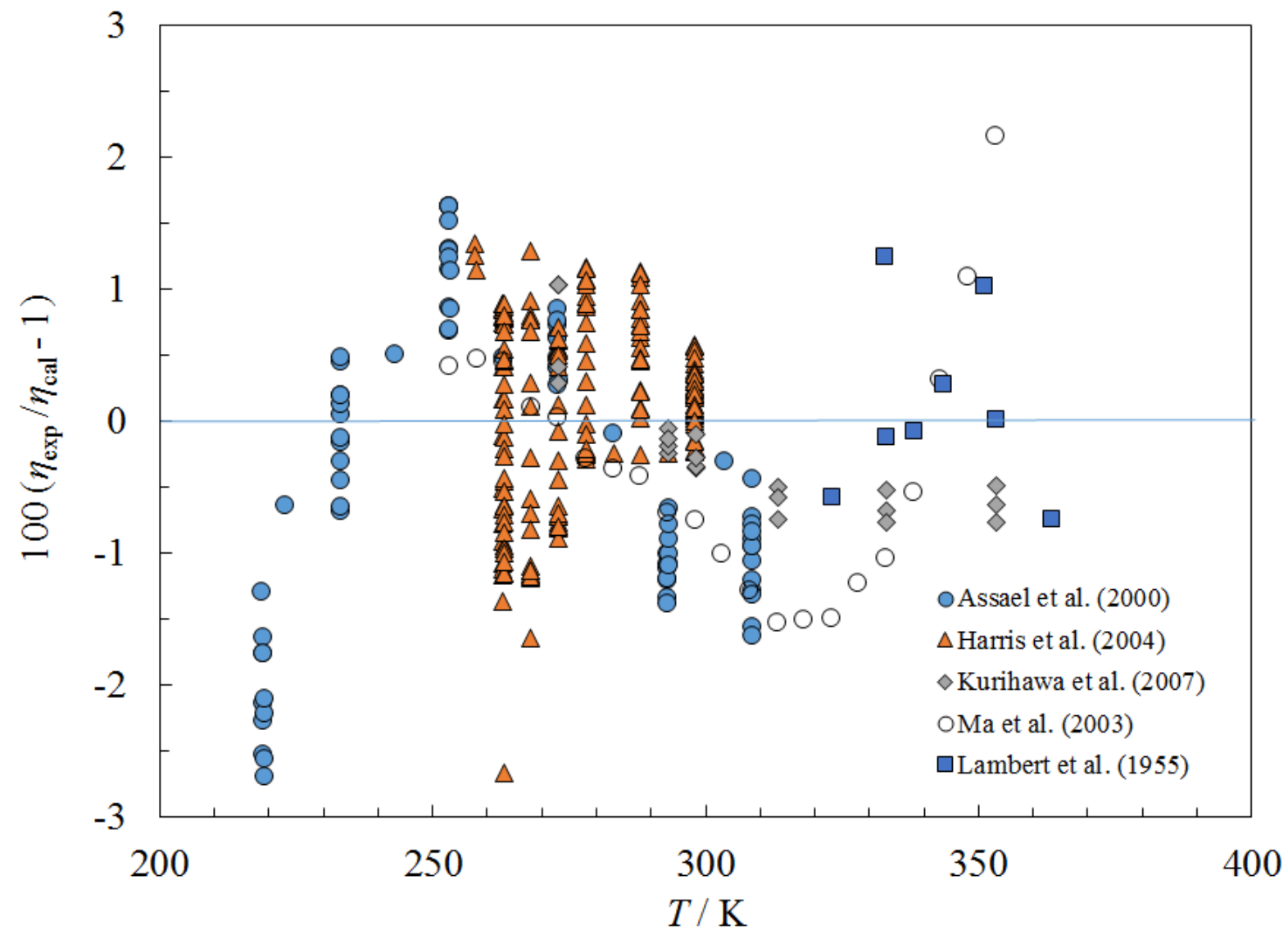

Figure 69. Percentage deviations between the model and the experimental viscosity data for cyclopentane as a function of temperature. 


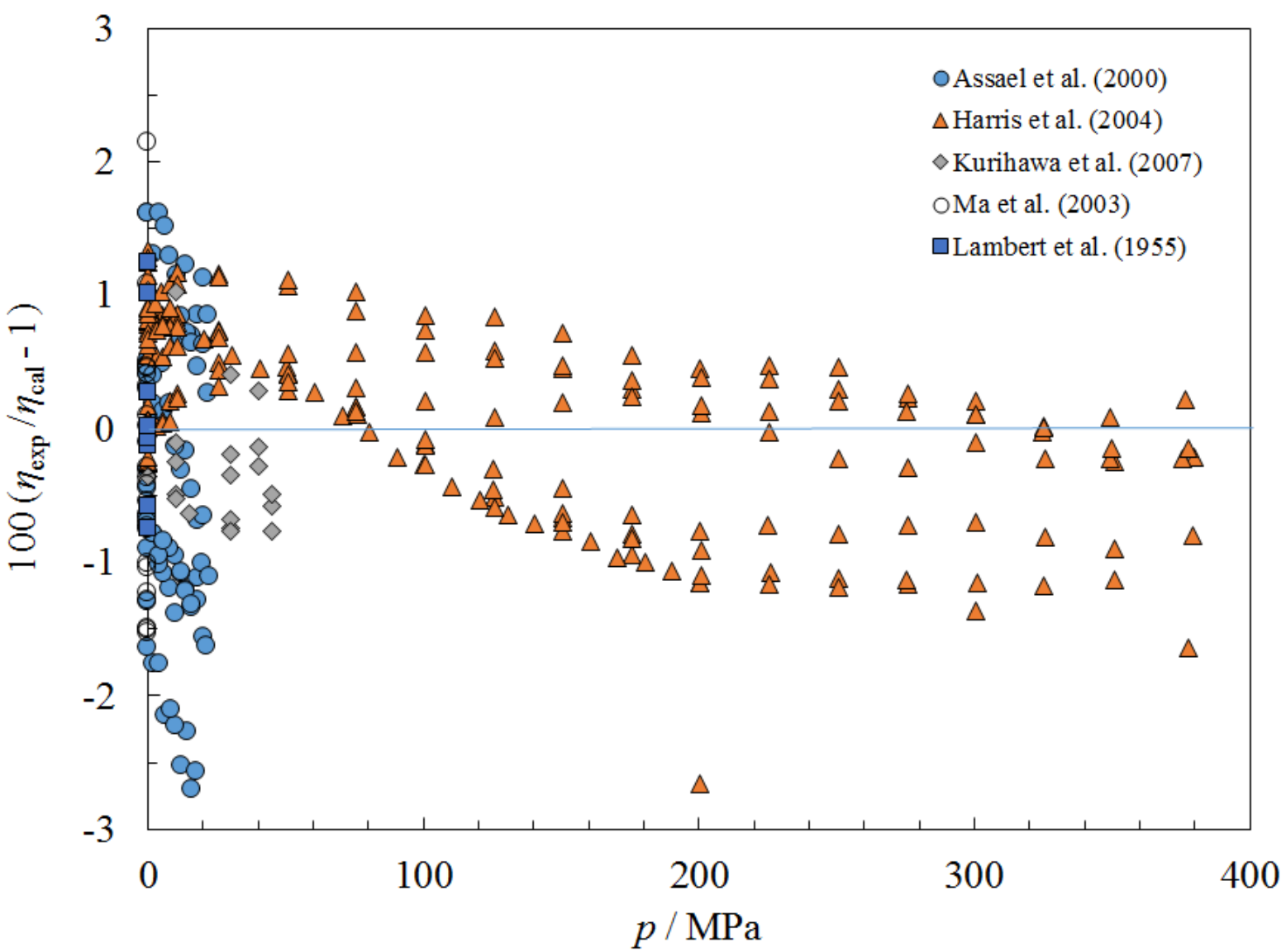

Figure 70. Percentage deviations between the model and the experimental viscosity data for cyclopentane as a function of pressure.

\subsubsection{Methylcyclohexane}

The equation of state of Lemmon (E.W. Lemmon, 2007a) was used to provide the density and the critical parameters. Lennard-Jones parameters were obtained from the method of Chung et al. (Chung et al., 1988). Data from several sources (Baylaucq, Boned, Dauge, \& Lagourette, 1997; Et-Tahir, Boned, Lagourette, \& Xans, 1995; Evans, 1938; Golubev, 1959; Pereiro, 2004; Zeberg-Mikkelsen, Barrouhou, \& Boned, 2003) were used to obtain the coefficients in Table 2. Figures 71 and 72 show deviations from the experimental data used in fitting the coefficients as a function of temperature and of pressure. There were no data in the gas phase; estimated uncertainty is $10 \%$. In the liquid phase, the estimated uncertainty is $5 \%$ at pressures to $100 \mathrm{MPa}$. Thermal conductivity (R. A. Perkins, Hammerschmidt, \& Huber, 2008) and surface tension (Mulero \& Cachadiña, 2014) are covered in separate papers and are not discussed here. 


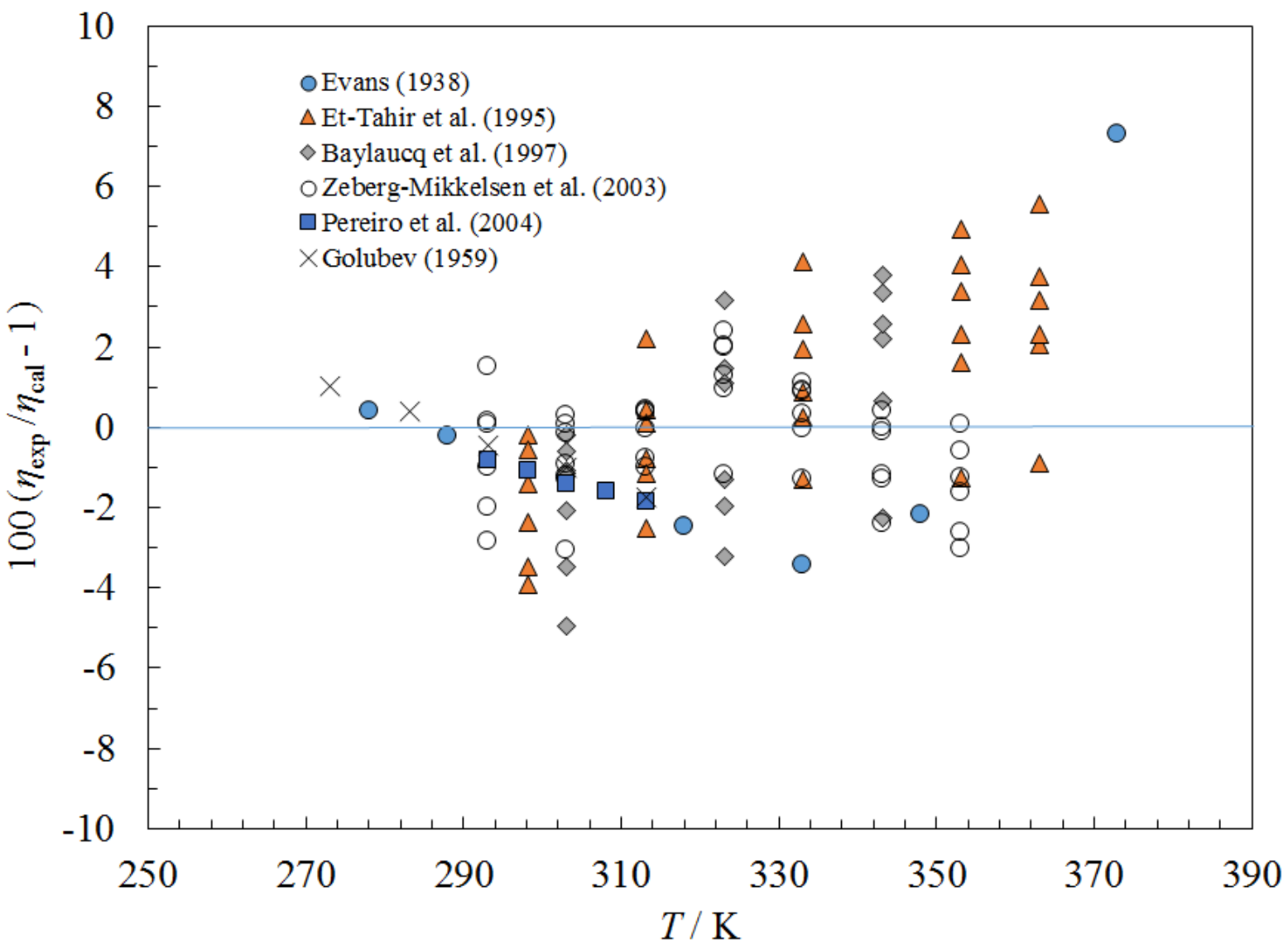

Figure 71. Percentage deviations between the model and the experimental viscosity data for methylcyclohexane as a function of temperature. 


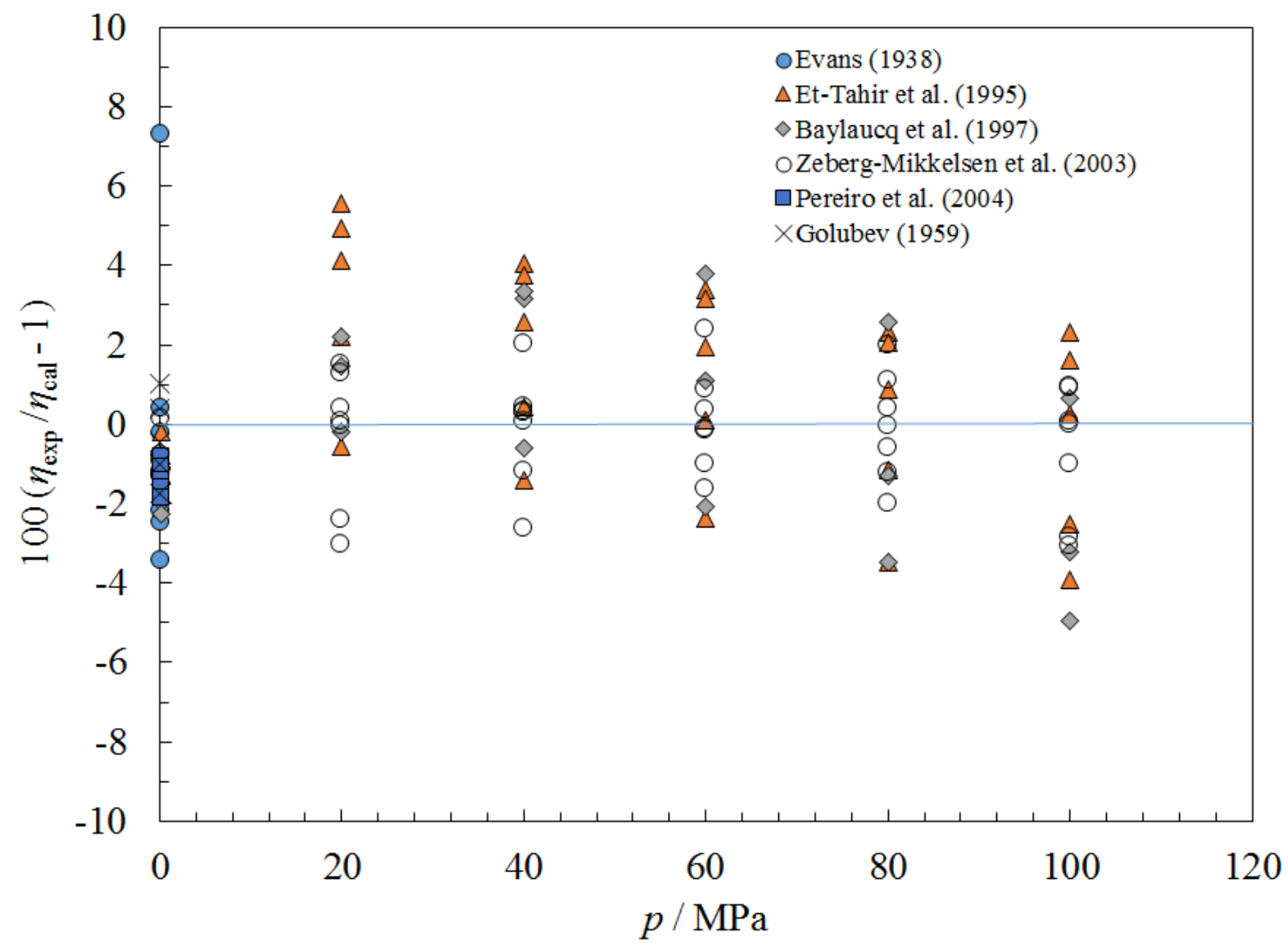

Figure 72. Percentage deviations between the model and the experimental viscosity data for methylcyclohexane as a function of pressure. 


\subsubsection{Propylcyclohexane}

The equation of state of Lemmon (E.W. Lemmon, 2007b) was used to provide the density and the critical parameters. Lennard-Jones parameters were obtained from the method of Chung et al. (Chung et al., 1988). Extremely limited kinematic viscosity data from several sources (DeLorenzi, Fermeglia, \& Torriano, 1994; Geist \& Cannon, 1946; Knothe \& Steidley, 2005; Koelbel, Siemes, \& Luther, 1949) were located and converted to dynamic viscosity with the equation of state, and then used to obtain the coefficients in Table 2. A deviation plot as a function of temperature is shown in Figure 73. There were no data in the gas phase; estimated uncertainty is $10 \%$. In the liquid phase, the estimated uncertainty at atmospheric pressure is $5 \%$, and is higher elsewhere. Thermal conductivity (R. A. Perkins et al., 2008) and surface tension (Mulero \& Cachadiña, 2014) are covered in separate papers and are not discussed here.

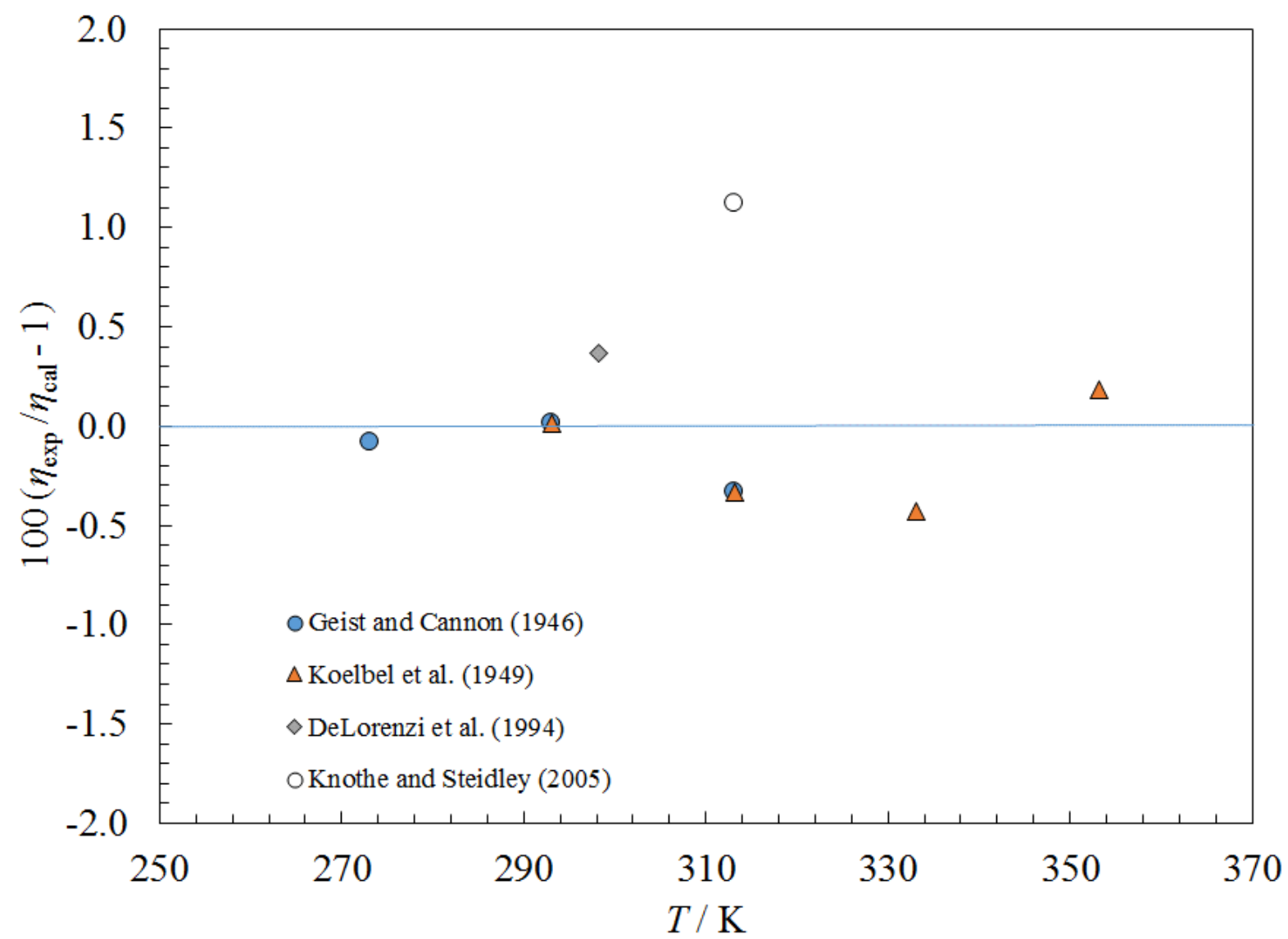

Figure 73. Percentage deviations between the model and the experimental viscosity data for propylcyclohexane as a function of temperature. 


\subsubsection{Cyclobutene (cyclobutylene)}

The equation of state of Gao et al. (K. Gao, J. Wu, \& E. W. Lemmon, 2017i) was used to provide the density and the critical parameters. Lennard-Jones parameters were calculated by the method of Chung et al. (Chung et al., 1988); values are presented in Table 1. We were unable to locate any experimental data for viscosity or thermal conductivity; the model is completely predictive. Coefficients are given in Tables 2-4, and are based on expected behavior with respect to propane. The estimated uncertainty for viscosity is $20 \%$ and the estimated uncertainty for thermal conductivity is also $20 \%$. Yaws (Yaws, 2014) presents a correlation for surface tension, but it incorporates a slightly different critical temperature $(446.33 \mathrm{~K})$ than what the equation of state of Gao et al. (K. Gao et al., 2017i) uses, so we had to adjust the coefficients (given in Table 5) slightly. The estimated uncertainty is $10 \%$.

\subsection{Ethers}

\subsubsection{Dimethyl ether}

The equation of state of $\mathrm{Wu}$ et al. (Wu, Zhou, \& Lemmon, 2011) was used to provide the density and the critical parameters. Lennard-Jones parameters were taken from Reid et al. (Reid, 1987) The viscosity correlation is covered in a separate publication (Meng, Zhang, Wu, \& Liu, 2012) and is not discussed here. However, the implementation of the ECS method for thermal conductivity requires a value for viscosity for use in the critical enhancement term, so we provide parameters for the viscosity in Table 2 so that users can match the sample calculation numbers in the Appendix. One may also implement a dedicated viscosity model; however, the numbers in the Appendix were obtained with the ECS model. The thermal conductivity data of $\mathrm{Wu}$ et al. (Wu, Li, Zheng, \& Assael, 2009) and of Wang et al. (Y. Wang, Wu, \& Liu, 2006) were used to obtain the coefficients in Table 3. Parameters for the critical enhancement are presented in Table 4. Deviation plots as a function of temperature and of pressure are given in Figures 74 and 75 . The estimated uncertainty in the gas phase is $3 \%$ and in the liquid at pressures up to $30 \mathrm{MPa}$ is $4 \%$. Surface tension is covered in a separate publication and is not discussed here (Mulero et al., 2012). 


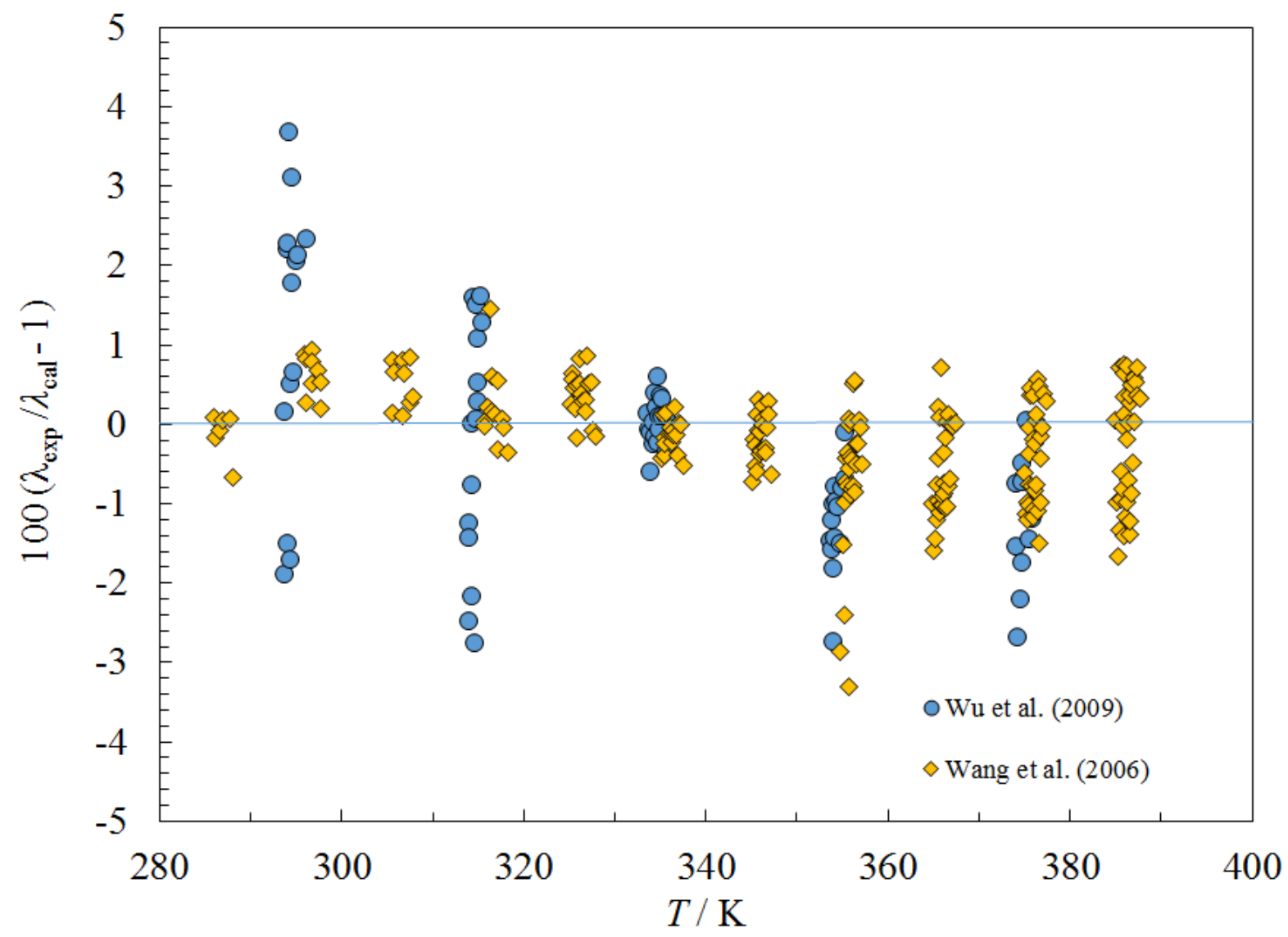

Figure 74. Percentage deviations between the model and the experimental thermal conductivity data for dimethyl ether as a function of temperature. 


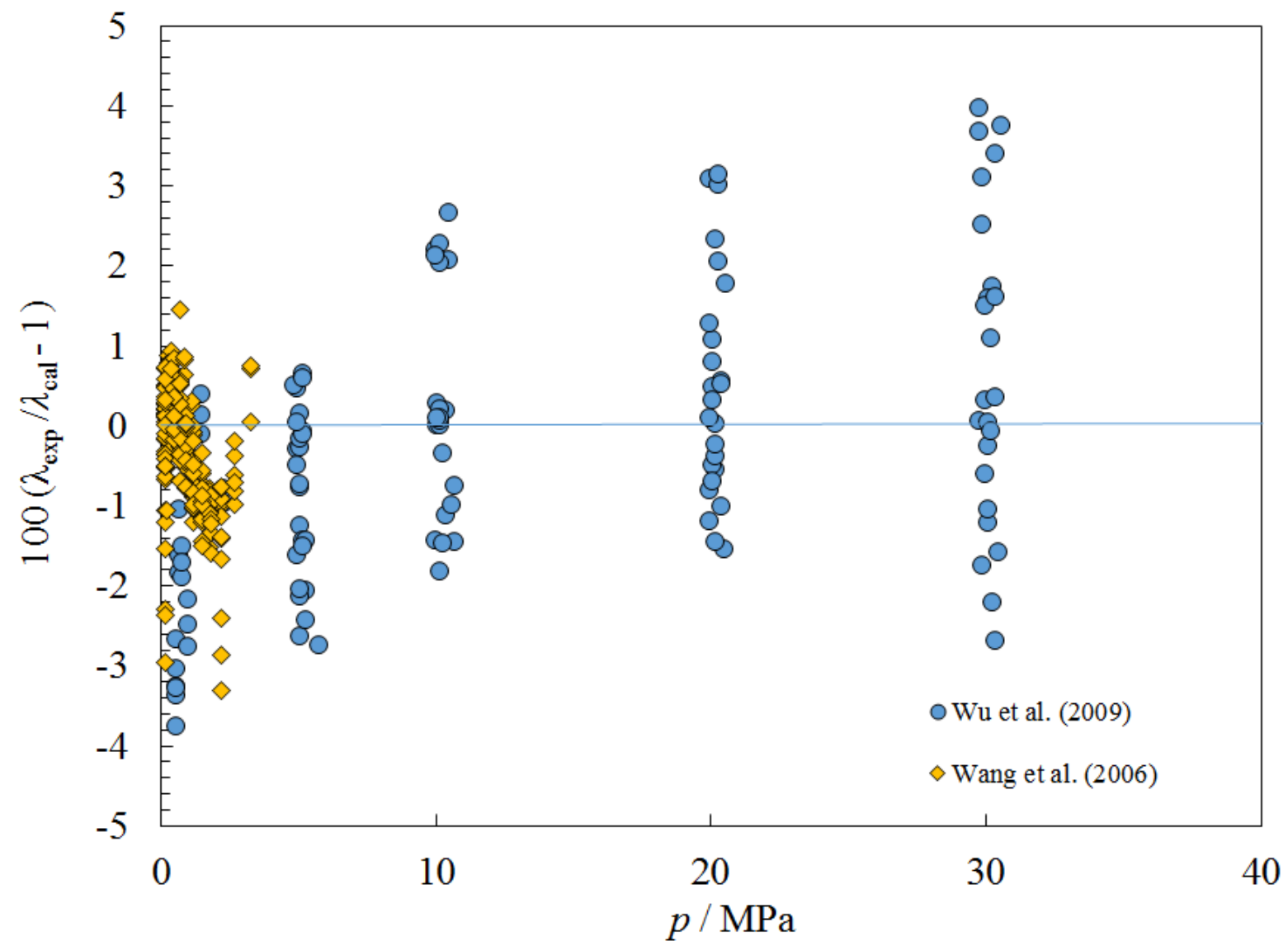

Figure 75. Percentage deviations between the model and the experimental thermal conductivity data for dimethyl ether as a function of pressure. 


\subsubsection{Diethyl ether}

The equation of state of Thol et al. (Thol, Piazza, \& Span, 2014) was used to provide the density and the critical parameters. Lennard-Jones parameters were estimated with the method of Chung et al. (Chung et al., 1988). The parameters in Table 2 were obtained by fitting the data of Meng et al. (Meng, Zhengg, Wu, \& Liu, 2008) for the liquid phase and Titani (Titani, 1933) and Pal and Bhattacharyya (A.K. Pal \& Bhattacharyya, 1969) for the gas phase. Figures 76 and 77 show the deviations in viscosity as a function of temperature and pressure, respectively. We estimate the uncertainty in the liquid phase to be $3 \%$ at temperatures from $243 \mathrm{~K}$ to $373 \mathrm{~K}$ at pressures up to $20 \mathrm{MPa}$, higher at lower temperatures. In the gas phase we estimate the uncertainty is $10 \%$. For thermal conductivity, we fit the data of Li et al. (Li, Wu, \& Dang, 2010) in the liquid phase and Vines (Vines, 1953) and Vines and Bennett (Vines \& Bennett, 1954) to obtain the coefficients in Table 3. Parameters for the critical enhancement are presented in Table 4. Figures 78 and 79 show the deviations in thermal conductivity as a function of temperature and pressure, respectively. We estimate the uncertainty in the liquid phase to be $3 \%$ at temperatures from 233 $\mathrm{K}$ to $373 \mathrm{~K}$ at pressures to $30 \mathrm{MPa}$. In the gas phase we estimate the uncertainty is $10 \%$. Surface tension is covered in a separate publication and is not discussed here (Mulero \& Cachadiña, 2014).

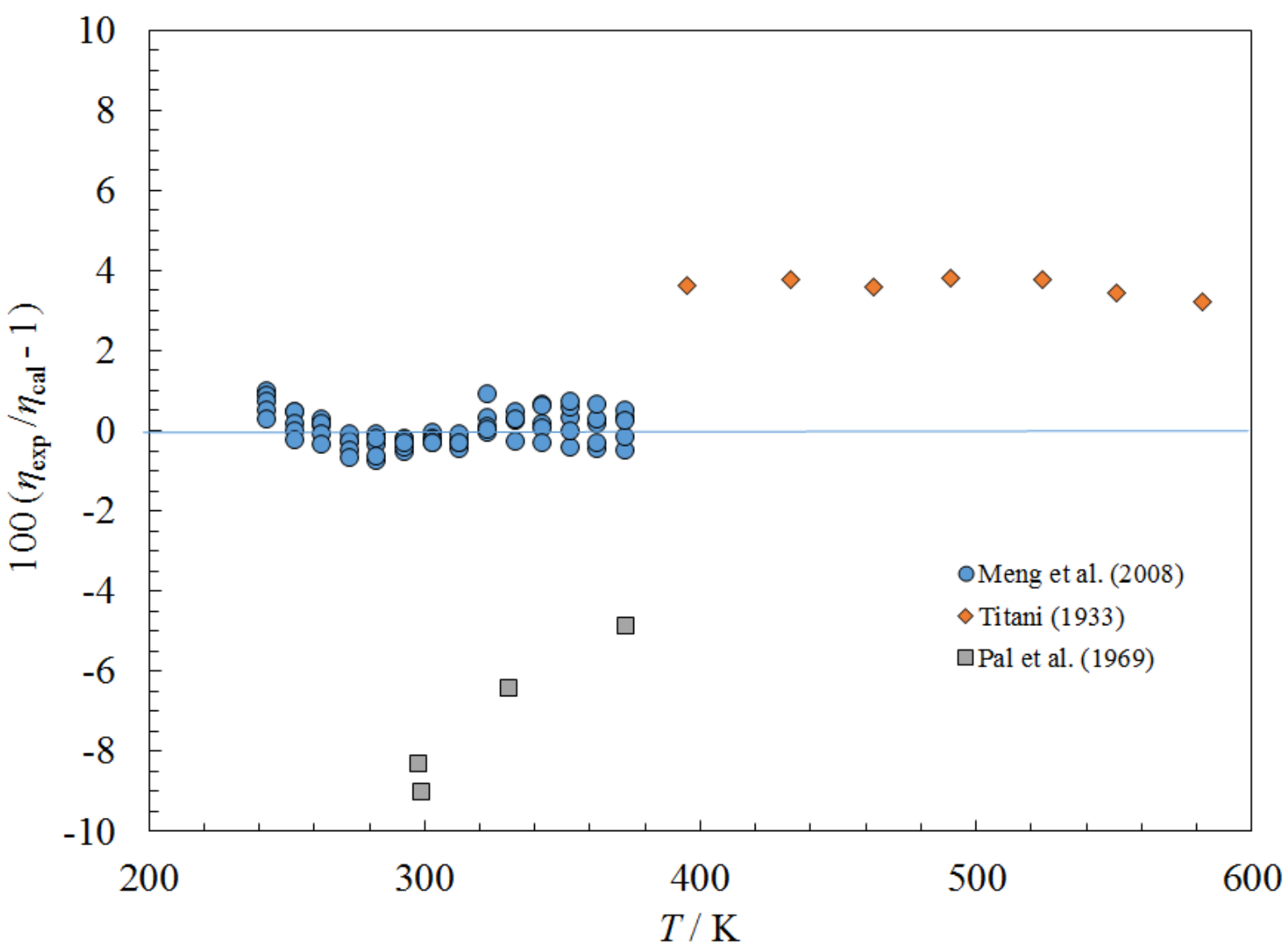

Figure 76. Percentage deviations between the model and the experimental viscosity data for diethyl ether as a function of temperature. 


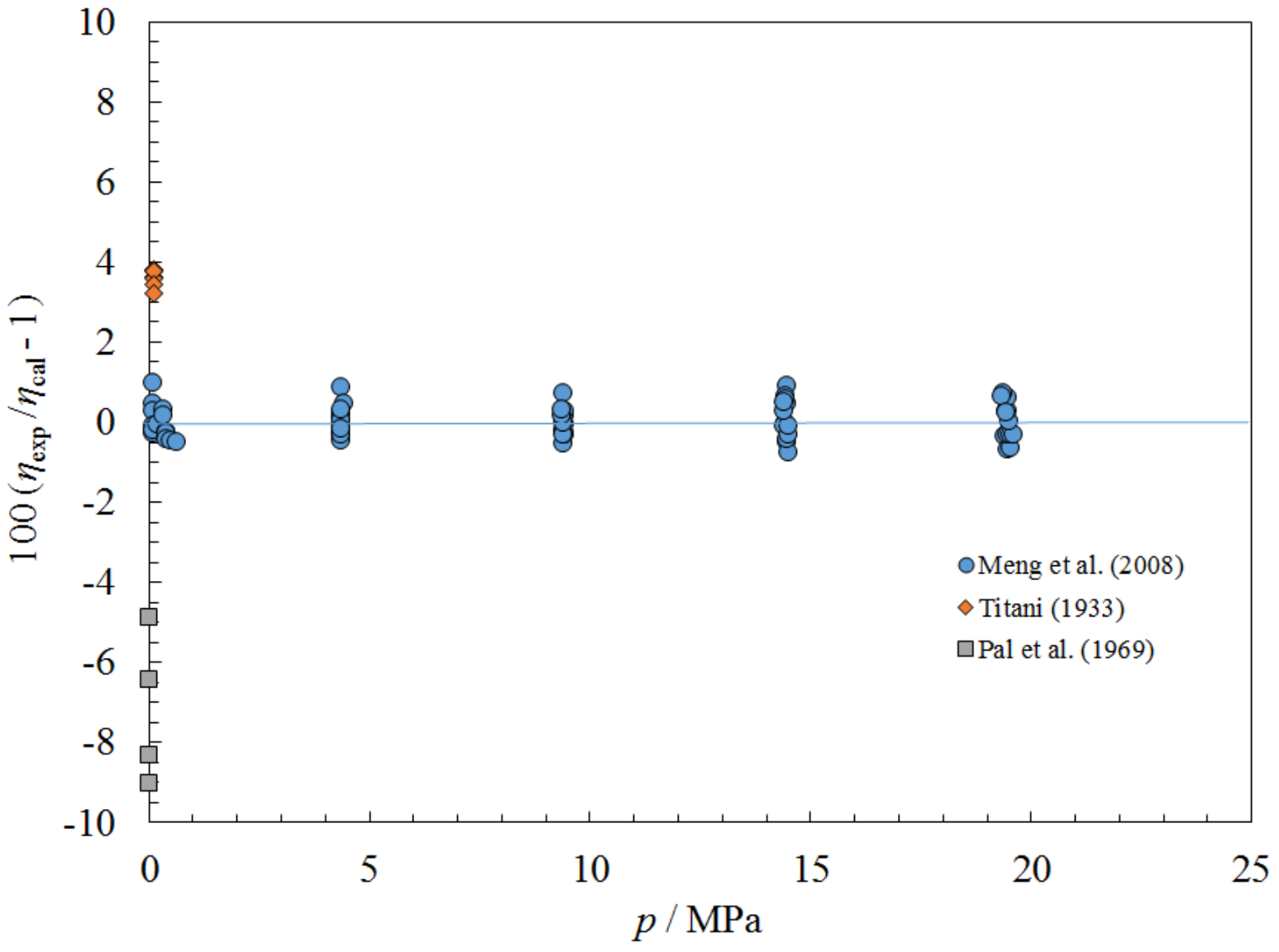

Figure 77. Percentage deviations between the model and the experimental viscosity data for diethyl ether as a function of pressure. 


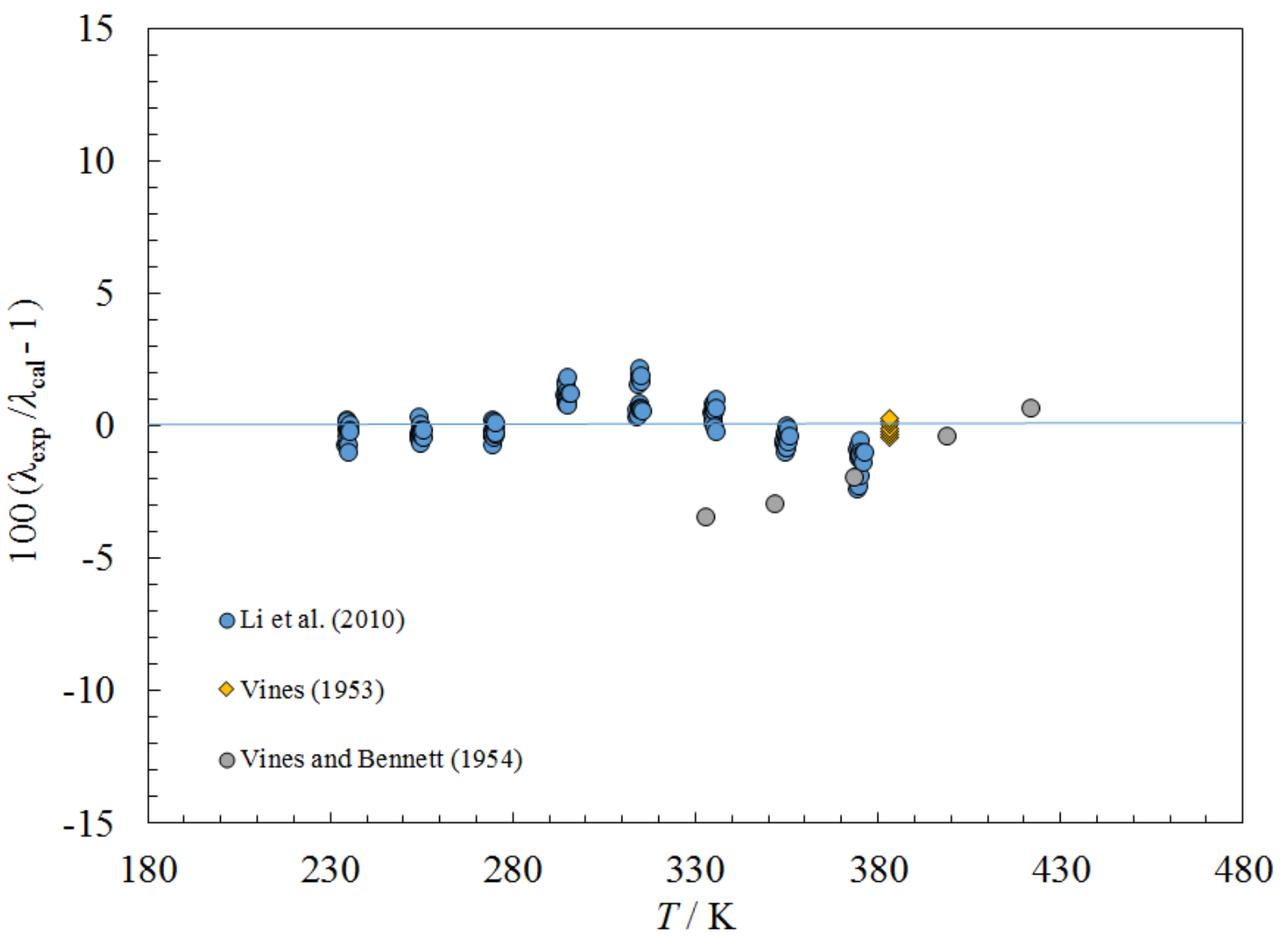

Figure 78. Percentage deviations between the model and the experimental thermal conductivity data for diethyl ether as a function of temperature. 


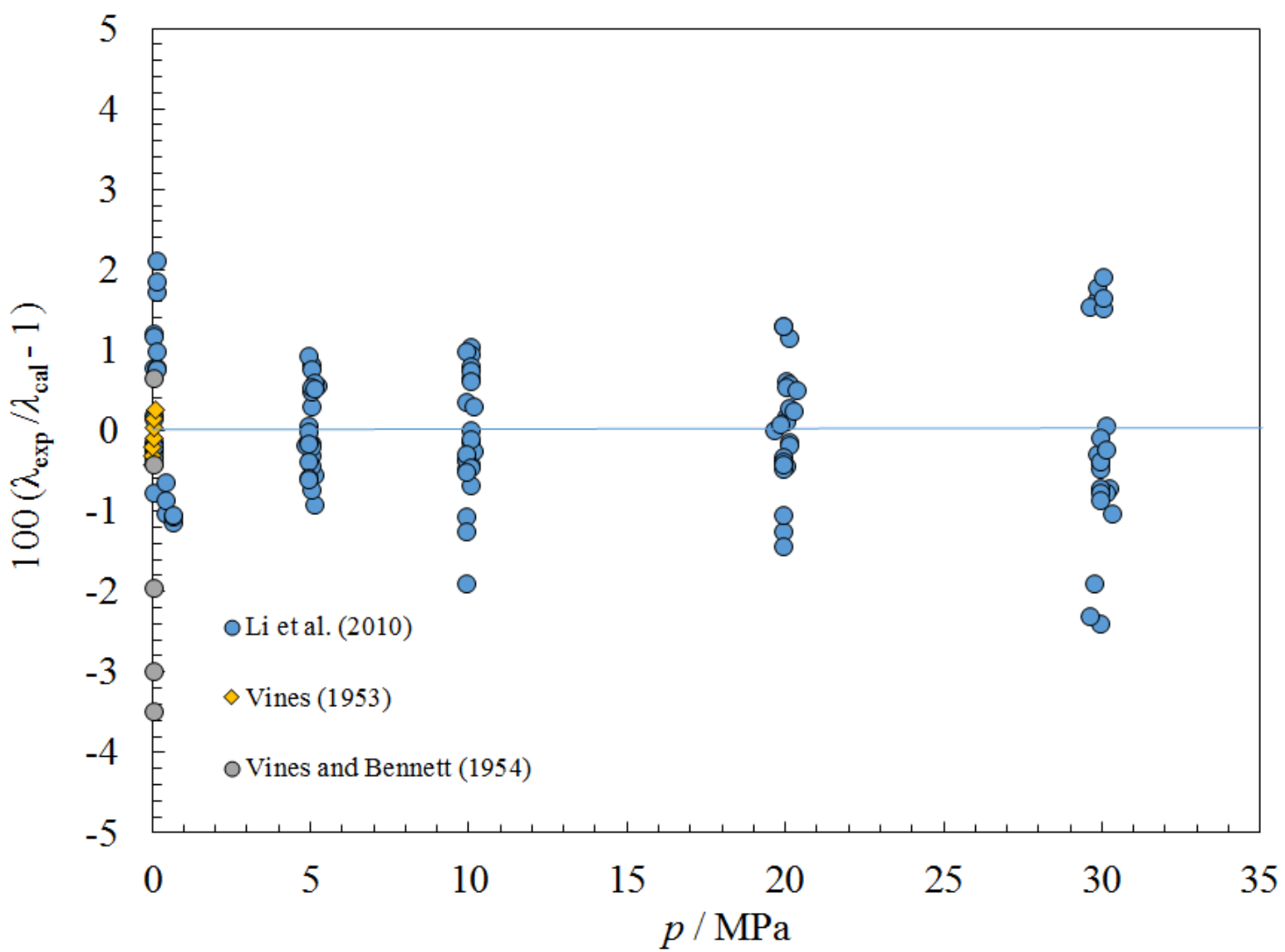

Figure 79. Percentage deviations between the model and the experimental thermal conductivity data for diethyl ether as a function of pressure. 


\subsection{Siloxanes}

\subsubsection{Hexamethyldisiloxane (MM)}

The recent equation of state of Thol et al. (Thol, Dubberke, et al., 2016) was used to provide density and the critical parameters for hexamethyldisiloxane. The Lennard-Jones parameters are from Maczek and Edwards (Maczek \& Edwards, 1979) and are based on fitting their gas-phase viscosity measurements. Several experimental data sets in the liquid phase were located (Abbas, Ihmels, Enders, \& Gmehling, 2011; Hurd, 1946; Wilcock, 1946) and fit to provide the viscosity coefficients in Table 2 with nitrogen as a reference fluid. The data of Abbas et al. (Abbas et al., 2011) are considered the most reliable; the average absolute percentage deviation for this set is 0.9 $\%$, and the authors claim an experimental uncertainty of $1 \%$. All available viscosity data are for the liquid at atmospheric pressure. Based on comparisons with data, we estimate the uncertainty in the liquid at atmospheric pressure to be on the order of $3 \%$, and also in the vapor phase to be 3 $\%$. Deviations are shown in Figure 80. There are no high pressure viscosity data available for comparisons; we estimate that the uncertainty will be larger as the pressure increases, especially for low temperatures, rising to $10 \%$ at $10 \mathrm{MPa}$. We recommend the equation be used only for pressures up to $10 \mathrm{MPa}$ due to the lack of high pressure data. An additional concern is that the corresponding states method used here works best when the reference fluid is chemically similar to the reference fluid, and we do not have a reference fluid that is chemically similar to hexamethyldisiloxane. Abbas et al. (Abbas et al., 2011) also measured the thermal conductivity of this fluid over the range $295 \mathrm{~K}$ to $507 \mathrm{~K}$ at pressures up to $10 \mathrm{MPa}$. The data of Abbas et al. (Abbas et al., 2011) and the data of Bates (Bates, 1949) were fit to obtain the coefficients in Table 3. Parameters for the critical enhancement are presented in Table 4. We estimate the uncertainty of the correlation for the liquid phase is $3 \%$ for $T<490 \mathrm{~K}$ at pressures to $10 \mathrm{MPa}, 10 \%$ at higher temperatures and pressures. Figures 81 and 82 show deviations for thermal conductivity as a function of temperature and pressure, respectively. Data are unavailable for comparisons in the gas phase, and we estimate the uncertainty as on the order of $25 \%$. Surface tension is discussed in a separate publication (Mulero \& Cachadiña, 2014). 


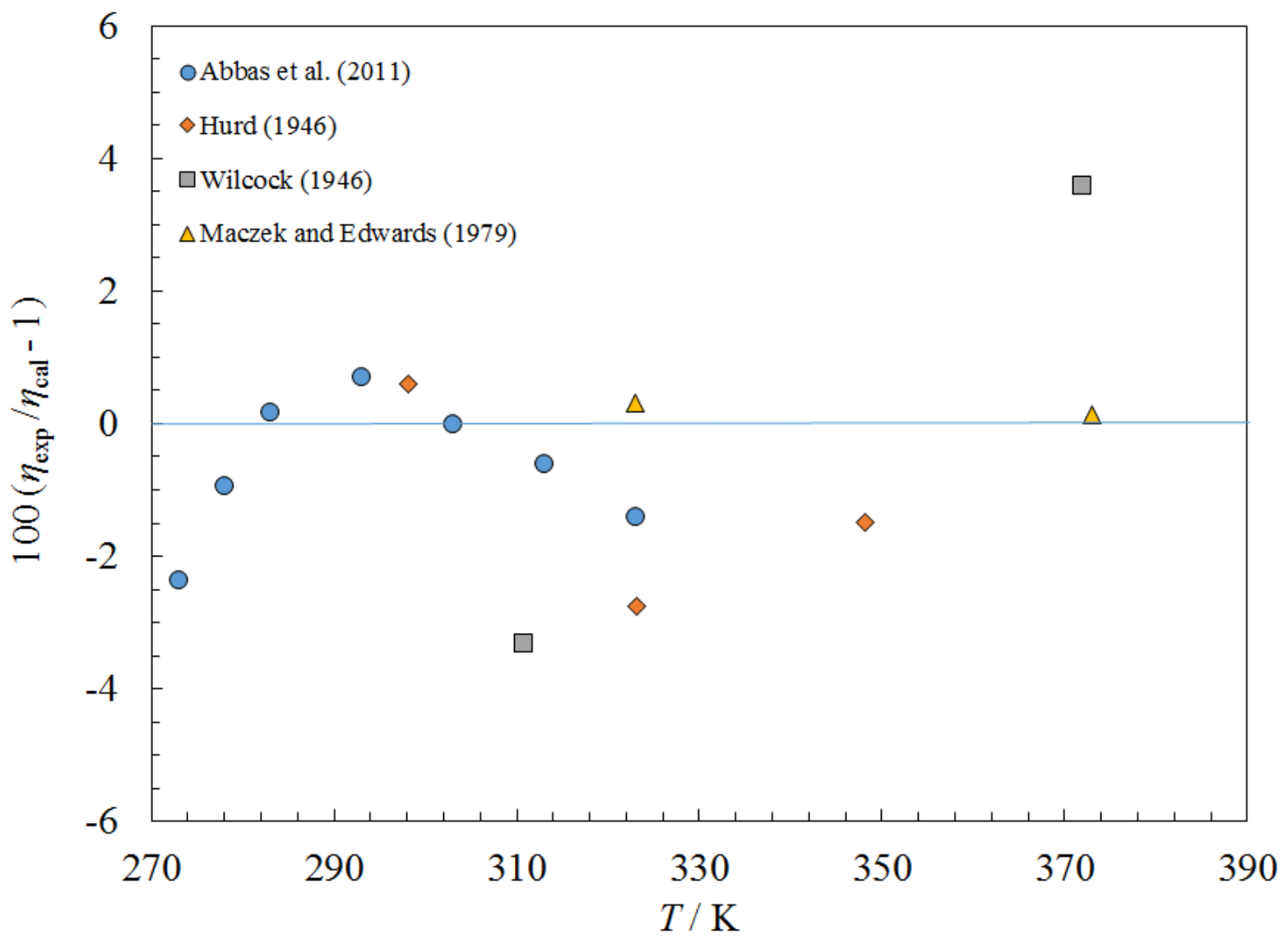

Figure 80. Percentage deviations between the model and the experimental viscosity data for hexamethyldisiloxane (MM). 


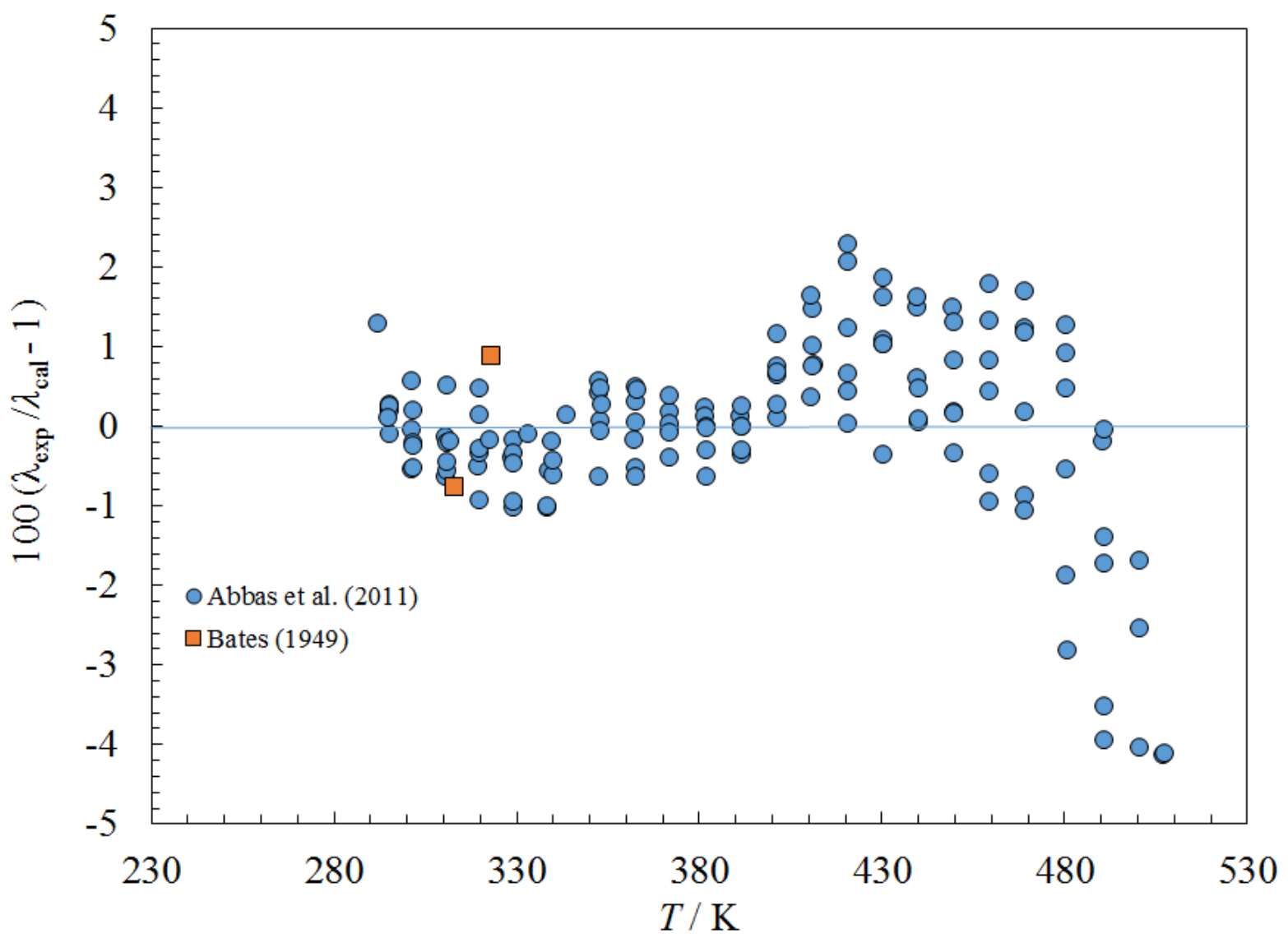

Figure 81. Percentage deviations between the model and the experimental thermal conductivity data for hexamethyldisiloxane (MM) as a function of temperature. 


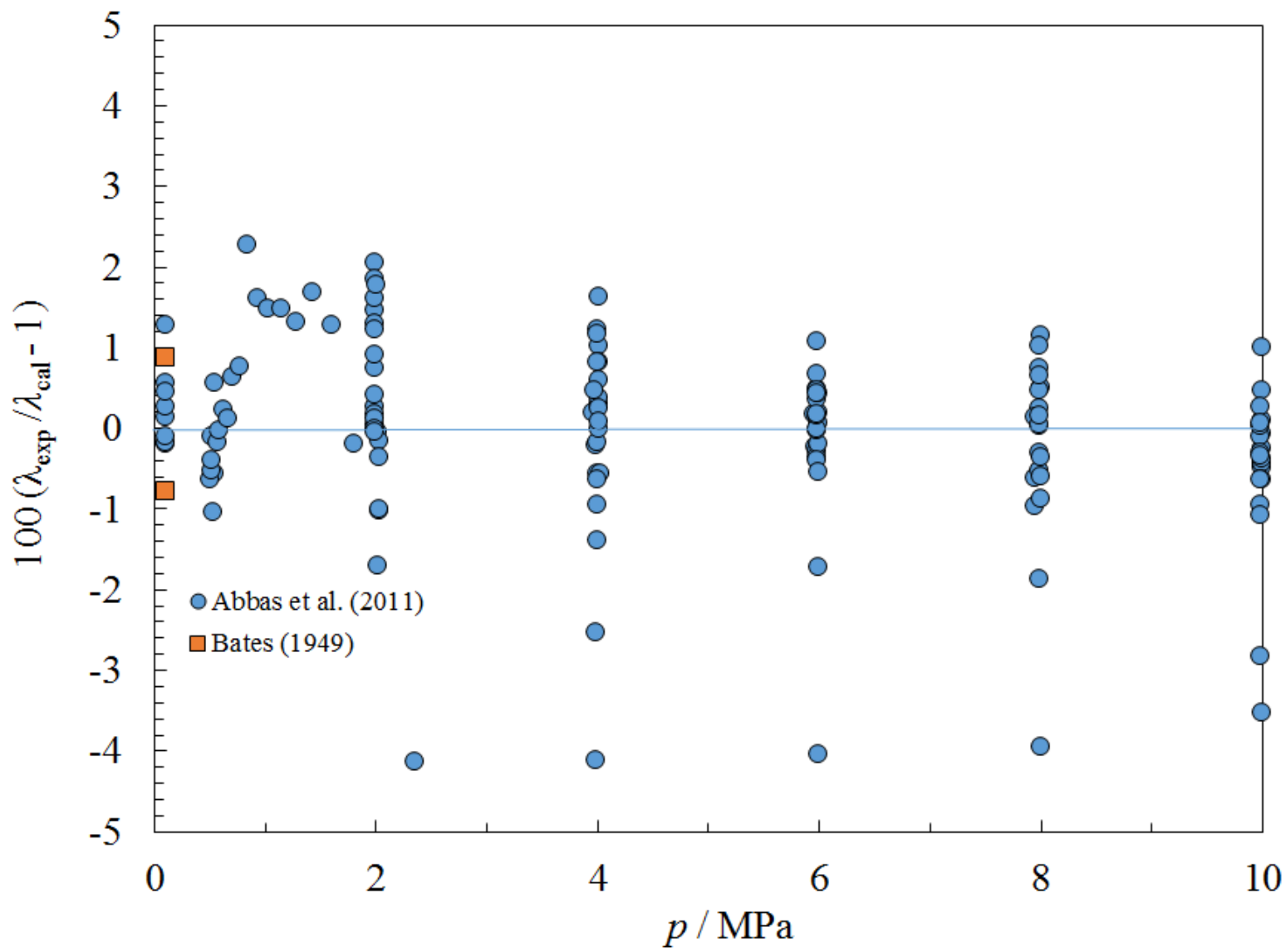

Figure 82. Percentage deviations between the model and the experimental thermal conductivity data for hexamethyldisiloxane (MM) as a function of pressure. 


\subsubsection{Octamethyltrisiloxane (MDM)}

We used the recent equation of state of Thol et al. (Thol, Dubberke, Baumhögger, Vrabec, \& Span, 2017) to provide the density and the critical parameters. The method of Chung et al. (Chung et al., 1988) was used to estimate the Lennard-Jones parameters, as this gave better agreement with the gas-phase data of Maczek and Edwards (Maczek \& Edwards, 1979) than the parameters provided in that work. For the liquid phase, three sets of data are available (Abbas et al., 2011; Hurd, 1946; Wilcock, 1946), of which the data of Abbas et al. (Abbas et al., 2011) are the most extensive although all points are limited to atmospheric pressure. The coefficients resulting from fitting the liquid-phase viscosity data are in Table 2, and deviations with all data are plotted in Figure 83. Based on comparisons with data, we estimate the uncertainty in the liquid at atmospheric pressure to be on the order of $3 \%$, and the vapor phase to be $5 \%$. There are no high-pressure viscosity data available for comparisons; we estimate that the uncertainty will be larger as the pressure increases, especially for low temperatures, rising to $10 \%$ at $10 \mathrm{MPa}$. We recommend the equation be used only for pressures up to $10 \mathrm{MPa}$. Abbas et al. (Abbas et al., 2011) also measured the thermal conductivity of this fluid over the range $305 \mathrm{~K}$ to $500 \mathrm{~K}$ at pressures up to $10 \mathrm{MPa}$. The data of Abbas et al. (Abbas et al., 2011) were fit to obtain the coefficients in Table 3. Parameters for the critical enhancement are presented in Table 4. We estimate the uncertainty of the correlation for the liquid phase is $5 \%$ for $T<500 \mathrm{~K}$ at pressures to $10 \mathrm{MPa}, 10 \%$ at higher temperatures and pressures. Figures 84 and 85 show deviation plots for thermal conductivity as a function of temperature and of pressure. Data are unavailable for comparisons in the gas phase, and we estimate the uncertainty as on the order of $25 \%$. Surface tension is discussed in a separate publication (Mulero \& Cachadiña, 2014). 


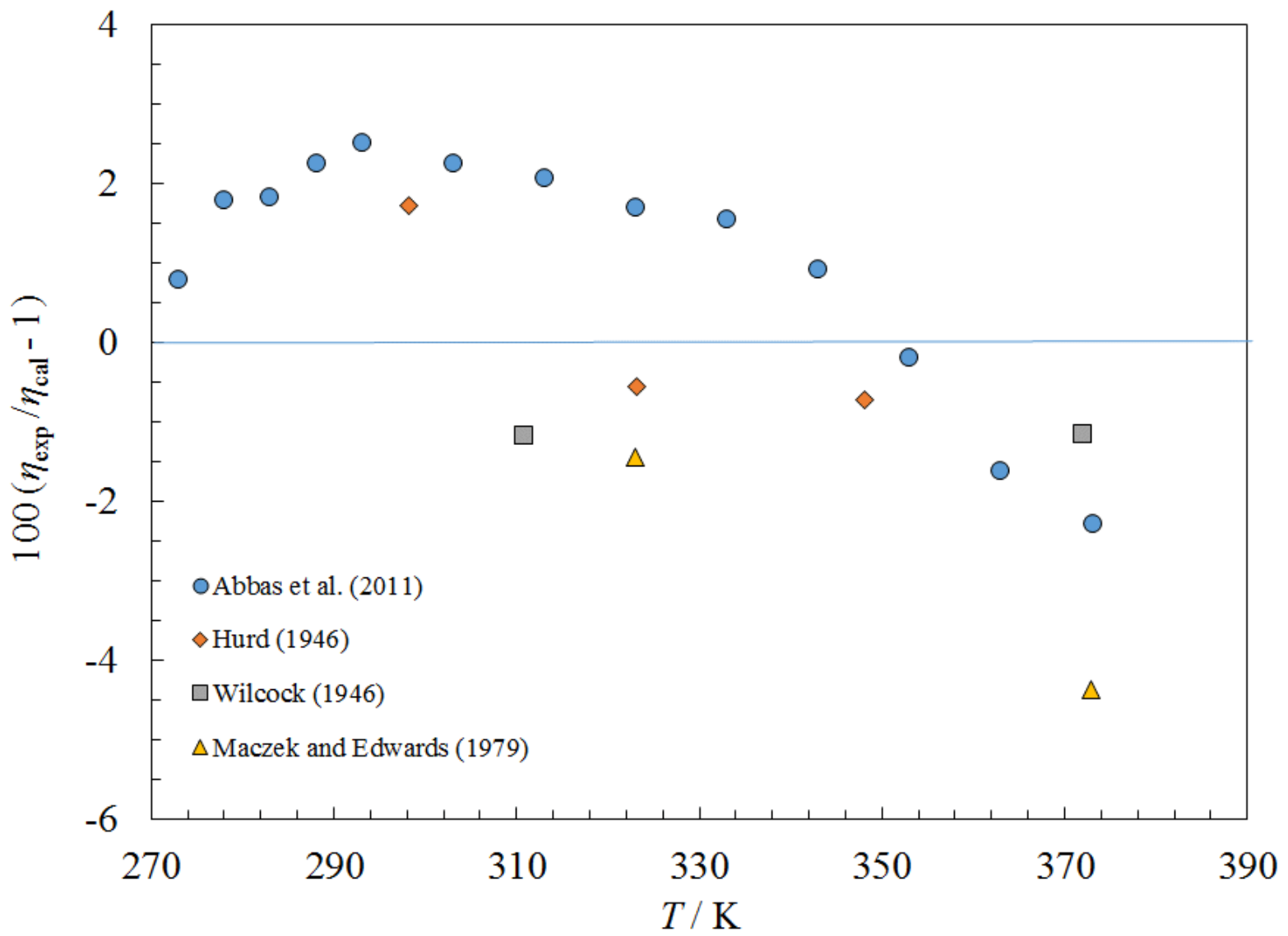

Figure 83. Percentage deviations between the model and the experimental viscosity data for octamethyltrisiloxane (MDM). 


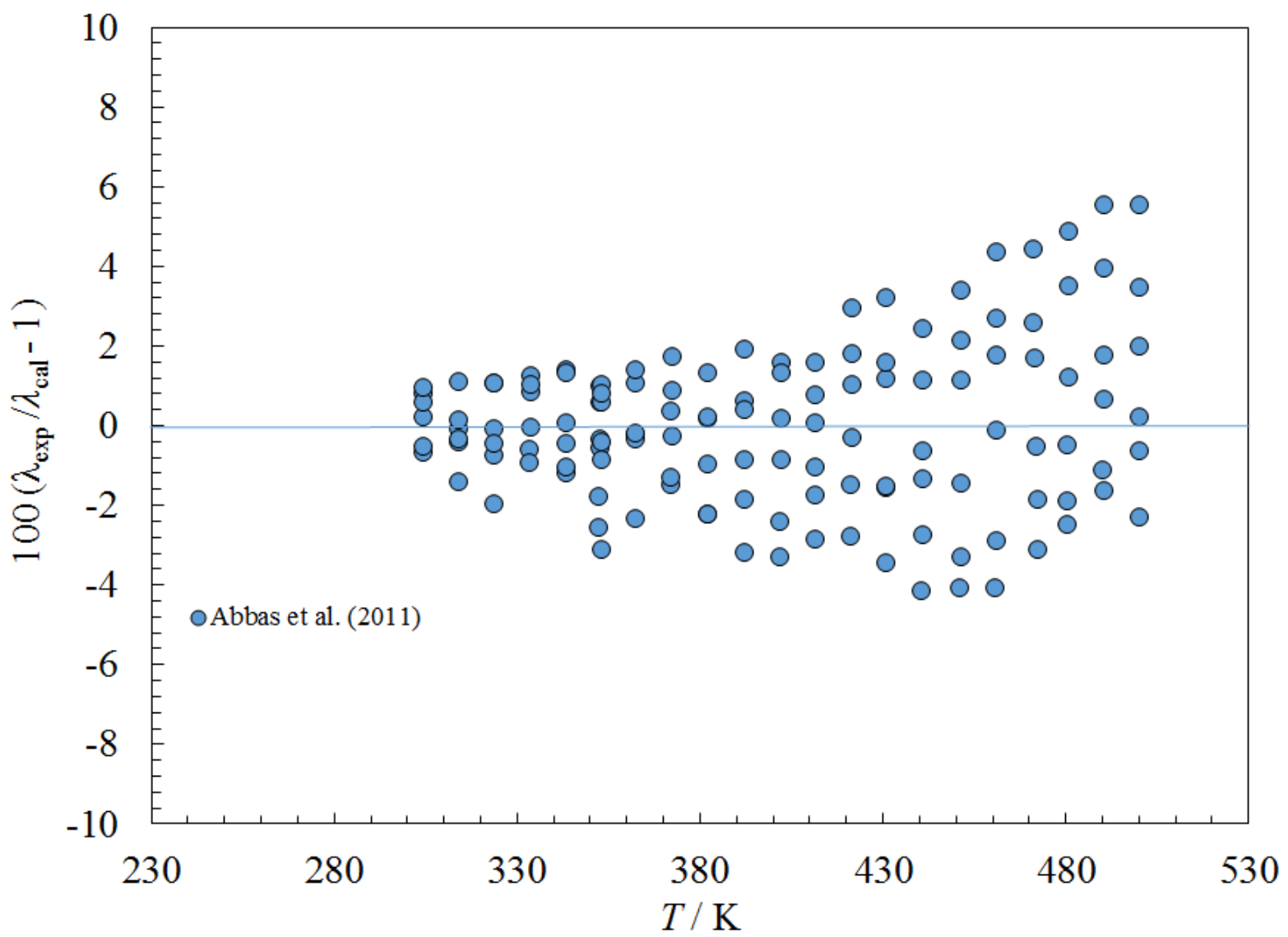

Figure 84. Percentage deviations between the model and the experimental thermal conductivity data for octamethyltrisiloxane (MDM) as a function of temperature. 


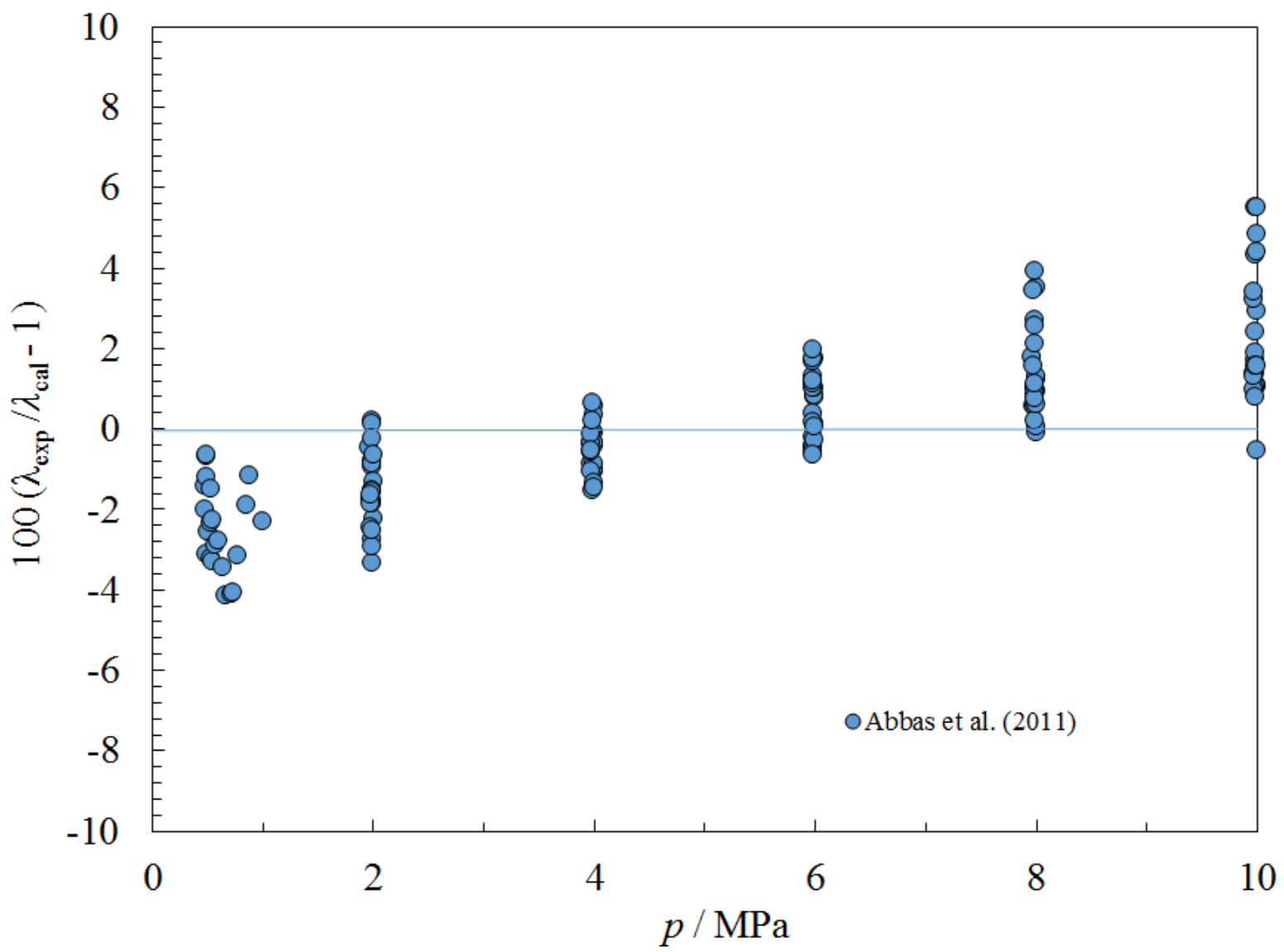

Figure 85. Percentage deviations between the model and the experimental thermal conductivity data for octamethyltrisiloxane (MDM) as a function of pressure. 


\subsubsection{Decamethyltetrasiloxane (MD2M)}

We used the equation of state of Thol et al. (Thol et al., 2017) to provide the density and the critical parameters. The method of Chung et al. (Chung et al., 1988) was used to estimate the Lennard-Jones parameters. We were unable to locate gas-phase viscosity data. For the liquid phase, three sets of data are available (Abbas et al., 2011; Hurd, 1946; Wilcock, 1946), of which the data of Abbas et al. (Abbas et al., 2011) are the most extensive although all points are limited to atmospheric pressure. The coefficients resulting from fitting the liquid-phase viscosity data are in Table 2 and deviations are plotted in Figure 86. Based on comparisons with data, we estimate the uncertainty in the liquid at atmospheric pressure to be on the order of $3 \%$, and in the vapor phase to be $10 \%$. There are no high-pressure viscosity data available for comparisons; we estimate that the uncertainty will be larger as the pressure increases, especially for low temperatures, rising to $10 \%$ at $10 \mathrm{MPa}$. We recommend the equation be used only for pressures up to $10 \mathrm{MPa}$. Abbas et al. (Abbas et al., 2011) also measured the thermal conductivity of this fluid over the range 296 $\mathrm{K}$ to $498 \mathrm{~K}$ at pressures up to $10 \mathrm{MPa}$. The data of Abbas et al. (Abbas et al., 2011) were fit to obtain the coefficients in Table 3. Parameters for the critical enhancement are presented in Table 4. We estimate the uncertainty of the correlation for the liquid phase is $5 \%$ for $T<500 \mathrm{~K}$ at pressures to $10 \mathrm{MPa}, 10 \%$ at higher temperatures and pressures. Figures 87 and 88 show deviation plots for thermal conductivity as a function of temperature and pressure. We attempted to remove the systematic deviations with pressure by trying different reference fluids, but were unsuccessful. Data are unavailable for comparisons in the gas phase, and we estimate the uncertainty as on the order of $25 \%$. Surface tension is discussed in a separate publication (Mulero \& Cachadiña, 2014). 


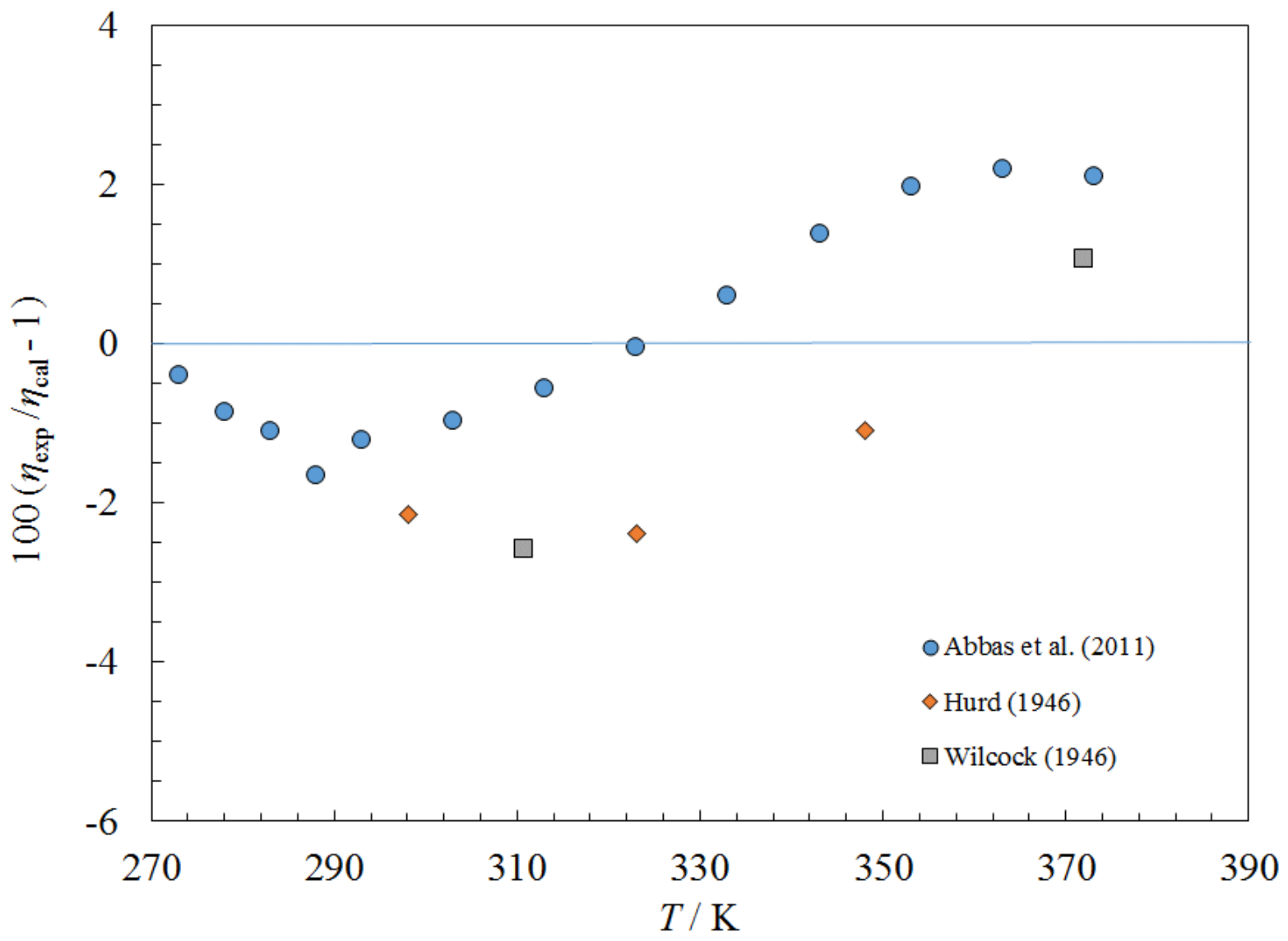

Figure 86. Percentage deviations between the model and the experimental viscosity data for decamethyltetrasiloxane (MD2M). 


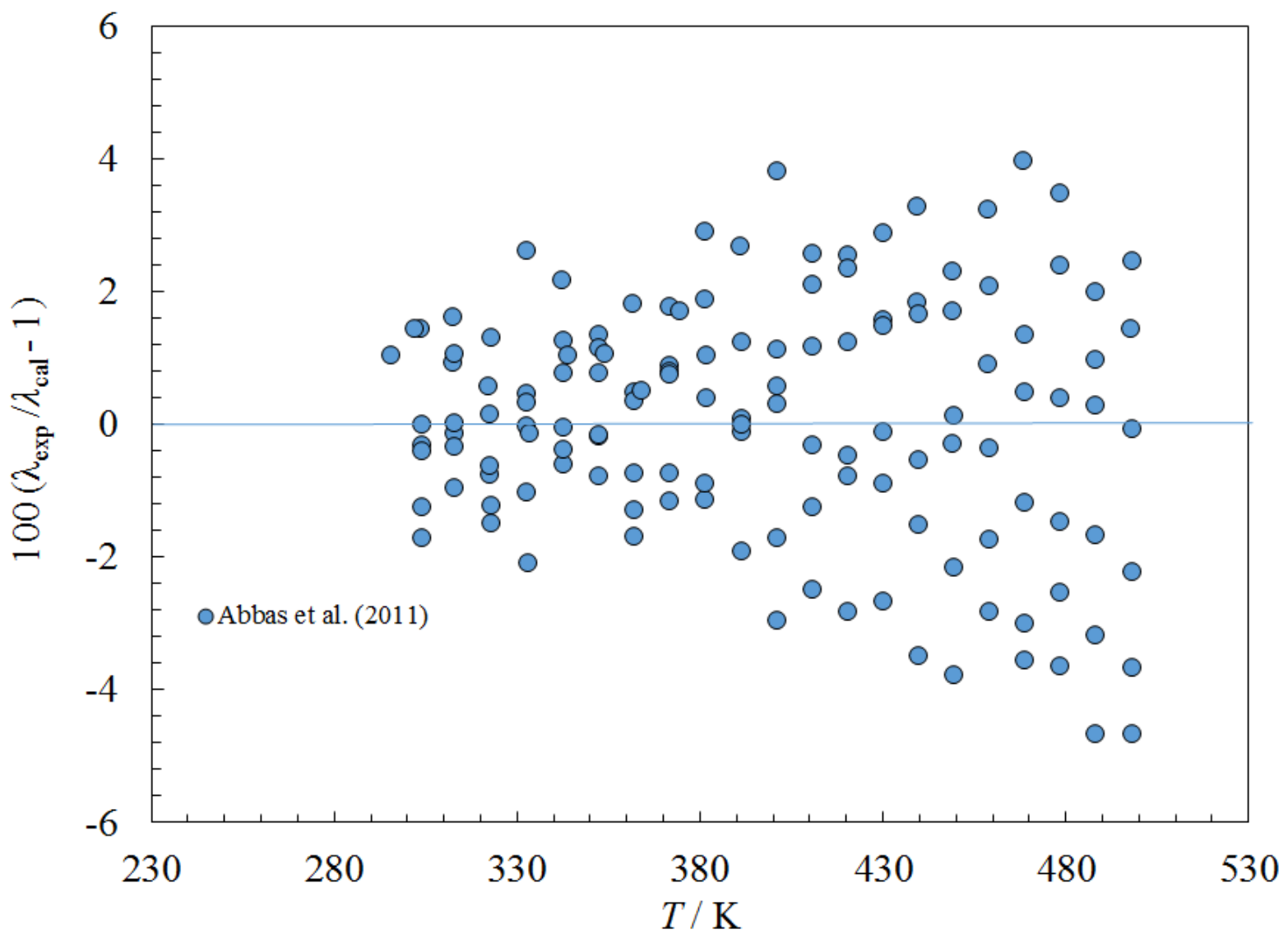

Figure 87. Percentage deviations between the model and the experimental thermal conductivity data for decamethyltetrasiloxane (MD2M) as a function of temperature. 


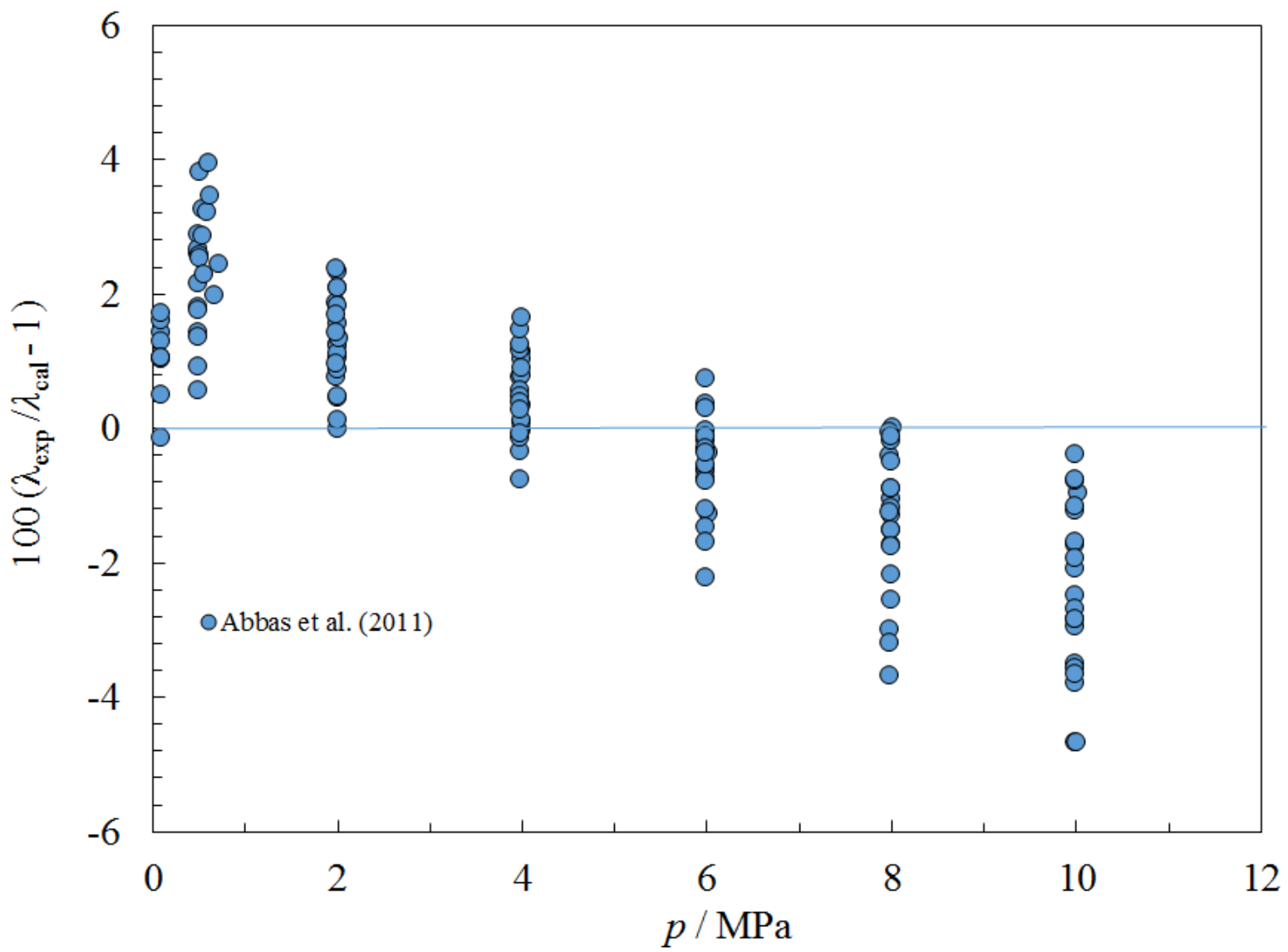

Figure 88. Percentage deviations between the model and the experimental thermal conductivity data for decamethyltetrasiloxane (MD2M) as a function of pressure. 


\subsubsection{Dodecamethylpentasiloxane (MD3M)}

We used the equation of state of Thol et al. (Thol, Javed, Baumhögger, Span, \& Vrabec, 2018) to provide the density and the critical parameters. The method of Chung et al. (Chung et al., 1988) was used to estimate the Lennard-Jones parameters. Experimental data are more limited for this siloxane than for the other siloxanes discussed so far; there are only 5 liquid viscosity points (Hurd, 1946; Wilcock, 1946), all at atmospheric pressure covering a limited temperature range, $298 \mathrm{~K}$ to $372 \mathrm{~K}$. These data were fit to provide the coefficients in Table 2. Figure 89 gives a deviation plot. The uncertainty of the liquid phase at atmospheric pressure is estimated to be $3 \%$, rising to $10 \%$ at pressures to $10 \mathrm{MPa}$. There are no data in the vapor phase available for comparison, but based on other members of the siloxane family, we estimate $10 \%$ uncertainty for the vapor-phase viscosity. Similarly, there are very few data for thermal conductivity, only two liquid-phase points at atmospheric pressure from Bates (Bates, 1949). We supplemented this data with experimental data for MD2M at atmospheric pressure that was scaled to match the Bates value at $323 \mathrm{~K}$, and this set was fit to provide the parameters in Table 3. Parameters for the critical enhancement are presented in Table 4 . We estimated the uncertainty of the thermal conductivity of the liquid phase to be $5 \%$ for $T<400 \mathrm{~K}$ at pressures to $10 \mathrm{MPa}, 10 \%$ at higher temperatures and pressures. A deviation plot is given in Figure 90. Data are unavailable for comparisons in the gas phase and we estimate the uncertainty as on the order of $25 \%$. Surface tension is discussed in a separate publication (Mulero \& Cachadiña, 2014).

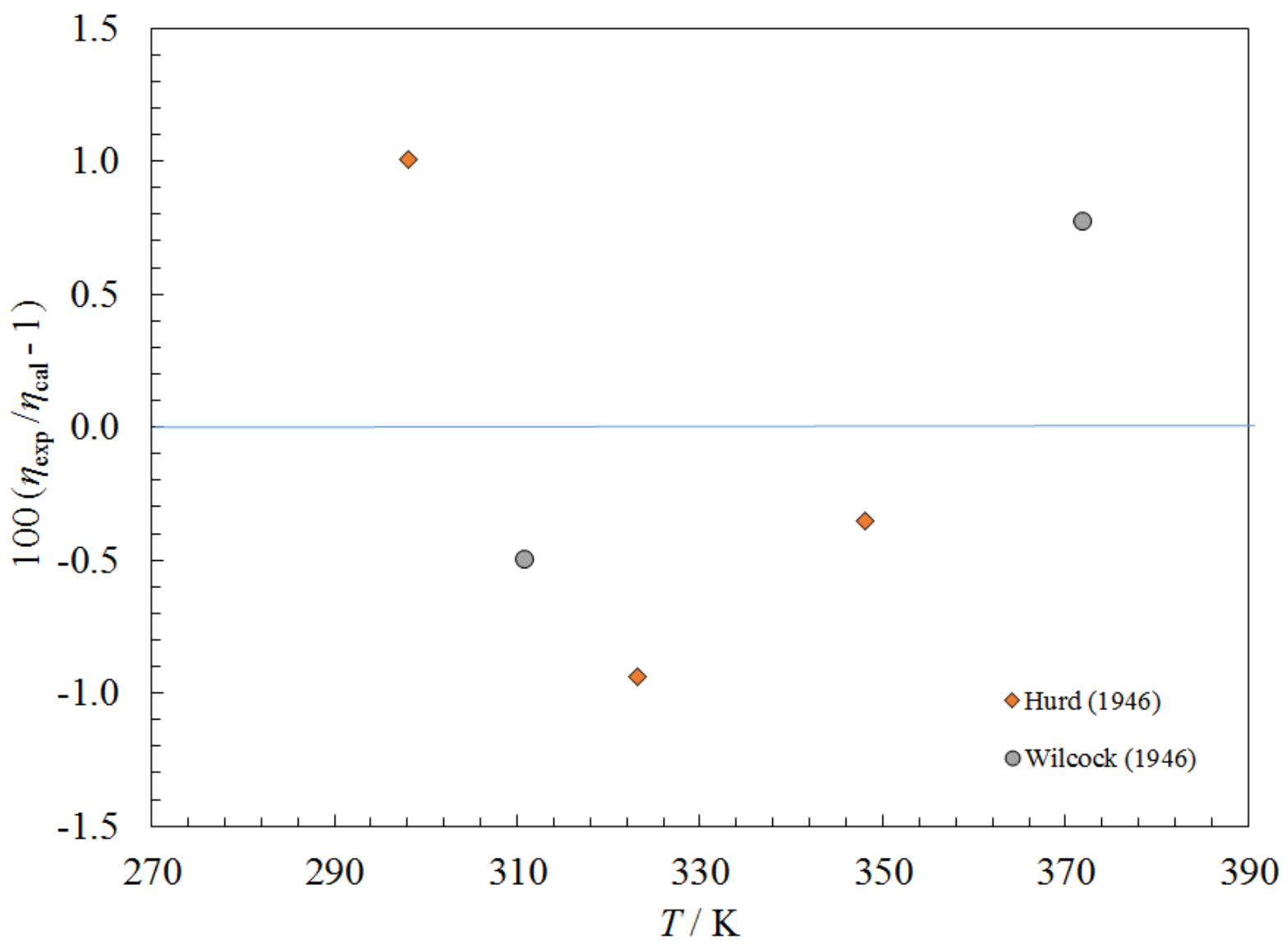

Figure 89. Percentage deviations between the model and the experimental viscosity data for dodecamethylpentasiloxane (MD3M). 


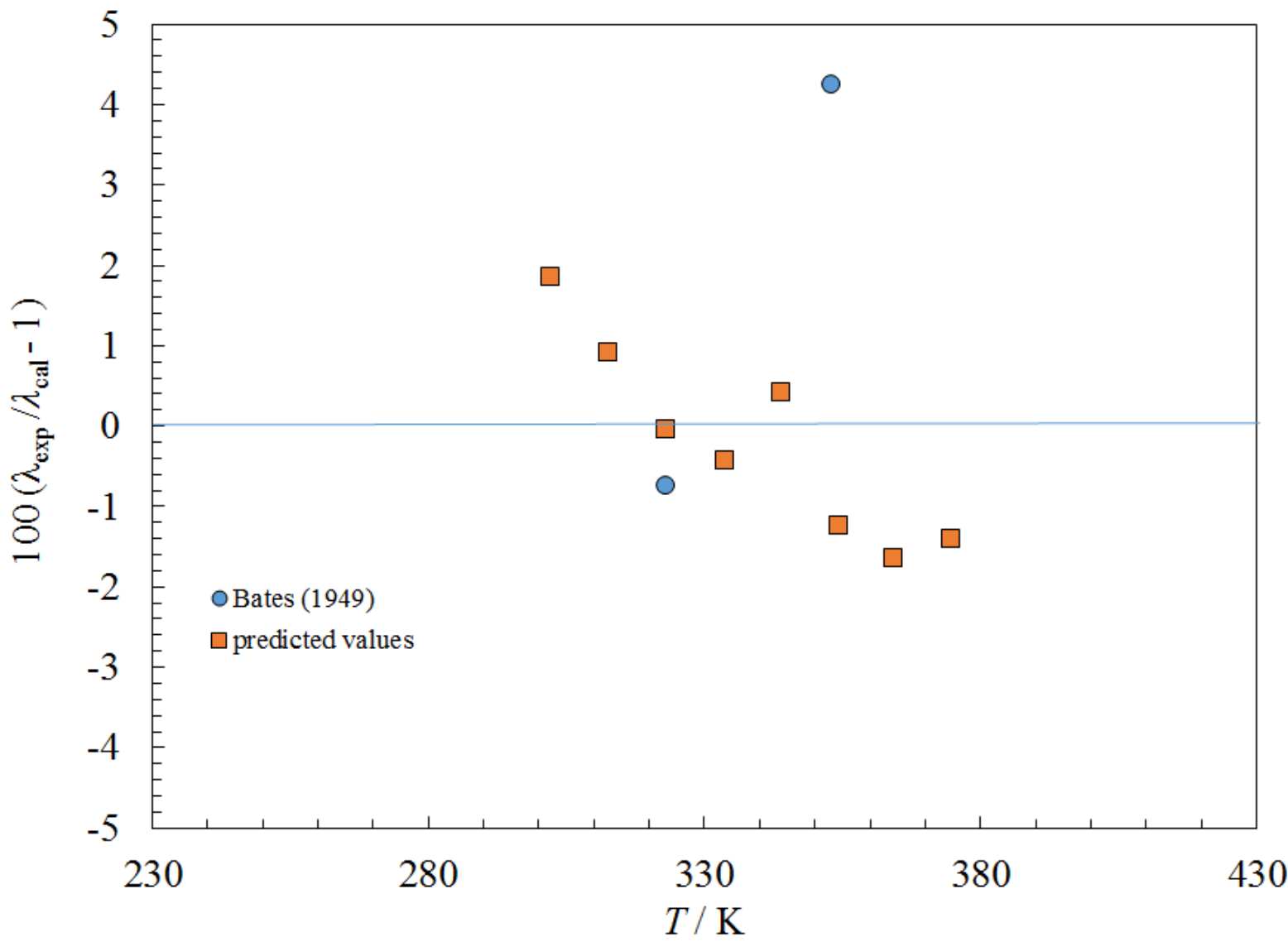

Figure 90. Percentage deviations between the model and the experimental thermal conductivity data for dodecamethylpentasiloxane (MD3M). 


\subsubsection{Tetradecamethylhexasiloxane (MD4M)}

We used the equation of state of Thol et al. (Thol, Javed, et al., 2018) to provide the density and the critical parameters. The method of Chung et al. (Chung et al., 1988) was used to estimate the Lennard-Jones parameters. Experimental data are very limited for this siloxane; there are only 5 liquid viscosity points (Hurd, 1946; Wilcock, 1946), all at atmospheric pressure covering a limited temperature range, $298 \mathrm{~K}$ to $372 \mathrm{~K}$. These data were fit to provide the coefficients in Table 2, and Figure 91 shows a deviation plot. The uncertainty of the liquid phase at atmospheric pressure is estimated to be $3 \%$, rising to $10 \%$ at pressures to $10 \mathrm{MPa}$. There are no data in the vapor phase available for comparison but based on other members of the siloxane family, we estimate $10 \%$ uncertainty for the vapor-phase viscosity. There were no data available for liquid-phase thermal conductivity, nor were there gas-phase thermal conductivity data. For the liquid phase, the coefficients in Table 3 were selected such that the liquid thermal conductivity of MD4M behaves in a manner consistent with similar siloxanes MD3M, MD2M, and MM. Parameters for the critical enhancement are presented in Table 4. Due to lack of data, we estimate the uncertainty for the liquid phase at saturation to be $20 \%$ and larger at higher temperature and pressures. Vapor-phase data were not found, and we estimate the uncertainty in the vapor phase to be $25 \%$. Surface tension is discussed in a separate publication (Mulero \& Cachadiña, 2014).

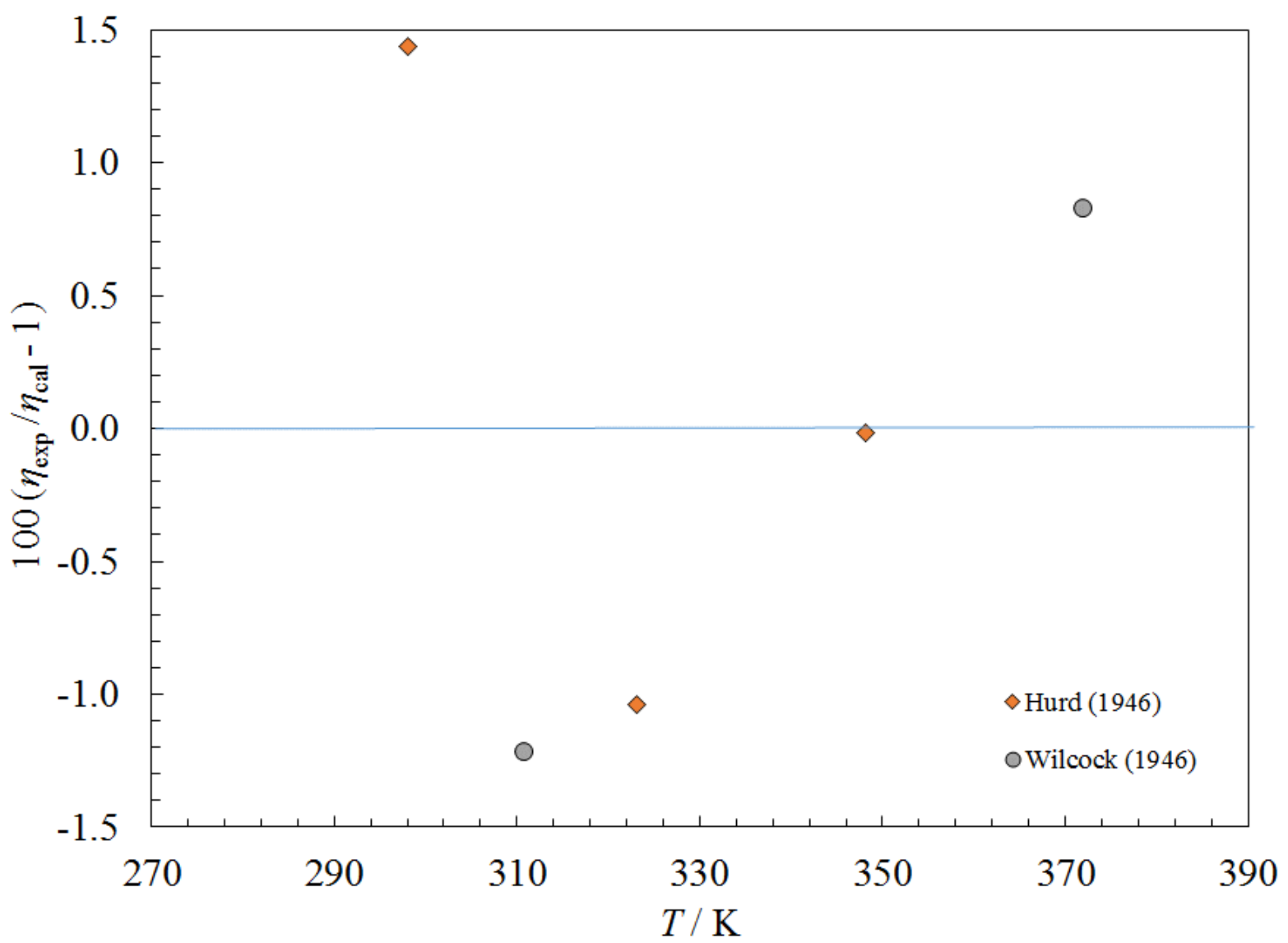

Figure 91. Percentage deviations between the model and the experimental viscosity data for tetradecamethylhexasiloxane (MD4M). 


\subsubsection{Octamethylcyclotetrasiloxane (D4)}

We used the equation of state of Thol et al. (Thol, Rutkai, et al., 2016) to provide the density and the critical parameters. The method of Chung et al. (Chung et al., 1988) was used to estimate the Lennard-Jones parameters. Several sets (Abbas et al., 2011; Hurd, 1946; Marsh, 1968; Palczewska-Tulinska \& Oracz, 2005; Wilcock, 1946) of liquid viscosity data, all limited to atmospheric pressure, were used to obtain the coefficients in Table 2. No gas-phase data were found. Figure 92 shows comparisons of the correlation with experimental viscosity data. The uncertainty of the liquid phase at atmospheric pressure is estimated to be $3 \%$ at temperatures less than $425 \mathrm{~K}$, rising to $10 \%$ at higher temperatures and pressures to $10 \mathrm{MPa}$. There are no data in the vapor phase available for comparison, but based on other members of the siloxane family, we estimate $10 \%$ uncertainty for the vapor-phase viscosity. For the liquid phase, the data from two liquid-phase data sets were fit to obtain the coefficients in Table 3. The data of Abbas extend from $296 \mathrm{~K}$ to $513 \mathrm{~K}$ at pressures to $10 \mathrm{MPa}$. No vapor-phase data were found. Figures 93 and 94 show percentage deviations between the model and the experimental thermal conductivity data as a function of temperature and of pressure. We estimate the uncertainty for the liquid phase at temperatures to $513 \mathrm{~K}$ and pressures to $10 \mathrm{MPa}$ to be $5 \%$ and larger at higher temperature and pressures. Vapor-phase data were not found and we estimate the uncertainty in the vapor phase to be $25 \%$. Surface tension is discussed in a separate publication (Mulero \& Cachadiña, 2014). 


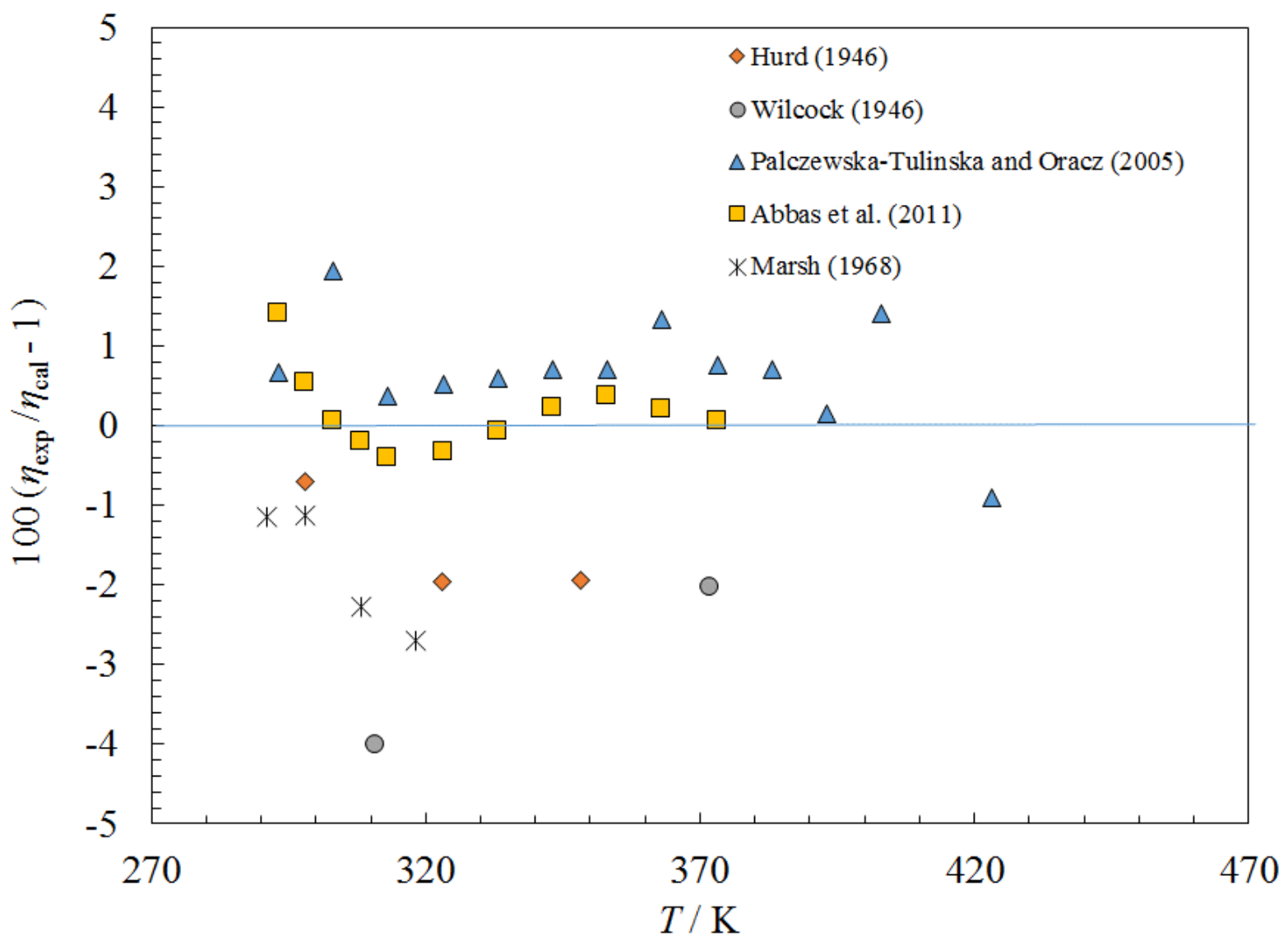

Figure 92. Percentage deviations between the model and the experimental viscosity data for octamethylcyclotetrasiloxane (D4). 


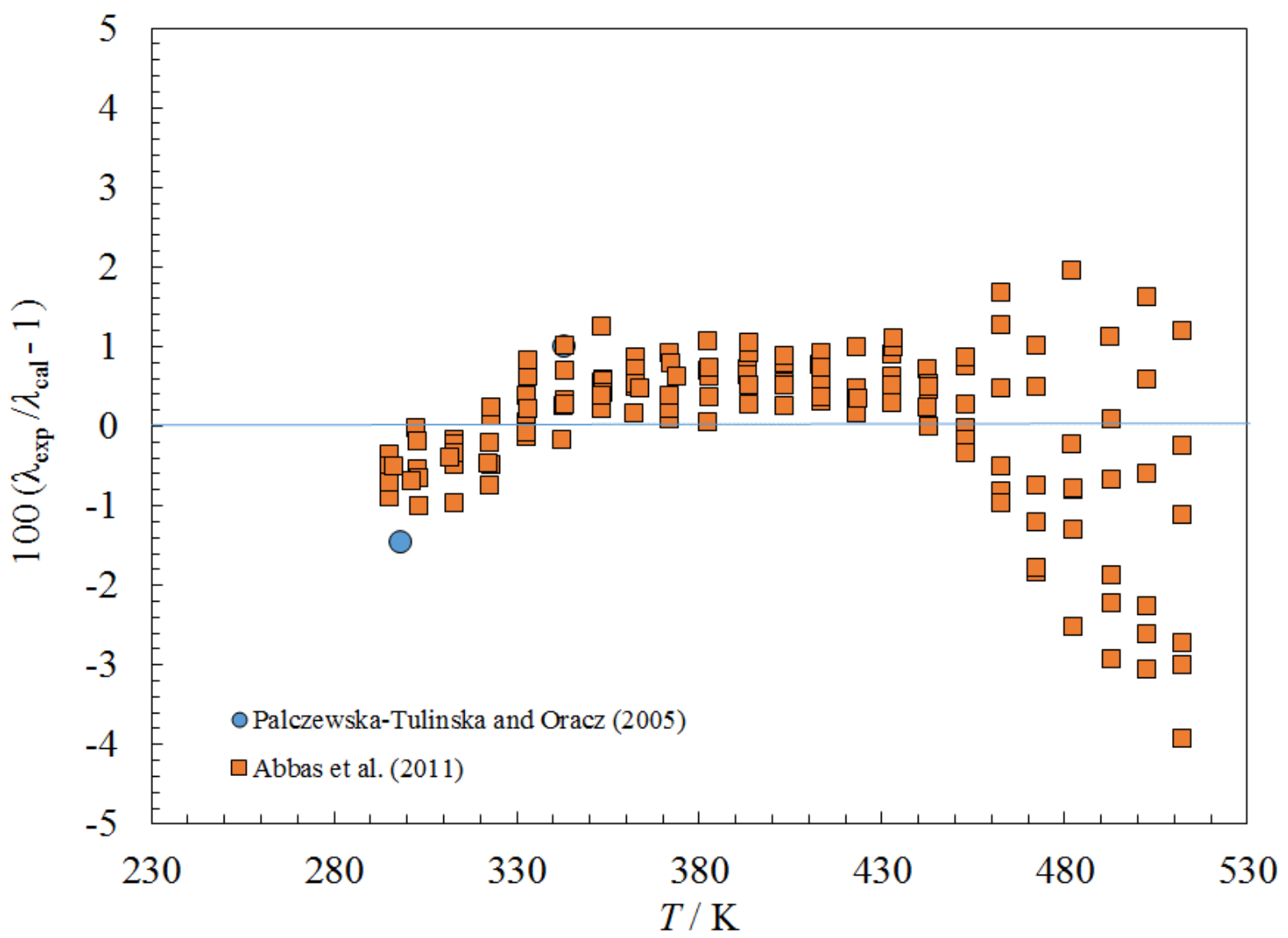

Figure 93. Percentage deviations between the model and the experimental thermal conductivity data for octamethylcyclotetrasiloxane (D4) as a function of temperature. 


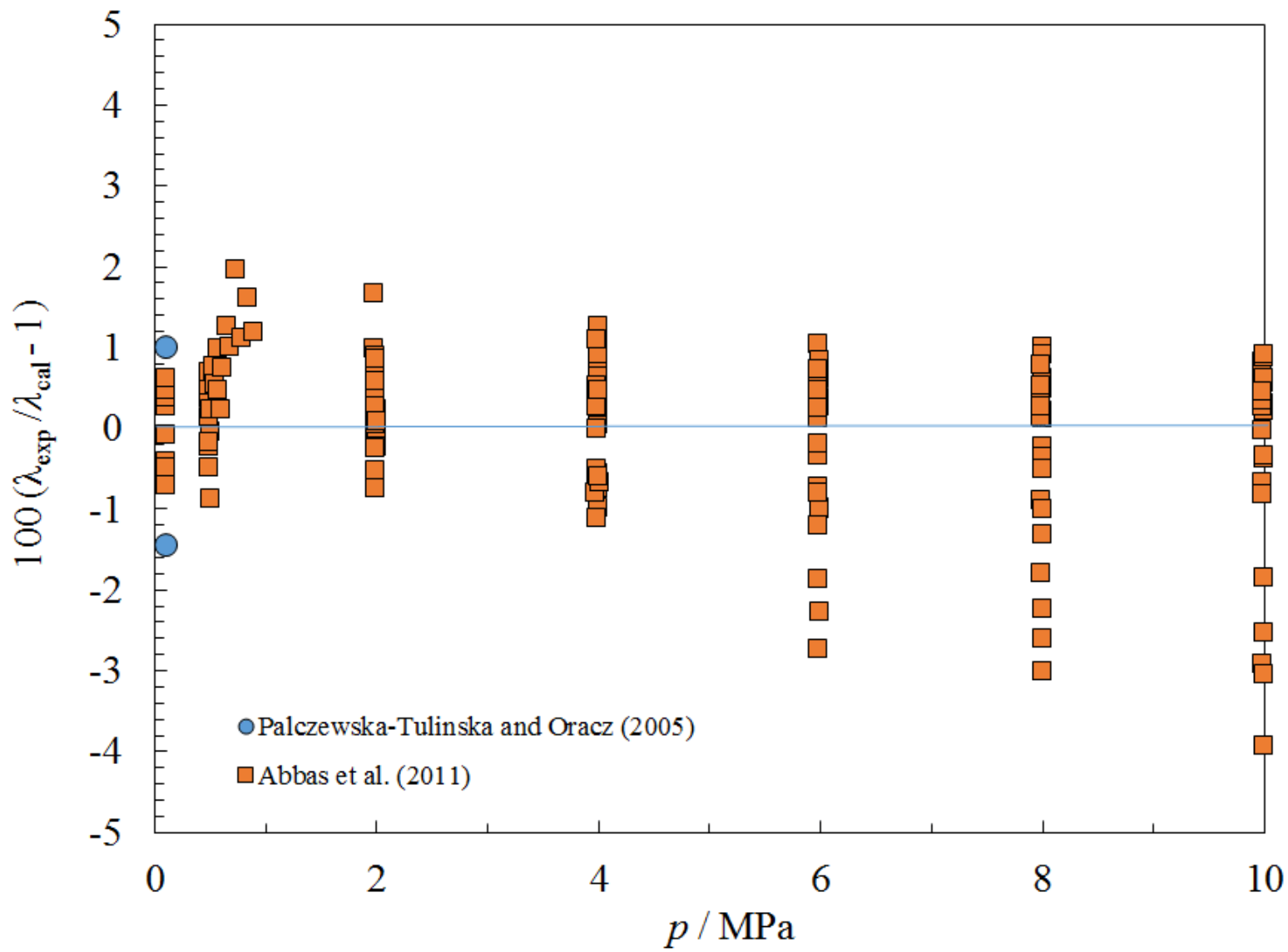

Figure 94. Percentage deviations between the model and the experimental thermal conductivity data for octamethylcyclotetrasiloxane (D4) as a function of pressure. 


\subsubsection{Decamethylcyclopentasiloxane (D5)}

We used the equation of state of Thol et al. (Thol, Javed, et al., 2018) to provide the density and the critical parameters. The method of Chung et al. (Chung et al., 1988) was used to estimate the Lennard-Jones parameters. Several sets (Abbas et al., 2011; Hurd, 1946; Palczewska-Tulinska \& Oracz, 2005; Wilcock, 1946) of liquid viscosity data, all limited to atmospheric pressure, were used to obtain the coefficients in Table 2. No gas-phase data were found. Figure 95 shows comparisons of the correlation with experimental viscosity data. The uncertainty of the liquid phase at atmospheric pressure is estimated to be $5 \%$ at temperatures between $300 \mathrm{~K}$ and $500 \mathrm{~K}$, rising to $10 \%$ at temperatures outside of this range and pressures to $10 \mathrm{MPa}$. There are no data in the vapor phase available for comparison, but based on other members of the siloxane family, we estimate $10 \%$ uncertainty for the vapor-phase viscosity. For the liquid phase, the data from two liquid-phase data sets (Abbas et al., 2011; Palczewska-Tulinska \& Oracz, 2005) were fit to obtain the coefficients in Table 3. Parameters for the critical enhancement are presented in Table 4 . The data of Abbas et al. (Abbas et al., 2011) extend from $294 \mathrm{~K}$ to $513 \mathrm{~K}$ at pressures to $10 \mathrm{MPa}$. No vapor-phase data were found. Figures 95 and 96 show percentage deviations between the model and the experimental thermal conductivity data. For previous fluids we have examined, the results of Abbas et al. (Abbas et al., 2011) and the results of Palczewske-Tulinska and Oracz (PalczewskaTulinska \& Oracz, 2005) have been in agreement. As shown in Figures 96 and 97, they do not agree for D5, and we do not have an explanation for the discrepancy. We estimate the uncertainty for the liquid phase at temperatures to $500 \mathrm{~K}$ and pressures to $10 \mathrm{MPa}$ to be $5 \%$ and larger at higher temperatures and pressures. Vapor-phase data were not found and we estimate the uncertainty in the vapor phase to be $25 \%$. Surface tension is discussed in a separate publication (Mulero \& Cachadiña, 2014). 


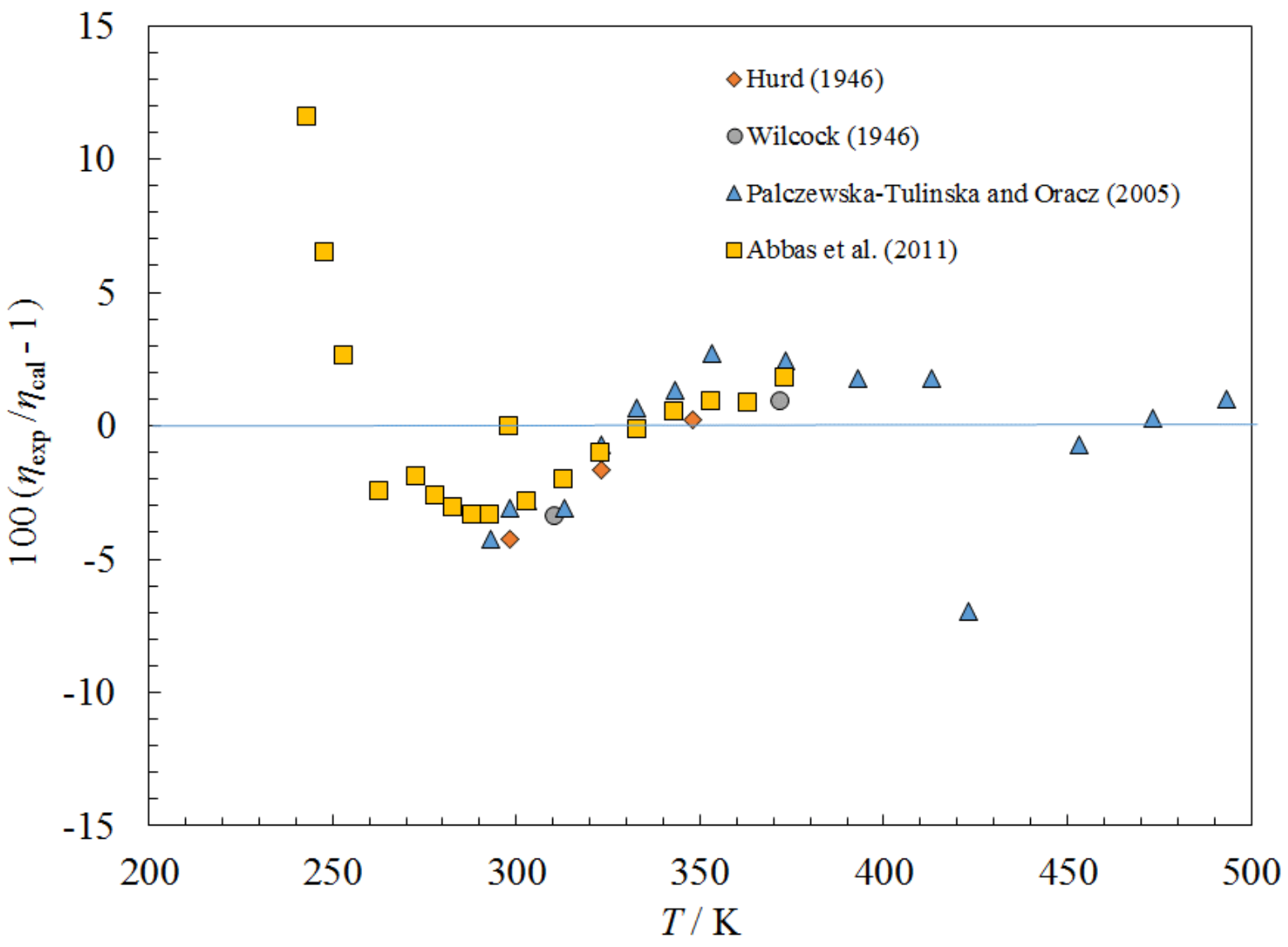

Figure 95. Percentage deviations between the model and the experimental viscosity data for decamethylcyclopentasiloxane (D5). 


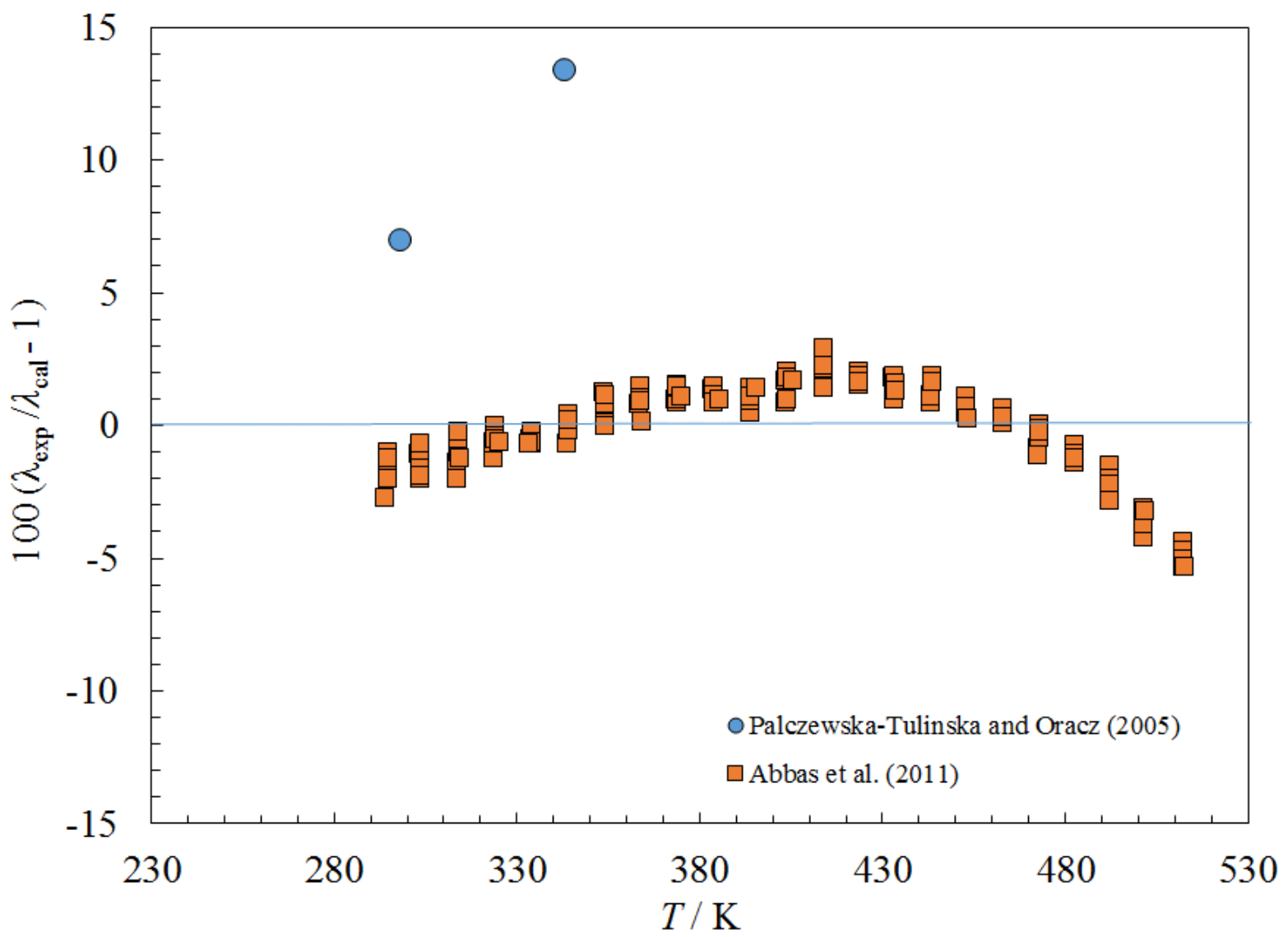

Figure 96. Percentage deviations between the model and the experimental thermal conductivity data for decamethylcyclopentasiloxane (D5) as a function of temperature. 


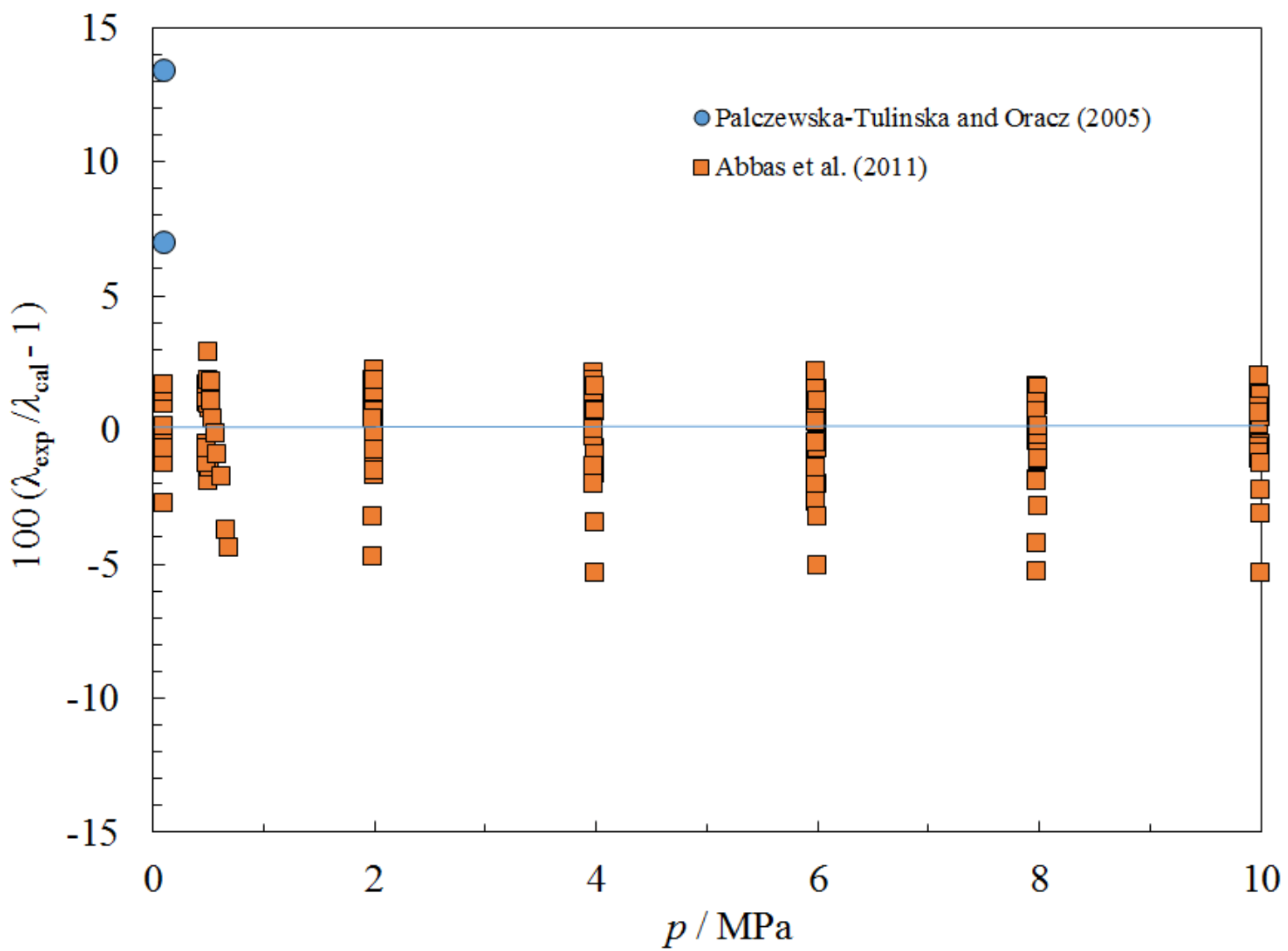

Figure 97. Percentage deviations between the model and the experimental thermal conductivity data for decamethylcyclopentasiloxane (D5) as a function of pressure. 


\subsubsection{Dodecamethylcyclohexasiloxane (D6)}

We used the equation of state of Colonna et al. (Colonna, Nannan, \& Guardone, 2008) to provide the density and the critical parameters. The method of Chung et al. (Chung et al., 1988) was used to estimate the Lennard-Jones parameters. Very limited liquid viscosity data were available (Hurd, 1946; Wilcock, 1946), limited to atmospheric pressure, that were used to obtain the coefficients in Table 2. No gas-phase data were found. Figure 98 shows comparisons of the correlation with experimental viscosity data. The uncertainty of the liquid phase at atmospheric pressure is estimated to be $5 \%$ at temperatures between $300 \mathrm{~K}$ and $373 \mathrm{~K}$, rising to $10 \%$ at temperatures outside of this range and pressures to $10 \mathrm{MPa}$. There are no data in the vapor phase available for comparison, but based on other members of the siloxane family, we estimate $10 \%$ uncertainty for the vapor-phase viscosity. For thermal conductivity, we did not find any experimental data in either phase. As an estimate, we increased the thermal conductivity values of Abbas et al. (Abbas et al., 2011) for D5 by $2 \%$ and fit them for the temperature range $280 \mathrm{~K}$ to $580 \mathrm{~K}$ to obtain the coefficients in Table 3. Parameters for the critical enhancement are presented in Table 4. Figure 99 shows deviations with the scaled data of Abbas et al. (Abbas et al., 2011). We estimate the uncertainty for the liquid phase at temperatures to $500 \mathrm{~K}$ and pressures to $10 \mathrm{MPa}$ to be $10 \%$ and larger at higher temperature and pressures. Vapor-phase data were not found, and we estimate the uncertainty in the vapor phase to be $25 \%$. Surface tension is discussed in a separate publication (Mulero \& Cachadiña, 2014).

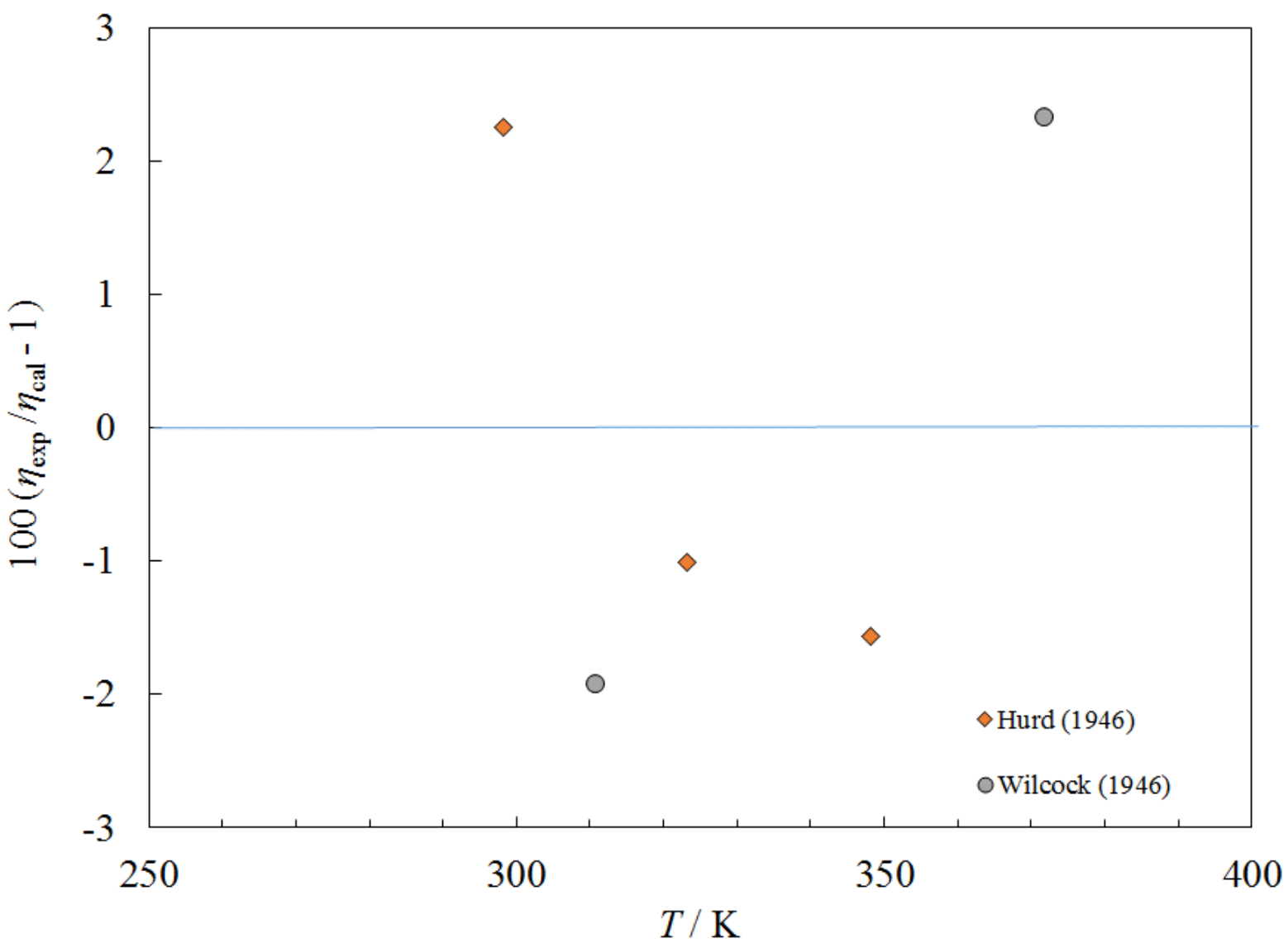

Figure 98. Percentage deviations between the model and the experimental viscosity data for dodecamethylcyclohexasiloxane (D6). 


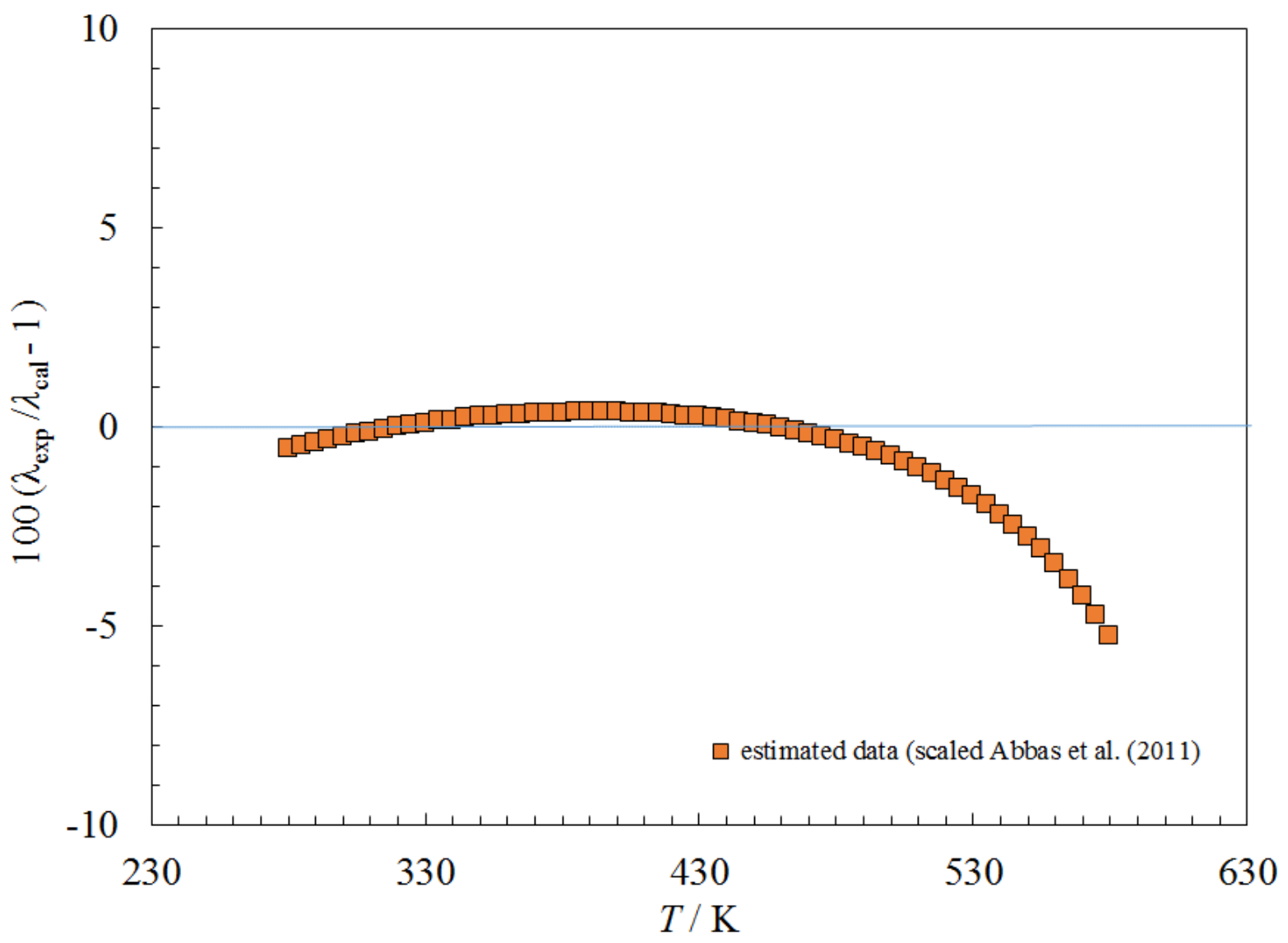

Figure 98. Percentage deviations between the model and the experimental thermal conductivity data for dodecamethylcyclohexasiloxane (D6). 


\subsection{Fluorinated and Chlorinated Compounds, Refrigerants}

\subsubsection{R40 (Methyl chloride)}

The equation of state of Thol et al. (Thol et al., 2014) was used to provide the density and the critical parameters. Lennard-Jones parameters were estimated with the method of Chung et al. (Chung et al., 1988) We fit the gas-phase viscosity data of Benning and Markwood (Benning \& Markwood, 1939) and Bhattacharyya (Bhattacharyya, 1970), and the liquid-phase data of Awberry and Griffiths (Awbery \& Griffiths, 1936) and Rutherford (Rutherford, 1984) to obtain the coefficients in Table 2. All liquid data were at low pressures, less than $1 \mathrm{MPa}$. Figure 100 shows deviation plots as a function of temperature. The estimated uncertainty in the gas phase for viscosity is $4 \%$, and $10 \%$ in the liquid. Gas-phase thermal conductivity data of Senftleben (Senftleben, 1964) and Vines and Bennett (Vines \& Bennett, 1954), and very limited liquid data of Griffiths et al.(Griffiths, Awberry, \& Powell, 1939), were used to obtain the coefficients in Table 3, and a deviation plot is shown in Figure 100. An additional liquid set of Kardos (Kardos, 1934) is shown in Figure 101 for comparison purposes only; it was not used in the fit. Parameters for the critical enhancement are presented in Table 4. All data were at low pressures, less than 1 $\mathrm{MPa}$. The estimated uncertainty in the gas phase is $3 \%, 10 \%$ in the liquid. Surface tension is discussed in a separate publication (Mulero \& Cachadiña, 2014).

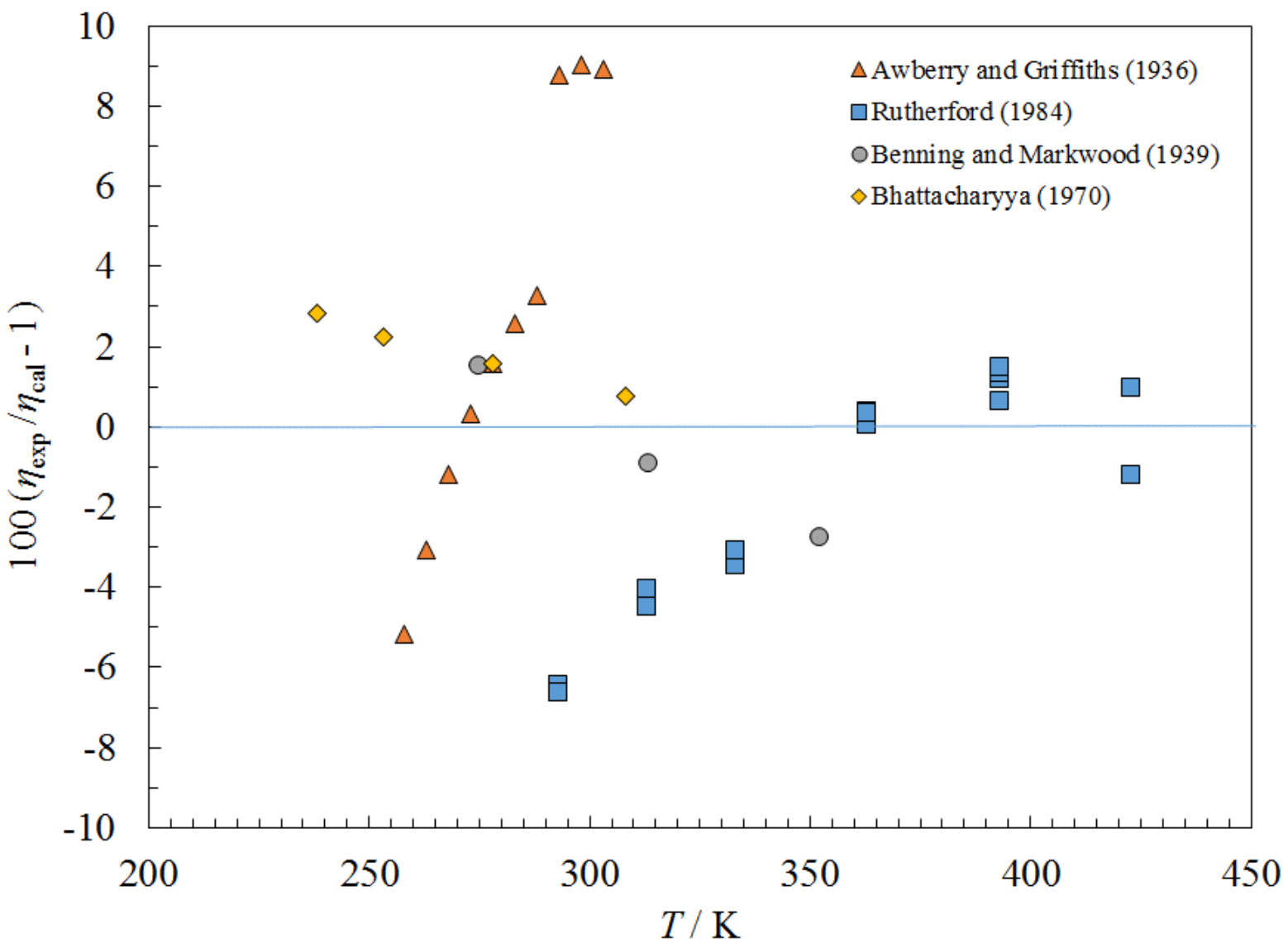

Figure 100. Percentage deviations between the model and the viscosity data for R40 as a function of temperature. 


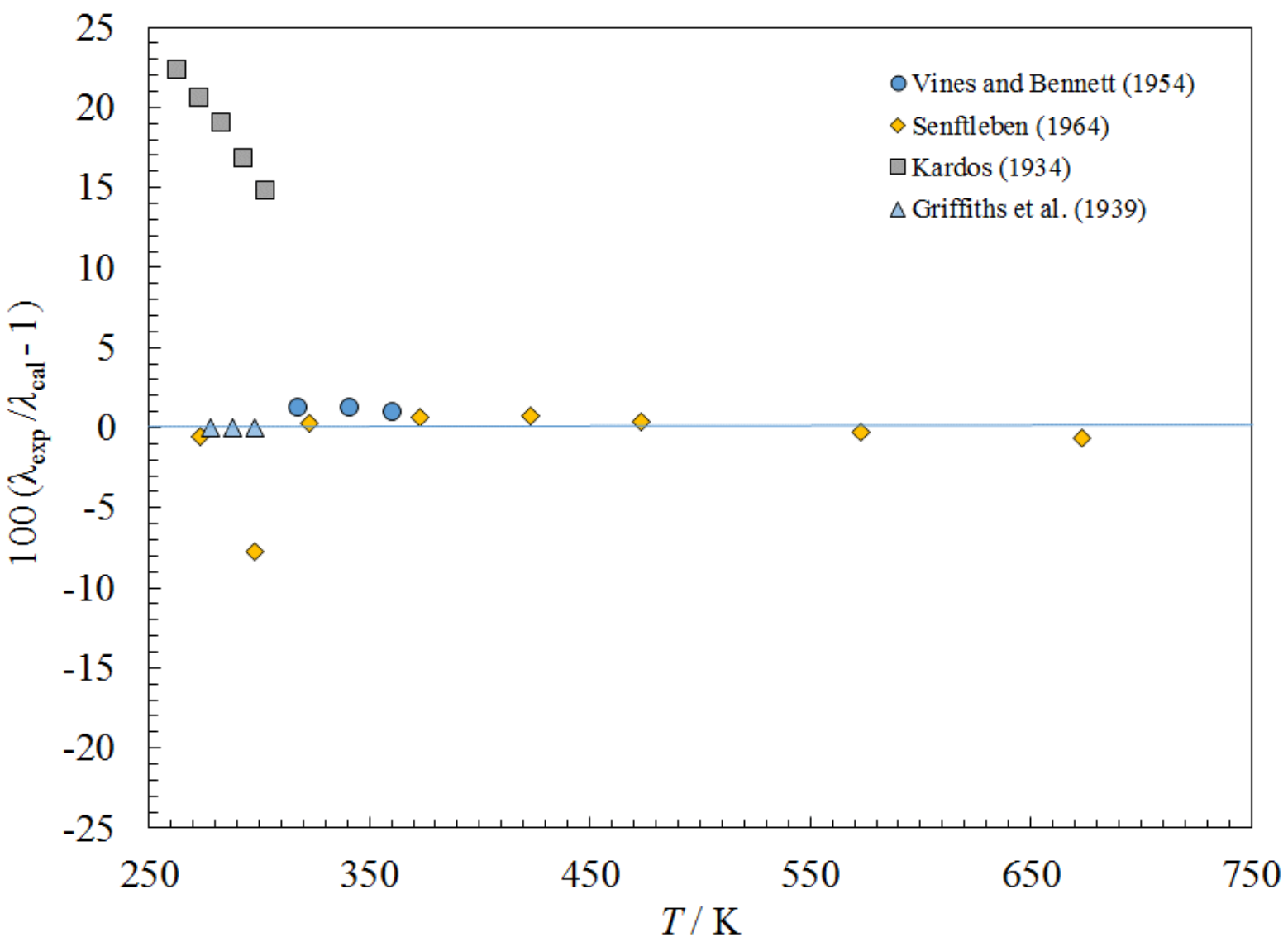

Figure 101. Percentage deviations between the model and the thermal conductivity data for R40 as a function of temperature.

\subsubsection{R1140 (Vinyl chloride)}

The equation of state of Thol and Span (2014) was used to provide the density and the critical parameters. Lennard-Jones parameters were obtained from the method of Chung et al. (Chung et al., 1988). Viscosity data were extremely limited, and none were above atmospheric pressure. Only one source of liquid-phase data was found (Miller, 1966), and these data were used to obtain the liquid-phase coefficients in Table 2. For viscosity of the vapor phase, we used one data point from a manufacturers' SDS (MathesonTriGas, 2008) to obtain $F_{\mathrm{c}}$ in Table 2. A deviation plot is given in Figure 102. Due to the extremely limited data, we estimate the uncertainty in viscosity for gas and liquid phases is $10 \%$. We were unable to locate any liquid-phase thermal conductivity data, so we use a totally predictive method for the extended corresponding states model with R134a as a reference fluid. One set of gas-phase thermal conductivity data was found (Senftleben, 1964) and used to obtain the dilute-gas coefficients in Table 3. Parameters for the critical enhancement are presented in Table 4. Comparisons are shown in Figure 103. We estimate that the uncertainty in the gas and liquid phases is $10 \%$. Two sets of surface tension data (Matheson Gas Data Book, unabridged ed., 1974; Miller, 1966) were used to obtain the surface tension coefficients in Table 5, and a deviation plot is shown in Figure 104. The estimated uncertainty for the surface tension is $5 \%$. 


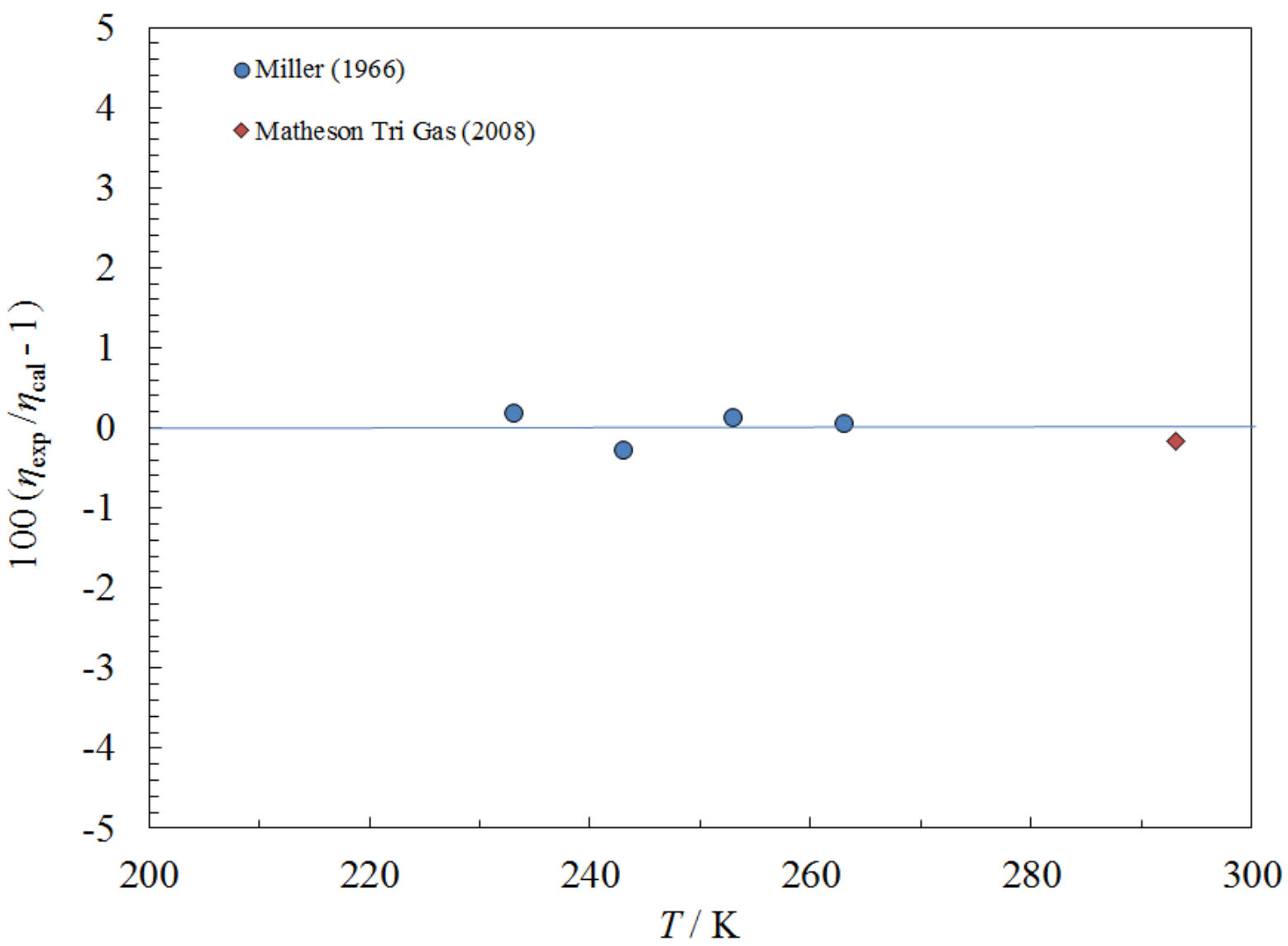

Figure 102. Percentage deviations between the model and the experimental viscosity data for vinyl chloride. 


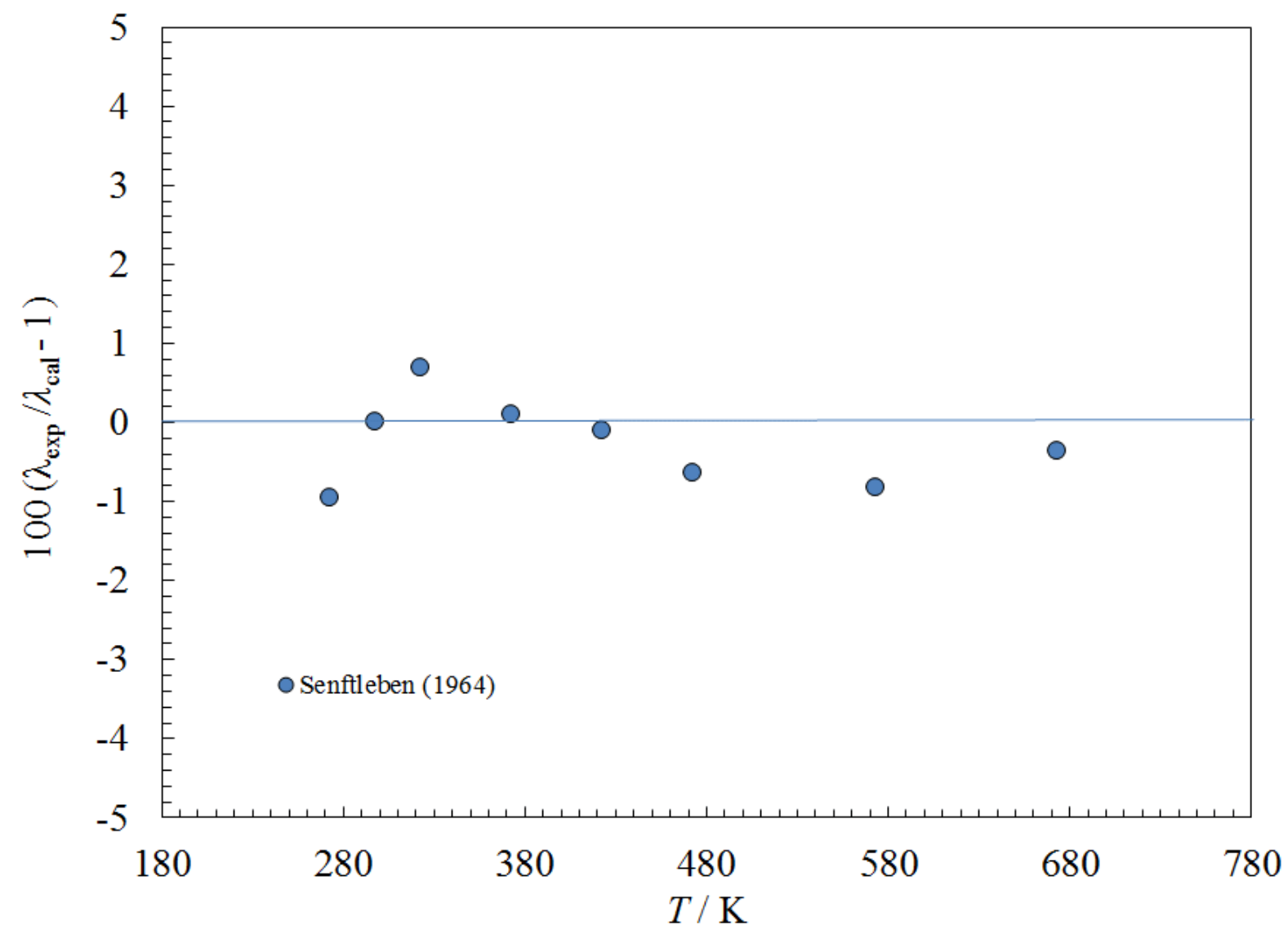

Figure 103. Percentage deviations between the model and the experimental thermal conductivity data for vinyl chloride. 


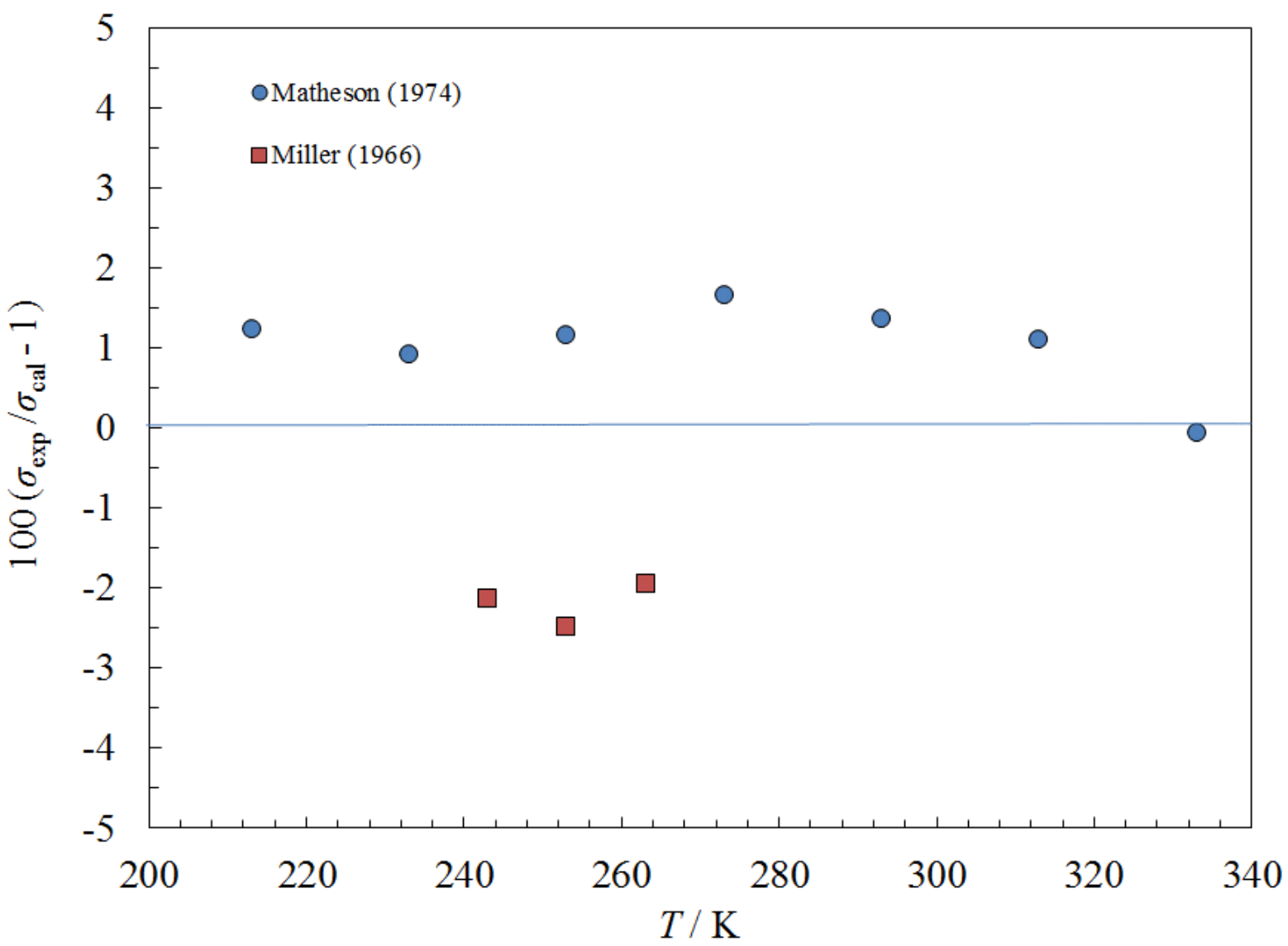

Figure 104. Percentage deviations between the model and the experimental surface tension data for vinyl chloride.

\subsubsection{R1123 (Trifluoroethylene)}

The equation of state of Akasaka et al. (R. Akasaka, Fukushima, \& Lemmon, 2016) was used to provide the density and the critical parameters. Lennard-Jones parameters were estimated with the method of Chung et al. (Chung et al., 1988). We were unable to locate any viscosity or thermal conductivity data, so the parameters in Tables 2-4 are totally predictive. We estimate the uncertainty for viscosity and thermal conductivity is $20 \%$. In addition, data were unavailable for surface tension and a predictive method by Chae et al. (Chae, Schmidt, \& Moldover, 1990) was used to obtain the parameters in Table 5. The estimated uncertainty for surface tension is $10 \%$. 


\subsubsection{R143a (1,1,1-Trifluoroethane)}

The equation of state of Lemmon and Jacobsen (E.W. Lemmon \& Jacobsen, 2000) was used to provide the density and the critical parameters. Lennard-Jones parameters were obtained by fitting the gas-phase viscosity data of Takahashi et al. (Takahashi, Shibasaki-Kitakawa, \& Yokoyama, 1999) and Wang et al. (X. Wang, Wu, \& Liu, 2009). Liquid-phase data of Ripple and Defibaugh (Ripple \& Defibaugh, 1997) and Avelino et al. (Avelino, Fareleira, \& Oliveira, 2006) were used to obtain the coefficients in Table 2. Deviation plots as a function of temperature and of pressure are shown in Figures 105 and 106. The estimated uncertainty for viscosity in the gas phase is $2 \%$, and in the liquid at pressures to $10 \mathrm{MPa}$ is also $2 \%$. For thermal conductivity, there were enough data covering wide ranges of temperature and pressure to make a preliminary fluid-specific correlation instead of an extended corresponding states model. The dilute-gas thermal conductivity data of Haynes (Haynes, 1994) from NIST were used to obtain the dilute-gas coefficients in Table 6 (located in the Appendix), and the wide-ranging fluid measurements of both Haynes (Haynes, 1994) and of Le Neindre et al. (Le Neindre, Garrabos, \& Kim, 2001) were used to obtain the residual coefficients in Table 6 . The value of $q_{\mathrm{d}}{ }^{-1}$ in Table 4 was obtained by fitting, while the other coefficients in Table 4 are from the generalized model of Perkins et al. (R. A. Perkins et al., 2013) Measurements of other researchers (Lee, Kim, \& Ro, 2001; Tanaka, Nakata, \& Makita, 1991; Yata, Hori, Kobayashi, \& Minamiyama, 1996) and the data used in regression (Haynes, 1994; Le Neindre et al., 2001) are shown in the deviation plots in Figures 107 and 108. As seen in the deviation plots, there are still discrepancies in the data, and future work would be useful to determine the most reliable data sets. We estimate the uncertainty in the gas phase is $5 \%$, and the uncertainty in the liquid and supercritical phases is also $5 \%$ at pressures up to $70 \mathrm{MPa}$. Surface tension is discussed in a separate publication (Mulero \& Cachadiña, 2014). 


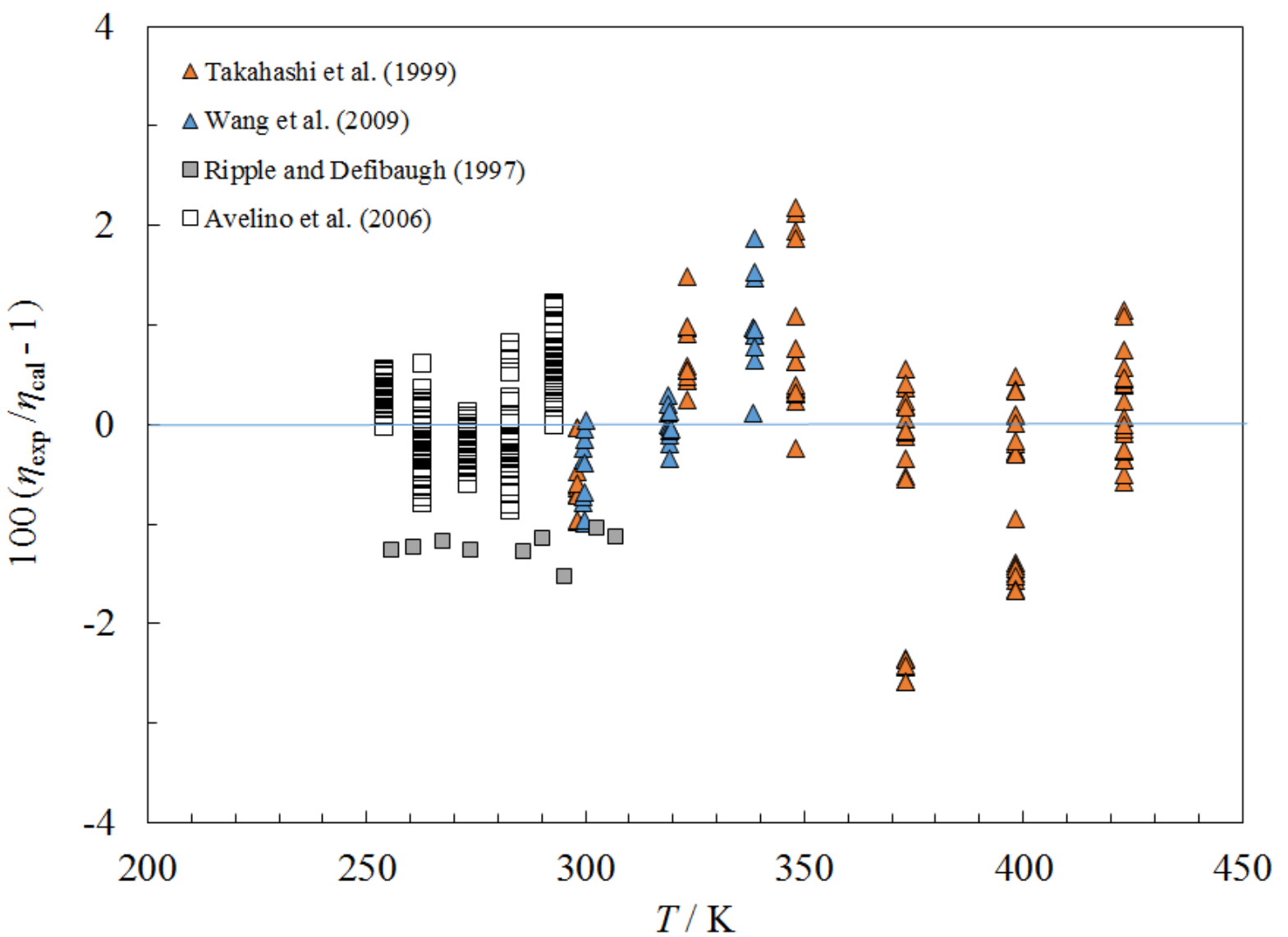

Figure 105. Percentage deviations between the model and the viscosity data for R143a as a function of temperature. 


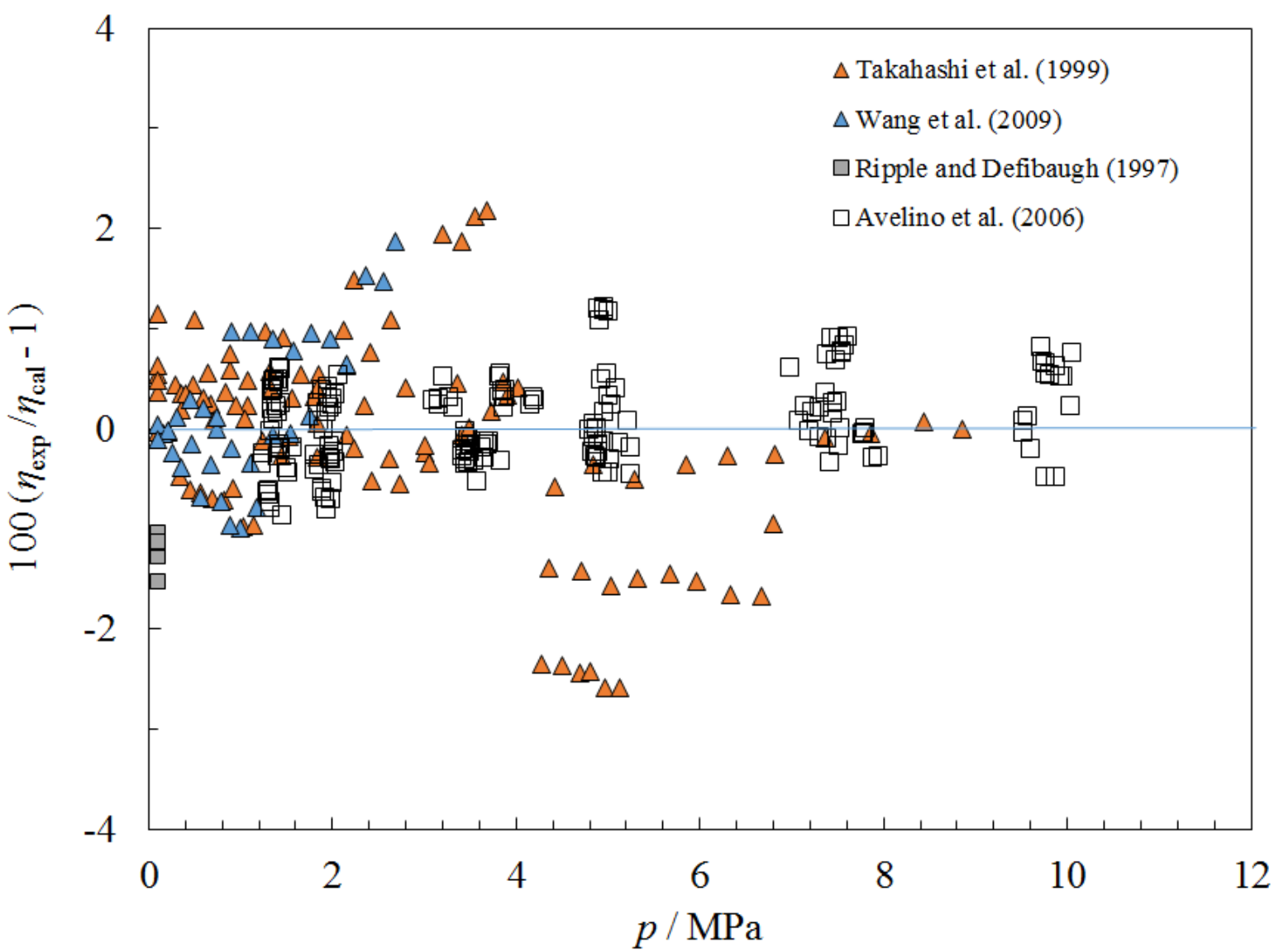

Figure 106. Percentage deviations between the model and the viscosity data for R143a as a function of pressure. 


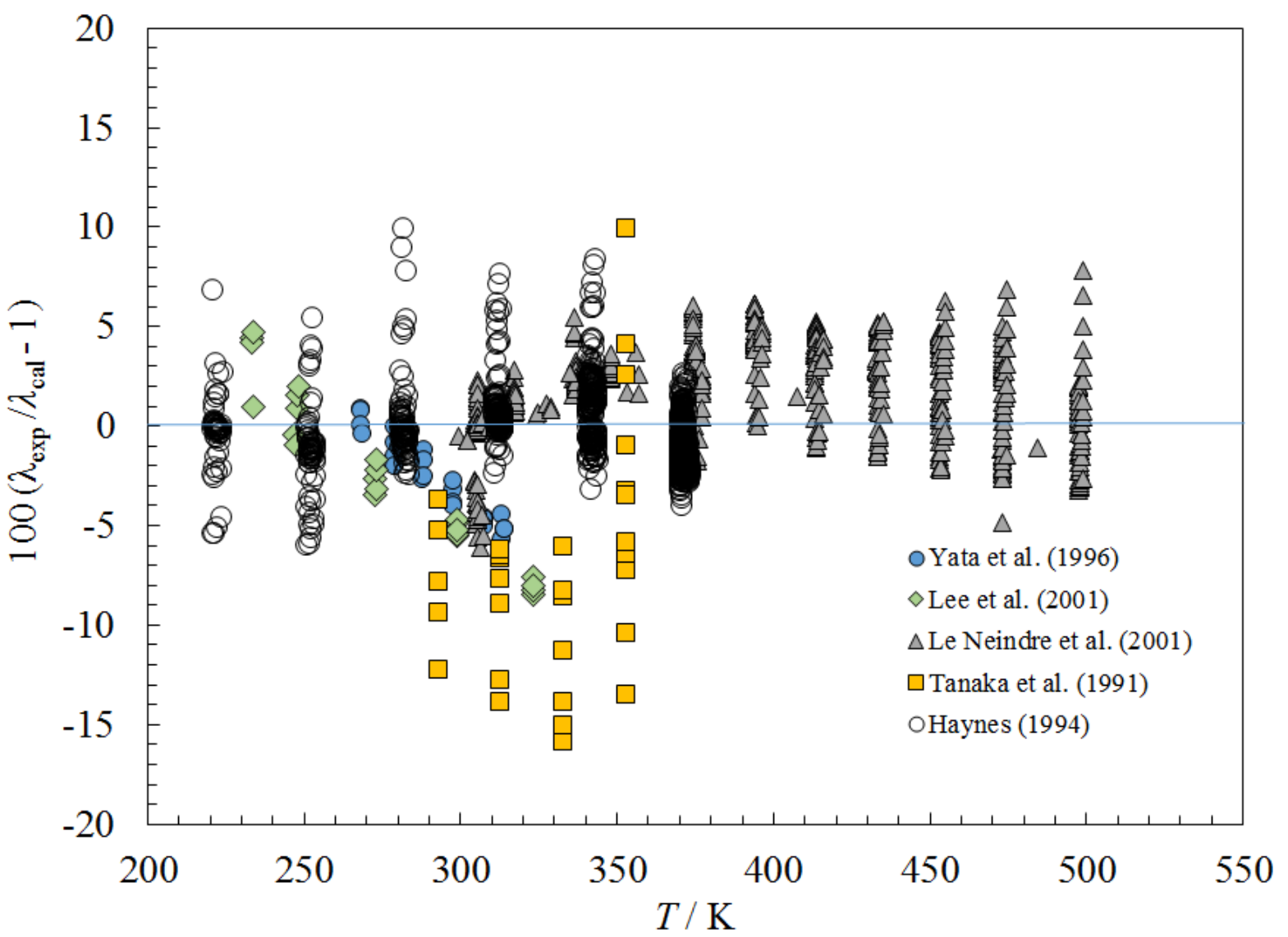

Figure 107. Percentage deviations between the model and the thermal conductivity data for R143a as a function of temperature. 


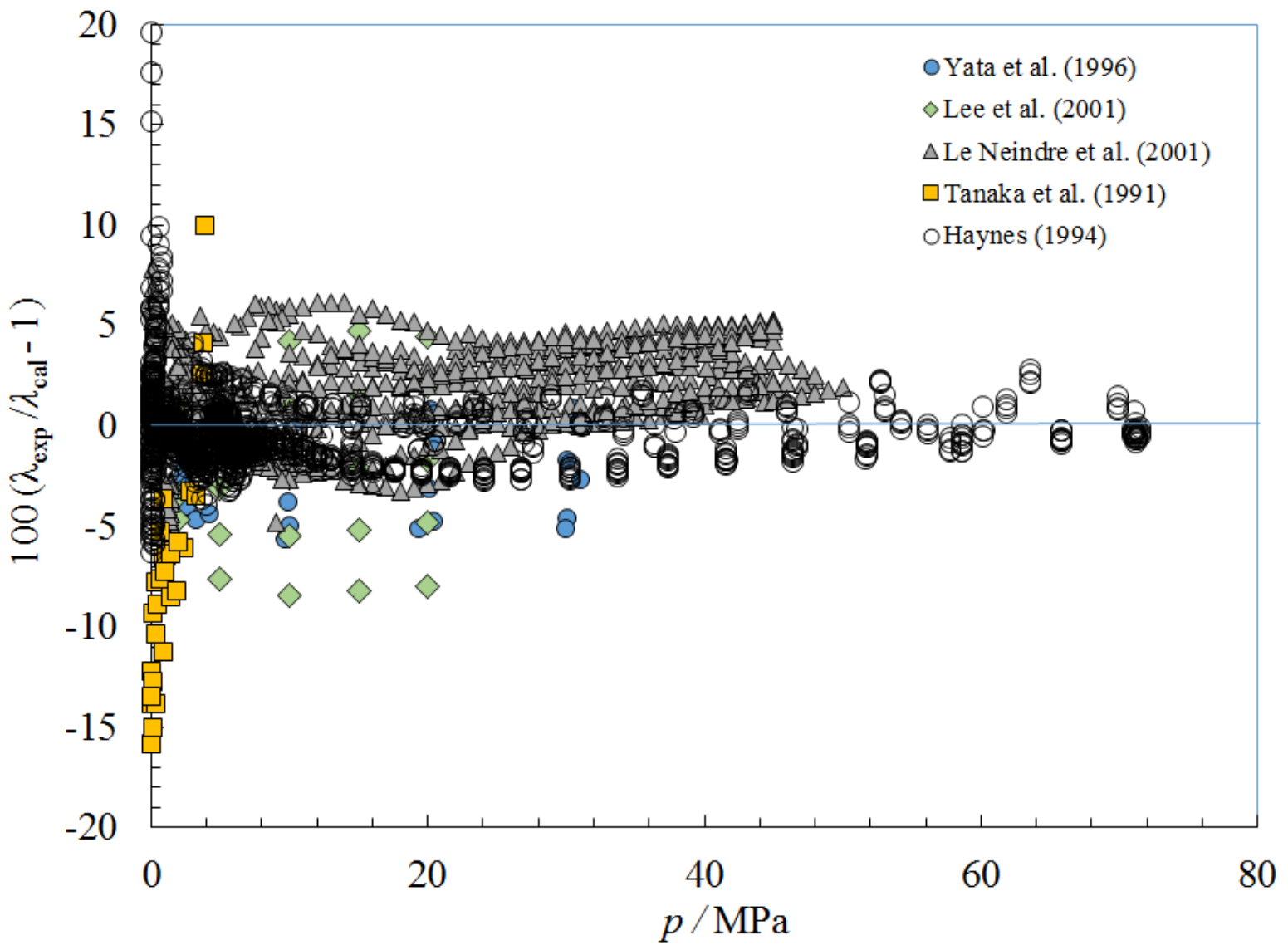

Figure 108. Percentage deviations between the model and the thermal conductivity data for R143a as a function of pressure.

\subsubsection{R1243zf (3,3,3-Trifluoropropene)}

We could not find any experimental data for R1243zf thermal conductivity or viscosity, so the method is completely predictive. The critical parameters are from the EOS of Akasaka (R. Akasaka, 2017), and the Lennard-Jones parameters are estimated by the method of Chung et al. (Chung et al., 1988). The dipole moment was estimated to be $8.106 \times 10^{-30} \mathrm{C} \cdot \mathrm{m}(2.43 \mathrm{D})$ (Kazakov, 2017). Using the method of Chung et al. (Chung et al., 1988) results in a value of 1.01 for $F_{\mathrm{c}}$ as reported in Table 2. R134a was selected as a reference fluid since it is similar chemically to R1243zf, and the coefficients presented in Tables 2 and 3 were selected so that the value of the saturated liquid viscosity of R1243zf is similar to that of R1234yf at its normal boiling point, and such that the thermal conductivity of the saturated liquid at its normal boiling point for R1233zf is also similar to that of R1234yf. Parameters for the critical enhancement are presented in Table 4. The estimated uncertainty for both thermal conductivity and viscosity is on the order of $10 \%$ at saturation, and higher at increased pressures. Surface tension is covered in Kondou (Kondou \& Koyama, 2015) 


\subsubsection{R150 (1,2-Dichloroethane)}

For density, we use the recently developed equation of state of Thol (Thol, Koeste, et al., 2018) to provide density and values for the critical point. For viscosity of the gas phase, the only data we found were those of Paniego and Pinto (Paniego \& Pinot, 1969). We used the LennardJones parameters estimated by the method of Chung et al. (Chung et al., 1988) to provide the parameters that are reported in Table 1, and fit the data of Paniego and Pinto to obtain the $F_{\mathrm{c}}$ reported in Table 2. In the liquid phase, we selected Thorpe and Rodger (Thorpe \& Rodger, 1894), Batschinski (Batschinski, 1913), Ni et al. (Ni, Su, Wang, \& Qiu, 2010), and Malhotra et al. (Malhotra, Price, Woolf, \& Easteal, 1990) for the determination of the parameters reported in Table 2. Although generally it is best to use a reference fluid that is most chemically similar to the fluid of interest, in this case we found better results with propane as a reference fluid especially at higher pressures, rather than R134a, so propane was selected even though it is not halogenated. Figures 109 and 110 show deviations between the model and viscosity data. The data of Malhotra et al. extend to very high pressures (up to $330 \mathrm{MPa}$ ). This greatly exceeds the limits of the EOS (50 $\mathrm{MPa}$ ), and we recommend the correlation be limited to $50 \mathrm{MPa}$. We estimate the uncertainty for viscosity in the gas phase to be $5 \%$, and in the liquid phase also $5 \%$ along the saturation boundary, rising to $10 \%$ at pressures up to $50 \mathrm{MPa}$ for temperatures above $270 \mathrm{~K}$. The thermal conductivity was fit to the data of Vines and Bennett (Vines \& Bennett, 1954), Mashirov and Tarzimanov (Mashirov \& Tarzimanov, 1974), and Qun-Fang et al. (Qun-Fang, Ruisen, Dan-Yan, \& Yu-Chun, 1997); the resulting coefficients are reported in Table 3, and the parameters for the critical enhancement are in Table 4. All data are in the gas phase except for the limited data of Qun-Fang et al. (Qun-Fang et al., 1997) that are for the saturated liquid. No data off of the saturation boundary were located. A deviation plot with experimental thermal conductivity data is given in Figure 111. We estimate the uncertainty in the gas and liquid phase is $5 \%$. For surface tension, we fit the data set (A. I. Vogel, 1948) recommended by the compilation of Jasper (Jasper, 1972) to the functional form of Eq. (22), and the coefficients are presented in Table 5 with the critical temperature as given in Table 1 . The estimated uncertainty is $3 \%$. Figure 112 shows deviations between the data and the surface tension correlation. 


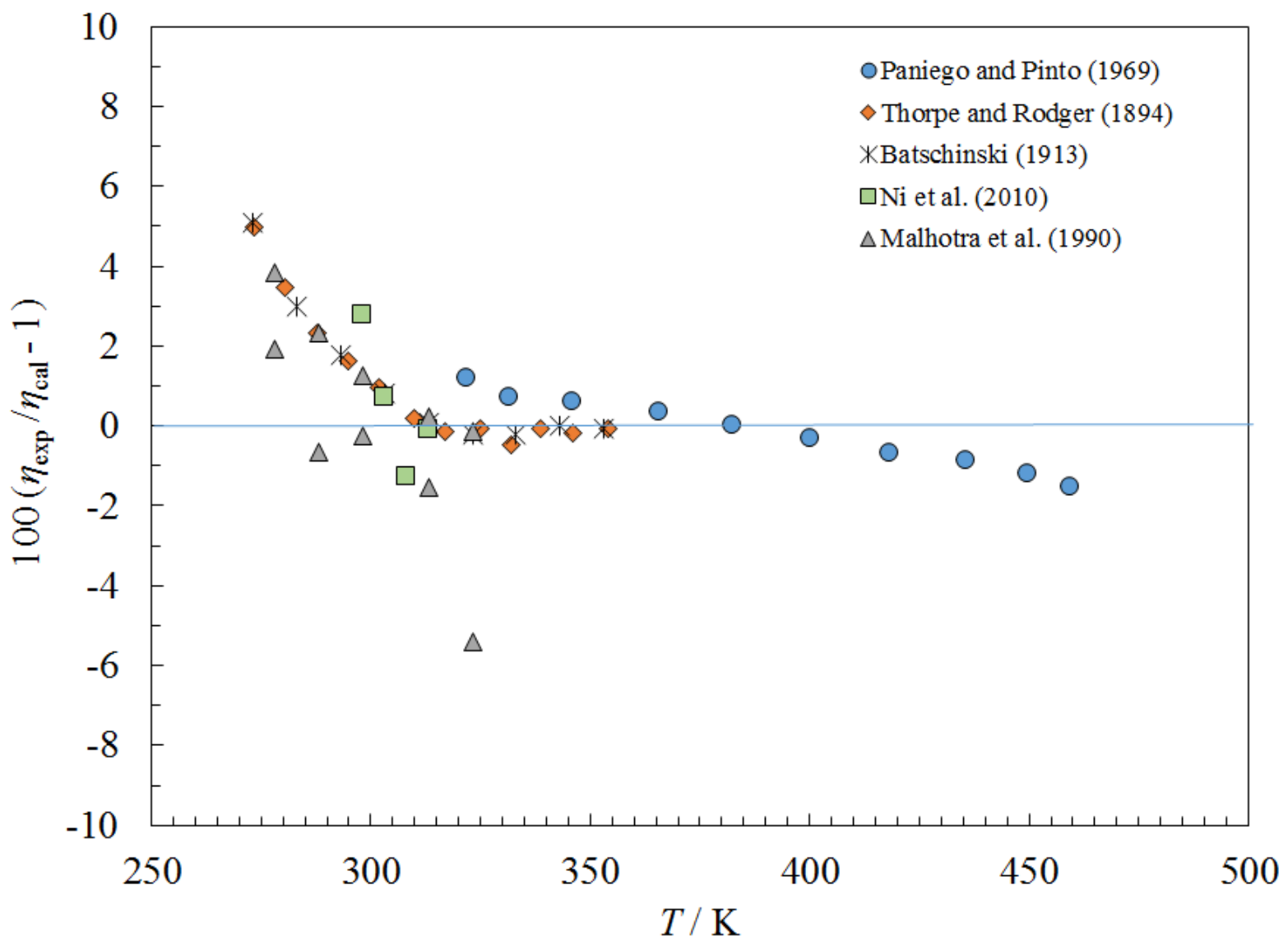

Figure 109. Percentage deviations between the model and the experimental viscosity data for $\mathrm{R} 150$ as a function of temperature. 


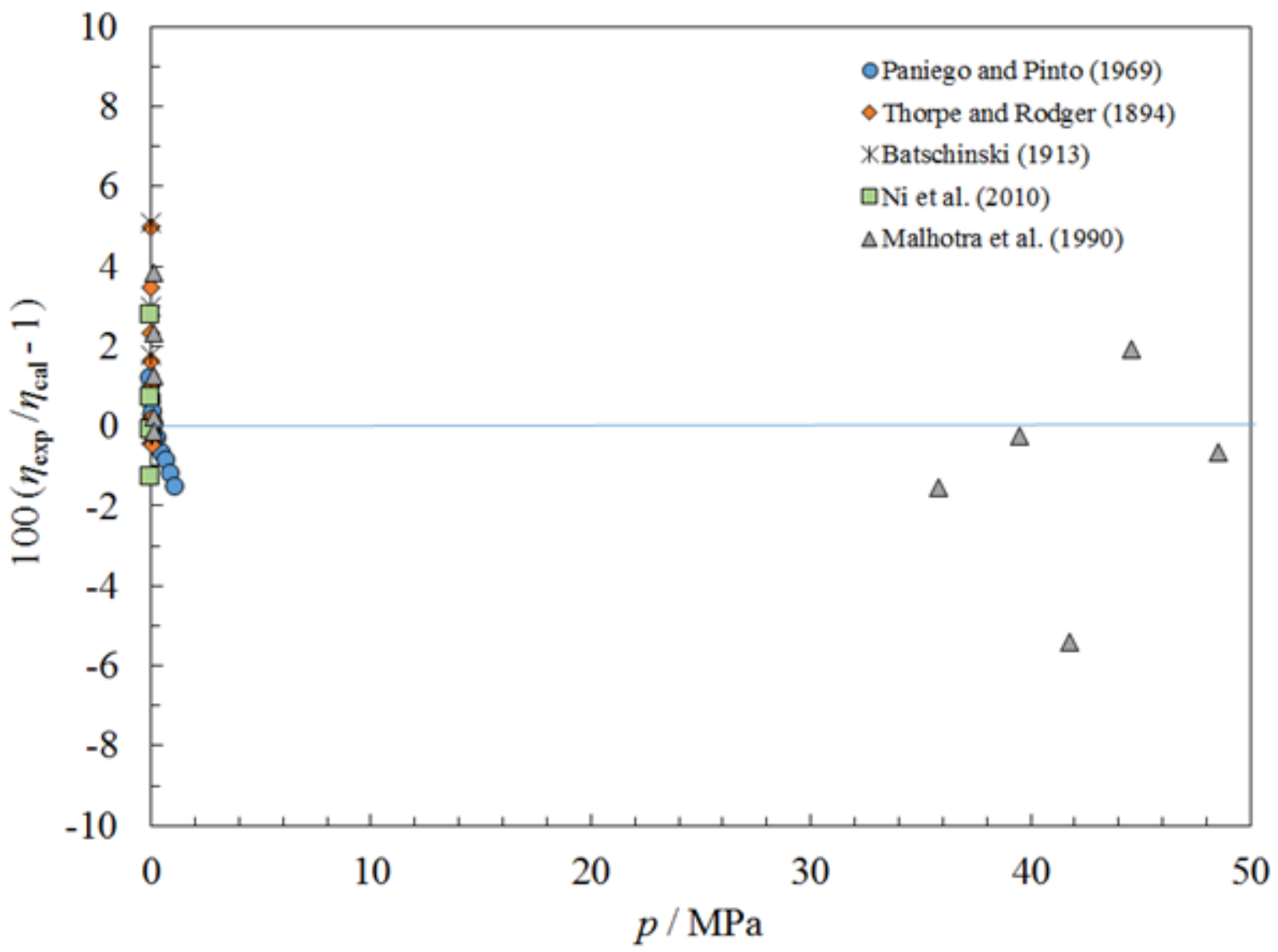

Figure 110. Percentage deviations between the model and the experimental viscosity data for $\mathrm{R} 150$ as a function of pressure. 


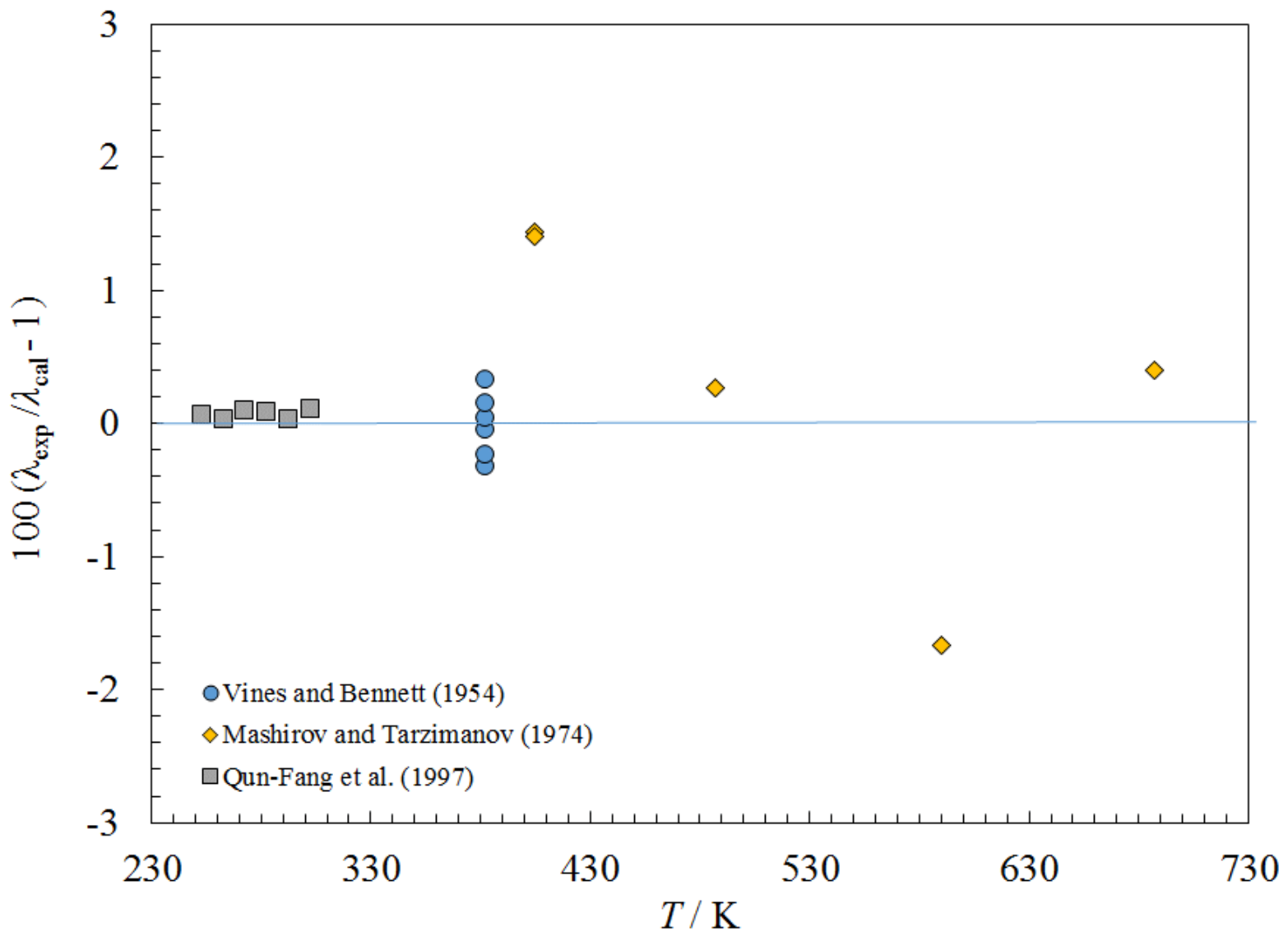

Figure 111. Percentage deviations between the model and the experimental thermal conductivity data for R150. 


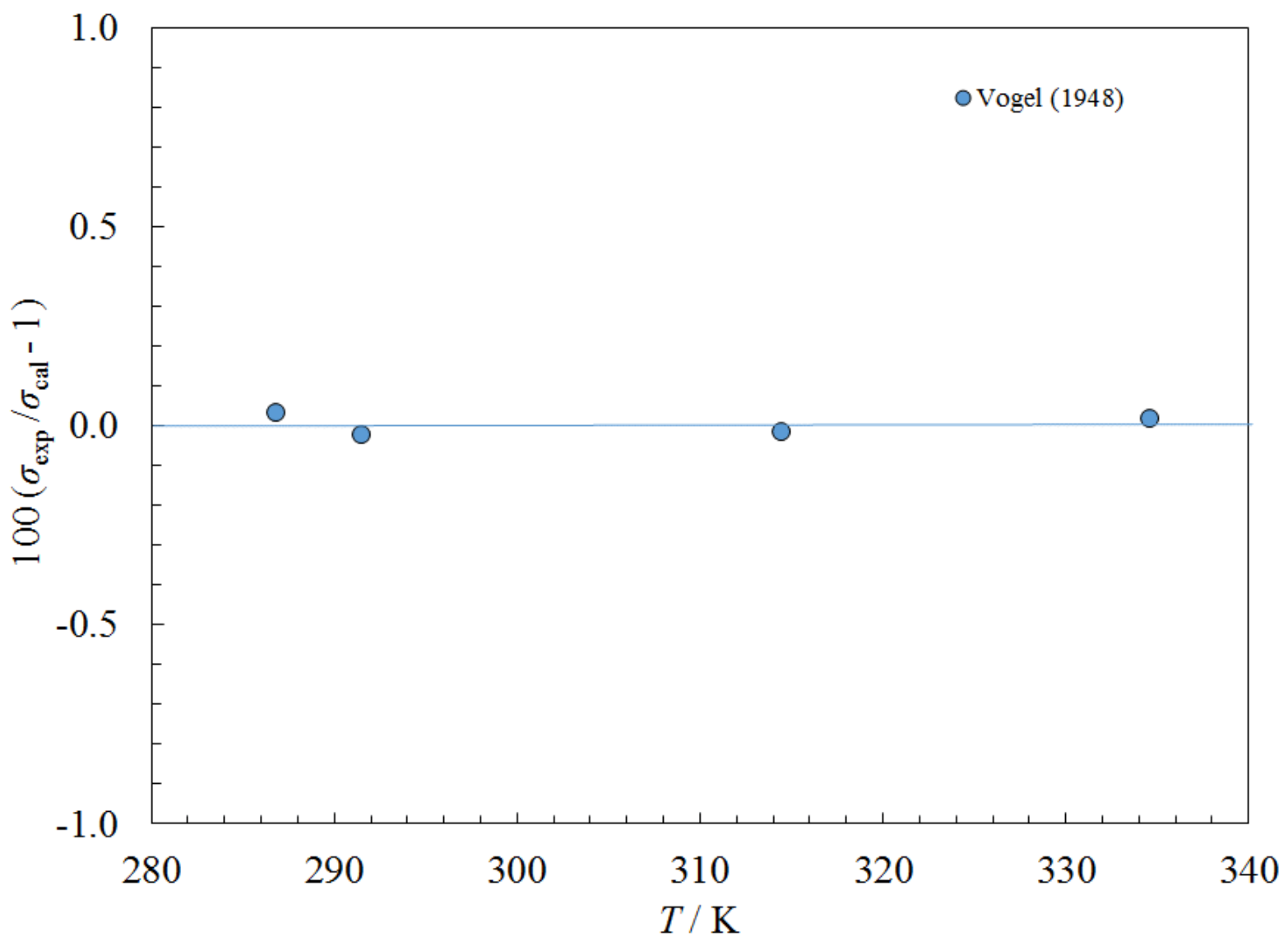

Figure 112. Percentage deviations between the model and the experimental surface tension data for R150.

\subsubsection{RE143a (Methyl trifluoromethyl ether)}

The equation of state of Akasaka and Kayukawa (R. Akasaka \& Kayukawa, 2012) was used to provide the density and the critical parameters. Lennard-Jones parameters were estimated with the method of Chung et al. (Chung et al., 1988) with parameters as given in Table 1. We were unable to locate any gas-phase viscosity data, and only one liquid point (Uchida et al., 2004) was found. Parameter values are given in Table 2. The coefficient $c_{0}$ was adjusted until agreement with the single viscosity data point was obtained with R134a as a reference fluid. The estimated uncertainty for the gas phase is $20 \%, 5 \%$ for the saturated liquid and higher at higher pressures. Similarly, only a single liquid-phase thermal conductivity data point was found (Uchida et al., 2004), and parameters are given in Table 3. Parameters for the critical enhancement are presented in Table 4 . The dilute-gas thermal conductivity coefficient was adjusted to be similar to other fluorinated ethers (RE347mcc, RE245cb). The estimated uncertainty for thermal conductivity in the gas phase is $20 \%, 5 \%$ for the saturated liquid and higher at higher pressures and near the critical point. A single surface tension point was found (Uchida et al., 2004), and the parameters in Table 5 were obtained by scaling the surface tension curve of another fluorinated ether (RE245cb) until agreement with the single datum was obtained. The estimated uncertainty is $10 \%$. 


\subsubsection{Chlorobenzene}

The equation of state of Thol et al. (Thol, Alexandrov, Span, \& Lemmon, 2018) was used to provide the density and the critical parameters. Lennard-Jones parameters were obtained from the method of Chung et al. (Chung et al., 1988). Liquid-phase data from three sources (Abdullaev, Akhundov, \& Ishkhanov, 1983; Abdullaev \& Djafarova, 1980; Singh \& Sinha, 1985) were used to obtain the coefficients in Table 2, and Figure 113 shows deviations with those data sources and the gas-phase data of Titani (Titani, 1927) as a function of temperature, while Figure 114 shows the deviations as a function of pressure. We estimate that the uncertainty in the gas and the liquid at pressures to $40 \mathrm{MPa}$ is $5 \%$, rising to $10 \%$ at $100 \mathrm{MPa}$ (the upper limit of the EOS). The liquidphase data of Bachmann (Bachmann, 1969), Guseinov and Mirzoev (Guseinov \& Mirzoev, 1975), Kashiwagi et al. (Kashiwagi, Oishi, Tanaka, Kabota, \& Makita, 1982), Abdullaev and Iskenderov (Abdullaev \& Iskenderov, 1980), and Nieto de Castro et al. (Nieto de Castro, Dix, Fareleira, Li, \& Wakeham, 1989) and the gas-phase data of Guseinov and Mirzoev (Guseinov \& Mirzoev, 1975) were used to obtain the coefficients in Table 3. Although we used multiple data sets in the regression, the most reliable data are those of Nieto de Castro et al. (Nieto de Castro et al., 1989) that unfortunately cover a more restricted temperature range. Figures 115 and 116 show percentage deviations between these data and the correlation as a function of temperature and of pressure, respectively. The estimated uncertainty in the gas phase is $20 \%, 3 \%$ in the liquid at temperatures below $360 \mathrm{~K}$ and pressures to $100 \mathrm{MPa}$, rising to $20 \%$ at higher temperatures due to inconsistencies in the data sets. The data of Guseinov and Mirzoev (Guseinov \& Mirzoev, 1975) do not agree with the data of Abdullaev and Iskenderov (Abdullaev \& Iskenderov, 1980); additional measurements are needed to resolve this discrepancy. Two sets of surface tension data that cover different temperature ranges (Jaeger, 1917; Ramsay \& Shields, 1893) were used to obtain the coefficients in Table 5. There is a large amount of scatter that is shown in Figure 117, and we estimate the uncertainty is $5 \%$. 


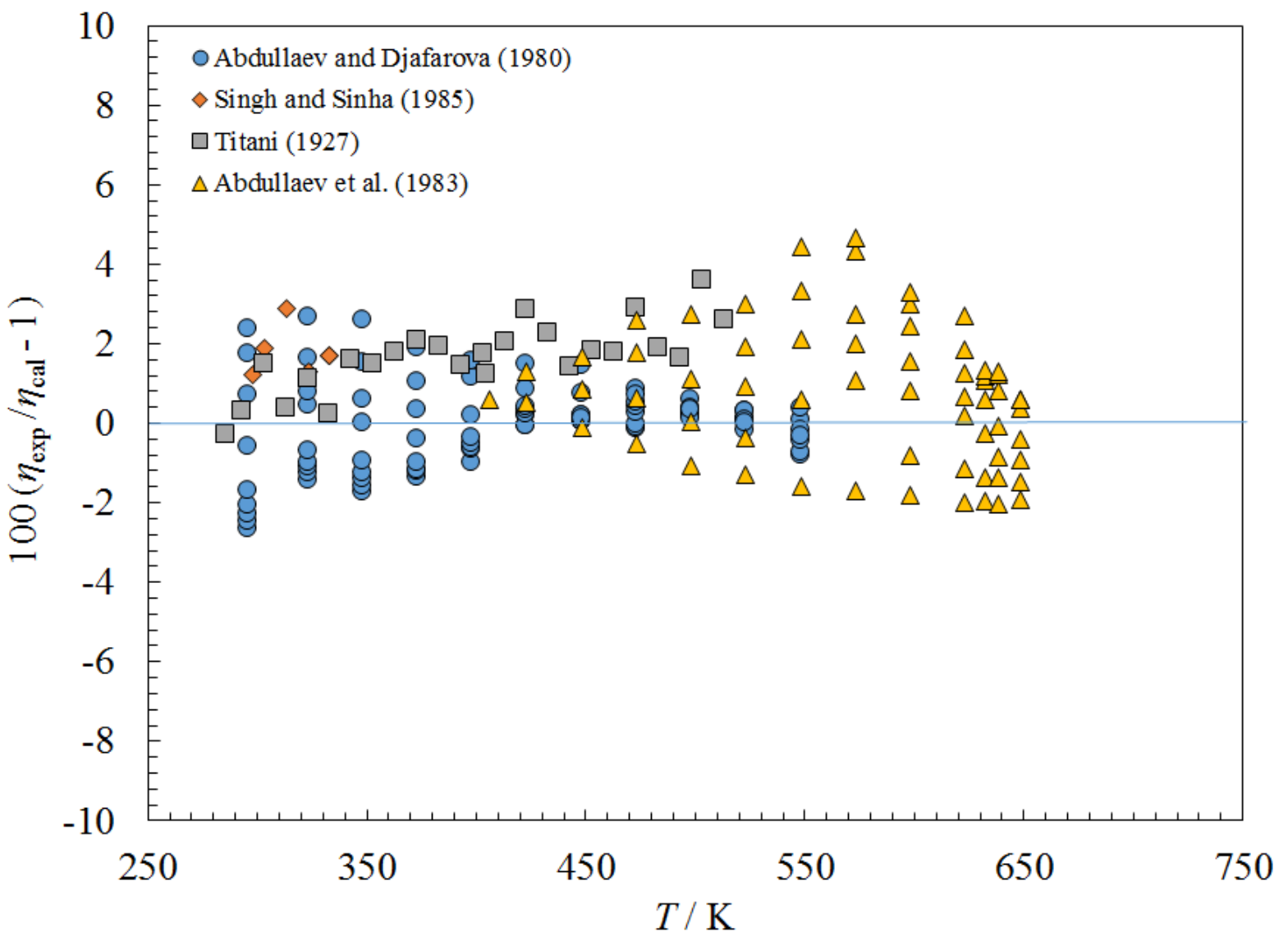

Figure 113. Percentage deviations between the model and the experimental viscosity data for chlorobenzene as a function of temperature. 


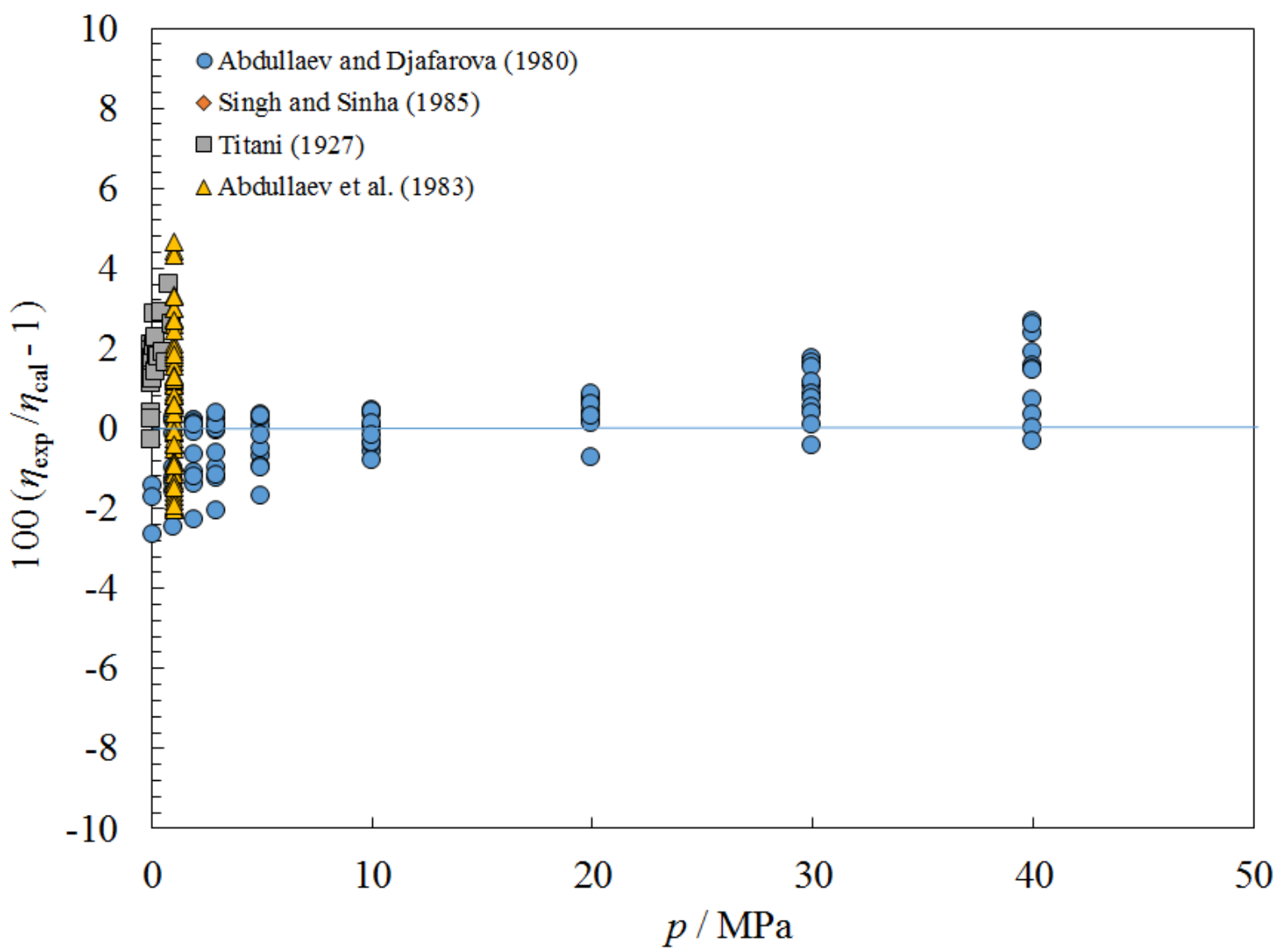

Figure 114. Percentage deviations between the model and the experimental viscosity data for chlorobenzene as a function of pressure. 


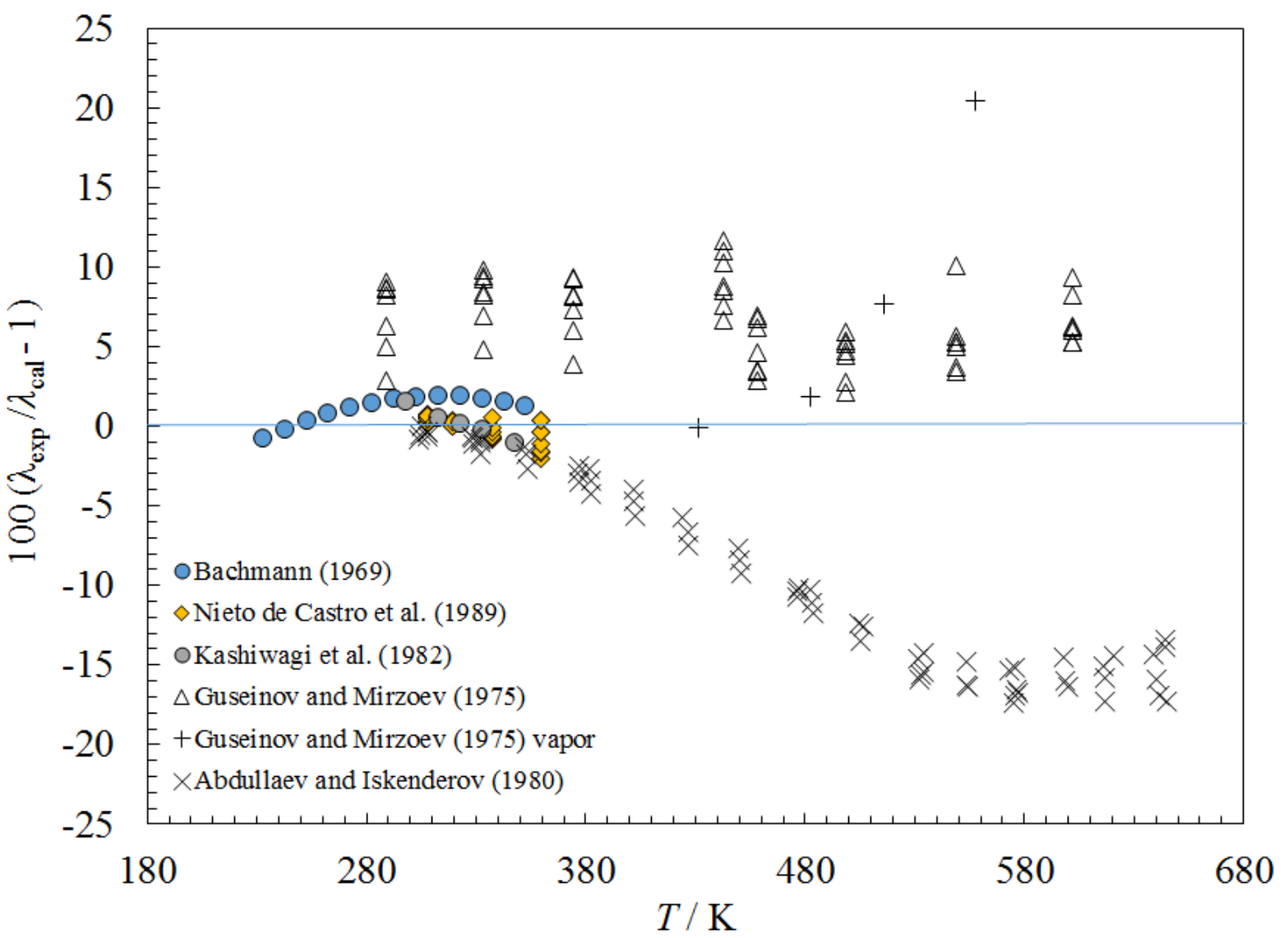

Figure 115. Percentage deviations between the model and the experimental thermal conductivity data for chlorobenzene as a function of temperature. 


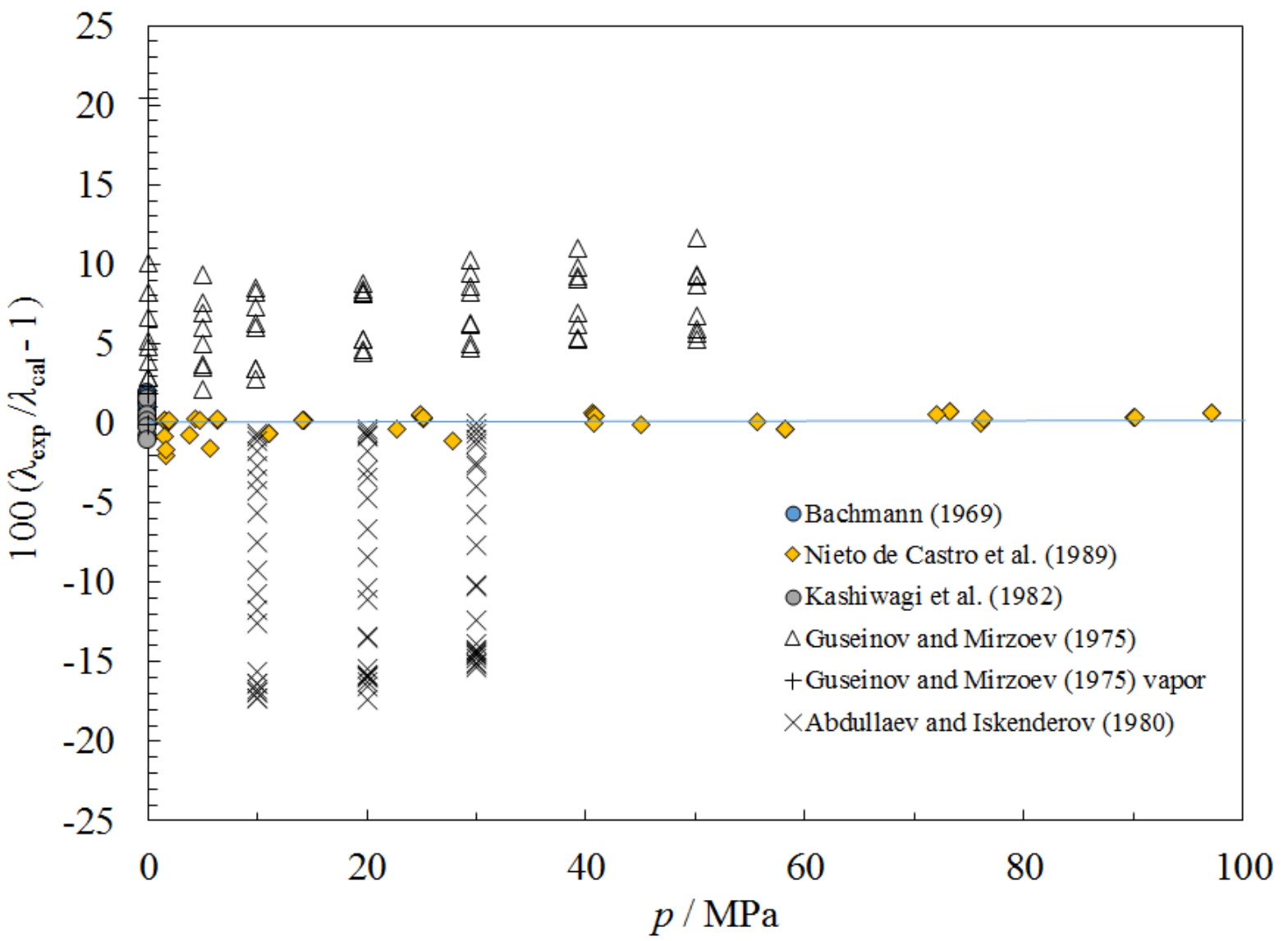

Figure 116. Percentage deviations between the model and the experimental thermal conductivity data for chlorobenzene as a function of pressure. 


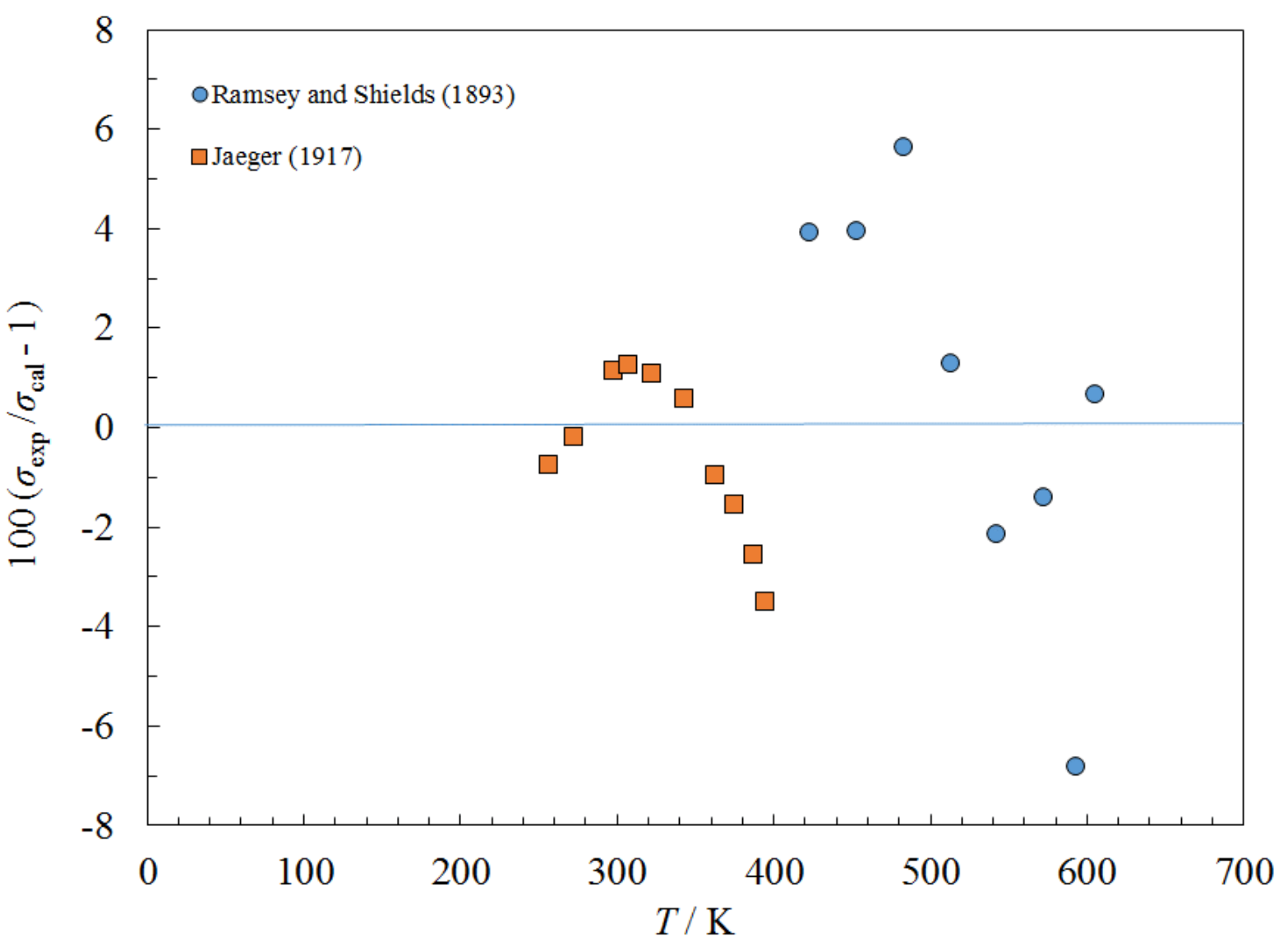

Figure 117. Percentage deviations between the model and the experimental surface tension data for chlorobenzene.

\subsubsection{R1234ze(Z) (Cis-1,3,3,3-tetrafluoropropene)}

The equation of state of Akasaka and Lemmon (R. Akasaka \& Lemmon, 2018) was used to provide the density and the critical parameters. Lennard-Jones parameters were calculated by the method of Chung et al. (Chung et al., 1988). Values are presented in Table 1. No published experimental data were found for viscosity; however, we obtained some preliminary unpublished values (Miyara, 2018a) and used those data to obtain the coefficients in Table 2. The preliminary data were along the saturation boundary in both the gas and liquid phases; no high-pressure data were available. Figure 118 shows deviation plots for the viscosity from the experimental data. Estimated uncertainty for viscosity in the saturated liquid phase is $3 \%, 4 \%$ for vapor from $300 \mathrm{~K}$ to $450 \mathrm{~K}$, higher at higher pressures. Similarly, for thermal conductivity published experimental data were unavailable, but we obtained some preliminary unpublished values (Miyara, 2018a) and used those data to obtain the coefficients in Table 3 . The preliminary data were along the saturation boundary in both the gas and liquid phases; no high-pressure data were available. Figure 119 shows deviation plots for the thermal conductivity from the experimental data. Not shown are 3 vapor-phase points at $\sim 354 \mathrm{~K}$ that are approximately $14 \%$ lower than the estimated values and appear to be anomalous. Estimated uncertainty for the thermal conductivity in the saturated liquid phase is $3 \%, 4 \%$ for vapor from $300 \mathrm{~K}$ to $430 \mathrm{~K}$, higher at higher pressures. We note that all data 
used for these correlations were preliminary and may be subject to additional analysis and possible change. Surface tension is discussed in Kondou et al. (Kondou \& Koyama, 2015).

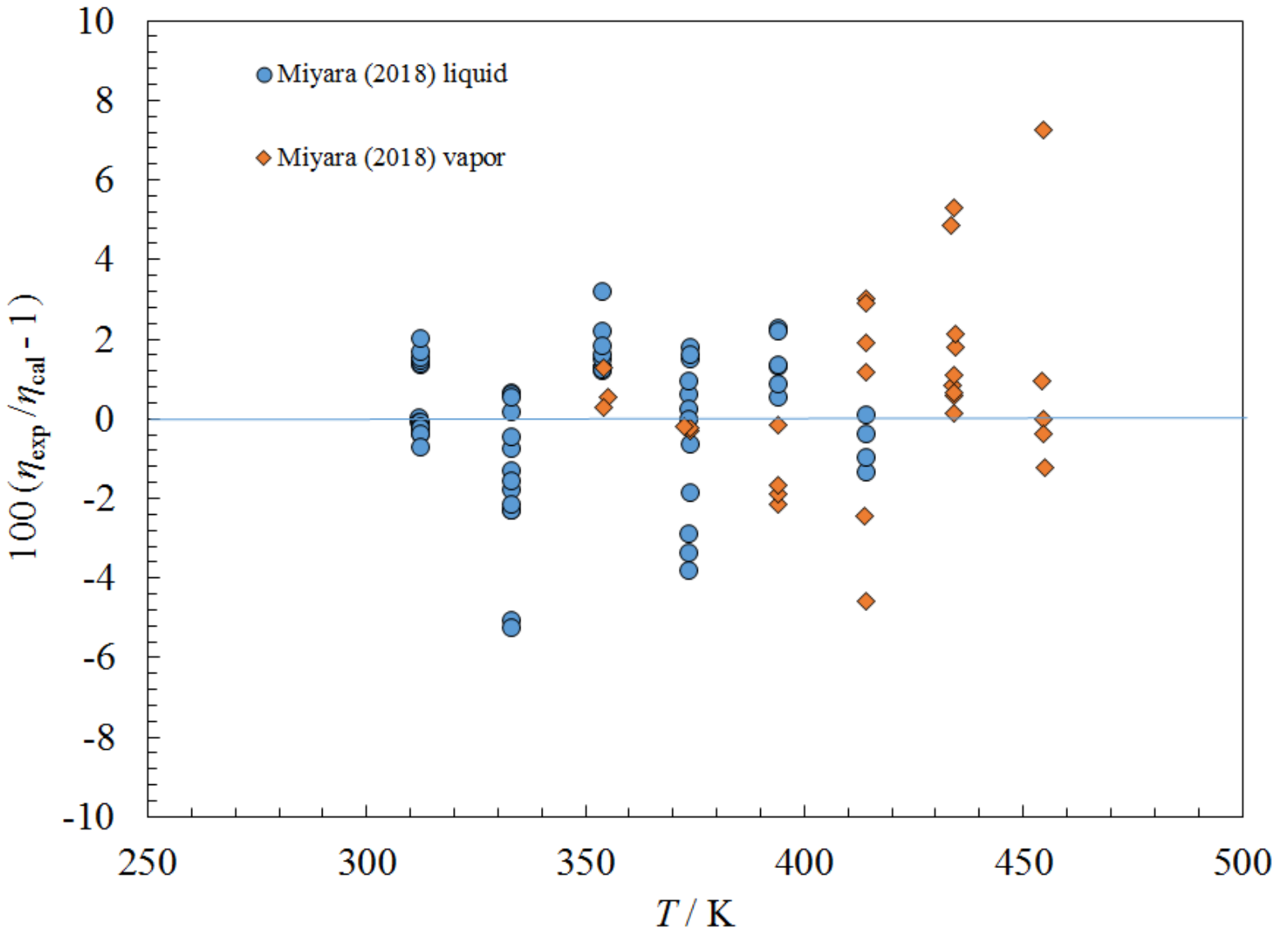

Figure 118. Percentage deviations between the model and the experimental viscosity data for R1234ze(Z). 


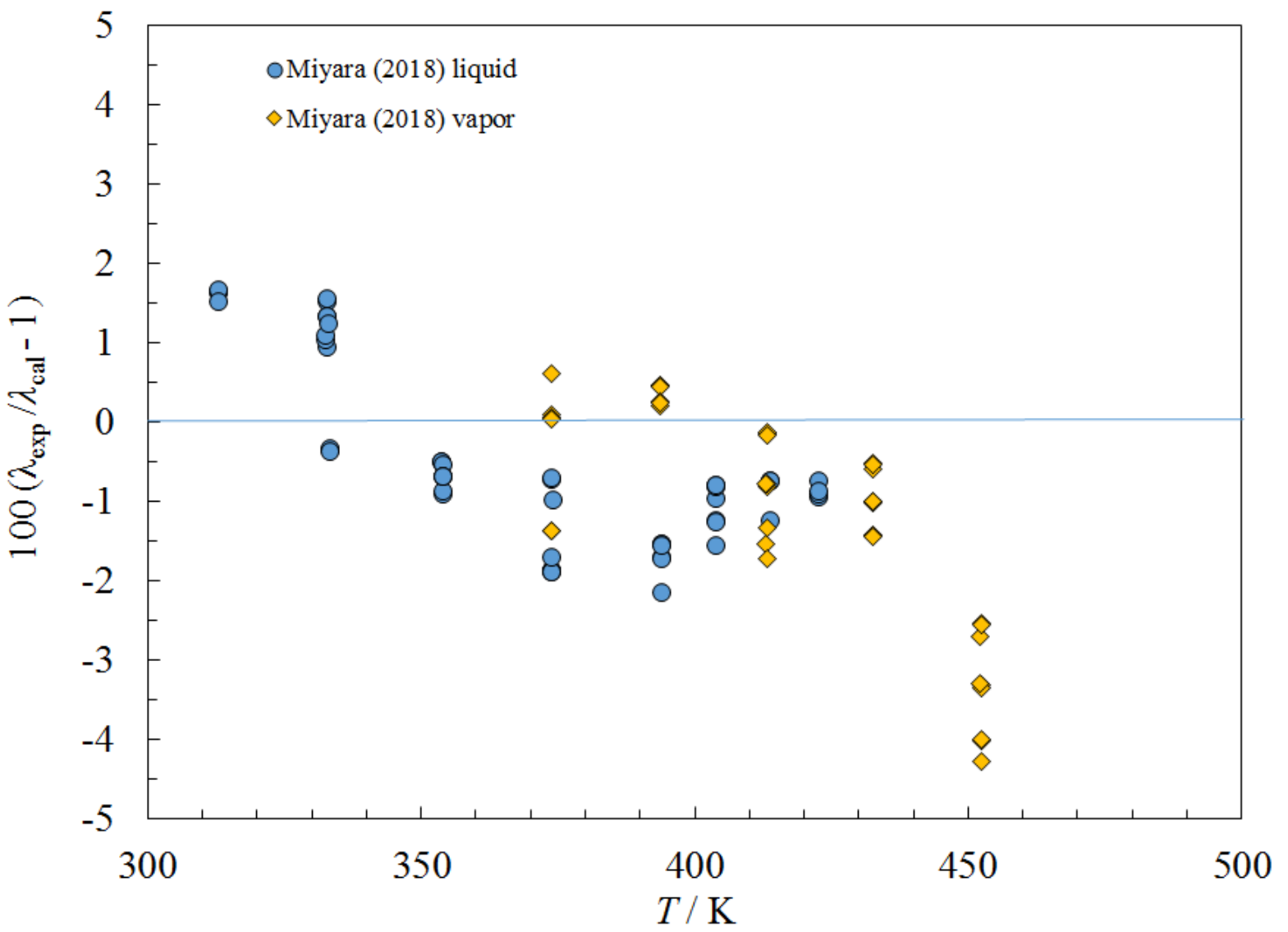

Figure 119. Percentage deviations between the model and the experimental thermal conductivity data for R1234ze(Z).

\subsubsection{R1233zd(E) (Trans-1-chloro-3,3,3-trifluoro-1-propene)}

The equation of state of Mondejar et al. (Mondéjar, McLinden, \& Lemmon, 2015) was used to provide the density and the critical parameters. Lennard-Jones parameters were calculated by the method of Chung et al. (Chung et al., 1988); values are presented in Table 1. Preliminary experimental gas-phase viscosity data (Miyara, 2018c) were used to obtain the coefficients in Table 2, and preliminary liquid-phase data (Meng, 2017; Miyara, 2018c) were used to obtain the liquid-phase coefficients in Table 2. Figures 120 and 121 show viscosity deviations as a function of temperature and of pressure. It should be noted that all viscosity data are unpublished and preliminary, and may be subject to change. The reader should consult the literature for the final publications for these data sets. The estimated uncertainty is $4 \%$ for the gas, and $4 \%$ for the liquid at pressures to $40 \mathrm{MPa}$. The thermal conductivity (R. Perkins \& Huber, 2017) and surface tension (Kondou \& Koyama, 2015) are covered in separate publications. 


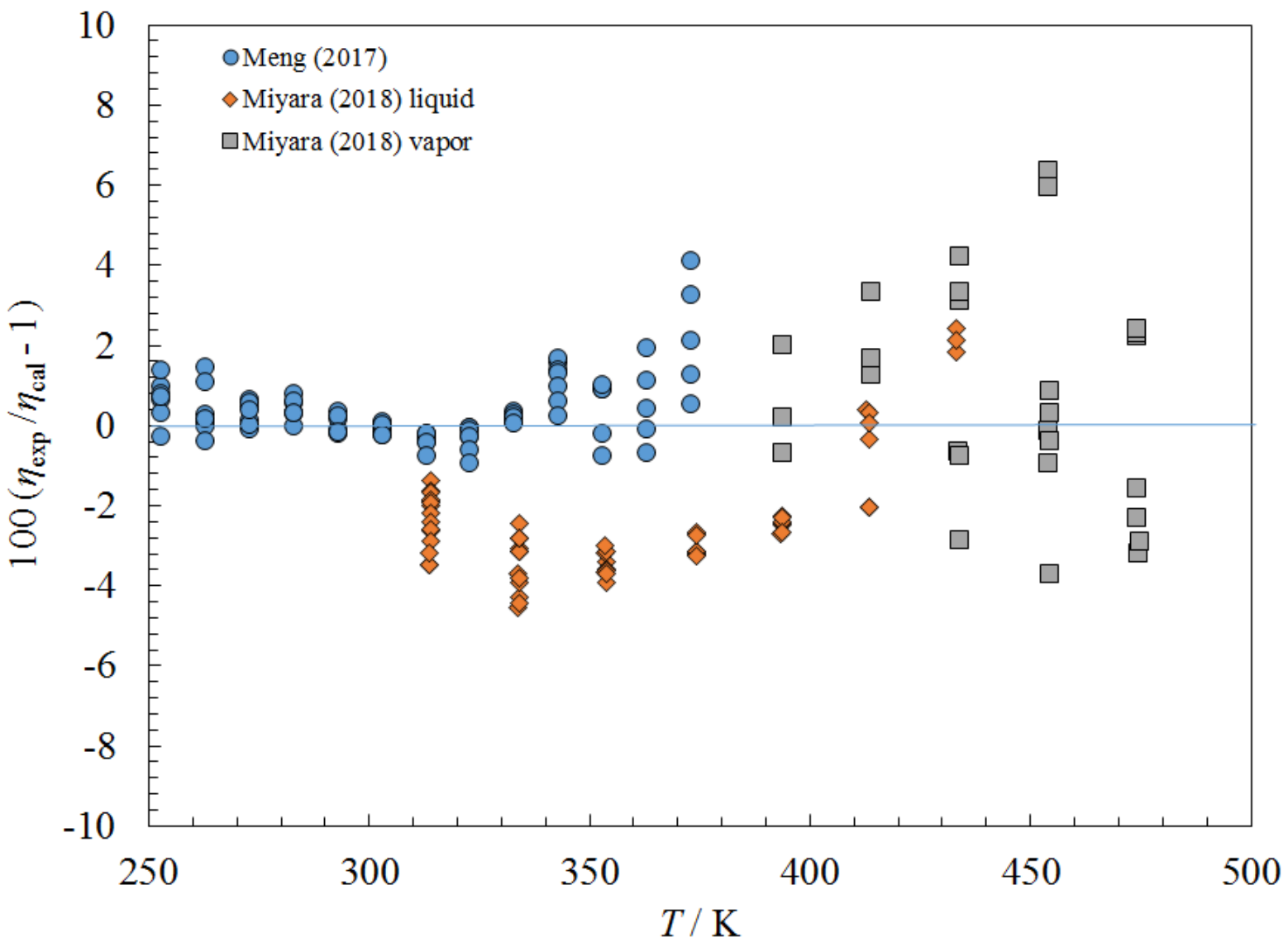

Figure 120. Percentage deviations between the model and the experimental viscosity data for R1233zd(E) as a function of temperature. 


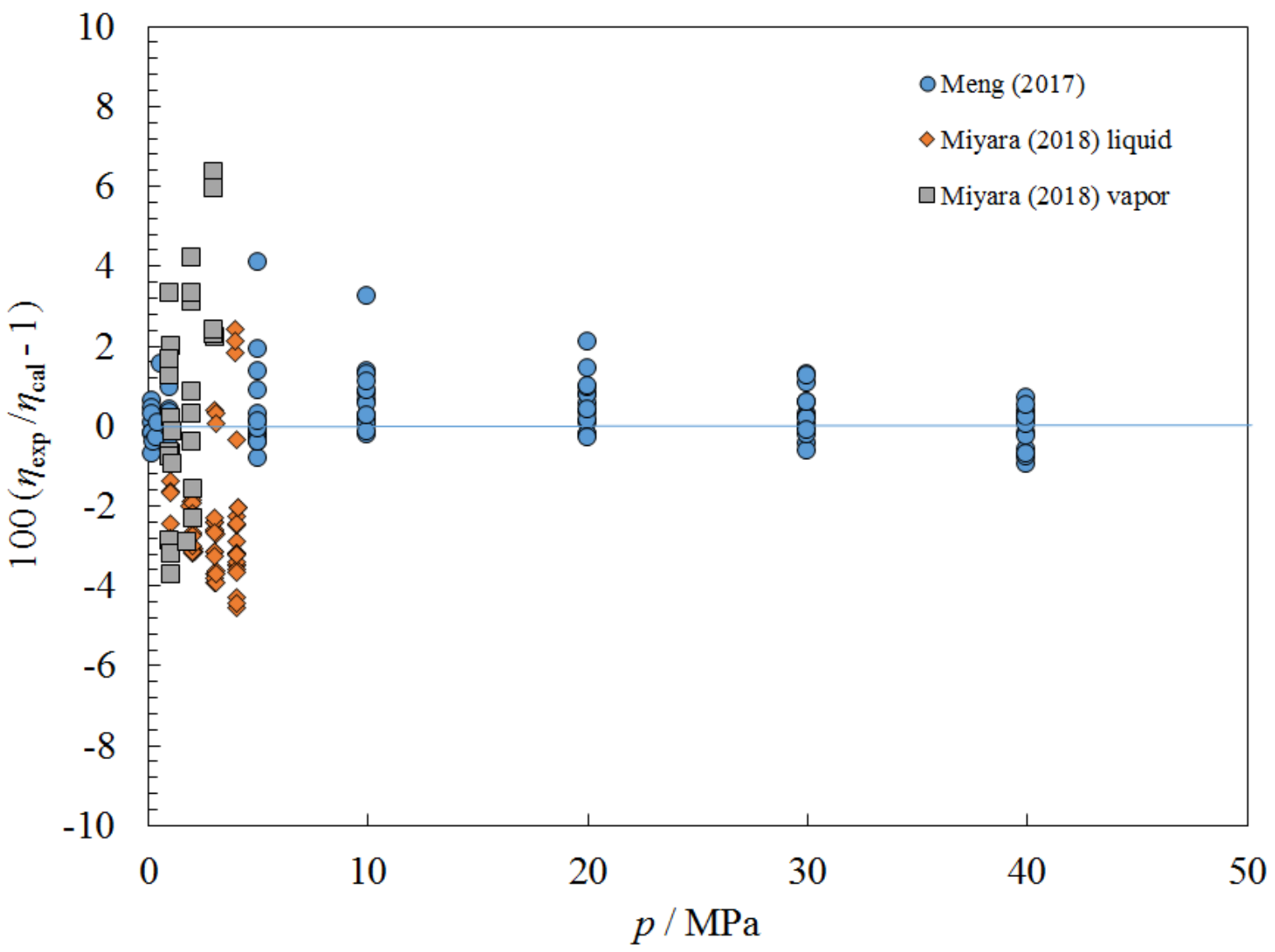

Figure 121. Percentage deviations between the model and the experimental viscosity data for $\mathrm{R} 1233 \mathrm{zd}(\mathrm{E})$ as a function of pressure.

\subsubsection{R245ca (1,1,2,2,3-Pentafluoropropane)}

The equation of state of Zhou and Lemmon (Zhou \& Lemmon, 2016) was used to provide the density and the critical parameters. This fluid is a fluorinated propane, and is an isomer of R245fa. Lennard-Jones parameters were estimated with the method of Chung et al. (Chung et al., 1988) with parameters as given in Table 1. The saturated liquid data of Laesecke and Hafer (Laesecke \& Hafer, 1998) were used to obtain the coefficients in Table 2, and deviations are shown in Figure 122. No data off of the saturation boundary were found, or in the gas phase. The estimated uncertainty in the gas phase is $10 \%$, and $2 \%$ for the saturated liquid from $248 \mathrm{~K}$ to $333 \mathrm{~K}$, higher outside of that range. The gas-phase thermal conductivity of Perkins et al. (R. Perkins et al., 2001) and Heinemann et al. (Heinemann, Klaen, Yourd, \& Dohrn, 2000) were used to obtain the thermal conductivity coefficients in Table 3 . Parameters for the critical enhancement are presented in Table 4. Since liquid-phase thermal conductivity data were not available, we scaled the saturated liquid thermal conductivity in reduced temperature for R245fa and used it to obtain the coefficients in Table 3. Deviations are shown in Figure 123. The estimated uncertainty in the gas phase is $5 \%$, $10 \%$ in the saturated liquid, higher elsewhere. Surface tension is discussed in Mulero et al. (Mulero et al., 2012) 


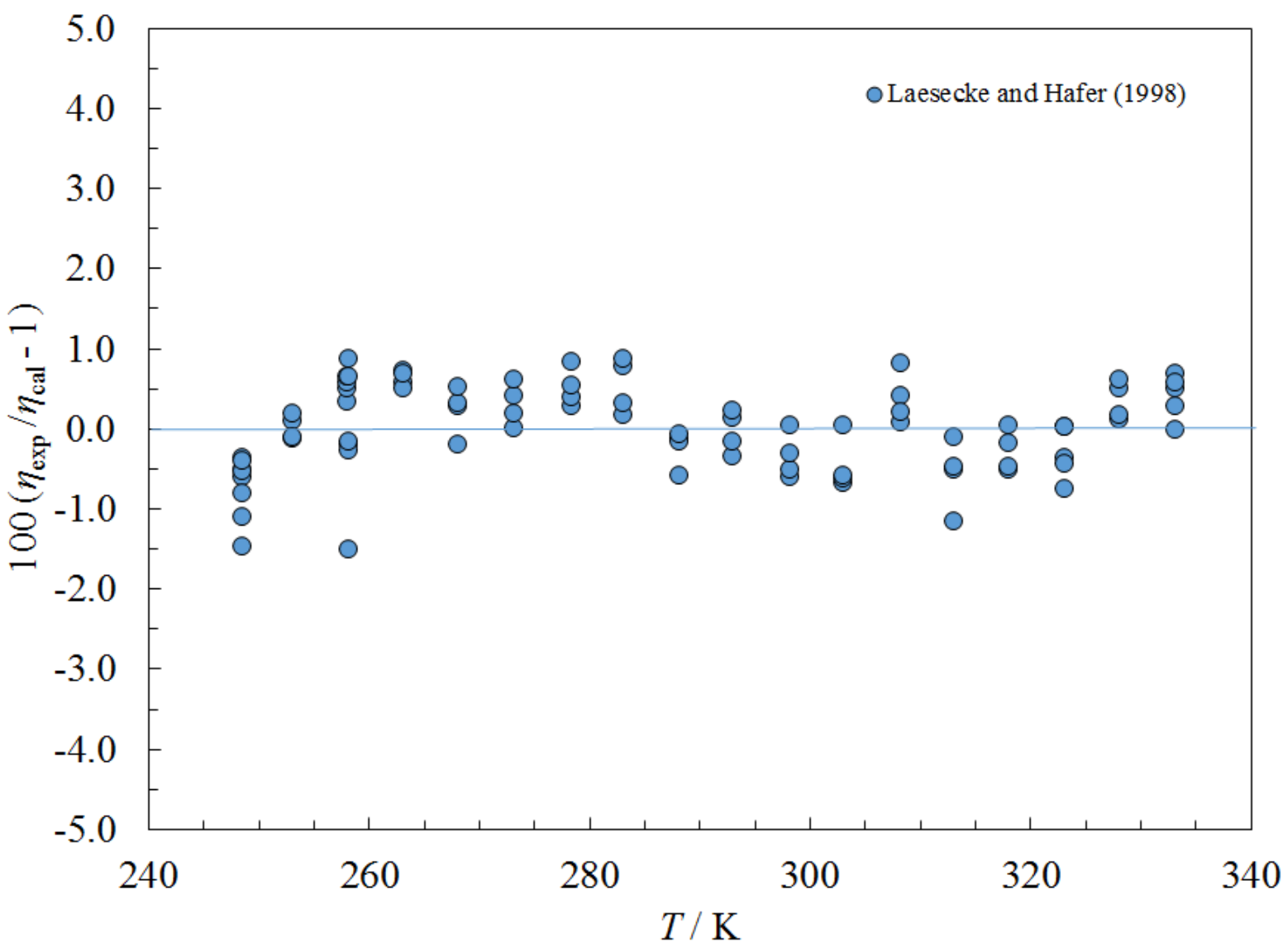

Figure 122. Percentage deviations between the model and the experimental viscosity data for R245ca as a function of temperature. 


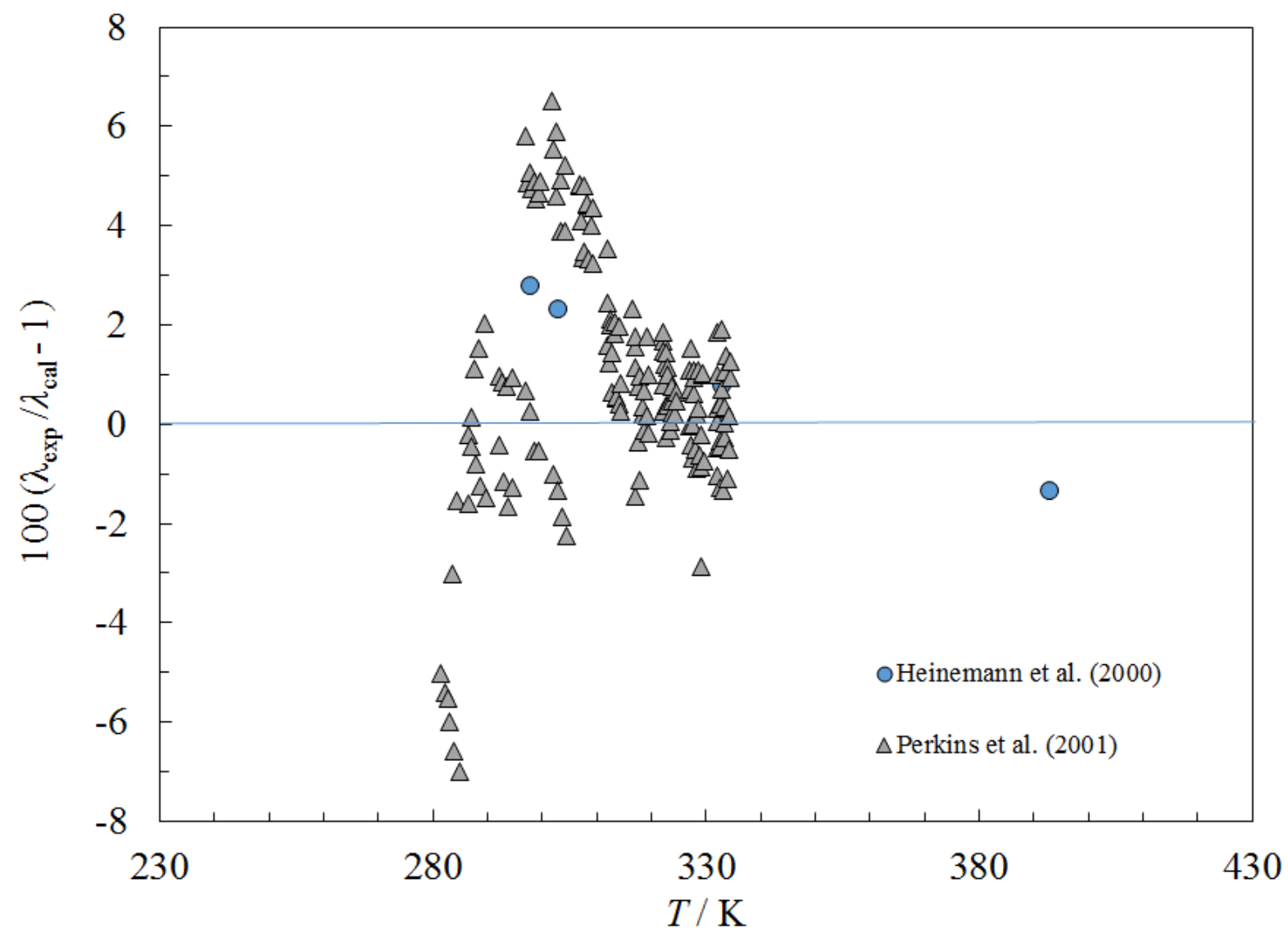

Figure 123. Percentage deviations between the model and the experimental thermal conductivity data for R245ca as a function of temperature.

\subsubsection{R365mfc (1,1,1,3,3-Pentafluorobutane)}

The equation of state of Lemmon and Span (E.W. Lemmon \& Span, 2015) was used to provide the density and the critical parameters. Lennard-Jones parameters were estimated with the method of Chung et al. (Chung et al., 1988), with parameters as given in Table 1. The saturated liquid data of Fröba et al. (Fröba, Krzeminski, \& Leipertz, 2004) were used to obtain the coefficients in Table 2, and deviations are shown in Figure 124. The data were converted from kinematic to dynamic viscosity using densities from the equation of state of Lemmon and Span (E.W. Lemmon \& Span, 2015). The estimated uncertainty in the gas phase is $10 \%$, and $2 \%$ for the saturated liquid from $253 \mathrm{~K}$ to $373 \mathrm{~K}$, higher elsewhere. The gas-phase thermal conductivity data of Marrucho et al. (Marrucho, Oliveira, \& Dohrn, 2002) and the saturated liquid data of Fröba et al. (Fröba et al., 2004) were used to obtain the thermal conductivity coefficients in Table 3. Parameters for the critical enhancement are presented in Table 4. Deviations are shown in Figure 125. The estimated uncertainty in the gas phase is $2 \%, 4 \%$ in the saturated liquid, higher elsewhere. Surface tension is discussed in Mulero et al. (Mulero et al., 2012) 


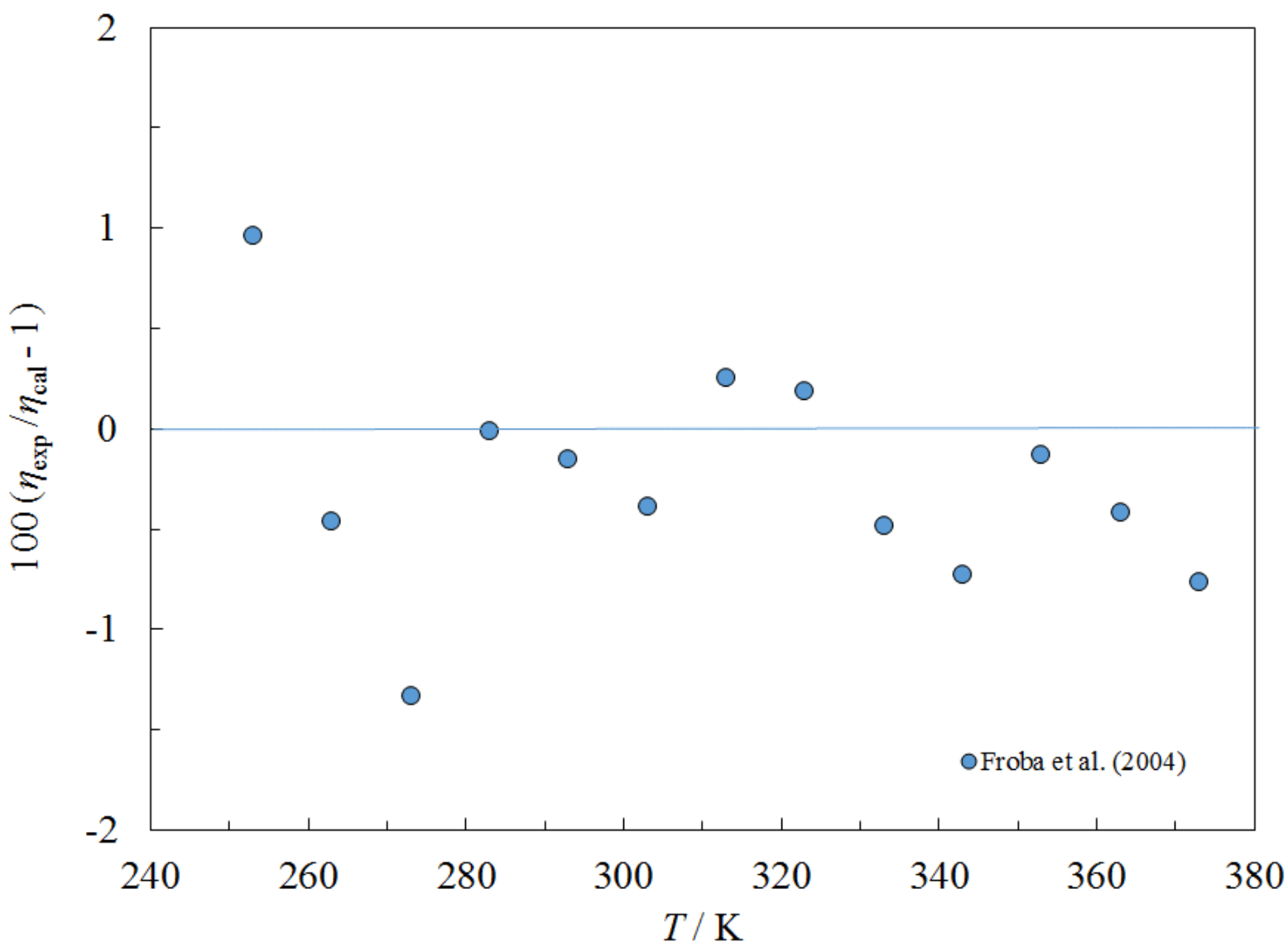

Figure 124. Percentage deviations between the model and the experimental viscosity data for R365mfc as a function of temperature. 


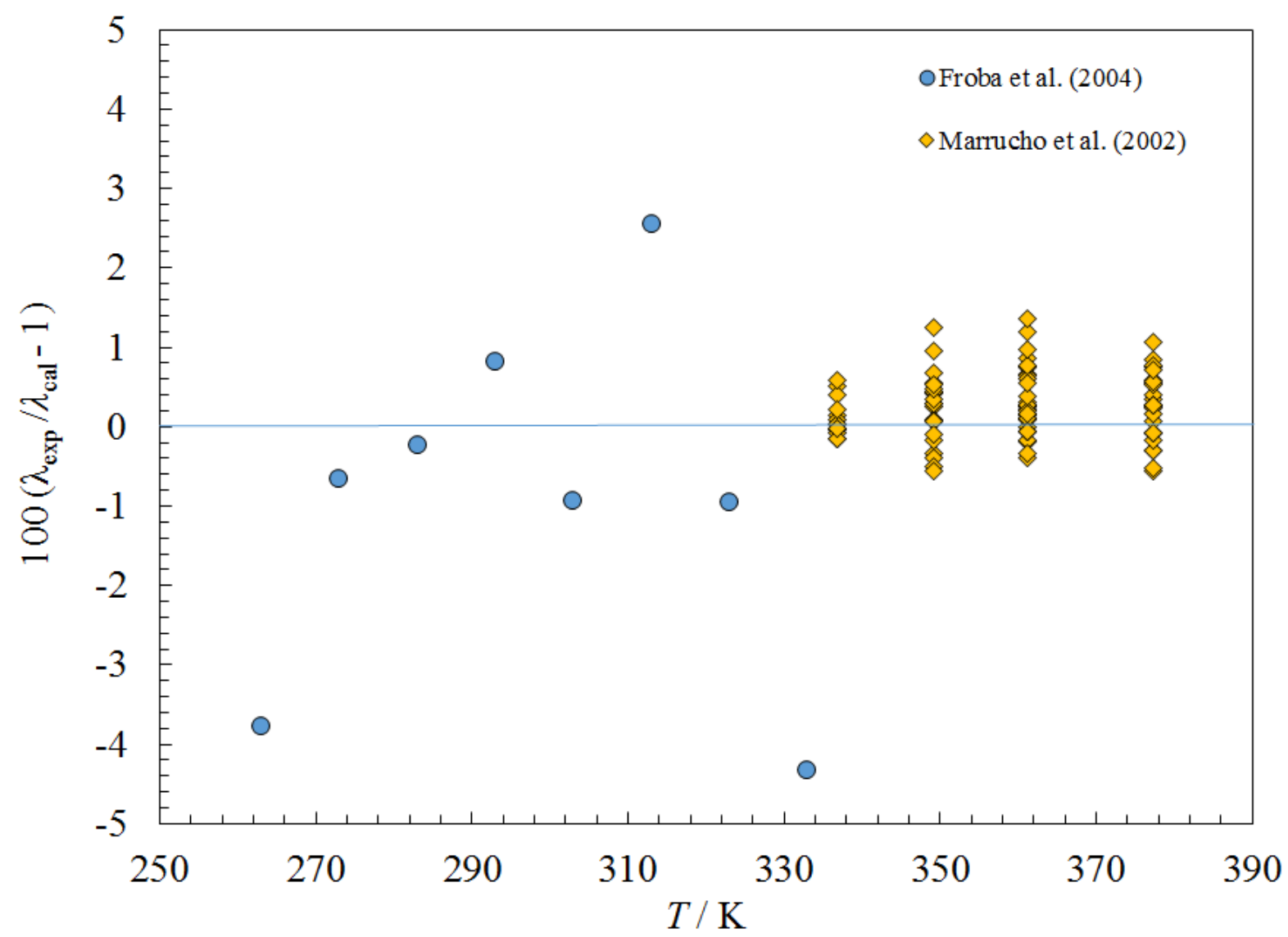

Figure 125. Percentage deviations between the model and the experimental thermal conductivity data for R365mfc as a function of temperature.

\subsubsection{R1224yd(Z) ((Z)-1-Chloro-2,3,3,3-tetrafluoropropene)}

The equation of state of Akasaka et al. (R. Akasaka, Fukushima, \& Lemmon, 2017) was used to provide the density and the critical parameters, and an acentric factor of 0.322 . LennardJones parameters were calculated by the method of Chung et al. (Chung et al., 1988); values are presented in Table 1 The dipole moment was estimated to be $4.903 \times 10^{-30} \mathrm{C} \cdot \mathrm{m}(1.47 \mathrm{D})$ (Kazakov, 2017). Using the method of Chung et al. (Chung et al., 1988) results in a value of $0.92 \mathrm{for} F_{\mathrm{c}}$ as reported in Table 2. Preliminary unpublished data from Miyara (Miyara, 2018b) were used to obtain the residual coefficients in Table 2. Figures 126 and 127 show comparison with the experimental data. Experimental data were unavailable for thermal conductivity, and a totally predictive model was used for the coefficients in Tables 3 and 4 . We estimate that the uncertainty for viscosity is $4 \%$ and for thermal conductivity is approximately $20 \%$ along the saturation boundary and higher as the pressure increases. $\mathrm{R} 1224 \mathrm{yd}(\mathrm{Z})$ is a fluorinated propene that also contains one chlorine atom. It is very similar to the compound R1233zd(E), trans-1-chloro-3,3,3trifluoro-1-propene, also a fluorinated propene with one chlorine atom. Since there are no surface tension data available, as a very rough estimate, we adopted the same surface tension parameters for $n$ and $\sigma_{0}$ as used for the surface tension of R1233zd(E) (Kondou \& Koyama, 2015) These parameters are given in Table 5 . We estimate the uncertainty for the surface tension is $10 \%$. 


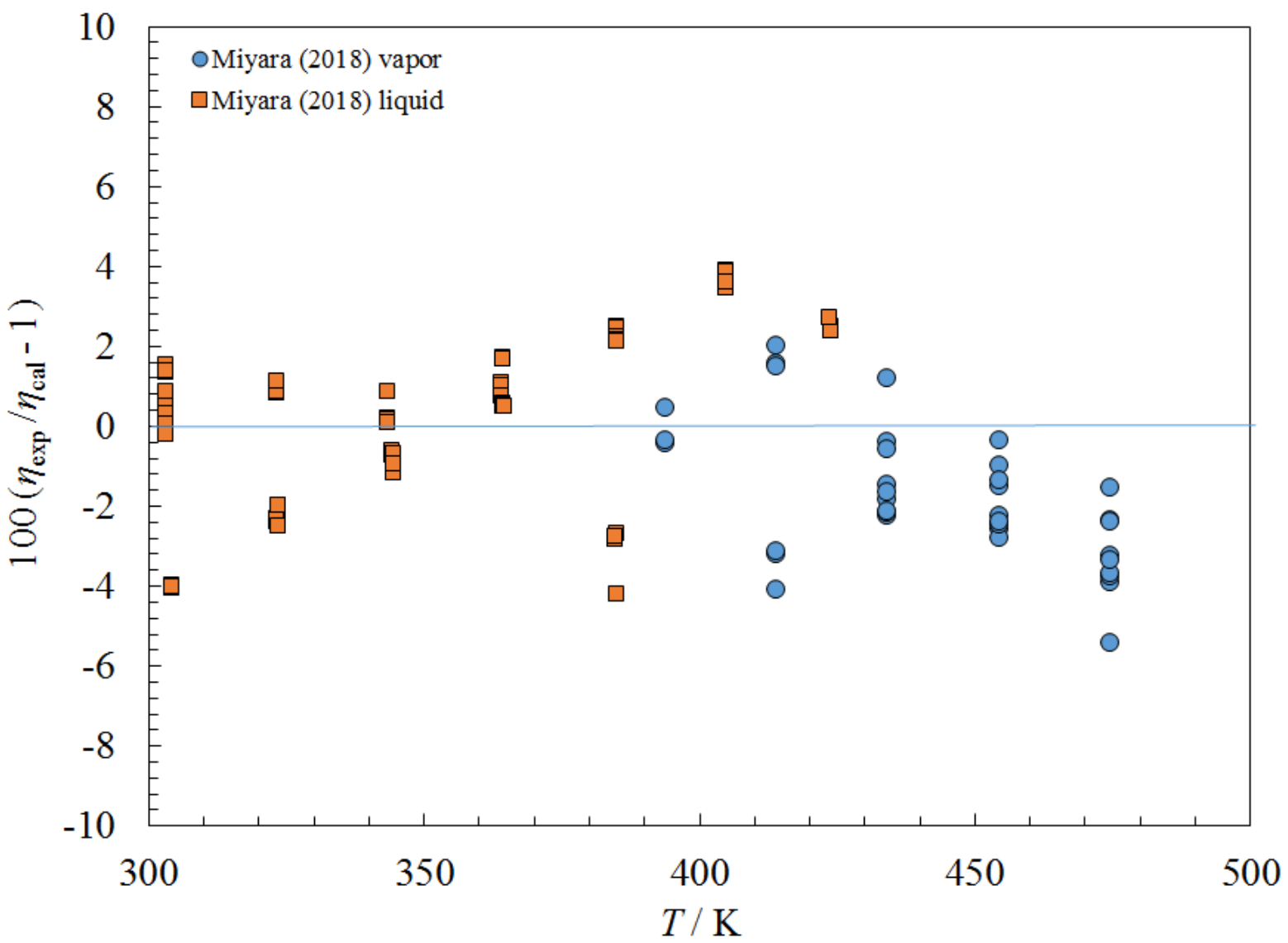

Figure 126. Percentage deviations between the model and the experimental viscosity data for R1224yd(Z) as a function of temperature. 


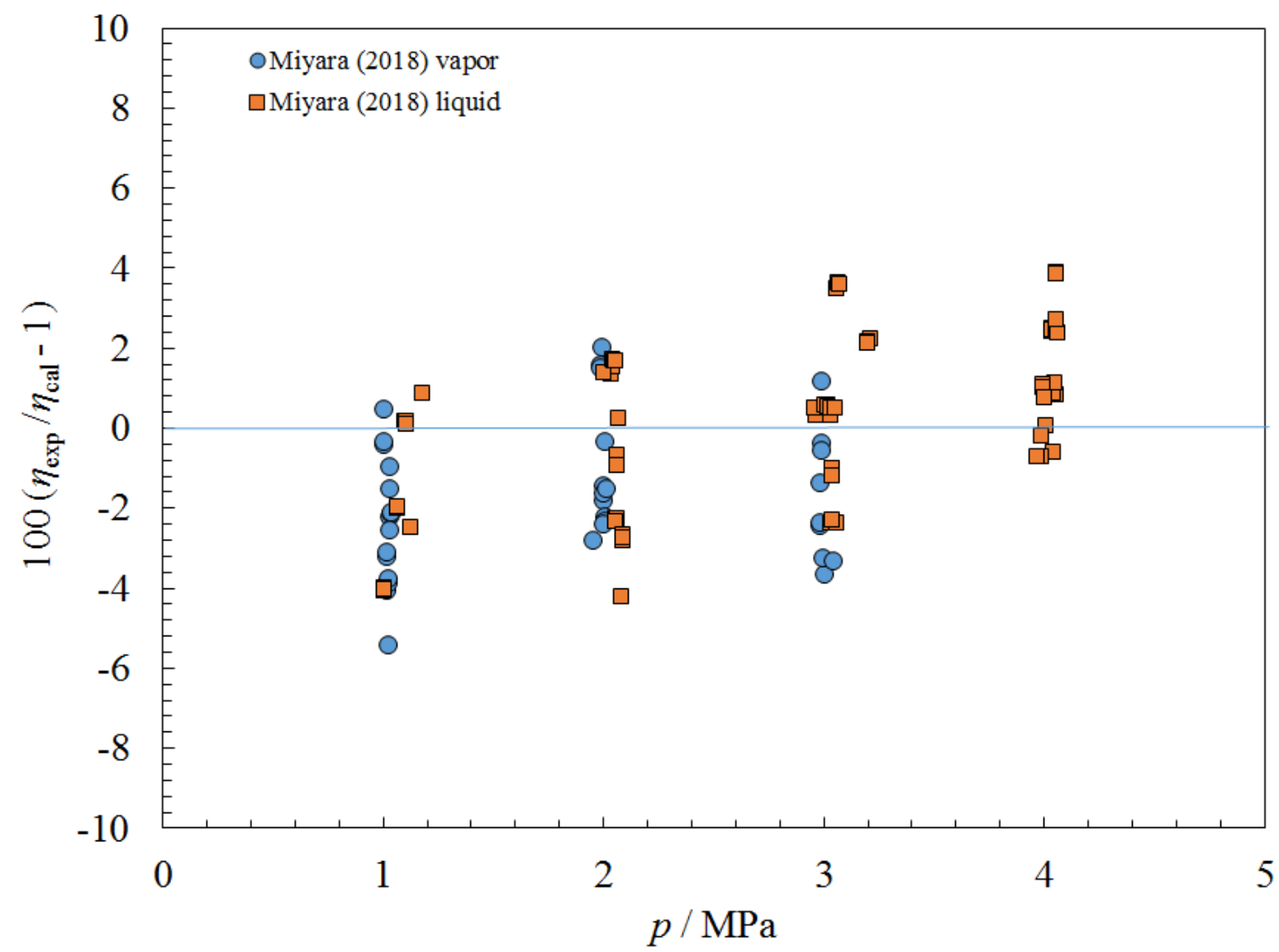

Figure 127. Percentage deviations between the model and the experimental viscosity data for $\mathrm{R} 1224 \mathrm{yd}(\mathrm{Z})$ as a function of pressure.

\subsubsection{RE245cb2 (1,1,1,3,3-Pentafluorobutane)}

The equation of state of Zhou and Lemmon (Zhou \& Lemmon, 2018a) was used to provide the density and the critical parameters. This fluid is a fluorinated ether, methyl-pentafluoroethylether. Lennard-Jones parameters were estimated with the method of Chung et al. (Chung et al., 1988), with parameters as given in Table 1. The liquid-phase viscosity data of Matsuo et al. (Matsuo, Tanaka, \& Sotani, 2002) were used to obtain the coefficients in Table 2, and deviation plots as a function of temperature and pressure are given in Figures 128 and 129. The estimated uncertainty in the gas phase for viscosity is $10 \%$, for the liquid at pressures up to $20 \mathrm{MPa}$ is $3 \%$ over the temperature range $298 \mathrm{~K}$ to $348 \mathrm{~K}$. The liquid-phase thermal conductivity data of Matsuo et al. (Matsuo et al., 2002) and the gas-phase data from Matsuo et al. (Matsuo, Tanaka, Tanada, Yamamoto, \& Sekiya, 1998) and Takada et al. (Takada, Matsuo, Tanaka, \& Sekiya, 1998) were used to obtain the coefficients in Table 3 and deviation plots as a function of temperature and pressure are given in Figures 130 and 131. Parameters for the critical enhancement are presented in Table 4 . The estimated uncertainty in the gas phase for thermal conductivity is $5 \%$, for the 
liquid at pressures up to $10 \mathrm{MPa}$ is $1 \%$ for temperatures from $275 \mathrm{~K}$ to $323 \mathrm{~K}$, higher elsewhere. The surface tension is discussed in (Mulero \& Cachadiña, 2014) and is not discussed here.

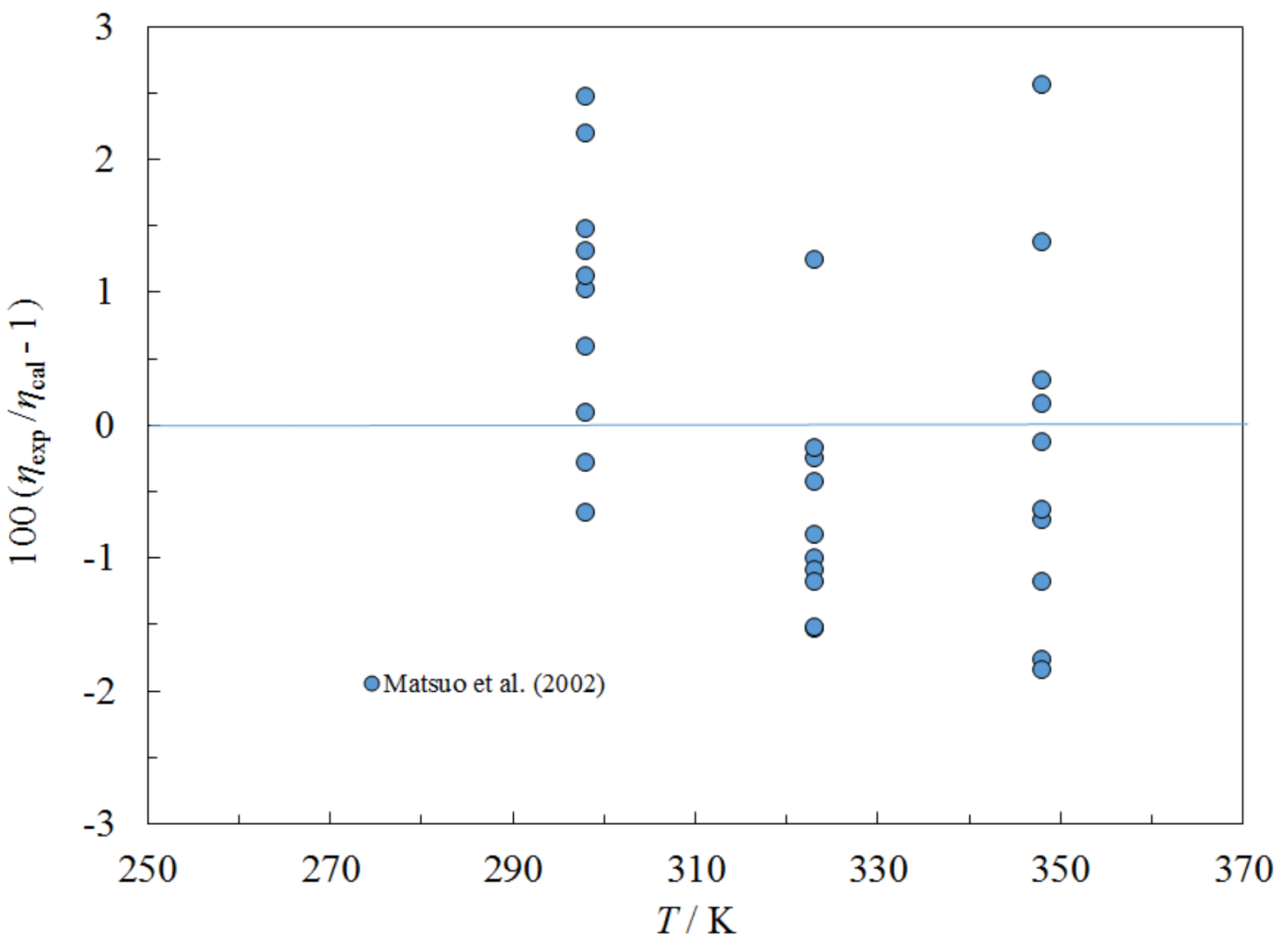

Figure 128. Percentage deviations between the model and the experimental viscosity data for RE245cb2 as a function of temperature. 


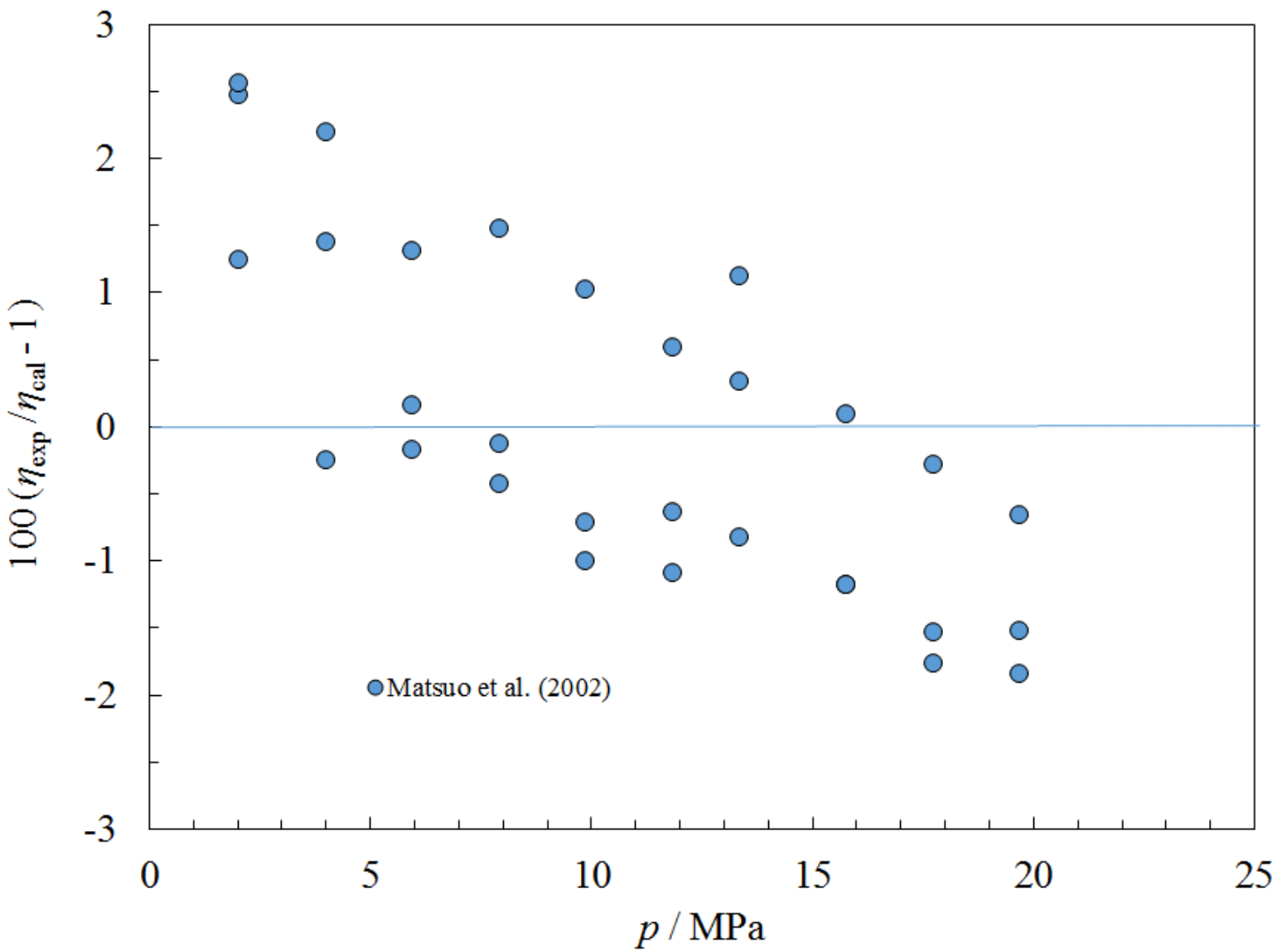

Figure 129. Percentage deviations between the model and the experimental viscosity data for RE245cb2 as a function of pressure. 


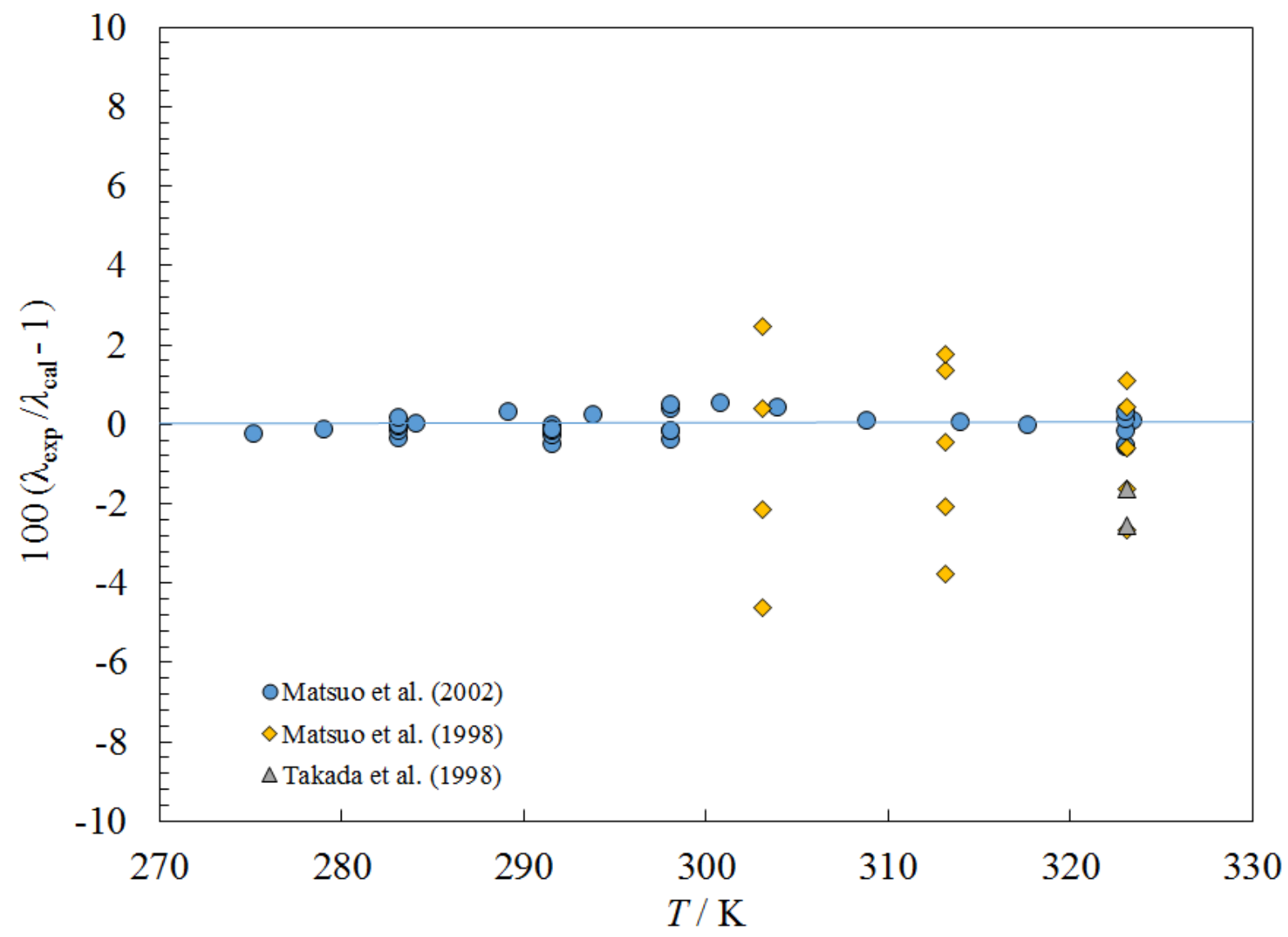

Figure 130. Percentage deviations between the model and the experimental thermal conductivity data for RE245cb2 as a function of temperature. 


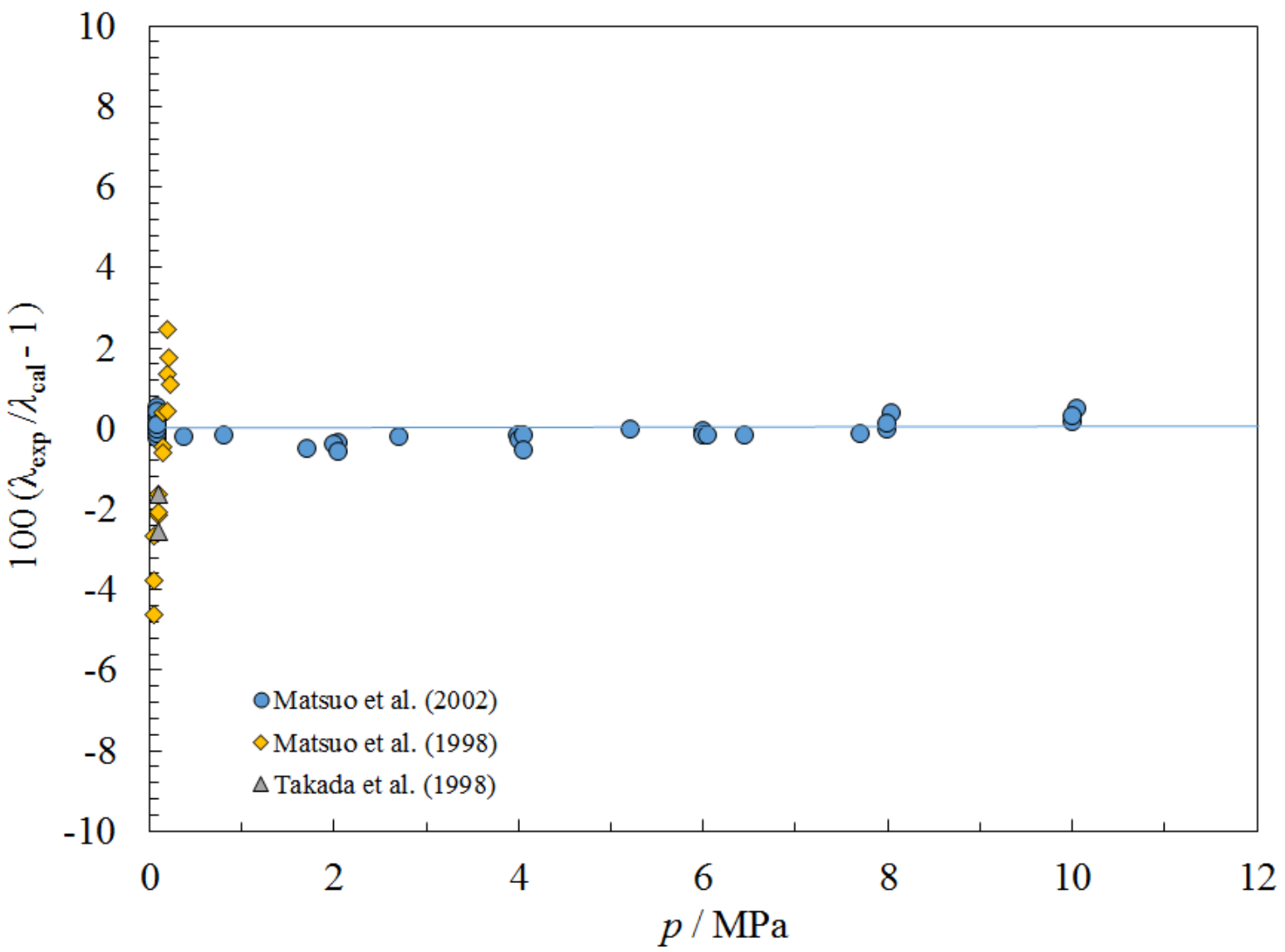

Figure 131. Percentage deviations between the model and the experimental thermal conductivity data for RE245cb2 as a function of pressure.

\subsubsection{RE245fa2 (2,2,2-Trifluoroethyl-difluoromethyl ether)}

The equation of state of Zhou and Lemmon (Zhou \& Lemmon, 2018a) was used to provide the density and the critical parameters. This fluid is a fluorinated ether, and is an isomer of RE$245 \mathrm{cb} 2$. Lennard-Jones parameters were estimated with the method of Chung et al. (Chung et al., 1988), with parameters as given in Table 1. The saturated-liquid viscosity data of Ripple and Matar were used to obtain the coefficients in Table 2, and a deviation plot as a function of temperature is given in Figure 132. The estimated uncertainty in the gas phase for viscosity is 10 $\%$, for the liquid along the saturation boundary is $2 \%$ at temperatures from $250 \mathrm{~K}$ to $332 \mathrm{~K}$. There were no data off of the saturation boundary, so it is difficult to assess uncertainty but we estimate $10 \%$ at pressures up to $20 \mathrm{MPa}$. The gas-phase data of Perkins et al. (R. Perkins et al., 2001) were used to obtain the gas-phase thermal conductivity coefficients in Table 3. Parameters for the critical enhancement are presented in Table 4. Liquid-phase data for thermal conductivity were unavailable; the coefficients in Table 3 were selected to give thermal conductivity values of liquid RE-245fa2 similar to RE-245cb2. A deviation plot is given in Figure 133. The data of Takada et al. (Takada et al., 1998) and of Matsuo et al. (Matsuo et al., 1998) are shown for comparison only; they were not used in the regression. The estimated uncertainty in the gas phase is $4 \%$, and $10 \%$ in the liquid. The surface tension is discussed in (Mulero \& Cachadiña, 2014) and is not discussed here. 


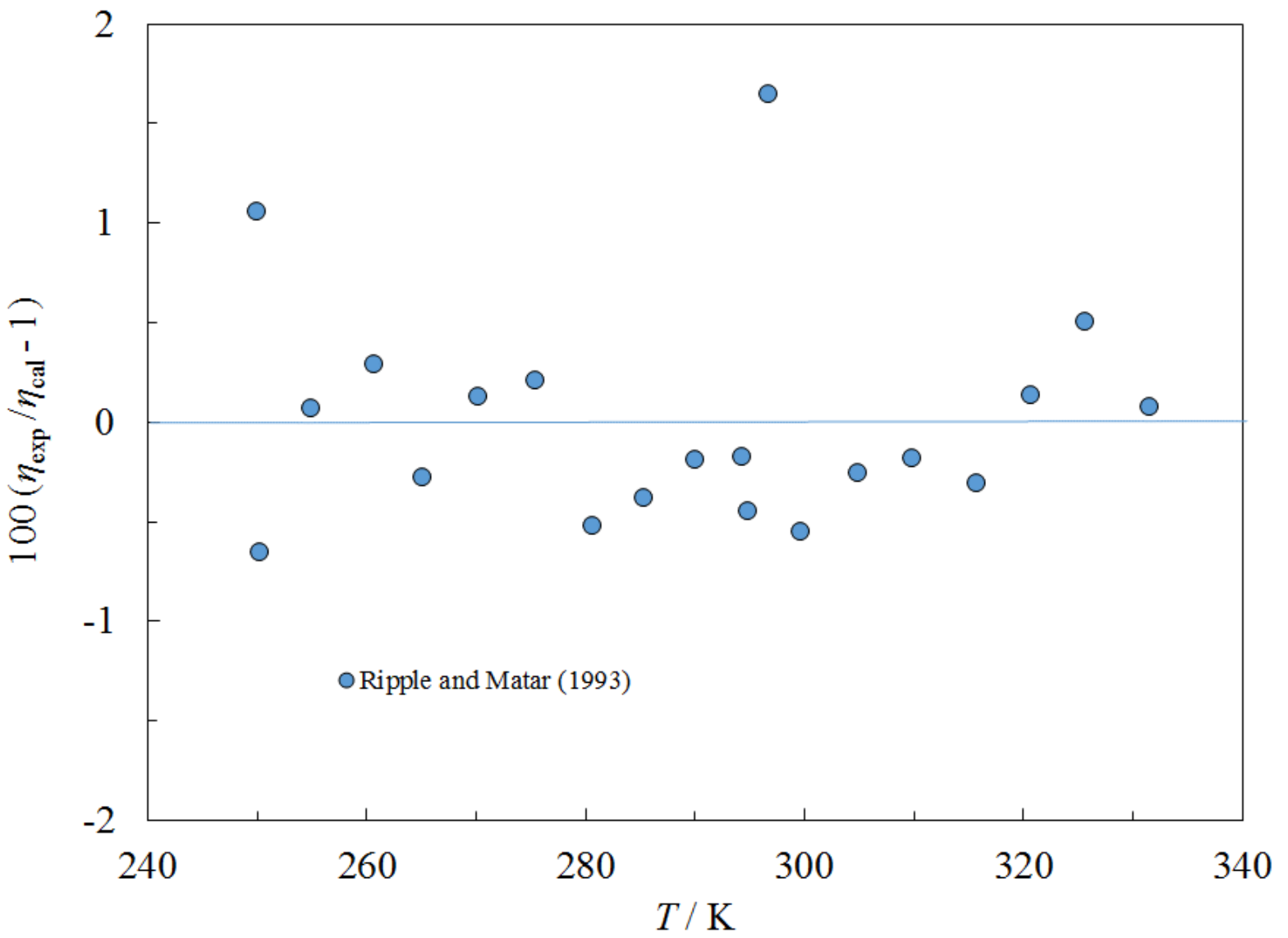

Figure 132. Percentage deviations between the model and the experimental viscosity data for RE245fa2 as a function of temperature. 


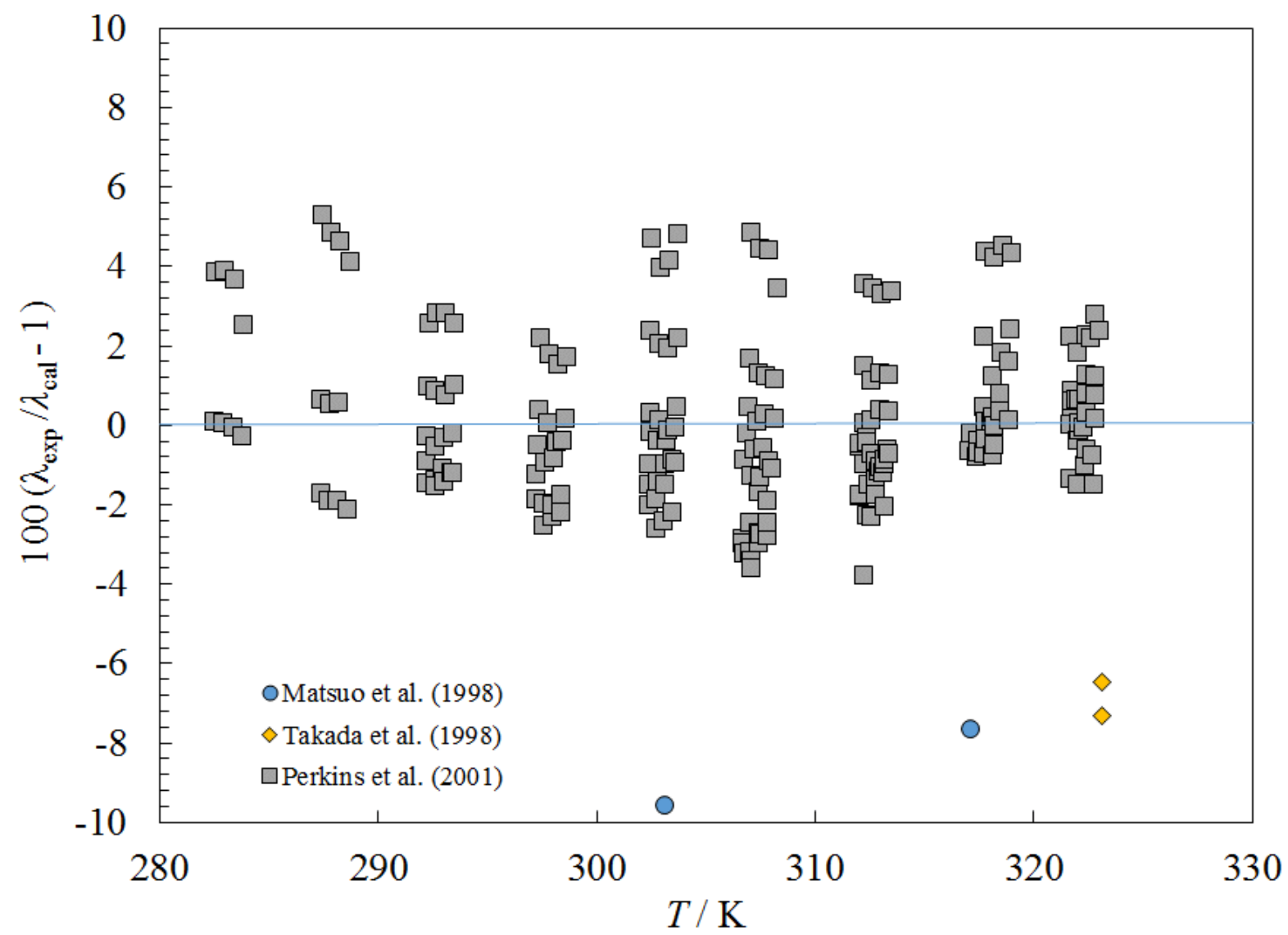

Figure 133. Percentage deviations between the model and the experimental thermal conductivity data for RE245fa2 as a function of temperature.

\subsubsection{R236fa (1,1,1,3,3,3-Hexafluoropropane)}

The equation of state of Pan et al. (Pan, Rui, Zhao, \& Qiu, 2012) was used to provide the density and the critical parameters. Lennard-Jones parameters were estimated with a method that scales R134a as described in Huber and Ely (Huber \& Ely, 1992b). Liquid-phase data of Laesecke and Defibaugh (Laesecke \& Defibaugh, 1996) and of Meng et al. (Meng, Zhang, \& Wu, 2011) were used to obtain the coefficients in Table 2, and Figures 134 and 135 show deviations from the data as a function of temperature and pressure. Gas-phase data of Wang et al.(X. Wang, Wang, Song, Lv, \& Liu, 2014) are also shown. We estimate that the uncertainty of the viscosity in the liquid phase at pressures up to $40 \mathrm{MPa}$ from $303 \mathrm{~K}$ to $373 \mathrm{~K}$ is $2 \%$, and the uncertainty in the gas phase is $5 \%$. Data from Perkins et al. (R. Perkins et al., 2001) and Pan et al. (Pan, Li, \& Wu, 2011) were used to obtain the coefficients in Table 3, and Figures 136 and 137 show deviations as a function of temperature and pressure. Parameters for the critical enhancement are presented in Table 4. The estimated uncertainty in the gas and liquid phases at pressures to $30 \mathrm{MPa}$ is $3 \%$. Surface tension is discussed in Mulero et al. (Mulero et al., 2012). 


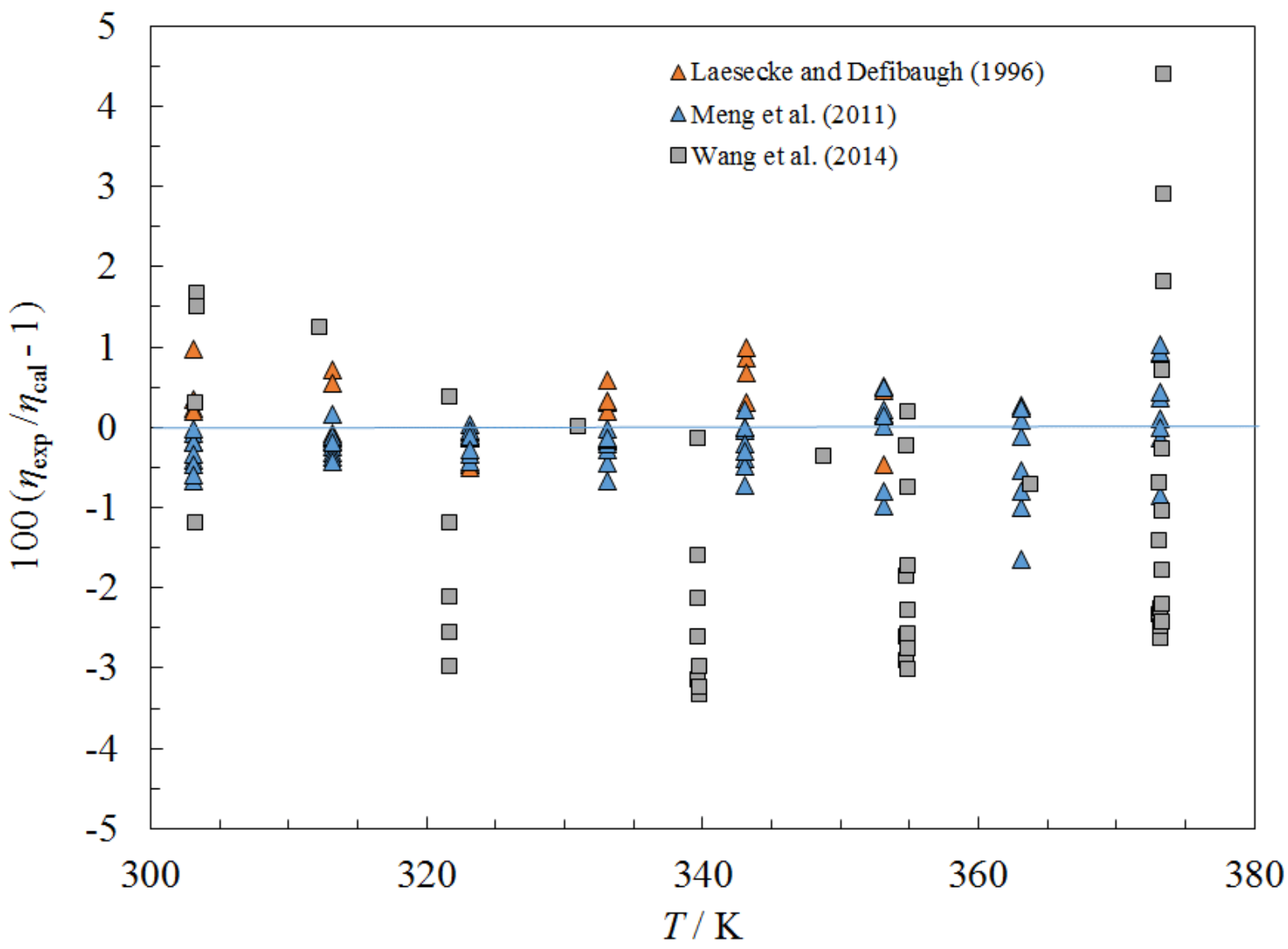

Figure 134. Percentage deviations between the model and the viscosity data for R236fa as a function of temperature. 


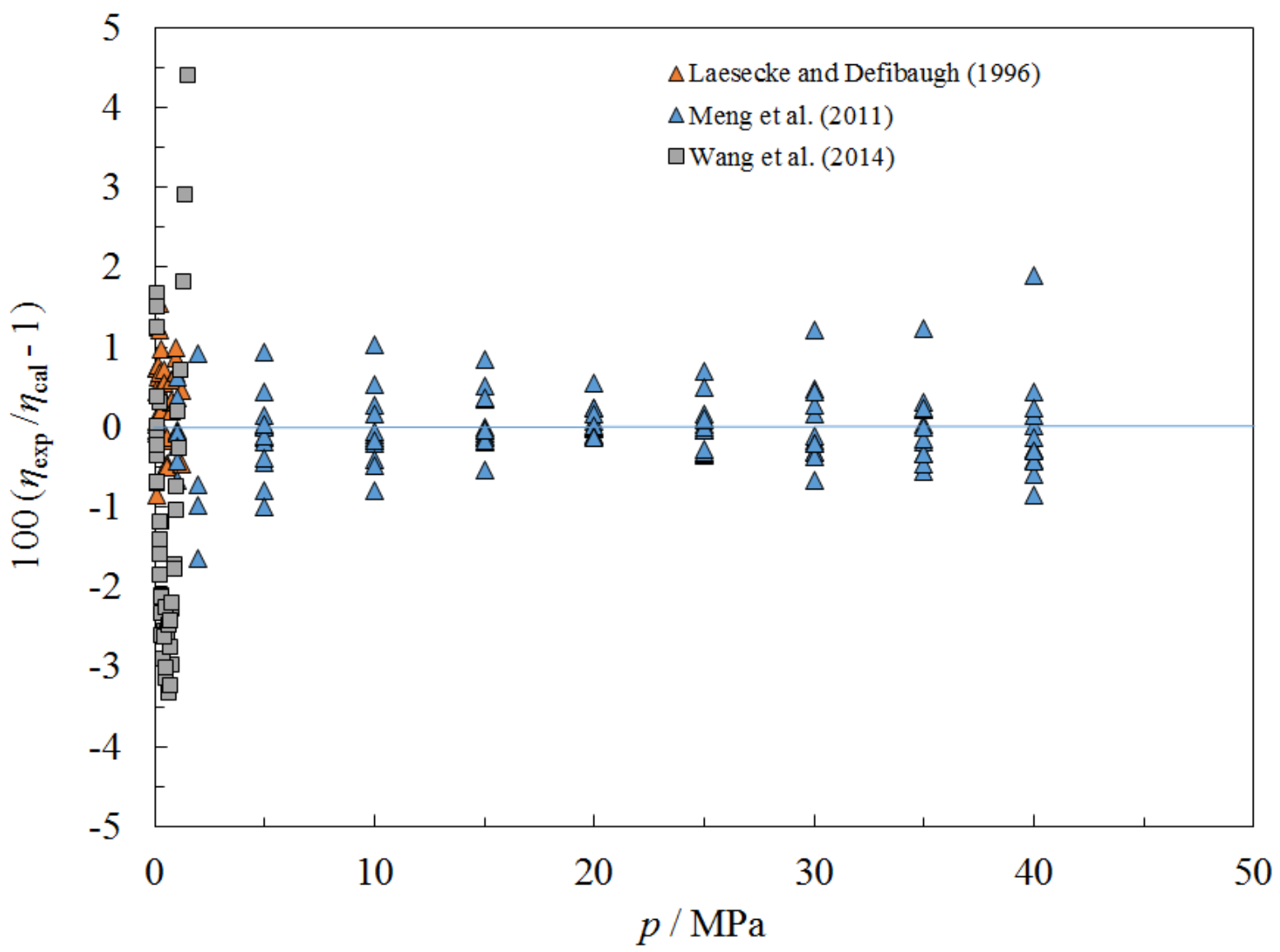

Figure 135. Percentage deviations between the model and the viscosity data for R236fa as a function of pressure. 


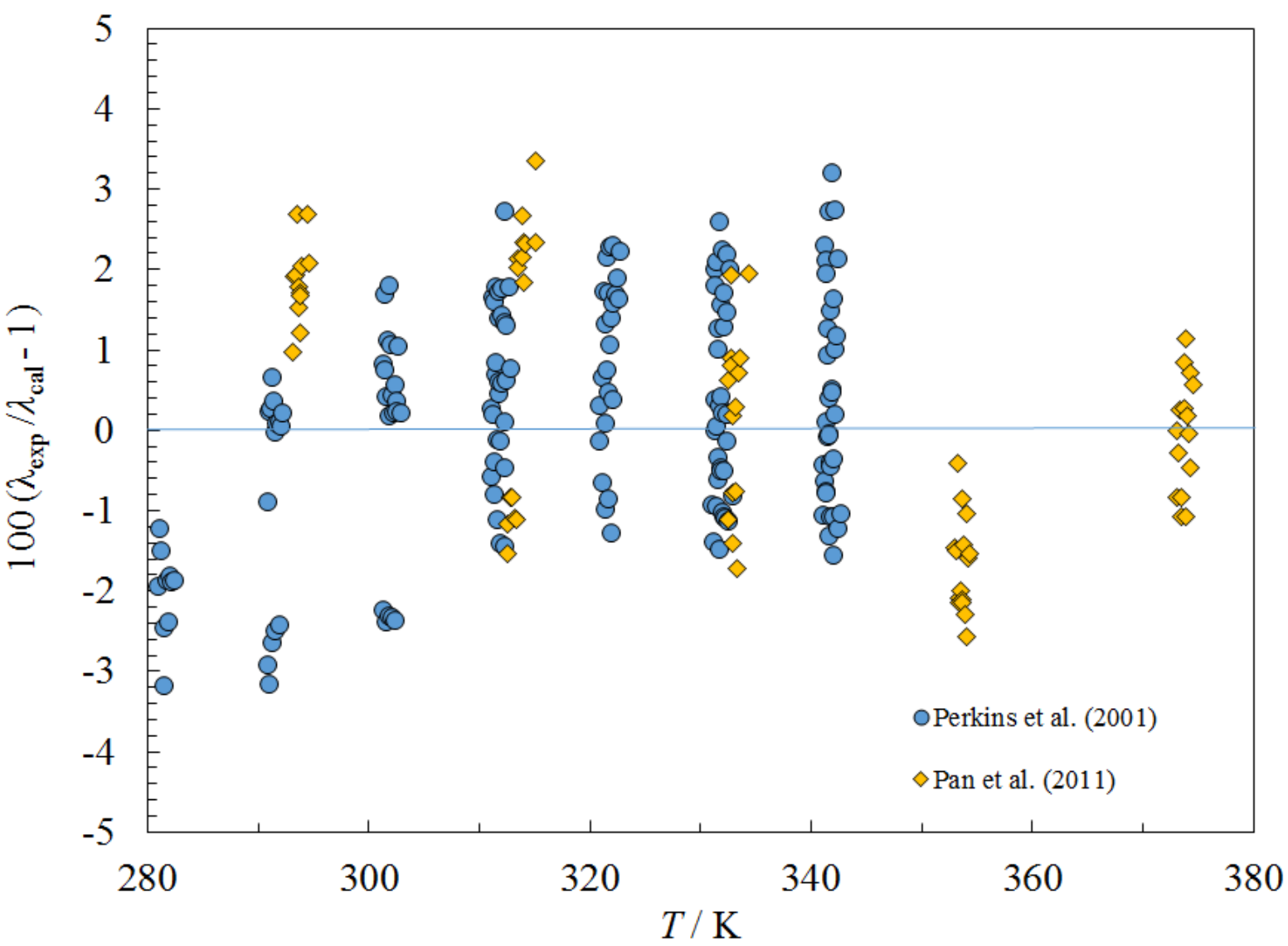

Figure 136. Percentage deviations between the model and the thermal conductivity data for R236fa as a function of temperature. 


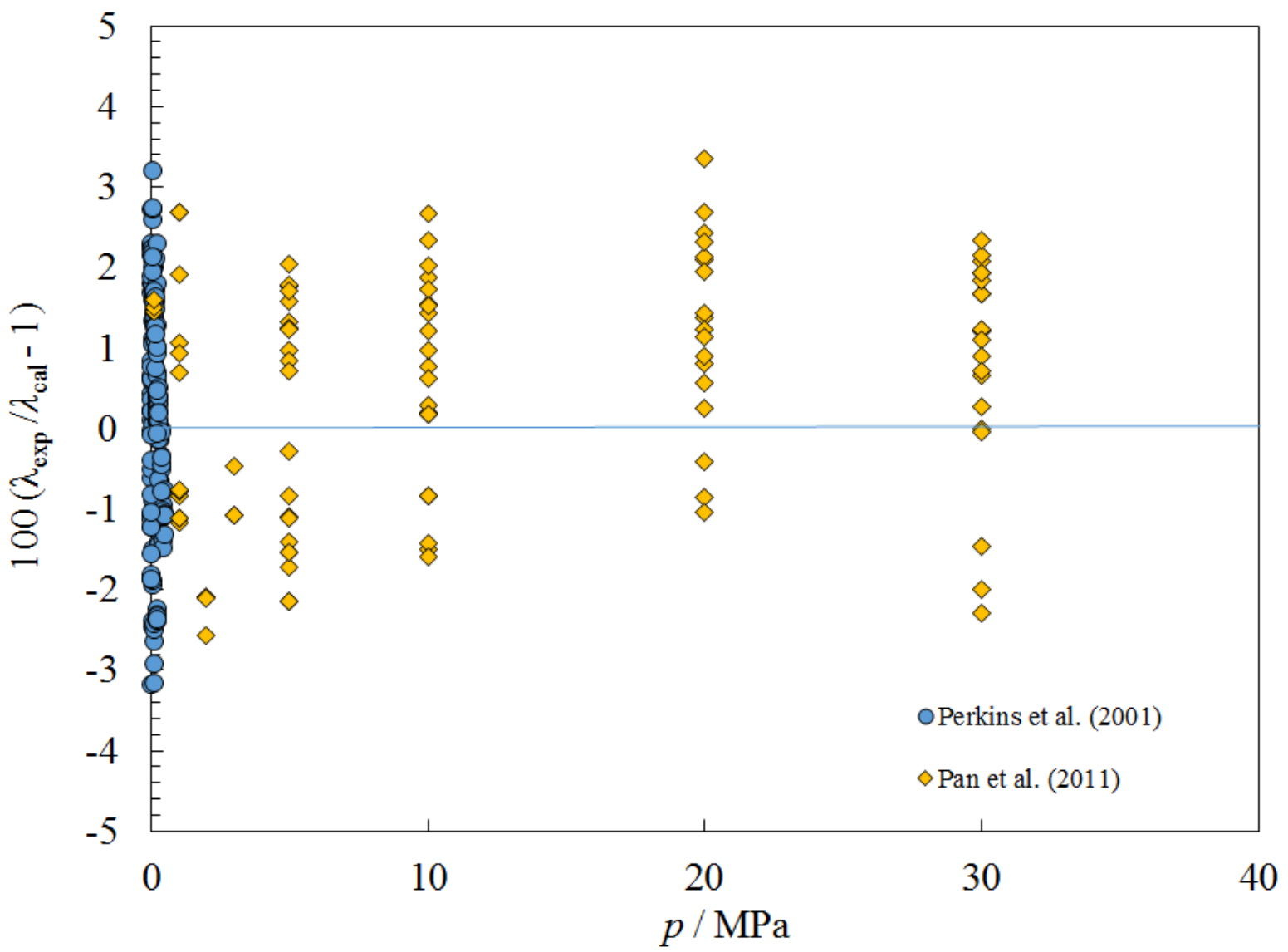

Figure 137. Percentage deviations between the model and the thermal conductivity data for R236fa as a function of pressure.

\subsubsection{R236ea (1,1,1,2,3,3-Hexafluoropropane)}

The equation of state of Rui et al. (Rui, Pan, \& Wang, 2013) was used to provide the density and the critical parameters. Lennard-Jones parameters were estimated with a method that scales R134a as described in Huber and Ely (Huber \& Ely, 1992b). This fluid is an isomer of R246fa. Liquid-phase data of Laesecke and Defibaugh (Laesecke \& Defibaugh, 1996) were used to obtain the coefficients in Table 2, and Figure 138 shows deviations from the data as a function of temperature. All viscosity data are at saturation. We estimate that the uncertainty of the viscosity in the liquid phase at saturation is $2 \%$, rising to $5 \%$ at pressures up to $60 \mathrm{MPa}$, and the uncertainty in the gas phase is $10 \%$. Data from Perkins et al. (R. Perkins et al., 2001; R. A. Perkins, 2002) were used to obtain the coefficients in Table 3, and Figures 139 and 140 show deviations as a function of temperature and pressure. Parameters for the critical enhancement are presented in Table 4 . The estimated uncertainty in the gas phase is $4 \%$, and in the liquid phase at pressures to $70 \mathrm{MPa}$ is $2 \%$. Surface tension is discussed in Mulero et al. (Mulero et al., 2012). 


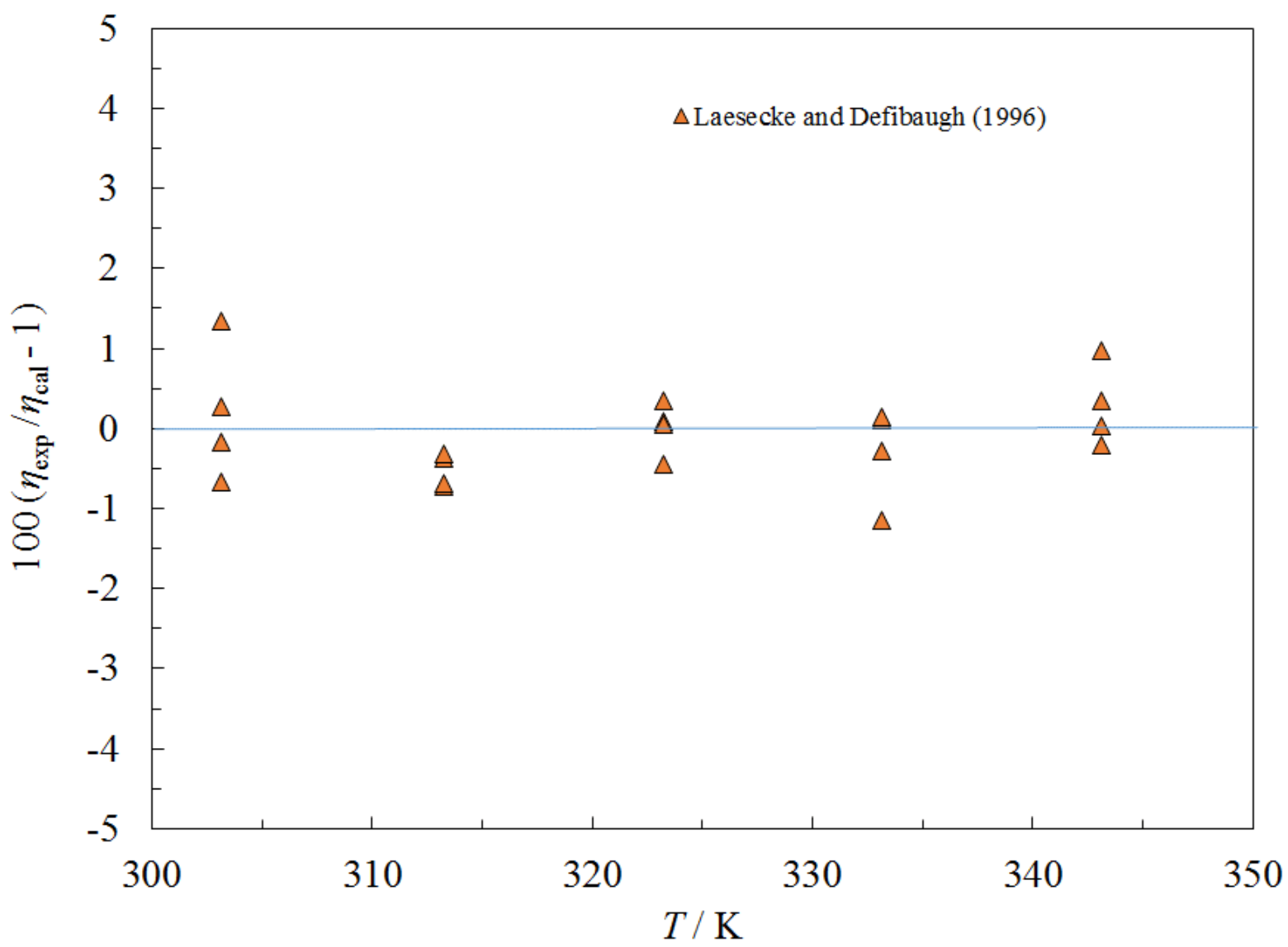

Figure 138. Percentage deviations between the model and the viscosity data for R236ea as a function of temperature. 


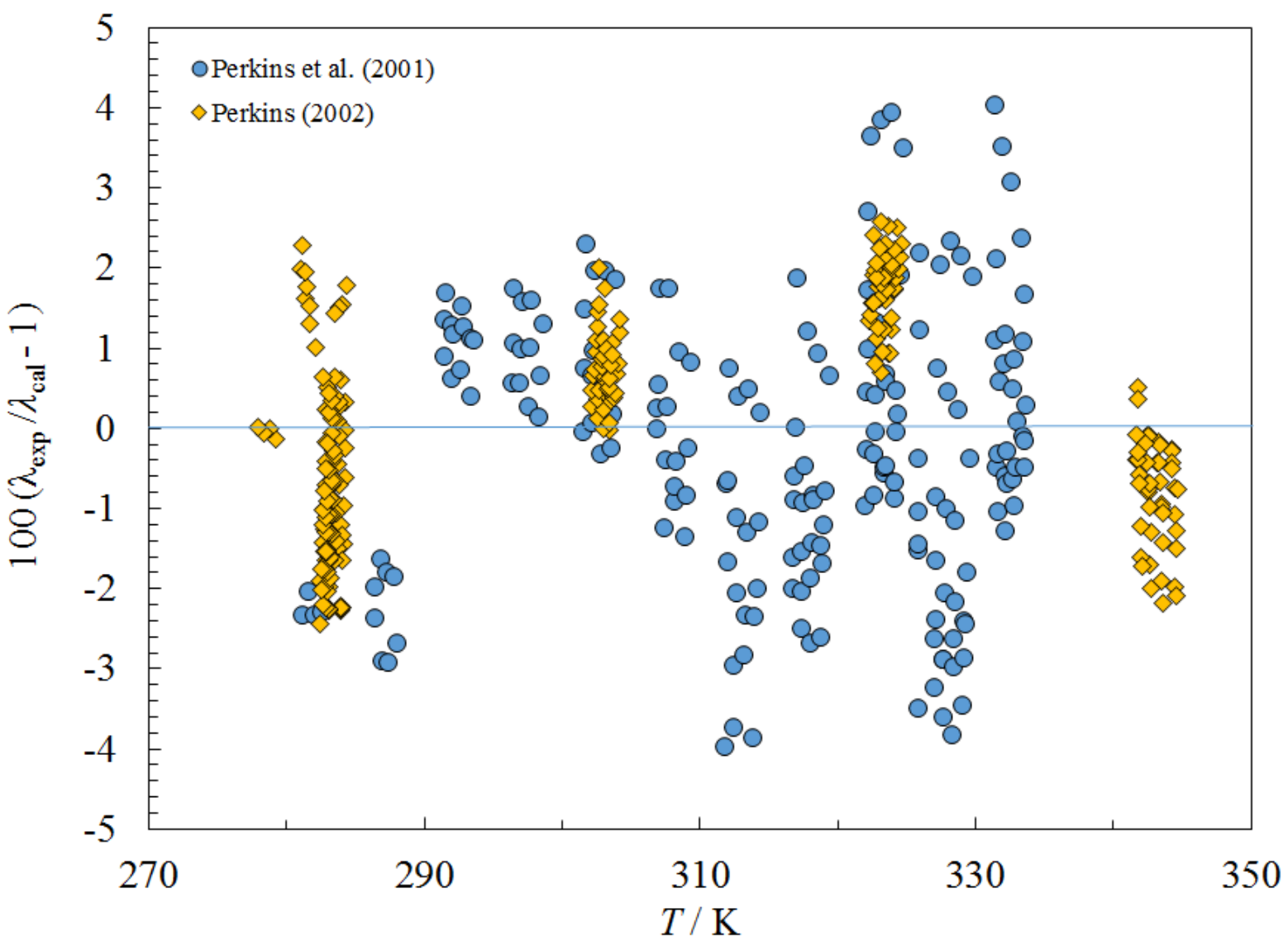

Figure 139. Percentage deviations between the model and the thermal conductivity data for R236ea as a function of temperature. 


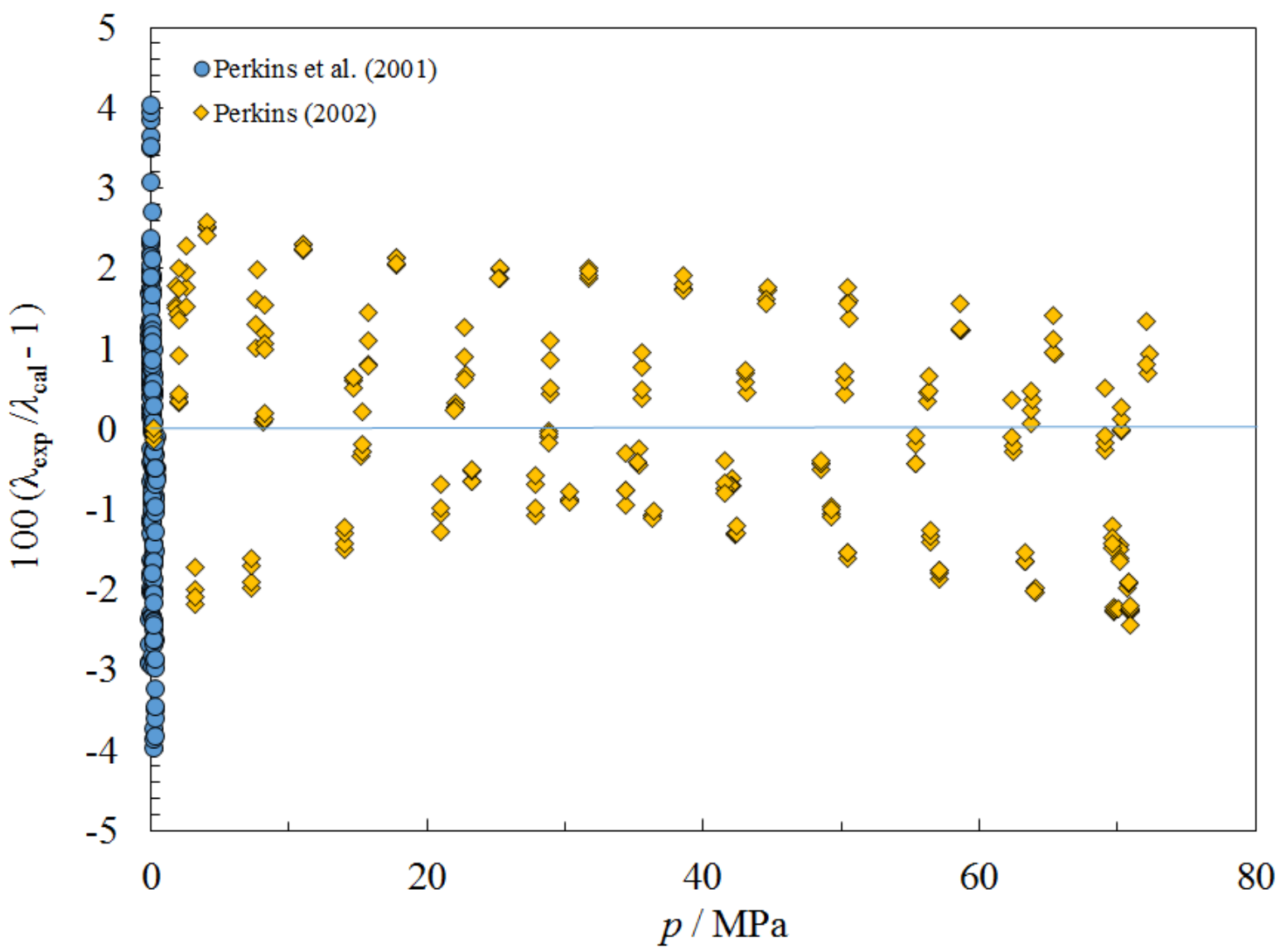

Figure 140. Percentage deviations between the model and the thermal conductivity data for R236ea as a function of pressure.

\subsubsection{R1336mzz(Z) ((Z)-1,1,1,4,4,4-Hexafluoro-2-butene)}

The equation of state of McLinden and Akasaka (M. O. McLinden \& Akasaka, 2018) was used to provide the density and the critical parameters, and an acentric factor of 0.386 . LennardJones parameters were calculated by the method of Chung et al. (Chung et al., 1988); values are presented in Table 1. No published experimental data were found for viscosity, but we obtained preliminary unpublished results (Miyara, 2018d) and used those data to obtain the coefficients in Table 2. The data were in the saturated liquid and saturated vapor phases; a deviation plot is shown in Figure 141. Estimated uncertainty for the viscosity of the saturated liquid from $300 \mathrm{~K}$ to $435 \mathrm{~K}$ is $3 \%$, higher elsewhere. For the vapor phase the estimated uncertainty is $6 \%$. Experimental data from Alam et al. (Alam, Islam, Kariya, \& Miyara, 2017) were used to obtain the coefficients in Table 3, and Figures 142 and 143 show deviations as a function of temperature and pressure, respectively. Liquid-phase data were only available near saturation pressure; there are no highpressure data. We estimate that the uncertainty for thermal conductivity in the gas phase and at saturation in the liquid is $3 \%$, and higher at higher pressures and near the critical point. $\mathrm{R} 1336 \mathrm{mzz}(\mathrm{Z})$ is a fluorinated butene. A similar compound is R1234ze(Z), cis-1,3,3,3tetrafluoropropene, also a fluorinated propene. Since there are no surface tension data available, as a very rough estimate, we adopted the same surface tension parameter for $n$ as used for the 
surface tension of R1234ze(Z) (Kondou \& Koyama, 2015), and adjusted the value of $\sigma_{0}$ slightly for $\mathrm{R} 1336 \mathrm{mzz}(\mathrm{Z})$ to account for the fact that it is a slightly larger molecule with a higher $T_{\mathrm{c}}$. These parameters are given in Table 5 . We estimate the uncertainty for surface tension is $10 \%$.

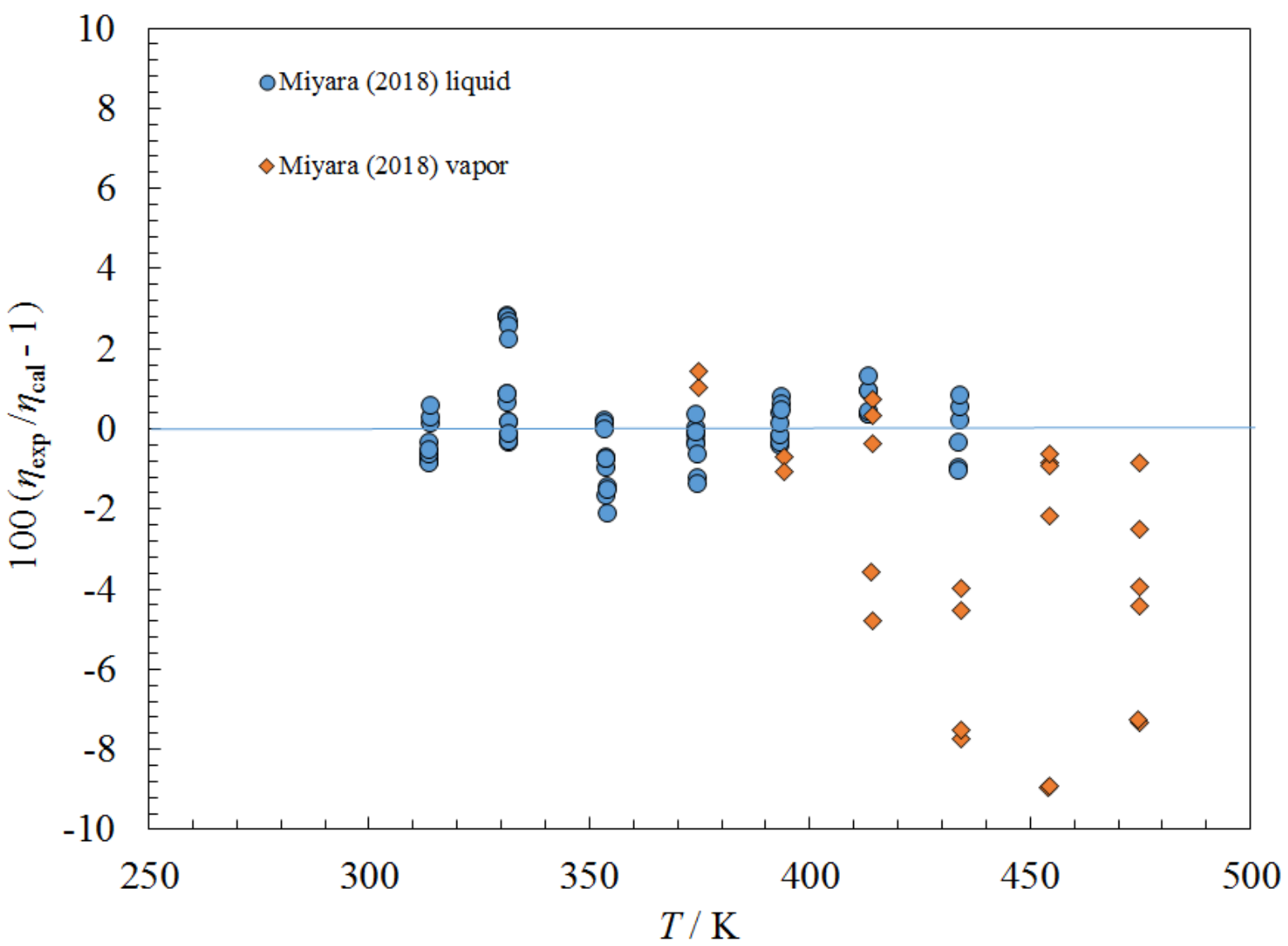

Figure 141. Percentage deviations between the model and the viscosity data for $\mathrm{R} 1336 \mathrm{mzz}(\mathrm{Z})$ as a function of temperature. 


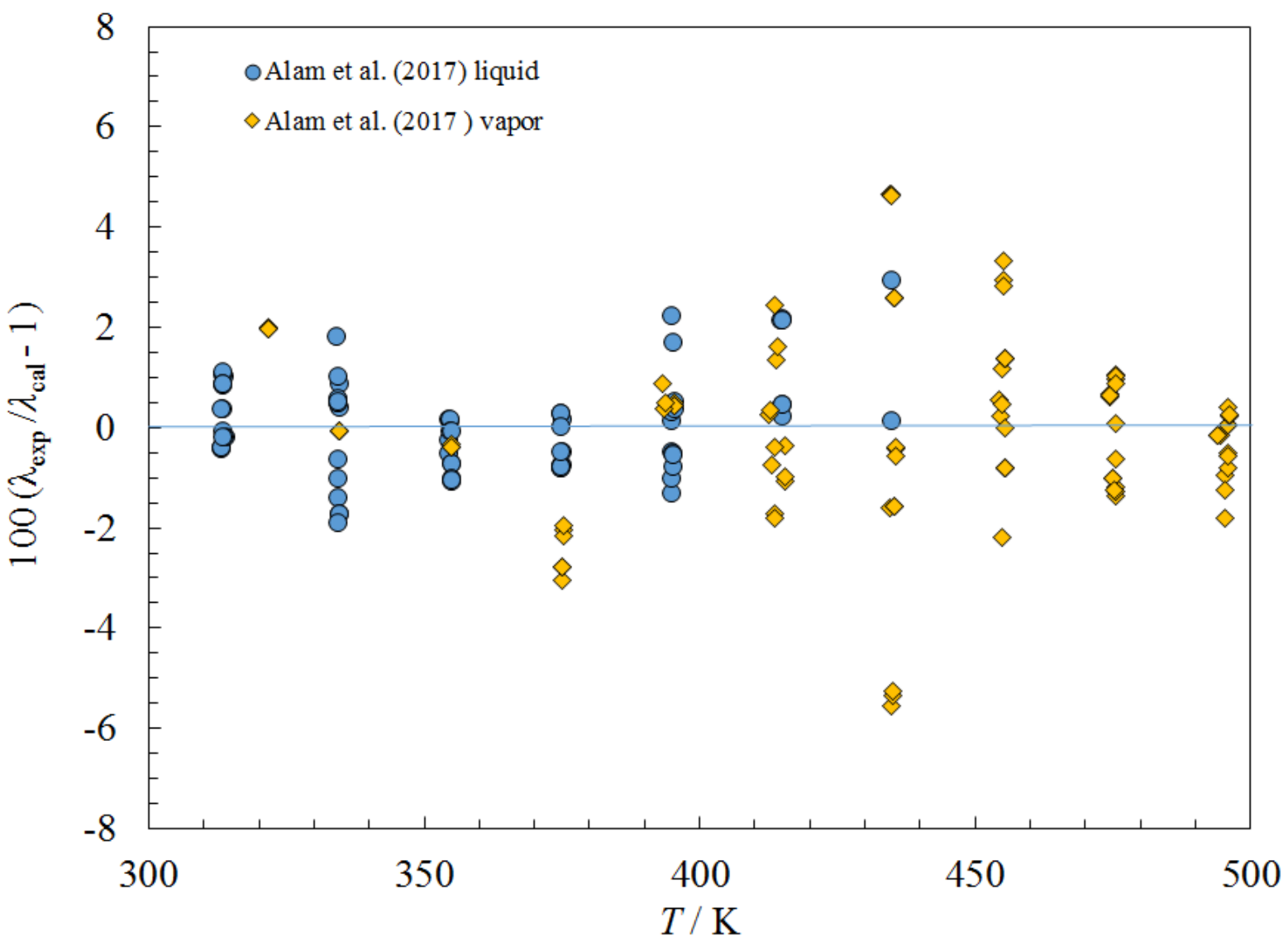

Figure 142. Percentage deviations between the model and the thermal conductivity data for $\mathrm{R} 1336 \mathrm{mzz}(\mathrm{Z})$ as a function of temperature. 


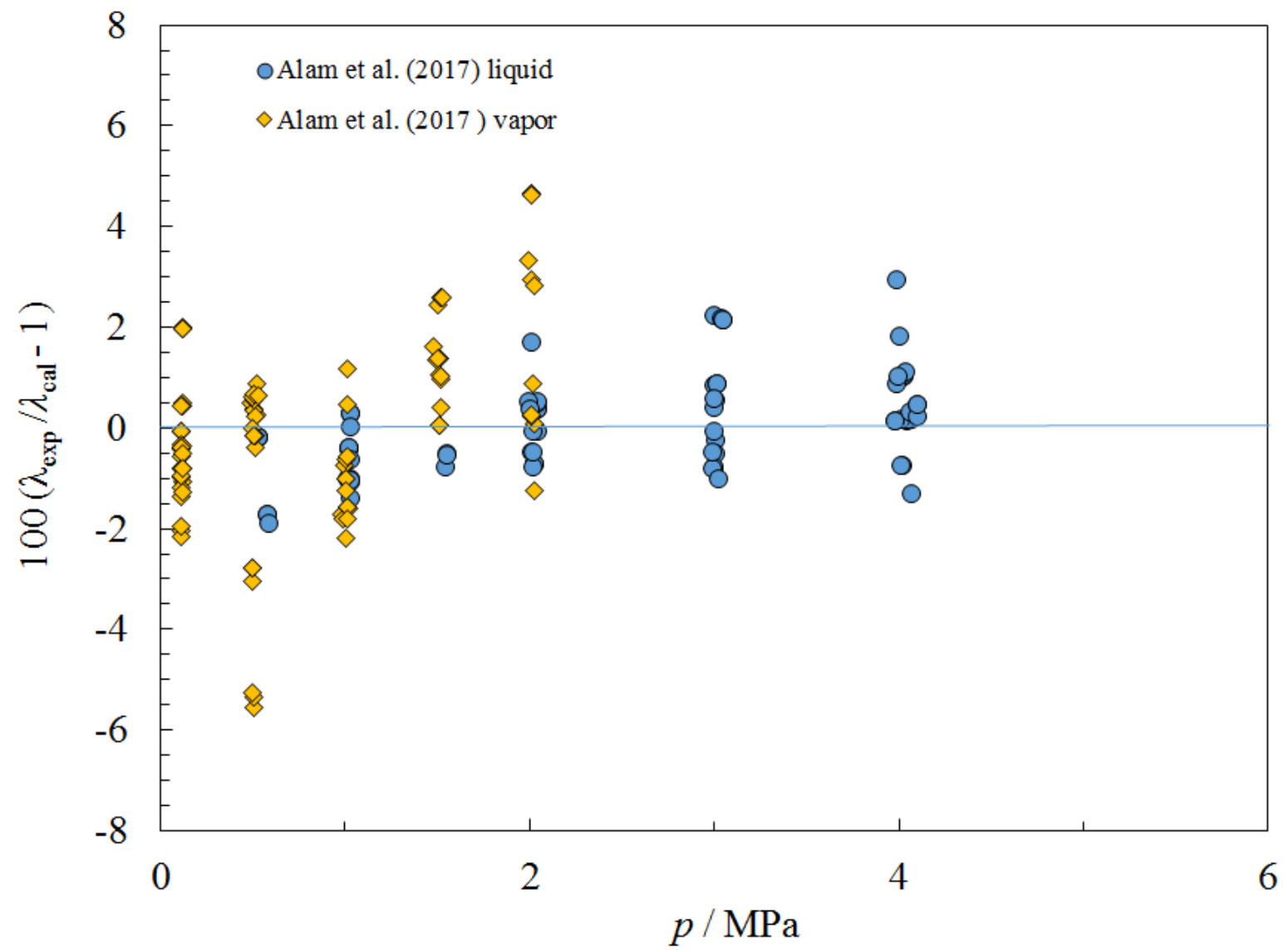

Figure 143. Percentage deviations between the model and the thermal conductivity data for $\mathrm{R} 1336 \mathrm{mzz}(\mathrm{Z})$ as a function of pressure.

\subsubsection{R114 (1,2-Dichloro-1,1,2,2-tetrafluoroethane)}

The equation of state of Platzer et al. (Platzer, Polt, \& Maurer, 1990) was used to provide the density and the critical parameters. Lennard-Jones parameters were calculated by fitting the vapor-phase viscosity data of Kamien and Witzell (Kamien \& Witzell, 1959). The liquid-phase viscosity data of Kumagai and Yokoyama (Kumagai \& Yokoyama, 2000), Arneman and Kruse (Arnemann \& Kruse, 1991), and Geller (V.Z. Geller, 1980) were used to obtain the coefficients in Table 2. Deviation plots as a function of temperature and pressure, respectively, are shown in Figures 142 and 143 . The estimated uncertainty is $5 \%$ at pressures to $20 \mathrm{MPa}$ (the limit of the EOS). Gas-phase thermal conductivity data of Keyes (Keyes, 1954) and the liquid-phase data of Yata et al. (Yata, Minamiyama, \& Tanaka, 1984), Fellows et al. (Fellows, Richard, \& Shankland, 1990), and Slusarev (Slusarev, 1979) were used to obtain the coefficients in Table 3, and deviation plots for thermal conductivity are shown in Figures 144 and 145. Parameters for the critical enhancement are presented in Table 4 . The estimated uncertainty for thermal conductivity at temperatures to $380 \mathrm{~K}$ and pressures to $20 \mathrm{MPa}$ is $8 \%, 10 \%$ elsewhere. Surface tension is discussed in Mulero et al. (Mulero et al., 2012). 


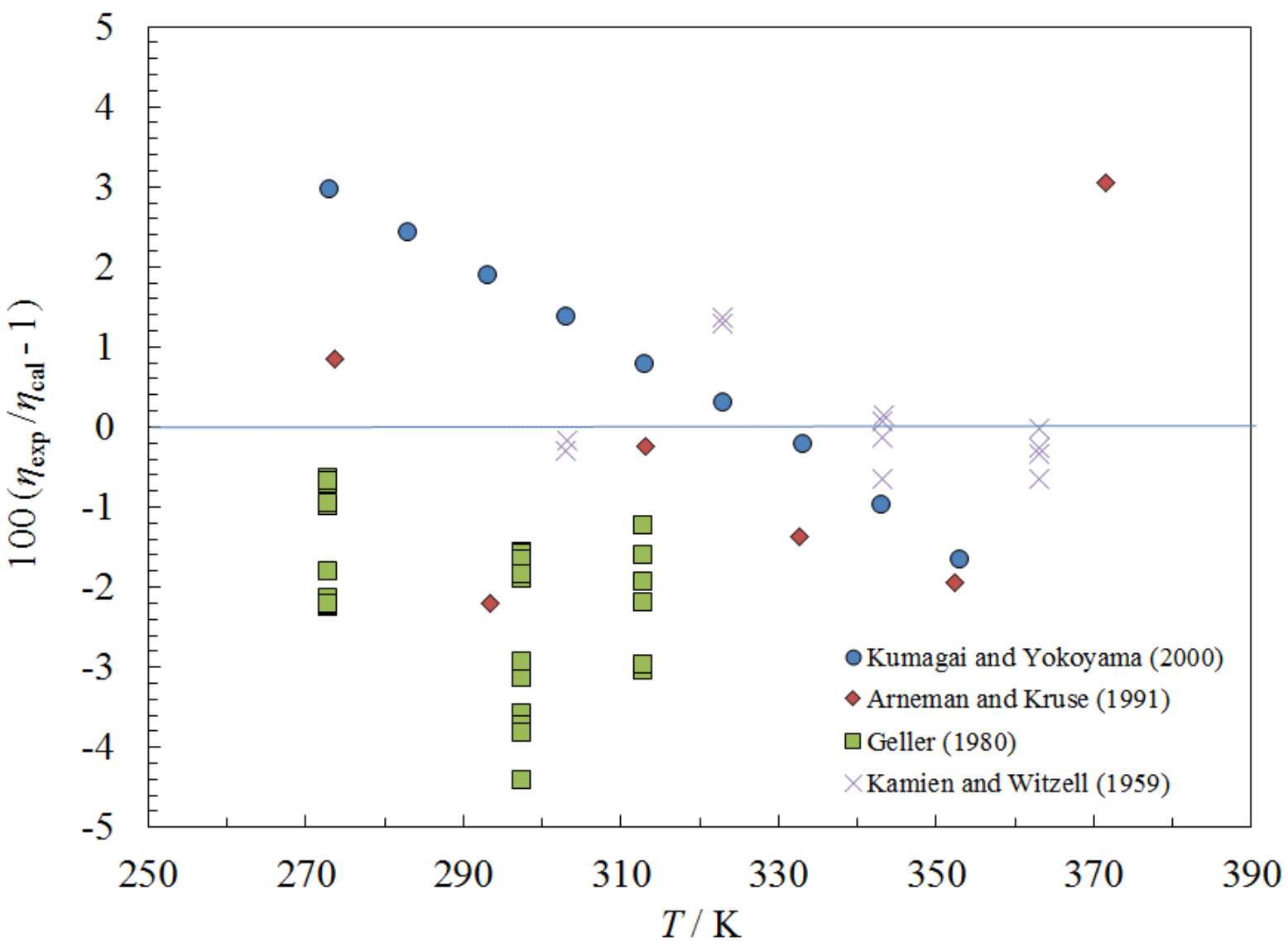

Figure 142. Percentage deviations between the model and the experimental viscosity data for R114 as a function of temperature. 


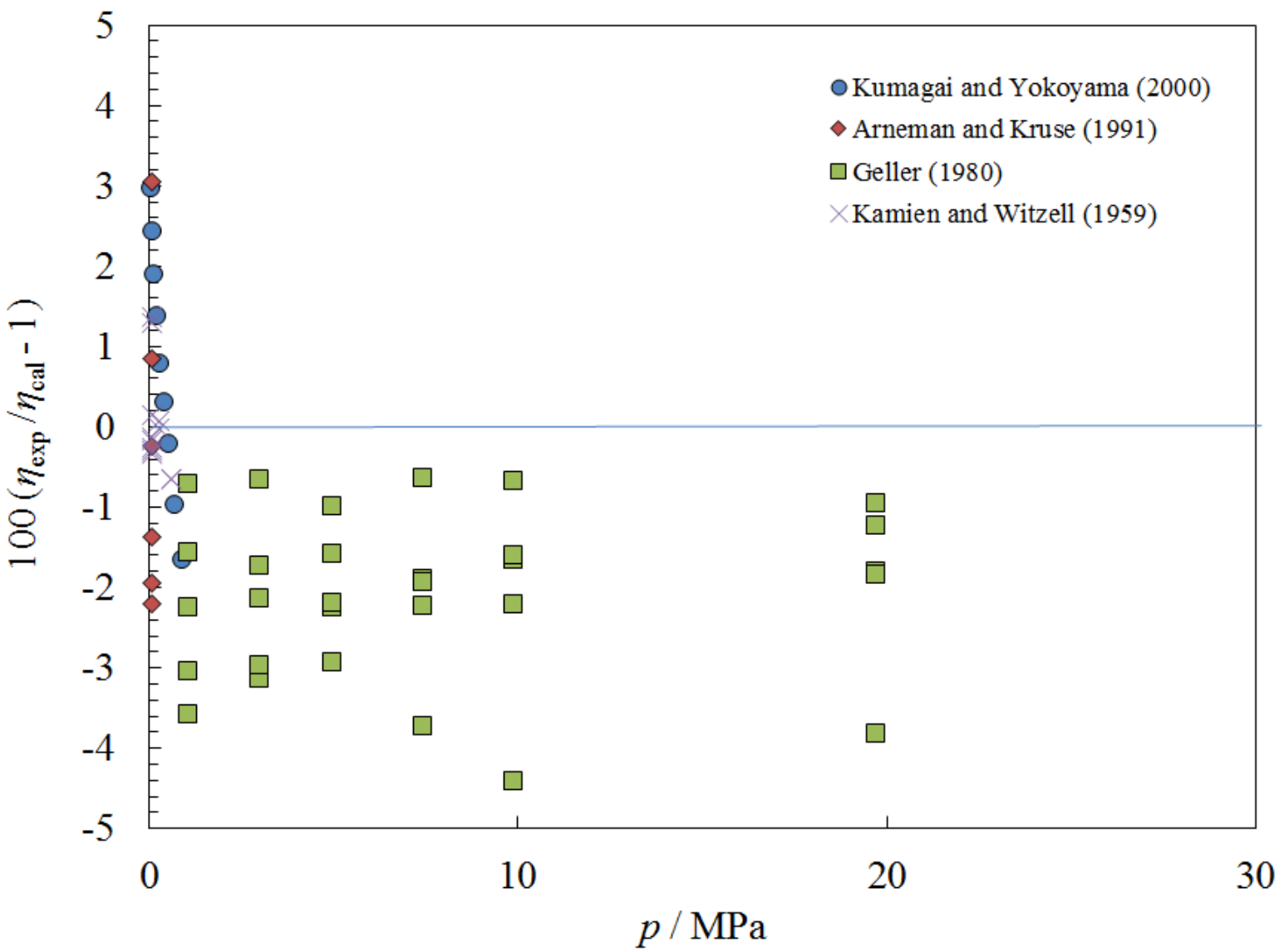

Figure 143. Percentage deviations between the model and the experimental viscosity data for R114 as a function of pressure. 


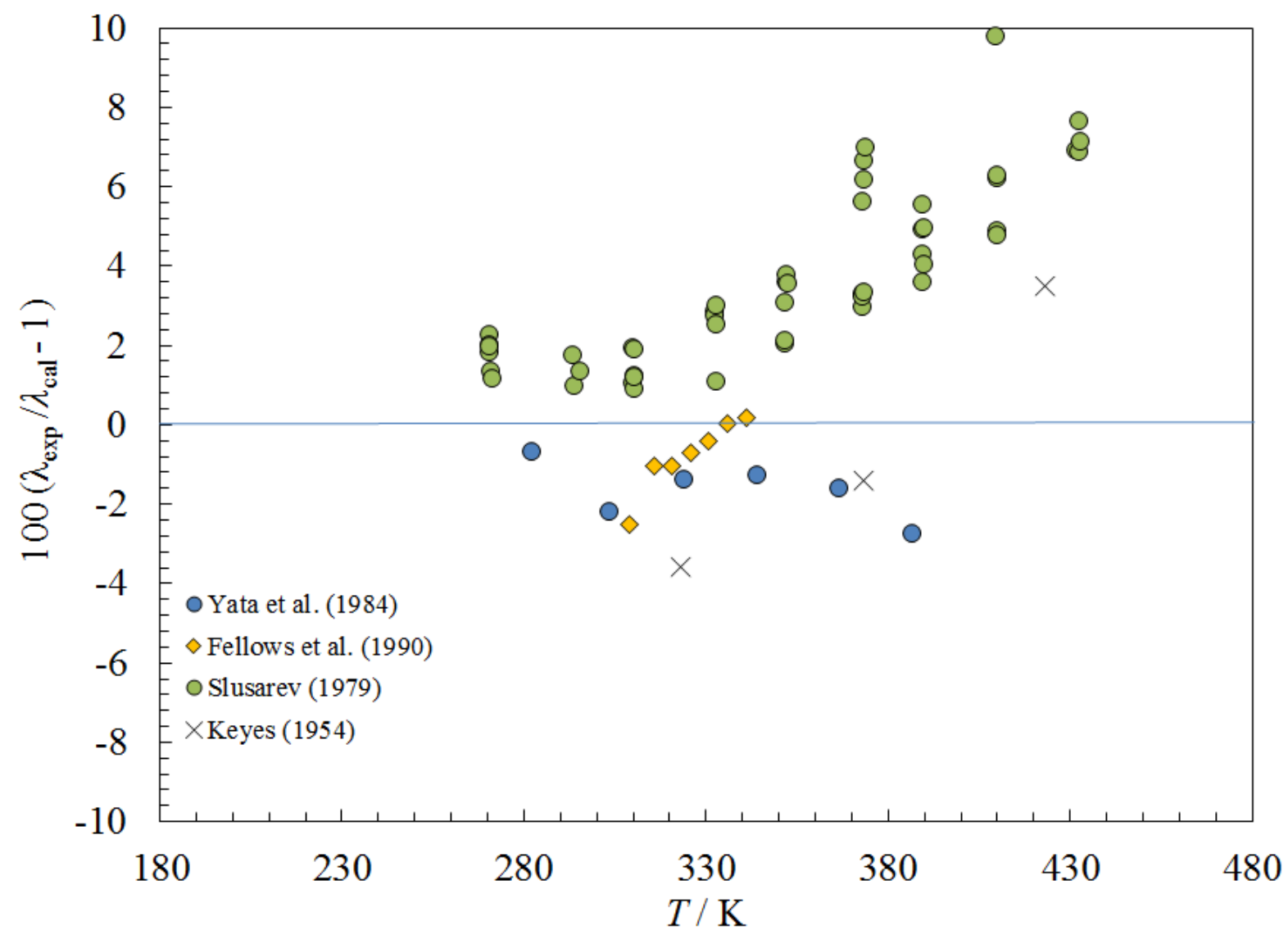

Figure 144. Percentage deviations between the model and the experimental thermal conductivity data for R114 as a function of temperature. 


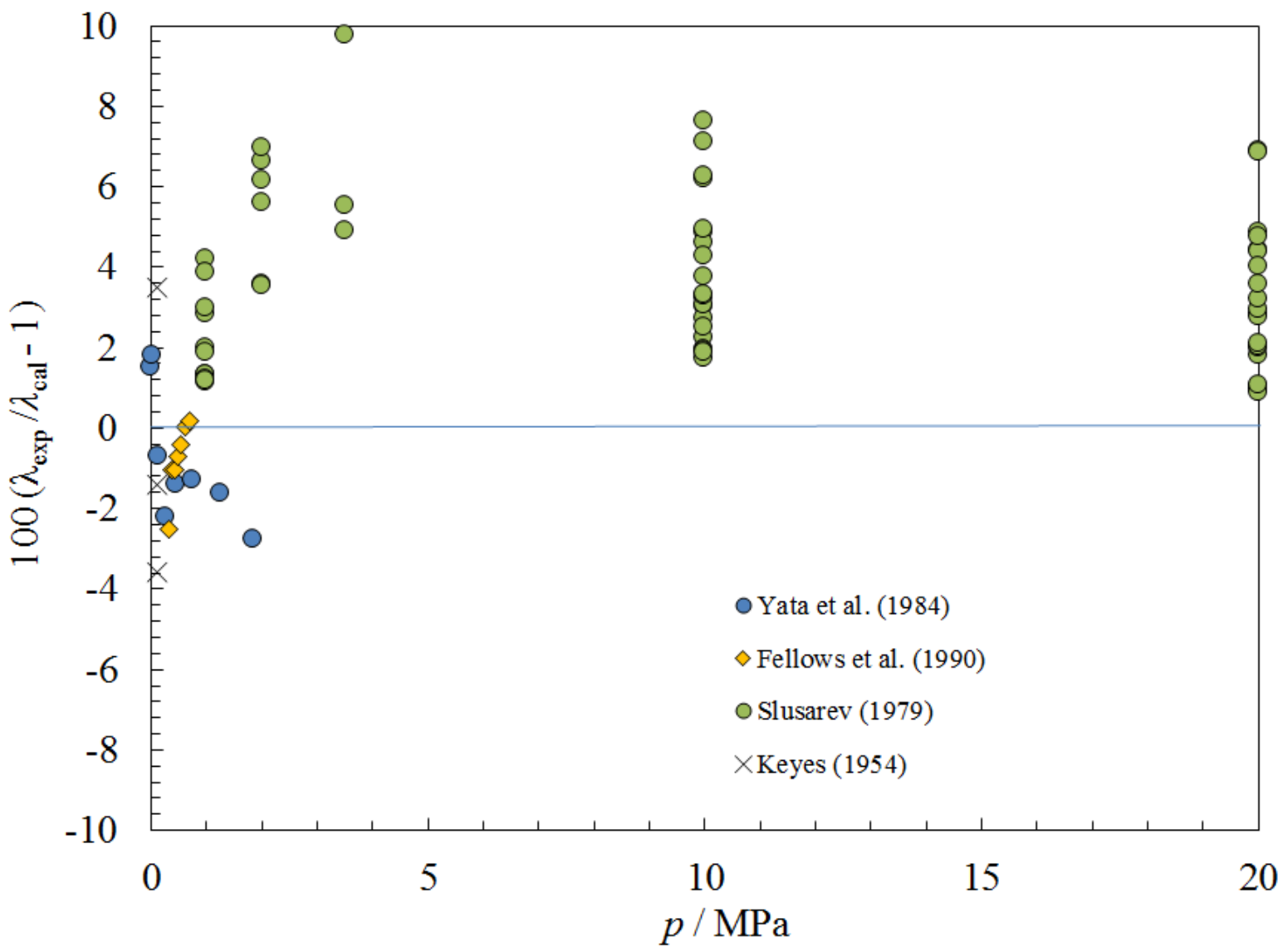

Figure 145. Percentage deviations between the model and the experimental thermal conductivity data for R114 as a function of pressure.

\subsubsection{R218 (Octafluoropropane)}

The equation of state of Lemmon and Span (E.W. Lemmon \& Span, 2006) was used to provide the density and the critical parameters. Lennard-Jones parameters were estimated with a method described in Huber and Ely (Huber \& Ely, 1992b). Only one vapor-phase viscosity point was found (Dunlop, 1994), and this was used along with the liquid-phase viscosity data of Geller (V.Z. Geller, 1980) to obtain the coefficients in Table 2. Deviation plots as a function of temperature and pressure, respectively, are shown in Figures 146 and 147. The estimated uncertainty in the gas phase is $10 \%$, and is $5 \%$ in the liquid at pressures to $20 \mathrm{MPa}$ (the limit of the EOS). Gas-phase thermal conductivity data of Tsvetkov et al. (Tsvetkov, Laptev, \& Vasilkov, 1977) and Clifford et al. (Clifford, Gray, \& Scott, 1981) and the liquid-phase data of Baryshev et al. (Baryshev, Artamonov, \& Geller, 1980) and Grebenkov et al. (Grebenkov, Kotelevsky, \& Saplitza, 1993) were used to obtain the coefficients in Table 3, and deviation plots for thermal conductivity are shown in Figures 148 and 149. Parameters for the critical enhancement are presented in Table 4. There is a lot of scatter in the data, especially for Baryshev et al.(Baryshev et al., 1980); only the saturated liquid values from Baryshev et al. (Baryshev et al., 1980) were used in the regression. Based primarily on the data of Grebenkov et al. (Grebenkov et al., 1993), we estimate the uncertainty in the gas and liquid phases is $10 \%$ at pressures to the limit of the EOS, 20 MPa. Surface tension is discussed in Mulero et al. (Mulero et al., 2012). 


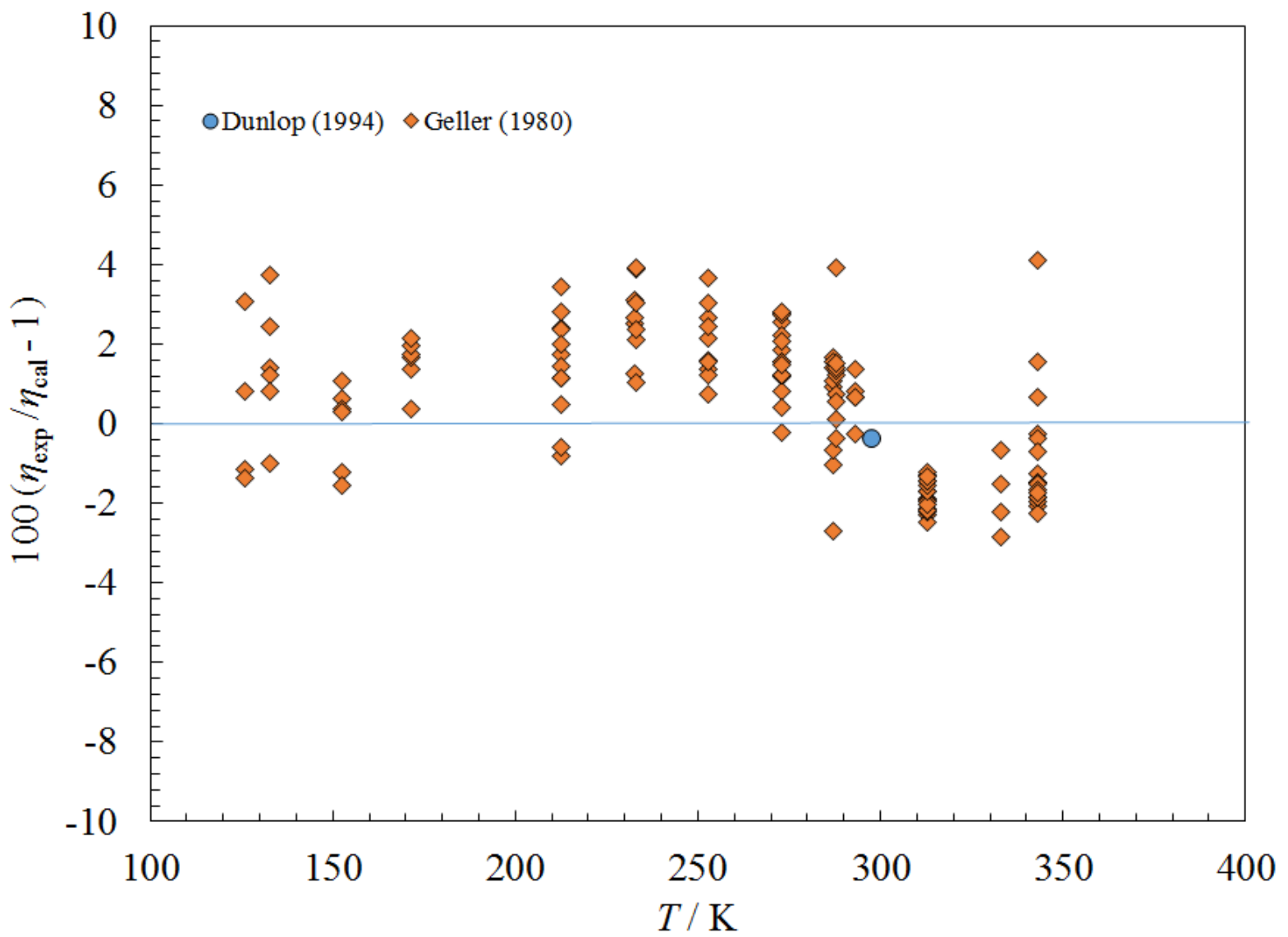

Figure 146. Percentage deviations between the model and the experimental viscosity data for R218 as a function of temperature. 


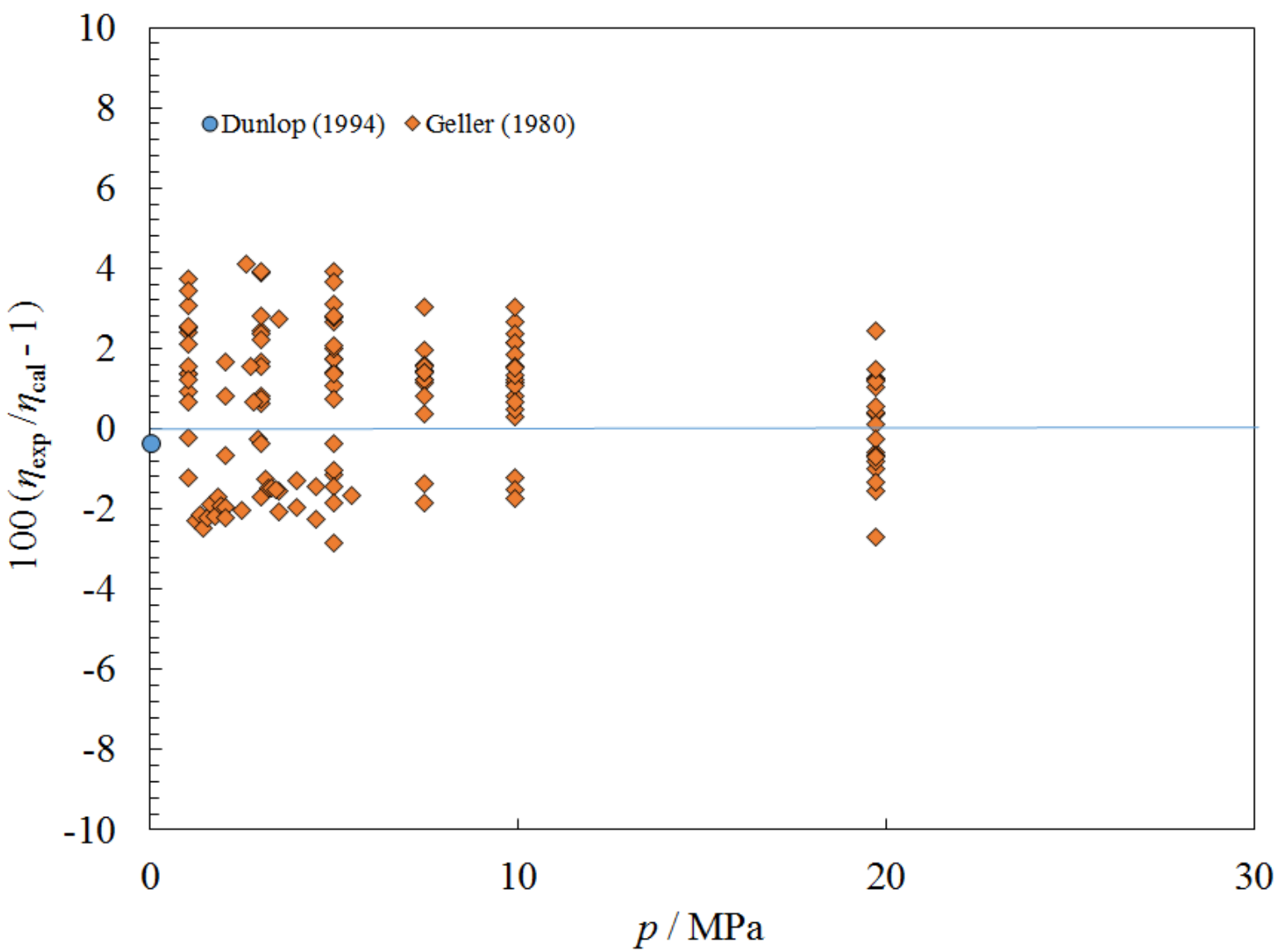

Figure 147. Percentage deviations between the model and the experimental viscosity data for R218 as a function of pressure. 


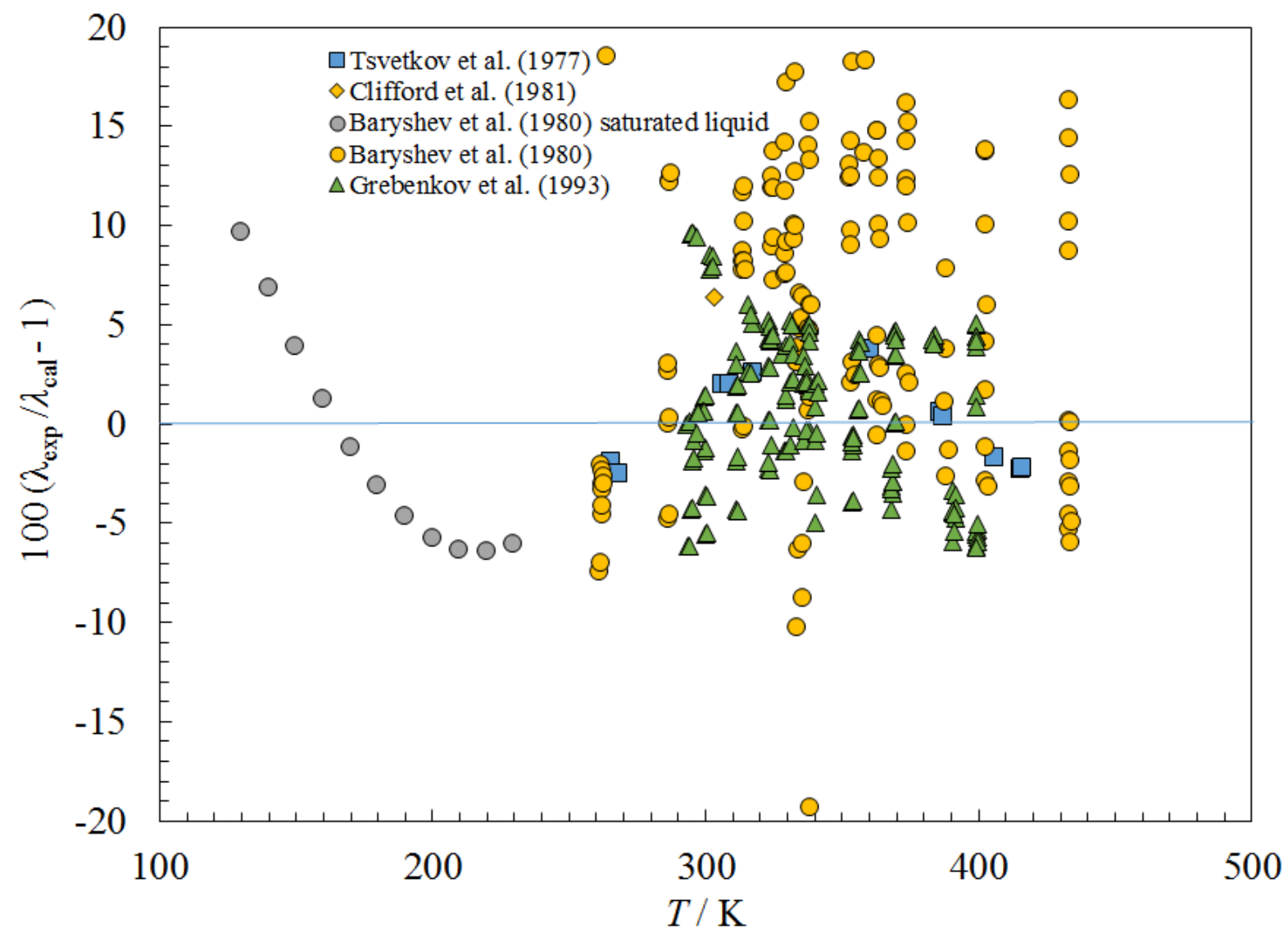

Figure 148. Percentage deviations between the model and the experimental thermal conductivity data for R218 as a function of temperature. 


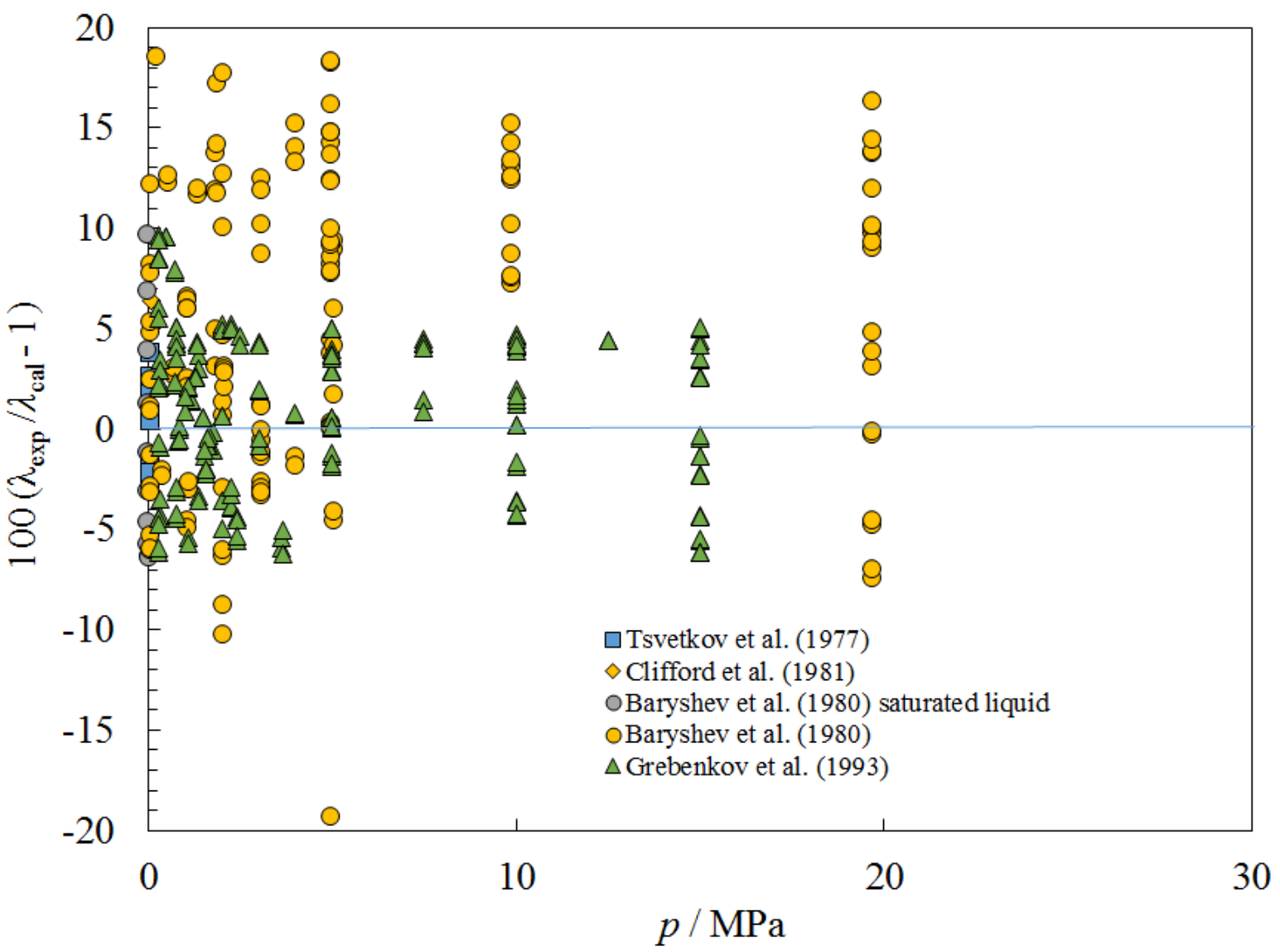

Figure 149. Percentage deviations between the model and the experimental experimental thermal conductivity data for R218 as a function of pressure.

\subsubsection{R13I1 ( $\left.\mathrm{CF}_{3} \mathrm{I}\right)$}

The equation of state of Lemmon and Span (E.W. Lemmon \& Span, 2015) was used to provide the density and the critical parameters. Lennard-Jones parameters were obtained from the method of Chung et al. (Chung et al., 1988). In the liquid phase, we fit the data of Duan et al. (Duan, Shi, Han, \& Zhu, 1999) to obtain the coefficients in Table 2. Figure 150 shows deviations from the experimental data used in fitting the coefficients as a function of temperature. The data are limited to the liquid saturation boundary. We estimate the uncertainty of the viscosity correlation in the liquid phase over the temperature range $250 \mathrm{~K}$ to $350 \mathrm{~K}$ is $5 \%$ at pressures to 50 $\mathrm{MPa}$. There are no data for the gas phase, and we estimate the uncertainty for the viscosity of the gas phase is $10 \%$. For thermal conductivity, the gas-phase data of Duan et al. (Duan, Sun, Shi, Zhu, \& Han, 1997) were fit to obtain the coefficients in Table 3, and Figure 151 shows percentage deviations with the experimental data. Parameters for the critical enhancement are presented in Table 4. There were no data found for the liquid phase, and a totally predictive model with R134a as a reference fluid was used. We estimate that the uncertainty for thermal conductivity in the liquid phase and vapor phase is $10 \%$. Surface tension is discussed in Mulero et al. (Mulero et al., 2012). 


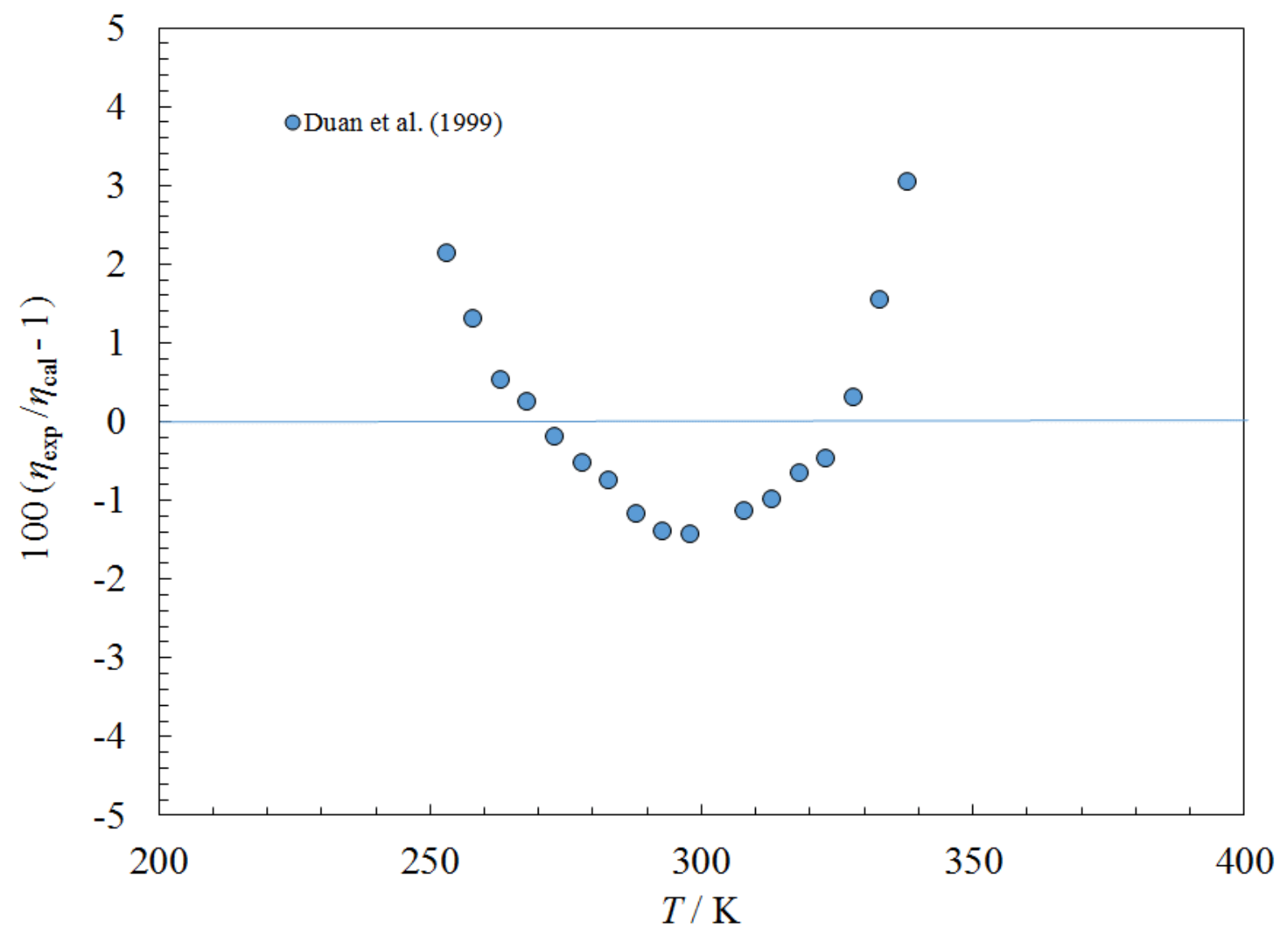

Figure 150. Percentage deviations between the model and the experimental viscosity data for $\mathrm{CF}_{3} \mathrm{I}$ as a function of temperature. 


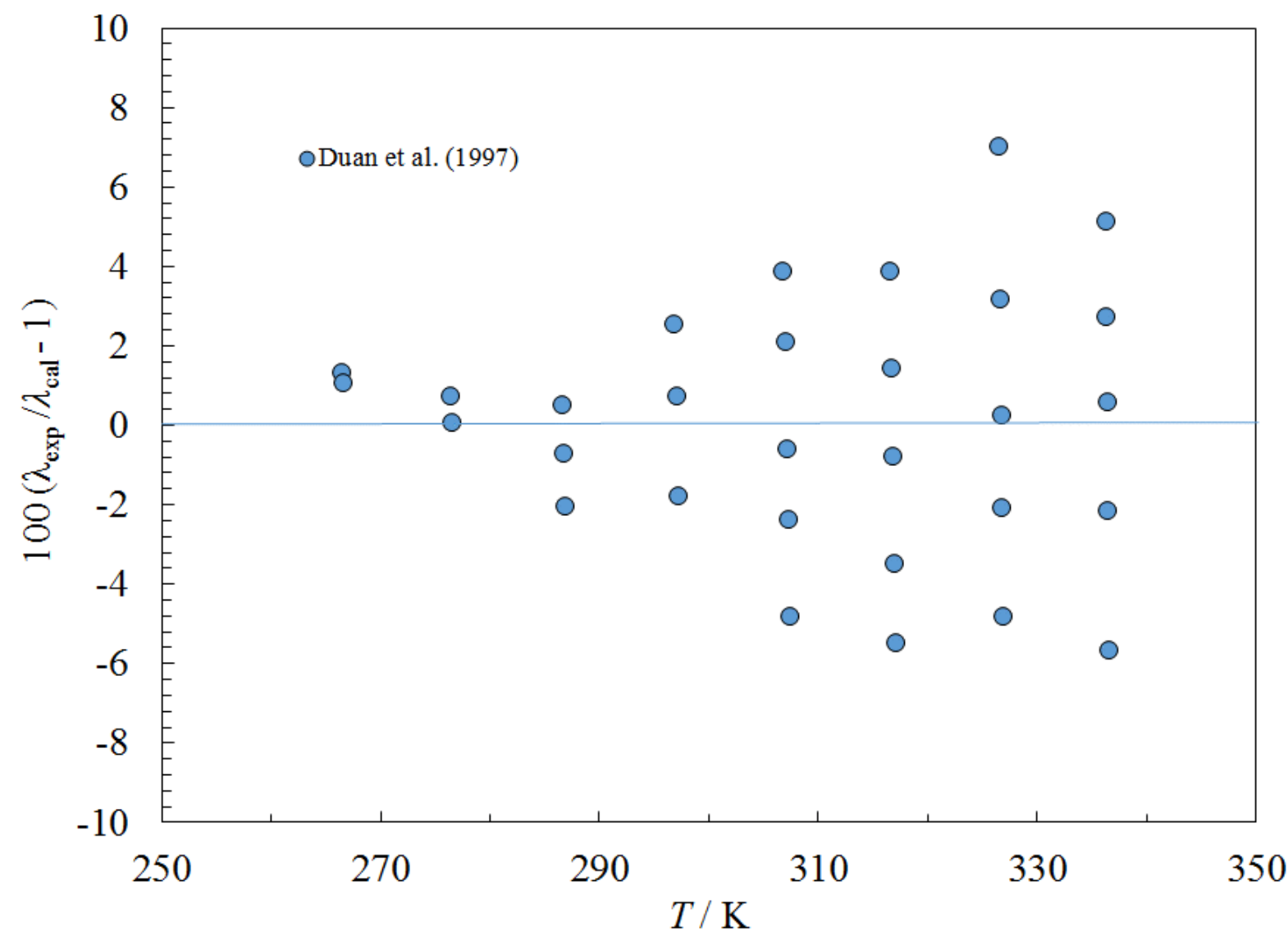

Figure 151. Percentage deviations between the model and the experimental thermal conductivity data for $\mathrm{CF}_{3} \mathrm{I}$ as a function of temperature.

\subsubsection{RC318 (Octafluorocyclobutane)}

The equation of state of Platzer et al. (Platzer et al., 1990) was used to provide the density and the critical parameters. Lennard-Jones parameters were obtained from the method of Chung et al. (Chung et al., 1988). Viscosity data from several sources (Finney, Fury, \& Jonas, 1977; V. Z. Geller, Karabanov, Gunchuk, Zakharzhevskiy, \& Lapardin, 1976; Kamien \& Witzell, 1959; Karbanov, 1978; P.M. Kessel'man, Porichanskii, \& Karbanov, 1976) were used to obtain the coefficients in Table 2, and Figures 152 and 153 give deviation plots as a function of temperature and pressure, respectively. The estimated uncertainty in the gas phase is $5 \%$ based on comparisons with Kamien and Witzell (Kamien \& Witzell, 1959). The data in the liquid phase exhibit a large amount of scatter, but we estimate the uncertainty of the viscosity in the liquid phase at temperatures above $235 \mathrm{~K}$ and pressures to $100 \mathrm{MPa}$ is $6 \%$. Thermal conductivity data in the gas phase from Perkins et al. (R. Perkins et al., 2001) and liquid and supercritical data from Vojtenko (Vojtenko, 1980) and Kessel'man et al. (P. M. Kessel'man et al., 1977) were used to obtain the coefficients in Table 3, and Figures 154 and 155 give deviation plots as a function of temperature and pressure, respectively. Parameters for the critical enhancement are presented in Table 4. Based on the data of Perkins et al. (R. Perkins et al., 2001), we estimate the uncertainty of thermal conductivity in the gas phase is $5 \%$. The data of Vojtenko (Vojtenko, 1980) have more scatter than Kesselman et al. (P. M. Kessel'man et al., 1977), and we use Kesselman et al. (P. M. 
Kessel'man et al., 1977) to estimate the uncertainty in the liquid phase at pressures to $60 \mathrm{MPa}$ as $5 \%$. Surface tension is discussed in Mulero et al. (Mulero et al., 2012).

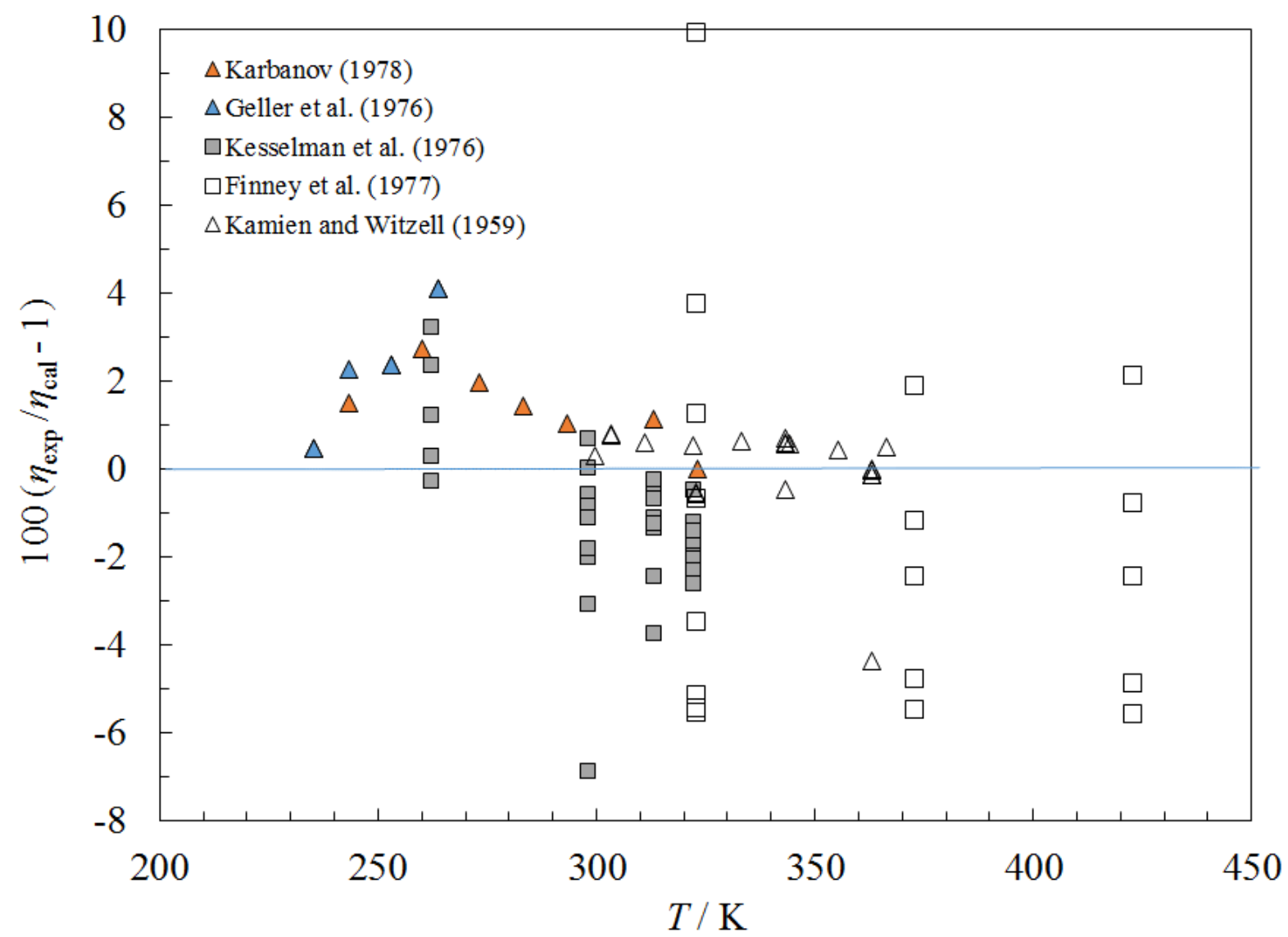

Figure 152. Percentage deviations between the model and the experimental viscosity data for RC318 as a function of temperature. 


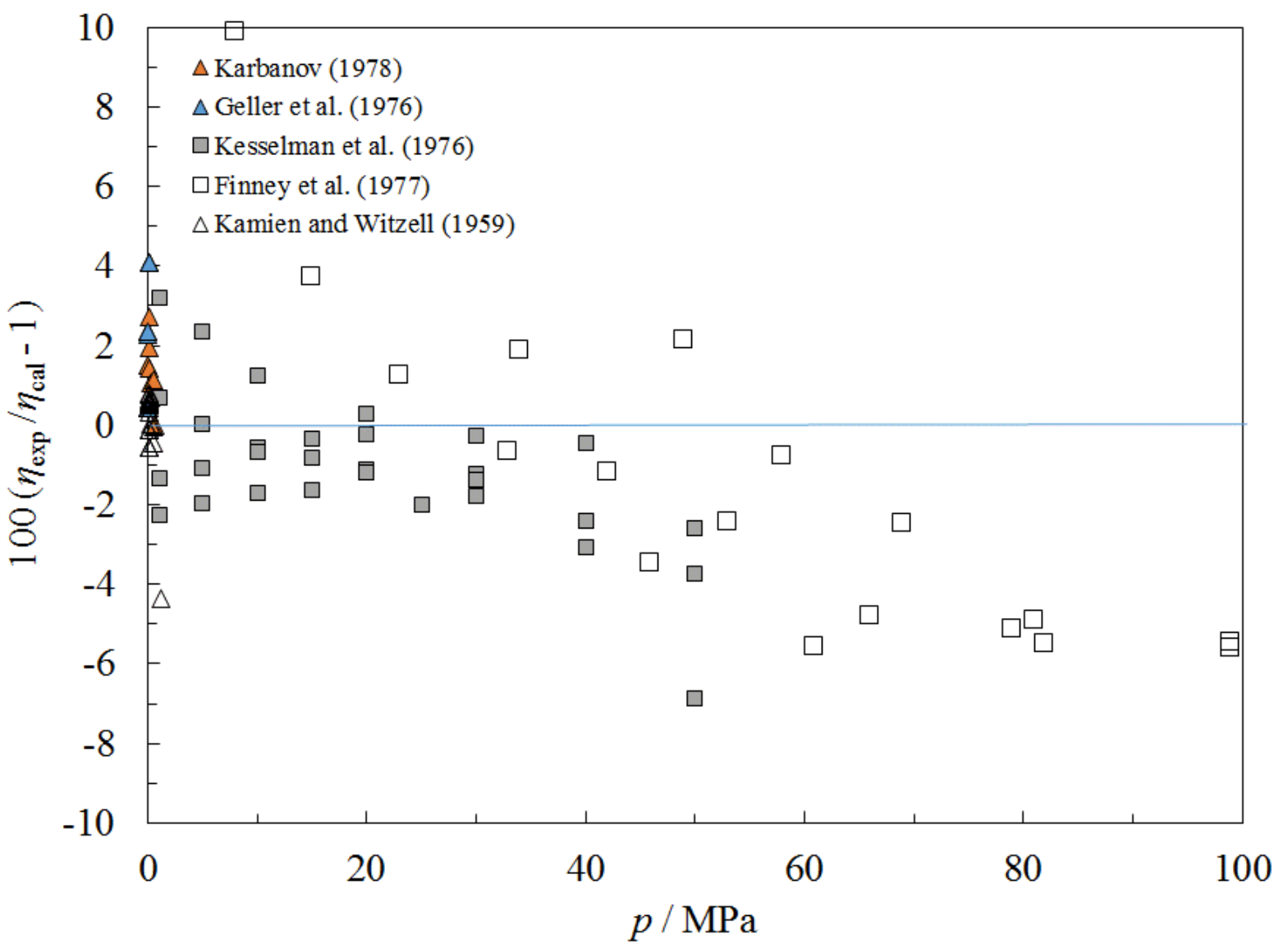

Figure 153. Percentage deviations between the model and the experimental viscosity data for RC318 as a function of pressure. 


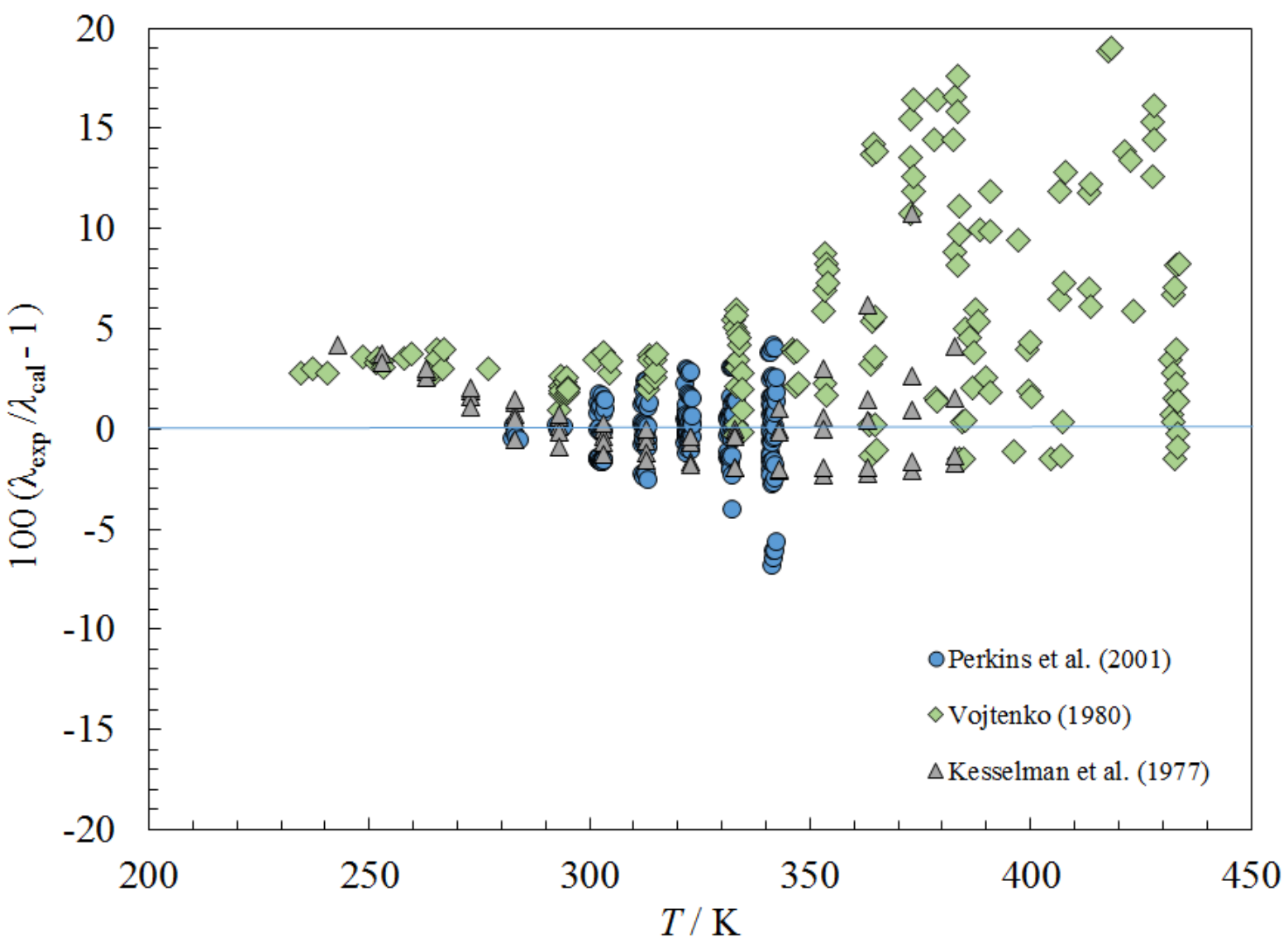

Figure 154. Percentage deviations between the model and the experimental thermal conductivity data for RC318 as a function of temperature. 


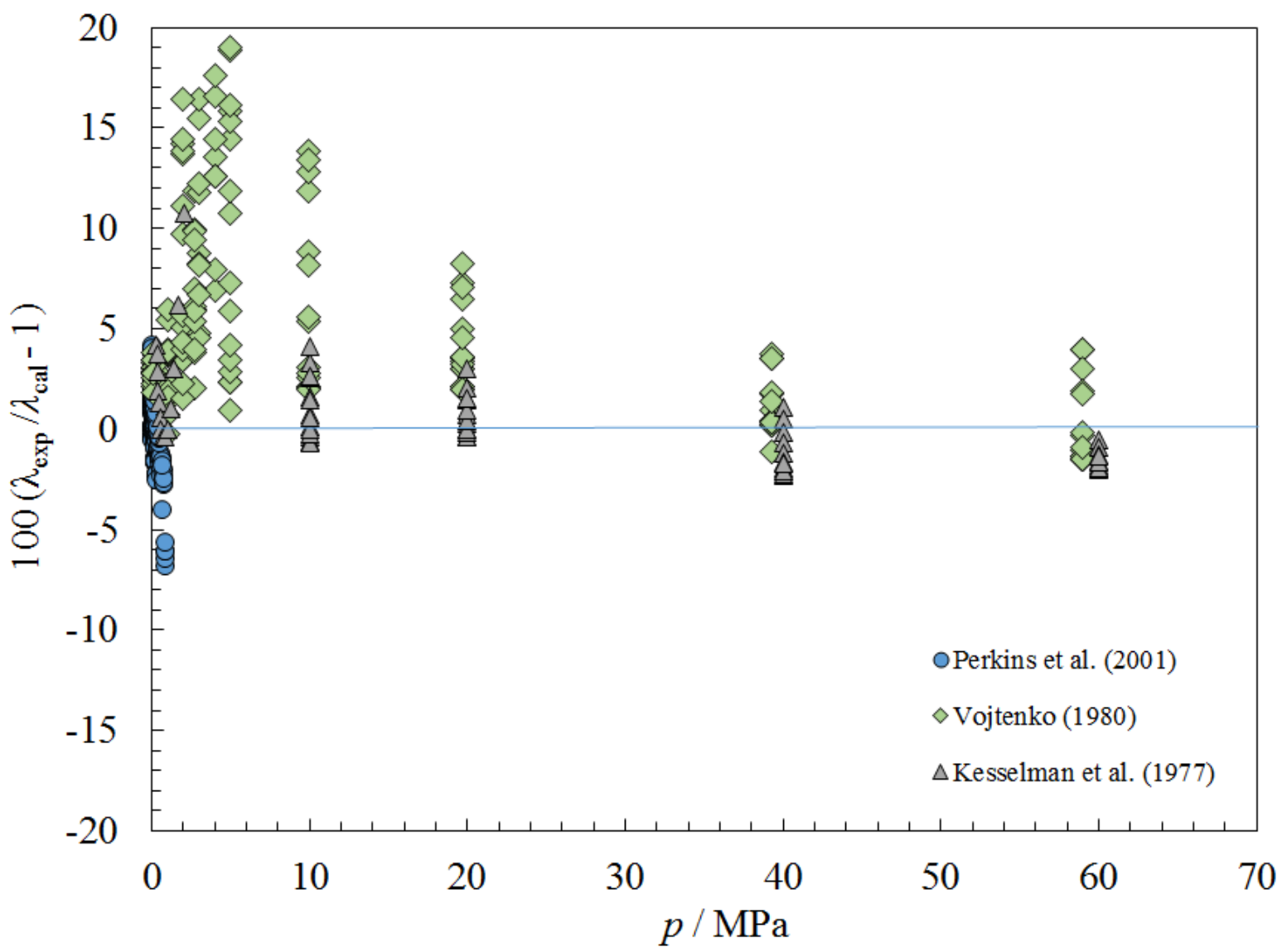

Figure 155. Percentage deviations between the model and the experimental thermal conductivity data for RC318 as a function of pressure.

\subsubsection{RE347mec (1,1,1,2,2,3,3-Heptafluoro-3-methoxypropane)}

This fluid is also known as HFE-7000. The equation of state of Zhou and Lemmon (Zhou \& Lemmon, 2018a) was used to provide the density and the critical parameters. Lennard-Jones parameters were estimated with the method of Chung et al. (Chung et al., 1988). The parameters in Table 2 were obtained by fitting the data of Hu et al. (Hu, Meng, Wei, Li, \& Wu, 2015), Nakazawa et al. (Nakazawa et al., 2002), and Rausch et al. (Rausch, Kretschmer, Will, Liepertz, $\&$ Fröba, 2015). The data of Rausch et al. (Rausch et al., 2015) are kinematic viscosities and were converted to dynamic viscosity using the densities from the EOS (Zhou \& Lemmon, 2018a). Figures 156 and 157 show the deviations in viscosity as a function of temperature and pressure, respectively. We estimate the uncertainty in the liquid phase to be $2 \%$ at temperatures above 250 $\mathrm{K}$ and pressures to $20 \mathrm{MPa}$, higher at lower temperatures. There are no experimental data in the gas phase. We estimate the uncertainty in the gas phase is $20 \%$. For thermal conductivity, there were enough data covering wide ranges of temperature and pressure to make a preliminary fluidspecific correlation instead of an extended corresponding states model. Preliminary data from Perkins (R. A. Perkins, 2018) were used to obtain the coefficients in Table 6. The value of $q_{\mathrm{d}}{ }^{-1}$ in Table 4 was obtained by fitting experimental data, and the other coefficients in Table 4 are from the generalized model of Perkins et al. (R. A. Perkins et al., 2013). Figures 158 and 159 show deviations as a function of temperature and pressure. Measurements in the liquid phase were 
obtained with a transient hot-wire method, and the gas-phase measurements were obtained with a steady-state hot-wire technique. Measurements are in progress on this fluid, and a separate publication with a finalized model is anticipated in late 2018 or early 2019 . The present model should be considered preliminary. The estimated uncertainty is $2 \%$ for the liquid at pressures to $70 \mathrm{MPa}, 5 \%$ in the vapor, and larger near the critical point. Mulero and Cachadiña (Mulero \& Cachadiña, 2014) published a correlation for surface tension.

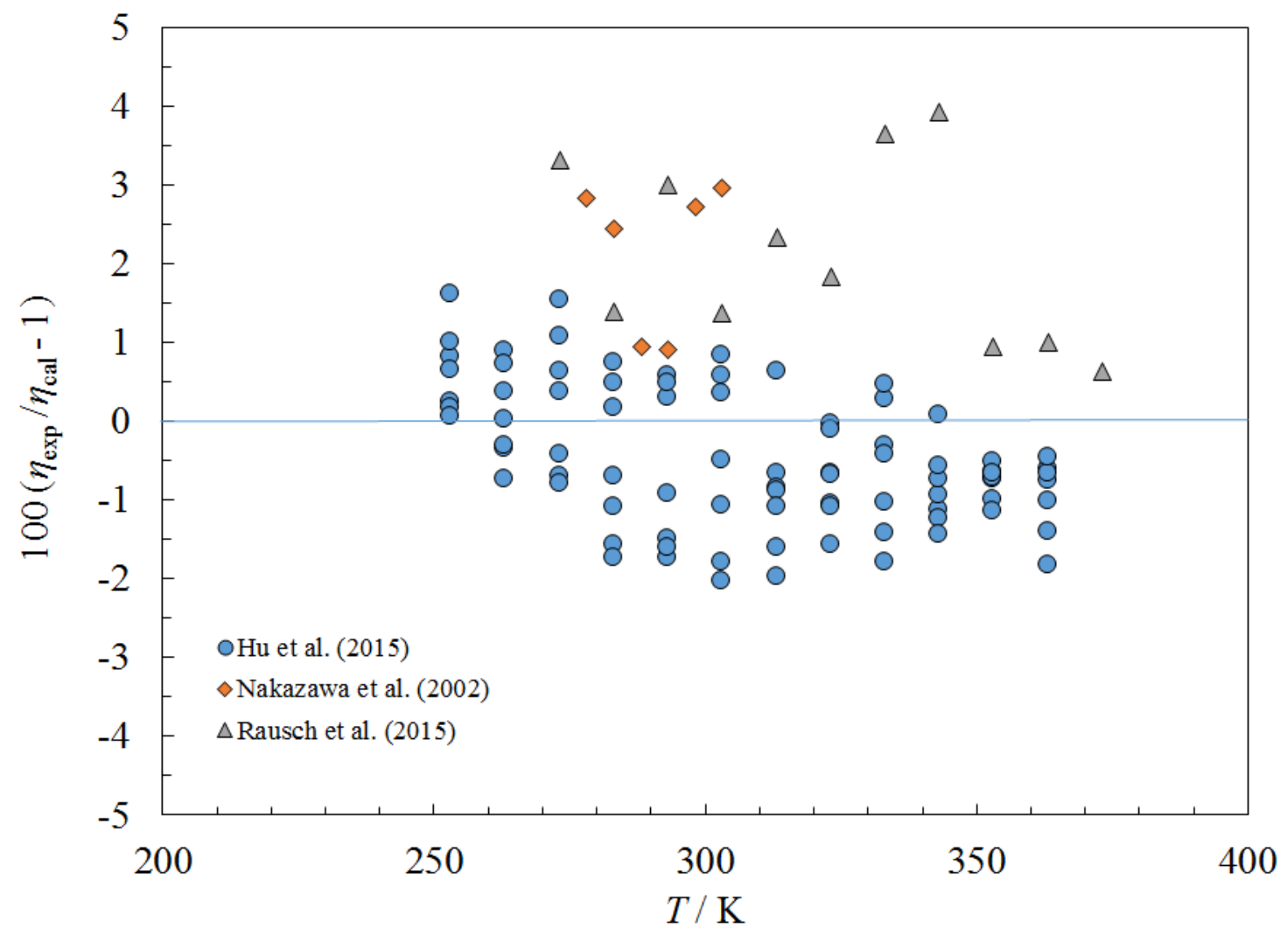

Figure 156. Percentage deviations between the model and the experimental viscosity data for RE347mcc as a function of temperature. 


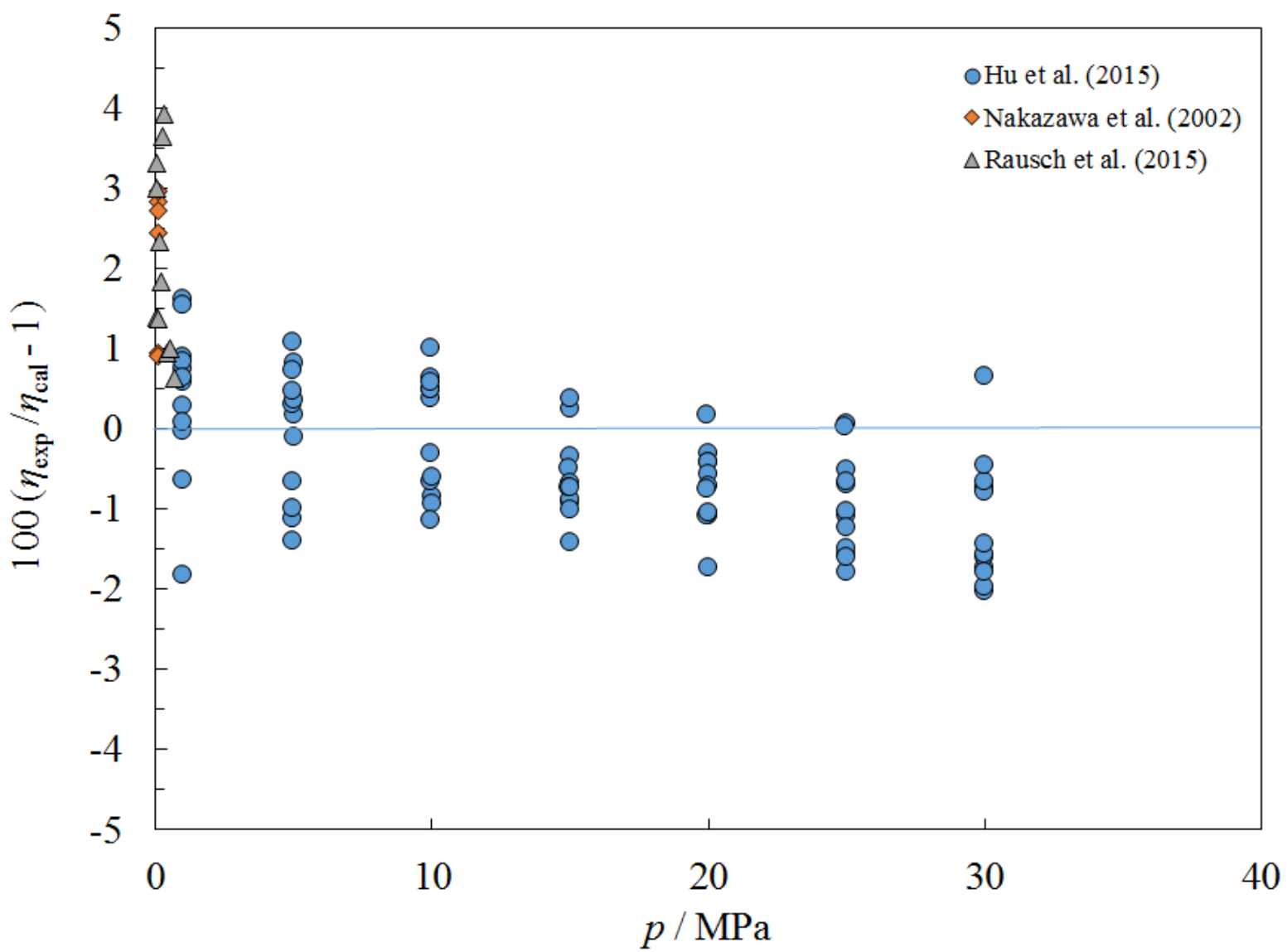

Figure 157. Percentage deviations between the model and the experimental viscosity data for RE347mcc as a function of pressure. 


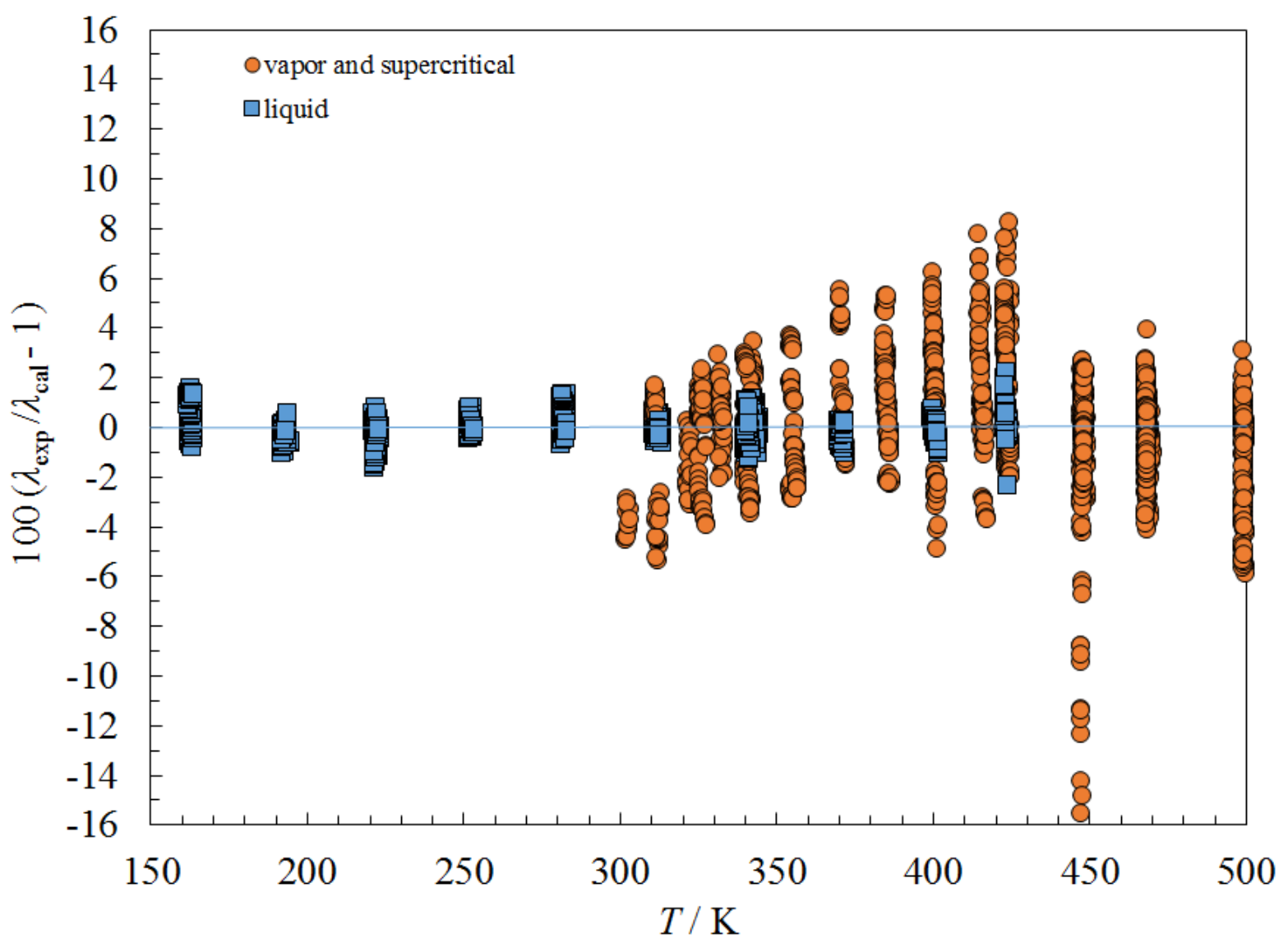

Figure 158. Percentage deviations between the model and the experimental thermal conductivity data for RE $347 \mathrm{mcc}$ as a function of temperature. 


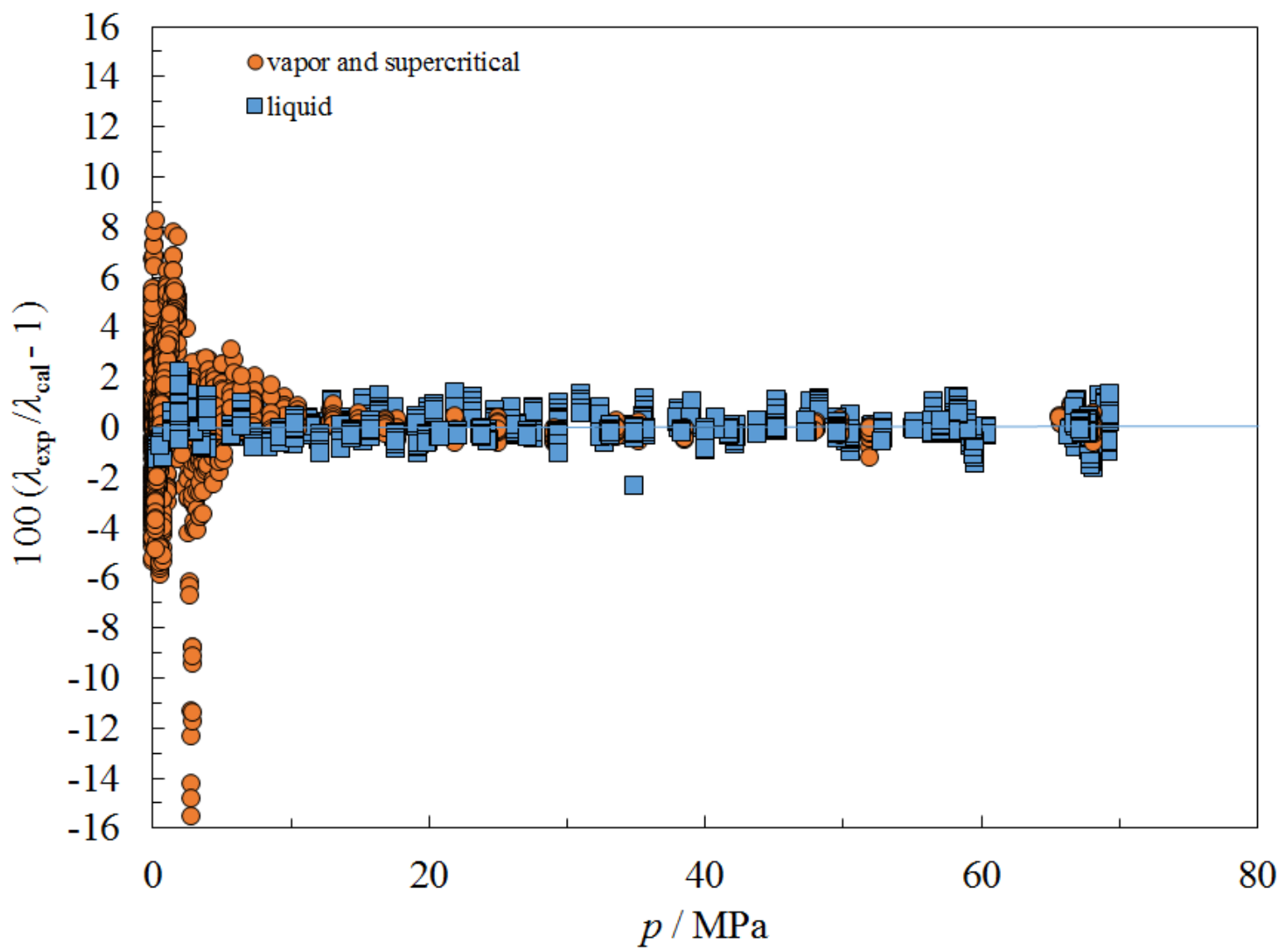

Figure 159. Percentage deviations between the model and the experimental thermal conductivity data for RE347mcc as a function of pressure.

\subsubsection{Perfluorobutane $\left(\mathrm{C}_{4} \mathrm{~F}_{10}\right)$}

A newly developed Helmholtz-form EOS for $\mathrm{C}_{4} \mathrm{~F}_{10}$ (K. Gao, J. Wu, \& E. W. Lemmon, 2017j) was used to provide densities. The Lennard-Jones coefficients were estimated by scaling the values from McCoubrey and Singh (J. C. McCoubrey \& Singh, 1960) for perfluoropentane, using the relationships from Chung et al. (Chung et al., 1988) $\left(\varepsilon / k_{\mathrm{B}}\right.$ scales with $T_{\mathrm{c}}, \sigma$ scales with $\left.V_{\mathrm{c}}{ }^{1 / 3}\right)$. No viscosity data were found. The coefficients in Table 2 were adjusted such that the value of the viscosity of the saturated liquid at the normal boiling point of perfluorobutane is $90 \%$ of the value of perfluoropentane at its normal boiling point. Due to the lack of data, the estimated uncertainty of the viscosity is 20 to $50 \%$. For thermal conductivity, we used the same value of $a_{\mathrm{o}}$ as was used for perfluoropentane. For the liquid, we used family behavior to obtain the thermal conductivity coefficients in Table 3. Parameters for the critical enhancement are presented in Table 4. We estimate the uncertainty of the thermal conductivity of the liquid and vapor phases to be $20 \%$ to $50 \%$. Surface tension is discussed in Mulero et al. (Mulero et al., 2012). 


\subsubsection{Perfluoropentane $\left(\mathrm{C}_{5} \mathrm{~F}_{12}\right)$}

A newly developed Helmholtz-form EOS for $\mathrm{C}_{5} \mathrm{~F}_{12}$ (K. Gao, J. Wu, \& A. W. Lemmon, 2017a) was used to provide densities. The Lennard-Jones coefficients were taken from McCoubrey and Singh (J. C. McCoubrey \& Singh, 1960). Viscosity data are extremely limited; only one set of liquid data at atmospheric pressure by Burger and Cady (Burger \& Cady, 1951) was found, and used to obtain the coefficients in Table 2. One additional source by Cochran et al. (Cochran, North, $\&$ Pethrick, 1974) was located but not used, since the purity was given as only $90 \%$ and the values differed significantly from those of Burger and Cady (Burger \& Cady, 1951). Figure 160 shows percentage deviations between the model and the experimental viscosity data used in regression. Deviations from Cochran et al. (Cochran et al., 1974) range from $38 \%$ to $58 \%$ and are not shown. For temperatures above $180 \mathrm{~K}$ and atmospheric pressure, we estimate the uncertainty is $5 \%$, rising to $10 \%$ at pressures to $30 \mathrm{MPa}$. Uncertainty in the gas phase is $5 \%$. We were unable to locate experimental thermal conductivity data in the gas phase, so we selected a value of 0.00125 for $a_{0}$ in Eq. (16). This was selected because it results in the gas-phase thermal conductivity of a related fluid, perfluoropropane, to be within $\sim 10 \%$ of experimental data. Only one valid liquid-phase thermal conductivity data point was found (Irving \& Jamieson, 1975), and this, along with family behavior, was used to obtain the coefficients in Table 3. Parameters for the critical enhancement are presented in Table 4 . We estimate the uncertainty of the thermal conductivity of the liquid and vapor phases to be $10 \%$. Surface tension is discussed in a separate publication (Mulero et al., 2012). 


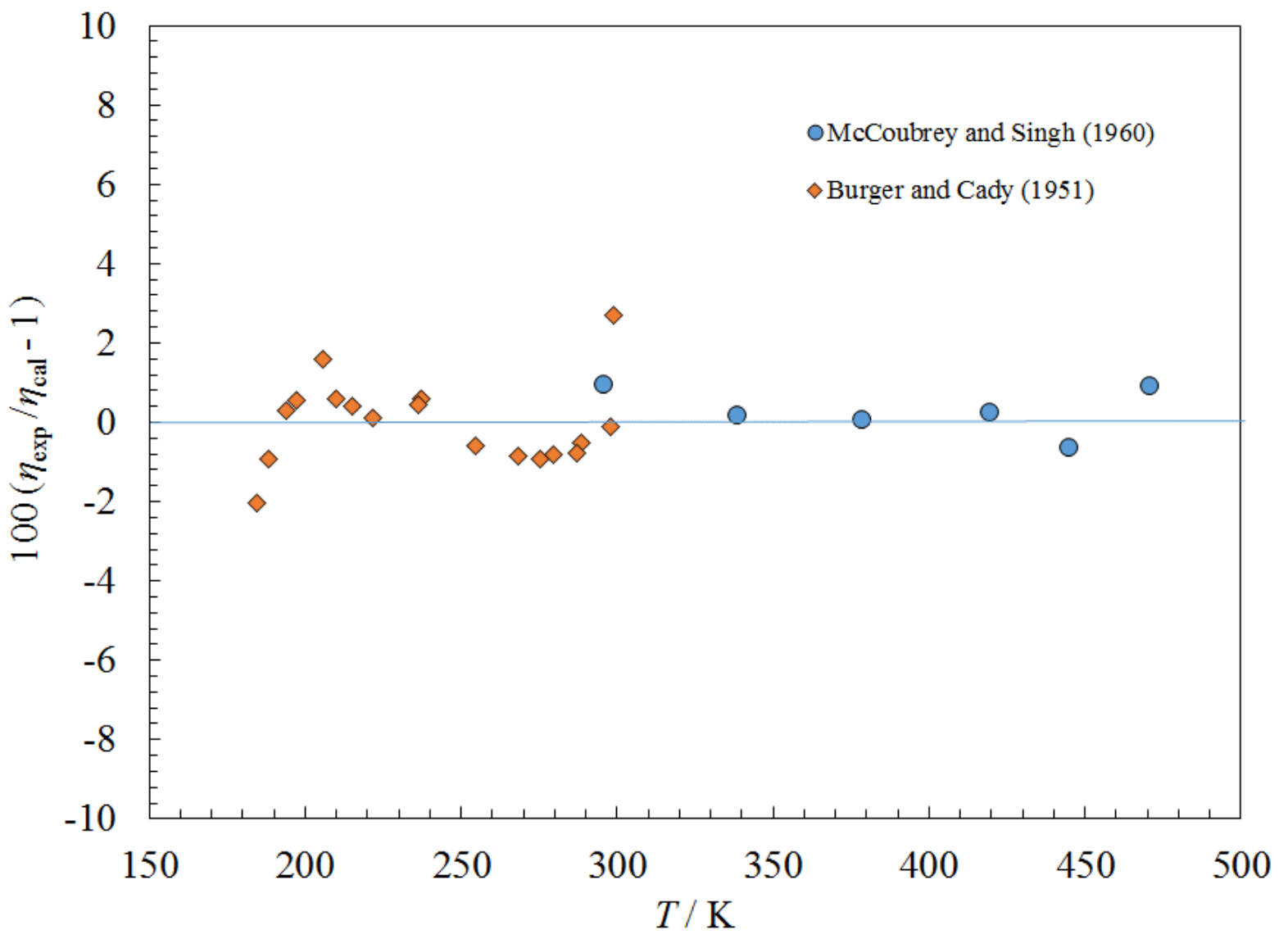

Figure 160. Percentage deviations between the model and the experimental viscosity data for perfluoropentane as a function of temperature.

\subsubsection{Perfluorohexane $\left(\mathrm{C}_{6} \mathrm{~F}_{14}\right)$}

A newly developed Helmholtz-form EOS for $\mathrm{C}_{6} \mathrm{~F}_{14}$ (K. Gao, J. Wu, \& E. W. Lemmon, 2017k) was used to provide densities. The Lennard-Jones coefficients were taken from McCoubrey and Singh (J. C. McCoubrey \& Singh, 1960). Several sources of liquid-phase viscosity data were found (Cochran et al., 1974; M.G. Freire, Ferreira, Fonseca, Marrucho, \& Coutinho, 2008; Stiles \& Cady, 1952), although none at pressures above saturation. In contrast to the situation for perfluoropentane discussed earlier, the sample of perfluorohexane was $99 \%$ pure and was used in the regression. The data of Freire et al. (M.G. Freire et al., 2008) are kinematic viscosities and were converted to absolute viscosity with densities from the EOS. These data appeared to differ significantly from the other sources and were not used in the regression to obtain the parameters in Table 2, but are shown in the deviation plot given in Figure 161. One source of gas-phase viscosity data was found (J. C. McCoubrey \& Singh, 1960). We estimate the uncertainty of the viscosity in the liquid phase at atmospheric pressure above $230 \mathrm{~K}$ is $5 \%$, rising to $10 \%$ at pressures to $40 \mathrm{MPa}$; the uncertainty in the gas phase is also $5 \%$. For thermal conductivity, we used the same value of $a_{0}$ as was used for perfluoropentane. For the liquid, we fit the data of Irving and Jamieson (Irving \& Jamieson, 1975) to obtain the coefficients in Table 3, and a deviation plot is shown in Figure 162. Parameters for the critical enhancement are presented in Table 4. The data are extremely limited, and we estimate the uncertainty in both the gas and liquid phases to be $10 \%$. For surface tension, we fit the available surface tension data (Ermakov \& Skripov, 1969; M. G. 
Freire, Carvalho, Queimada, Marrucho, \& Coutinho, 2006; Kennan \& Pollack, 1988; Skripov \& Firsov, 1968; Stiles \& Cady, 1952) to obtain the coefficients in Table 5, and a deviation plot is shown in Figure 163. There is a large amount of scatter in the data, and we estimate the uncertainty for surface tension is $6 \%$. 


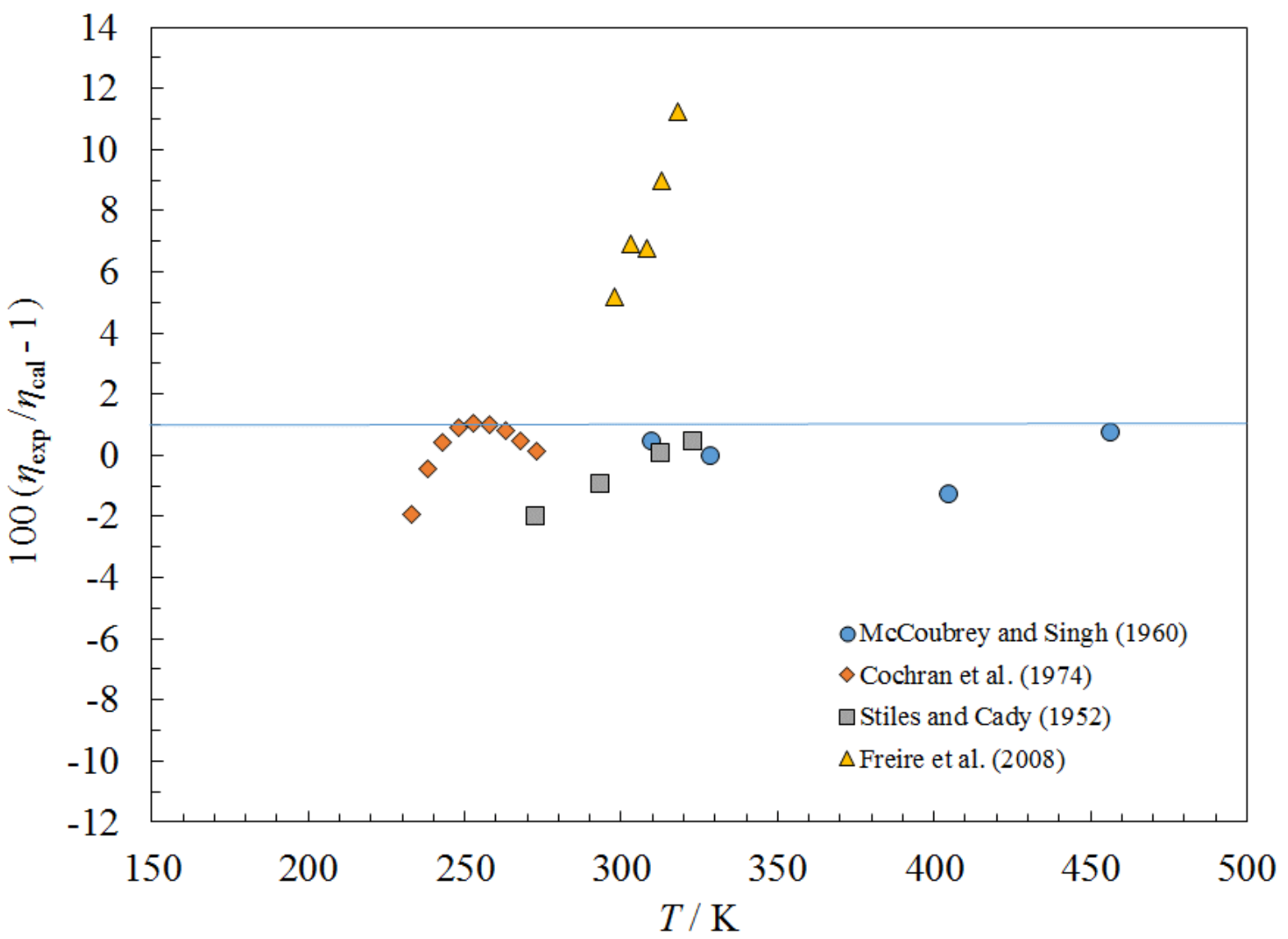

Figure 161. Percentage deviations between the model and the experimental viscosity data for perfluorohexane as a function of temperature. 


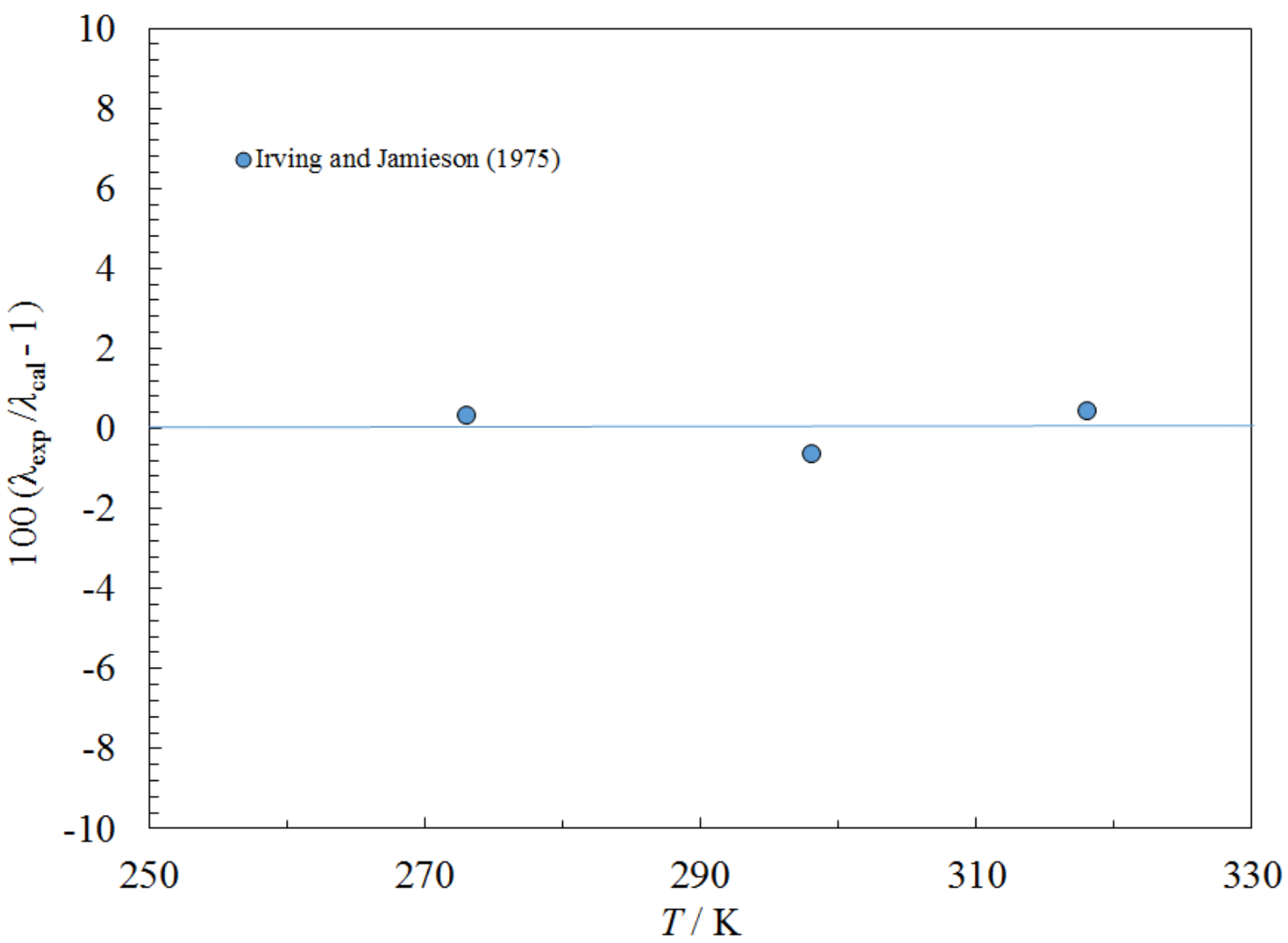

Figure 162. Percentage deviations between the model and the experimental thermal conductivity data for perfluorohexane as a function of temperature. 


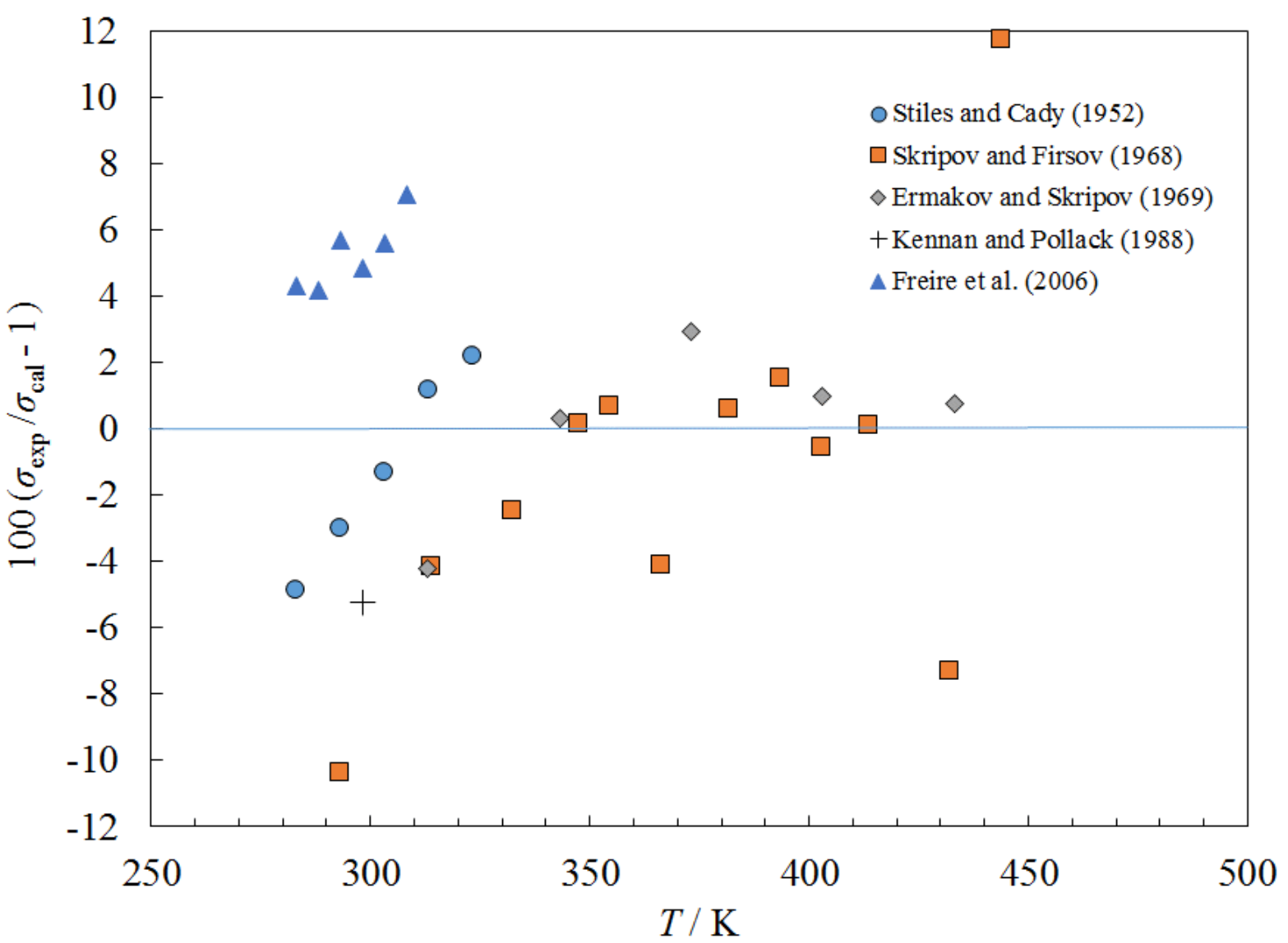

Figure 163. Percentage deviations between the model and the experimental surface tension data for perfluorohexane as a function of temperature.

\subsection{Fatty acid methyl esters (FAMES)}

\subsubsection{Methyl palmitate}

The equation of state of Huber et al. (Huber, Lemmon, Kazakov, Ott, \& Bruno, 2009) was used to provide the density and the critical parameters. Lennard-Jones parameters were estimated with the method of Chung et al. (Chung et al., 1988). Liquid-phase data at atmospheric pressure of Pratas et al. (Pratas et al., 2011) were used to obtain the coefficients in Table 2, and Figure 164 shows deviations from the data as a function of temperature. Other data sets (Ceriani et al., 2007; Yao, Hammond, \& Wang, 2008) are shown in the deviation plot but were not used in the fit. We estimate that the uncertainty of the viscosity in the liquid phase at atmospheric pressure from $308 \mathrm{~K}$ to $363 \mathrm{~K}$ is $2 \%$. Data in the vapor phase were unavailable, and we estimate the uncertainty in the gas phase is $20 \%$. Data for thermal conductivity were unavailable. As an estimate, we used the coefficients for the dedicated polynomial model for methyl oleate in Perkins and Huber (R. A. Perkins \& Huber, 2011) for methyl palmitate, changing only the critical parameters. The estimated uncertainty for thermal conductivity in the liquid phase at atmospheric pressure is $5 \%$ and in the vapor phase is $20 \%$. A separate publication covers surface tension (Mulero \& Cachadiña, 2014); it is not covered here. 


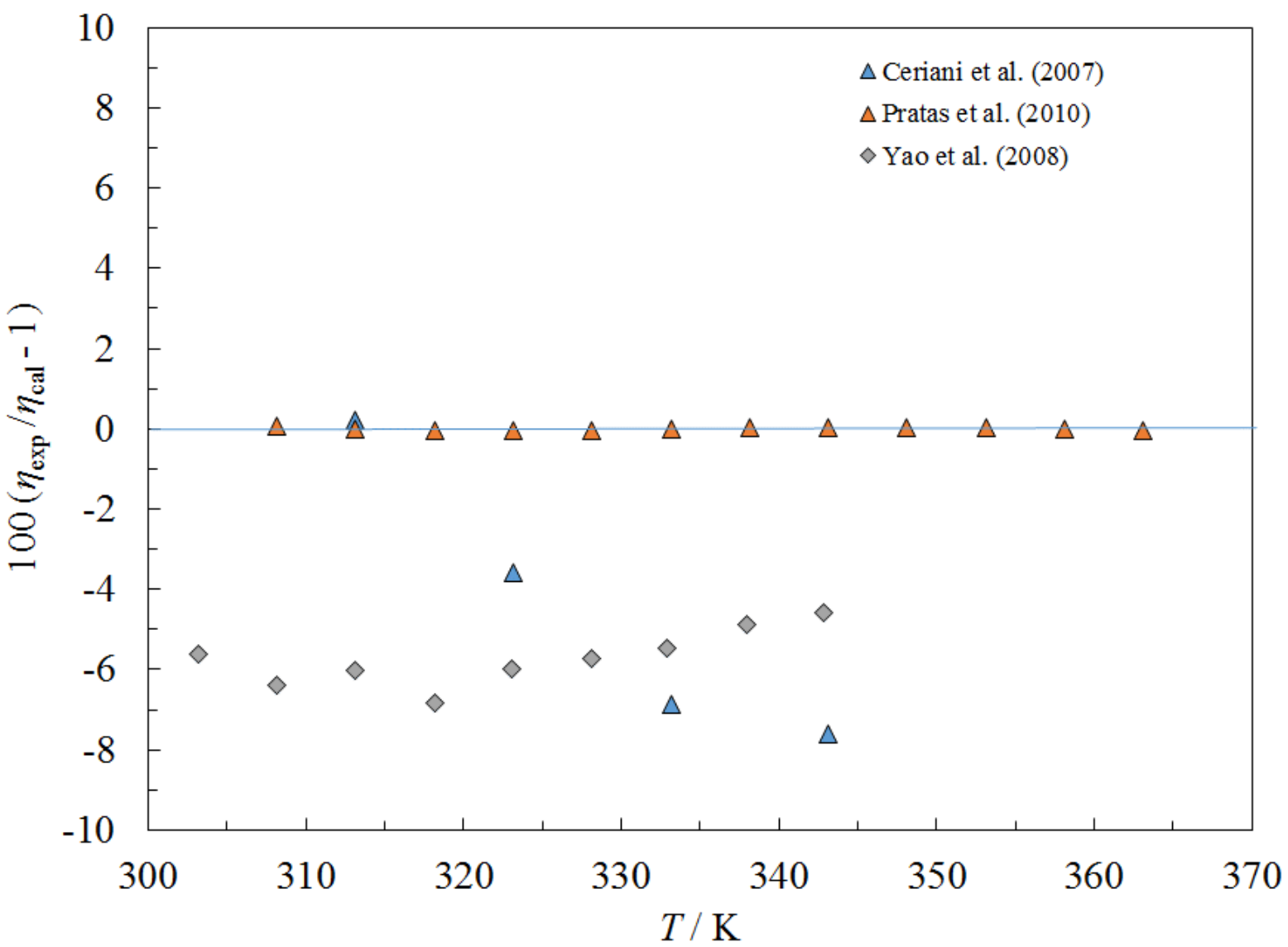

Figure 164. Percentage deviations between the model and the viscosity data for methyl palmitate as a function of temperature.

\subsubsection{Methyl linolenate}

The equation of state of Huber et al. (Huber et al., 2009) was used to provide the density and the critical parameters. Lennard-Jones parameters were estimated with the method of Chung et al. (Chung et al., 1988). Liquid-phase data at atmospheric pressure of Pratas et al. (Pratas et al., 2011) were used to obtain the coefficients in Table 2, and Figure 165 shows deviations from the data as a function of temperature. In previous versions of REFPROP, the coefficients were obtained by fitting the 2007 data of Ceriani et al. (Ceriani et al., 2007), which are also shown in the deviation plot. Based on comparison with other related fluids, such as methyl oleate, we feel the Pratas et al. (Pratas et al., 2011) data are superior and have used them in our present analysis. We estimate that the uncertainty of the viscosity in the liquid phase at atmospheric pressure from $278 \mathrm{~K}$ to $373 \mathrm{~K}$ is $3 \%$. Data in the vapor phase were unavailable, and we estimate the uncertainty in the gas phase is $20 \%$. Data for thermal conductivity were unavailable. As was done for methyl palmitate, for an estimate, we used the coefficients for the model for methyl oleate in Perkins and Huber (R. A. Perkins \& Huber, 2011) for methyl linolenate, changing only the critical parameters. The estimated uncertainty for thermal conductivity in the liquid phase at atmospheric pressure is $5 \%$ and in the vapor phase is $20 \%$. Similarly, surface tension data were unavailable so we used the coefficients for methyl oleate from Mulero and Cachadiña (Mulero \& Cachadiña, 2014), again changing only the critical point, as an estimate. 


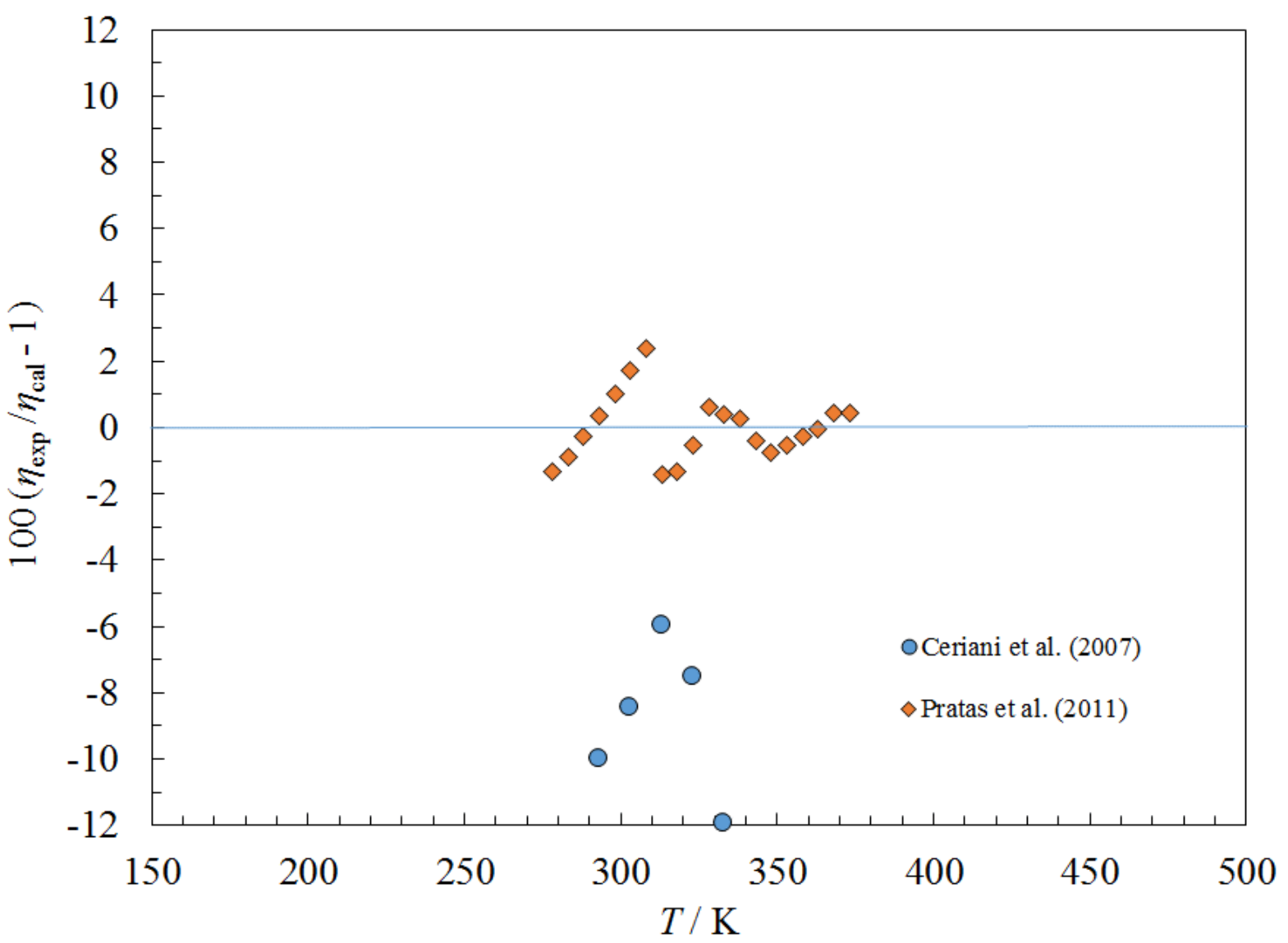

Figure 165. Percentage deviations between the model and the viscosity data for methyl linolenate as a function of temperature.

\subsubsection{Methyl linoleate}

The equation of state of Huber et al. (Huber et al., 2009) was used to provide the density and the critical parameters. Lennard-Jones parameters were estimated with the method of Chung et al. (Chung et al., 1988). Liquid-phase data at atmospheric pressure of Pratas et al. (Pratas et al., 2011) were used to obtain the coefficients in Table 2, and Figure 166 shows deviations from the data as a function of temperature. In previous versions of REFPROP, the coefficients were obtained by fitting the 2007 data of Ceriani et al. (Ceriani et al., 2007), which are also shown in the deviation plot. Based on comparison with other related fluids, such as methyl oleate, we feel the Pratas et al. (Pratas et al., 2011) data are superior and have used them in our present analysis. We estimate that the uncertainty of the viscosity in the liquid phase at atmospheric pressure from $278 \mathrm{~K}$ to $353 \mathrm{~K}$ is $2 \%$. Data in the vapor phase were unavailable, and we estimate the uncertainty in the gas phase is $20 \%$. Separate publications cover the thermal conductivity (R. A. Perkins \& Huber, 2011) and surface tension (Mulero \& Cachadiña, 2014); they are not covered here. 


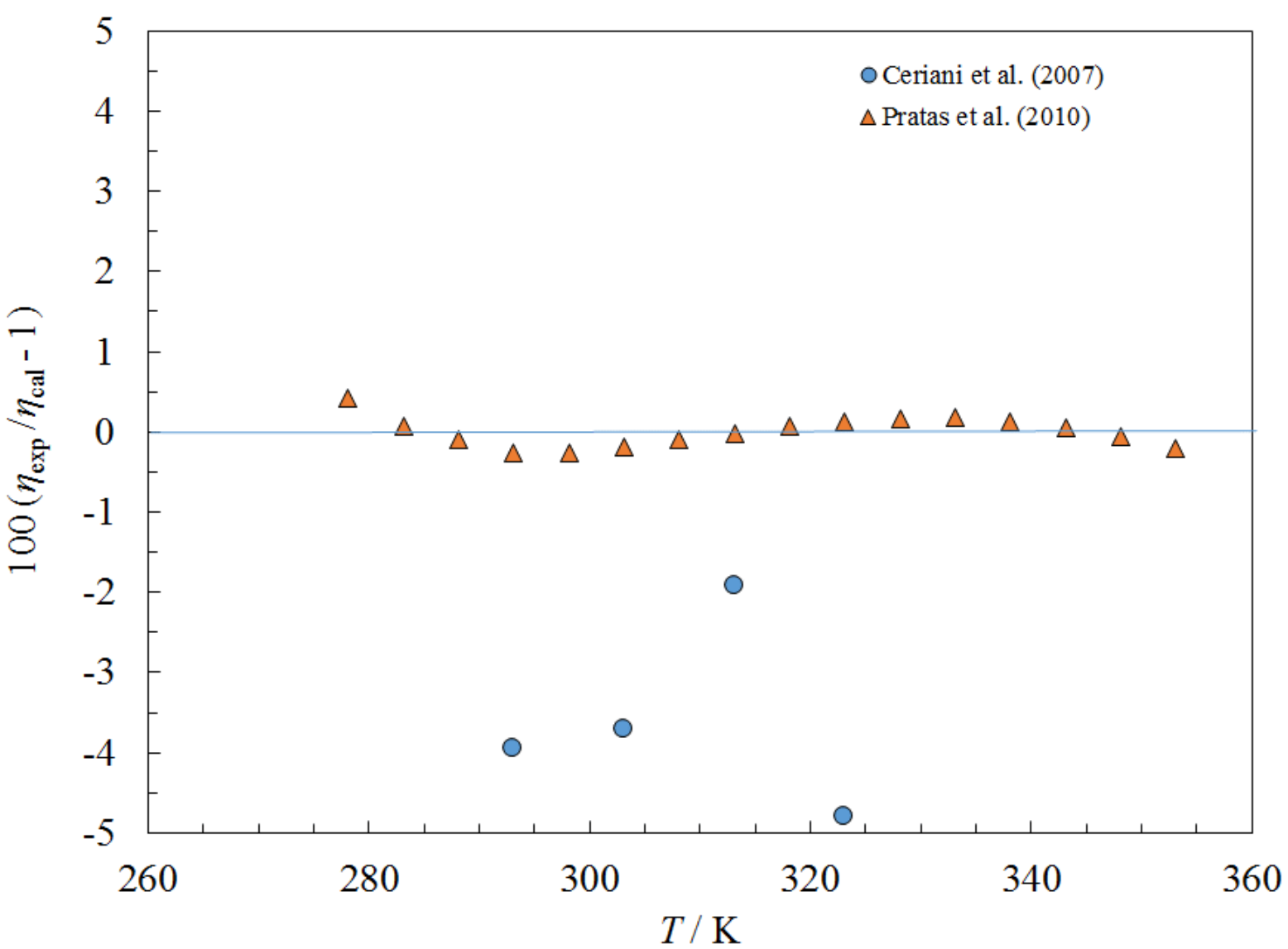

Figure 166. Percentage deviations between the model and the viscosity data for methyl linoleate as a function of temperature.

\subsubsection{Methyl oleate}

The equation of state of Huber et al. (Huber et al., 2009) was used to provide the density and the critical parameters. Lennard-Jones parameters were estimated with the method of Chung et al. (Chung et al., 1988). Liquid-phase data at atmospheric pressure of Pratas et al. (Pratas et al., 2011) and Yao et al. (Yao et al., 2008) were used to obtain the coefficients in Table 2, and Figure 167 shows deviations from the data as a function of temperature. In previous versions of REFPROP, the coefficients were obtained by fitting the 2007 data of Ceriani et al. (Ceriani et al., 2007), which are also shown in the deviation plot. We feel the Pratas et al. (Pratas et al., 2011) data are superior and have used them in our present analysis. We estimate that the uncertainty of the viscosity in the liquid phase at atmospheric pressure from $273 \mathrm{~K}$ to $353 \mathrm{~K}$ is $2 \%$. Data in the vapor phase were unavailable, and we estimate the uncertainty in the gas phase is $20 \%$. Separate publications cover the thermal conductivity (R. A. Perkins \& Huber, 2011) and surface tension (Mulero \& Cachadiña, 2014); they are not covered here. 


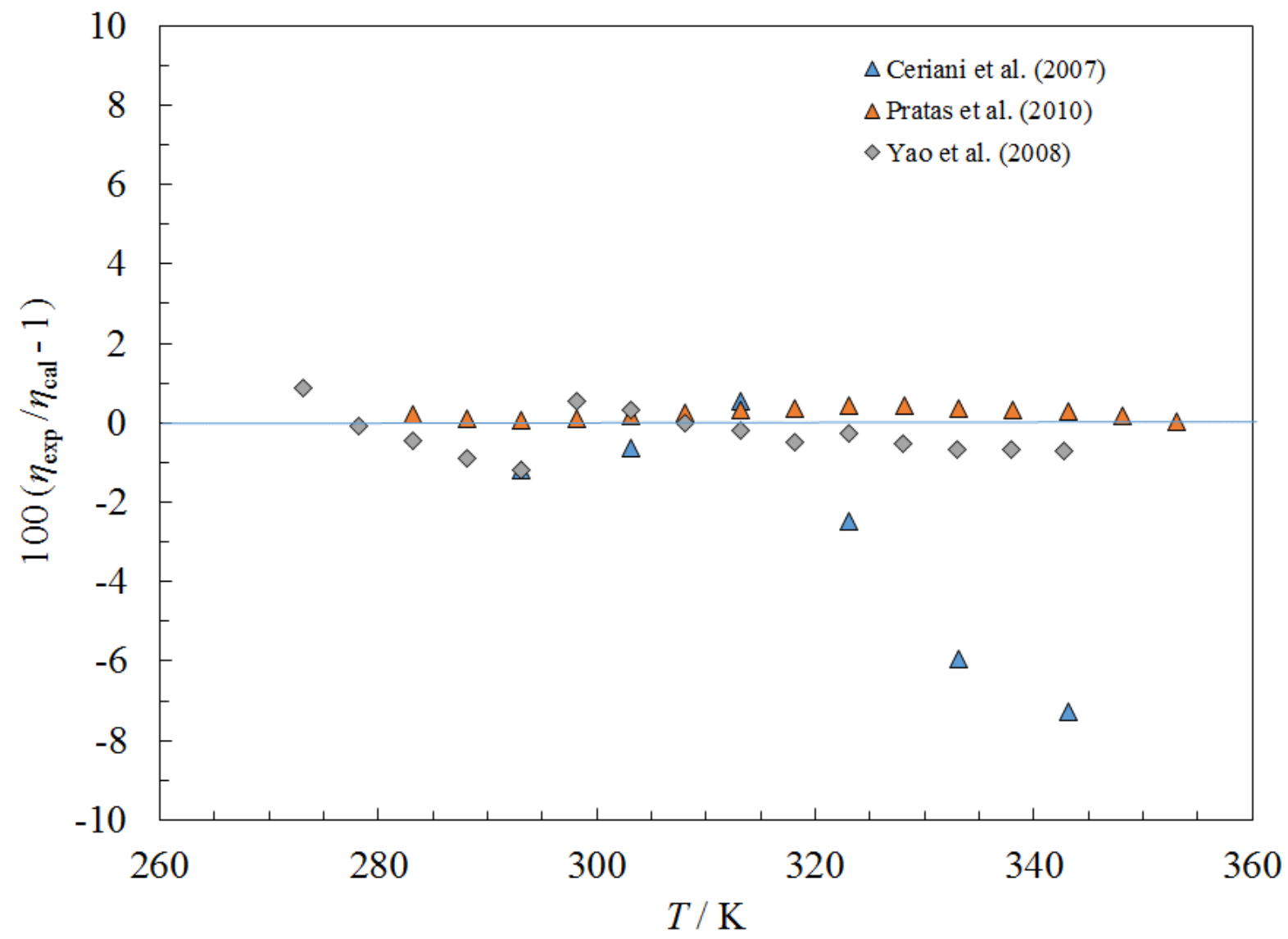

Figure 167. Percentage deviations between the model and the viscosity data for methyl oleate as a function of temperature.

\subsubsection{Methyl stearate}

The equation of state of Huber et al. (Huber et al., 2009) was used to provide the density and the critical parameters. Lennard-Jones parameters were estimated with the method of Chung et al. (Chung et al., 1988). Liquid-phase data at atmospheric pressure of Pratas et al. (Pratas et al., 2011) were used to obtain the coefficients in Table 2, and Figure 168 shows deviations from the data as a function of temperature. We estimate that the uncertainty of the viscosity in the liquid phase at atmospheric pressure from $313 \mathrm{~K}$ to $363 \mathrm{~K}$ is $2 \%$. Data in the vapor phase were unavailable, and we estimate the uncertainty in the gas phase is $20 \%$. Data for thermal conductivity were unavailable. As an estimate, we used the coefficients for the model for methyl oleate in Perkins and Huber (R. A. Perkins \& Huber, 2011) for methyl stearate, changing only the critical parameters. A separate publication covers surface tension (Mulero \& Cachadiña, 2014); it is not covered here. 


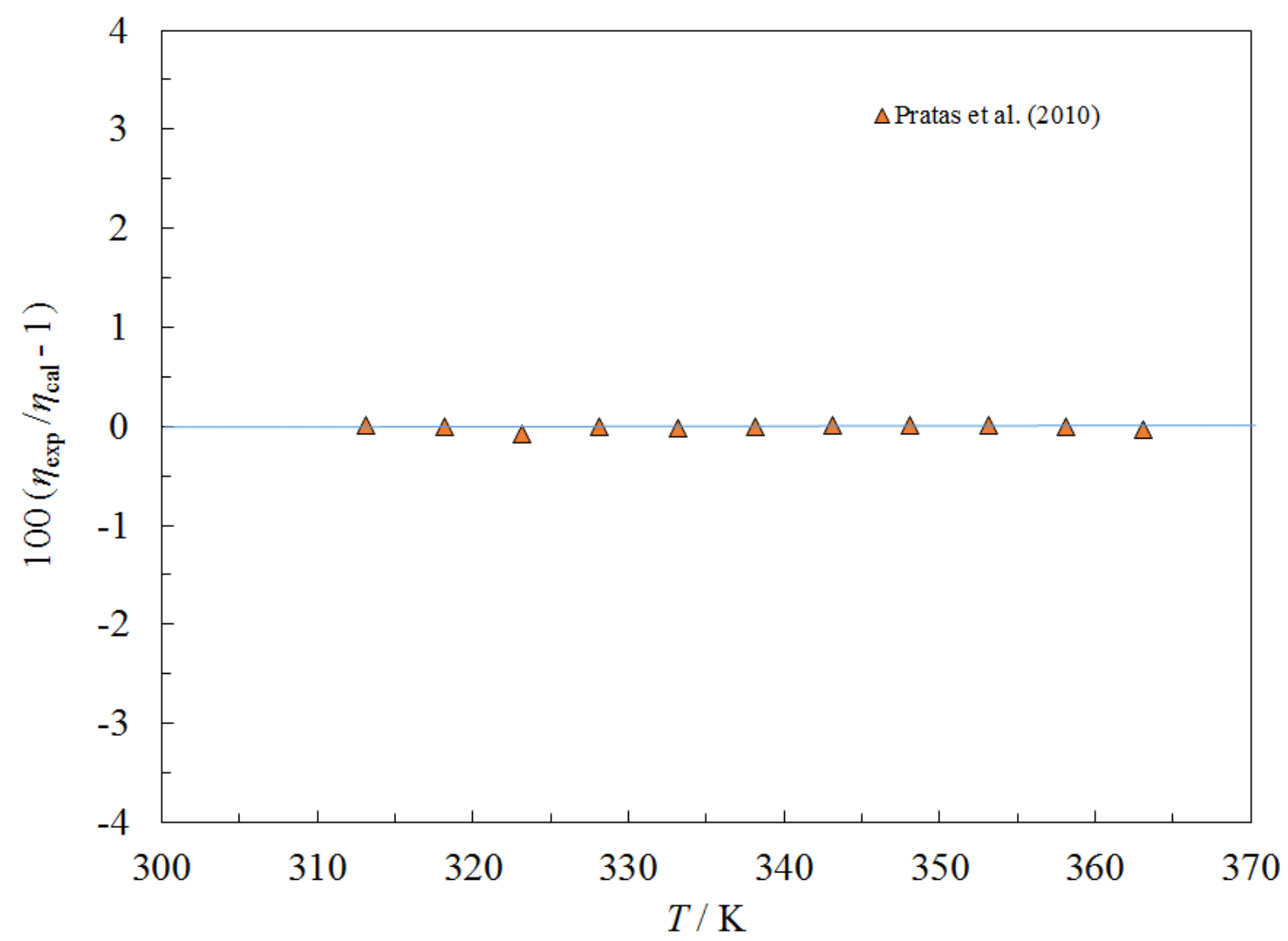

Figure 168. Percentage deviations between the model and the viscosity data for methyl stearate as a function of temperature.

\subsection{Noble Gases}

\subsubsection{Neon}

The equation of state of Thol et al. (Thol, Beckmüller, et al., 2018) was used to provide the density and the critical parameters. Lennard-Jones parameters were obtained from Kestin et al. (J. Kestin, S.T. Ro, \& W.A. Wakeham, 1972a). Viscosity data from five sources (Förster, 1963; Kestin et al., 1972a; Reynes \& Thodos, 1966; Slusar, Rudenko, \& Tret'yakov, 1973; E. Vogel, 1984) were used to obtain the coefficients in Table 2, and Figures 169 and 170 show deviations with those data sources as a function of temperature and of pressure, respectively. The data of Slusar et al. (Slusar et al., 1973) are along the saturated liquid and vapor boundary, and Förster (Förster, 1963) also is along the liquid saturation boundary. The coefficient $F_{\mathrm{c}}$ in Table 2 was adjusted to ensure that the value of the dilute-gas viscosity at $25{ }^{\circ} \mathrm{C}$ and $0.1 \mathrm{MPa}$ agreed with the reference value presented in Assael et al. (Assael et al., 2018) to within its stated uncertainty. We estimate the uncertainty for the viscosity for both the saturated liquid and vapor is $10 \%$. The uncertainty in the supercritical region at atmospheric pressure is $2 \%$, rising to $4 \%$ at $80 \mathrm{MPa}$. Due to the internal contribution to the dilute-gas thermal conductivity in Eq. (15) being identically zero for noble gases, it is not possible to fit coefficients for $f_{\text {int }}$ and the value is Table 3 is a placeholder only. Gas-phase thermal conductivity data from Millat et al. (Millat, Ross, Wakeham, \& Zalaf, 1988), Saxena and Tondon (Saxena \& Tondon, 1971), and Vargaftik and Yakush (Vargaftik \& 
Yakush, 1971) are shown in Figure 171 for comparison purposes. We were unable to locate any liquid-phase thermal conductivity data except for 5 saturated liquid data points over a small temperature range from Bewilogua and Yamashira (Bewilogua \& Yamashira, 1972); these were used to obtain the liquid-phase parameters in Table 3. Parameters for the critical enhancement are presented in Table 4. Figure 169 shows a deviation plot with the selected experimental data. We estimate the uncertainty of the thermal conductivity in the liquid phase is $10 \%$ at saturation, and $5 \%$ for the gas at atmospheric pressure. Surface tension is discussed in Mulero et al. (Mulero et al., 2012).

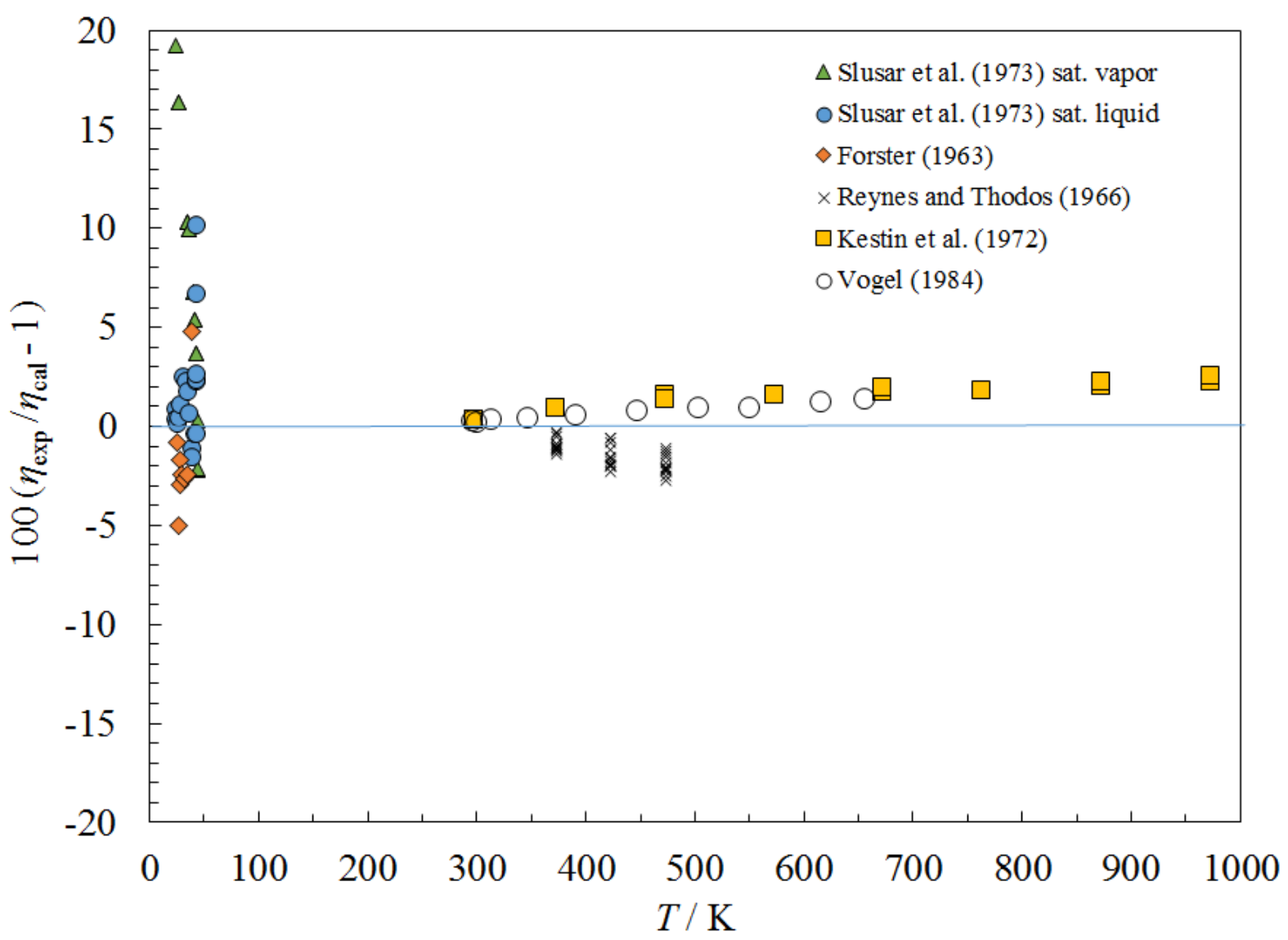

Figure 169. Percentage deviations between the model and the experimental viscosity data for neon as a function of temperature. 


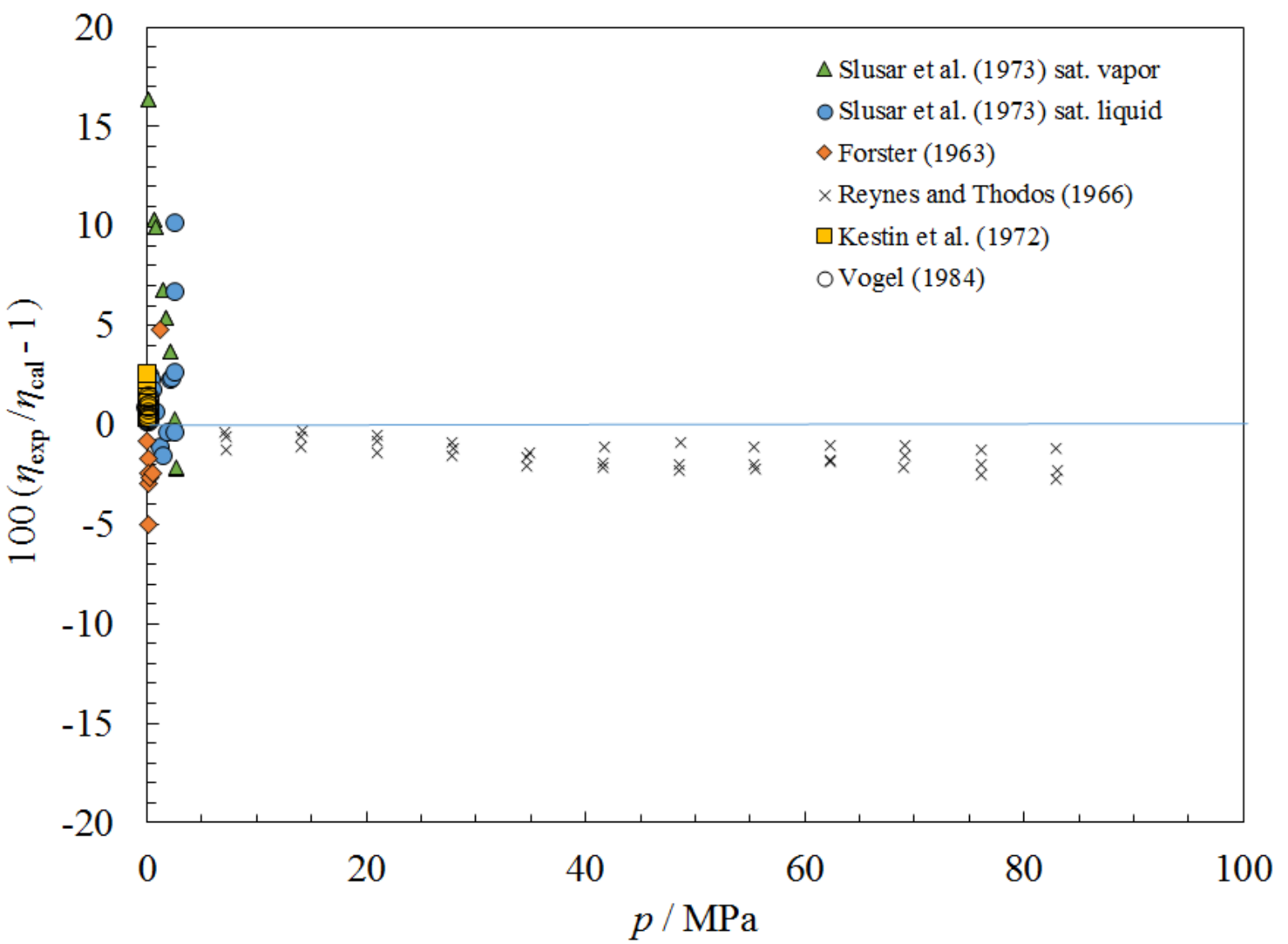

Figure 170. Percentage deviations between the model and the experimental viscosity data for neon as a function of pressure. 


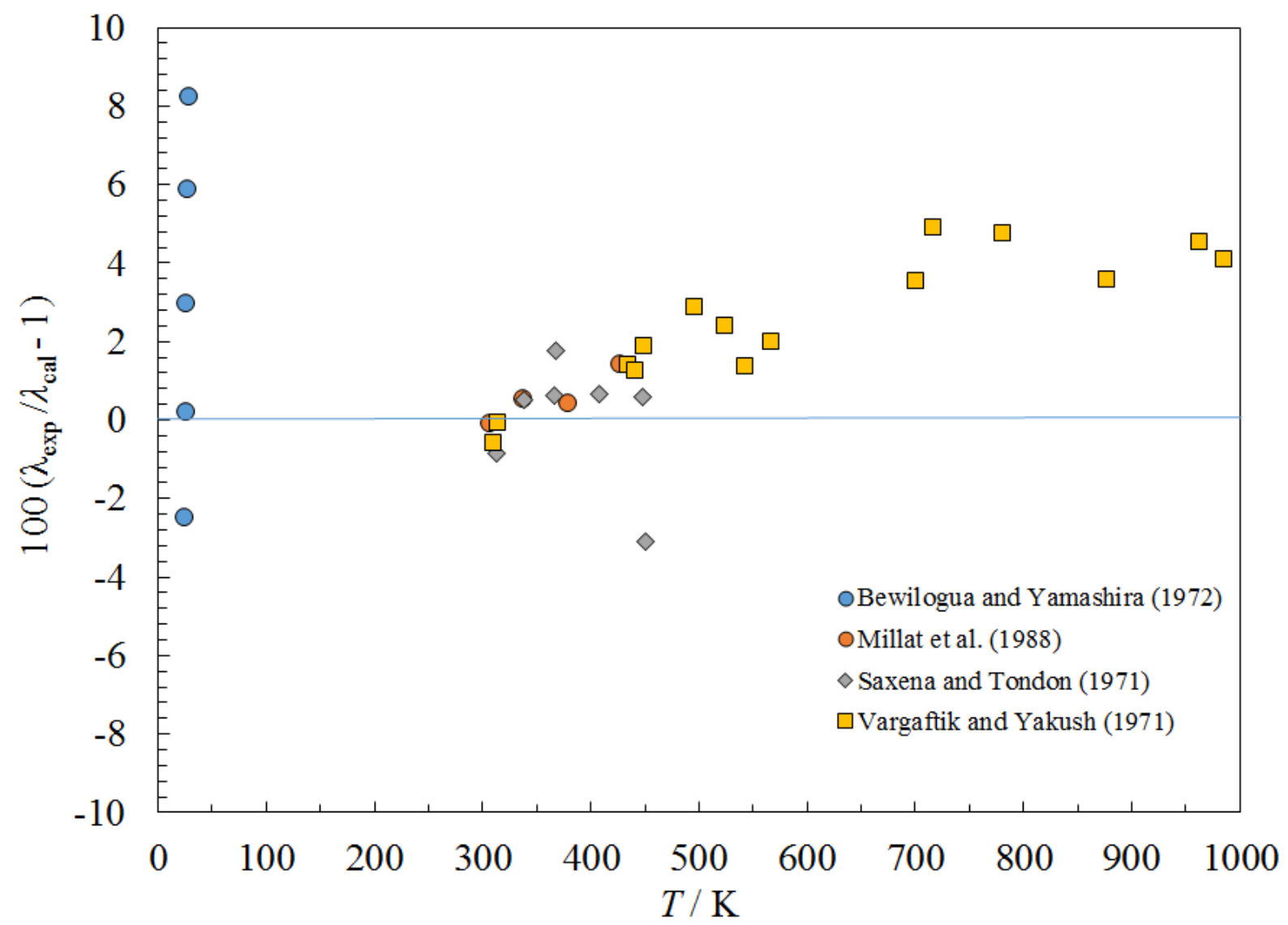

Figure 171. Percentage deviations between the model and the experimental thermal conductivity data for neon as a function of temperature.

\subsubsection{Krypton}

The equation of state of Lemmon and Span (E.W. Lemmon \& Span, 2006) was used to provide the density and the critical parameters. Lennard-Jones parameters were obtained from Reid et al. (Reid, 1987). At the time of the release of REFPROP v10, we were unable to locate any liquid-phase viscosity data, and the corresponding states model was used in a totally predictive mode. After the release of REFPROP v10 we became aware of the saturated liquid-phase viscosity data of Boon et al. (Boon, Legros, \& Thomaes, 1967). Comparisons with selected gas-phase data (Evers, Loesch, \& Wagner, 2002; Kestin, Khalifa, \& Wakeham, 1977; J. Kestin, S.T. Ro, \& W. A. Wakeham, 1972b; Kestin, Wakeham, \& Watanabe, 1970) and the liquid-phase data of Boon et al. (Boon et al., 1967) are shown in Figure 172. The estimated uncertainty in the gas phase is $3 \%$ at atmospheric pressure, and the estimated uncertainty for the liquid phase at saturation is also $3 \%$. The coefficient $F_{\mathrm{c}}$ in Table 2 was adjusted to ensure that the value of the dilute-gas viscosity at 25 ${ }^{\circ} \mathrm{C}$ and 0.1 MPa matched the reference value presented in Assael et al. (Assael et al., 2018) to within its stated uncertainty. This is also in better agreement with the 2002 data of Evers et al. (Evers et al., 2002), which are systematically lower than the earlier measurements made in the 1970's. Due to the internal contribution to the dilute-gas thermal conductivity in Eq. (15) being identically zero for noble gases, it is not possible to fit coefficients for $f_{\text {int }}$ and the value is Table 3 
is a placeholder only. The liquid-phase data of Ikenberry and Rice (Ikenberry \& Rice, 1963) were used to obtain the coefficients in Table 3. Additional gas-phase data from several sources (Hammerschmidt, 1995; Makhrov \& Miroshnichenko, 1984; Saxena \& Gupta, 1970; Saxena \& Tondon, 1971; Vargaftik \& Yakush, 1971; Voshinin, Kerzhentsev, Studnikov, \& Yakush, 1975; Zaitseva, 1959) are used for comparison purposes only. Parameters for the critical enhancement are presented in Table 4. Deviations of thermal conductivity as a function of temperature and pressure are shown in Figures 173 and 174. We estimate the uncertainty of thermal conductivity in the liquid phase at pressures to $50 \mathrm{MPa}$ is $4 \%$; for the vapor phase at temperatures up to $400 \mathrm{~K}$ it is also $4 \%$, rising to $10 \%$ at $1000 \mathrm{~K}$. Surface tension is discussed in Mulero et al. (Mulero et al., 2012).

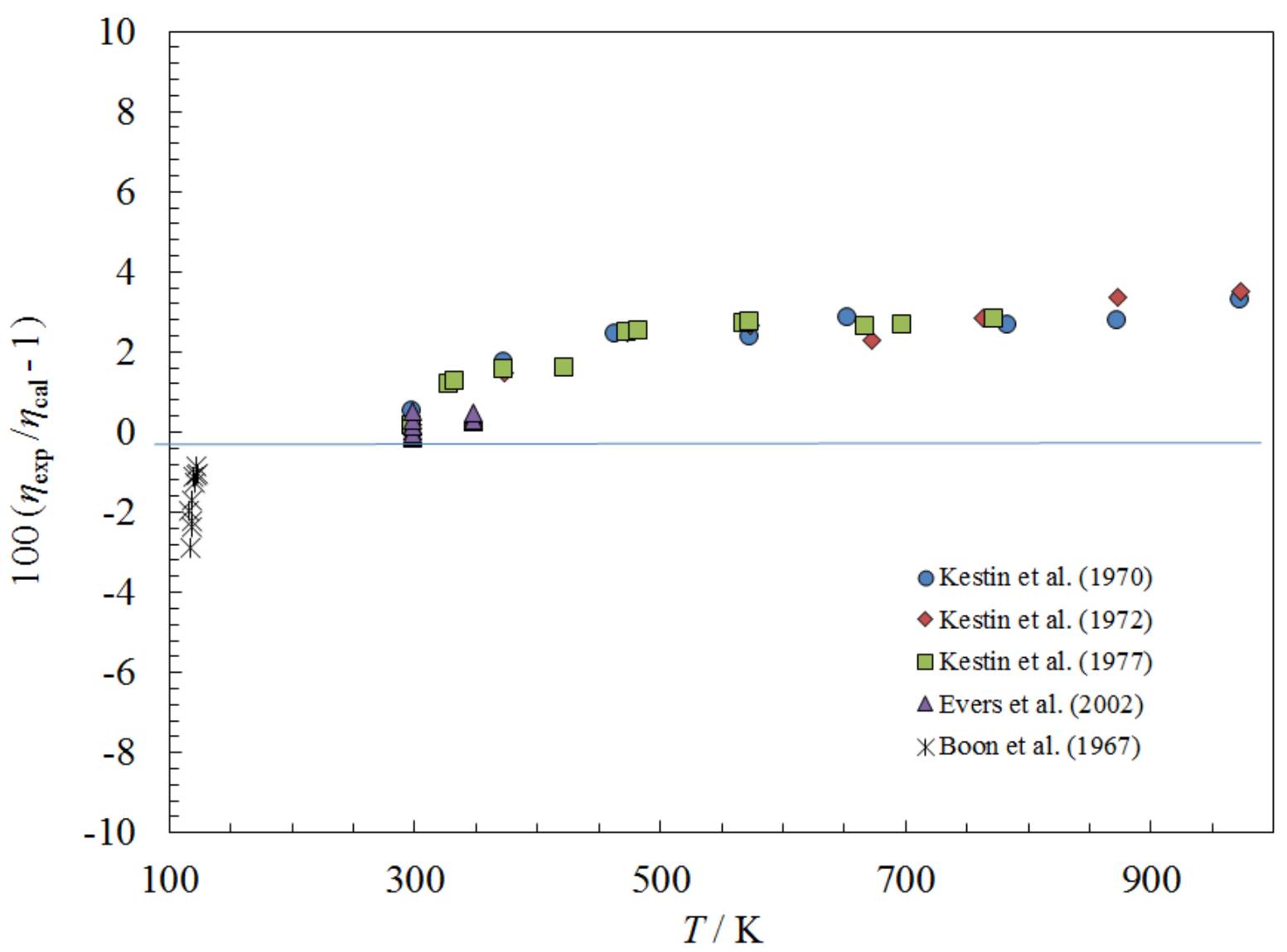

Figure 172. Percentage deviations between the model and the experimental viscosity data for krypton as a function of temperature. 


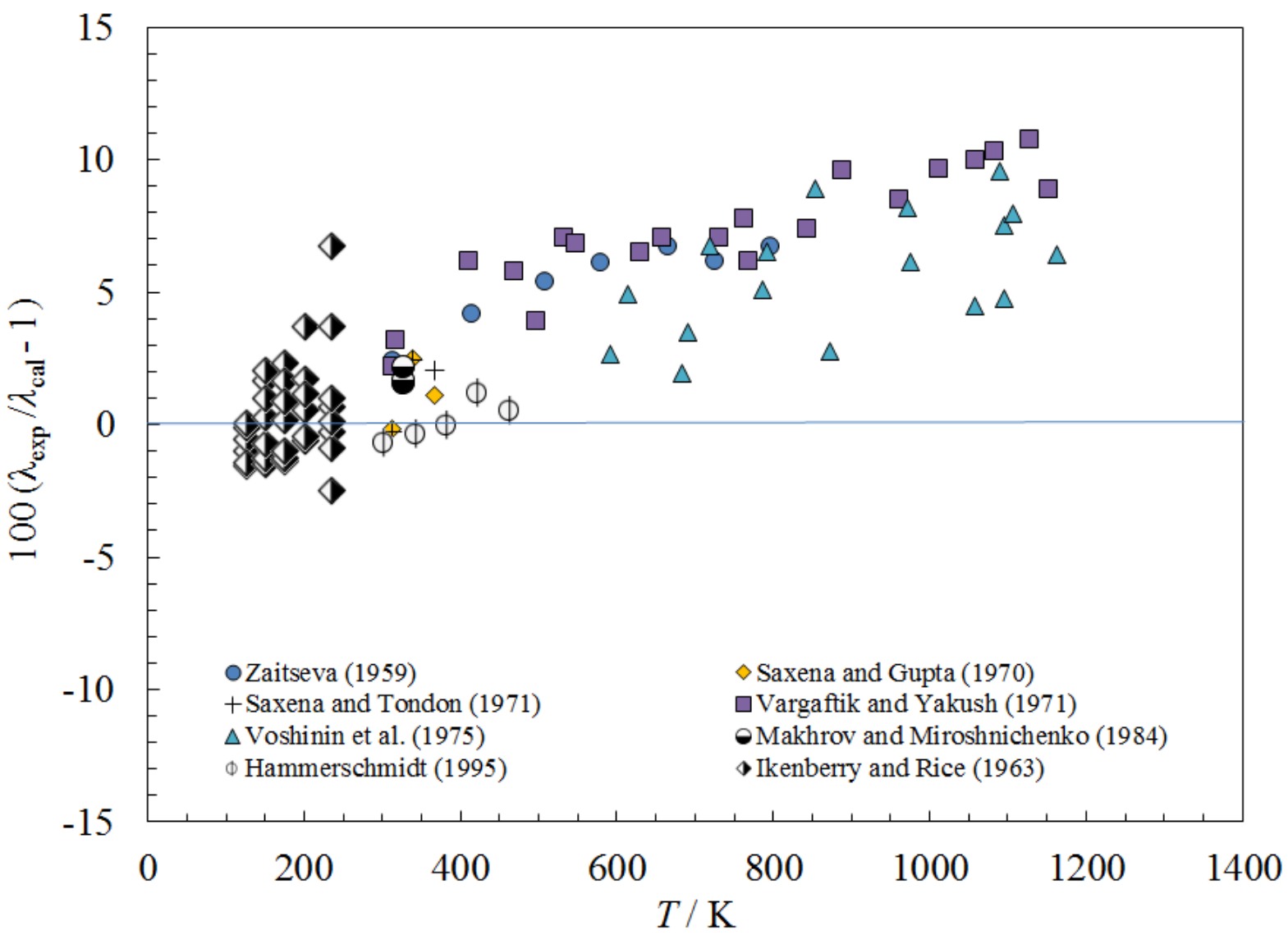

Figure 173. Percentage deviations between the model and the experimental thermal conductivity data for krypton as a function of temperature. 


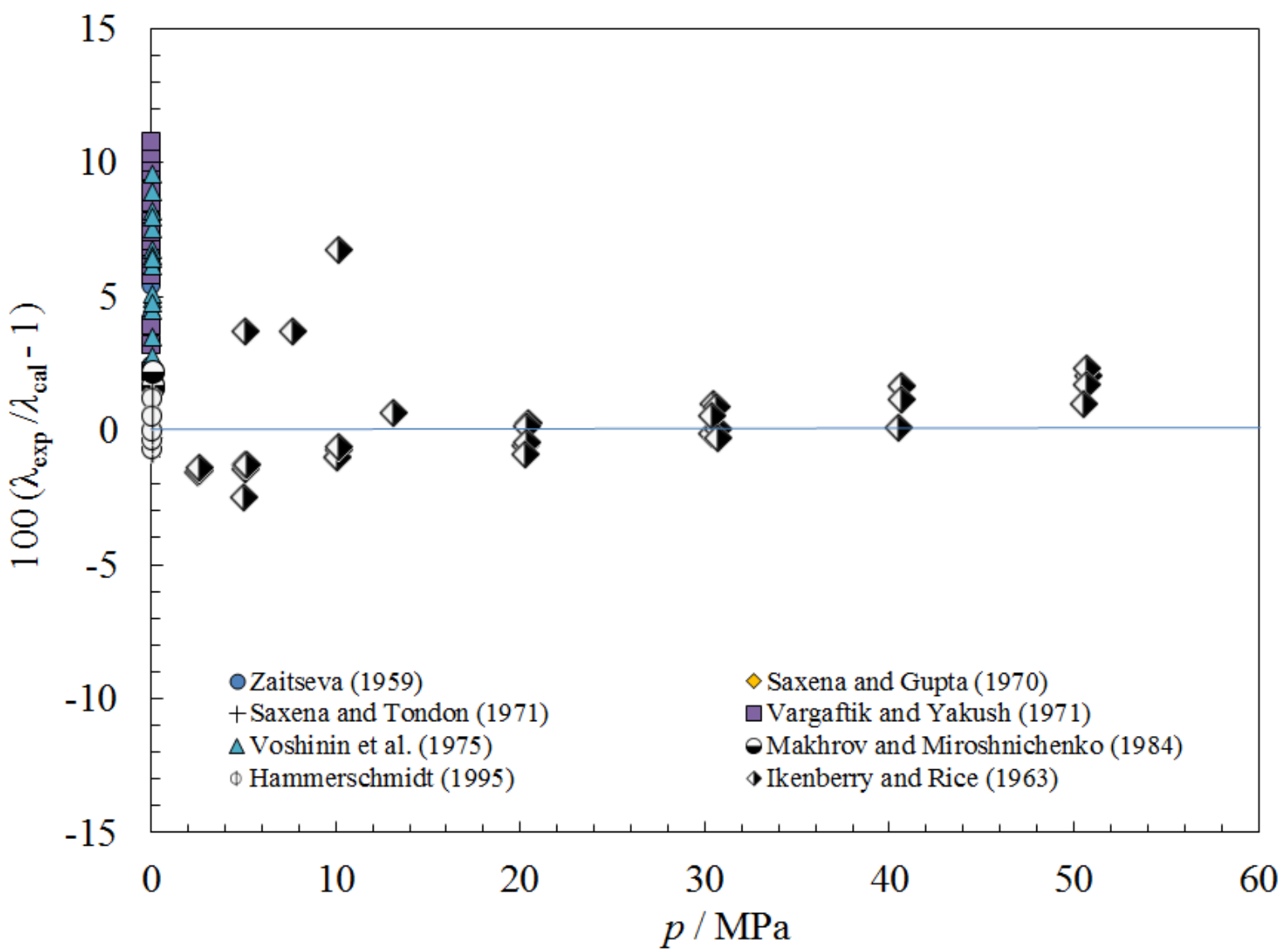

Figure 174. Percentage deviations between the model and the experimental thermal conductivity data for krypton as a function of pressure.

\subsubsection{Xenon}

The equation of state of Lemmon and Span (E.W. Lemmon \& Span, 2006) was used to provide the density and the critical parameters. Lennard-Jones parameters were obtained by fitting the viscosity data of Vogel (E. Vogel, 1984). Limited liquid-phase viscosity data from Ulybin and Makarushkin (Ulybin \& Makarushkin, 1977) were used to obtain the coefficients in Table 2, and Figures 175 and 176 show deviations with data sources as a function of temperature and of pressure, respectively. We estimate the uncertainty for the viscosity for the liquid at pressures to $60 \mathrm{MPa}$ and temperatures above $170 \mathrm{~K}$ is $5 \%$. The data of Vogel in the vapor phase have an uncertainty of $0.2 \%$ to $0.3 \%$, but our correlation has an uncertainty of $0.7 \%$ in the vapor phase. The coefficient $F_{\mathrm{c}}$ in Table 2 was adjusted to ensure that the value of the dilute-gas viscosity at $25^{\circ} \mathrm{C}$ and $0.1 \mathrm{MPa}$ matched the reference value presented Assael et al. (Assael et al., 2018) to within its stated uncertainty. Very limited liquid-phase thermal conductivity data of Ikenberry and Rice (Ikenberry \& Rice, 1963) were used to obtain the parameters in Table 3. Due to the internal contribution to the dilute-gas thermal conductivity in Eq. (15) being identically zero for noble gases, it is not possible to fit coefficients for $f_{\text {int }}$ and the value is Table 3 is a placeholder only. Parameters for the critical enhancement are presented in Table 4. Deviations with several gasphase data sets (Bakulin, Ulybin, \& Zherdev, 1975; Shashkov, Nesterov, Sudnik, \& Aleinikova, 1976; Stefanov, Zarkova, \& Oliver, 1976; Vargaftik \& Yakush, 1971) and the liquid data used in 
fitting are shown in Figures 177 and 178. We estimate the uncertainty of the thermal conductivity in the liquid phase is $3 \%$ at pressures to $50 \mathrm{MPa}$ and temperatures from $170 \mathrm{~K}$ to $235 \mathrm{~K}$, and $10 \%$ for the gas phase. Surface tension is discussed in Mulero et al. (Mulero et al., 2012).

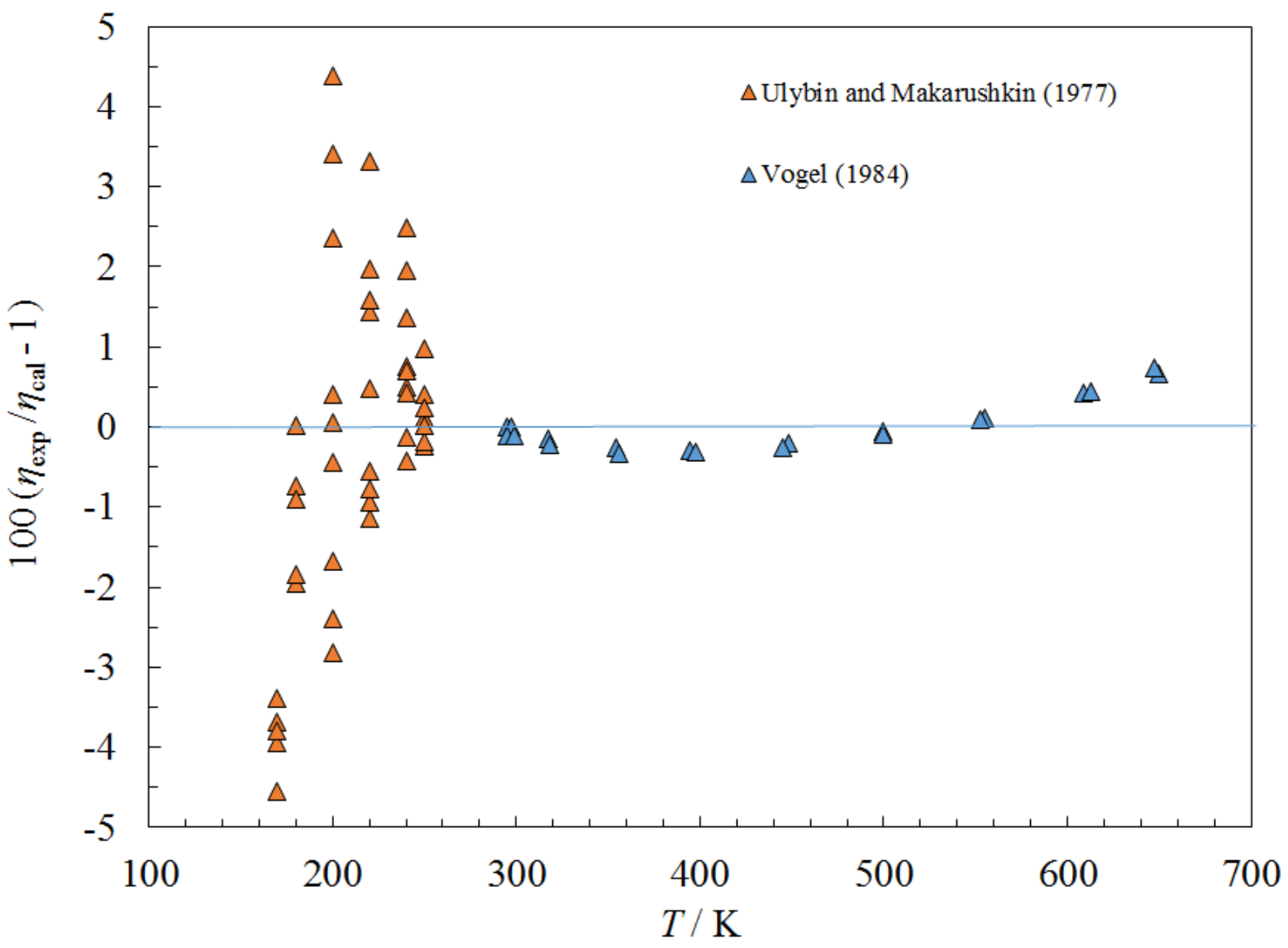

Figure 175. Percentage deviations between the model and the experimental viscosity data for xenon as a function of temperature. 


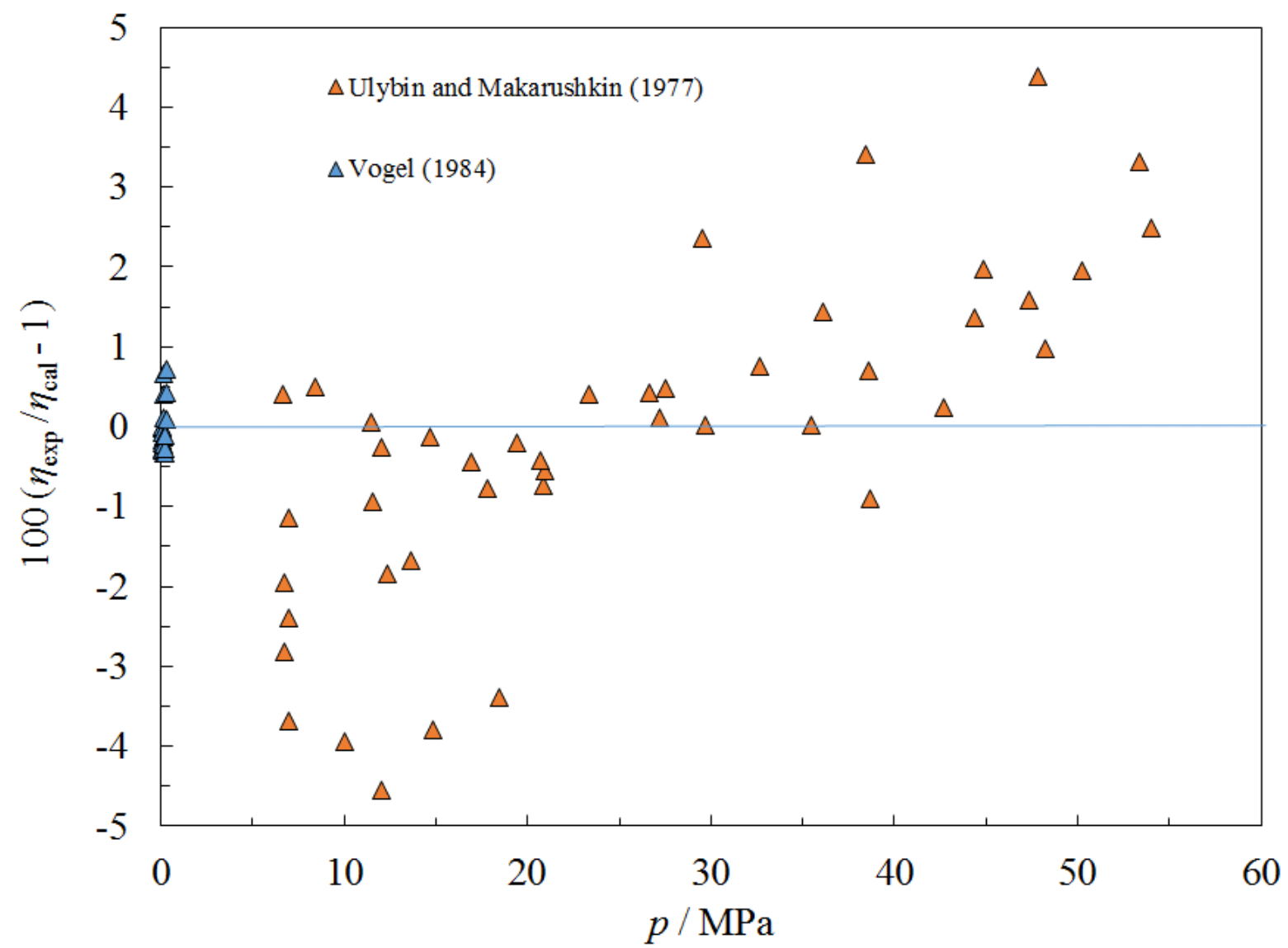

Figure 176. Percentage deviations between the model and the experimental viscosity data for xenon as a function of pressure. 


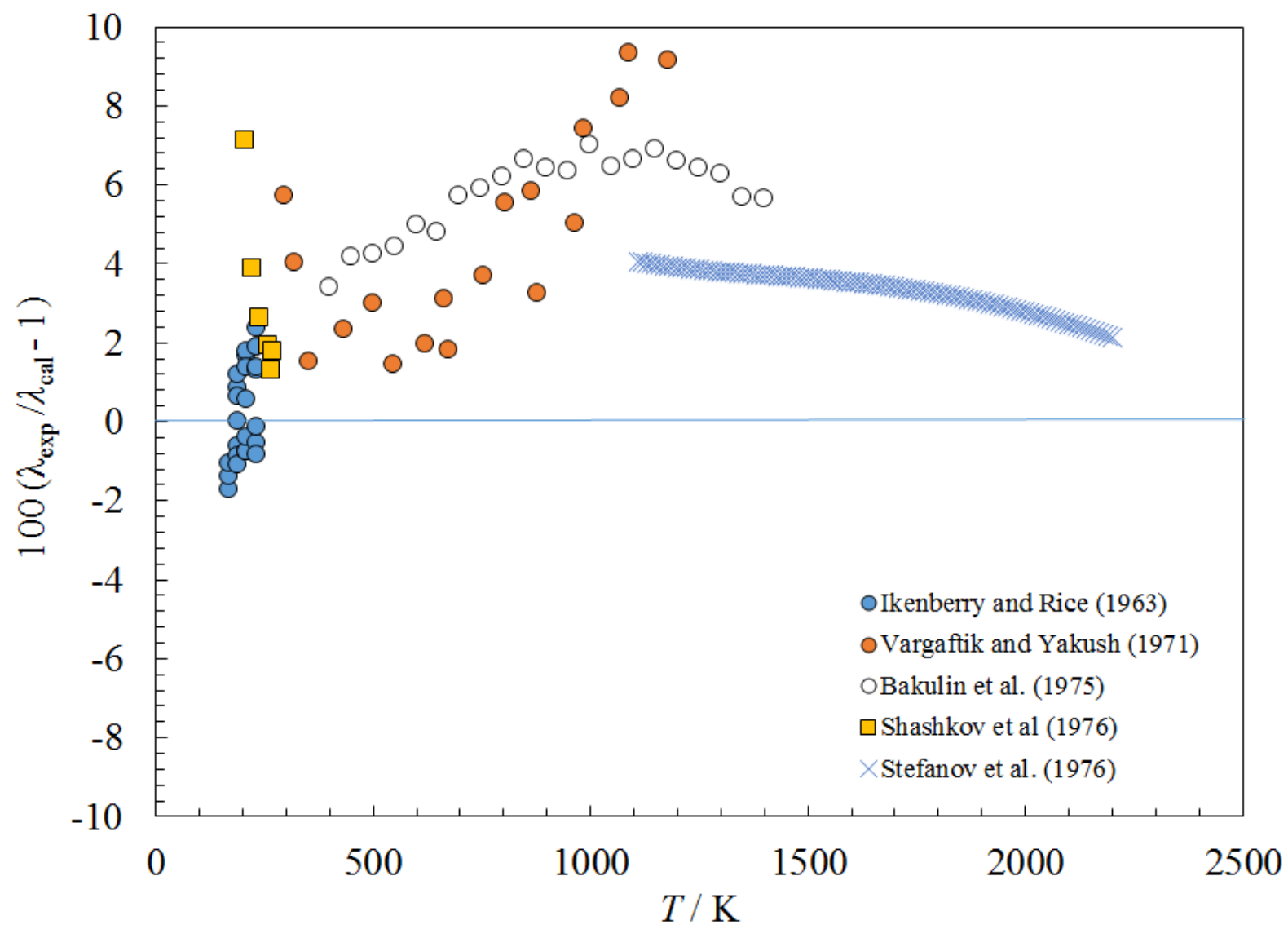

Figure 177. Percentage deviations between the model and the experimental thermal conductivity data for xenon as a function of temperature. 


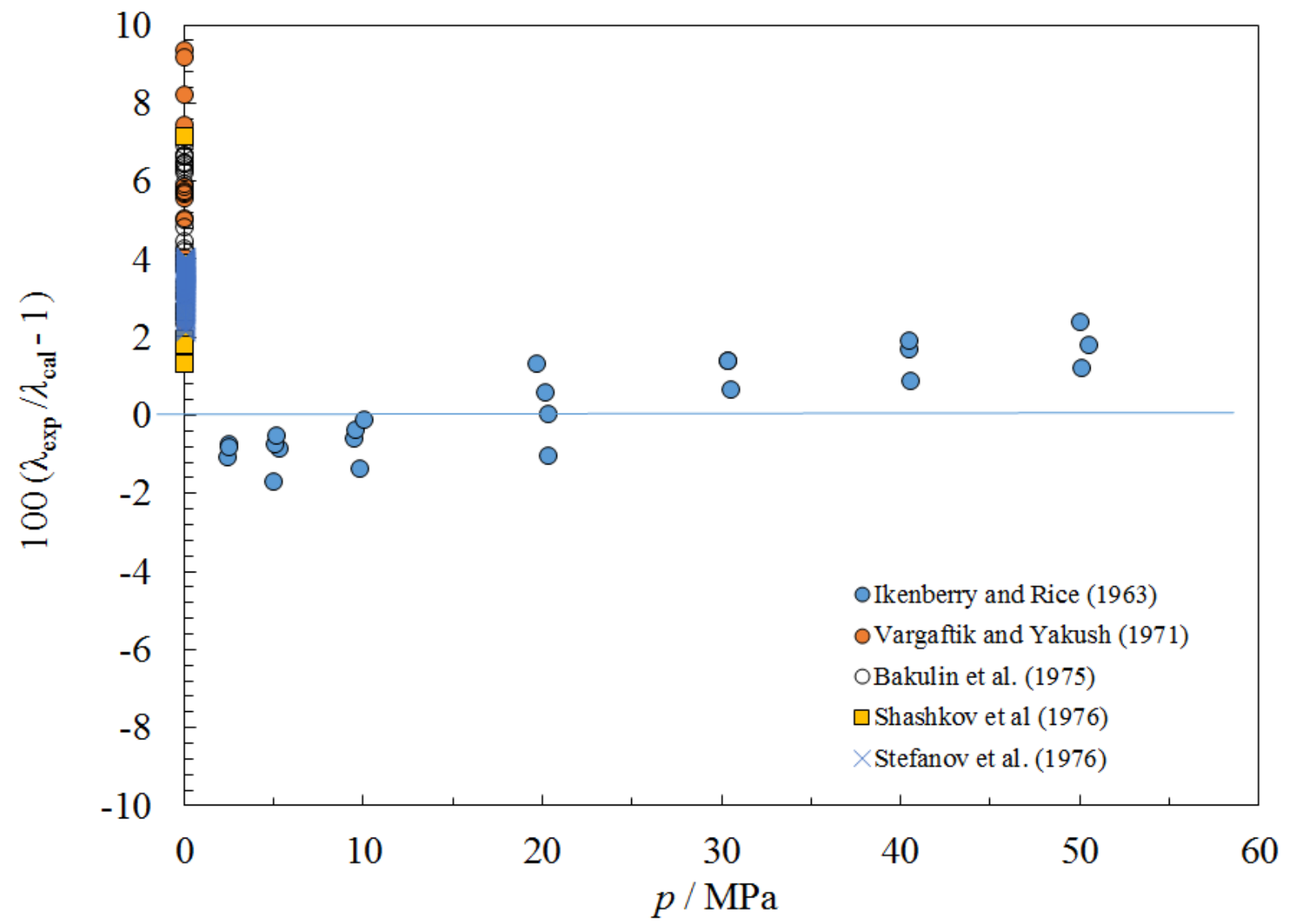

Figure 178. Percentage deviations between the model and the experimental thermal conductivity data for xenon as a function of pressure. 


\subsection{Sulfides}

\subsubsection{Hydrogen sulfide}

The equation of state of Lemmon and Span (E.W. Lemmon \& Span, 2006) was used to provide the density and the critical parameters. Lennard-Jones parameters were estimated with the method of Chung et al. (Chung et al., 1988) with parameters as given in Table 1. Two sets of experimental dilute-gas thermal conductivity data were found (Barua, Manna, \& Mukhopadhyay, 1968; Correia, Schramm, \& Schäfer, 1968), as well as ab-initio calculations (Hellmann, Bich, Vogel, \& Vesovic, 2012). The ab-initio data were used to obtain the dilute-gas coefficients in Table 3 . The estimated uncertainty of the gas-phase thermal conductivity is $2 \%$, and comparisons with data are shown in Figure 179. We were unable to locate any liquid-phase experimental data for thermal conductivity, so a completely predictive model with propane as a reference was used; coefficients are in Table 3. Parameters for the critical enhancement are presented in Table 4. It is very difficult to estimate the uncertainty since there are no data available, and the fluid is not very close in chemical structure to the reference fluid, propane. Thus we estimate the uncertainty for liquid-phase thermal conductivity at $50 \%$. We do not cover the viscosity or surface tension here, as there are existing correlations for these properties for hydrogen sulfide (Mulero et al., 2012; Schmidt, Quiñones-Cisneros, Carroll, \& Kvamme). However, the implementation of the ECS method for thermal conductivity requires a value for viscosity for use in the critical enhancement term, so we provide parameters for the viscosity in Table 2 so that users can match the sample calculation numbers in the Appendix. One may also implement a dedicated viscosity model; however, the numbers in the Appendix were obtained with the ECS model. 


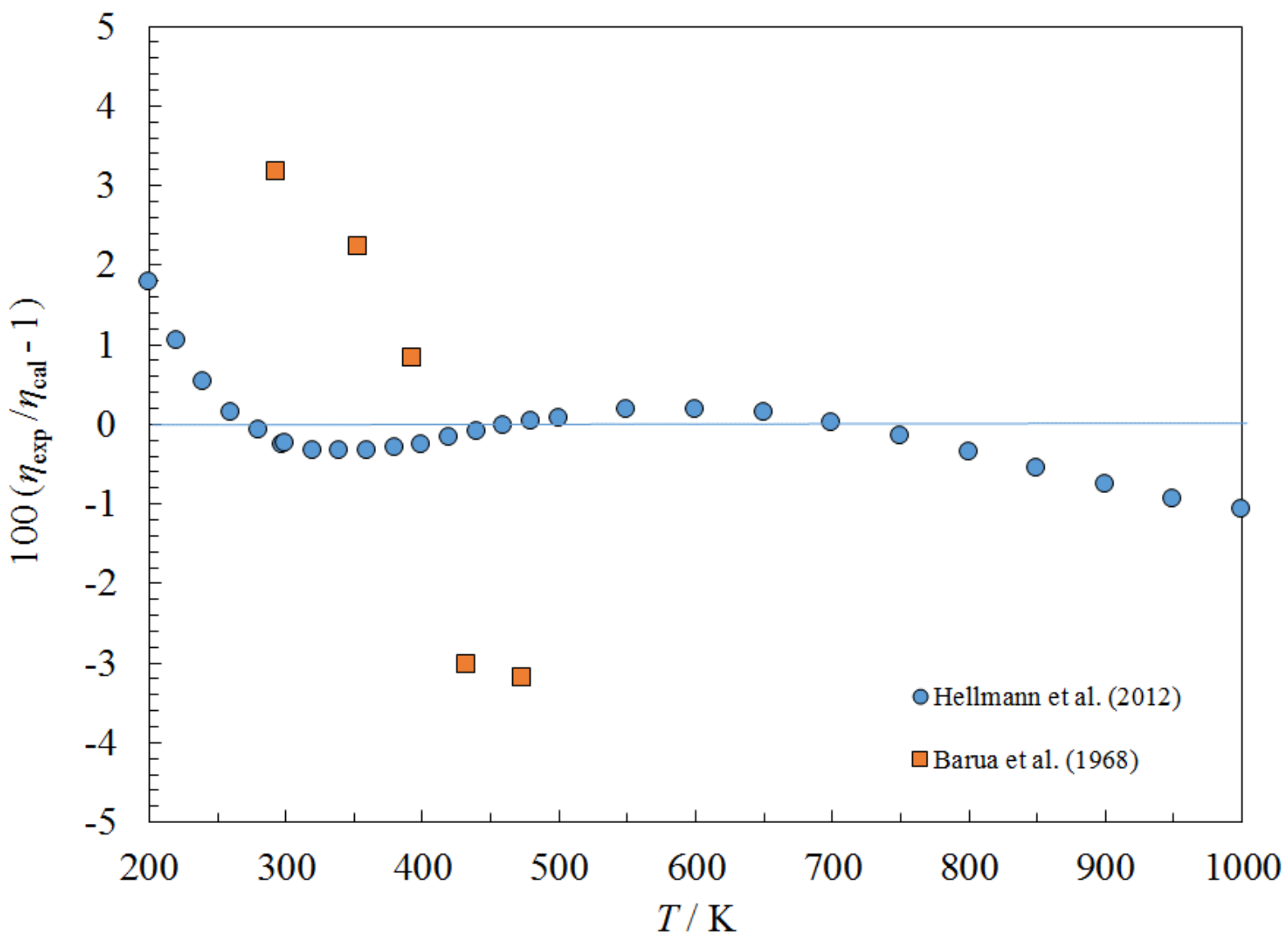

Figure 179. Percentage deviations between the model and the experimental thermal conductivity data for hydrogen sulfide as a function of temperature.

\subsubsection{Carbonyl sulfide}

The equation of state of Lemmon and Span (E.W. Lemmon \& Span, 2006) was used to provide the density and the critical parameters. Lennard-Jones parameters were obtained from Hirschfelder et al. (Hirschfelder et al., 1964) Comparisons with the gas-phase data of Smith (Smith, 1922) are shown in Figure 180. There were no data available in the liquid phase for viscosity; propane was used as a reference fluid, and a completely predictive model was used. Due to lack of experimental data and the differences in the chemical structure between the reference fluid and carbonyl sulfide, the estimated uncertainty for the liquid phase is $50 \%$. No data at all were located for the thermal conductivity. The corresponding states coefficients in Table 3 were adjusted so that predictions are similar to those of the method of Sastri and Rao (Sastri \& Rao, 1999). Parameters for the critical enhancement are presented in Table 4 . The estimated uncertainty is $50 \%$ in the liquid phase, $25 \%$ in the gas. Surface tension is discussed in Mulero et al. (Mulero et al., 2012). 


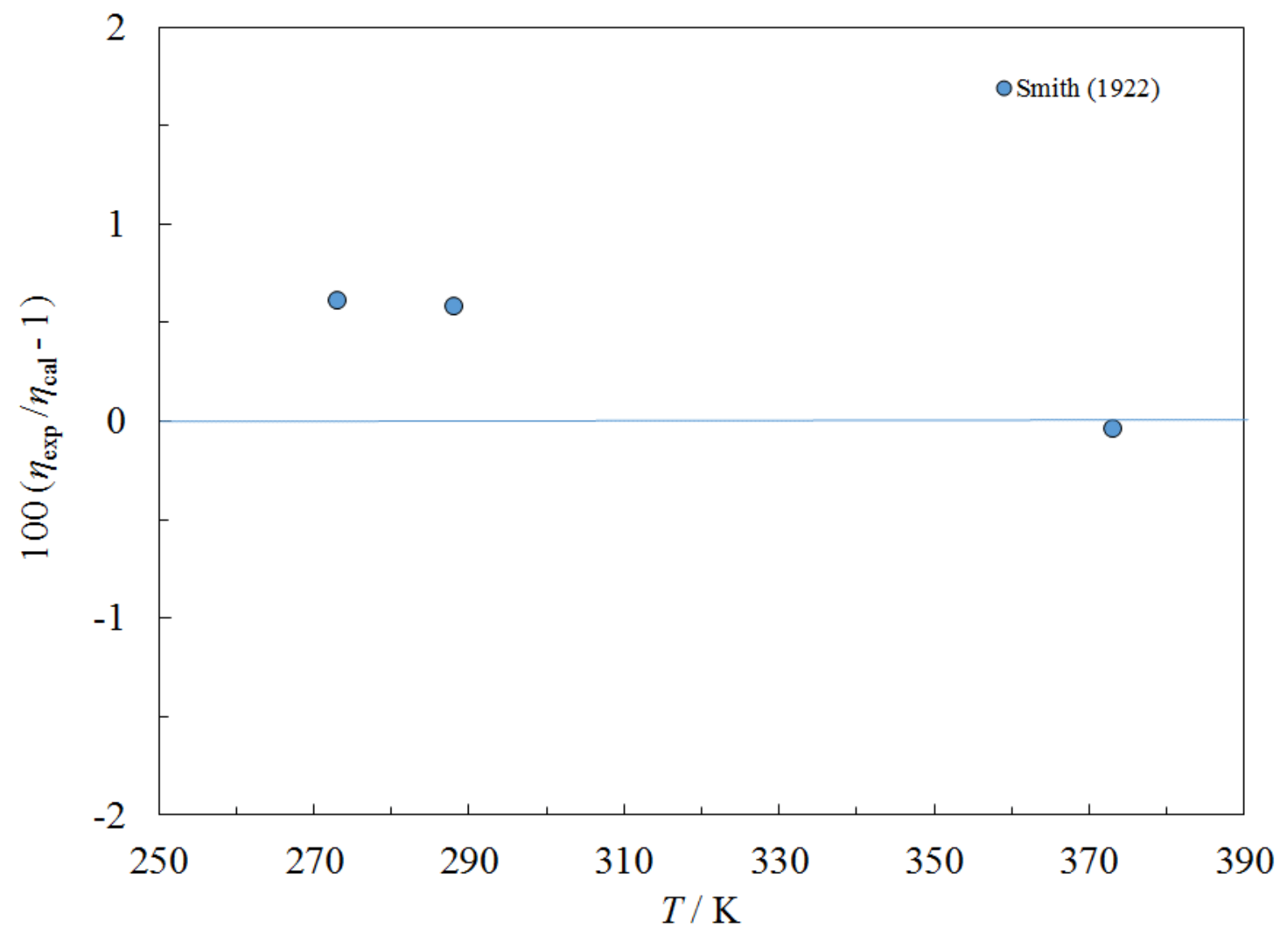

Figure 180. Percentage deviations between the model and the experimental viscosity data for carbonyl sulfide as a function of temperature. 


\subsection{Oxides}

\subsubsection{Carbon monoxide}

The equation of state of Lemmon and Span (E.W. Lemmon \& Span, 2006) was used to provide the density and the critical parameters. Lennard-Jones parameters were calculated by fitting the data of Vogel (E. Vogel, 2012). Extremely limited liquid-phase data were found- only 1 set at saturation from Rudenko and Shubnikov (Rudenko \& Shubnikov, 1934) was used to obtain the coefficients in Table 2, and Figure 181 shows deviations from the data as a function of temperature. We estimate the uncertainty along the saturation boundary in the liquid phase is $2 \%$, and the uncertainty in the gas phase is $1 \%$ or better. For thermal conductivity, the gas-phase data of Johnston and Grilly (Johnston \& Grilly, 1946) were used to obtain the gas phase coefficients in Table 3. We also used the theoretical calculations of Hellmann (2011) to guide the hightemperature behavior. For the liquid phase, only four points at saturation from Borovik et al. (Borovik, Matveev, \& Panina, 1940) were found and used to obtain the coefficients in Table 3. Parameters for the critical enhancement are presented in Table 4. Figure 182 shows deviation plots with the thermal conductivity data. For the dilute gas, we estimate the uncertainty is $2 \%$, and $4 \%$ for the liquid at saturation. Due to lack of experimental data at pressures above saturation, these correlations are recommended for use at pressures below $10 \mathrm{MPa}$. This correlation also should not be used above the limits of the EOS, $500 \mathrm{~K}$. Surface tension is discussed in Mulero et al. (Mulero et al., 2012). 


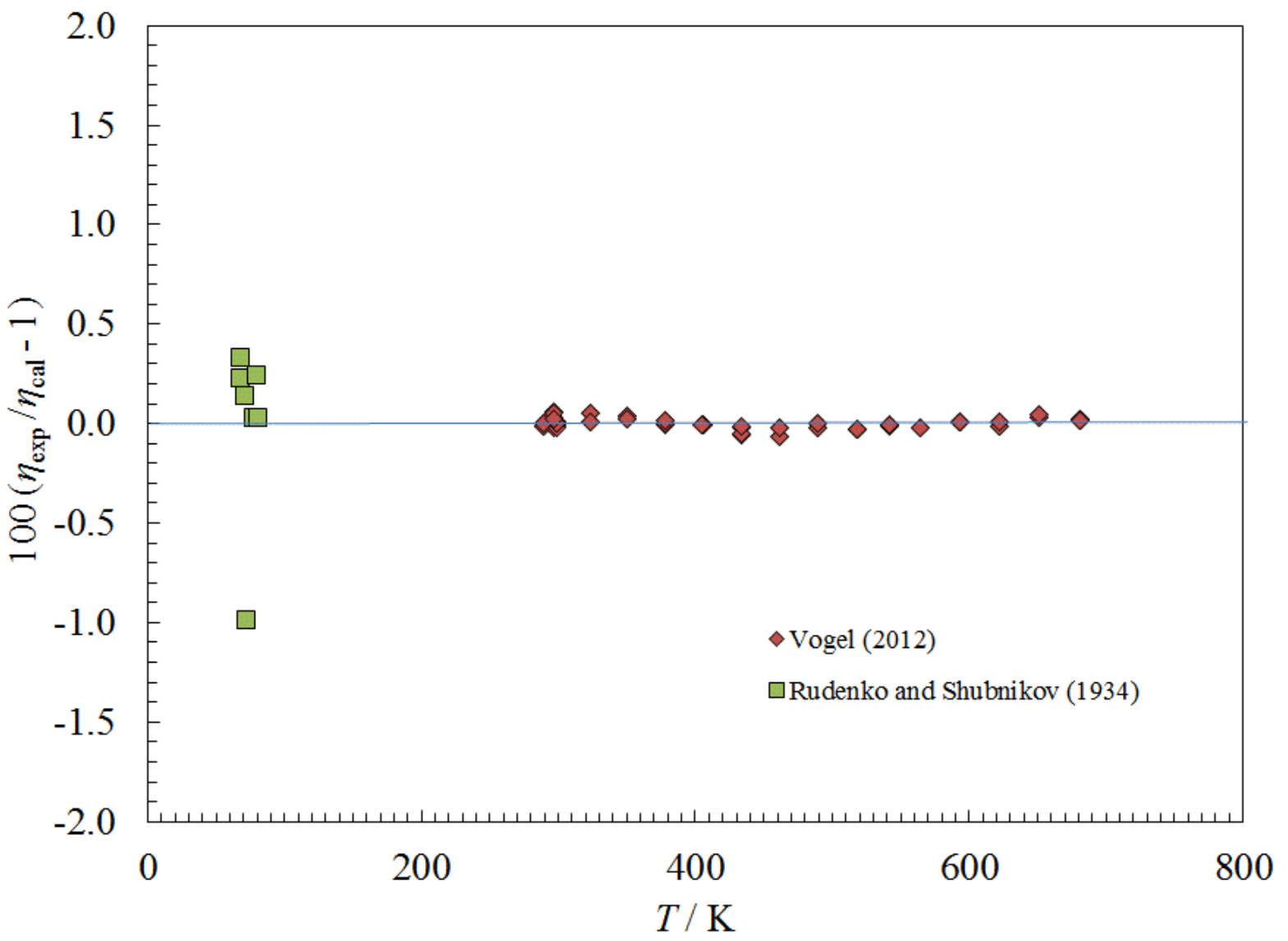

Figure 181. Percentage deviations between the model and the viscosity data for carbon monoxide as a function of temperature. 


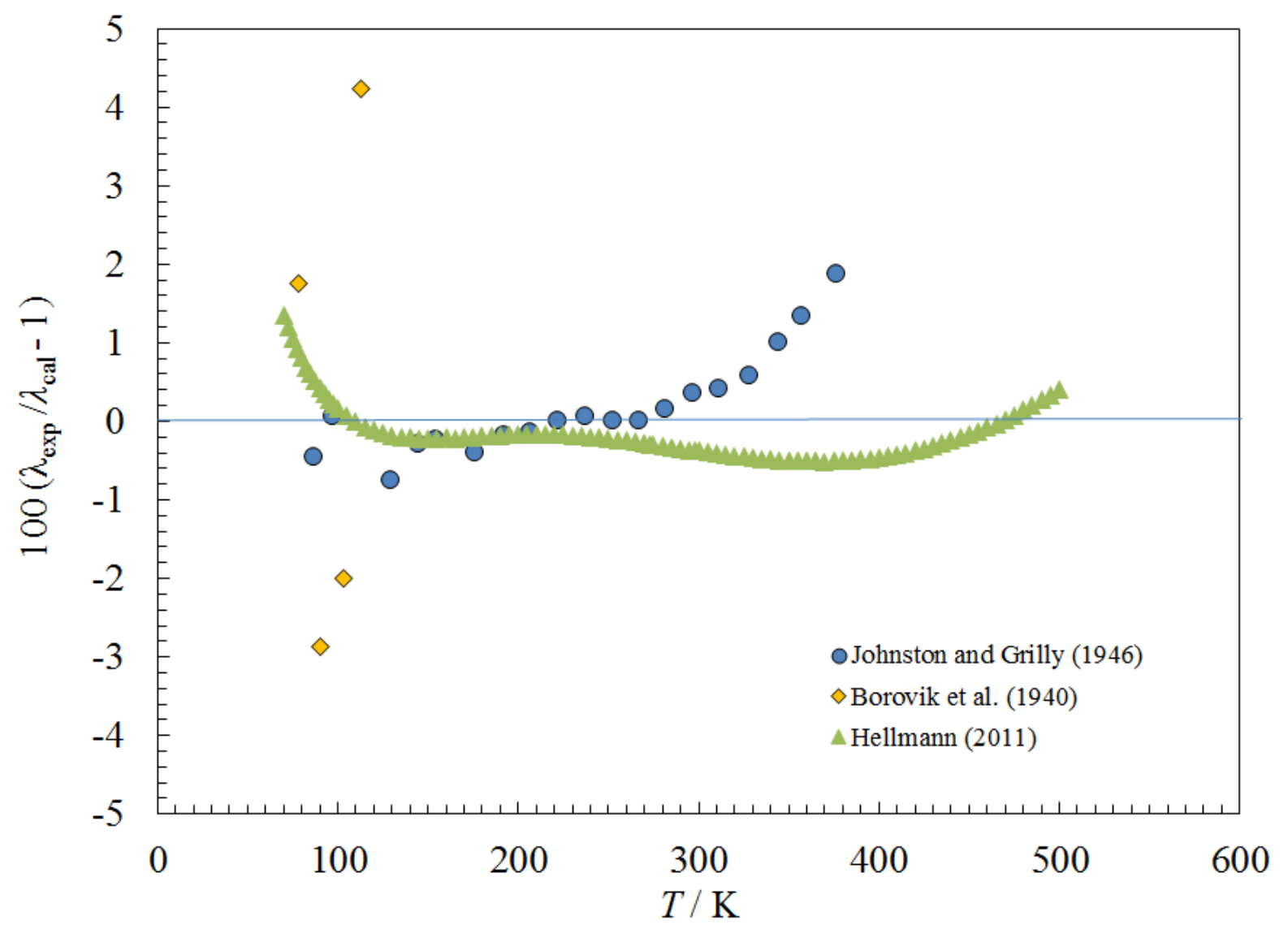

Figure 182. Percentage deviations between the model and the thermal conductivity data for carbon monoxide as a function of temperature.

\subsubsection{Nitrous oxide}

The equation of state of Lemmon and Span (E.W. Lemmon \& Span, 2006) was used to provide the density and the critical parameters. Lennard-Jones parameters were obtained from Reid et al. (Reid, 1987). Although there are numerous gas-phase viscosity data (Clifford et al., 1981; E. J. R. Harris, Hope, Gough, \& Smith, 1979; Hurly, 2004; Kestin \& Wakeham, 1979) available, there are very limited liquid-phase viscosity data. Takahashi et al. (Takahashi, ShibasakiKitakawa, Yokoyama, \& Takahashi, 1996) has only one isotherm at $298 \mathrm{~K}$ that is subcritical. To augment the dense-fluid data, we used predicted values from Horvath (Horvath, 1975) to supplement the experimental data in the liquid phase. The Horvath numbers are not experimental data; they are obtained using an estimation method in Jossi et al. (Jossi, Stiel, \& Thodos, 1962) and were used along with Takahashi et al. (Takahashi et al., 1996) to determine the coefficients in Table 2. Figures 183 and 184 show deviations with the experimental data. We estimate the uncertainty in the gas phase is $5 \%$, and in the liquid phase along the saturation boundary is $10 \%$, higher elsewhere. Thermal conductivity data from Johnston and Grilly (Johnston \& Grilly, 1946), Keyes (Keyes, 1954), Choy and Raw (Choy \& Raw, 1966), and Richter and Sage (Richter \& Sage, 1963) were used to obtain the coefficients in Table 3. Parameters for the critical enhancement are presented in Table 4. Deviation plots are shown in Figures 185 and 186. The estimated uncertainty 
for thermal conductivity in the gas phase is $5 \%$, and is $5 \%$ in the liquid phase at temperatures above $277 \mathrm{~K}$ and pressures below $35 \mathrm{MPa}$; higher elsewhere. Surface tension is discussed in Mulero et al. (Mulero et al., 2012).

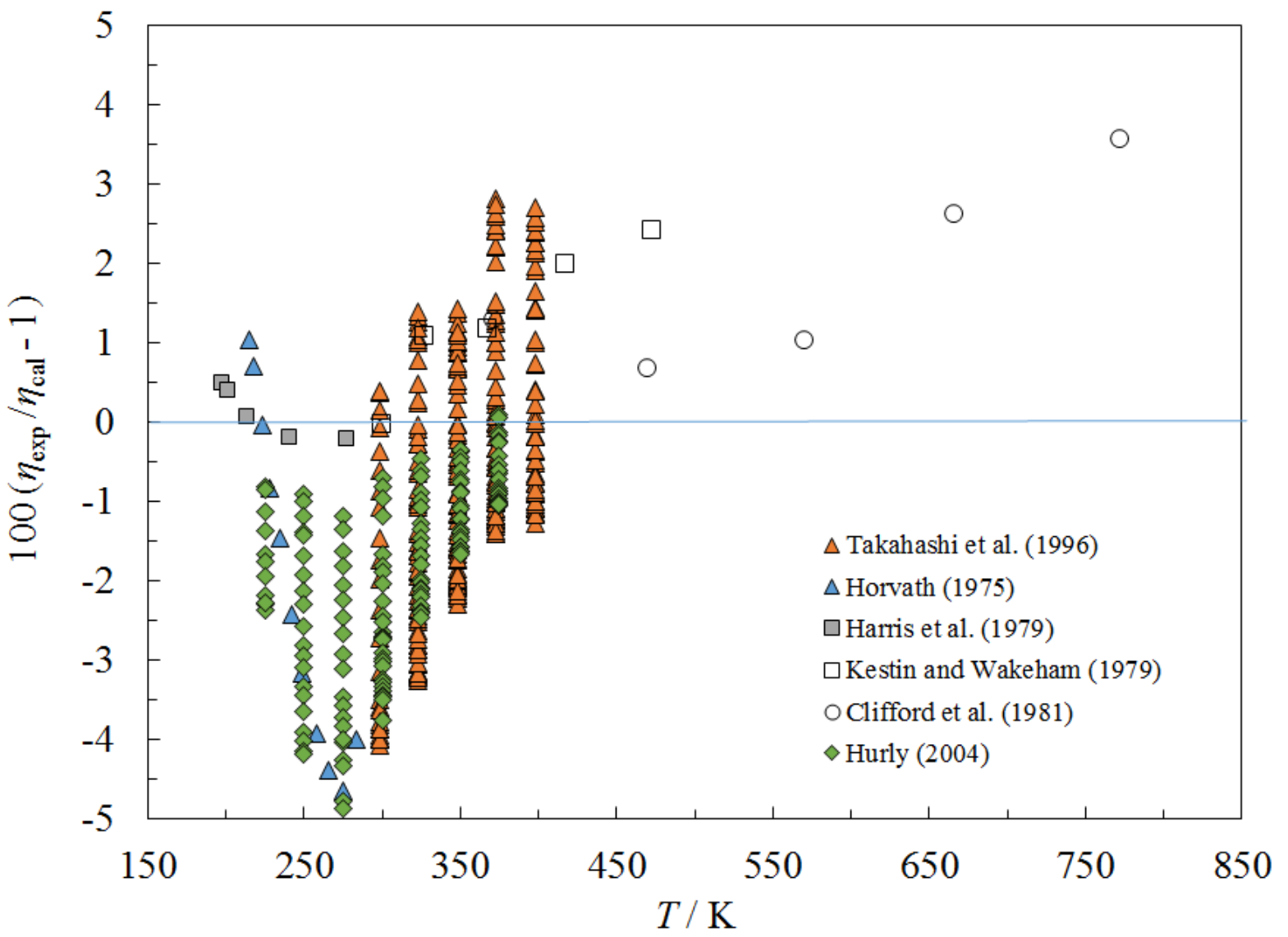

Figure 183. Percentage deviations between the model and the experimental viscosity data for nitrous oxide as a function of temperature. 


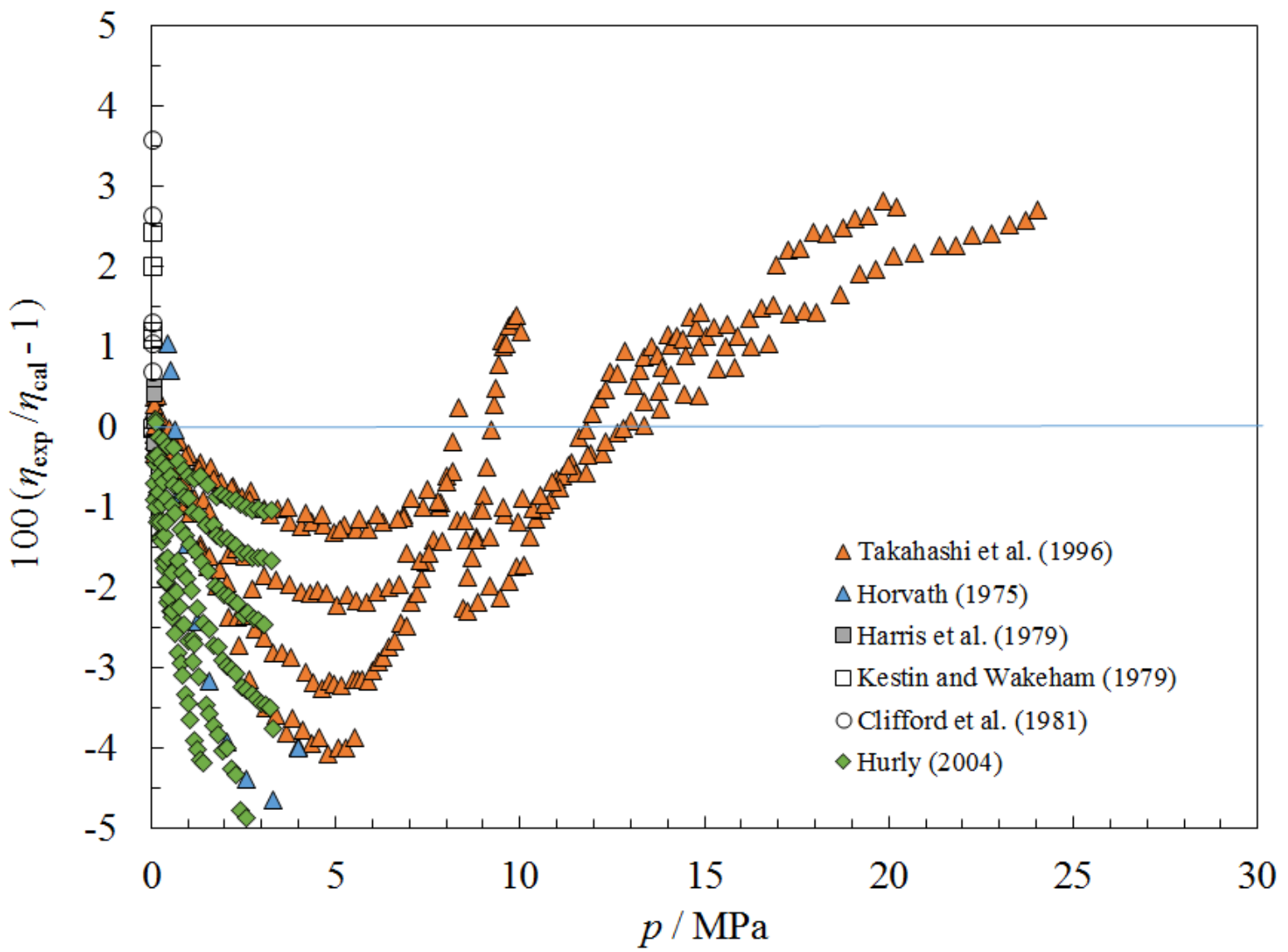

Figure 184. Percentage deviations between the model and the experimental viscosity data for nitrous oxide as a function of pressure. 


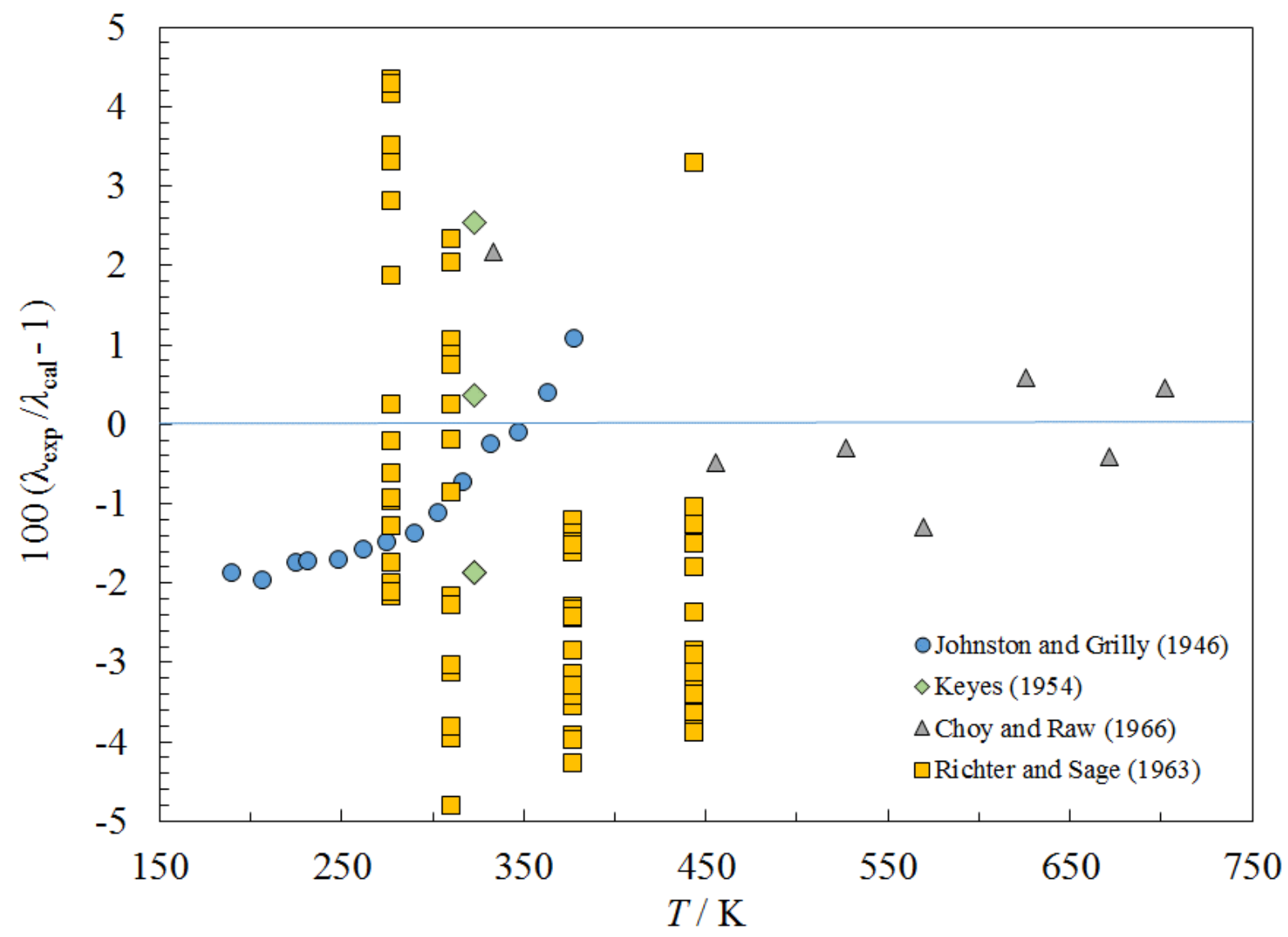

Figure 185. Percentage deviations between the model and the experimental thermal conductivity data for nitrous oxide as a function of temperature. 


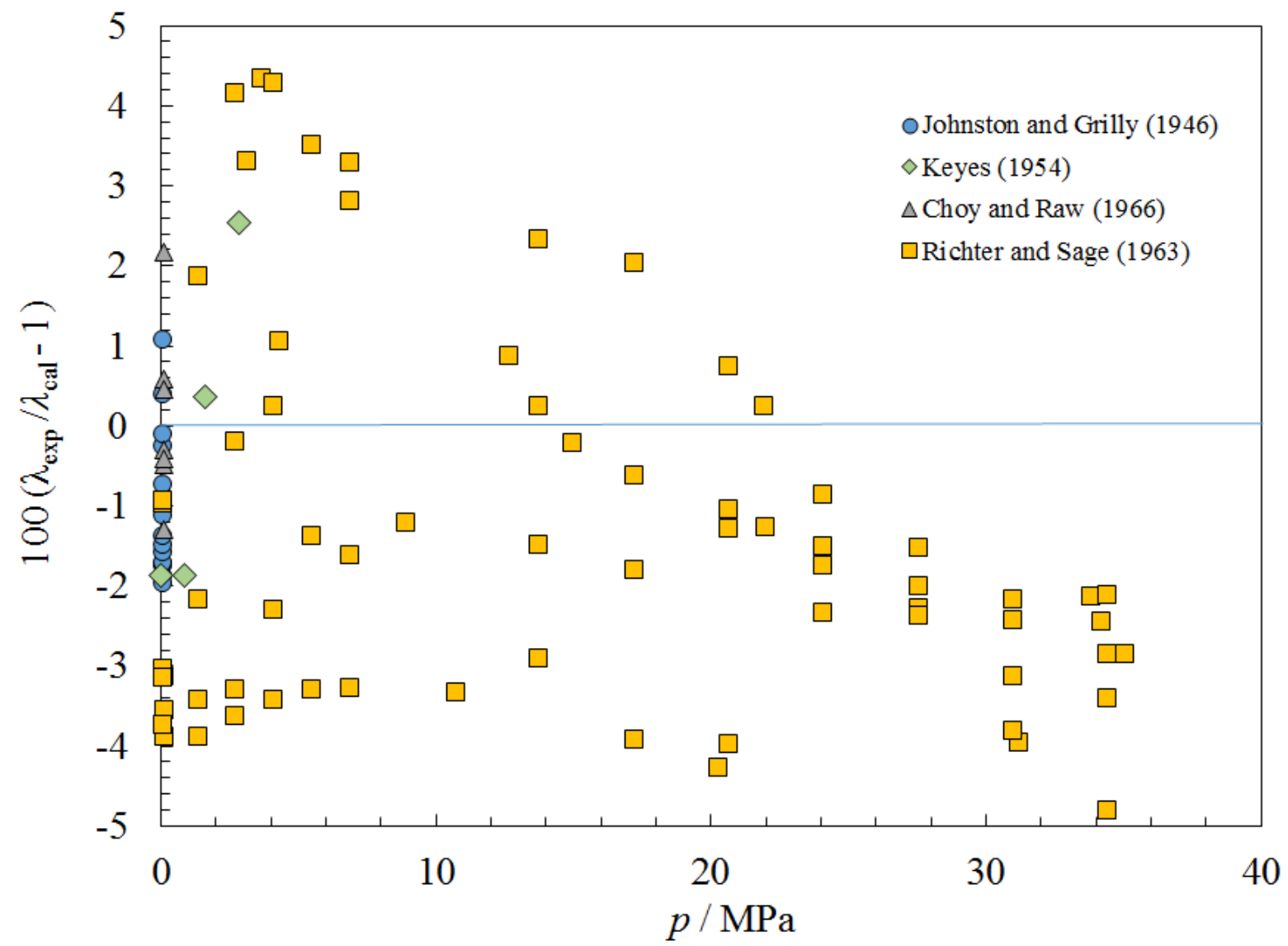

Figure 186. Percentage deviations between the model and the experimental thermal conductivity data for nitrous oxide as a function of pressure. 


\subsubsection{Ethylene oxide (oxirane)}

The equation of state of Thol et al. (Thol, Rutkai, Koster, Kortmann, \& Span, 2015) was used to provide the density and the critical parameters. Lennard-Jones parameters were obtained from the method of Chung et al. (Chung et al., 1988). Extremely limited liquid-phase viscosity data of Timmermans and Hennaut-Roland (Timmermans \& Hennaut-Roland, 1937) and Maass and Boomer (Maass \& Boomer, 1922) were used to provide the coefficients in Table 2, and Figure 187 shows a deviation plot. The data were limited to atmospheric pressure. We estimate the uncertainty in the liquid phase is $5 \%$, and the correlation is limited to $10 \mathrm{MPa}$ (the limit of the EOS). There are no viscosity data available for the gas phase, and we estimate the uncertainty of the gas phase viscosity is $10 \%$. We were unable to locate liquid-phase thermal conductivity data, and use a predictive method with propane as a reference fluid. The coefficients were selected to give predictions in between those of the Sastri Rao method as implemented in TDE (Diky et al., 2016), and the Missenard method as implemented in DIPPR Diadem (DIPPR, 2016). Gas-phase coefficients in Table 3 were obtained by fitting the data of Vines and Bennett (Vines \& Bennett, 1954) and Senftleben (Senftleben, 1964); a deviation plot is shown in Figure 188. Parameters for the critical enhancement are presented in Table 4. We estimate the uncertainty of the thermal conductivity in the gas phase is $10 \%$, and $20 \%$ to $50 \%$ in the liquid phase at pressures to $10 \mathrm{MPa}$. A separate publication covers surface tension (Mulero \& Cachadiña, 2014); it is not covered here.

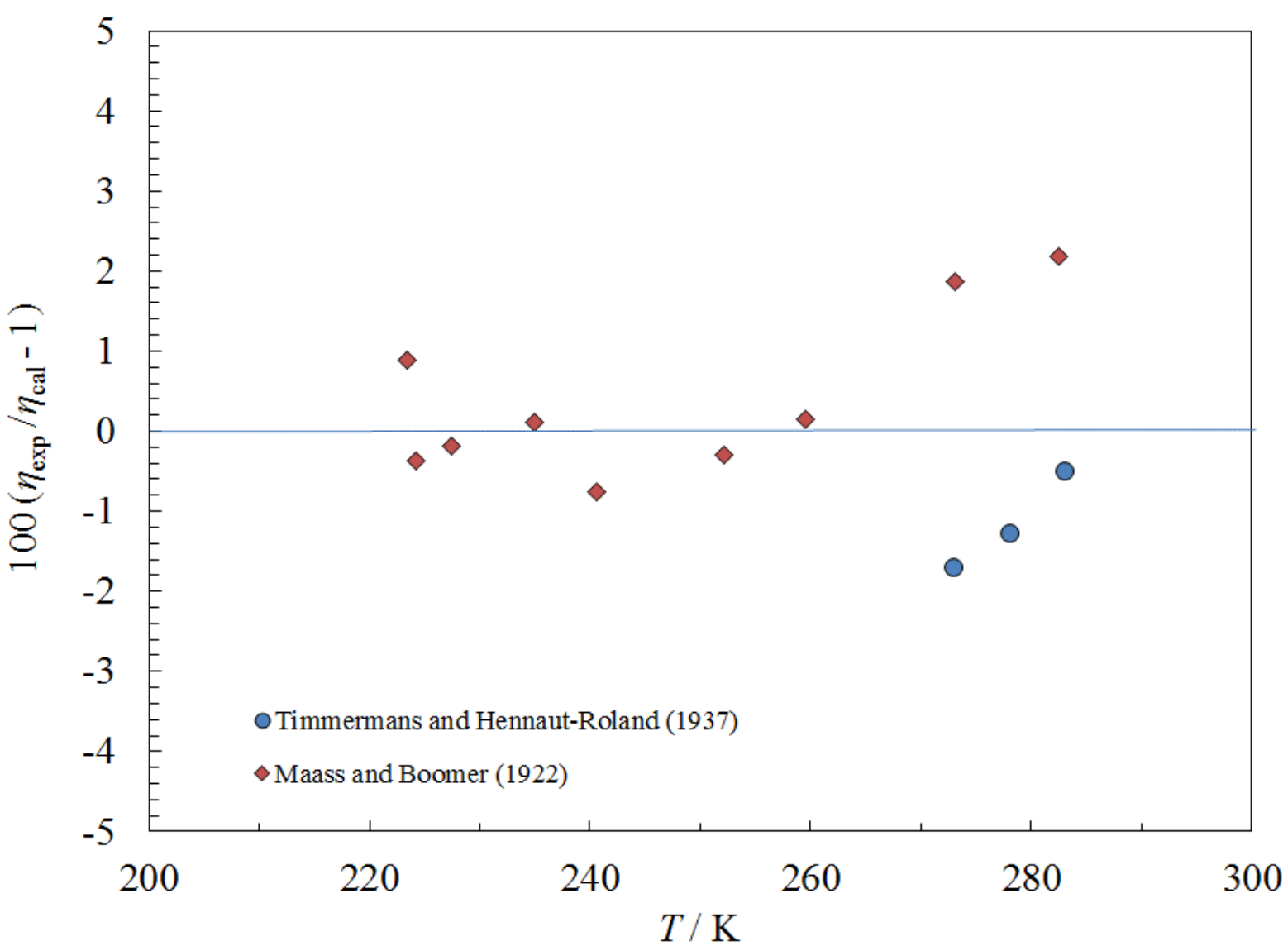

Figure 187. Percentage deviations between the model and the experimental viscosity data for ethylene oxide as a function of pressure. 


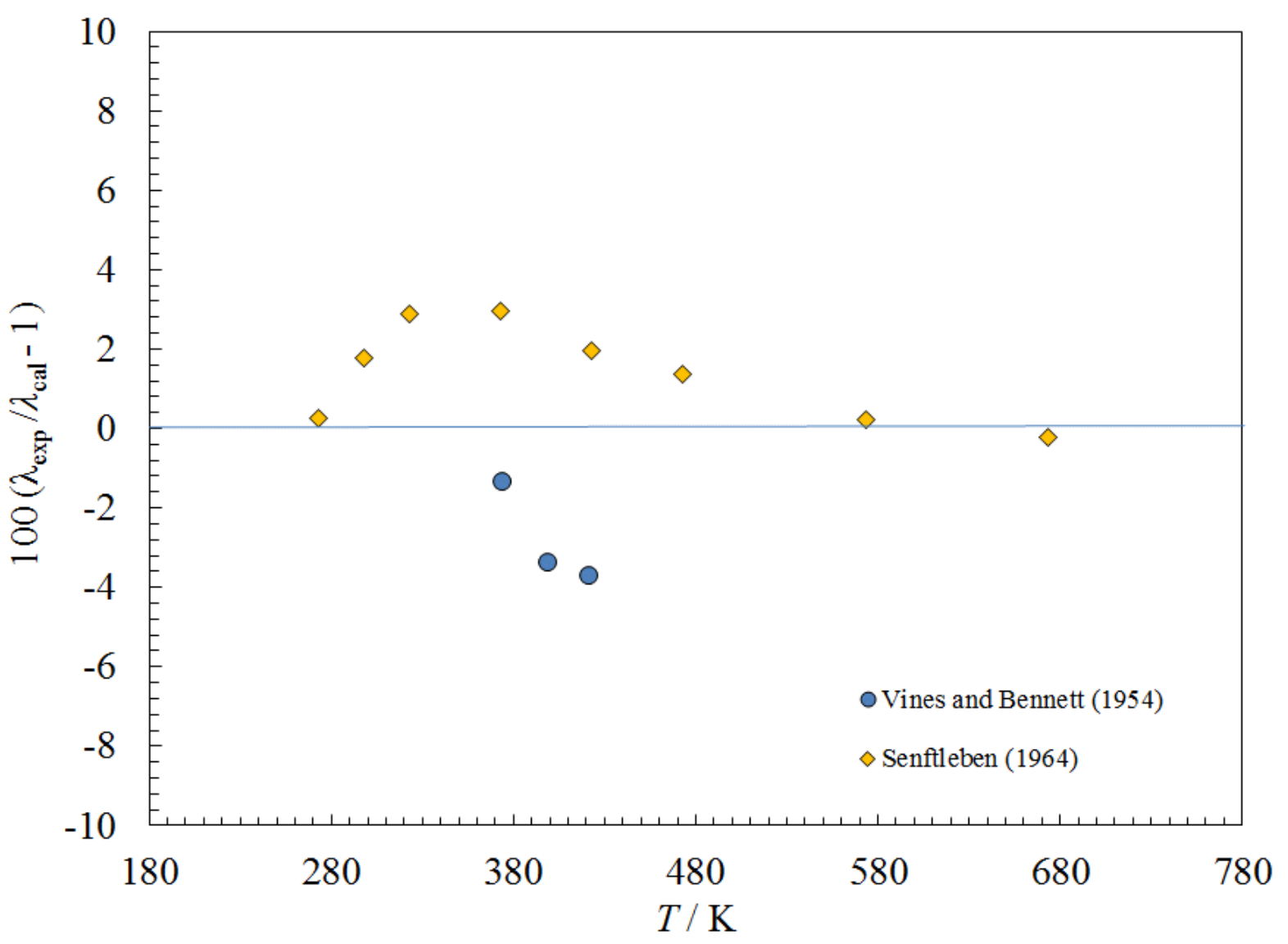

Figure 188. Percentage deviations between the model and the experimental thermal conductivity data for ethylene oxide as a function of temperature.

\subsubsection{Propylene oxide}

The equation of state of Gao et al. (K. Gao, J. Wu, \& E. W. Lemmon, 2017m) was used to provide the density and the critical parameters. Lennard-Jones parameters were calculated by the method of Chung et al. (Chung et al., 1988); values are presented in Table 1. Experimental data for liquid viscosity at atmospheric pressure from Zimakov and Sokalava (Zimakov \& Sokolava, 1953) were used to obtain the coefficients in Table 2. Figure 189 shows the deviations. The estimated uncertainty for viscosity at atmospheric pressure is $2 \%$ from $272 \mathrm{~K}$ to $293 \mathrm{~K}, 5 \%$ at atmospheric pressure outside of that range, and as high as $10 \%$ at higher pressures. Very limited liquid thermal conductivity data (only 2 points at atmospheric pressure) from Jamiesen and Tudhope (Jamiesen \& Tudhope, 1964) were used to obtain the coefficients in Table 3. Figure 190 shows the deviations. Parameters for the critical enhancement are presented in Table 4. The estimated uncertainty for thermal conductivity at atmospheric pressure is $5 \%$ (the uncertainty of the experimental data), $10 \%$ at higher pressures and higher close to the critical point. We were unable to locate surface tension data; the coefficients in Table 5 are predictive based on similarities with ethylene oxide, with an estimated uncertainty of $10 \%$. 


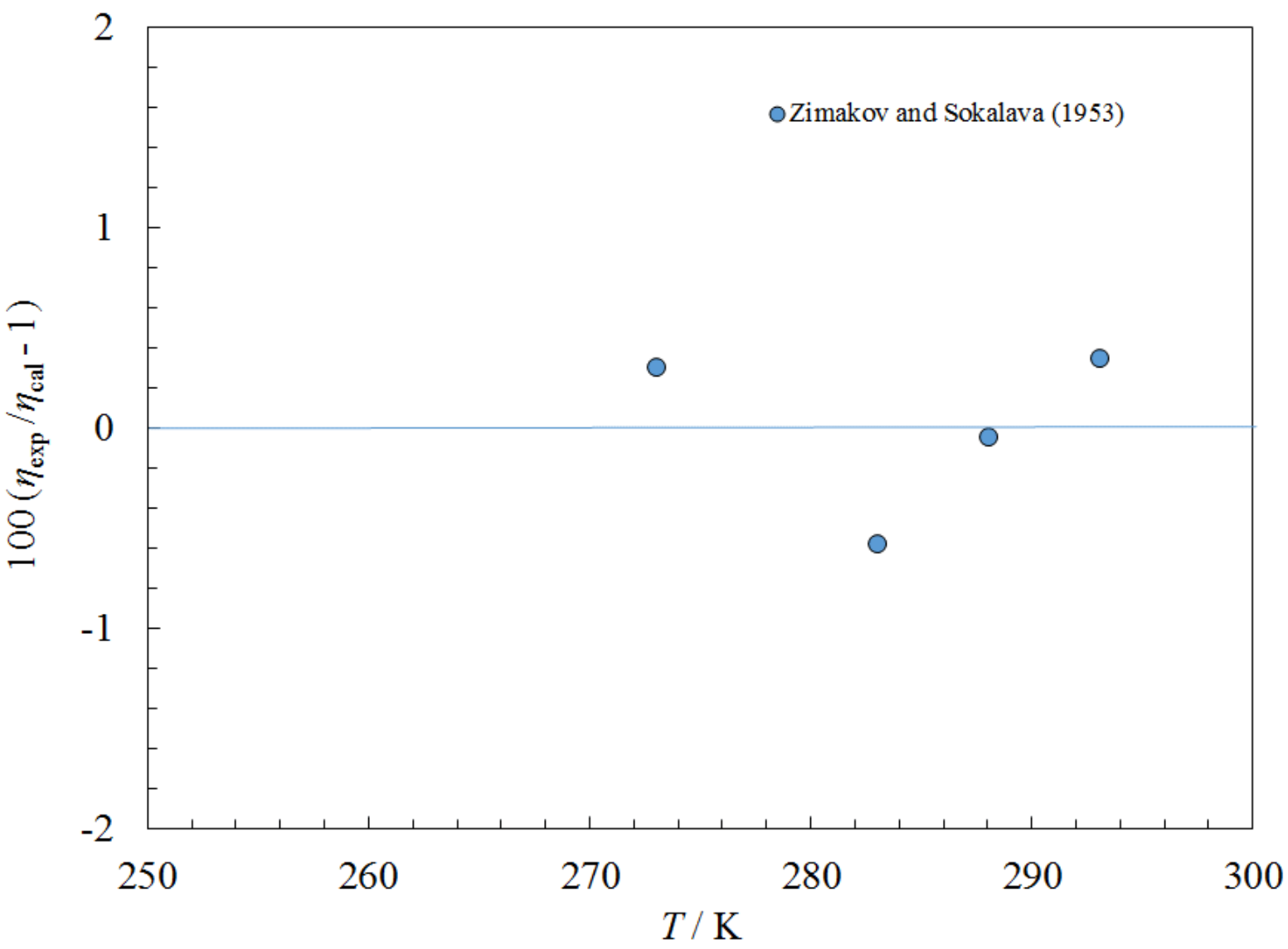

Figure 189. Percentage deviations between the model and the experimental viscosity data for propylene oxide as a function of temperature. 


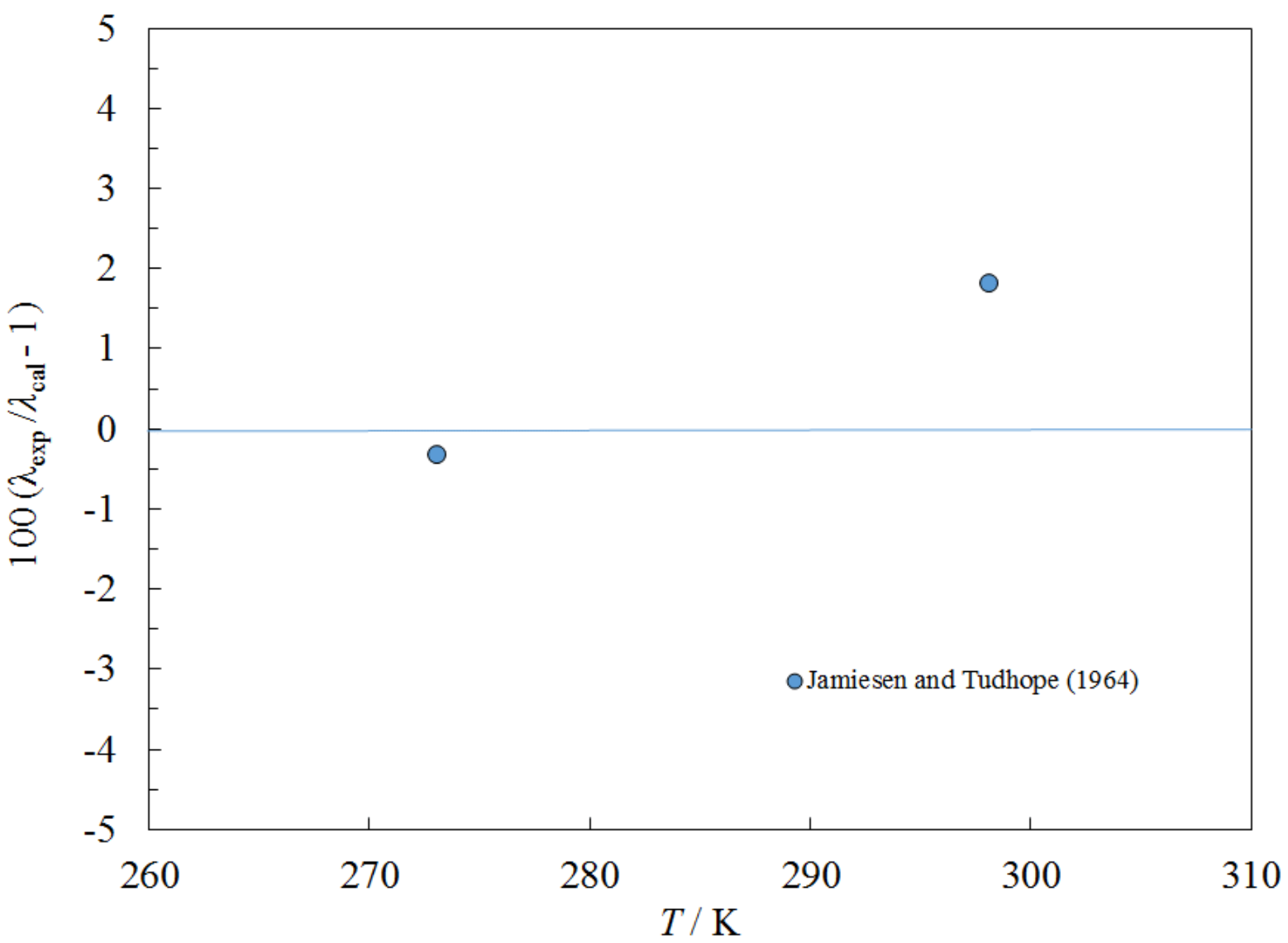

Figure 190. Percentage deviations between the model and the experimental thermal conductivity data for propylene oxide as a function of temperature.

\subsubsection{Sulfur dioxide}

The equation of state of Gao et al. (K. Gao, Wu, Zhang, \& Lemmon, 2016) was used to provide the density and the critical parameters. Lennard-Jones parameters were obtained from Hirschfelder, Curtiss, and Bird (Hirschfelder et al., 1964). Liquid-phase viscosity data, limited to atmospheric pressure, from Hartl et al. (Hartl, Neuder, \& Gores, 2009) and from Awbery and Griffiths (Awbery \& Griffiths, 1936) were used to determine the coefficients in Table 2. Figure 191 shows comparisons with these data and also with gas-phase viscosity data from additional references (Bhattacharyya, 1970; Clifford et al., 1981; A. K. Pal \& Barua, 1967; Stewart \& Maass, 1932). We estimate the uncertainty in the liquid phase along the saturation boundary is $5 \%$, rising to $10 \%$ at $35 \mathrm{MPa}$ (the limit of the EOS). We estimate the uncertainty in the gas phase is $5 \%$. The data of Baker and de Haas (Baker \& de Haas, 1964) were used to obtain the gas-phase coefficients in Table 3, and the saturated liquid thermal conductivity data of Kardos (Kardos, 1934) were used to obtain the liquid-phase coefficients. Parameters for the critical enhancement are presented in Table 4. A deviation plot is shown in Fig. 192. The data were very limited, and we estimate that the uncertainty in the gas phase is $5 \%$, and $5 \%$ in the liquid along the saturation boundary, rising to $10 \%$ at $35 \mathrm{MPa}$. Surface tension is discussed in Mulero et al. (Mulero et al., 2012). 


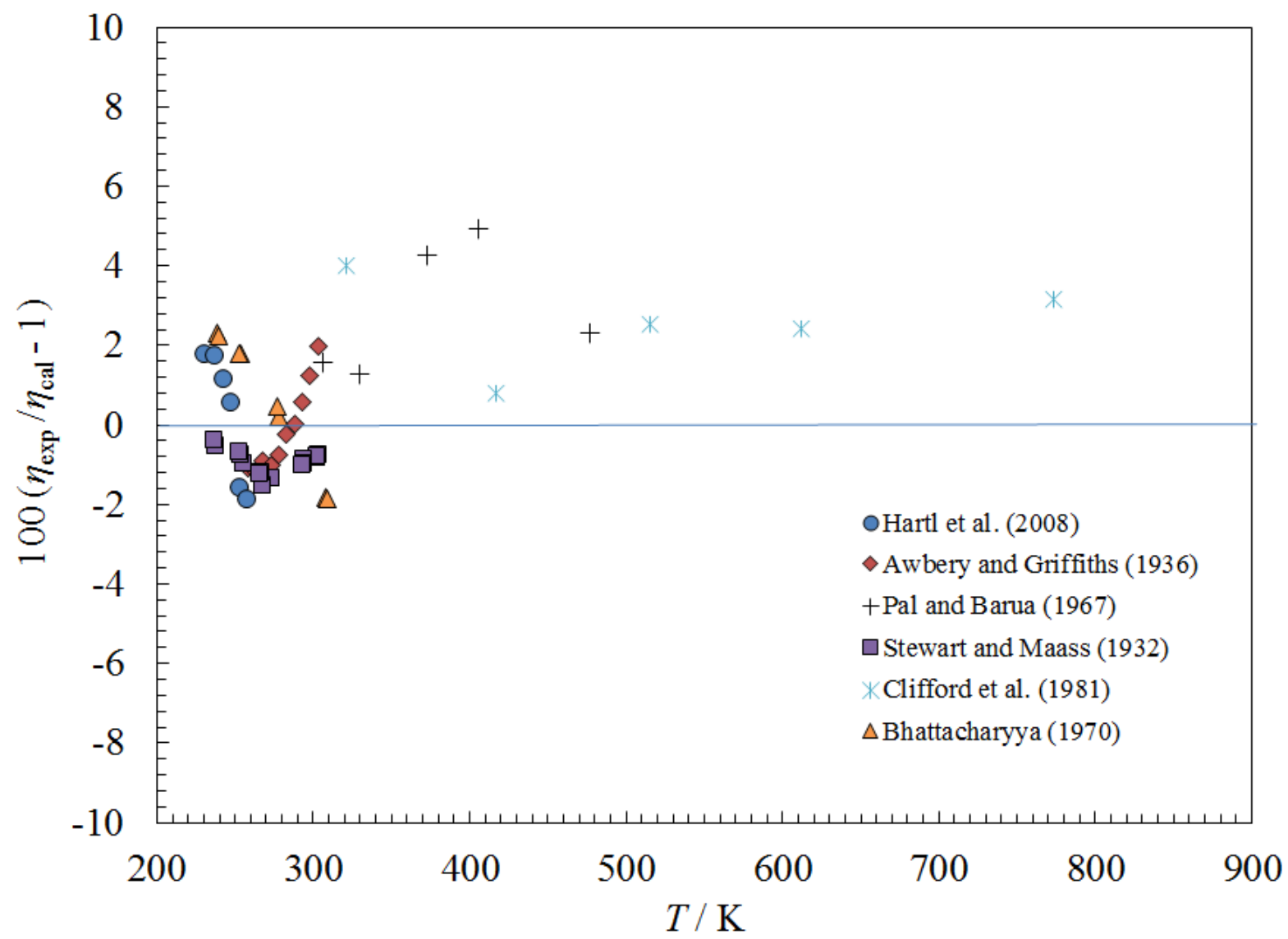

Figure 191. Percentage deviations between the model and the experimental viscosity data for sulfur dioxide as a function of temperature. 


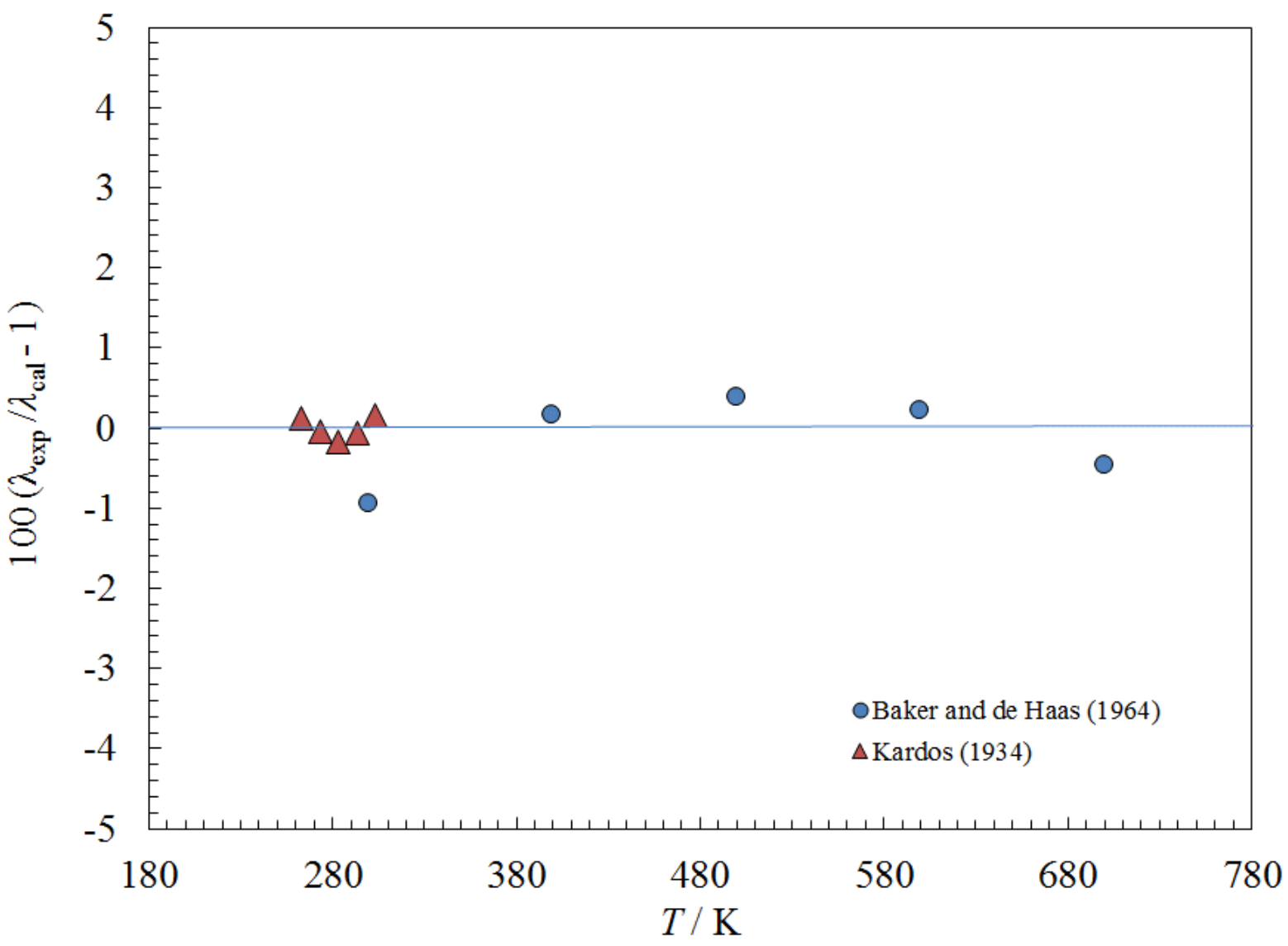

Figure 192. Percentage deviations between the model and the experimental thermal conductivity data for sulfur dioxide as a function of temperature.

\subsection{Miscellaneous Fluids}

\subsubsection{Chlorine}

We used the recently developed equation of state of Herrig, et al. (Herrig, Thol, \& Span, 2018a) to provide density and critical properties. Lennard-Jones parameters were taken from Hirschfelder et al. (Hirschfelder et al., 1964); the values are presented in Table 1. For viscosity of the gas phase, the only data we found were those of Trautz and Ruf (Trautz \& Ruf, 1934); in the liquid phase the only data available are those of Steacie and Johnson (Steacie \& Johnson, 1925) that were obtained along the liquid saturation boundary(Steacie \& Johnson, 1925). The coefficients obtained from fitting the experimental viscosity data are presented in Table 2. Figure 193 gives the viscosity deviations as a function of temperature. Due to the very limited data, we estimate the uncertainty to be on the order of $10 \%$ for both the gas and liquid phases. For thermal conductivity, we could not locate any liquid-phase data, although there are recommended values presented in the compilation of Ho et al. (Ho, Powell, \& Liley, 1972) that are based on a correlation for diatomic substances and stated as being provisional. Only one limited data set (Chaikin \& Markevich, 1958) was located for the gas phase. Our recommended parameters are given in Table 3, and deviations are shown in Figure 194. Parameters for the critical enhancement are presented in Table 4. We estimated the uncertainty in the gas phase to be on the order of $10 \%$. For the liquid phase, it is 
difficult to estimate an uncertainty due to lack of data and we can only estimate that the uncertainty is on the order of $50 \%$. For surface tension, we fit the data set (Johnson \& McIntosh, 1909) recommended by the compilation of Jasper (Jasper, 1972) to the functional form of Eq. (22), and the coefficients are presented in Table 5. A deviation plot is given in Figure 195. We estimate the uncertainty for surface tension to be $5 \%$.

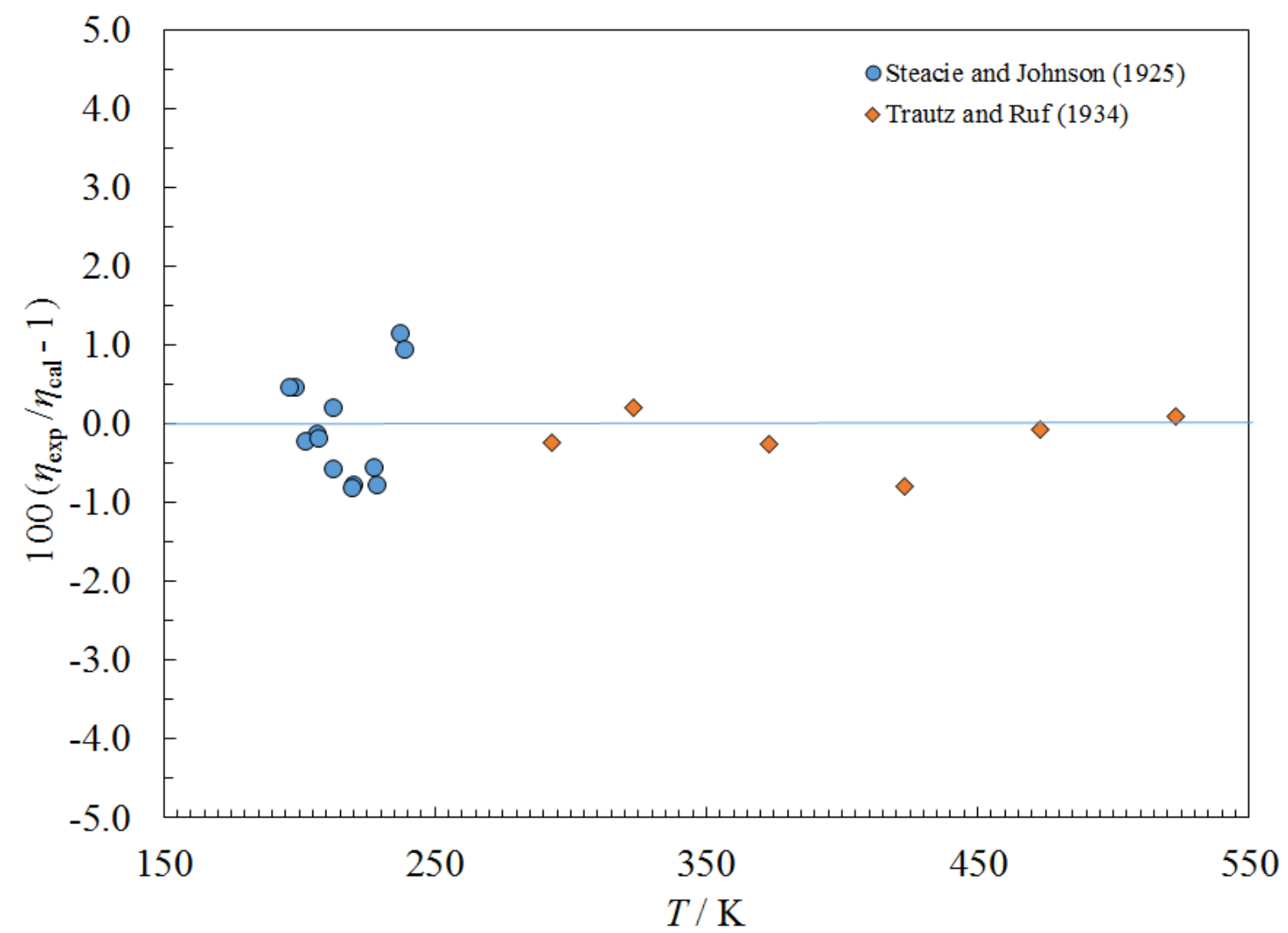

Figure 193. Percentage deviations between the model and the experimental viscosity data for chlorine. 


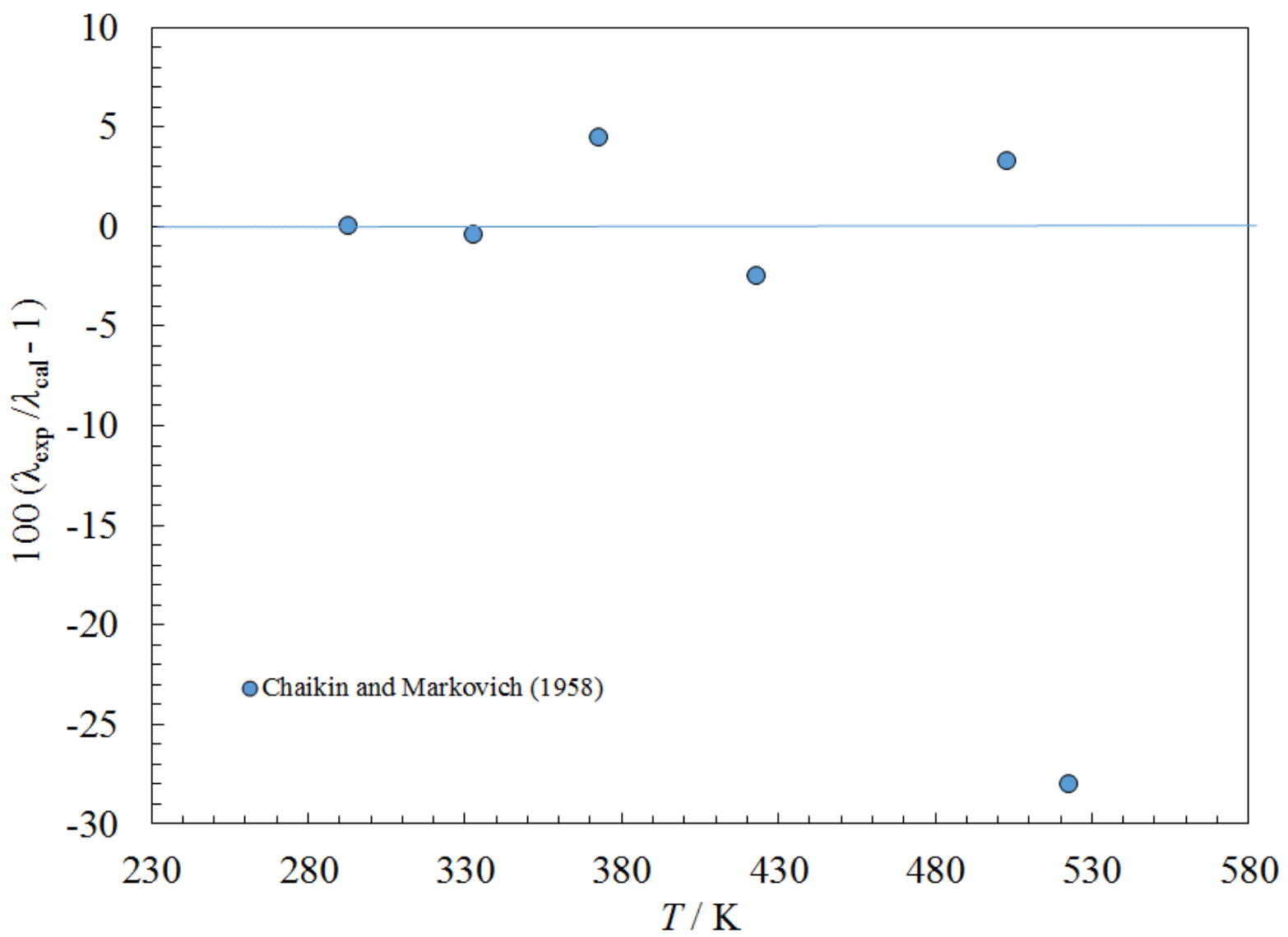

Figure 194. Percentage deviations between the model and the experimental thermal conductivity data for chlorine. 


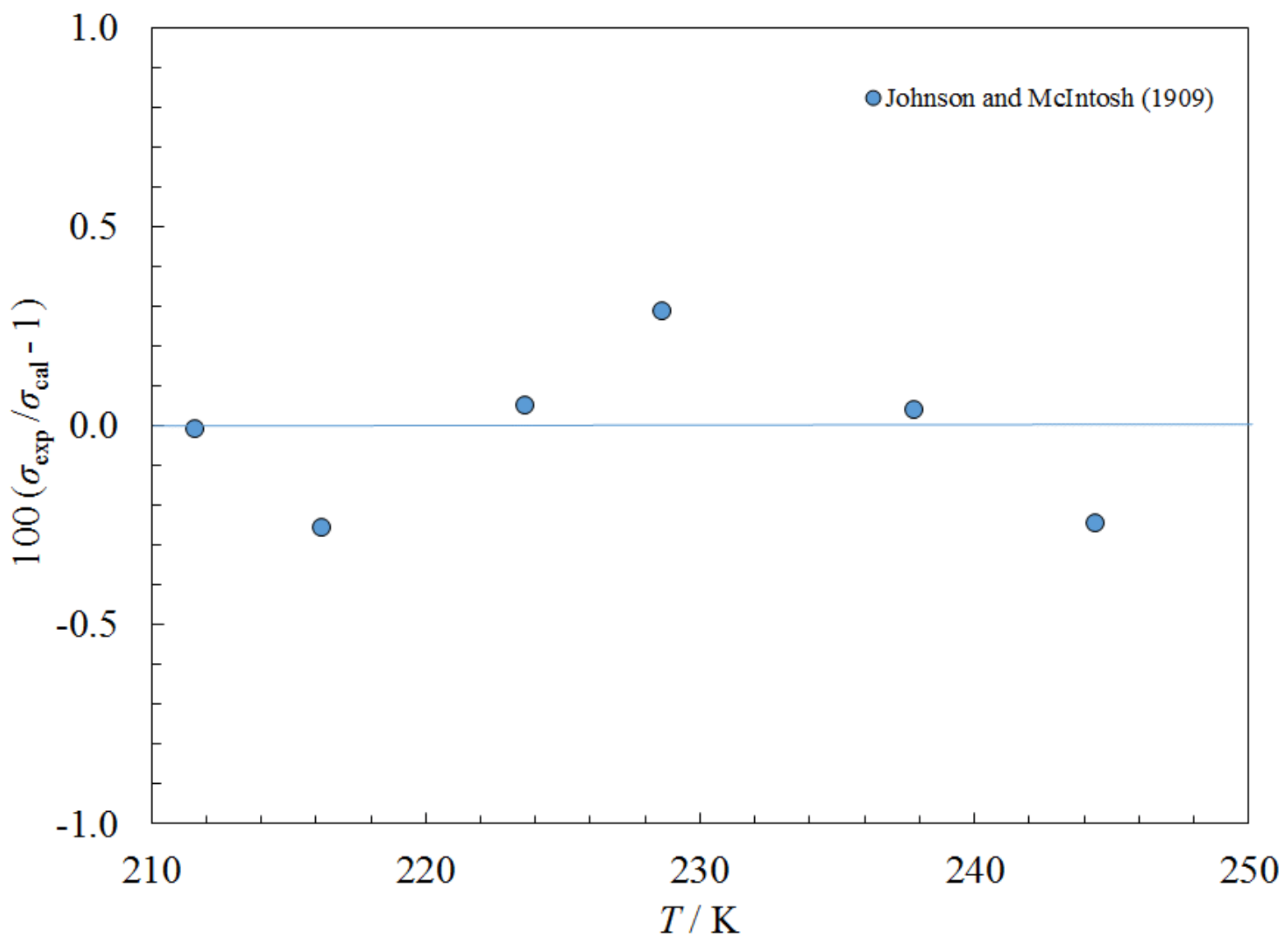

Figure 195. Percentage deviations between the model and the experimental surface tension data for chlorine.

\subsubsection{Fluorine}

We used the equation of state of de Reuck (de Reuck, 1990) to provide density. LennardJones parameters were taken from Reid et al. (Reid, 1987). There are few experimental data for this fluid, so we relied heavily on predictions provided by Hanley and Prydz (Hanley \& Prydz, 1972), who reviewed existing data and found most to be unreliable. We used recommended values for the saturated liquid viscosity and dilute gas viscosity from Hanley and Prydz (Hanley \& Prydz, 1972) and the data of Elverum and Doescher (Elverum \& Doescher, 1952) to obtain the coefficients in Table 2, and Figure 196 shows a deviation plot. We estimate the uncertainty in the viscosity in the gas phase and along the liquid saturation boundary to be $20 \%$ (based on Hanley and Prydz (Hanley \& Prydz, 1972) estimates of their data). Similarly we used Hanley and Prydz (Hanley \& Prydz, 1972) dilute-gas and saturated liquid values to obtain the coefficients in Table 3, with deviations shown in Fig. 197. Parameters for the critical enhancement are presented in Table 4. Again, we estimate the uncertainty in the thermal conductivity in the gas phase and along the liquid saturation boundary to be $20 \%$. Surface tension is discussed in Mulero et al. (Mulero et al., 2012). 


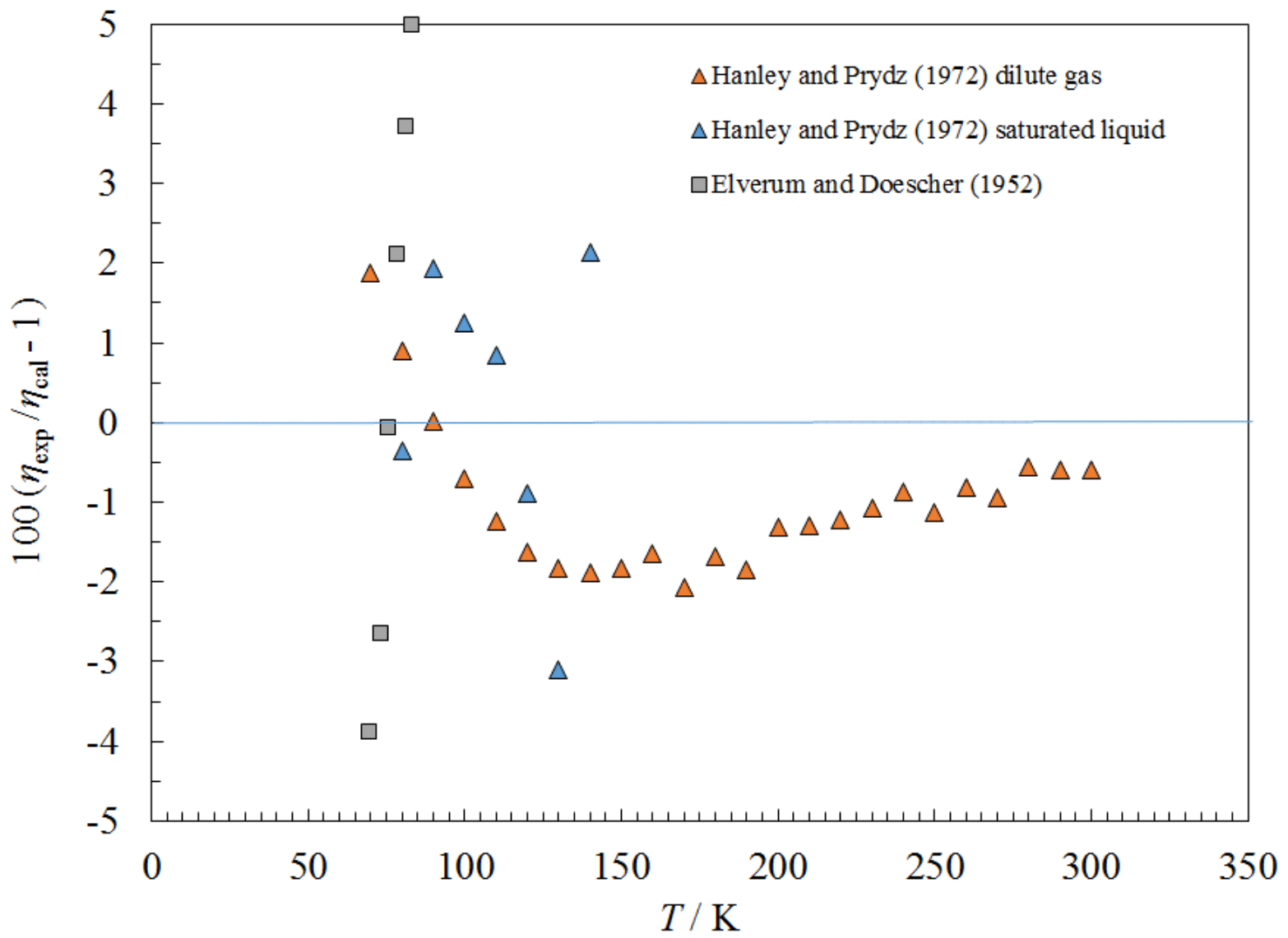

Figure 196. Percentage deviations between the model and the experimental viscosity data for fluorine. 


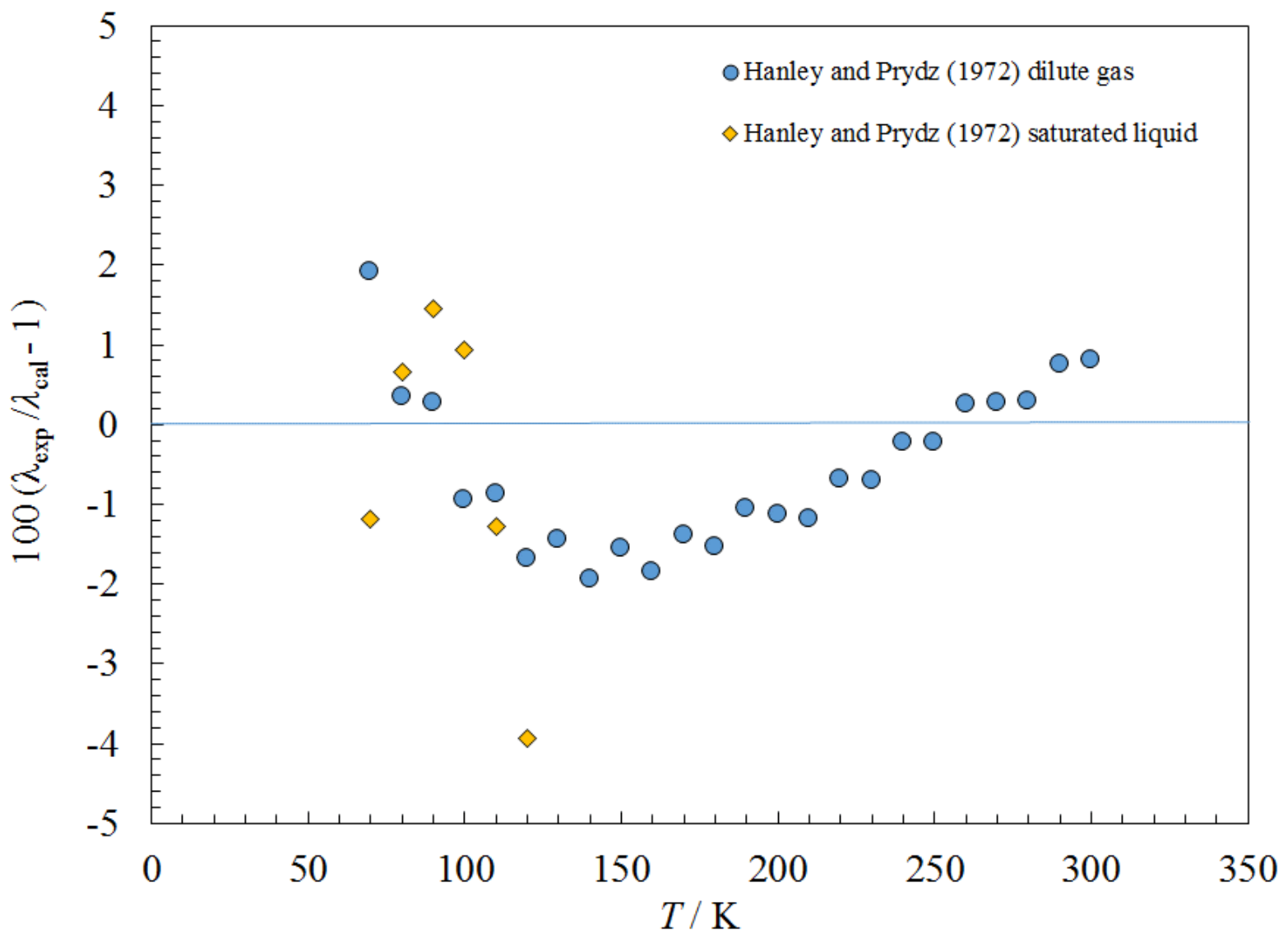

Figure 197. Percentage deviations between the model and the experimental thermal conductivity data for fluorine. 


\subsubsection{Hydrogen chloride (HCl)}

The equation of state of Thol et al. (Thol, Dubberke, Baumhögger, Span, \& Vrabec, 2018) was used to provide the density and the critical parameters. Lennard-Jones parameters were estimated with the method of Chung et al. (Chung et al., 1988). There are two sources of liquid viscosity that cover different temperature ranges (Krynicki \& Hennel, 1963; Steele, McIntosh, \& Archibald, 1906), and these were used to obtain the parameters in Table 2. Both sets are along the saturation boundary. Figure 192 shows the deviations in viscosity as a function of temperature. As indicated in Figure 198, the data sets are not in very good agreement. We estimate the uncertainty in the liquid phase to be $10 \%$ at temperatures above $240 \mathrm{~K}$ along the saturation boundary, much higher at lower temperatures and higher pressures. We estimate $100 \%$ uncertainty for pressures to $50 \mathrm{MPa}$ and temperatures below $240 \mathrm{~K}$ due to the lack of data and the dissimilarities between the propane reference fluid and hydrogen chloride. There were no data located in the gas phase, and we estimate the uncertainty is $50 \%$. For thermal conductivity, we fit the data of Jamiesen et al. (Jamiesen, Irving, \& Tudhope, 1975) in the liquid phase along saturation and Hansen et al. (Hansen, Tsao, Aminabhavi, \& Yaws, 1995) in the vapor phase to obtain the coefficients in Table 3. Parameters for the critical enhancement are presented in Table 4. Figure 199 shows the deviations in thermal conductivity as a function of temperature. The lowest temperature point of Jamiesen does not follow the same trend as the others, and we suspect that it may have larger errors. We estimate the uncertainty in the liquid phase along the saturation boundary and in the gas phase to be $10 \%$. The uncertainty at higher pressures and lower temperature may be significantly higher, but is difficult to assess due to severe lack of data for this fluid. A separate publication covers surface tension (Mulero \& Cachadiña, 2014); it is not covered here. 


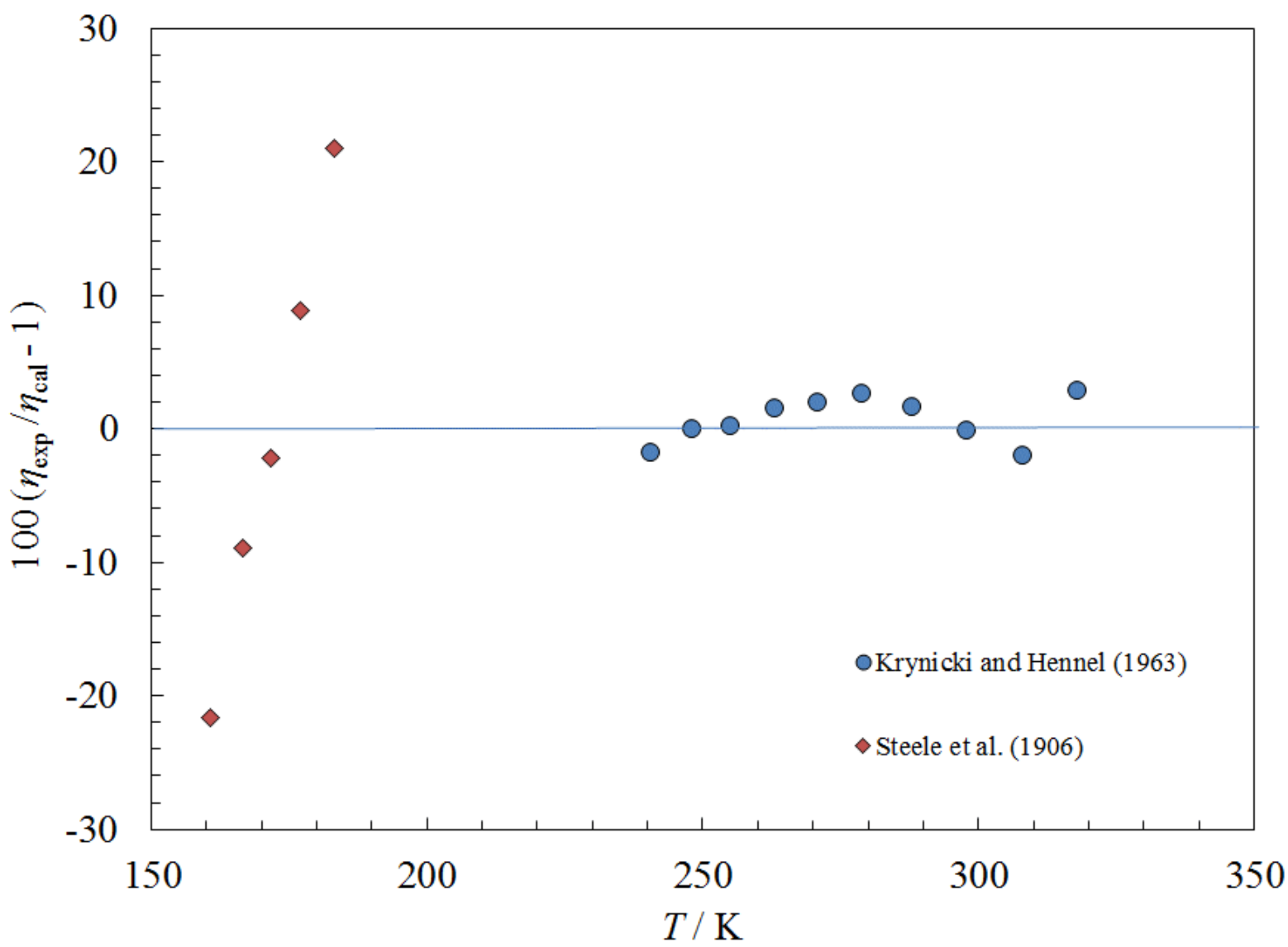

Figure 198. Percentage deviations between the model and the experimental viscosity data for hydrogen chloride as a function of temperature. 


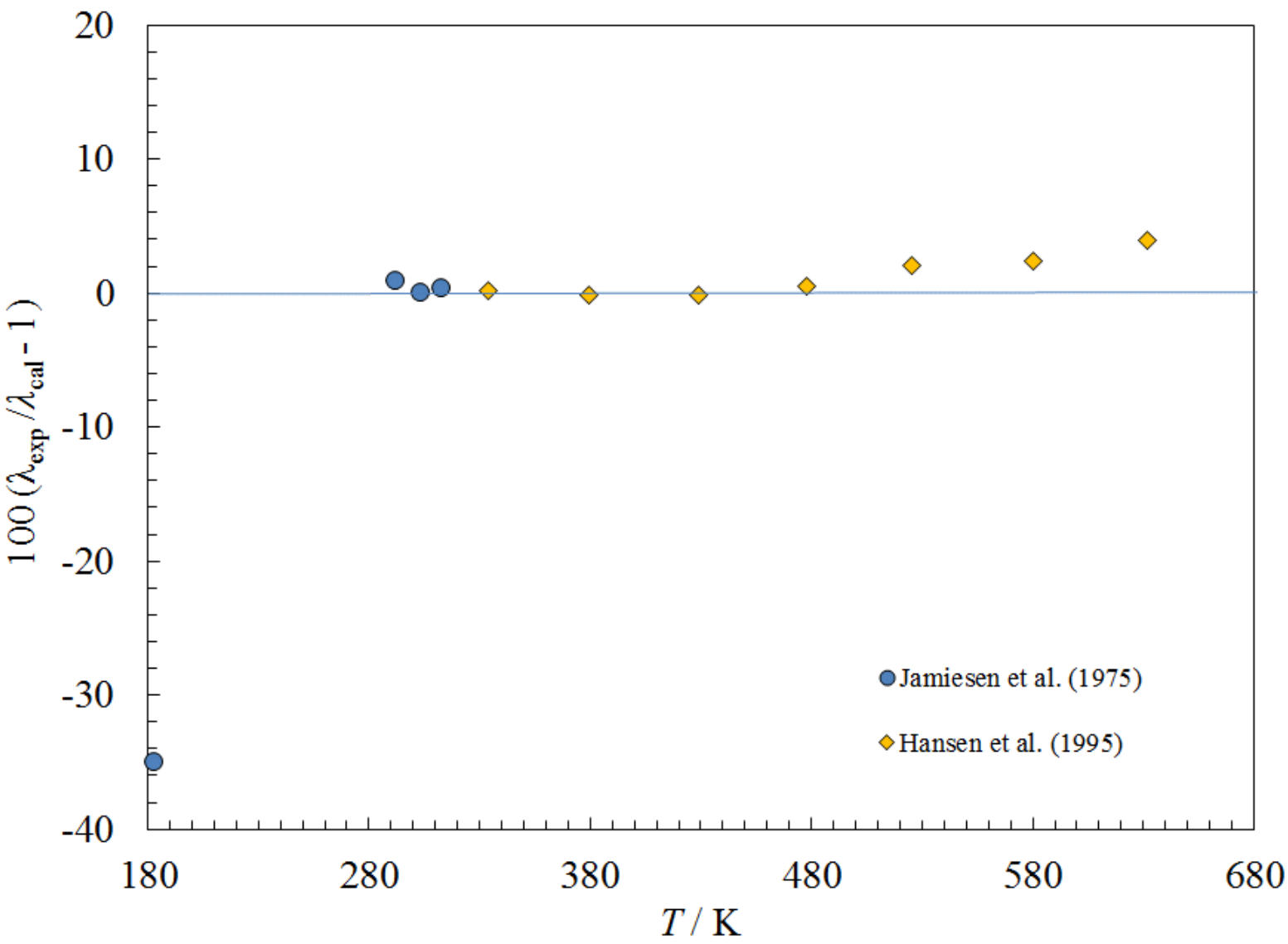

Figure 199. Percentage deviations between the model and the experimental thermal conductivity data for hydrogen chloride as a function of temperature.

\subsubsection{Dimethyl carbonate}

We use the equation of state of Zhou et al. (Zhou, Wu, \& Lemmon, 2011) to provide density and values for the critical point. Lennard-Jones parameters were computed using the method of Chung et al. (Chung et al., 1988). No data sources for vapor-phase viscosity were found, but several sets of liquid-phase data were used (Bi, Wu, Liu, \& Meng, 2003; Chen et al., 2015; Comunas, Baylaucq, Boned, \& Fernandez, 2001; Meng, Zheng, Wu, \& Liu, 2009; Rodriguez, Canosa, Dominguez, \& Tojo, 2004; C. Yang, Xu, \& Ma, 2004) to obtain the coefficients in Table 2 that enabled coverage from 283 to $383 \mathrm{~K}$ and pressures up to $100 \mathrm{MPa}$. The limit of the equation of state is $60 \mathrm{MPa}$, but it behaves in a physically reasonable manner at pressures to $100 \mathrm{MPa}$. Figures 200 and 201 show comparisons with liquid-phase viscosity data as a function of temperature and of pressure. The set of Comunas et al. (Comunas et al., 2001) extends to $100 \mathrm{MPa}$, while the set of Meng et al. (Meng et al., 2009) has an upper limit of $20 \mathrm{MPa}$. All other data sets are at atmospheric or saturation pressure. Based on comparisons with these data sets, we estimate the uncertainty in the liquid phase along the saturation boundary for viscosity is $2 \%$, rising to $4 \%$ at pressures to $60 \mathrm{MPa}$. Similarly, no vapor-phase data for thermal conductivity were found, but two liquid-phase datasets (Jin, Wu, Liu, \& Pan, 2004; L. Song, Wu, \& Liu, 2008) were used to fit the coefficients in Table 3. Parameters for the critical enhancement are presented in Table 4. 
Deviations are shown in Figures 202 and 203. The data of Jin et al. (Jin et al., 2004) are all at atmospheric pressure, while those of Song et al. (L. Song et al., 2008) extend to $30 \mathrm{MPa}$. Both were made on a transient single hot-wire method by the same laboratory. Based on comparisons with the data of Song et al. (L. Song et al., 2008) and Jin et al. (Jin et al., 2004), we estimate the uncertainty in the liquid phase to be $4 \%$, at pressures to $30 \mathrm{MPa}$. A separate publication covers surface tension (Mulero \& Cachadiña, 2014); it is not covered here.

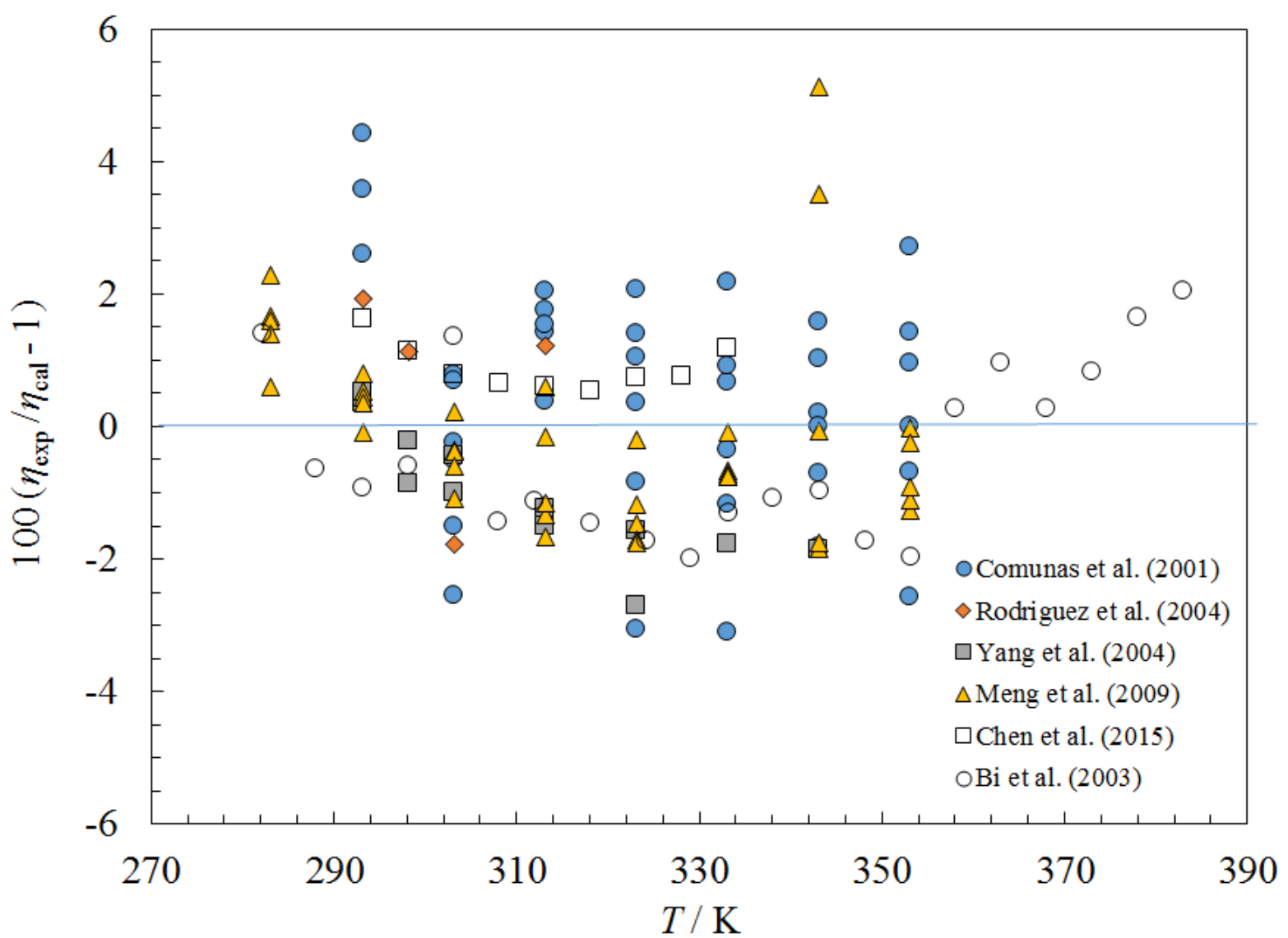

Figure 200. Percentage deviations between the model and the experimental viscosity data for dimethyl carbonate as a function of temperature 


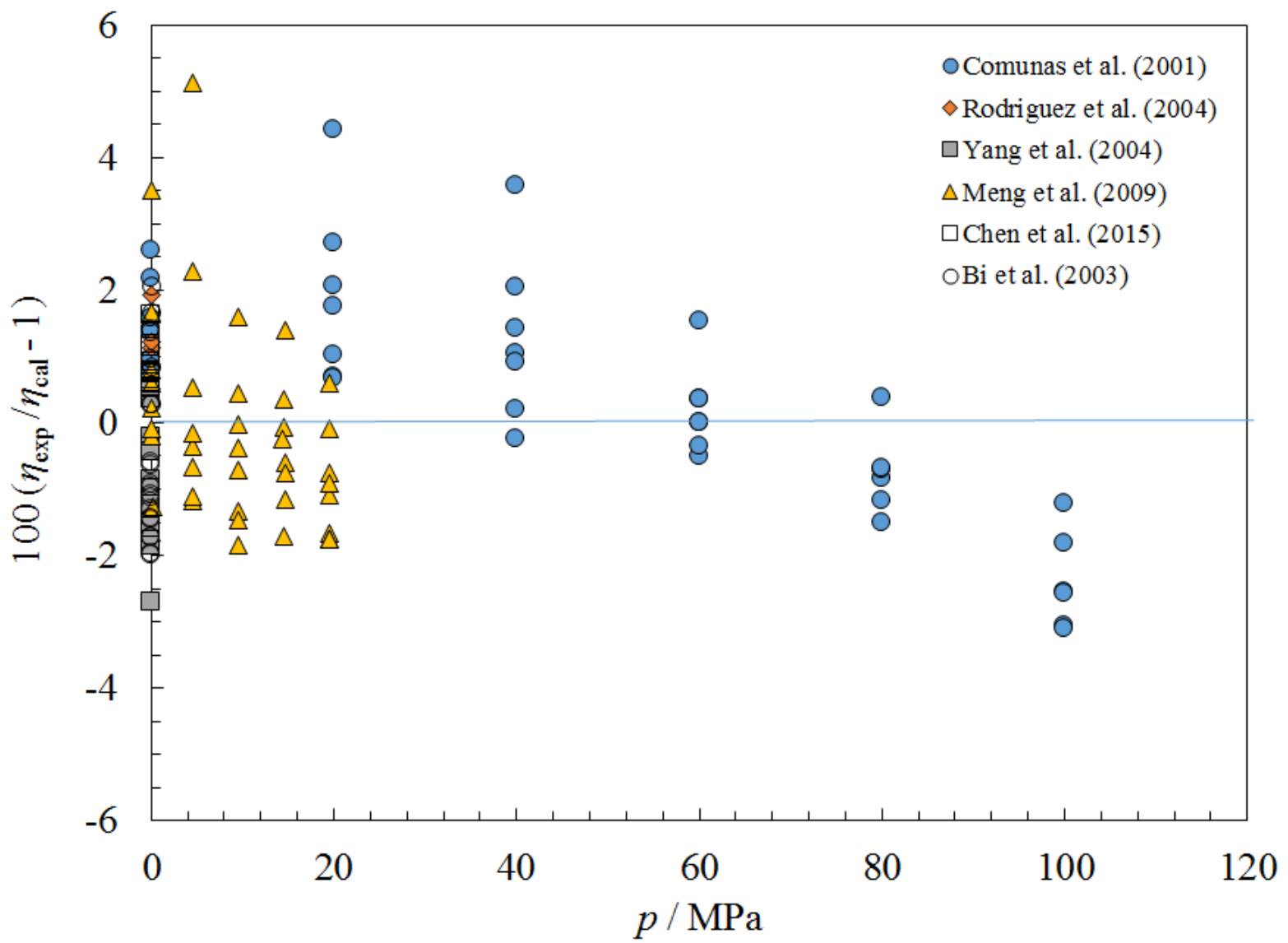

Figure 201. Percentage deviations between the model and the experimental viscosity data for dimethyl carbonate as a function of pressure 


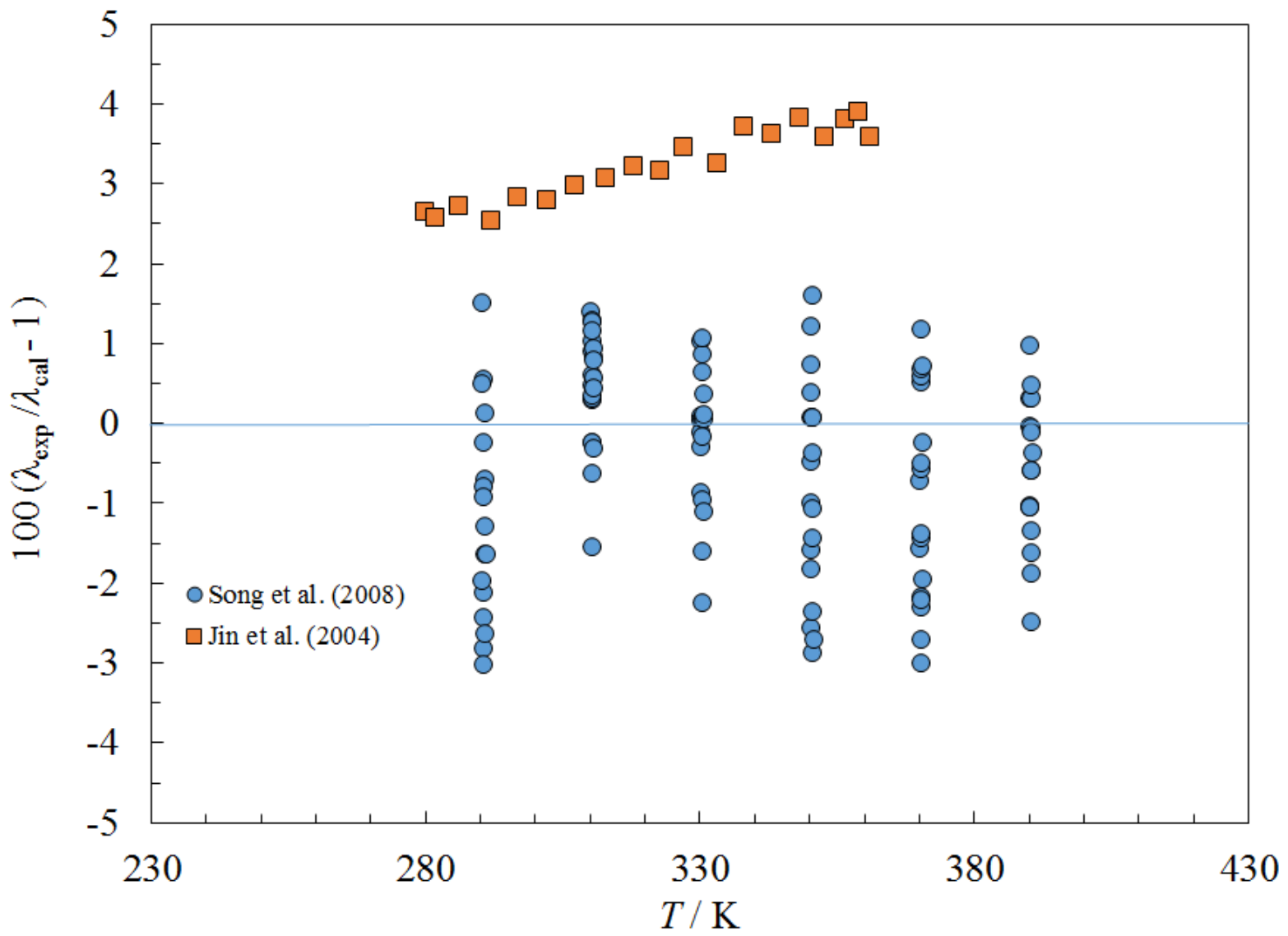

Figure 202. Percentage deviations between the model and the experimental thermal conductivity data for dimethyl carbonate as a function of temperature. 


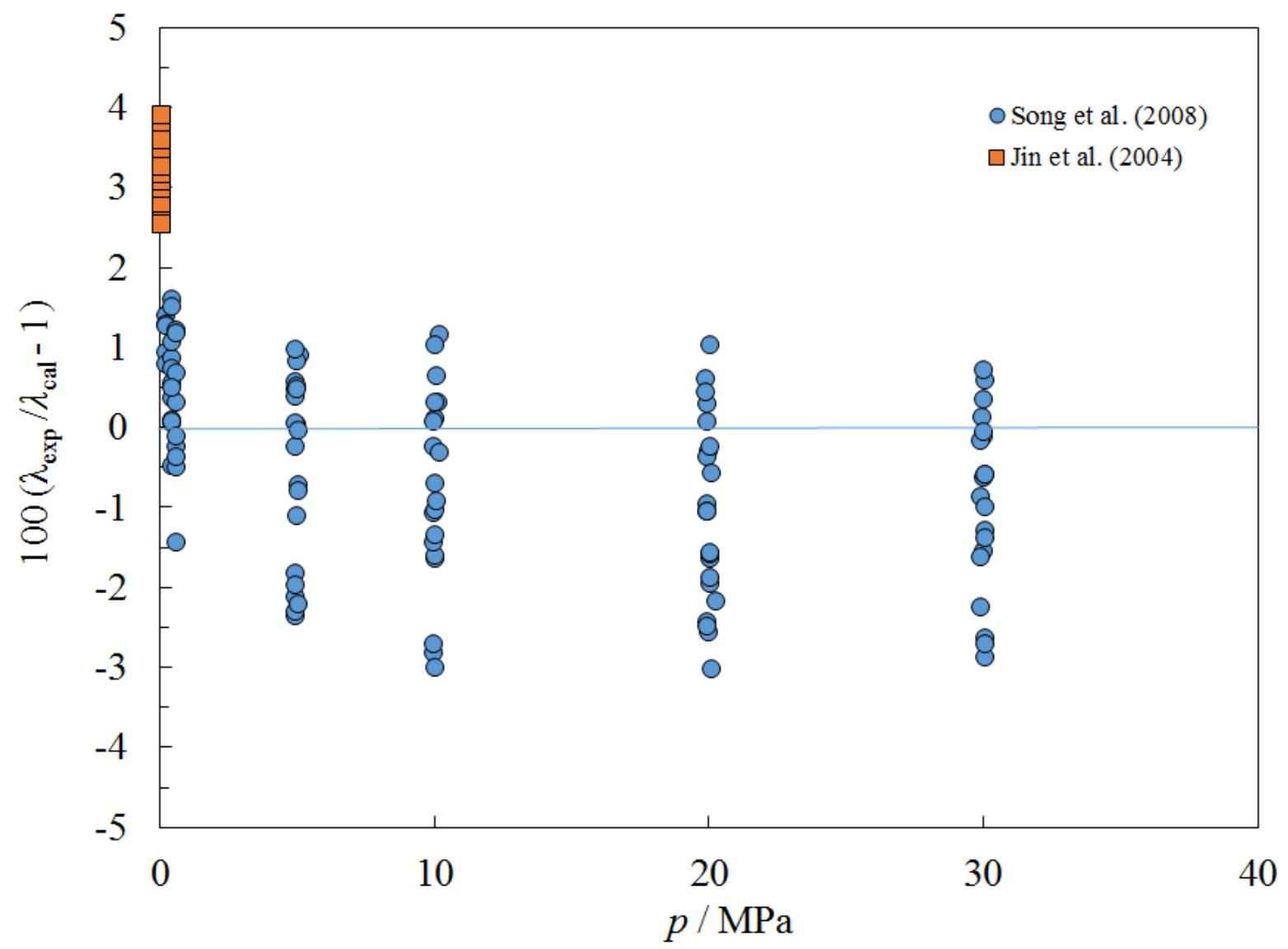

Figure 203. Percentage deviations between the model and the experimental thermal conductivity data for dimethyl carbonate as a function of pressure.

\subsubsection{Acetone}

The equation of state of Lemmon and Span (E.W. Lemmon \& Span, 2006) was used to provide the density and the critical parameters. Lennard-Jones parameters were obtained from Hirschfelder et al. (Hirschfelder et al., 1964). Gas-phase viscosity from three sources (Craven \& Lambert, 1951; Ling \& Van Winkle, 1958; Titani, 1933) was located but only used for comparisons. In the liquid phase, we fit the data of Atoyan and Mamedov (Atoyan \& Mamedov, 1976), Yang et al. (L. Yang, Luo, Lian, \& Liu, 2010), and Bagdasaryan (Bagdasaryan, 1964) to obtain the coefficients in Table 2. Figure 204 shows deviations from experimental viscosity data as a function of temperature, and Figure 205 shows the deviation as a function of pressure. Some additional data sets that were not used in the regression (Batschinski, 1913; Liu \& Kiran, 2007; Thorpe \& Rodger, 1894) are also shown. The figures demonstrate that there is a large amount of disagreement between data sets that exceeds the estimated uncertainty of most of the measurements, which is generally about $3 \%$. Given the poor data situation, we estimate that the uncertainty of the viscosity in the liquid phase at saturation is $5 \%$, rising to $15 \%$ at pressures to $100 \mathrm{MPa}$. The equation should not be used above $100 \mathrm{MPa}$. The estimated uncertainty in the gas phase is also $5 \%$. For thermal conductivity, the gas-phase data of Medzhidov and Safarov 
(Medzhidov \& Safarov, 1983) and the liquid-phase data of Mallan et al. (Mallan, Michaelian, \& Lockhart, 1972) and Qun-Fang et al. (Qun-Fang et al., 1997) were used to obtain the coefficients in Table 3. Parameters for the critical enhancement are presented in Table 4. There were no data above saturation pressure. Figure 206 shows the percentage deviations between the model and the experimental thermal conductivity data as a function of temperature. The estimated uncertainty in the gas phase is $5 \%$, and in the liquid phase at saturation is $5 \%$, rising to $20 \%$ at pressures to 100 MPa. Surface tension is discussed in Mulero et al. (Mulero et al., 2012).

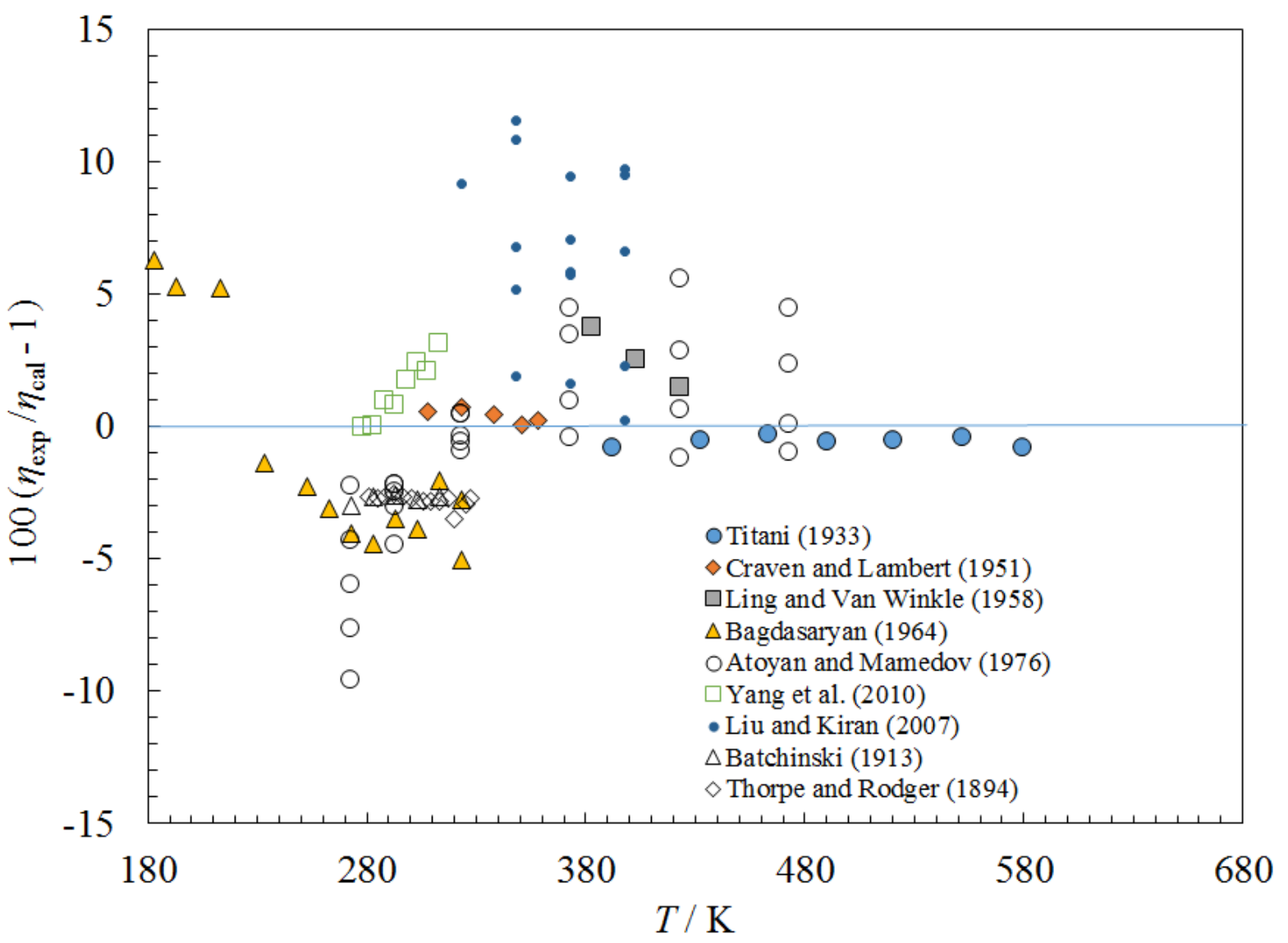

Figure 204. Percentage deviations between the model and the experimental viscosity data for acetone as a function of temperature. 


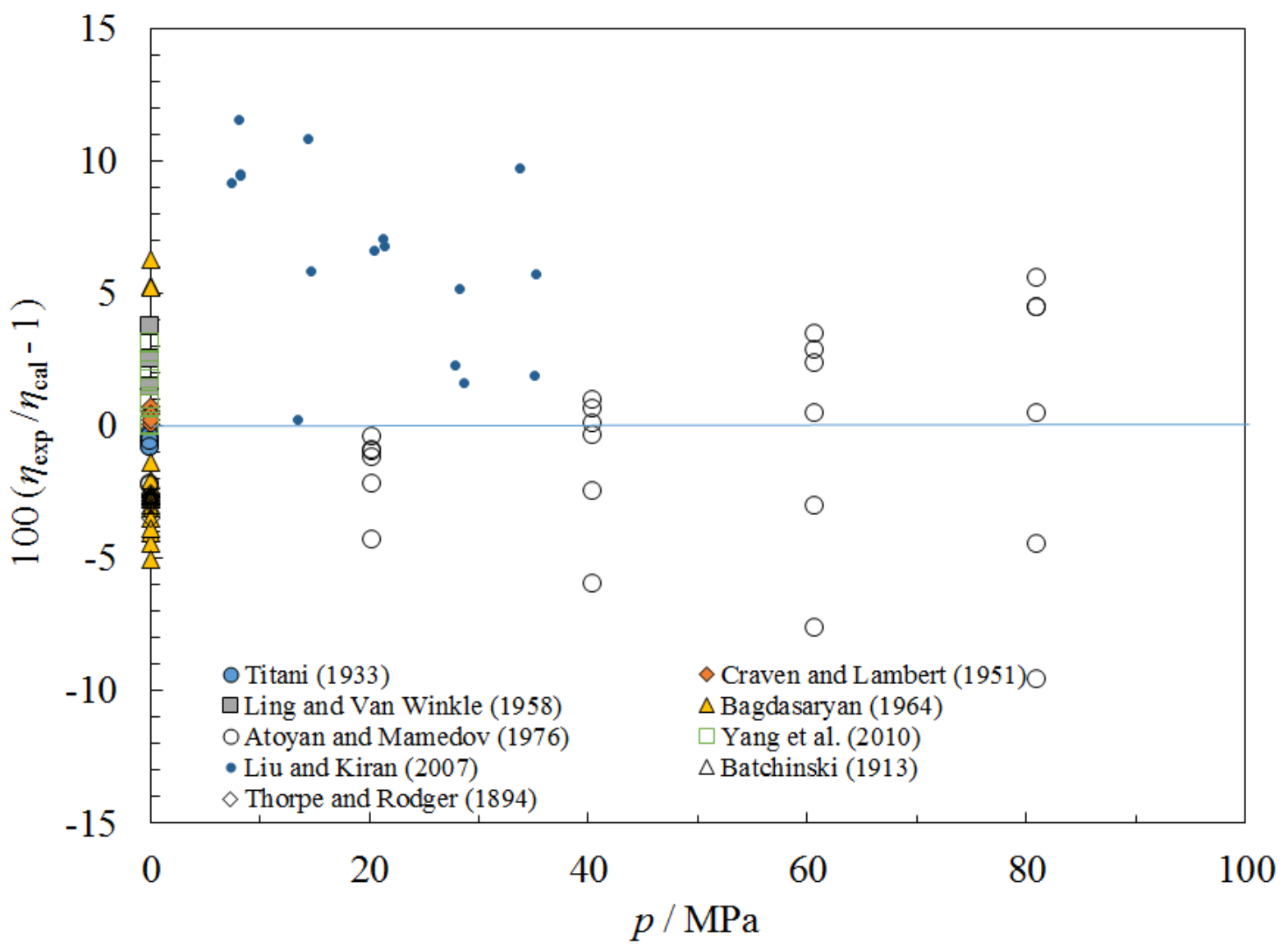

Figure 205. Percentage deviations between the model and the experimental viscosity data for acetone as a function of pressure. 


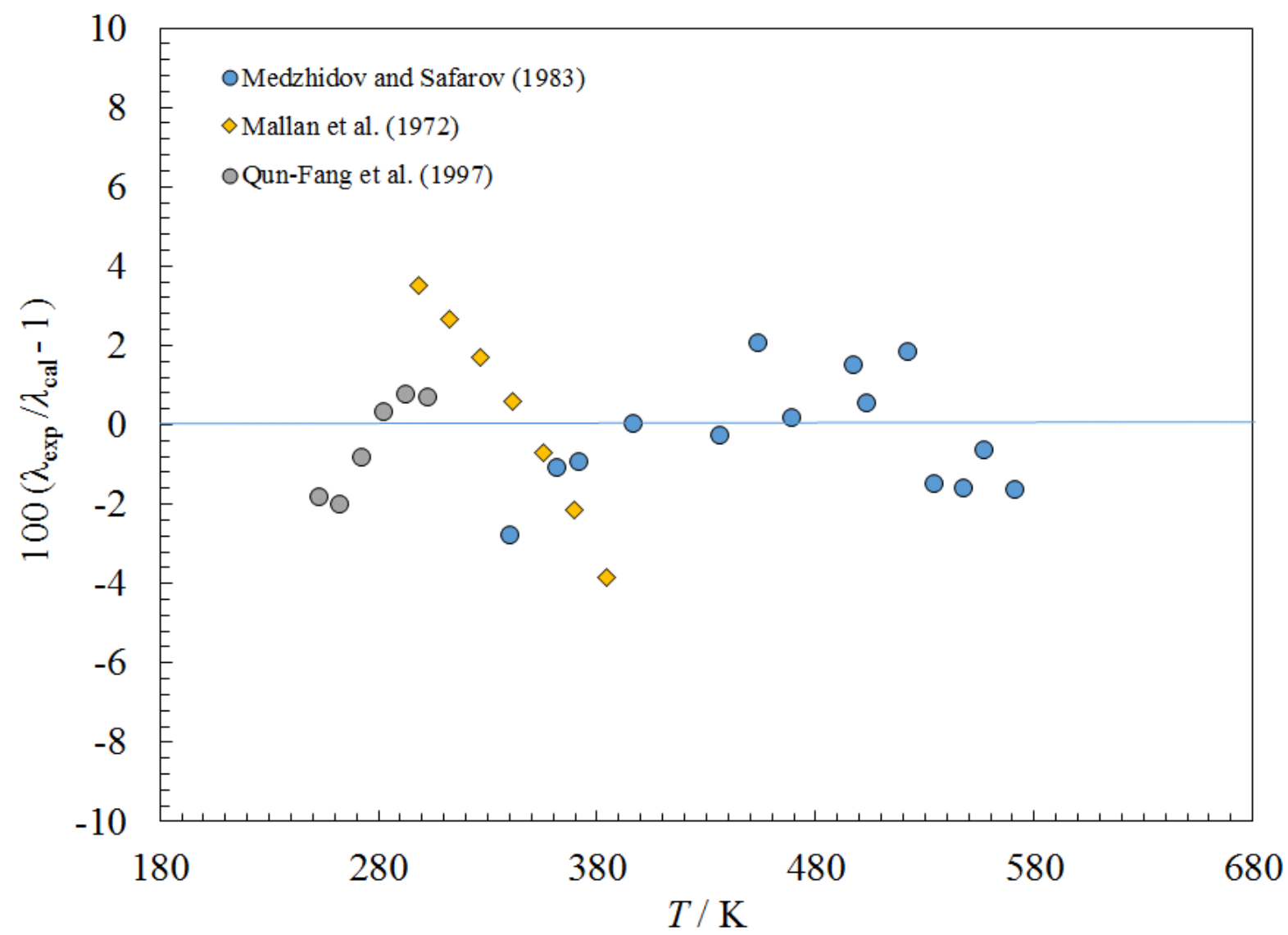

Figure 206. Percentage deviations between the model and the experimental thermal conductivity data for acetone as a function of temperature. 


\subsubsection{Monoethanolamine (MEA)}

The equation of state of Herrig et al. (Herrig, Thol, \& Span, 2018b) was used to provide the density and the critical parameters. Lennard-Jones parameters were computed using the method of Chung et al. (Chung et al., 1988). Data sources for vapor-phase viscosity were not found. Data from several limited liquid-phase data sets at atmospheric pressure (Blanco, Garcia-Abuin, Gomez-Diaz, Navaza, \& Villaverde, 2013; R. M. DiGuilio, Lee, Schaeffer, Brasher, \& Teja, 1992; J.-H. Song, Park, Yoon, Lee, \& Lee, 1996; Yin, Zhu, \& Ma, 2016) were used to obtain the coefficients in Table 2. Figure 207 shows deviations from experimental viscosity data as a function of temperature. We estimate that the uncertainty of the viscosity in the liquid phase at atmospheric pressure over the temperature range from $293 \mathrm{~K}$ to $424 \mathrm{~K}$ is $3 \%$, and higher at lower temperatures and higher pressures. The estimated uncertainty in the gas phase is $20 \%$. For thermal conductivity, gas-phase data were not found, and very limited liquid data at saturation from DiGuilio et al. (R. M. DiGuilio, McGregor, \& Teja, 1992) were used to obtain the coefficients in Table 3. Parameters for the critical enhancement are presented in Table 4 . There were no data above saturation pressure. Figure 208 shows the percentage deviations between the model and the experimental thermal conductivity. The estimated uncertainty for the thermal conductivity of the liquid phase at saturation is $2 \%$ over the temperature range $298 \mathrm{~K}$ to $447 \mathrm{~K}$, higher outside of that range. The estimated uncertainty in the gas phase is $20 \%$. Surface tension data from Han et al. (Han, Jin, Eimer, \& Melaaen, 2012), Blanco et al. (Blanco et al., 2013), and Jayarathna et al. (Jayarathna et al., 2013) were used to obtain the surface tension coefficients in Table 5, and Figure 209 shows deviations from the data. The estimated uncertainty for surface tension over the temperature range from $293 \mathrm{~K}$ to $313 \mathrm{~K}$ is $2 \%$. 


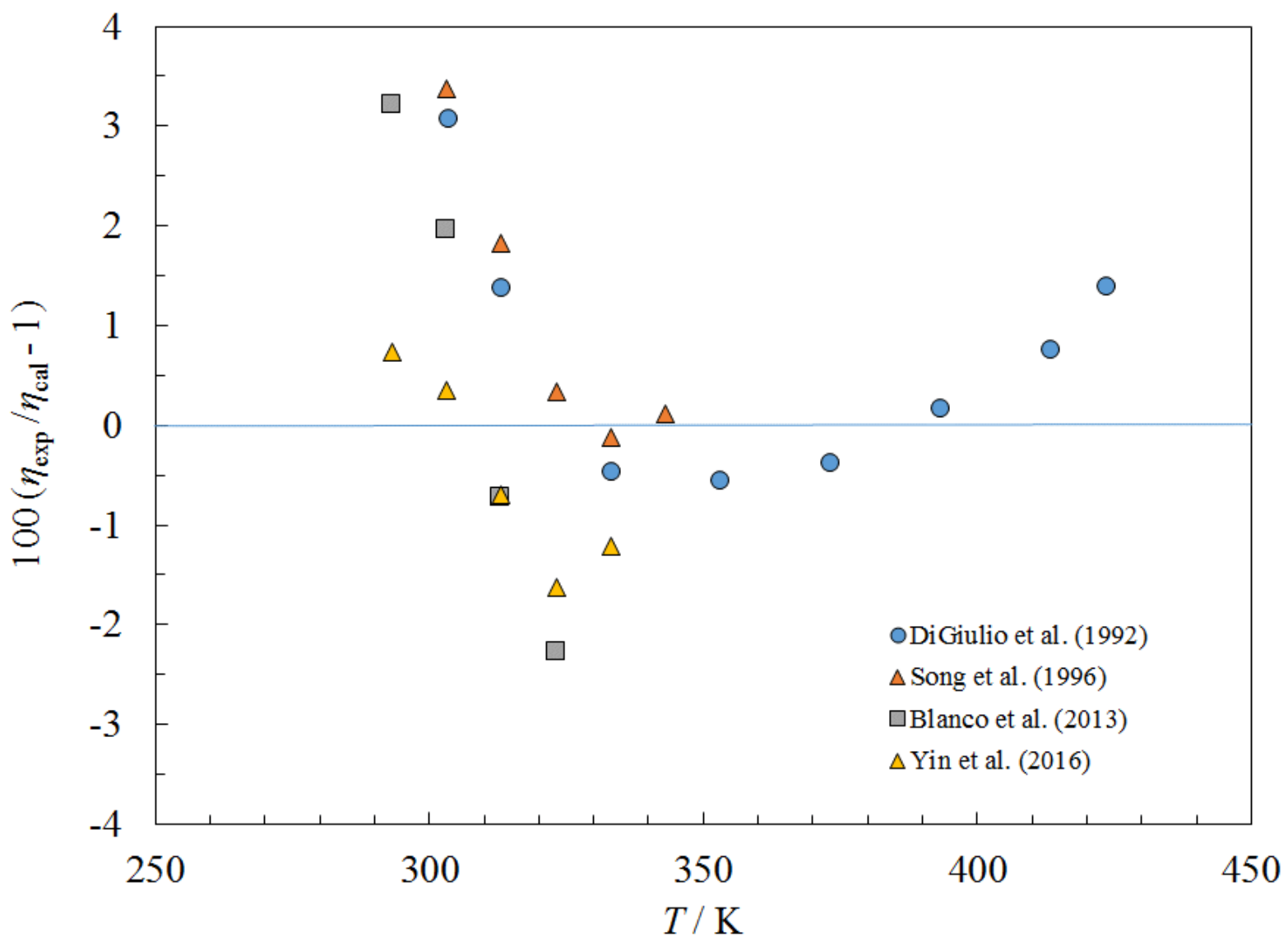

Figure 207. Percentage deviations between the model and the experimental viscosity data for monoethanolamine as a function of temperature. 


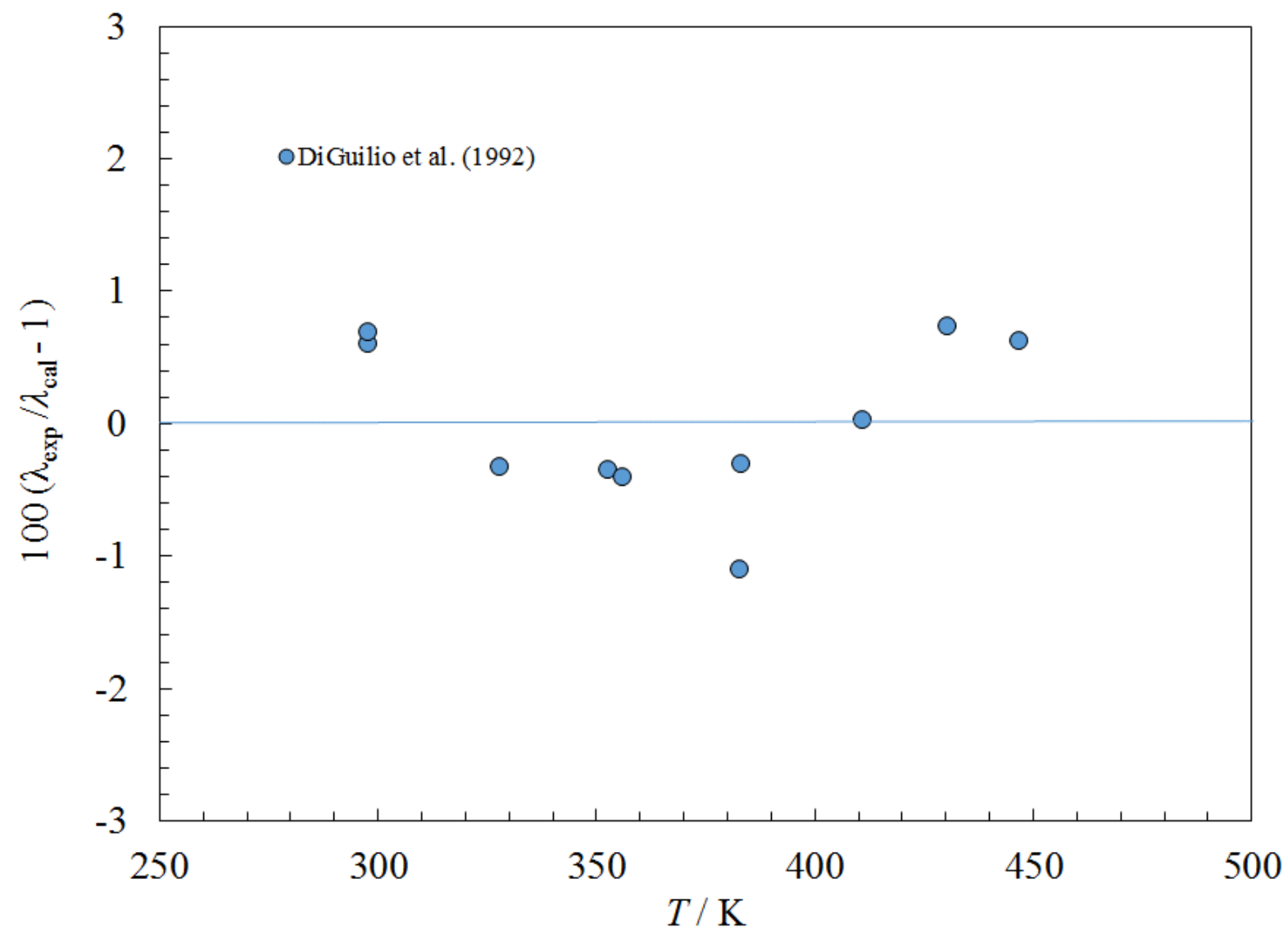

Figure 208. Percentage deviations between the model and the experimental thermal conductivity data for monoethanolamine as a function of temperature. 


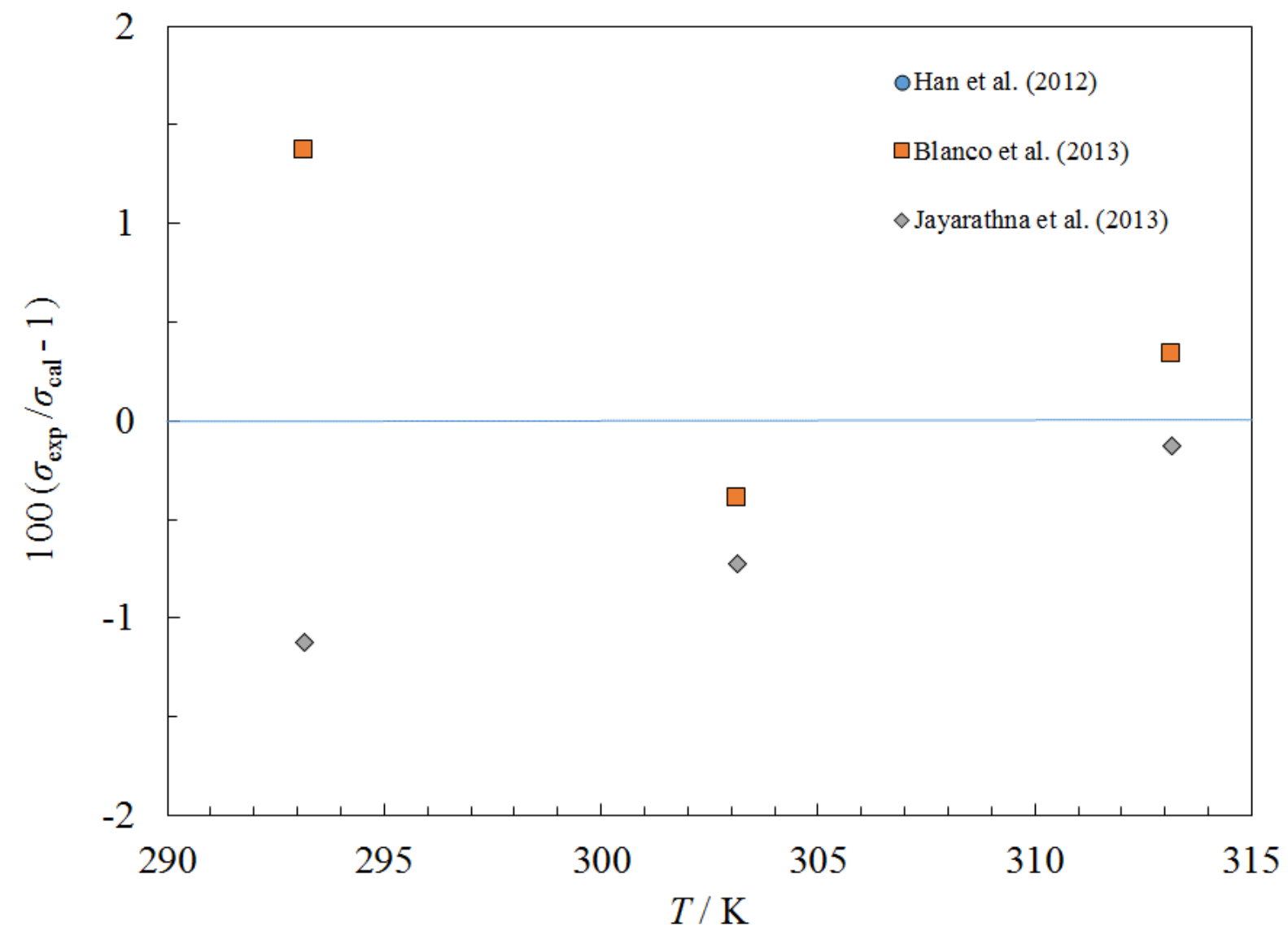

Figure 209. Percentage deviations between the model and the experimental surface tension data for monoethanolamine as a function of temperature.

\subsubsection{Diethanolamine (DEA)}

The equation of state of Herrig et al. (Herrig, Thol, Kortmann, Lemmon, \& Span, 2018) was used to provide the density and the critical parameters. Lennard-Jones parameters were computed using the method of Chung et al. (Chung et al., 1988). Data sources for vapor-phase viscosity were not found. Data from several limited liquid-phase data sets at atmospheric pressure (Aguila-Hernandez, Trejo, Garcia-Flores, \& Molnar, 2008; Blanco et al., 2013; R. M. DiGuilio, Lee, et al., 1992; Haghtalab \& Shojaeian, 2014; Teng, Maham, Hepler, \& Mather, 1994) were used to obtain the coefficients in Table 2 . Figure 210 shows deviations from experimental viscosity data as a function of temperature. There are large discrepancies in the data, and additional viscosity data are necessary to resolve the discrepancies. We estimate that the uncertainty of the viscosity in the liquid phase at atmospheric pressure over the temperature range from $305 \mathrm{~K}$ to $423 \mathrm{~K}$ is $40 \%$, and higher at lower temperatures and higher pressures. The model cannot be used lower than about $305 \mathrm{~K}$. Due to deficiencies in the model, it cannot reproduce the very steep rise in viscosity at low temperatures. The estimated uncertainty in the gas phase is $20 \%$. For thermal 
conductivity, gas-phase data were not found, and very limited liquid data at saturation from DiGuilio et al. (R. M. DiGuilio, McGregor, et al., 1992) were used to obtain the coefficients in Table 3. Parameters for the critical enhancement are presented in Table 4. There were no data above saturation pressure. Figure 211 shows the percentage deviations between the model and the experimental thermal conductivity. The estimated uncertainty for the thermal conductivity of the liquid phase at saturation is $2 \%$ over the temperature range $295 \mathrm{~K}$ to $442 \mathrm{~K}$, higher outside of that range. The estimated uncertainty in the gas phase is $20 \%$. Surface tension data from Fu and Zhong (Fu \& Zhong, 2010), Blanco et al. (Blanco et al., 2013), and Lopez et al. (Lopez, Garcia-Abuin, Gomez-Diaz, La Rubia, \& Navaza, 2013) were used to obtain the surface tension coefficients in Table 5, and Fig. 212 shows deviations with the data. The estimated uncertainty for surface tension over the temperature range from $293 \mathrm{~K}$ to $333 \mathrm{~K}$ is $2 \%$.

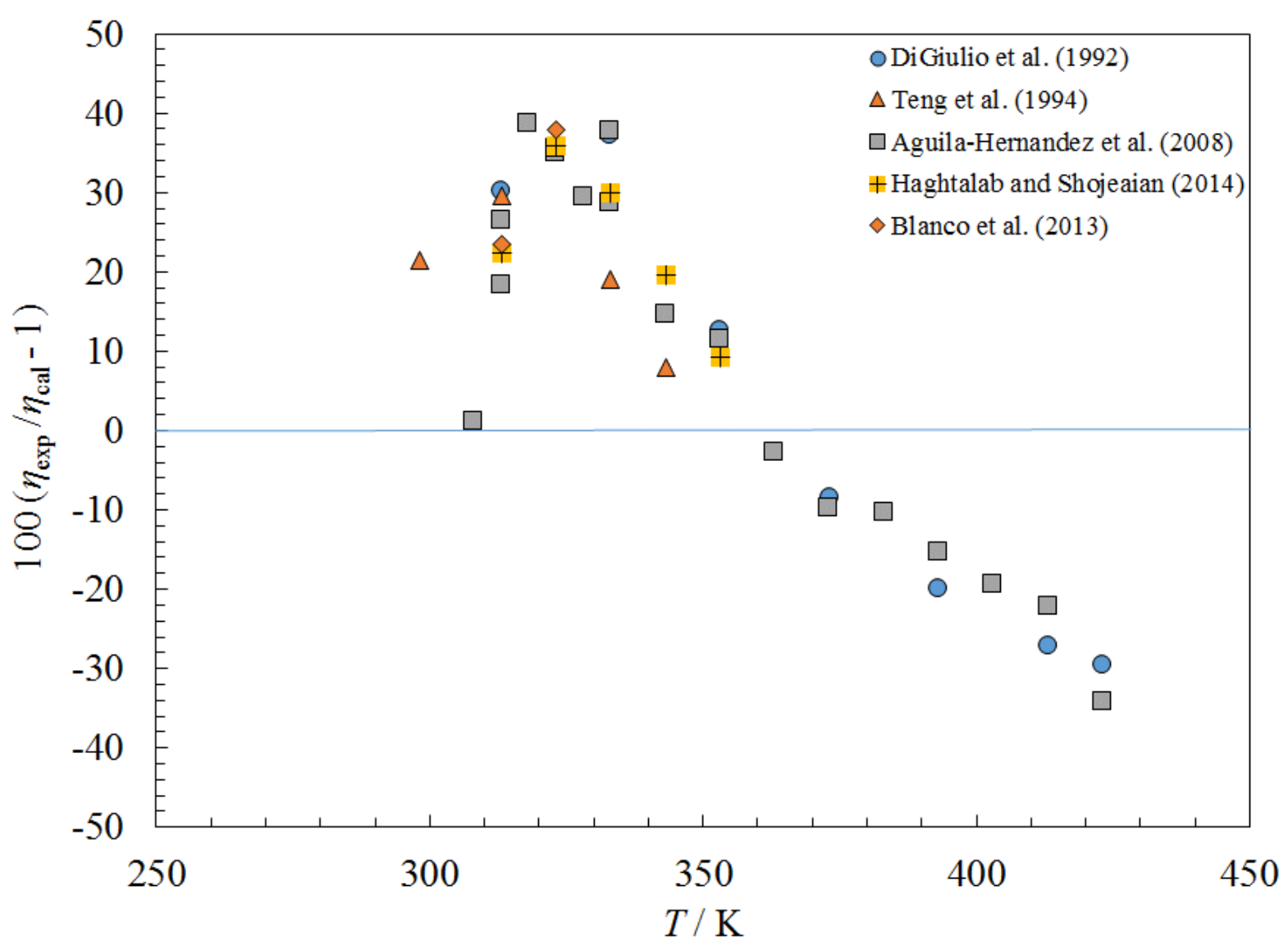

Figure 210. Percentage deviations between the model and the experimental viscosity data for diethanolamine as a function of temperature. 


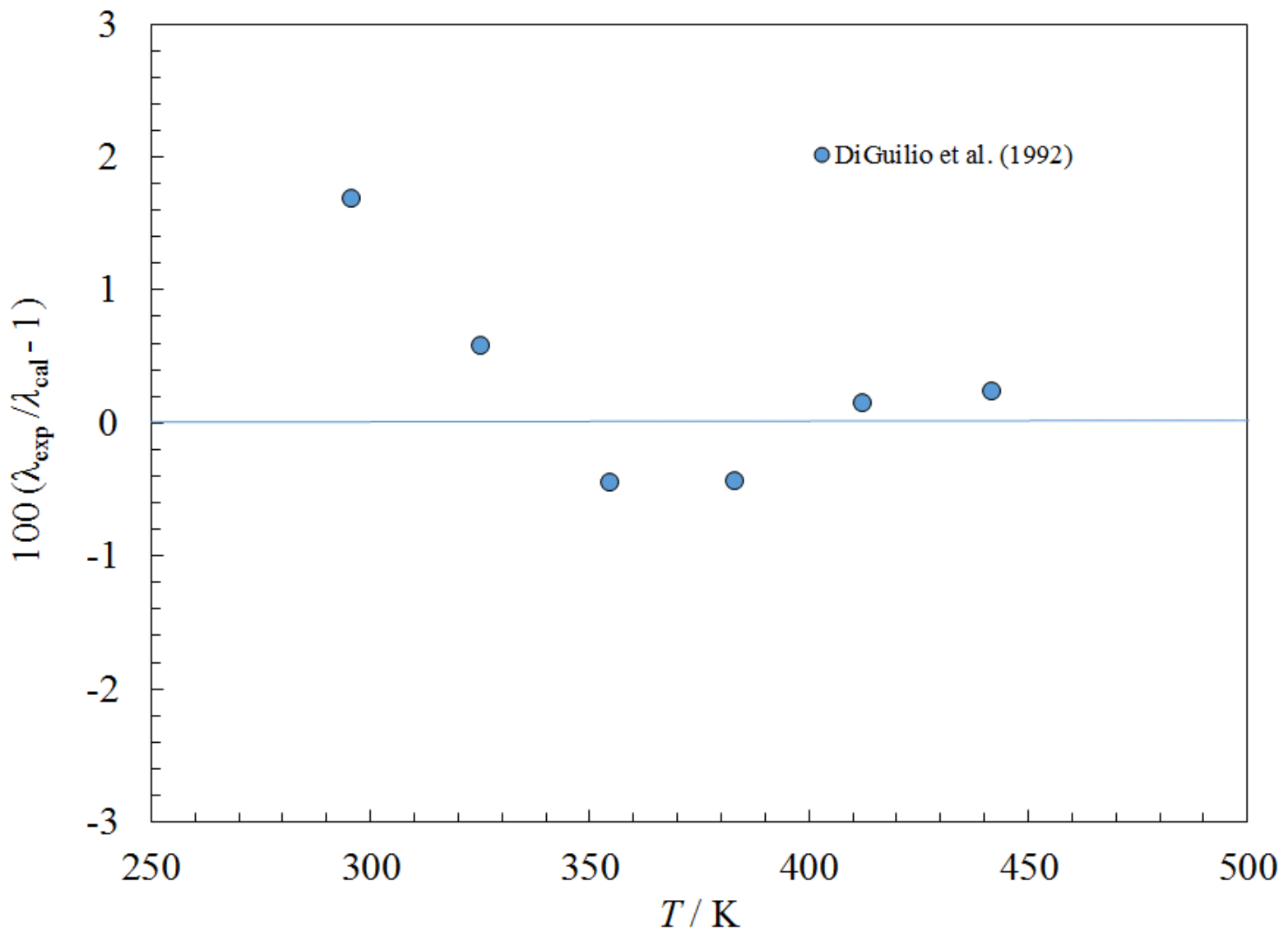

Figure 211. Percentage deviations between the model and the experimental thermal conductivity data for diethanolamine as a function of temperature. 


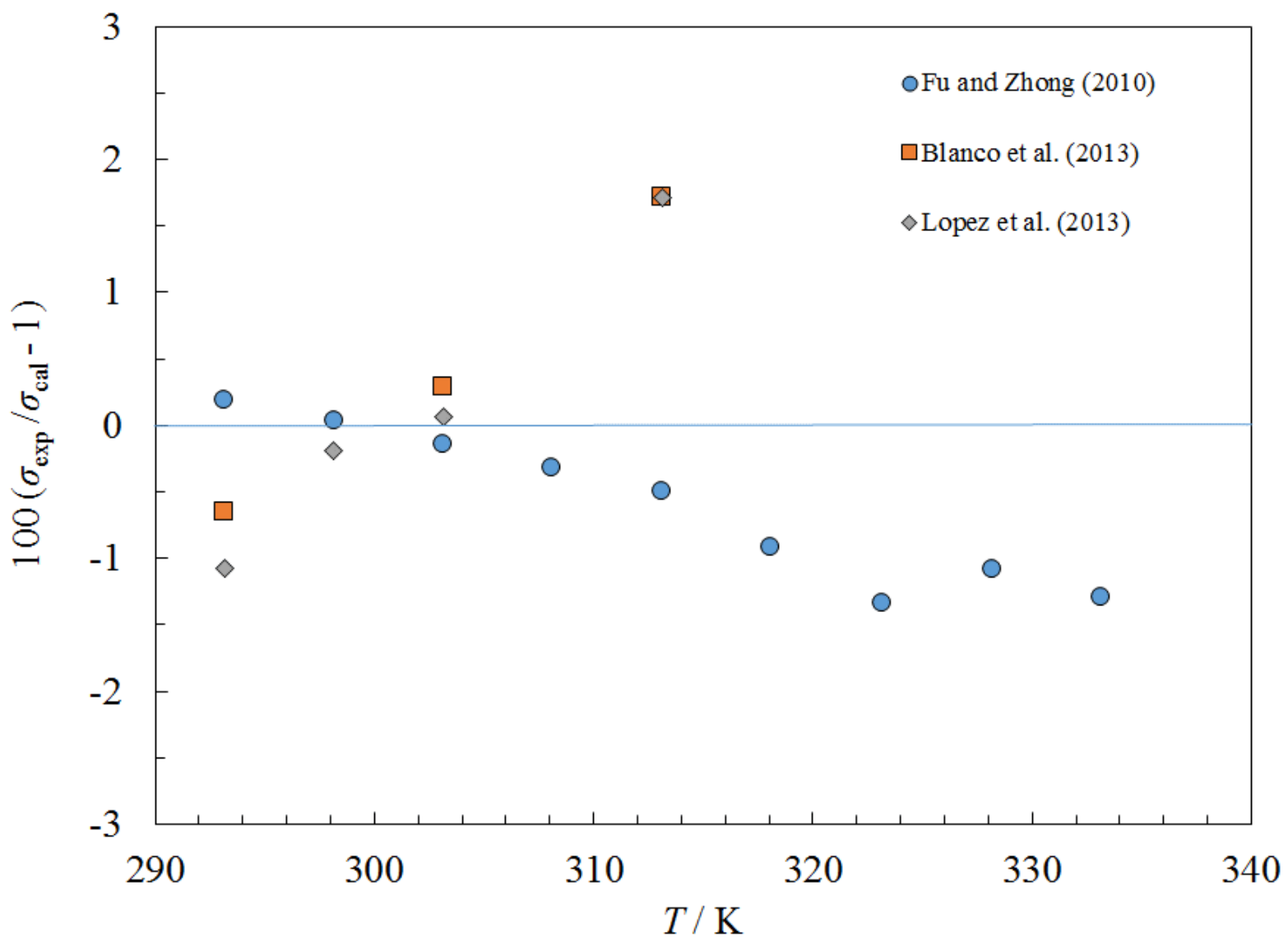

Figure 212. Percentage deviations between the model and the experimental surface tension data for diethanolamine as a function of temperature.

\subsubsection{Ethylene glycol}

The equation of state of Zhou and Lemmon (Zhou \& Lemmon, 2018b) was used to provide the density and the critical parameters. Lennard-Jones parameters were computed using the method of Chung et al. (Chung et al., 1988). Data sources for vapor-phase viscosity were not found. Data from several liquid-phase data sets at atmospheric pressure (Carvalho, Fonseca, Moita, Santos, \& Coutinho, 2015; Losetty, Wilfred, \& Shekar, 2016; Sun, Niu, Gao, Shen, \& Wei, 2015; Zhao et al., 2015) were used to obtain the coefficients in Table 2. Figure 213 shows deviations from experimental viscosity data as a function of temperature, and Figure 214 as a function of pressure. We estimate the uncertainty is $4 \%$ for the liquid phase between $288 \mathrm{~K}$ and $373 \mathrm{~K}$ at atmospheric pressure. The data of Sagdeev et al. (Sagdeev, Fomina, Mukhamedzyanov, \& Abdulagatov, 2012) extend to very high pressures (245 MPa) and we show comparisons up to $50 \mathrm{MPa}$. The highpressure data were not used in the regression, as we were unable to find parameters that fit the high-pressure data, and we limit the use of the equation to $10 \mathrm{MPa}$. Future work should be done with a fluid-specific model rather than corresponding states to fit this fluid, or perhaps a highviscosity reference fluid could be used. The present result should be considered very preliminary. We estimate the uncertainty of the viscosity in the liquid phase above atmospheric pressure and in the vapor phase to be on the order of $20 \%$. For thermal conductivity, gas-phase data were not 
found, and very limited liquid data at atmospheric pressure (R. DiGuilio \& Teja, 1990; Yu. L. Rastorguev \& Gazdiev, 1969) were used to obtain the coefficients in Table 3. Parameters for the critical enhancement are presented in Table 4 . There were no data above atmospheric pressure. Figure 215 shows the percentage deviations between the model and the experimental thermal conductivity. The estimated uncertainty for the thermal conductivity of the liquid phase at atmospheric pressure is $2 \%$ over the temperature range $298 \mathrm{~K}$ to $452 \mathrm{~K}$, higher outside of that range. The estimated uncertainty in the gas phase is $20 \%$. Surface tension data from three sources (Azizian \& Bashavard, 2005; Azizian \& Hemmati, 2003; Rafati, Bagheri, \& Najafi, 2011) were used to obtain the surface tension coefficients in Table 5, and Fig. 216 shows deviations with the data. The estimated uncertainty for surface tension over the temperature range from $283 \mathrm{~K}$ to $323 \mathrm{~K}$ is $1 \%$.

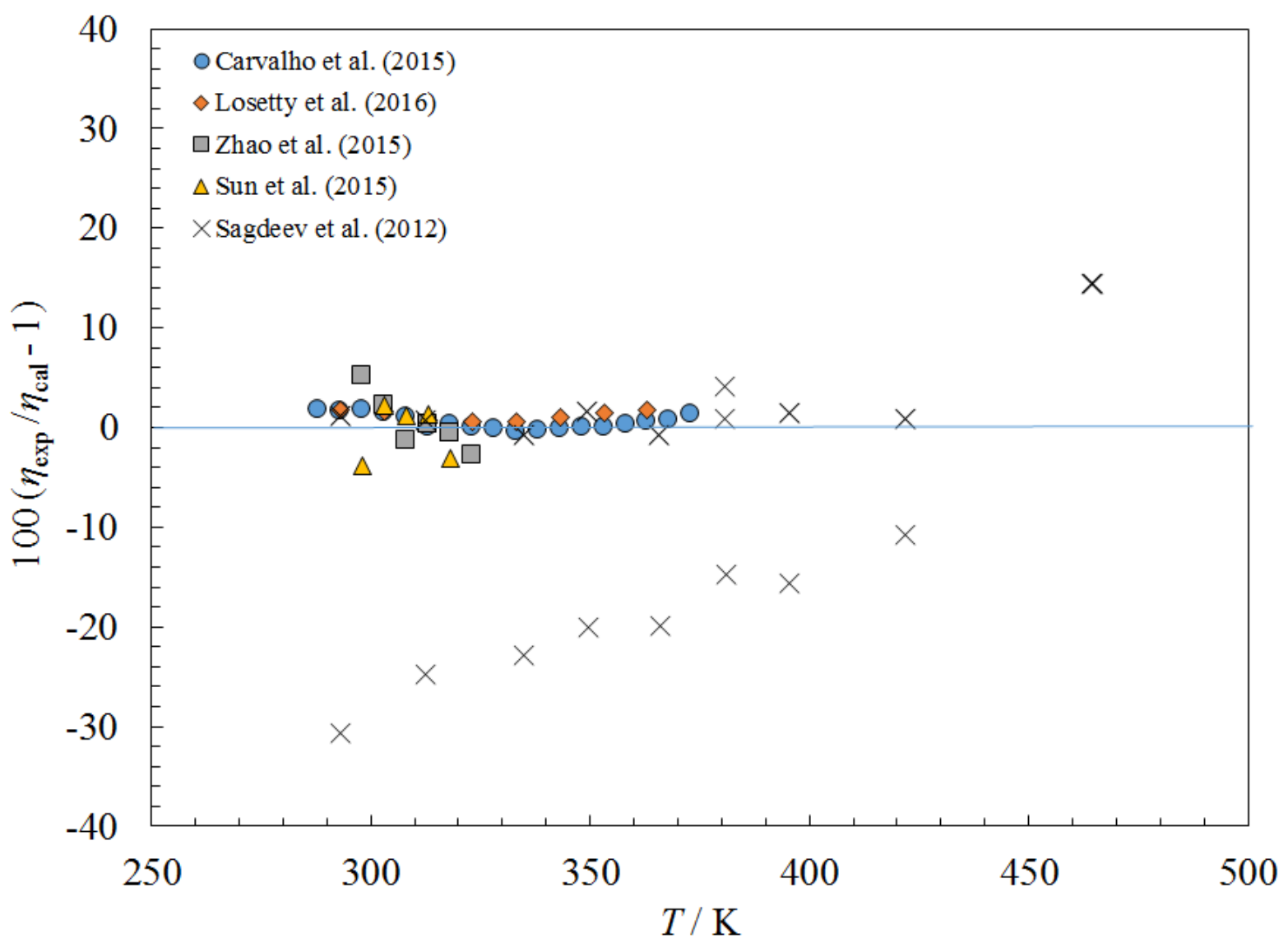

Figure 213. Percentage deviations between the model and the experimental viscosity data for ethylene glycol as a function of temperature. 


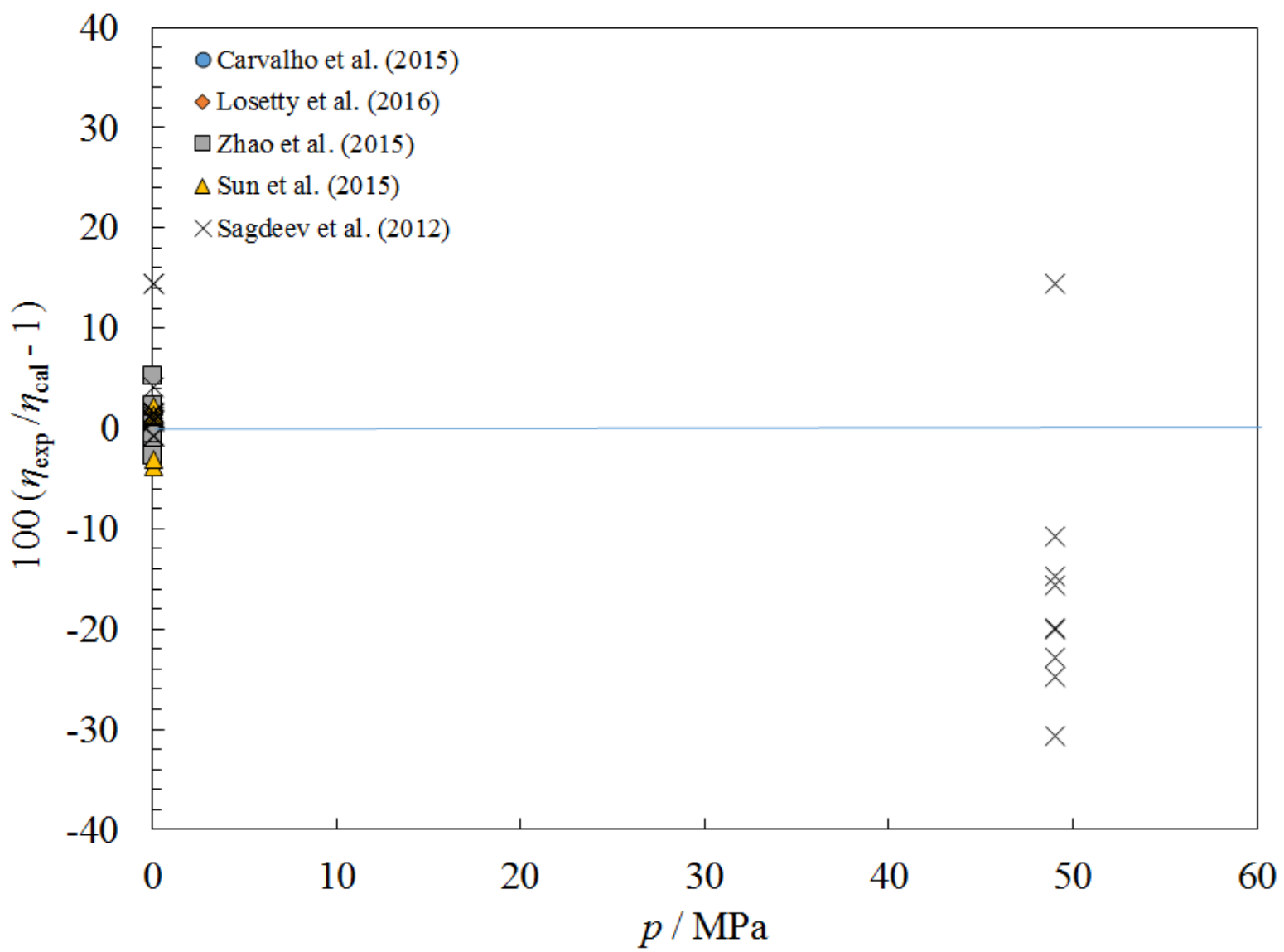

Figure 214. Percentage deviations between the model and the experimental viscosity data for ethylene glycol as a function of pressure. 


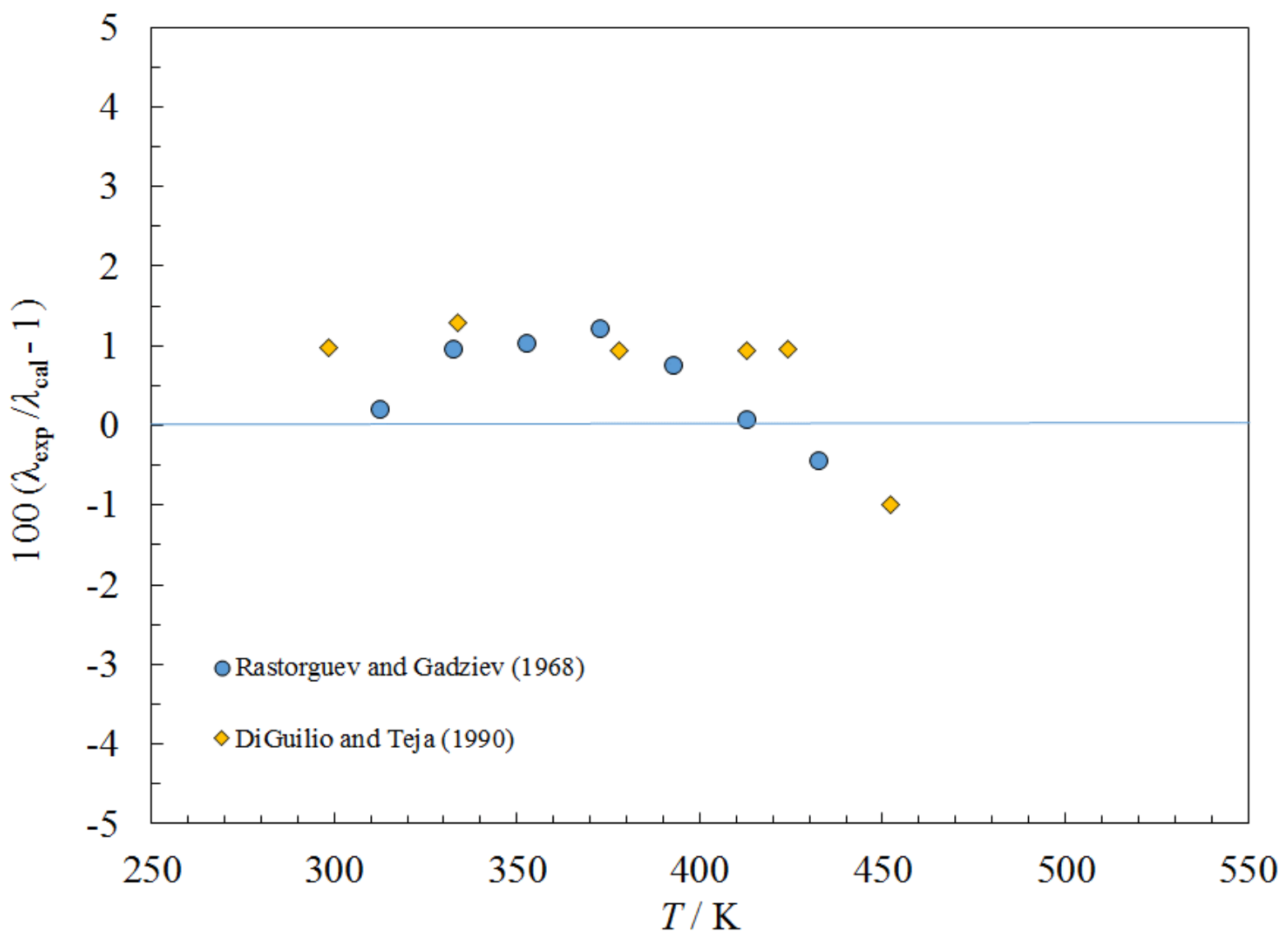

Figure 215. Percentage deviations between the model and the experimental thermal conductivity data for ethylene glycol as a function of temperature. 


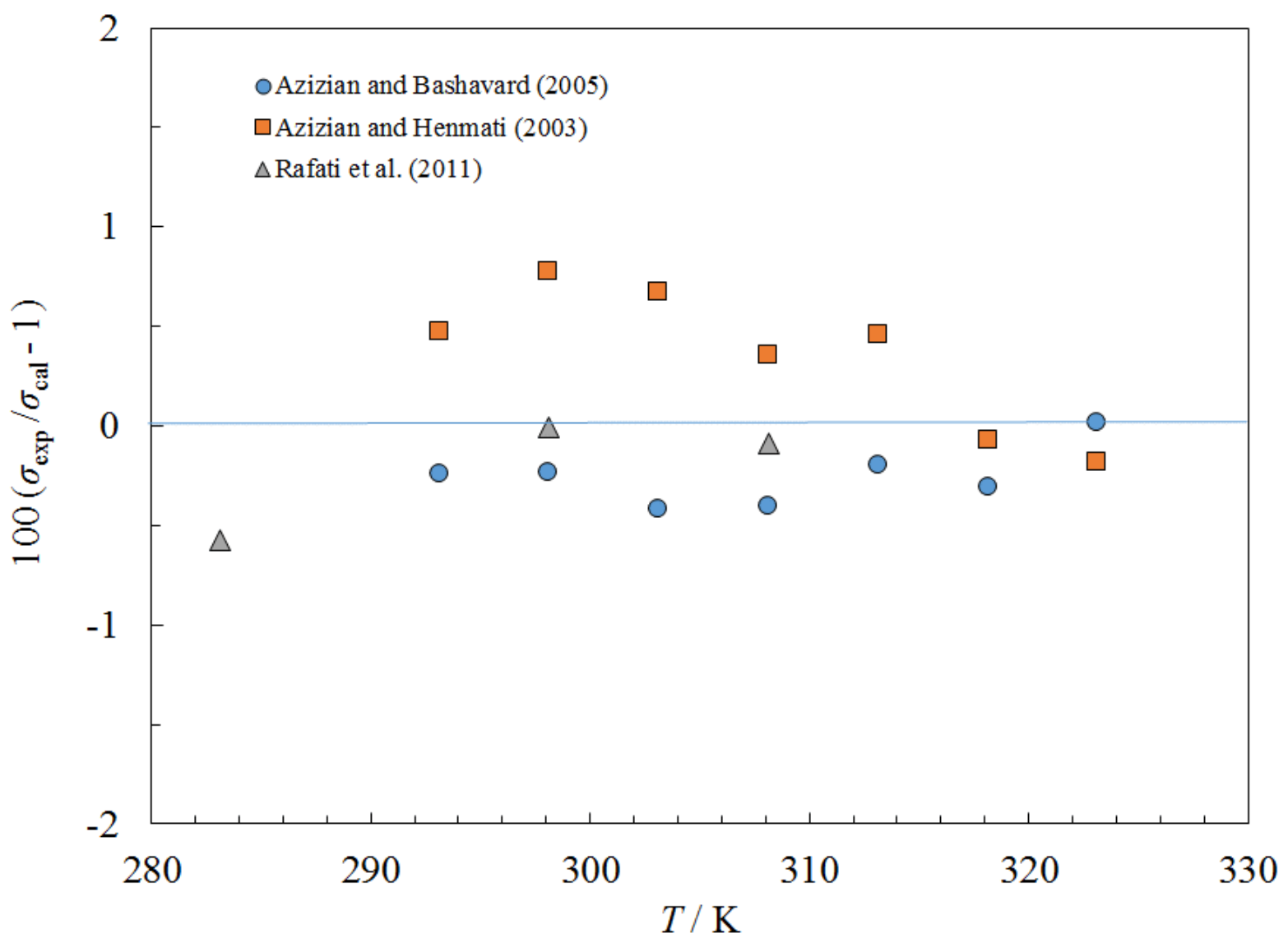

Figure 216. Percentage deviations between the model and the experimental surface tension data for ethylene glycol as a function of temperature. 


\section{Conclusions}

We describe the data used, and the coefficients obtained for models for the viscosity, thermal conductivity, and surface tension for selected fluids implemented in version 10.0 of the NIST computer program REFPROP. This document serves as documentation on the models developed. In addition, these models should be considered preliminary, intended to serve as an interim measure to allow calculations of viscosity, thermal conductivity, and surface tension until better models become available. Comparisons with available experimental data are also given.

\section{Acknowledgements}

M. Huber acknowledges the amazing detective work done by the staff of the Department of Commerce Boulder Laboratories Library in locating obscure journal articles used in the course of this work. She also acknowledges Dr. Ian Bell for extremely careful checking of the coefficients and for checking the sample calculations in excruciating detail. 


\section{References}

Abbas, R., Ihmels, E. C., Enders, S., \& Gmehling, J. (2011). Measurement of Transport Properties for Selected Siloxanes and their Mixtures Used as Working Fluids for Organic Rankine Cycles. Ind. Eng. Chem. Res., 50, 8756-8763.

Abdullaev, F. G., Akhundov, T. S., \& Ishkhanov, Y. B. (1983). Experimental study of the dynamic viscosity coefficients of benzene and chlorbenzene at high temperatures. Izv. Vyssh. Uchebn. Zaved., Neft Gaz, 53-59.

Abdullaev, F. G., \& Djafarova, N. I. (1980). Experimental study of the dynamic viscosity coefficient of chlorbenzene. Izv. Vyssh. Uchebn. Zaved., Neft Gaz, 23, 89-90.

Abdullaev, F. G., \& Iskenderov, A. I. (1980). Experimental study of the thermal conductivity of chlorobenzene in the wide temperature and pressure ranges. Izv. Vyssh. Uchebn. Zaved., Neft Gaz, 23, 53-55.

Adzumi, H. (1937). Studies on the Flow of Gaseous Mixtures Through Capillaries I. The Viscosity of Binary Gaseous Mixtures. Bull. Chem. Soc. Jpn., 12, 199-226.

Aguila-Hernandez, J., Trejo, A., Garcia-Flores, B. E., \& Molnar, R. (2008). Viscometric and volumetric behaviour of binary mixtures of sulfolane and $\mathrm{N}$-methylpyrrolidone with monoethanolamine and diethanolamine in the range 303-373 K. Fluid Phase Equilib., 267, 172-180.

Akasaka, R. (2017). [Helmholtz equation of state for R1243zf, unpublished, implemented in REFPROP v10 (second preliminary equation for R1243zf)].

Akasaka, R., Fukushima, M., \& Lemmon, E. W. (2016). A Helmholtz energy equation of state for trifluoroethylene (R-1123). Paper presented at the International Refrigeration and Air Conditioning Conference at Purdue, July 11-14, 2016.

Akasaka, R., Fukushima, M., \& Lemmon, E. W. (2017). A Helmholtz Energy Equation of State for Cis-1-chloro-2,3,3,3-tetrafluoropropene (R-1224yd(Z)). Paper presented at the European Conference on Thermophysical Properties, Graz, Austria.

Akasaka, R., \& Kayukawa, Y. (2012). A Fundamental Equation of State for Trifluoromethyl Methyl Ether (HFE-143m) and Its Application to Refrigeration Cycle Analysis. Int. J. Refrig., 35(4), 1003-1013.

Akasaka, R., \& Lemmon, E. W. (2018). Fundamental Equations of State for cis-1,3,3,3Tetrafluoropropene (R-1234ze(Z)) and 3,3,3-Trifluoropropene (R-1243zf). J. Chem. Eng. Data, submitted.

Alam, M. J., Islam, M. A., Kariya, K., \& Miyara, A. (2017). Measurement of thermal conductivity of cis-1,1,1,4,4,4-hexafluoro-2-butene $(\mathrm{R}-1336 \mathrm{mzz}(\mathrm{Z}))$ by the transient hotwire method. Int. J. Refrig., 84, 220-227.

Arnemann, M., \& Kruse, H. (1991). Liquid viscosities of the non-azeotropic binary refrigerant mixtures R22/Rlg14, R22/R152A, R22/R142B. Measurements and calculations. Paper presented at the Actes Congr. Int. Froid, 18th

Assael, M. J., Bogdanou, I., Mylona, S. K., Huber, M. L., Perkins, R. A., \& Vesovic, V. (2013). Reference Correlation of the Thermal Conductivity of n-Heptane from the Triple Point to $600 \mathrm{~K}$ and up to $250 \mathrm{MPa}$. J. Phys. Chem. Ref. Data, 42(2), 023101. doi:10.1063/1.4794091

Assael, M. J., \& Dalaouti, N. K. (2000). Measurement of the viscosity of cyclopentane from 210 to $310 \mathrm{~K}$ and pressures up to $25 \mathrm{MPa}$. High Temp. - High Pressures, 32, 179-184. 
Assael, M. J., Kalyva, A. E., Monogenidou, S. A., Huber, M. L., Perkins, R. A., Friend, D. G., \& May, E. F. (2018). Reference values and reference correlations for the thermal conductivity and viscosity of fluids. J. Phys. Chem. Ref. Data, 47, 021501.

Assael, M. J., Koutian, A., Huber, M. L., \& Perkins, R. A. (2016). Reference Correlations of the Thermal Conductivity of Ethylene and Propylene. J. Phys. Chem. Ref. Data, 45(3), 033104.

Assael, M. J., Mylona, S. K., Huber, M. L., \& Perkins, R. A. (2012). Reference Correlation of the Thermal Conductivity of Toluene from the Triple Point to $1000 \mathrm{~K}$ and up to 1000 MPa. J. Phys. Chem. Ref. Data, 41(2), 023101.

Atoyan, V. A., \& Mamedov, I. A. (1976). Complex study of acoustic and viscous properties of dimethyl ketone in a wide range of temperatures and pressures. Zh. Fiz. Khim., 50, 29762978.

Audonnet, F., \& Padua, A. A. H. (2001). Simultaneous measurement of density and viscosity of n-pentane from 298 to $383 \mathrm{~K}$ and up to $100 \mathrm{MPa}$ using a vibrating-wire instrument. Fluid Phase Equilib., 181, 147-161.

Avelino, H. M. T., Fareleira, J. M. N. A., \& Oliveira, C. M. B. P. (2006). Viscosity of Compressed Liquid 1,1,1-Trifluoroethane (HFC-143a) and Pentafluoroethane (HFC125). J. Chem. Eng. Data, 51, 1672-1677.

Awbery, J. H., \& Griffiths, E. (1936). The Viscosities of Some Liquid Refrigerants. Proc. Phys. Soc., 48, 372-380.

Azizian, S., \& Bashavard, N. (2005). Equilibrium Surface Tensions of Benzyl Alcohol + Ethylene Glycol Mixtures. J. Chem. Eng. Data, 50, 709-712.

Azizian, S., \& Hemmati, M. (2003). Surface Tension of Binary Mixtures of Ethanol + Ethylene glycol from 20 to $50{ }^{\circ}$ C. J. Chem. Eng. Data, 48, 662-663.

Bachmann, R. (1969). Liquid Thermal Conductivities of Derivatives of Benzene Measured By An Unsteady-State Hot Wire Method. Waerme- Stoffuebertrag, 2(3), 129-134.

Bagdasaryan, S. S. (1964). Theory of nuclei of a new phase and the limits of the liquid state. $Z h$. Fiz. Khim., 38, 1816-1820.

Baker, C. B., \& de Haas, N. (1964). Gas Thermal Conductivity Studies at High Temperature. III. Results for SO2. Phys. Fluids, 7, 1400-1402.

Bakulin, S. S., Ulybin, S. A., \& Zherdev, E. N. (1975). Experimental study of the thermal conductivity of xenon at temperatures from 400 to 1400 K. Teplofiz. Vys. Temp., 13, 760763.

Barua, A. K., Manna, A., \& Mukhopadhyay, P. (1968). Thermal Conductivity and Rotational Relaxation in Some Polar Gases. J. Chem. Phys., 49, 2422-2425. doi:https://doi.org/10.1063/1.1670416

Baryshev, V. P., Artamonov, S. D., \& Geller, V. Z. (1980). Thermal conductivity of freon-218. Inzh.-Fiz. Zh., 38, 244.

Bates, O. K. (1949). Thermal Conductivity of Liquid Silicones. Ind. Eng. Chem., 41, 1966-1968.

Batschinski, A. J. (1913). Investigations of the internal friction of fluids. Z. Phys. Chem., 84, 643-706.

Baylaucq, A., Boned, C., Dauge, P., \& Lagourette, B. (1997). Measurements of the Viscosity and Density of Three Hydrocarbons and the Three Associated Binary Mixtures Versus

Pressure and Temperature. Int. J. Thermophys., 18, 1089-1107.

Beckmueller, R., Thol, M., Lemmon, E. W., \& Span, R. (2018). Fundamental Equation of State for n-Octane. for submission to Int. J. Thermophys. 
Bell, I. H., Wronski, J., Quoilin, S., \& Lemort, V. (2014). Pure and Pseudo-pure Fluid Thermophysical Property Evaluation and the Open-Source Thermophysical Property Library CoolProp. Ind. Eng. Chem. Res., 53, 2498-2508. doi:dx.doi.org/10.1021/ie4033999

Benning, A. F., \& Markwood, W. H. (1939). The Viscosities of "Freon" Refrigerants. ASHRAE J., 37, 243-247.

Bewilogua, L., \& Yamashira, T. (1972). Thermal Conductivity of Liquid-Neon Isotopes. J. Low Temp. Phys., 8, 255-260.

Bhattacharyya, P. K. (1970). Viscosity of Binary Polar-Gas Mixtures: $\mathrm{CH}_{3} \mathrm{Cl}-\mathrm{H}_{2} \mathrm{~S}$ and $\mathrm{CH}_{3} \mathrm{Cl}$ $\mathrm{SO}_{2}$. J. Chem. Phys., 53, 893-895.

Bi, S., Wu, J., Liu, Z., \& Meng, X. (2003). Experimental Investigation of the Viscosity of Saturated Liquid Dimethyl Carbonate. Xi'an Jiaotong Daxue Xuebao, 37(9), 903-905.

Blackham, T. M., Lemmon, A. K., \& Lemmon, E. W. (2018). Helmholtz equation of state for isooctane. for submission to Int. J. Thermophysics.

Blanco, A., Garcia-Abuin, A., Gomez-Diaz, D., Navaza, J., \& Villaverde, O. (2013). Density, Speed of Sound, Viscosity, Surface Tension, and Excess Volume of N-Ethyl-2pyrrolidone + Ethanolamine (or Diethanolamine or Triethanolamine) from $\mathrm{T}=(293.15$ to 323.15) K. J. Chem. Eng. Data, 58, 653-659.

Boon, J. P., Legros, J. C., \& Thomaes, G. (1967). On the principle of corresponding states for the viscosity of simple liquids. Physica, 33, 547-557.

Borovik, E., Matveev, A., \& Panina, E. J. (1940). J. Tech. Phys. (USSR), 10, 988-998.

Brazier, D. W., \& Freeman, G. R. (1969). The effect of pressure on the density, dielectric constant, and viscosity of several hydrocarbons and other organic liquids. Can. J. Chem., 47, 893-899.

Briggs, S. W., \& Comings, E. W. (1943). Effect of Temperature on Liquid -Liquid Equilibrium. Benzene-Acetone-Water System and Docosane-Diphenylhexane-Furfural System. Ind. Eng. Chem., 35, 411-417.

Burger, L. L., \& Cady, G. H. (1951). Physical properties of perfluoropentanes. J. Am. Chem. Soc., 73, 4243-4246.

Carvalho, P. J., Fonseca, C. H. G., Moita, M.-L. C. J., Santos, A. F. S., \& Coutinho, J. A. P. (2015). Thermophysical properties of glycols and glymes. J. Chem. Eng. Data, 60, 37213737.

Ceriani, R., Goncalves, C. B., Rabelo, J., Caruso, M., Cunha, A. C. C., Cavaleri, F. W., .. . Meirelles, A. J. A. (2007). Group Contribution Model for Predicting Viscosity of Fatty Compounds. J. Chem. Eng. Data, 52, 965-972.

Chae, H. B., Schmidt, J. W., \& Moldover, M. R. (1990). Alternative Refrigerants R123a, R134, R141b, R142b, and R152a: Critical Temperature, Refractive Index, Surface Tension, and Estimates of Liquid, Vapor, and Critical Densities. J. Phys. Chem., 94, 8840-8845.

Chaikin, A. M., \& Markevich, A. M. (1958). Zh. Fiz. Khim., 32, 116-120.

Chavanne, G. \& Van Risseghem, H., (1922). Bull. Soc. Chim. Belg., 31, 87-94.

Chen, F., Yang, Z., Chen, Z., Hu, J., Chen, C., \& Cai, J. (2015). Density, viscosity, speed of sound, excess property and bulk modulus of binary mixtures of a-butyrolactone with acetonitrile, dimethyl carbonate, and tetrahydrofuran at temperatures (293.15 to 333.15) K. J. Mol. Liq., 209, 683-692. 
Chichester, J. C., \& Huber, M. L. (2008). Documentation and Assessment of the Transport Property Models for Mixtures Implememented in NIST REFPROP (Version 8.0). NISTIR 6650

Choy, P., \& Raw, C. J. G. (1966). Thermal Conductivity of Some Polyatomic Gases at Moderately High Temperatures. J. Chem. Phys., 45, 1413-1417.

Chung, T. H., Ajlan, L., Lee, L. L., \& Starling, K. E. (1988). Generalized multiparameter correlation for nonpolar and polar fluid transport properties. Ind. Eng. Chem. Res., 27, 671-679.

Clifford, A. A., Gray, P., \& Scott, A. C. (1981). Viscosities of Gaseous Nitric Oxide, Nitrous Oxide and Sulphur Dioxide at High Temperatures. J. Chem. Soc. Faraday Trans. 1, 77, 997-1001.

Cochran, M. A., North, A. M., \& Pethrick, R. A. (1974). Ultrasonic Studies of Perfluoro-nAlkanes. J. Chem. Soc. Faraday Trans. II, 70, 1274-1279.

Colonna, P., Nannan, N. R., \& Guardone, A. (2008). Multiparameter Equations of State for Siloxanes Fluid Phase Equilib., 263, 115-130.

Comunas, M. J. P., Baylaucq, A., Boned, C., \& Fernandez, J. (2001). High-Pressure Measurements of the Viscosity and Density of Two Polyethers and Two Dialkyl Carbonates. Int. J. Thermophys., 22, 749-769.

Correia, P., Schramm, B., \& Schäfer, K. (1968). Die Wärmeleitfähigkeit von Gasen in einem größeren Temperaturbereich. Ber. Bunsenges. Phys. Chem., 72, 393-399.

Craven, P. M., \& Lambert, J. D. (1951). The Viscosities of Organic Vapors. Proc. R. Soc. (London), 205, 439-449.

de Reuck, K. M. (1990). International Thermodynamic Tables of the Fluid State-11 Fluorine. Oxford, UK: International Union of Pure and Applied Chemistry, Pergamon Press.

DeLorenzi, L., Fermeglia, M., \& Torriano, G. (1994). Densities and Viscosities of 1,1,1Trichloroethane + Paraffins and + Cycloparaffins at 298.15 K. J. Chem. Eng. Data, 39, 483-487.

Diaz Pena, M., Cabello, A., \& Cheda, J. A. R. (1975). Viscosidad del n-Octano e isomeros en fase de vapor. An. Quim., 71, 637.

DiGuilio, R., \& Teja, A. S. (1990). Thermal Conductivity of Poly(ethylene glycols) and Their Binary Mixtures. J. Chem. Eng. Data, 35, 117-121.

DiGuilio, R. M., Lee, R. J., Schaeffer, S. T., Brasher, L. L., \& Teja, A. S. (1992). Densities and viscosities of the ethanolamines. J. Chem. Eng. Data, 37, 239-242.

DiGuilio, R. M., McGregor, W. L., \& Teja, A. S. (1992). Thermal conductivities of the ethanolamines. J. Chem. Eng. Data, 37, 242-245.

Diky, V., Chirico, R. D., Frenkel, M., Bazyleva, A., Magee, J. W., Paulechka, E., ... Kroenlein, K. (2016). Thermo Data Engine (TDE) Version 10.1, NIST Standard Reference Database 103b. Gaithersburg, MD: NIST.

Diky, V., Chirico, R. D., Frenkel, M., Bazyleva, A., Magee, J. W., Paulechka, E., ... Kroenlein, K. (2017). ThermoDataEngine (TDE), version 10.2. NIST Standard Reference Database 103b . Gaithersburg, MD: NIST.

DIPPR , Brigham Young University. (2016). The DIPPR Information and Data Evalutation Mangager for the Design Institute for Physical Properties, V8.1.0.

Duan, Y.-Y., Shi, L., Han, L.-Z., \& Zhu, M.-S. (1999). Viscosity of saturated liquid trifluoroiodomethane from 253 to 338 K. Fluid Phase Equilib., 162, 303-312. 
Duan, Y.-Y., Sun, L.-Q., Shi, L., Zhu, M.-S., \& Han, L.-Z. (1997). Thermal Conductivity of Gaseous Trifluoroiodomethane (CF3I). J. Chem. Eng. Data, 42, 890-893.

Dunlop, P. J. (1994). Viscosities of a Series of Gaseous Fluorocarbons at 25 C. J. Chem. Phys., 100, 3149-3151.

Dymond, J. H., Glen, N. F., \& Isdale, J. D. (1985). Transport Properties of Nonelectrolyte Liquid Mixtures--VII. Viscosity Coeff. for Isooctane and for Equimolar Mixtures of Isooctane + n-Octane and Isooctane + n-Dodecane from 25 to $100 \mathrm{deg}$. C at Pressures up to $500 \mathrm{MPa}$ or to the freezing pressure. Int. J. Thermophys., 6, 233-250.

Elverum, G., \& Doescher, R. N. (1952). Physical Properties of Liquid Fluorine. J. Chem. Phys., 20, 1834-1836.

Ely, J. F., \& Hanley, H. J. M. (1981). Prediction of transport properties. 1. Viscosity of fluids and mixtures. Ind. Eng. Chem. Fundam., 20(4), 323-332.

Ely, J. F., \& Hanley, H. J. M. (1983). Prediction of transport properties. 2. Thermal conductivity of pure fluids and mixtures. Ind. Eng. Chem. Fundam., 22(1), 90-97.

Ely, J. F., \& Magee, J. W. (1989). Experimental Measurement and Prediction of Thermophysical Property Data of Carbon Dioxide Rich Mixtures. Paper presented at the Proceedings of the 68th GPA Annual Convention, San Antonio, TX (March 13-14, 1989).

Ermakov, G. V., \& Skripov, V. P. (1969). Thermodynamic Properties of Fluorocarbons. Zh. Fiz. Khim., 43, 1308-1309.

Estrada-Baltazar, A., Iglesias-Silva, G.A., \& Barrufet, M. A. (1998). Liquid Viscosities of Pentane and Pentane + Decane from 298.15 K to $373.15 \mathrm{~K}$ and up to $25 \mathrm{MPa}$. J. Chem. Eng. Data, 43, 601-604.

Et-Tahir, A., Boned, C., Lagourette, B., \& Xans, P. (1995). Determination of the viscosity of various hydrocarbons and mixtures of hydrocarbons versus temperature and pressure. Int. J. Thermophys., 16, 1309-1334.

Evans, E. B. (1938). The viscosity of hydrocarbons. Parts VII and VIII. J. Inst. Pet., 24, 537.

Evers, C., Loesch, H. W., \& Wagner, W. (2002). An absolute viscometer-densimeter and measurements of the viscosity of nitrogen, methane, helium, neon, argon, and krypton over a wide range of density and temperature. Int. J. Thermophys., 23, 1411-1439.

Fellows, B. R., Richard, R. G., \& Shankland, I. R. (1990). Thermal conductivity data for some environmentally acceptable fluorocarbons. Int. J. Thermophys., 21, 311-325.

Filipov, L. P. \& Laushkina, L. A. (1984). Study of Thernmal Conductance and Heat Capacity of Liquids I. Ismers of Alkanes. Zh. Fiz. Khim., 58, 1068-1071.

Finney, R. J., Fury, M., \& Jonas, J. (1977). Density and temperature dependence of self-diffusion and shear viscosity of perfluorocyclobutane in the dense fluid region. J. Chem. Phys., 66, 760-765.

Förster, S. (1963). Viscosity Measurements in Liquid Neon, Argon, and Nitrogen. Cryogenics, 3, 176-177.

Freire, M. G., Carvalho, P. J., Queimada, A. J., Marrucho, I. M., \& Coutinho, J. A. P. (2006). Surface Tension of Liquid Fluorocompounds. J. Chem. Eng. Data, 51, 1820-1824.

Freire, M. G., Ferreira, A. G. M., Fonseca, I. M. A., Marrucho, I. M., \& Coutinho, J. A. P. (2008). Viscosities of Liquid Fluorocompounds. J. Chem. Eng. Data, 53, 538-542.

Fröba, A. P., Krzeminski, K., \& Leipertz, A. (2004). Thermophysical Properties of 1,1,1,3,3Pentafluorobutane (R365mfc). Int. J. Thermophys., 25, 987-1004.

Fu, D., \& Zhong, Z.-K. (2010). Experimental Study on the Surface Tension of DiethanolamineN-Methyldiethanolamine-Water Mixtures. Acta Chim. Sinica, 68, 1241-1246. 
Galkov, G. I., \& Gerf, S. F. (1941). The Viscosity Coefficient of Propene Along the Saturated Line. Zh. Tekh. Fiz., 11, 613-615.

Gao, K., Wu, J., \& Lemmon, E. W. (2017a). [Helmholtz equation of state for perfluoropentane, unpublished, implemented in REFPROP v10].

Gao, K., Wu, J., \& Lemmon, E. W. (2017b). [Helmholtz equation of state for 1-butyne, unpublished, implemented in REFPROP v10].

Gao, K., Wu, J., \& Lemmon, E. W. (2017c). [Helmholtz equation of state for 1-pentene, unpublished, implemented in REFPROP v10].

Gao, K., Wu, J., \& Lemmon, E. W. (2017d). [Helmholtz equation of state for 1,3-butadiene, unpublished, implemented in REFPROP v10].

Gao, K., Wu, J., \& Lemmon, E. W. (2017e). [Helmholtz equation of state for 2,2dimethylbutane, unpublished, implemented in REFPROP v10].

Gao, K., Wu, J., \& Lemmon, E. W. (2017f). [Helmholtz equation of state for 2,3dimethylbutane, unpublished, implemented in REFPROP v10].

Gao, K., Wu, J., \& Lemmon, E. W. (2017g). [Helmholtz equation of state for 3-methylpentane, unpublished, implemented in REFPROP v10].

Gao, K., Wu, J., \& Lemmon, E. W. (2017h). [Helmholtz equation of state for acetylene, unpublished, implemented in REFPROP v10].

Gao, K., Wu, J., \& Lemmon, E. W. (2017i). [Helmholtz equation of state for cyclobutene, unpublished, implemented in REFPROP v10].

Gao, K., Wu, J., \& Lemmon, E. W. (2017j). [Helmholtz equation of state for perfluorobutane, unpublished, implemented in REFPROP v10].

Gao, K., Wu, J., \& Lemmon, E. W. (2017k). Helmholtz equation of state for perfluorohexane, unpublished, implemented in REFPROP v10.

Gao, K., Wu, J., \& Lemmon, E. W. (20171). [Helmholtz equation of state for propadiene, unpublished, implemented in REFPROP v10].

Gao, K., Wu, J., \& Lemmon, E. W. (2017m). [Helmholtz equation of state for propylene oxide, unpublished, implemented in REFPROP v10].

Gao, K., Wu, J., Zhang, P., \& Lemmon, E. W. (2016). A Helmholtz Energy Equation of State for Sulfur Dioxide. J. Chem. Eng. Data, 61, 2859-2872.

Gao, W., Zhao, X., \& Liu, Z. (2009). Surface Tension of 2,2-Dimethylbutane from (233 to 378) K. J. Chem. Eng. Data, 54, 1761-1763.

Gedanitz, H., Dávila, M. J., \& Lemmon, E. W. (2015). Speed of Sound Measurements and a Fundamental Equation of State for Cyclopentane. J. Chem. Eng. Data, 60(5), 1311-1337.

Geist, J. M., \& Cannon, M. R. (1946). Viscosities of Pure Hydrocarbons. Ind. Eng. Chem. Anal. Ed, 18, 611-613.

Geller, V. Z. (1980). Investigation of the Viscosity of Freons of the Methane, Ethane, and Propane Types. Summary of Experimental Data. Teplofiz. Svoistva Veshchestv. Mater., 15, 89-114.

Geller, V. Z., Karabanov, E. M., Gunchuk, B. V., Zakharzhevskiy, V. Y., \& Lapardin, N. I. (1976). The Investigation of the Viscosity Coefficient of Some of the Liquid Gases About the Saturated Line. Gaz. Prom., 3, 32.

Gerf, S. F., \& Galkov, G. I. (1940). Viscosity of Liquified Pure Gases and Their Mixtures. Zh. Tekh. Fiz., 10, 725-732.

Giller, E. B., \& Drickamer, H. G. (1949). Viscosity of Normal Paraffins near the Freezing Point. Ind. Eng. Chem., 41, 2067-2069. 
Golubev, I. F. (1959). Viscosity of Gases and Gas Mixtures. Moscow: Fizmat Press.

Gonzalez, M. H., \& Lee, A. L. (1968). Viscosity of 2,2-Dimethylpropane. J. Chem. Eng. Data, 13(1), 66-69. doi:10.1021/je60036a021

Grebenkov, A. J., Kotelevsky, J. G., \& Saplitza, V. V. (1993). Transport properties of ozone-safe refrigerants. I. Thermal conductivity of R134a, R152a, and R218 in the liquid and vapour phases. Thermophysical processes in Refrigeration systems and properties of working Media, SPTIHP Publisher, St. Petersburg

Griffiths, E., Awberry, J. H., \& Powell, R. W. (1939). Report of the Food Investigation Board for the years 1936-1938, Department of Scientific and Industrial Research, 1939.

Guseinov, K. D., \& Mirzoev, B. M. (1975). Experimental study of the thermal conductivity of gaseous benzene homologous. Izv. Vyssh. Uchebn. Zaved., Neft Gaz, 18, 27-28.

Haepp, H. J. (1976). Messung der Viscositat von Kohlendioxid und Propylen. WarmeStoffubertrag, 9, 281-290.

Haghtalab, A., \& Shojaeian, A. (2014). Volumetric and viscometric behaviour of the binary systems of N-methyldiethanolamine and diethanolamine with 1-butyl-3methylimidazolium acetate at various temperatures. J. Chem. Thermodyn., 68, 128-137.

Hammerschmidt, U. (1995). Thermal conductivity of a wide range of alternative refrigerants measured with an improved guarded hot-plate apparatus. Int. J. Thermophys., 16, 12031211.

Han, J., Jin, J., Eimer, D. A., \& Melaaen, M. C. (2012). Density of Water (1) + Monoethanolamine (2) $+\mathrm{CO}_{2}$ (3) from (298.15 to 413.15) K and Surface Tension of Water (1) + Monoethanolamine (2) from (303.15 to 333.15) K. J. Chem. Eng. Data, 57, 1095-1103.

Hanley, H. J. M., \& Prydz, R. (1972). The viscosity and thermal conductivity coefficients of gaseous and liquid fluorine. J. Phys. Chem. Ref. Data, 1(4), 1101-1113.

Hansen, K. C., Tsao, L.-H., Aminabhavi, T. M., \& Yaws, C. L. (1995). Gaseous Thermal Conductivity of Hydrogen Chloride, Hydrogen Bromide, Boron Trichloride, and Boron Trifluoride in the Temperature Range from 55 to $380{ }^{\circ}$ C. J. Chem. Eng. Data, 40, 18-20.

Harris, E. J. R., Hope, C. G., Gough, D. W., \& Smith, E. B. (1979). Viscosity of Gaseous Carbon Dioxide, Sulfur Hexafluoride, and Nitrous Oxide at Low Temperatures. J. Chem. Soc., Faraday Trans. 1, 75, 892-897.

Harris, K. R., Newitt, P. J., \& Woolf, L. A. (2004). Temperature and Density Dependence of the Viscosity of Cyclopentane. J. Chem. Eng. Data, 49, 138-142.

Hartl, R., Neuder, R., \& Gores, H. J. (2009). Temperature Dependence of Association Constants of LiAlCl4 in Liquid Sulfur Dioxide. Acta Chim. Slov., 56, 1009-1114.

Haynes, W. M. (1994). Thermophysical Properties of HFC-143a and HFC-152a. Final report, ARTI MCLR Project Number 650-50800

Heinemann, T., Klaen, W., Yourd, R., \& Dohrn, R. (2000). Experimental Determination of the Vapor Phase Thermal Conductivity of Blowing Agents for Polyurethane Rigid Foam. $J$. Cell. Plast., 36, 45-56.

Hellmann, R. (2011). [personal communication from R. Hellmann (Univer. Rostock, Germany) regarding $\mathrm{CO}$ to $\mathrm{M}$. Huber ].

Hellmann, R., Bich, E., Vogel, E., \& Vesovic, V. (2012). Thermophysical Properties of Dilute Hydrogen Sulfide Gas. J. Chem. Eng. Data, 57, 1312-1317. 
Herrig, S., Thol, M., Kortmann, M., Lemmon, E. W., \& Span, R. (2018). [Helmholtz equation of state for diethanolamine, unpublished, implemented in REFPROP v10 (personal communication to E.W. Lemmon, NIST, Boulder)].

Herrig, S., Thol, M., \& Span, R. (2018a). Helmholtz equation of state for chlorine. for submission to J. Phys. Chem.

Herrig, S., Thol, M., \& Span, R. (2018b). [Helmholtz equation of state for monoethanolamine, unpublished, implemented in REFPROP v10 (personal communication to E.W. Lemmon, NIST, Boulder)].

Hirschfelder, J. O., Curtiss, C. F., \& Bird, R. B. (1964). Molecular theory of gases and liquids (Corr. print. with notes attached ed.). New York: Wiley.

Ho, C. Y., Powell, R. W., \& Liley, P. E. (1972). Thermal Conductivity of the Elements. J. Phys. Chem. Ref. Data, 1(2), 279-421.

Horvath, A. L. (1975). Physical Properties of Inorganic Compounds. New York: Crane Russak.

Hu, X., Meng, X., Wei, K., Li, W., \& Wu, J. (2015). Compressed Liquid Viscosity Measurements of HFE-7000, HFE-7100, HFE-7200, and HFE-7500 at Temperatures from (253 to 373) $\mathrm{K}$ and Pressures up to $30 \mathrm{MPa}$. J. Chem. Eng. Data, 60, 3562-3570.

Huber, M. L., \& Ely, J. F. (1992a). Prediction of the Thermal Conductivity of Refrigerants and Refrigerant Mixtures. Fluid Phase Equilib., 80, 249-261.

Huber, M. L., \& Ely, J. F. (1992b). Prediction of the Viscosity of Refrigerants and Refrigerant Mixtures. Fluid Phase Equilib., 80, 239-248.

Huber, M. L., Laesecke, A., \& Perkins, R. A. (2003). Model for the viscosity and thermal conductivity of refrigerants, including a new correlation for the viscosity of R134a. Ind. Eng. Chem. Res., 42(13), 3163-3178. doi:10.1021/ie0300880

Huber, M. L., Laesecke, A., \& Perkins, R. A. (2004). Transport properties of n-dodecane. Energy \& Fuels, 18, 968-975.

Huber, M. L., Laesecke, A., \& Xiang, H. W. (2004). Viscosity correlations for minor constituent fluids in natural gas: n-octane, n-nonane and n-decane. Fluid Phase Equilib., 224, 263 270.

Huber, M. L., Lemmon, E. W., Kazakov, A. F., Ott, L. S., \& Bruno, T. J. (2009). Model for the Thermodynamic Properties of a Biodiesel Fuel. Energy \& Fuels, 23, 3790-3797.

Huber, M. L., \& Perkins, R. A. (2005). Thermal conductivity correlations for minor constituent fluids in natural gas: n-octane, n-nonane and n-decane. Fluid Phase Equilib., 227, 47-55.

Hurd, C. B. (1946). Studies on Siloxanes. I. The Specific Volume and Viscosity in Relation to Temperature and Constitution. J. Am. Chem. Soc., 68, 364-370.

Hurly, J. J. (2004). Viscosity and Speed of Sound of Gaseous Nitrous Oxide and Nitrogen Trifluoride Measured with a Greenspan Viscometer. Int. J. Thermophys., 25, 625-641.

Ikenberry, L. D., \& Rice, S. A. (1963). On the Kinetic Theory of Dense Fluids. XIV Experimental and Theoretical Studies of Thermal Conductivity in Liquid Ar, Kr, Xe, and $\mathrm{CH}_{4}$. J. Chem. Phys., 39, 1561-1571.

Irving, J. B., \& Jamieson, D. T. (1975). Thermal Conductivity of 13 Fluorocarbon Liquids. $J$. Fluorine Chem., 5, 449-456.

Jaeger, F. M. (1917). Uber die Temperaturabhangigkeit der molekuran freien uberflackenenergie von flussigkeiten im temperaturbereich von -80 bis +1650 C. Z. Anorg. Allg. Chem., 101, $1-214$.

Jamiesen, D. T., Irving, J. B., \& Tudhope, J. S. (1975). Liquid Thermal Conductivity - A Data Survey to 1973. Edinburgh: Crown Publishing. 
Jamiesen, D. T., \& Tudhope, J. S. (1964). A simple device for measuring the thermal conductivity of liquids with moderate accuracy. J. Inst. Petrol., 50(486), 150-153.

Jasper, J. (1972). The surface tension of pure liquid compounds. J. Phys. Chem. Ref. Data, 1(4), 841-1009.

Jayarathna, S. A., Jayarathna, C. K., Kottage, D. A., Dayarathna, S., Eimer, D. A., \& Melaaen, M. C. (2013). Density and Surface Tension Measurements of Partially Carbonated Aqueous Monoethanolamine Solutions. J. Chem. Eng. Data, 58, 343-348.

Jin, X., Wu, J., Liu, Z., \& Pan, J. (2004). The thermal conductivity of dimethyl carbonate in the liquid phase. Fluid Phase Equilib., 220, 37-40.

Johnson, F. M. G., \& McIntosh, D. (1909). Liquid Chlorine. J. Am. Chem. Soc., 31(10), 11381144.

Johnston, H. L., \& Grilly, E. R. (1946). The Thermal Conductivites of Eight Common Gases between 80 and 380 K. J. Chem. Phys., 14, 233-238.

Jossi, J. A., Stiel, L. I., \& Thodos, G. (1962). The Viscosity of Pure Substances in the Dense Gaseous and Liquid Phases. AIChE J., 8(1), 59-63.

Kamien, C. Z., \& Witzell, O. W. (1959). Effect of pressure and temperature on the viscosity of refrigerants in the vapor phase. ASHRAE J., 65, 663-674.

Karapet'yants, M. K., \& Kuo-sen, Y. (1963). Temperature Variation of the Viscosity of nAlkanes. Russ. J. Phys. Chem., 37, 1106.

Karbanov, E. M. (1978). Investigation of the Dynamic Viscosity of Some Freons of Ethane Type and of the Bromide Freons. (Ph.D.), Grozny Petroleum Institute, Grozny, USSR.

Kardos, A. (1934). Die Wirmeleitfihigkeit verschiedener Fliissigkeiten. Z. ges. Kalte-Ind., 41, 29-35.

Kashiwagi, H., Oishi, M., Tanaka, Y., Kabota, H., \& Makita, T. (1982). Thermal Conductivity of Fourteen Liquids in the Temperature Range 298-373 K. Int. J. Thermophys., 3, 101-115.

Kazakov, A. (2017). [personal communication to M.L. Huber regarding dipole moments of R1336mzz(Z) and R1224yd(Z)].

Kennan, R. P., \& Pollack, G. L. (1988). Solubility of xenon in perfluoroalkanes: Temperature dependence and thermodynamics. J. Chem. Phys., 89, 517-521.

Kessel'man, P. M., Porichanskii, E. G., \& Karbanov, E. M. (1976). Study of dynamic viscosity coefficients for freons in the wide temperature and pressure interval. Kholod. Tekh. Tekhnol, 22, 48-50.

Kessel'man, P. M., Porichanskii, E. G., Romanov, V. K., Ponomareva, O. P., Svetlichnyi, P. I., Zhelezny, V. P., Slyusarev, V.V., \& Karbanov, E. M. (1977). Complex study of the thermophysical properties of refrigerant S318. Teplofiz. Svoistva Veshchestv Mater., 410.

Kestin, J., Khalifa, H. E., \& Wakeham, W. A. (1977). The Viscosity of Gaseous Mixtures Containing Krypton. J. Chem. Phys., 67, 4254-4259.

Kestin, J., Ro, S. T., \& Wakeham, W. A. (1972a). Viscosity of the Binary Gaseous Mixture He$\mathrm{Ne}$ and $\mathrm{Ne}-\mathrm{N}_{2}$ in the Temperature Range 25-700 ${ }^{\circ} \mathrm{C}$. J. Chem. Phys., 56, 5837-5842.

Kestin, J., Ro, S. T., \& Wakeham, W. A. (1972b). Viscosity of the Binary Gaseous Mixture Neon-Krypton. J. Chem. Phys., 56, 4086-4091.

Kestin, J., \& Wakeham, W. A. (1979). The Viscosity of Three Polar Gases. Ber. Bunsen-Ges. Phys. Chem., 83, 573-576. 
Kestin, J., Wakeham, W. A., \& Watanabe, K. (1970). Viscosity, Thermal Conductivity, and Diffusion Coefficient of Ar-Ne and Ar-Kr Gaseous Mixtures in the Temperature Range 25-700 C. J. Chem. Phys., 53, 3773-3780.

Keyes, F. G. (1954). Thermal Conductivity of Gases. Trans. ASME, 76, 809-816.

Klein, S. A., McLinden, M. O., \& Laesecke, A. (1997). An improved extended corresponding states method for estimation of viscosity of pure refrigerants and mixtures. Int. J. Refrig., 20, 208-217.

Knothe, G., \& Steidley, K. R. (2005). Kinematic viscosity of biodiesel fuel components and related compounds. Influence of compound structure and comparison to petrodiesel fuel components. Fuel, 84, 1059-1065.

Koelbel, H., Siemes, W., \& Luther, H. (1949). Viscosity Relations of Hydrocarbons III. Viscosity as function of molar mass of homologous hydrocarbons. Brennst.-Chem., 30, 362-371.

Kondou, C., \& Koyama, S. (2015). Surface tension of low GWP refrigerants R1243zf, R1234ze(Z), and R1233zd(E). Int. J. Refrig., 53, 80-89. doi: DOI: 10.1016/j.ijrefrig.2015.01.005

Krynicki, K., \& Hennel, J. W. (1963). Viscosity of liquid ammonia and hydrogen chloride. Acta Phys. Pol., 24(8), 269.

Kumagai, A., \& Yokoyama, C. (2000). Revised Viscosities of Saturated Liquid Halocarbon Refrigerants from 273 to $353 \mathrm{~K}$. Int. J. Thermophys., 21, 909-912.

Kurihara, K., Kandil, M. E., Marsh, K. N., \& Goodwin, A. R. H. (2007). Measurement of the Viscosity of Liquid Cyclopentane Obtained with a Vibrating Wire Viscometer at Temperatures between (273 and 353) K and Pressures below $45 \mathrm{MPa}$. J. Chem. Eng. Data, 52, 803-807.

Laesecke, A., \& Defibaugh, D. R. (1996). Viscosity of 1,1,1,2,3,3-Hexafluoropropane and 1,1,1,3,3,3- Hexafluoropropane at Saturated-Liquid Conditions from $262 \mathrm{~K}$ to $353 \mathrm{~K} . J$. Chem. Eng. Data, 41, 59-62.

Laesecke, A., \& Hafer, R. F. (1998). Viscosity of Fluorinated Propane Isomers. 2. Measurements of Three Compounds and Model Comparisons. J. Chem. Eng. Data, 43, 84-92.

Lambert, J. D., Cotton, K. J., Pailthorpe, M. W., Robinson, A. M., Scrivins, J., Vale, W. R. F., \& Young, R. M. (1955). Transport Properties of Gaseous Hydrocarbons. Proc. Royal Soc. London A, 231, 280-290.

Le Neindre, B., Garrabos, Y., \& Kim, N. S. (2001). Measurements of the Thermal Conductivity of HFC-143a in the Temperature Range from 300 to $500 \mathrm{~K}$ at Pressures up to $50 \mathrm{MPa}$. Int. J. Thermophys., 22(3), 723-748.

Lee, S. H., Kim, M. S., \& Ro, S. T. (2001). Themal conductivity of 1,1,1-trifluoroethane (R143a) and R404A in the liquid phase. J. Chem. Eng. Data, 46, 1013-1015.

Lemmon, E. W. (2007a). [Helmholtz equation of state for methylcyclohexane, unpublished, implemented in REFPROP v10].

Lemmon, E. W. (2007b). [Helmholtz equation of state for propylcyclohexane, unpublished, implemented in REFPROP v10].

Lemmon, E. W., Bell, I. H., Huber, M. L., \& McLinden, M. O. (2018). NIST Standard Reference Database 23, NIST Reference Fluid Thermodynamic and Transport Properties Database (REFPROP): Version 10.0. Gaithersburg, MD.

Lemmon, E. W., \& Huber, M. L. (2004). Thermodynamic Properties of n-Dodecane. Energy \& Fuels, 18, 960-967. 
Lemmon, E. W., \& Ihmels, E. C. (2005). Thermodynamic Properties of the Butenes. Part II. Short Fundamental Equations of State. Fluid Phase Equilib., 228-229, 173-187.

Lemmon, E. W., \& Jacobsen, R. T. (2000). An International Standard Formulation for the Thermodynamic Properties of 1,1,1-Trifluoroethane (HFC-143a) for Temperatures from 161 to $450 \mathrm{~K}$ and Pressures to $50 \mathrm{MPa}$. J. Phys. Chem. Ref. Data, 29(4), 521-552.

Lemmon, E. W., \& Jacobsen, R. T. (2004). Viscosity and Thermal Conductivity Equations for Nitrogen, Oxygen, Argon, and Air. Int. J. Thermophys., 25, 21-69.

Lemmon, E. W., McLinden, M. O., Overhoff, U., \& Wagner, W. (2017). A Reference Equation of State for Propylene for Temperatures from the Melting Line to $575 \mathrm{~K}$ and Pressures up to $1000 \mathrm{MPa}$. J. Phys. Chem. Ref. Data (to be submitted).

Lemmon, E. W., McLinden, M. O., \& Wagner, W. (2009). Thermodynamic Properties of Propane. III. A Reference Equation of State for Temperatures from the Melting Line to $650 \mathrm{~K}$ and Pressures up to $1000 \mathrm{MPa}$. J. Chem. Eng. Data, 54, 3141-3180.

Lemmon, E. W., \& Span, R. (2006). Short Fundamental Equations of State for 20 Industrial Fluids. J. Chem. Eng. Data, 51, 785-850.

Lemmon, E. W., \& Span, R. (2015). Thermodynamic Properties of R-227ea, R-365mfc, R-115, and R-13I1. J. Chem. Eng. Data, 60, 3745-3758. doi:DOI: 10.1021/acs.jced.5b00684

Lesche, H., Klemp, D., \& Nickel, B. (1984). Dichte und Viskositat des Isopentans von $115 \mathrm{~K}$ bis 293 K. Z. Phys. Chem. (Leipzig), 141, 239-249.

Li, X., Wu, J., \& Dang, Q. (2010). Thermal Conductivity of Liquid Diethyl Ether, Diisopropyl Ether, and Di-n-butyl Ether from (233 to 373) K at Pressures up to $30 \mathrm{MPa}$. J. Chem. Eng. Data, 55, 1241-1246.

Ling, T. D., \& Van Winkle, M. (1958). Properties of binary mixtures as a function of composition. Ind. Eng. Chem. Chem. Eng. Data Ser., 3, 88-95.

Liu, K., \& Kiran, E. (2007). Viscositry, Density and Excess Volume of Acetone + Carbon Dioxide Mixtures at High Pressures. Ind. Eng. Chem. Res., 46, 5453-5462.

Lopez, A. B., Garcia-Abuin, A., Gomez-Diaz, D., La Rubia, M. D., \& Navaza, J. M. (2013). Density, speed of sound, viscosity, refractive index and surface tension of N-methyl-2pyrrolidone + diethanolamine (or triethanolamine) from $\mathrm{T}=(293.15$ to 323.15$) \mathrm{K} . J$. Chem. Thermodyn., 61, 1-6.

Losetty, V., Wilfred, C. D., \& Shekar, M. C. (2016). Synthesis and study of ionic interactions by volumetric, transport, FT-IR and computational methods of alkyl imidazolium acetate ionic liquid with molecular solvents (DMSO, DMF and EG) at T $=(293.15-363.15) \mathrm{K} . J$. Mol. Liq., 224, 480-491.

Lusternik, V. E., \& Zdanov, A. G. (1973). Viscosity of alkanes, alkenes and alkynes in gaseous phase. Teplofiz. Svoistva Veshchestv Mater., Rabinovich, V. A., Ed., Standards Publ.: Moscow, 7.

Ma, P. S., Zhou, Q., Yang, C., \& Xia, S. (2004). Measurement of viscosities and densities of liquids by vibrating wire method. Huagong Xuebao, 55, 1608-1613.

Ma, R. F., Shi, L., Duan, Y. Y., Han, L. Z., \& Liu, N. X. (2003). Saturated liquid viscosity of cyclopentane and isopentane. J. Chem. Eng. Data, 48(6), 1418-1421.

doi:10.1021/je0202174

Maass, O., \& Boomer, E. H. (1922). Vapor densities at low pressures and over an extended temperature range: I the properties of ethylene oxide compared to oxygen compounds of similar molecular weight. J. Am. Chem. Soc., 44, 1709-1728. 
Maass, O., \& Wright, K. (1921). Some Physical Properties of Hydrocarbons Containing Two and Three Carbon Atoms. J. Am. Chem. Soc., 43, 1098-1111.

Maczek, A. O. S., \& Edwards, C. J. C. (1979). The viscosity and binary diffusion coefficients of some gaseous hydrocarbons, fluorocarbons and siloxanes. Paper presented at the Symp. Transp. Prop. Fluids Fluid Mixtures: Their Meas. Estim., Correl. Use, Paper no. 3.5, Glasgow, Scotland.

Makhrov, V. V., \& Miroshnichenko, V. I. (1984). Experimental study of the thermal conductivity and temperature jump in non-reacting gases at low pressures. Inzh.-Fiz. Zh., 47, 376-383.

Malhotra, R., Price, W. E., Woolf, L. A., \& Easteal, A. J. (1990). Thermodynamic and transport properties of 1,2-dichloroethane. Int. J. Thermophys., 11, 835-861.

Mallan, G. M., Michaelian, M. S., \& Lockhart, F. J. (1972). Liquid thermal conductivities of organic compounds and petroleum fractions. J. Chem. Eng. Data, 17, 412-415.

Marrucho, I. M., Oliveira, N. S., \& Dohrn, R. (2002). Vapor-Phase Thermal Conductivity, Vapor Pressure, and Liquid Density of R365mfc. J. Chem. Eng. Data, 47, 554-558.

Marsh, K. N. (1968). Mutual Diffusion in Octamethylcyclotetrasiloxane Mixtures. Trans. Faraday Soc., 64, 894.

Marsh, K. N., Perkins, R. A., \& Ramires, M. L. V. (2002). Measurement and Correlation of the Thermal Conductivity of Propane from 86 to $600 \mathrm{~K}$ at Pressures to $70 \mathrm{MPa}$. J. Chem. Eng. Data, 47, 932-940.

Mashirov, V. E., \& Tarzimanov, A. A. (1974). Thermal conductivity of vaporous compounds. Izv. Vyssh. Uchebn. Zaved., Neft Gaz, 17, 98-99.

Matheson Gas Data Book, unabridged ed. (1974). East Rutherford, NJ: Matheson Company, Inc.

MathesonTriGas. (2008). SDS for vinyl Chloride, http://www2.mathesongas.com/pdfs/msds/MAT24940.pdf.

Matsuo, S., Tanaka, Y., \& Sotani, T. (2002). Experimental study on the transport properties of fluorinated ethers. Fluid Phase Equilib., 194-197, 1205-1213.

Matsuo, S., Tanaka, Y., Tanada, N., Yamamoto, H., \& Sekiya, A. (1998). Gaseous Thermal Conductivities of Fluorinated Methyl Ethyl Ethers. J. Chem. Eng. Data, 43, 473-476.

McCoubrey, J. C., McCrea, J. N., \& Ubbelohde, A. R. (1951). The configuration of flexible polymethylene molecules in the gas phase. J. Chem. Soc., 1961-1971.

McCoubrey, J. C., \& Singh, N. M. (1960). Viscosity of some fluorocarbons in the vapor phase. Trans. Faraday Soc., 56, 486-489.

McCoubrey, J. C., \& Singh, N. M. (1963). The Vapor Phase Viscosities of the Pentanes. J. Phys. Chem., 67, 517-518.

McLinden, M. O., \& Akasaka, R. (2018). [Helmholtz equation of state for R1336mzz(Z), unpublished, implemented in REFPROP v10].

McLinden, M. O., Klein, S. A., \& Perkins, R. A. (2000). An Extended Corresponding States Model for the Thermal Conductivity of Pure Refrigerants and Refrigerant Mixtures. Int. J. Refrig., 23(1), 43-63.

Medzhidov, K., \& Safarov, M. M. (1983). Thermal conductivity of ketones as a function of temperature. Teplofiz. Vys. Temp., 21, 1229-1231.

Meng, X. (2017). [personal communication on the viscosity of R1233zd(E) to M. Huber].

Meng, X., Zhang, J., \& Wu, J. (2011). Compressed Liquid Viscosity of 1,1,1,3,3-

Pentafluoropropane (R245fa) and 1,1,1,3,3,3-Hexafluoropropane (R236fa). J. Chem. Eng. Data, 56, 4956-4964. 
Meng, X., Zhang, J., Wu, J., \& Liu, Z. (2012). Experimental Measurement and Modeling of the Viscosity of Dimethyl Ether. J. Chem. Eng. Data, 57, 988-993.

Meng, X., Zheng, P., Wu, J., \& Liu, Z.-G. (2009). Viscosity and Density Measurements of Dimethyl Carbonate. J. Eng. Thermophys., 30(5), 36-40.

Meng, X., Zheng, P., Wu, J., \& Liu, Z. (2008). Density and viscosity measurements of diethyl ether from 243 to $373 \mathrm{~K}$ and up to $20 \mathrm{MPa}$. Fluid Phase Equilib., 271, 1-5.

Millat, J., Ross, M., Wakeham, W. A., \& Zalaf, M. (1988). The Thermal Conductivity of Neon, Methane, and Tetrafluoromethane. Physica, 148A, 124-152.

Miller, S. A. (1966). Acetylene, Its Properties, Manufacture and Uses, volume II. New York: Academic Press.

Miyara, A. (2018a). [personal communication on preliminary viscosity and thermal conductivity data for R1234ze(Z) to M. Huber].

Miyara, A. (2018b). [personal communication on the viscosity of r1224yd(Z) to M. Huber].

Miyara, A. (2018c). [personal communication on the viscosity of R1233zd(E) to M. Huber].

Miyara, A. (2018d). [personal communication regarding preliminary measurements on the viscosity of R1336mzz(Z) to $M$. Huber].

Mohr, P. J., Newell, D. B., \& Taylor, B. N. (2016). CODATA Recommended Values of the Fundamental Physical Constants: 2014. J. Phys. Chem. Ref. Data, 45, 043102. doi:http://dx.doi.org/10.1063/1.4954402

Mondéjar, M. E., McLinden, M. O., \& Lemmon, E. W. (2015). Thermodynamic properties of trans-1-chloro-3,3,3-trifluoropropene $(\mathrm{R} 1233 \mathrm{zd}(\mathrm{E})$ : Vapor pressure, $(\mathrm{p}, \rho, \mathrm{T})$ behavior, and speed of sound measurements, and equation of state. J. Chem. Eng. Data, 60, 2477-2489. doi:10-1021/acs.jced/5b00348

Morehouse, F. R., \& Maass, O. (1931). The Preparation and Physical Properties of Ethyl and Methyl Acetylene. Can. J. Res., 5, 306-312.

Mukhamedzyanov, G. K., \& Usmanov, A. G. (1967). Thermal conductivity of higher saturated hydrocarbons. Izv. Vyssh. Uchebn. Zaved., Neft Gaz, 10, 76-80.

Mulero, A., \& Cachadiña, I. (2014). Recommended Correlations for the Surface Tension of Several Fluids Included in the REFPROP Program. J. Phys. Chem. Ref. Data, 43, 023104. doi:doi: 10.1063/1.4878755

Mulero, A., Cachadiña, I., \& Parra, M. I. (2012). Recommended Correlations for the Surface Tension of Common Fluids. J. Phys. Chem. Ref. Data, 41, 043105.

Nakazawa, N., Kawamura, M., Sekiya, A., Ootake, K., Tamai, R., Kurokawa, Y., \& Murata, J. (2002). Liquid viscosities of fluorinated dialkylethers. Trans. JSRAE, 19, 33-41.

Naziev, Y. M., \& Abasov, A. A. (1968). Determination of Thermal Conductivity of Olefinic Hydrocarbons in the Gas Phase. Chemtech, 20(12), 756-759.

Naziev, Y. M., \& Aliev, M. A. (1973). Study of the thermophysical properties of n-undecane; nheptadecane and n-octadecane at various temperatures and pressures. Izv. Vyssh. Uchebn. Zaved., Neft Gaz, 16, 73-76.

Neduzij, I. A., \& Khmara, Y. I. (1968). Investigation of the Viscosity Coefficients of Propene, Isobutylene, Butadiene-1,3, Toluene and Cyclohexane Along the Saturated Line. Teplofiz. Kharakt. Veshchestv, 158-160.

Nemzer, B. V. (1985). Surface tension of saturated high molecular weight petroleum hydrocarbons(nC21H44-nC24H50). Izv. Vyssh. Uchebn. Zaved., Neft Gaz, 28, 64. 
Neufeld, P. D., Janzen, A. R., \& Aziz, R. A. (1972). Empirical Equations to Calculate 16 of the Trasnport Collision Integrals Omega $(1, \mathrm{~s})^{*}$ for the Lennard-Jones (12-6) Potential. $J$. Chem. Phys., 57, 1100-1102.

Ni, B., Su, L., Wang, H., \& Qiu, H. (2010). Thermophysical properties of the binary mixtures of 1,2-Dichloroethane with Chlorobenzene and Bromobenzene from 298.15 to $313.15 \mathrm{~K} . J$. Chem. Eng. Data, 55, 4541-4545.

Nieto de Castro, C. A., Dix, M., Fareleira, J. M. N. A., Li, S. F. Y., \& Wakeham, W. A. (1989). Thermal conductivity of chlorobenzene at pressures up to $430 \mathrm{MPa}$. Physica A, 156, 534546.

Olchowy, G. A., \& Sengers, J. V. (1989). A simplified representation for the thermal conductivity of fluids in the critical region. Int. J. Thermophys., 10(2), 417-426.

Oliveira, C. M. B. P., \& Wakeham, W. A. (1992). The viscosity of five liquid hydrocarbons at pressures up to $250 \mathrm{MPa}$. Int. J. Thermophys., 13, 773-790.

Padua, A. A. H., Fareleira, J. M. N. A., Calado, J. C. G., \& Wakeham, W. A. (1996). Density and Viscosity Measurements of 2,2,4-Trimethylpentane (Isooctane) from $198 \mathrm{~K}$ to $348 \mathrm{~K}$ and up to $100 \mathrm{MPa}$. J. Chem. Eng. Data, 41, 1488.

Pal, A. K., \& Barua, A. K. (1967). Viscosity and intermolecular potentials of hydrogen sulfide, sulfur dioxide, and ammonia. Trans. Faraday Soc., 63, 341-346.

Pal, A. K., \& Bhattacharyya, P. K. (1969). Viscosity of binary polar-gas mixtures. J. Chem. Phys., 51, 828-831.

Palczewska-Tulinska, M., \& Oracz, P. (2005). Selected Physicochemical Properties of Hexamethylcyclotrisiloxane, Octamethylcyclotetrasiloxane, and Decamethylcyclopentasiloxane. J. Chem. Eng. Data, 50, 1711-1719.

Pan, J., Li, X., \& Wu, J. (2011). Thermal conductivity of liquid 1,1,1,3,3,3-hexafluoropropane (HFC-236fa) from $253 \mathrm{~K}$ to $373 \mathrm{~K}$ and pressure up to $30 \mathrm{MPa}$. Fluid Phase Equilib., 304, 64-67.

Pan, J., Rui, X., Zhao, X., \& Qiu, L. (2012). An equation of state for the thermodynamic properties of 1,1,1,3,3,3-hexafluoropropane (HFC-236fa). Fluid Phase Equilib., 321, 1016.

Paniego, A. R., \& Pinot, J. M. G. (1969). Viscosity of the Halogen Derivatives in the Vapor Phase. Anales de Fisica, 64(11), 343-349.

Parkinson, C., \& Gray, P. (1972). Thermal Conductivities of Gaseous Mixtures Containing Hydrocarbons. Part 1. n-Butane, iso-butane, iso-pentane and neo-pentane, and their binary mixtures with argon J. Chem. Soc., 68(6), 1065-1076.

Parkinson, C., Mukhopadhyay, P., \& Gray, P. (1972). Thermal Conductivities of Gaseous Mixtures Containing Hydrocarbons Part 2.1-Cyclopropane, Propene, But-1 -ene and trans-But-2-ene, and Their Binary Mixtures with Argon. J. Chem. Soc. Faraday Trans., $68,1077-1085$.

Pereiro, A. B., Rodriguez, A., Canosa, J., \& Tojo, J. (2004). Density, Viscosity, and Speed of Sound of Dialkyl Carbonates with Cyclopentane and Methyl Cyclohexane at Several Temperatures. J. Chem. Eng. Data, 49, 1392-1399.

Perkins, R.A., Cusco, L., Howley, J., Laesecke, A., Matthes, S., \& Ramires, M. V. L. (2001). Thermal Conductivities of Alternatives to CFC-11 for Foam Insulation. J. Chem. Eng. Data, 46, 428-432. 
Perkins, R. A., \& Huber, M. L. (2017). Measurement and Correlation of the Thermal Conductivity of trans-1-Chloro-3,3,3-trifluoropropene (R1233zd(E)). J. Chem. Eng. Data, 62, 2659-2665.

Perkins, R. A. (2002). [personal communication from R.A. Perkins to M.L. Huber regarding liquid thermal conductivity of R236ea].

Perkins, R. A. (2018). [personal communication to M.L. Huber, preliminary data for RE347mcc].

Perkins, R. A., Hammerschmidt, U., \& Huber, M. L. (2008). Measurement and correlation of the thermal conductivity of methylcyclohexane and propylcyclohexane from (300 to 600) $\mathrm{K}$ at pressures to $60 \mathrm{MPa}$. J. Chem. Eng. Data, 53(9), 2120-2127. doi:10.1021/je800255r

Perkins, R. A., \& Huber, M. L. (2011). Measurement and Correlation of the Thermal Conductivities of Biodiesel Constituent Fluids: Methyl Oleate and Methyl Linoleate. Energy \& Fuels, 25, 2383-2388.

Perkins, R. A., Laesecke, A., Howley, J., Ramires, M.L.V., Gurova, A.N. and Cusco, L. (2000). Experimental Thermal Conductivity Values for the IUPAC Round-robin Sample of 1,1,1,2-Tetrafluoroethane (R134a), NISTIR 6605

Perkins, R. A., Sengers, J. V., Abdulagatov, I. M., \& Huber, M. L. (2013). Simplified Model for the Critical Thermal-Conductivity Enhancement in Molecular Fluids. Int. J. Thermophys., 34, 191-212.

Platzer, B., Polt, A., \& Maurer, G. (1990). Thermophysical properties of refrigerants. Berlin: Springer-Verlag.

Polt, A., Platzer, B., \& Maurer, G. (1992). Parameter der thermischen Zustandsgleichung von Bender für 14 mehratomige reine Stoffe. Chem. Tech. (Leipzig), 44, 216-224.

Pratas, M. J., Freitas, S., Oliveira, M. B., Monteiro, S. C., Lima, A. S., \& Coutinho, J. A. P. (2011). Densities and Viscosities of Minority Fatty Acid Methyl and Ethyl Esters Present in Biodiesel. J. Chem. Eng. Data, 56, 2175-2180.

Quayle, O. R., Day, A. R., \& Brown, G. M. (1944). A Study of Organic Parachors. VII. A Series of Saturated Hydrocarbons. J. Am. Chem. Soc., 66, 938-941.

Queimada, A. J., Caco, A. I., Marrucho, I. M., \& Coutinho, J. A. P. (2005). Surface tension of decane binary and ternary mixtures with eicosane, docosane and tetracosane. J. Chem. Eng. Data, 50, 1043-1046.

Queimada, A. J., Quiñones-Cisneros, S. E., Marrucho, I. M., Coutinho, J. A. P., \& Stenby, E. H. (2005). Viscosity and Liquid Density of Asymmetric n-Alkane Mixtures: Measurement and Modeling. Int. J. Thermophys., 26, 47-61.

Queimada, A. J., Silva, F. A. E., Caco, A. I., Marrucho, I. M., \& Coutinho, J. A. P. (2003). Measurement and modeling of surface tensions of asymmetric systems: heptane, eicosane, docosane, tetracosane and their mixtures. Fluid Phase Equilib., 214, 211-221.

Qun-Fang, L., Ruisen, L., Dan-Yan, N., \& Yu-Chun, H. (1997). Thermal Conductivities of Some Organic Solvents and Their Binary Mixtures. J. Chem. Eng. Data, 42, 971-974.

Rafati, A. A., Bagheri, A., \& Najafi, M. (2011). Surface tension of non-ideal binary and ternary liquid mixtures at various temperatures and $\mathrm{p}=81.5 \mathrm{kPa}$. J. Chem. Thermodyn., 43, 248254.

Ramsay, W., \& Shields, J. (1893). The variation of molecular surface-energy with temperature. Philos. Trans. R. Soc. London, Ser. A, 184, 647-675.

Rastorguev, Y. L., Bogatov, G. F., \& Grigov'ev, B. A. (1974). A Study of the Thermal Conductivity of Higher n-Alkanes. Khim. Tekhnol. Topl. Masel, 9, 54. 
Rastorguev, Y. L., \& Gazdiev, M. A. (1969). Study of the thermal conductivity of manyatomic alcohols. Inzh.-Fiz. Zh., 17, 72-79.

Rausch, M. H., Kretschmer, L., Will, S., Leipertz, A., \& Fröba, A. P. (2015). Density, Surface Tension, and Kinematic Viscosity of Hydrofluoroethers HFE-7000, HFE-7100, HFE7200, HFE-7300, and HFE-7500. J. Chem. Eng. Data, 60, 3759-3765.

Reid, R. C., Prausnitz, J.M., \& Poling, B.E. (1987). The Properties of Gases and Liquids (Fourth ed.). New York: McGraw-Hill.

Reynes, E. G., \& Thodos, G. (1966). Viscosity of Helium, Neon, and Nitrogen in the Dense Gaseous Region. J. Chem. Eng. Data, 11, 137-140.

Richter, G. N., \& Sage, B. H. (1963). Thermal Conductivity of Fluids. Nitrous Oxide. J. Chem. Eng. Data, 8, 221-225.

Riesco, N., \& Vesovic, V. (2016). Extended Hard-Sphere Model for Predicting the Viscosity of long-chain n-alkanes. Fluid Phase Equilib., 425, 385-392.

Ripple, D., \& Defibaugh, D. (1997). Viscosity of the saturated liquid phase of three fluorinated ethanes: R152a, R143a, and R125. J. Chem. Eng. Data, 42, 360-364.

Rodriguez, A., Canosa, J., Dominguez, A., \& Tojo, J. (2004). Viscosities of dimethyl carbonate with alcohols at several temperatures UNIFAC-VISCO interaction parameters (-OCOO/alcohol). Fluid Phase Equilib., 216, 167-174.

Romeo, R., \& Lemmon, E. W. (2017). [Helmholtz equation of state for docosane, personal communication to E.W. Lemmon, NIST, Boulder].

Rudenko, N. S., \& Shubnikov, L. V. (1934). Viscosity of liquid nitrogen, carbon monoxide, argon and oxygen and its dependence on temperature. Phys. Z., 6, 470-477.

Rui, X., Pan, J., \& Wang, Y. (2013). An Equation of State for Thermodynamic Properties of 1,1,1,2,3,3-Hexafluoropropane (R236ea). Fluid Phase Equilib., 341, 78-85.

Rutherford, W. M. (1984). Viscosity of Liquid-Phase Methyl Chloride. J. Chem. Eng. Data, 29, 163-165.

Ryabtsev, N. I., \& Kazaryan, V. A. (1970). The Measurement of Thermal Conductivity of iButylene. Gazov. Delo, 36-38.

Sagdeev, D. I., Fomina, M. G., Mukhamedzyanov, G. K., \& Abdulagatov, I. M. (2012). Experimental study of the density and viscosity of polyethylene glycols and their mixtures at temperatures from $293 \mathrm{~K}$ to $465 \mathrm{~K}$ and at high pressures up to $245 \mathrm{MPa}$. Fluid Phase Equilib., 315, 64-76.

Sakiadis, B. C. C., \& Coates, J. (1955). Studies of Thermal Conductivty of Liquids, Pts. I and II. AIChE J., 1, 275-288.

Sastri, S. R. S., \& Rao, K. K. (1999). A new temperature-thermal conductivity relationship for predicting saturated liquid thermal conductivity. J. Chem. Eng. Data, 74, 161-169.

Saxena, S. C., \& Gupta, G. P. (1970). Experimental Data and Procedures for Predicting Thermal Conductivity of Multicomponent Mixtures of Nonpolar Gases. J. Chem. Eng. Data, 15, 98-107.

Saxena, S. C., \& Tondon, P. K. (1971). Experimental Data and Procedures for Predicting Thermal Conductivity of Binary Mixtures of Nonpolar Gases. J. Chem. Eng. Data, 16, 212-220.

Schmidt, K. A. G., Quiñones-Cisneros, S. E., Carroll, J. J., \& Kvamme, B. (2008). Hydrogen Sulfide Viscosity Modeling. Energy \& Fuels, 22, 3424-3434.

Senftleben, H. (1964). New values of thermal conductivity and specific heat at different temperatures for a series of gases. Z. Angew. Phys., 17, 86-87. 
Shashkov, A. G., Nesterov, N. A., Sudnik, V. M., \& Aleinikova, V. I. (1976). Experimental study of the thermal conductivity of rare gas at low temperatures. Inzh.-Fiz. Zh., 30, 671679.

Singh, R. P., \& Sinha, C. P. (1985). Viscosities and activation energies of viscous flow of ternary mixtures of toluene, chlorobenzene, 1-hexanol and benzyl alcohol. J. Chem. Eng. Data, $30,470-474$.

Skripov, V. P., \& Firsov, V. V. (1968). Surface tension of Perfluoroalkanes. Russ. J. Phys. Chem., 42, 653-656.

Slusar, V. P., Rudenko, N. S., \& Tret'yakov, V. M. (1973). Experimental study of the viscosity of simple substances along the saturation curve and under pressure. Ukr. Fiz. Zh, 18, 190194.

Slusarev, V. V. (1979). The Investigation of Thermal Conductivity of Freons of the Ethane Type of Fluorocarbons. (PhD), Odessa Technological Institute of Food Industry Odessa, USSR.

Smith, C. J. (1922). On the Viscosity and Molecular Dimensions of Gaseous Carbon Oxysulfide. Philos. Mag., 44, 389-392.

Song, J.-H., Park, S.-B., Yoon, J.-H., Lee, H., \& Lee, K.-H. (1996). Densities and Viscosities of Monoethanolamine + Ethylene Glycol + Water. J. Chem. Eng. Data, 41, 1152-1154.

Song, L., Wu, J., \& Liu, Z.-G. (2008). Thermal conductivity of dimethyl carbonate in liquid phase. Huagong Xuebao, 59, 551-556.

Span, R., Lemmon, E. W., Jacobsen, R. T., Wagner, W., \& Yokozeki, A. (2000). A Reference Equation of State for the Thermodynamic Properties of Nitrogen for Temperatures from 63.151 to $1000 \mathrm{~K}$ and Pressures to $2200 \mathrm{MPa}$. J. Phys. Chem. Ref. Data, 29(6), 13611433.

Steacie, E. W. R., \& Johnson, F. M. G. (1925). The viscosities of the liquid halogens. J. Am. Chem. Soc., 47, 754-762.

Steele, B. D., McIntosh, D., \& Archibald, E. H. (1906). The halogen hydrides as conducting solvents: I the vapour pressures, densities, surface energies and viscosities of the pure solvents. Philos. Trans. R. Soc. London, Ser. A, 205, 99-110.

Stefanov, B., Zarkova, L., \& Oliver, D. A. (1976). Measurements of the thermal conductivity of gases and vapor up to $2500 \mathrm{~K}$. Teplofiz. Vys. Temp., 14, 56-66.

Stewart, W. W., \& Maass, O. (1932). The Coefficient of Viscosity of Sulphur Dioxide over a Low Tempersture Range. Can. J. Res., 6, 453-457.

Stiles, V. E., \& Cady, G. H. (1952). Physical properties of perfluoro-n-hexane and perfluoro-2methylpentane. J. Am. Chem. Soc., 74, 3771-3773.

Sun, S., Niu, Y., Gao, F., Shen, J., \& Wei, X. (2015). Solubility Properties and Spectral Characterization of Dilute $\mathrm{SO}_{2}$ in Binary Mixtures of Urea + Ethylene Glycol. J. Chem. Eng. Data, 60, 161-170.

Takada, N., Matsuo, S., Tanaka, Y., \& Sekiya, A. (1998). Gaseous thermal conductivities of new hydrofluoroethers (HFEs). J. Fluorine Chem., 91, 81-85.

Takahashi, M., Shibasaki-Kitakawa, N., \& Yokoyama, C. (1999). Viscosity of gaseous HFC143a (1,1,1-trifluoroethane) under high pressures. Int. J. Thermophys., 20, 435-443.

Takahashi, M., Shibasaki-Kitakawa, N., Yokoyama, C., \& Takahashi, S. (1996). Viscosity of Gaseous Nitrous Oxide from $298.15 \mathrm{~K}$ to $398.15 \mathrm{~K}$ at Pressures up to $25 \mathrm{MPa}$. J. Chem. Eng. Data, 41, 1495-1498. 
Tanaka, Y., Nakata, M., \& Makita, T. (1991). Thermal conductivity of gaseous HFC-134a, HFC143a, HCFC-141b, and HCFC-142 b. Int. J. Thermophys., 12, 949-963.

Teng, T. T., Maham, Y., Hepler, L. G., \& Mather, A. E. (1994). Viscosity of Aqueous Solutions of N-Methyldiethanolamine and of Diethanolamine. J. Chem. Eng. Data, 39, 290-293.

Thol, M., Alexandrov, I. S., Span, R., \& Lemmon, E. W. (2018). Helmholtz equation of state for chlorobenzene. for submission to J. Chem. Eng. Data.

Thol, M., Beckmüller, R., Weiss, R., Harvey, A. H., Lemmon, E. W., Jacobsen, R. T., \& Span, R. (2018). Thermodynamic Properties for Neon for Temperatures from the Triple Point to $700 \mathrm{~K}$ at Pressures to $700 \mathrm{MPa}$. for submission to Int. J. Thermophys.

Thol, M., Dubberke, F. H., Baumhögger, E., Span, R., \& Vrabec, J. (2018). Helmholtz equation of state for Hydrogen Chloride. for submission to J. Chem. Eng. Data.

Thol, M., Dubberke, F. H., Baumhögger, E., Vrabec, J., \& Span, R. (2017). Speed of Sound Measurements and Fundamental Equations of State for Octamethyltrisiloxane and Decamethyltetrasiloxane. J. Chem. Eng. Data, 62, 2633-2648.

Thol, M., Dubberke, F. H., Rutkai, G., Windmann, T., Köster, A., Span, R., \& Vrabec, J. (2016). Fundamental equation of state correlation for hexamethyldisiloxane based on experimental and molecular simulation data. Fluid Phase Equilib., 418, 133-151. doi:http://dx.doi.org/10.1016/j.fluid.2015.09.047

Thol, M., Javed, M. A., Baumhögger, E., Span, R., \& Vrabec, J. (2018). Thermodynamic Properties of Dodecamethylpentasiloxane, Tetradecamethylhexasiloxane, and Decamethylcyclopentasiloxane. for submission to Fluid Phase Equilib.

Thol, M., Koeste, A., Rutkai, G., Span, R., Wagner, W., \& Vrabec, J. (2018). EOS for 1,2dichloroethane. for submission to Mol. Physics.

Thol, M., Piazza, L., \& Span, R. (2014). A New Functional Form for Equations of State for Some Polar and Weakly Associating Fluids. Int. J. Thermophys., 35(5), 783-811.

Thol, M., Rutkai, G., Köster, A., Dubberke, F. H., Windmann, T., Span, R., \& Vrabec, J. (2016). Thermodynamic Properties for Octamethylcyclotetrasiloxane. J. Chem. Eng. Data, 61, 2580-2595.

Thol, M., Rutkai, G., Koster, A., Kortmann, M., \& Span, R. (2015). Fundamental equation of state for ethylene oxide based on a hybrid dataset. Chem. Eng. Sci., 121, 87-99.

Thol, M., \& Span, R. (2014). [Helmholtz equation of state for vinyl chloride, unpublished, implemented in REFPROP v10 (personal communication to E.W. Lemmon)].

Thol, M., Uhde, T., Lemmon, E. W., \& Span, R. (2018). [Fundamental Equations of State for Hydrocarbons. Part I. n-Pentane, personal communication to E.W. Lemmon, NIST, Boulder].

Thorpe, T. E., \& Rodger, J. W. (1894). Bakerian Lecture. On the Relations between the Viscosity (Internal Friction) of Liquids and their Chemical Nature. Philos. Trans. R. Soc. London, Ser. A, 185, 397-710.

Tillner-Roth, R., \& Baehr, H. D. (1994). An international standard formulation of the thermodynamic properties of 1,1,1,2-tetrafluoroethane (HFC-134a) for temperatures from $170 \mathrm{~K}$ to $455 \mathrm{~K}$ at pressures up to $70 \mathrm{MPa}$. J. Phys. Chem. Ref. Data, 23, 657-729.

Timmermans, J., \& Hennaut-Roland, M. (1937). Works from International Bureau at PhysicalChemical Standards. VIII. Physical constants of 20 organic compounds. J. Chim. Phys. Phys.-Chim. Biol., 34, 693-735.

Titani, T. (1927). The Viscosity of Liquids Above Their Boiling Points Part I. Bull. Chem. Soc. Jpn., 2, 95-105. 
Titani, T. (1930). The Viscosity of Vapors of Organic Compounds. Part II. Bull. Chem. Soc. Jpn., 5, 98-108.

Titani, T. (1933). The Viscosity of Vapors of Organic Compounds. Part III. Bull. Chem. Soc. Jpn., 8, 255-276.

Tohidi, B., Burgass, R.W., Danesh, A., \& Todd, A. C. (2001). Viscosity and Density of Methane + Methylcyclohexane from (323 to 423) K and Pressures to $140 \mathrm{MPa}$. J. Chem. Eng. Data, 46, 385-390.

Trautz, M., \& Husseini, I. (1934). Die Reibung, Warmeleitung und Diffusion im Gasmischungen. Ann. Physik, 20, 121-126.

Trautz, M., \& Ruf, F. (1934). Die Reibung, Wärmeleitung und Diffusion in Gasmischungen. Ann. Phys., 20(5), 127-134.

Tsvetkov, O. B., Laptev, Y. A., \& Vasilkov, A. I. (1977). The Results of Measurements of Thermal Conductivity of Gaseous Freons with the Heating Wire Method. Mashinyi $i$ Apparatyi Cholodilnoj, Kriogennoh Techniki i Kondizionirovaniyi Vosducha, 2, 54-56.

Uchida, Y., Yasumoto, M., Yamada, Y., Ochi, K., Furuya, T., \& Otake, K. (2004). Critical Properties of Four HFE + HFC Binary Systems: Trifluoromethoxymethane (HFE-143m) + Pentafluoroethane (HFC-125), + 1,1,1,2-Tetrafluoroethane (HFC-134a), +1,1,1,2,3,3,3Heptafluoropropane (HFC-227ea), and +1,1,1,2,3,3-Hexafluoropropane (HFC-236ea). $J$. Chem. Eng. Data, 49, 1615-1621.

Ulybin, S. A., \& Makarushkin, V. I. (1977). Viscosity of xenon at temperatures from 170 to 1300 $\mathrm{K}$ and at pressures up to 1000 bar. Teplofiz. Vys. Temp., 15, 509-514.

Van Wijk, W. R., Van der Veen, J. H., Brinkman, H. C., \& Seeder, W. A. (1940). The influence of the temperature and the specific volume on the viscosity of liquids. III. Physica, 7, 4556. doi:10.1016/s0031-8914(40)90068-4

Vargaftik, N. B., \& Yakush, L. V. (1971). Experimental study of the thermal conductivity of neon, krypton, xenon in the wide temperature range. Inzh.-Fiz. Zh., 21, 491-499.

Vassiliou, C.-M., Assael, M. J., Huber, M. L., \& Perkins, R. A. (2015). Reference Correlations of the Thermal Conductivity of Cyclopentane, iso-Pentane, and n-Pentane. J. Phys. Chem. Ref. Data, 44, 033102. doi: http://dx.doi.org/10.1063/1.4927095

Vilim, O. (1960). Thermal Conductivity of Hydrocarbons. Collect. Czech. Chem. Commun., 25, 993.

Vines, R. G. (1953). The Thermal Conductivity of Organic Vapors: The Influence of Molecular Interaction. Aust. J. Chem., 6, 1-26.

Vines, R. G., \& Bennett, L. A. (1954). The thermal conductivity of organic vapors. The relation between thermal conductivity and viscosity and the significance of the Eucken factor. $J$. Chem. Phys., 22, 360-366.

Vogel, A. I. (1948). Physical Properties and Chemical Constitution. Part XIV. The Parachors and the Refractivities of the Halogens. J. Chem. Soc., 1948, 644-654.

Vogel, E. (1984). Precise Measurements of the Viscosity Coefficient of Nitrogen and the RareGases Between Room-Temperature and 650 K. Ber. Bunsenges. Phys. Chem, 88, 9971002.

Vogel, E. (2012). Towards Reference Viscosities of Carbon Monoxide and Nitrogen at Low Density Using Measurements between $290 \mathrm{~K}$ and $680 \mathrm{~K}$ as well as Theoretically Calculated Viscosities. Int. J. Thermophys., 33, 741-757.

Vogel, E., \& Herrmann, S. (2016). New Formulation for the Viscosity of Propane. J. Phys. Chem. Ref. Data, 46(4), 043103. 
Vogel, E., \& Holdt, B. (1991). Temperature and initial-density dependence of the vapour phase viscosity of n-alkanes ( $\mathrm{n}=5$ - 7). High Temp. - High Pressures, 23, 473-483.

Vogel, E., Holdt, B., \& Strehlow, T. (1988). The initial density dependence of organic vaporscyclohexane and neopentane Physica A, 148(1-2), 46-60.

Vogel, E., Küchenmeister, C., Bich, E., \& Laesecke, A. (1998). Reference Correlation of the Viscosity of Propane. J. Phys. Chem. Ref. Data, 27, 947-970.

Vojtenko, A. K. (1980). Investigation of the Thermal Conductivity of Some Freons in the Wide Range of Parameters Including the Critical Region. (Ph.D.), Grozny Petroleum Institute, Grozny.

Voshinin, A. A., Kerzhentsev, V. V., Studnikov, E. L., \& Yakush, L. V. (1975). Experimental study of the thermal conductivity of krypton at high temperatures. Inzh.-Fiz. Zh., 28, 821825.

Wang, X., Wang, W., Song, B., Lv, S., \& Liu, Z. (2014). Measurement and correlation of viscosity of HFC227ea and HFC236fa in the vapor phase. Int. J. Refrig., 46, 152-157.

Wang, X., Wu, J., \& Liu, Z.-G. (2009). Viscosity of R143a in gaseous phase. Int. J. Thermophys., 30(4), 1455-1458.

Wang, Y., Wu, J., \& Liu, Z. (2006). Thermal Conductivity of Gaseous Dimethyl Ether from (263 to 383) K. J. Chem. Eng. Data, 51, 164-168.

Watanabe, H. (2003). Thermal Conductivity and Thermal Diffusivity of Sixteen Isomers of Alkanes: $\mathrm{CnH} 2 \mathrm{n}+2$ (n= 6 to 8). J. Chem. Eng. Data, 48, 124-136.

Watanabe, H., \& Kato, H. (2004). Thermal conductivity and thermal diffusivity of twenty-nine liquids: Alkenes, cyclic (alkanes, alkenes, alkadienes, aromatics), and deuterated hydrocarbons. J. Chem. Eng. Data, 49(4), 809-825. doi:10.1021/je034162x

Wen, C., Meng, X., Wei, K., \& Wu, J. (2017). Compressed Liquid Viscosity of 2Methylpentane, 3-methylpentane, and 2,3-Dimethylbutane at Temperatures from (273 to 343 ) $\mathrm{K}$ and Pressures up to $40 \mathrm{MPa}$. J. Chem. Eng. Data, 62, 1146-1152.

Werth, S., Stöbener, K., Klein, P., Küfer, K.-H., Horsch, M., \& Hasse, H. (2015). Molecular modelling and simulation of the surface tension of real quadrupolar fluids. Chem. Eng. Sci., 121, 110-117.

Wibaut, J. P., Hoog, H., Langedijk, S. L., Overhoff, J., Smittenberg, J., Benninga, N., Bouman, G. P., van Dijk, H., Gaade, W., Geldof, H., Hackmann, J. Th., Jonker, E. W., Paap, T., \& Zuiderweg, F. J. (1939). Study on the Preparation and the Physical Constants of A Number of Alkanes and Cycloalkanes. Recl. Trav. Chim. Pays-Bas, 58, 329-377.

Wilcock, D. F. (1946). Vapor Pressure-Viscosity Relations in Methylpolysiloxanes. J. Am. Chem. Soc., 68, 691-696.

Wobser, R., \& Muller, F. (1941). Die Innere Reibung von Gasen und Dampfen und Ihre Messung. Kolloidchem. Beih, 52, 165-276.

Wu, J., Li, X., Zheng, H., \& Assael, M. J. (2009). Thermal Conductivity of Liquid Dimethyl Ether from (233 to 373) K at Pressures up to $30 \mathrm{MPa}$. J. Chem. Eng. Data, 54, 17201723.

Wu, J., Zhou, Y., \& Lemmon, E. W. (2011). An equation of state for the thermodynamic properties of dimethyl ether. J. Phys. Chem. Ref. Data, 40, 023104.

Yang, C., Xu, W., \& Ma, P. (2004). Excess Molar Volumes and Viscosities of Binary Mixtures of Dimethyl Carbonate with Chlorobenzene, Hexane, and Heptane from (293.15 to 353.15) K and at Atmospheric Pressure. J. Chem. Eng. Data, 49, 1802-1808. 
Yang, L., Luo, T.-L., Lian, H.-L., \& Liu, G.-J. (2010). Density and Viscosity of (2,2-DichloroN,N-di-2-propenylacetamide + Acetone) and (2,2-Dichloro-N,N-di-2-propenylacetamide + Ethanol) at $\mathrm{T}=(278.15$ to 313.15) K. J. Chem. Eng. Data, 55, 1364-1367.

Yao, L., Hammond, E., \& Wang, T. (2008). Melting points and viscosities of fatty acid esters that are potential targets for engineered oilseed. J. Am. Oil Chem. Soc., 85, 77-82.

Yata, J., Hori, M., Kobayashi, K., \& Minamiyama, T. (1996). Thermal conductivity of alternative refrigerants in the liquid phase. Int. J. Thermophys., 17, 561-571.

Yata, J., Minamiyama, T., \& Tanaka, S. (1984). Measurement of thermal conductivity of liquid fluorocarbons. Int. J. Thermophys., 5, 209-218.

Yaws, C. L. (2014). Thermophysical Properties of Chemicals and Hydrocarbons. Austin, U.S.A.: Elsevier Science.

Yin, Y., Zhu, C., \& Ma, Y. (2016). Volumetric and viscometric properties of binary and ternary mixtures of 1-butyl-3-methylimidazolium tetrafluoroborate, monoethanolamine and water. J. Chem. Thermodyn., 102, 413-428.

Zaitseva, L. S. (1959). Experimental study of the thermal conductivity of monoatomic gases in a wide temperature range. Zh. Tekh. Fiz., 29, 497-505.

Zambrano, J. R., Sobrino, M., Martin, M. C., Villamanan, M. A., Chamorro, C. R., \& Segovia, J. J. (2016). Contributing to accurate high pressure viscosity measurements: Vibrating wire viscometer and falling body viscometer techniques. J. Chem. Thermodyn., 96, 104-116. doi:10.1016/j.jct.2015.12.021

Zeberg-Mikkelsen, C. K., Barrouhou, M., \& Boned, C. (2003). Viscosity and Density Measurements of Binary Mixtures Composed of Methylcyclohexane + cis-Decalin Versus Temperature and Pressure. Int. J. Thermnophys., 24, 361-374.

Zhao, T., Zhang, J., Guo, B., Zhang, F., Sha, F., Xie, X., \& Wei, X. (2015). Density, viscosity and spectroscopic studies of the binary system of ethylene glycol + dimethyl sulfoxide at $\mathrm{T}=(298.15$ to 323.15) K. J. Mol. Liq., 207, 315-322.

Zhou, Y., \& Lemmon, E. W. (2016). Equation of State for the Thermodynamic Properties of 1,1,2,2,3-Pentafluoropropane (R-245ca). Int. J. Thermophys., 37, 27.

Zhou, Y., \& Lemmon, E. W. (2018a). Equations of State for RE245cb2, RE347mcc, RE245fa2, and R1216. to be submitted to J. Phys. Chem. Ref. Data.

Zhou, Y., \& Lemmon, E. W. (2018b). [Helmholtz equation of state for ethylene glycol, unpublished, implemented in REFPROP v10].

Zhou, Y., Wu, J. T., \& Lemmon, E. W. (2011). Thermodynamic Properties of Dimethyl Carbonate. J. Phys. Chem. Ref. Data, 40(4), 043106. doi:10.1063/1.3664084

Zimakov, P. V., \& Sokolava, A. V. (1953). Physical properties of propylene oxide. Zh. Fiz. Khim., 27, 1079-1081. 
Table 1. Critical parameters and Lennard-Jones parameters.

\begin{tabular}{|c|c|c|c|c|c|c|c|}
\hline Fluid & CAS & $T_{\mathrm{c}}(\mathrm{K})$ & $P_{\mathrm{c}}(\mathrm{MPa})$ & $\rho_{\mathrm{c}}\left(\mathrm{mol} \cdot \mathrm{dm}^{-3}\right)$ & $M\left(\mathrm{~g} \cdot \mathrm{mol}^{-1}\right)$ & $\varepsilon / k_{\mathrm{B}}(\mathrm{K})$ & $\sigma(\mathrm{nm})$ \\
\hline$n$-pentane & $109-66-0$ & 469.7 & 3.3675 & 3.21 & 72.14878 & 349.44 & 0.5790 \\
\hline isopentane & $78-78-4$ & 460.35 & 3.378 & 3.271 & 72.14878 & 365.56 & 0.5450 \\
\hline neopentane & $463-82-1$ & 433.74 & 3.196 & 3.27 & 72.14878 & 255.65 & 0.61887 \\
\hline isohexane & $107-83-5$ & 497.7 & 3.040 & 2.715 & 86.17536 & 395.2 & 0.580 \\
\hline 3-methylpentane & 96-14-0 & 506.0 & 3.1845 & 2.78 & 86.17536 & 401.81 & 0.575 \\
\hline 2,2-dimethylbutane & \begin{tabular}{|l|}
$75-83-2$ \\
\end{tabular} & 490 & 3.138 & 2.78 & 86.17536 & 389.1 & 0.575 \\
\hline 2,3-dimethylbutane & \begin{tabular}{|l|}
$79-29-8$ \\
\end{tabular} & 500.6 & 3.161 & 2.8 & 86.17536 & 397.5 & 0.574 \\
\hline isooctane & 540-84-1 & 544.0 & 2.572 & 2.12 & 114.22852 & 635.7 & 0.588 \\
\hline$n$-docosane & $629-97-0$ & 792.2 & 1.174 & 0.723 & 310.601 & 515.83 & 1.062 \\
\hline propylene & $115-07-1$ & 364.211 & 4.555 & 5.457 & 42.07974 & 298.9 & \begin{tabular}{|l|}
0.4678 \\
\end{tabular} \\
\hline isobutene & $115-11-7$ & 418.09 & 4.0098 & 4.17 & 56.10632 & 425.0 & 0.4776 \\
\hline trans-2-butene & 624-64-6 & 428.61 & 4.0273 & 4.213 & 56.10632 & 259.0 & 0.5508 \\
\hline cis-2-butene & 590-18-1 & 435.75 & 4.2255 & 4.244 & 56.10632 & 259.0 & 0.5508 \\
\hline 1-butene & $106-98-9$ & 419.29 & 4.0051 & 4.24 & 56.10632 & 319.0 & 0.5198 \\
\hline 1-pentene & $109-67-1$ & 465.74 & 3.598 & 3.45 & 70.1329 & 369.8 & 0.5354 \\
\hline propadiene & 463-49-0 & 398.0 & 5.2156 & 5.9 & 40.06386 & 316.0 & \begin{tabular}{|l|}
0.4477 \\
\end{tabular} \\
\hline 1,3-butadiene & 106-99-0 & 425.135 & 4.3053 & 4.53 & 54.09044 & 337.6 & \begin{tabular}{|l|}
0.4889 \\
\end{tabular} \\
\hline acetylene (ethyne) & \begin{tabular}{|l|}
$74-86-2$ \\
\end{tabular} & 308.3 & 5.9882 & 8.83 & 26.03728 & 244.8 & \begin{tabular}{|l|}
0.3914 \\
\end{tabular} \\
\hline propyne & \begin{tabular}{|l|}
$74-99-7$ \\
\end{tabular} & 402.38 & 5.626 & 6.1133 & 40.06386 & 246.85 & 0.478 \\
\hline 1-butyne & $107-00-6$ & 432 & 4.1416 & 4.65 & 54.09044 & 343.0 & 0.4847 \\
\hline cyclopropane & $75-19-4$ & 398.3 & 5.5797 & 6.1429 & 42.081 & 316.29 & 0.442 \\
\hline cyclopentane & $287-92-3$ & 511.72 & 4.5828 & 3.92 & 70.1329 & 406.33 & 0.518 \\
\hline methylcyclohexane & $108-87-2$ & 572.2 & 3.470 & 2.72 & 98.18606 & 454.38 & 0.5795 \\
\hline propylcyclohexane & $1678-92-8$ & 630.8 & 2.860 & 2.06 & 126.23922 & 500.91 & \begin{tabular}{|l|}
0.6358 \\
\end{tabular} \\
\hline cyclobutene & $822-35-5$ & 448 & 5.1495 & 5.17 & 54.09044 & 355.8 & 0.4679 \\
\hline dimethyl ether & $115-10-6$ & 400.378 & 5.3368 & 5.94 & 46.06844 & 395.0 & 0.4307 \\
\hline
\end{tabular}




\begin{tabular}{|c|c|c|c|c|c|c|c|}
\hline Fluid & CAS & $T_{\mathrm{c}}(\mathbf{K})$ & $P_{\mathrm{c}}(\mathrm{MPa})$ & $\rho_{\mathrm{c}}\left(\mathrm{mol} \cdot \mathrm{dm}^{-3}\right)$ & $M\left(\mathrm{~g} \cdot \mathrm{mol}^{-1}\right)$ & $\varepsilon / k_{\mathrm{B}}(\mathrm{K})$ & $\sigma(\mathbf{n m})$ \\
\hline diethyl ether & $60-29-7$ & 466.7 & 3.7202 & 3.5617 & 74.1216 & 370.6 & 0.530 \\
\hline hexamethyldisiloxane & $107-46-0$ & 518.7 & 1.93113 & 1.653 & 162.3768 & 357.0 & 0.719 \\
\hline octamethyltrisiloxane & $107-51-7$ & 565.3609 & 1.4375 & 1.134 & 236.53146 & 448.9 & 0.776 \\
\hline decamethyltetrasiloxane & $141-62-8$ & 599.40 & 1.144 & 0.864 & 310.6854 & 476.0 & 0.849 \\
\hline dodecamethylpentasiloxane & $141-63-9$ & 628.96 & 0.96112 & 0.7 & 384.839 & 499.5 & 0.911 \\
\hline tetradecamethylhexasiloxane & $107-52-8$ & 653.2 & 0.84037 & 0.57 & 458.99328 & 518.7 & 0.976 \\
\hline octamethylcyclotetrasiloxane & $556-67-2$ & 586.5 & 1.3472 & 1.043 & 296.61576 & 465.7 & 0.798 \\
\hline decamethylcyclopentasiloxane & $541-02-6$ & 618.3 & 1.0934 & 0.82 & 370.7697 & 491.0 & 0.864 \\
\hline dodecamethylcyclohexasiloxane & $540-97-6$ & 645.78 & 0.961 & 0.6272885478 & 444.924 & 512.8 & 0.945 \\
\hline $\mathrm{R} 40$ & $74-87-3$ & 416.3 & 6.6899 & 7.194233 & 50.48752 & 330.6 & 0.419 \\
\hline $\mathrm{R} 1140$ & $75-01-4$ & 424.964 & 5.5903 & 5.62 & 62.49822 & 337.46 & 0.455 \\
\hline R1123 & $359-11-5$ & 331.73 & 4.5426 & 6.0 & 82.02455 & 263.4 & 0.4452 \\
\hline $\mathrm{R} 143 \mathrm{a}$ & $420-46-2$ & 345.857 & 3.761 & 5.12845 & 84.041 & 301.76 & 0.4827 \\
\hline R1243zf & $677-21-4$ & 376.93 & 3.5179 & 4.3 & 96.05113 & 299.3 & 0.4975 \\
\hline R150 & $107-06-2$ & 561.6 & 5.22612 & 4.33 & 98.9592 & 445.96 & 0.4963 \\
\hline RE143a & $421-14-7$ & \begin{tabular}{|l|}
377.921 \\
\end{tabular} & 3.635 & 4.64814 & 100.0398 & 300.104 & 0.4847 \\
\hline chlorobenzene & $108-90-7$ & 632.35 & 4.5206 & 3.24 & 112.557 & 502.1 & 0.547 \\
\hline R1234ze(Z) & $29118-25-0$ & 423.27 & 3.5306 & 4.0 & 114.0416 & 336.11 & 0.5096 \\
\hline R1233zd(E) & $102687-65-0$ & 439.6 & 3.6237 & 3.68 & 130.4962 & 349.1 & 0.524 \\
\hline R245ca & $679-86-7$ & 447.57 & 3.9407 & 3.92 & 134.04794 & 355.41 & 0.5131 \\
\hline $\mathrm{R} 365 \mathrm{mfc}$ & $406-58-6$ & 460.0 & 3.266 & 3.2 & 148.07452 & 365.28 & 0.5490 \\
\hline R1224yd(Z) & $111512-60-8$ & 428.69 & 3.337 & 3.55 & 148.4867 & 340.42 & 0.530 \\
\hline RE245cb2 & $22410-44-2$ & \begin{tabular}{|l|}
406.813 \\
\end{tabular} & \begin{tabular}{|l|}
2.8864 \\
\end{tabular} & 3.329 & 150.047336 & 323.05 & 0.5418 \\
\hline RE245fa2 & $1885-48-9$ & 444.88 & 3.433 & 3.432258 & 150.047336 & 353.28 & 0.5363 \\
\hline R236fa & $690-39-1$ & \begin{tabular}{|l|}
398.07 \\
\end{tabular} & 3.2 & 3.626 & 152.0384 & 307.24 & 0.5644 \\
\hline R236ea & $431-63-0$ & 412.44 & 3.42 & 3.71617 & 152.0384 & 318.33 & 0.5604 \\
\hline R1336mzz(Z) & $692-49-9$ & 444.5 & 2.903 & 3.044 & 164.056 & 352.97 & 0.5582 \\
\hline R114 & 76-14-2 & 418.83 & 3.257 & 3.3932 & 170.921 & 174.0 & 0.648 \\
\hline R218 & 76-19-7 & \begin{tabular}{|l|}
345.02 \\
\end{tabular} & 2.640 & 3.34 & 188.01933 & 266.35 & 0.580 \\
\hline $\mathrm{CF}_{3} \mathrm{I}$ & $2314-97-8$ & 396.44 & 3.953 & 4.4306 & 195.9104 & 314.8 & 0.4926 \\
\hline RC318 & $115-25-3$ & 388.38 & 2.7775 & 3.09938 & 200.04 & 308.41 & 0.5549 \\
\hline
\end{tabular}




\begin{tabular}{|c|c|c|c|c|c|c|c|}
\hline Fluid & CAS & $T_{\mathrm{c}}(\mathrm{K})$ & $P_{\mathrm{c}}(\mathrm{MPa})$ & $\rho_{\mathrm{c}}\left(\mathrm{mol} \cdot \mathrm{dm}^{-3}\right)$ & $M\left(\mathrm{~g} \cdot \mathrm{mol}^{-1}\right)$ & $\varepsilon / k_{\mathrm{B}}(\mathrm{K})$ & $\sigma(\mathrm{nm})$ \\
\hline RE347mcc & $375-03-1$ & 437.7 & 2.4782 & 2.64 & 200.0548424 & 347.6 & 0.5853 \\
\hline perfluorobutane & $355-25-9$ & 386.326 & 2.3224 & 2.637 & 238.027 & 179 & 0.694 \\
\hline perfluoropentane & $678-26-2$ & 421.0 & 2.063 & 2.17 & 288.034 & 195 & 0.736 \\
\hline perfluorohexane & $355-42-0$ & 448.0 & \begin{tabular}{|l|}
1.7416 \\
\end{tabular} & 1.825 & 338.042 & 160 & 0.805 \\
\hline methyl palmitate & $112-39-0$ & 755 & 1.350 & 0.897 & 270.45066 & 599.54 & 0.8388 \\
\hline methyl linolenate & $301-00-8$ & 772 & 1.369 & 0.8473 & 292.45618 & 613.04 & 0.8549 \\
\hline methyl linoleate & $112-63-0$ & 799 & 1.341 & \begin{tabular}{|l|}
0.8084 \\
\end{tabular} & 294.47206 & 634.48 & 0.8684 \\
\hline methyl oleate & $112-62-9$ & 782 & 1.246 & 0.81285 & 296.48794 & 620.98 & 0.8668 \\
\hline methyl stearate & $112-61-8$ & 775 & 1.239 & 0.7943 & 298.50382 & 615.42 & 0.8735 \\
\hline neon & $7440-01-9$ & 44.4 & 2.66163 & 24.1 & 20.179 & 45.58 & 0.2707 \\
\hline krypton & $7439-90-9$ & 209.48 & 5.525 & 10.85 & 83.798 & 178.9 & 0.3655 \\
\hline xenon & $7440-63-3$ & 289.733 & 5.842 & 8.4 & 131.293 & 260.91 & 0.3929 \\
\hline hydrogen sulfide & 7783-06-4 & 373.1 & 9.0 & 10.19 & 34.08088 & 296.28 & 0.3732 \\
\hline carbonyl sulfide & $463-58-1$ & 378.77 & 6.370 & 7.41 & 60.0751 & 335. & 0.413 \\
\hline carbon monoxide & $630-08-0$ & 132.86 & 3.494 & 10.85 & 28.0101 & 103.697 & 0.3615 \\
\hline nitrous oxide & 10024-97-2 & 309.52 & 7.245 & 10.27 & 44.0128 & 232.4 & 0.3828 \\
\hline ethylene oxide & \begin{tabular}{|l|}
$75-21-8$ \\
\end{tabular} & 468.92 & \begin{tabular}{|l|}
3.7047 \\
\end{tabular} & 7.17 & 44.05256 & 372.37 & 0.420 \\
\hline propylene oxide & \begin{tabular}{|l|}
$75-56-9$ \\
\end{tabular} & 488.11 & 5.4366 & 5.155 & 58.07914 & 387.6 & 0.4683 \\
\hline sulfur dioxide & 7446-09-5 & 430.64 & \begin{tabular}{|l}
7.8866 \\
\end{tabular} & 8.078 & 64.0638 & 363 & 0.4026 \\
\hline chlorine & $7782-50-5$ & 416.8654 & \begin{tabular}{|l|}
7.6424 \\
\end{tabular} & 8.06 & 70.906 & 257 & 0.44 \\
\hline fluorine & $7782-41-4$ & 144.414 & 5.1724 & 15.603 & 37.99681 & 112.6 & 0.3357 \\
\hline hydrogen chloride & $7647-01-0$ & 324.68 & 8.3135 & 11.87 & 36.46094 & 257.8 & 0.355 \\
\hline dimethyl carbonate & $616-38-6$ & 557.0 & 4.9088 & 4.0 & 90.0779 & 442.3 & 0.510 \\
\hline acetone & $67-64-1$ & 508.1 & 4.6924 & 4.7 & 58.07914 & 519 & 0.4669 \\
\hline monoethanolamine & $141-43-5$ & 671.4 & 8.125 & 5.39 & 61.0831 & 533.15 & 0.4614 \\
\hline diethanolamine & $111-42-2$ & 736.5 & 4.95075 & 3.3 & 105.1356 & 584.85 & 0.5434 \\
\hline ethylene glycol & $107-21-1$ & 719.0 & 10.5087 & 5.88 & 62.06784 & 570.95 & 0.4482 \\
\hline
\end{tabular}


Table 2. Coefficients for the residual viscosity, Eq. (11), and $F_{\mathrm{c}}$ from Eq. (8).

\begin{tabular}{|c|c|c|c|c|c|c|}
\hline Fluid & Ref fluid & $F_{\mathbf{c}}$ & $c_{0}$ & $c_{1}$ & $c_{2}$ & $c_{3}$ \\
\hline$n$-pentane & propane & 1.0 & 0.455019 & 0.677221 & -0.277823 & 0.0372505 \\
\hline isopentane & propane & 0.95 & 0.939665 & 0.0093105 & 0.0135256 & -0.00262931 \\
\hline neopentane & propane & 1 & 1.06643 & 0.00804685 & 0 & 0 \\
\hline isohexane & propane & 1 & 1.26640 & -0.191045 & 0.0336982 & 0 \\
\hline 3-methylpentane & propane & 1 & 1.024 & -0.0248711 & 0.00554504 & 0 \\
\hline 2,2-dimethylbutane & propane & 1 & 0.968903 & 0.0318896 & 0 & 0 \\
\hline 2,3-dimethylbutane & propane & 1 & 1.25327 & -0.178928 & 0.0348391 & 0 \\
\hline isooctane & octane & 1 & 1.09755 & -0.0223075 & 0 & 0 \\
\hline$n$-docosane & dodecane & 1 & 1.20571 & -0.0689138 & 0 & 0 \\
\hline propylene & propane & 1 & 1.135460 & -0.0232208 & -0.0447703 & 0.0121815 \\
\hline isobutene & propane & 1 & 1.12449 & -0.147034 & 0.0366550 & 0 \\
\hline trans-2-butene & propane & 1 & 1.12449 & -0.147034 & 0.0366550 & 0 \\
\hline cis-2-butene & propane & 1 & 1.12449 & -0.147034 & 0.0366550 & 0 \\
\hline 1-butene & propane & 1 & 1.12449 & -0.147034 & 0.0366550 & 0 \\
\hline 1-pentene & propane & 0.96 & 0.97 & 0 & 0 & 0 \\
\hline propadiene & propane & 1 & 0.99 & 0 & 0 & 0 \\
\hline 1,3-butadiene & propane & 1 & 0.569829 & 0.169932 & -0.00650648 & 0 \\
\hline acetylene & propane & 0.97 & 0.98 & 0 & 0 & 0 \\
\hline propyne & propane & 1 & 0.98 & 0 & 0 & 0 \\
\hline 1-butyne & propane & 1 & 0.98 & 0 & 0 & 0 \\
\hline cyclopropane & propane & 0.95 & 1.0 & 0 & 0 & 0 \\
\hline cyclopentane & propane & 0.99 & 1.04775 & $-8.02057 \times 10^{-4}$ & 0 & 0 \\
\hline methylcyclohexane & propane & 1 & 1.2122 & -0.129599 & 0.0257019 & 0 \\
\hline propylcyclohexane & propane & 1 & 1.85997 & -0.587812 & 0.103092 & 0 \\
\hline cyclobutene & propane & 1 & 1 & 0 & 0 & 0 \\
\hline dimethyl ether & propane & 1 & 1.81678 & -0.696062 & 0.13901 & 0 \\
\hline diethyl ether & propane & 0.90 & 1.15039 & -0.1535 & 0.0330048 & 0 \\
\hline hexamethyldisiloxane & nitrogen & 1 & -0.413767 & 1.17675 & -0.241401 & 0 \\
\hline octamethyltrisiloxane & nitrogen & 1 & 1.46043 & -0.161196 & 0 & 0 \\
\hline
\end{tabular}




\begin{tabular}{|c|c|c|c|c|c|c|}
\hline Fluid & Ref fluid & $F_{\mathrm{c}}$ & $c_{0}$ & $c_{1}$ & $c_{2}$ & $c_{3}$ \\
\hline decamethyltetrasiloxane & nitrogen & 1 & 1.41415 & -0.14233 & 0 & 0 \\
\hline dodecamethylpentasiloxane & nitrogen & 1 & 1.45796 & -0.15796 & 0 & 0 \\
\hline tetradecamethylhexasiloxane & nitrogen & 1 & 1.45542 & -0.154807 & 0 & 0 \\
\hline octamethylcyclotetrasiloxane & nitrogen & 1 & 2.42579 & -0.871777 & 0.137283 & 0 \\
\hline decamethylcyclopentasiloxane & nitrogen & 1 & -2.49055 & 4.63356 & -1.89292 & 0.247782 \\
\hline dodecamethylcyclohexasiloxane & nitrogen & 1 & 1.06991 & 0.00448715 & 0 & 0 \\
\hline $\mathrm{R} 40$ & $\mathrm{R} 134 \mathrm{a}$ & 0.97 & 0.894605 & -0.0117296 & 0.0130264 & 0 \\
\hline R1140 (Vinyl chloride) & R134a & 1.06 & 0.991393 & -0.0190085 & 0 & 0 \\
\hline R1123 (trifluoroethylene) & R134a & 1 & 1 & 0 & 0 & 0 \\
\hline R143a (1,1,1-trifluoroethane) & R134a & 0.992 & 0.942896 & 0.0142114 & 0 & 0 \\
\hline $\begin{array}{l}\text { R1243zf (3,3,3- } \\
\text { Trifluoropropene) }\end{array}$ & R134a & 1.01 & 0.98 & 0 & 0 & 0 \\
\hline R150 (1,2-dichloroethane) & propane & 0.96 & 0.766881 & 0.136995 & -0.0161687 & 0 \\
\hline RE143a & R134a & 1 & 1.008 & 0 & 0 & 0 \\
\hline chlorobenzene & $\mathrm{R} 134 \mathrm{a}$ & 0.934 & 0.809284 & 0.0881819 & -0.0147911 & 0 \\
\hline R1234ze(Z) & R134a & 0.85 & 0.829337 & 0.0476201 & 0 & 0 \\
\hline $\mathrm{R} 1233 \mathrm{zd}(\mathrm{E})$ & $\mathrm{R} 134 \mathrm{a}$ & 0.93 & -0.0848988 & 1.22693 & -0.463275 & 0.0568798 \\
\hline R245ca & R134a & 1 & 0.850332 & 0.185603 & -0.0415622 & 0 \\
\hline $\mathrm{R} 365 \mathrm{mfc}$ & R134a & 1 & 0.670823 & 0.316208 & -0.0680077 & 0 \\
\hline R1224yd(Z) & R134a & 0.92 & 0.712387 & 0.186976 & \begin{tabular}{|l|}
-0.0316058 \\
\end{tabular} & 0 \\
\hline RE245cb2 & R134a & 1 & 1.0692 & -0.023595 & 0 & 0 \\
\hline RE245fa2 & R134a & 1 & 1.10656 & -0.0337904 & 0 & 0 \\
\hline R236fa & propane & 1 & 1.08017 & -0.026407 & \begin{tabular}{|l|}
0.00605762 \\
\end{tabular} & 0 \\
\hline R236ea & R134a & 1 & 1.19985 & -0.0906827 & 0.0128243 & 0 \\
\hline $\mathrm{R} 1336 \mathrm{mzz}(\mathrm{Z})$ & R134a & 0.94 & 0.615513 & 0.281144 & -0.0527921 & 0 \\
\hline R114 & propane & 1 & 1.36002 & -0.209356 & 0.0373222 & 0 \\
\hline R218 & propane & 1 & 1.06992 & 0.0100068 & -0.00126094 & 0 \\
\hline $\mathrm{CF}_{3} \mathrm{I}$ & R134a & 0.95 & 1.22725 & -0.0879263 & 0 & 0 \\
\hline RC318 & propane & 0.90 & 1.72536 & -0.454947 & 0.085819 & 0 \\
\hline RE347mcc & propane & 1 & 2.57345 & -1.73973 & 0.659016 & -0.0824925 \\
\hline perfluorobutane & R134a & 1 & 1.045 & 0 & 0 & 0 \\
\hline
\end{tabular}




\begin{tabular}{|c|c|c|c|c|c|c|}
\hline Fluid & Ref fluid & $F_{\mathrm{c}}$ & $c_{0}$ & $c_{1}$ & $c_{2}$ & $c_{3}$ \\
\hline perfluoropentane & R134a & 1 & 0.663775 & 0.38206 & -0.0882706 & 0 \\
\hline perfluorohexane & R134a & 1 & 0.673625 & 0.35383 & -0.0787347 & 0 \\
\hline methyl palmitate & propane & 1 & 1.441560 & -0.253261 & 0.0359069 & 0 \\
\hline methyl linolenate & propane & 1 & \begin{tabular}{|l|}
1.04783 \\
\end{tabular} & -0.0251965 & 0 & 0 \\
\hline methyl linoleate & propane & 1 & 1.49489 & -0.25538 & 0.0306593 & 0 \\
\hline methyl oleate & propane & 1 & 1.92477 & -0.515884 & 0.0703972 & 0 \\
\hline methyl stearate & propane & 1 & \begin{tabular}{|l|}
1.46654 \\
\end{tabular} & -0.260069 & 0.0354629 & 0 \\
\hline neon & nitrogen & 0.989544 & \begin{tabular}{|l|}
1.12101 \\
\end{tabular} & -0.0388911 & 0 & 0 \\
\hline krypton & \begin{tabular}{|l|} 
nitrogen \\
\end{tabular} & 1.008291 & 1 & 0 & 0 & 0 \\
\hline xenon & \begin{tabular}{|l|} 
nitrogen \\
\end{tabular} & 1.0012 & 0.806961 & 0.130263 & -0.0222093 & 0 \\
\hline hydrogen sulfide & propane & 1.0 & 1.0 & 0 & 0 & 0 \\
\hline carbonyl sulfide & propane & 1.0 & 1.0 & 0 & 0 & 0 \\
\hline carbon monoxide & \begin{tabular}{|l|} 
nitrogen \\
\end{tabular} & 1 & \begin{tabular}{|l|}
1.07369 \\
\end{tabular} & -0.0283067 & 0 & 0 \\
\hline nitrous oxide & nitrogen & 1 & \begin{tabular}{|l|}
0.88769 \\
\end{tabular} & 0.0214265 & 0 & 0 \\
\hline ethylene oxide & propane & 1 & 1.29794 & -0.295066 & 0.0626748 & 0 \\
\hline propylene oxide & propane & 0.96 & 0.821761 & 0.0611306 & 0 & 0 \\
\hline sulfur dioxide & propane & 1 & 0.917778 & 0.0248405 & 0 & 0 \\
\hline chlorine & propane & 1 & 1.269 & -0.08947 & 0 & 0 \\
\hline fluorine & propane & 0.97 & 1.8426 & -0.748356 & 0.16319 & 0 \\
\hline hydrogen chloride & propane & 1 & 0.615877 & 0.55609 & -0.337867 & 0.0681029 \\
\hline dimethyl carbonate & propane & 1 & 0.811428 & 0.0616704 & 0 & 0 \\
\hline acetone & propane & 1 & \begin{tabular}{|l|}
1.25183 \\
\end{tabular} & -0.239533 & 0.0485815 & 0 \\
\hline monoethanolamine & propane & 0.88 & \begin{tabular}{|l|}
1.18676 \\
\end{tabular} & -0.260695 & 0.0789293 & 0 \\
\hline diethanolamine & propane & 0.74 & 0.996593 & 0.0399708 & 0 & 0 \\
\hline ethylene glycol & propane & 1 & 0.864168 & -0.00230208 & -0.00183225 & 0.00904878 \\
\hline
\end{tabular}


Table 3. Coefficients for the dilute gas and residual thermal conductivity, Eq. (16), Eq. (21).

\begin{tabular}{|c|c|c|c|c|c|c|c|}
\hline Fluid & Ref fluid & $a_{0}$ & $a_{1}$ & $a_{2}$ & $b_{0}$ & $b_{1}$ & $b_{2}$ \\
\hline neopentane & \begin{tabular}{|l} 
propane \\
\end{tabular} & 0.00130 & 0 & 0 & 1.057 & 0 & 0 \\
\hline isohexane & propane & 0.00115 & 0 & 0 & 1.09075 & -0.0103574 & 0 \\
\hline 3-methylpentane & propane & 0.00125 & 0 & 0 & 0.984496 & 0.0251364 & 0 \\
\hline 2,2-dimethylbutane & propane & 0.00116 & 0 & 0 & 0.962159 & 0.0268488 & 0 \\
\hline 2,3-dimethylbutane & propane & 0.00116 & 0 & 0 & 1.00438 & 0.014484 & 0 \\
\hline isooctane & octane & 0.00115 & 0 & 0 & 0.827544 & 0.0391177 & 0 \\
\hline$n$-docosane & dodecane & 0.00132 & 0 & 0 & 1.31627 & -0.083506 & 0.0127753 \\
\hline isobutene & propane & 0.00102143 & $6.64409 \times 10^{-7}$ & 0 & 0.838527 & 0.0648013 & 0 \\
\hline trans-2-butene & propane & 0.00102143 & $6.64409 \times 10^{-7}$ & 0 & 0.838527 & 0.0648013 & 0 \\
\hline cis-2-butene & propane & 0.00102143 & $6.64409 \times 10^{-7}$ & 0 & 0.838527 & 0.0648013 & 0 \\
\hline 1-butene & propane & 0.0009002390 & $1.13436 \times 10^{-6}$ & 0 & 0.838527 & 0.0648013 & 0 \\
\hline 1-pentene & propane & 0.00077636 & $1.5844 \times 10^{-6}$ & $-1.19304 \times 10^{-9}$ & 1.07723 & -0.00850457 & 0 \\
\hline propadiene & propane & 0.0012 & 0 & 0 & 0.95 & 0 & 0 \\
\hline 1,3-butadiene & propane & 0.0012 & 0 & 0 & 0.95 & 0 & 0 \\
\hline acetylene (ethyne) & propane & $6.46532 \times 10^{-4}$ & $9.98935 \times 10^{-7}$ & $1.22755 \times 10^{-10}$ & 0.9 & 0 & 0 \\
\hline propyne & propane & 0.0012 & 0 & 0 & 0.93 & 0 & 0 \\
\hline 1-butyne & propane & 0.0012 & 0 & 0 & 0.95 & 0 & 0 \\
\hline cyclopropane & propane & 0.00122 & 0 & 0 & 1.0 & 0 & 0 \\
\hline cyclobutene & propane & 0.0012 & 0 & 0 & 0.95 & 0 & 0 \\
\hline dimethyl ether & propane & $-9.8101 \times 10^{-4}$ & $1.22857 \times 10^{-5}$ & $-1.60844 \times 10^{-8}$ & 1.12497 & $-4.88562 \times 10^{-2}$ & 0 \\
\hline diethyl ether & propane & 0.00132 & 0 & 0 & 1.16276 & -0.0357361 & 0 \\
\hline hexamethyldisiloxane & nitrogen & 0.00132 & 0 & 0 & 1.1777 & 0.0473755 & 0 \\
\hline octamethyltrisiloxane & nitrogen & 0.00132 & 0 & 0 & 3.47746 & -1.50335 & 0.27515 \\
\hline decamethyltetrasiloxane & nitrogen & 0.00132 & 0 & 0 & 3.48369 & -1.34356 & 0.23613 \\
\hline dodecamethylpentasiloxane & nitrogen & 0.00132 & 0 & 0 & 1.72213 & 0 & 0 \\
\hline tetradecamethylhexasiloxane & nitrogen & 0.00132 & 0 & 0 & 1.91993 & 0 & 0 \\
\hline octamethylcyclotetrasiloxane & nitrogen & 0.00132 & 0 & 0 & 1.43353 & 0.0407501 & 0 \\
\hline decamethylcyclopentasiloxane & nitrogen & 0.00132 & 0 & 0 & 1.40287 & 0.0940128 & 0 \\
\hline dodecamethylcyclohexasiloxane & nitrogen & 0.00132 & 0 & 0 & 1.38744 & 0.127827 & 0 \\
\hline
\end{tabular}




\begin{tabular}{|c|c|c|c|c|c|c|c|}
\hline Fluid & Ref fluid & $a_{0}$ & $a_{1}$ & $a_{2}$ & $b_{0}$ & $b_{1}$ & $b_{2}$ \\
\hline $\mathrm{R} 40$ & $\mathrm{R} 134 \mathrm{a}$ & $2.78821 \times 10^{-4}$ & $2.10163 \times 10^{-6}$ & 0 & 0.971796 & -0.0356445 & 0 \\
\hline R1140 & R134a & $4.68338 \times 10^{-4}$ & $1.55637 \times 10^{-6}$ & 0 & 1 & 0 & 0 \\
\hline $\mathrm{R} 1123$ & R134a & 0.00125 & 0 & 0 & 1 & 0 & 0 \\
\hline R1243zf & $\mathrm{R} 134 \mathrm{a}$ & 0.00125 & 0 & 0 & 0.96 & 0 & 0 \\
\hline $\mathrm{R} 150$ & propane & $9.18633 \times 10^{-4}$ & $7.08996 \times 10^{-7}$ & 0 & \begin{tabular}{|l|}
1.35752 \\
\end{tabular} & -0.116398 & 0 \\
\hline RE143a & R134a & 0.001129 & 0 & 0 & 0.975 & 0 & 0 \\
\hline chlorobenzene & $\mathrm{R} 134 \mathrm{a}$ & 0.002 & 0 & 0 & 1.14085 & -0.11208 & 0.0189958 \\
\hline R1234ze(Z) & R134a & 0.00162 & 0 & 0 & \begin{tabular}{|l|}
1.10592 \\
\end{tabular} & -0.0471388 & 0 \\
\hline R245ca & R134a & $7.33395 \times 10^{-4}$ & $1.62265 \times 10^{-6}$ & 0 & \begin{tabular}{|l|}
1.04155 \\
\end{tabular} & -0.0118606 & 0 \\
\hline R365mfc & R134a & $4.11581 \times 10^{-3}$ & $-1.70975 \times 10^{-5}$ & $2.55262 \times 10^{-8}$ & 0.434182 & 0.229206 & 0 \\
\hline R1224yd(Z) & R134a & 0.00125 & 0 & 0 & 1.04 & 0 & 0 \\
\hline RE245cb2 & R134a & 0.001129 & 0 & 0 & 0.96324 & 0.027265 & 0 \\
\hline RE245fa2 & $\mathrm{R} 134 \mathrm{a}$ & 0.001668 & $-1.3154 \times 10^{-6}$ & 0 & \begin{tabular}{|l|}
0.61384 \\
\end{tabular} & 0.12385 & 0 \\
\hline R236fa & propane & 0.00100946 & $1.21255 \times 10^{-6}$ & 0 & 1.29118 & -0.0785568 & 0 \\
\hline R236ea & R134a & 0.0054277 & $-2.33425 \times 10^{-5}$ & $3.46098 \times 10^{-8}$ & 0.961712 & 0.0337897 & 0 \\
\hline R1336mzz(Z) & $\mathrm{R} 134 \mathrm{a}$ & 0.00109396 & $0.675562 \times 10^{-6}$ & 0 & 1.09323 & -0.0316036 & 0 \\
\hline R114 & propane & 0.00132 & 0 & 0 & 1.2005 & -0.0533827 & 0 \\
\hline R218 & propane & $5.99446 \times 10^{-4}$ & $2.29822 \times 10^{-6}$ & $-9.77006 \times 10^{-10}$ & 0.466251 & 0.54426 & -0.110279 \\
\hline $\mathrm{CF}_{3} \mathrm{I}$ & R134a & $1.28541 \times 10^{-3}$ & $5.32854 \times 10^{-7}$ & 0 & 1.0 & 0 & 0 \\
\hline $\mathrm{RC} 318$ & propane & $1.24931 \times 10^{-3}$ & $6.94039 \times 10^{-8}$ & 0 & 1.43669 & -0.113691 & 0 \\
\hline perfluorobutane & R134a & 0.00125 & 0 & 0 & 1.990 & -0.33 & 0 \\
\hline perfluoropentane & R134a & 0.00125 & 0 & 0 & \begin{tabular}{|l|}
1.99279 \\
\end{tabular} & -0.308118 & 0 \\
\hline perfluorohexane & R134a & 0.00125 & 0 & 0 & \begin{tabular}{|l|}
1.99965 \\
\end{tabular} & -0.290494 & 0 \\
\hline neon & nitrogen & 0.00132 & 0 & 0 & \begin{tabular}{|l|}
0.83 \\
\end{tabular} & 0 & 0 \\
\hline krypton & nitrogen & 0.00132 & 0 & 0 & 0.962573 & -0.0118156 & 0 \\
\hline xenon & nitrogen & 0.00132 & 0 & 0 & 0.906192 & 0.006383 & 0 \\
\hline hydrogen sulfide & propane & $1.5603 \times 10^{-4}$ & $1.78874 \times 10^{-6}$ & $-6.75136 \times 10^{-10}$ & 1.0 & 0 & 0 \\
\hline carbonyl sulfide & propane & 0.00125 & 0 & 0 & 0.95 & 0 & 0 \\
\hline carbon monoxide & nitrogen & $3.29558 \times 10^{-4}$ & $3.05976 \times 10^{-6}$ & $-3.13222 \times 10^{-9}$ & \begin{tabular}{|l|}
1.00037 \\
\end{tabular} & -0.0082682 & 0 \\
\hline nitrous oxide & nitrogen & $5.15648 \times 10^{-4}$ & $2.85508 \times 10^{-6}$ & $-2.46391 \times 10^{-9}$ & 0.923824 & 0.03315898 & 0 \\
\hline ethylene oxide & propane & $3.03522 \times 10^{-4}$ & $1.99873 \times 10^{-6}$ & 0 & 0.9 & -0.005 & 0 \\
\hline
\end{tabular}




\begin{tabular}{|l|l|l|l|l|l|l|l|}
\hline Fluid & Ref fluid & $\boldsymbol{a}_{\mathbf{0}}$ & $\boldsymbol{a}_{\mathbf{1}}$ & $\boldsymbol{a}_{2}$ & $\boldsymbol{b}_{\mathbf{0}}$ & $\boldsymbol{b}_{\mathbf{1}}$ & $\boldsymbol{b}_{2}$ \\
\hline propylene oxide & propane & 0.00132 & 0 & 0 & 1.04 & 0 \\
\hline sulfur dioxide & propane & $6.60505 \times 10^{-4}$ & $7.47059 \times 10^{-7}$ & 0 & 1.38755 & -0.128721 & 0 \\
\hline chlorine & propane & 0.0029 & 0 & 0 & 1.24341 & -0.0812555 & 0 \\
\hline fluorine & propane & 0.00105 & 0 & 0 & 0.753172 & 0.0658443 & 0 \\
\hline hydrogen chloride & propane & 0.0006 & 0 & 0 & 1.57373 & -0.17681 & 0 \\
\hline dimethyl carbonate & propane & 0.00132 & 0 & 0 & 1.12380 & -0.0154353 & 0 \\
\hline acetone & propane & $0.954299 \times 10^{-3}$ & $0.522303 \times 10^{-6}$ & 0 & 1.08482 & -0.0313081 & 0 \\
\hline monoethanolamine & propane & 0.00132 & 0 & 0 & 1.61924 & -0.210496 & 0 \\
\hline diethanolamine & propane & 0.00132 & 0 & 0 & 1.47408 & -0.123082 & 0 \\
\hline ethylene glycol & propane & 0.00132 & 0 & 0 & 1.79177 & -0.275354 & 0 \\
\hline
\end{tabular}


Table 4. Coefficients for the critical enhancement of thermal conductivity.

\begin{tabular}{|c|c|c|c|}
\hline Fluid & $\bar{\Gamma}_{0}$ & $\xi_{0}(\mathbf{m})$ & $q \mathrm{D}^{-1}(\mathrm{~m})$ \\
\hline neopentane & 0.057 & $0.230 \times 10^{-9}$ & $0.664 \times 10^{-9}$ \\
\hline isohexane & 0.059 & $0.238 \times 10^{-9}$ & $0.708 \times 10^{-9}$ \\
\hline 3-methylpentane & 0.059 & $0.237 \times 10^{-9}$ & $0.703 \times 10^{-9}$ \\
\hline 2,2-dimethylbutane & 0.059 & $0.240 \times 10^{-9}$ & $0.703 \times 10^{-9}$ \\
\hline 2,3-dimethylbutane & 0.058 & $0.238 \times 10^{-9}$ & $0.701 \times 10^{-9}$ \\
\hline isooctane & 0.059 & $0.256 \times 10^{-9}$ & $0.771 \times 10^{-9}$ \\
\hline$n$-docosane & 0.067 & $0.310 \times 10^{-9}$ & $1.114 \times 10^{-9}$ \\
\hline isobutene & 0.058 & $0.212 \times 10^{-9}$ & $0.611 \times 10^{-9}$ \\
\hline trans-2-butene & 0.057 & $0.210 \times 10^{-9}$ & $0.609 \times 10^{-9}$ \\
\hline cis-2-butene & 0.058 & $0.210 \times 10^{-9}$ & $0.607 \times 10^{-9}$ \\
\hline 1-butene & 0.057 & $0.211 \times 10^{-9}$ & $0.607 \times 10^{-9}$ \\
\hline 1-pentene & 0.058 & $0.223 \times 10^{-9}$ & $0.652 \times 10^{-9}$ \\
\hline propadiene & 0.055 & $0.195 \times 10^{-9}$ & $0.541 \times 10^{-9}$ \\
\hline 1,3-butadiene & 0.057 & $0.207 \times 10^{-9}$ & $0.593 \times 10^{-9}$ \\
\hline acetylene (ethyne) & 0.056 & $0.166 \times 10^{-9}$ & $0.470 \times 10^{-9}$ \\
\hline propyne & 0.058 & $0.186 \times 10^{-9}$ & $0.535 \times 10^{-9}$ \\
\hline 1-butyne & 0.054 & $0.199 \times 10^{-9}$ & $0.588 \times 10^{-9}$ \\
\hline cyclopropane & 0.057 & $0.191 \times 10^{-9}$ & $0.534 \times 10^{-9}$ \\
\hline cyclobutene & 0.056 & $0.200 \times 10^{-9}$ & $0.567 \times 10^{-9}$ \\
\hline dimethyl ether & 0.057 & $0.189 \times 10^{-9}$ & $0.540 \times 10^{-9}$ \\
\hline diethyl ether & 0.066 & $0.196 \times 10^{-9}$ & $0.645 \times 10^{-9}$ \\
\hline hexamethyldisiloxane & 0.062 & $0.268 \times 10^{-9}$ & $0.840 \times 10^{-9}$ \\
\hline octamethyltrisiloxane & 0.064 & $0.295 \times 10^{-9}$ & $0.956 \times 10^{-9}$ \\
\hline decamethyltetrasiloxane & 0.066 & $0.311 \times 10^{-9}$ & $1.049 \times 10^{-9}$ \\
\hline dodecamethylpentasiloxane & 0.066 & $0.330 \times 10^{-9}$ & $1.127 \times 10^{-9}$ \\
\hline tetradecamethylhexasiloxane & 0.070 & $0.347 \times 10^{-9}$ & $1.208 \times 10^{-9}$ \\
\hline octamethylcyclotetrasiloxane & 0.064 & $0.298 \times 10^{-9}$ & $0.983 \times 10^{-9}$ \\
\hline decamethylcyclopentasiloxane & 0.064 & $0.319 \times 10^{-9}$ & $1.068 \times 10^{-9}$ \\
\hline dodecamethylcyclohexasiloxane & 0.072 & $0.341 \times 10^{-9}$ & $1.170 \times 10^{-9}$ \\
\hline $\mathrm{R} 40$ & 0.056 & $0.180 \times 10^{-9}$ & $0.505 \times 10^{-9}$ \\
\hline R1140 (Vinyl chloride) & 0.059 & $0.195 \times 10^{-9}$ & $0.551 \times 10^{-9}$ \\
\hline R1123 (trifluoroethylene) & 0.075 & $0.153 \times 10^{-9}$ & $0.538 \times 10^{-9}$ \\
\hline R143a & 0.055 & $0.193 \times 10^{-9}$ & $0.230 \times 10^{-9}$ \\
\hline R1243zf & 0.056 & $0.205 \times 10^{-9}$ & $0.604 \times 10^{-9}$ \\
\hline R150 (1,2-dichloroethane) & 0.056 & $0.204 \times 10^{-9}$ & $0.603 \times 10^{-9}$ \\
\hline RE143a & 0.054 & $0.198 \times 10^{-9}$ & $0.588 \times 10^{-9}$ \\
\hline chlorobenzene & 0.098 & $0.160 \times 10^{-9}$ & $0.666 \times 10^{-9}$ \\
\hline R1234ze(Z) & 0.055 & $0.206 \times 10^{-9}$ & $0.620 \times 10^{-9}$ \\
\hline R245ca & 0.060 & $0.205 \times 10^{-9}$ & $0.624 \times 10^{-9}$ \\
\hline $\mathrm{R} 365 \mathrm{mfc}$ & 0.060 & $0.218 \times 10^{-9}$ & $0.669 \times 10^{-9}$ \\
\hline R1224yd(Z) & 0.058 & $0.214 \times 10^{-9}$ & $0.646 \times 10^{-9}$ \\
\hline
\end{tabular}




\begin{tabular}{|c|c|c|c|}
\hline Fluid & $\bar{\Gamma}_{0}$ & $\xi_{0}(\mathbf{m})$ & $q \mathrm{D}^{-1}(\mathrm{~m})$ \\
\hline RE245cb2 & 0.057 & $0.217 \times 10^{-9}$ & $0.660 \times 10^{-9}$ \\
\hline RE245fa2 & 0.061 & $0.212 \times 10^{-9}$ & $0.653 \times 10^{-9}$ \\
\hline R236fa & 0.060 & $0.209 \times 10^{-9}$ & $0.641 \times 10^{-9}$ \\
\hline R236ea & 0.060 & $0.208 \times 10^{-9}$ & $0.636 \times 10^{-9}$ \\
\hline $\mathrm{R} 1336 \mathrm{mzz}(\mathrm{Z})$ & 0.058 & $0.221 \times 10^{-9}$ & $0.681 \times 10^{-9}$ \\
\hline R114 & 0.059 & $0.223 \times 10^{-9}$ & $0.656 \times 10^{-9}$ \\
\hline $\mathrm{R} 218$ & 0.061 & $0.219 \times 10^{-9}$ & $0.659 \times 10^{-9}$ \\
\hline $\mathrm{CF}_{3} \mathrm{I}$ & 0.057 & $0.210 \times 10^{-9}$ & $0.598 \times 10^{-9}$ \\
\hline RC318 & 0.062 & $0.222 \times 10^{-9}$ & $0.677 \times 10^{-9}$ \\
\hline RE347mcc & 0.058 & $0.231 \times 10^{-9}$ & $0.5553 \times 10^{-9}$ \\
\hline perfluorobutane & 0.061 & $0.233 \times 10^{-9}$ & $0.715 \times 10^{-9}$ \\
\hline perfluoropentane & 0.062 & $0.244 \times 10^{-9}$ & $0.765 \times 10^{-9}$ \\
\hline perfluorohexane & 0.060 & $0.254 \times 10^{-9}$ & $0.812 \times 10^{-9}$ \\
\hline neon & 0.060 & $0.131 \times 10^{-9}$ & $0.331 \times 10^{-9}$ \\
\hline krypton & 0.058 & $0.168 \times 10^{-9}$ & $0.437 \times 10^{-9}$ \\
\hline xenon & 0.058 & $0.182 \times 10^{-9}$ & $0.479 \times 10^{-9}$ \\
\hline hydrogen sulfide & 0.058 & $0.164 \times 10^{-9}$ & $0.447 \times 10^{-9}$ \\
\hline carbonyl sulfide & 0.056 & $0.182 \times 10^{-9}$ & $0.500 \times 10^{-9}$ \\
\hline carbon monoxide & 0.059 & $0.164 \times 10^{-9}$ & $0.437 \times 10^{-9}$ \\
\hline nitrous oxide & 0.057 & $0.159 \times 10^{-9}$ & $0.446 \times 10^{-9}$ \\
\hline ethylene oxide & 0.028 & $0.176 \times 10^{-9}$ & $0.506 \times 10^{-9}$ \\
\hline propylene oxide & 0.056 & $0.194 \times 10^{-9}$ & $0.567 \times 10^{-9}$ \\
\hline sulfur dioxide & 0.059 & $0.167 \times 10^{-9}$ & $0.485 \times 10^{-9}$ \\
\hline chlorine & 0.056 & $0.179 \times 10^{-9}$ & $0.486 \times 10^{-9}$ \\
\hline fluorine & 0.056 & $0.145 \times 10^{-9}$ & $0.385 \times 10^{-9}$ \\
\hline hydrogen chloride & 0.054 & $0.154 \times 10^{-9}$ & $0.424 \times 10^{-9}$ \\
\hline dimethyl carbonate & 0.059 & $0.204 \times 10^{-9}$ & $0.620 \times 10^{-9}$ \\
\hline acetone & 0.052 & $0.196 \times 10^{-9}$ & $0.586 \times 10^{-9}$ \\
\hline monoethanolamine & 0.065 & $0.173 \times 10^{-9}$ & $0.559 \times 10^{-9}$ \\
\hline diethanolamine & 0.068 & $0.185 \times 10^{-9}$ & $0.662 \times 10^{-9}$ \\
\hline ethylene glycol & 0.073 & $0.166 \times 10^{-9}$ & $0.542 \times 10^{-9}$ \\
\hline
\end{tabular}


Table 5. Coefficients for the surface tension, Eq. (25).

\begin{tabular}{|c|c|c|c|c|c|c|}
\hline Fluid & $\sigma_{0}$ & $\sigma_{1}$ & $\sigma_{2}$ & no $_{0}$ & $n_{1}$ & $n_{2}$ \\
\hline 3-methylpentane & 0.052645 & 0 & 0 & 1.232 & 0 & 0 \\
\hline 2,2-dimethylbutane & 0.0504886 & 0 & 0 & 1.24897 & 0 & 0 \\
\hline 2,3-dimethylbutane & 0.05235 & 0 & 0 & 1.24897 & 0 & 0 \\
\hline$n$-docosane & 0.052917 & 0 & 0 & 1.2768 & 0 & 0 \\
\hline 1-pentene & 0.050798 & 0 & 0 & 1.16356 & 0 & 0 \\
\hline propadiene & 0.056 & 0 & 0 & 1.205 & 0 & 0 \\
\hline 1,3-butadiene & 0.045947 & 0 & 0 & 0.960983 & 0 & 0 \\
\hline acetylene & 0.0615167 & 0 & 0 & 1.19797 & 0 & 0 \\
\hline 1-butyne & 0.0564795 & 0 & 0 & 1.06959 & 0 & 0 \\
\hline cyclobutene & 0.0651302 & 0 & 0 & 1.23574 & 0 & 0 \\
\hline R1140 (vinyl chloride) & 0.0655789 & 0 & 0 & 1.16473 & 0 & 0 \\
\hline R1123 (trifluoroethylene) & 0.0612 & 0 & 0 & 1.26 & 0 & 0 \\
\hline R150 (1,2-dichloroethane) & 0.0785663 & 0 & 0 & 1.19315 & 0 & 0 \\
\hline RE143a & 0.0371 & 0 & 0 & 0.98412 & 0 & 0 \\
\hline chlorobenzene & 0.0610108 & 0.0309068 & 0 & 1.13941 & 3.64067 & 0 \\
\hline R1224yd(Z) & 0.06195 & 0 & 0 & 1.277 & 0 & 0 \\
\hline $\mathrm{R} 1336 \mathrm{mzz}(\mathrm{Z})$ & 0.06 & 0 & 0 & 1.22 & 0 & 0 \\
\hline perfluorohexane & 0.0230631 & 0.0703415 & 0 & 0.98534 & 2.6579 & 0 \\
\hline propylene oxide & 0.073 & 0 & 0 & 1.22 & 0 & 0 \\
\hline chlorine & 0.0783601 & 0 & 0 & 1.28083 & 0 & 0 \\
\hline monoethanolamine & 0.0776613 & 0 & 0 & 0.801525 & 0 & 0 \\
\hline diethanolamine & 0.0859443 & 0 & 0 & 1.15945 & 0 & 0 \\
\hline ethylene glycol & 0.0731084 & 0 & 0 & 0.776849 & 0 & 0 \\
\hline
\end{tabular}


Table 6. Coefficients for the dedicated polynomial correlations of thermal conductivity, Eqs. (22-24).

\begin{tabular}{|l|l|l|}
\hline Fluid & R143a & RE347mcc \\
\hline$\alpha_{0}$ & $-7.00852 \times 10^{-3}$ & 0 \\
\hline$\alpha_{1}$ & $6.56307 \times 10^{-5}$ & -0.0239098 \\
\hline$\alpha_{2}$ & $2.62499 \times 10^{-8}$ & 0.0960335 \\
\hline$\alpha_{3}$ & 0 & -0.060505 \\
\hline$\alpha_{4}$ & 0 & 0.012299 \\
\hline$\beta_{1,1}$ & -0.0812212 & -0.00842403 \\
\hline$\beta_{1,2}$ & -0.0166652 & 0.0545889 \\
\hline$\beta_{1,3}$ & 0.0874477 & -0.0530301 \\
\hline$\beta_{1,4}$ & -0.0351468 & 0.0201447 \\
\hline$\beta_{1,5}$ & 0.0039957 & -0.0025046 \\
\hline$\beta_{2,1}$ & 0.0762355 & 0.00931228 \\
\hline$\beta_{2,2}$ & -0.0227662 & -0.0367016 \\
\hline$\beta_{2,3}$ & -0.0175726 & 0.0392477 \\
\hline$\beta_{2,4}$ & 0.00379467 & -0.0155674 \\
\hline$\beta_{2,5}$ & 0.000776919 & 0.00220816 \\
\hline
\end{tabular}


Table 7. Sample points for checking computer calculations for the viscosity, Eq. (1).

\begin{tabular}{|c|c|c|c|c|c|}
\hline fluid & $\begin{array}{c}T \\
\mathbf{K}\end{array}$ & $\begin{array}{l}\rho \\
\mathrm{mol} \cdot \mathrm{dm}^{-3}\end{array}$ & $\begin{array}{l}\eta^{*}(T) \\
\text { Eq. (8) } \\
\mu \mathrm{Pa} \cdot \mathrm{s}\end{array}$ & $\begin{array}{l}\Delta \eta(T, \rho) \\
\boldsymbol{\mu P a} \cdot \mathbf{s}\end{array}$ & $\begin{array}{l}\eta(T, \rho) \\
\mu \mathrm{Pa} \cdot \mathrm{s}\end{array}$ \\
\hline$n$-pentane & 422.7 & 6.548 & 9.600623 & 70.43086 & 80.03148 \\
\hline isopentane & 414.3 & 6.562 & 9.87305 & 67.45612 & 77.32917 \\
\hline neopentane & 390.4 & 6.434 & 8.969835 & 83.14565 & 92.11549 \\
\hline isohexane & 447.9 & 5.581 & 10.42747 & 76.59299 & 87.02045 \\
\hline 3-methylpentane & 455.4 & 5.639 & 10.69816 & 72.62745 & 83.32561 \\
\hline 2,2-dimethylbutane & 441 & 5.585 & 10.52772 & 76.90141 & 87.42913 \\
\hline 2,3-dimethylbutane & 450.5 & 5.652 & 10.67738 & 79.46832 & 90.14569 \\
\hline isooctane & 489.6 & 4.325 & 9.990441 & 82.52528 & 92.51572 \\
\hline n-docosane & 713 & 1.568 & 8.180269 & 113.6723 & 121.8525 \\
\hline propylene & 327.8 & 10.92 & 9.427081 & 64.91978 & 74.34686 \\
\hline isobutene & 376.3 & 8.515 & 10.01926 & 63.78265 & 73.80192 \\
\hline trans-2-butene & 385.7 & 8.529 & 9.820665 & 66.02451 & 75.84518 \\
\hline cis-2-butene & 392.2 & 8.669 & 9.973505 & 66.29859 & 76.27209 \\
\hline 1-butene & 377.4 & 8.494 & 9.819935 & 63.86093 & 73.68086 \\
\hline 1-pentene & 419.2 & 6.898 & 10.25376 & 65.13526 & 75.38902 \\
\hline propadiene & 358.2 & 11.972 & 10.67221 & 67.35075 & 78.02296 \\
\hline 1,3-butadiene & 382.6 & 9.139 & 10.7457 & 50.61067 & 61.35637 \\
\hline acetylene & 277.5 & 17.772 & 9.610704 & 61.26215 & 70.87285 \\
\hline propyne & 362.1 & 12.571 & 10.60779 & 70.01691 & 80.62469 \\
\hline 1-butyne & 388.8 & 9.61 & 11.02208 & 75.55447 & 86.57655 \\
\hline cyclopropane & 358.5 & 12.343 & 10.66446 & 72.24535 & 82.90981 \\
\hline cyclopentane & 460.5 & 7.777 & 11.83852 & 91.46665 & 103.3052 \\
\hline methylcyclohexane & 515 & 5.48 & 11.95601 & 95.47905 & 107.4351 \\
\hline propylcyclohexane & 567.7 & 4.223 & 11.82404 & 112.9976 & 124.8216 \\
\hline cyclobutene & 403.2 & 10.301 & 12.04322 & 77.59229 & 89.63551 \\
\hline dimethyl ether* & 360.3 & 12.001 & 11.09644 & 72.25466 & 83.35110 \\
\hline diethyl ether & 420 & 7.267 & 10.09324 & 72.62748 & 82.72072 \\
\hline hexamethyldisiloxane & 466.8 & 3.375 & 10.18113 & 73.86883 & 84.04997 \\
\hline octamethyltrisiloxane & 508.8 & 2.366 & 10.28641 & 120.7424 & 131.0288 \\
\hline decamethyltetrasiloxane & 539.5 & 1.836 & 10.14153 & 120.6249 & 130.7664 \\
\hline dodecamethylpentasiloxane & 566.1 & 1.47 & 10.04151 & 132.881 & 142.9225 \\
\hline tetradecamethylhexasiloxane & 587.9 & 1.229 & 9.736868 & 132.6810 & 142.4179 \\
\hline octamethylcyclotetrasiloxane & 527.8 & 2.176 & 11.09375 & 182.4992 & 193.593 \\
\hline decamethylcyclopentasiloxane & 556.5 & 1.718 & 10.86474 & 173.662 & 184.5268 \\
\hline dodecamethylcyclohexasiloxane & 581.2 & 1.371 & 10.1672 & 114.6773 & 124.8445 \\
\hline R40 & 374.7 & 14.671 & 13.56871 & 77.30141 & 90.87011 \\
\hline R1140 & 382.5 & 11.537 & 14.13538 & 76.39917 & 90.53455 \\
\hline
\end{tabular}




\begin{tabular}{|c|c|c|c|c|c|}
\hline fluid & $\begin{array}{l}T \\
\mathbf{K}\end{array}$ & $\begin{array}{l}\rho \\
\mathbf{m o l} \cdot \mathbf{d m}^{-3}\end{array}$ & $\begin{array}{l}\eta^{*}(T) \\
\text { Eq. (8) } \\
\mu \mathrm{Pa} \cdot \mathrm{s}\end{array}$ & $\begin{array}{l}\Delta \eta(T, \rho) \\
\mu \mathbf{P a} \cdot \mathbf{s}\end{array}$ & $\begin{array}{l}\eta(T, \rho) \\
\mu \mathrm{Pa} \cdot \mathrm{s}\end{array}$ \\
\hline R1123 & 298.6 & 12.527 & 14.09982 & 101.6719 & 115.7717 \\
\hline R143a & 311.3 & 10.627 & 11.72793 & 91.49722 & 103.2252 \\
\hline R1243zf & 339.2 & 8.901 & 13.15103 & 90.41507 & 103.5661 \\
\hline R150 & 505.4 & 8.821 & 15.56212 & 107.1092 & 122.6713 \\
\hline RE143a & 340.1 & 9.488 & 14.01787 & 109.5854 & 123.6032 \\
\hline chlorobenzene & 569.1 & 6.658 & 14.1066 & 87.56647 & 101.6731 \\
\hline R1234ze(Z) & 380.9 & 8.511 & 12.17959 & 93.27073 & 105.4503 \\
\hline R1233zd(E) & 395.6 & 7.561 & 13.73952 & 99.35177 & 113.0913 \\
\hline R245ca & 402.8 & 8.056 & 15.75873 & 139.1505 & 154.9093 \\
\hline R365mfc & 414 & 6.494 & 14.66741 & 120.3 & 134.9674 \\
\hline R1224yd(Z) & 385.8 & 7.275 & 13.99604 & 99.99117 & 113.9872 \\
\hline RE245cb2 & 366.1 & 6.908 & 14.25515 & 117.6941 & 131.9492 \\
\hline RE245fa2 & 400.4 & 7.154 & 15.21611 & 130.3583 & 145.5744 \\
\hline R236fa & 358.3 & 7.465 & 13.26679 & 128.6758 & 141.9426 \\
\hline R236ea & 371.2 & 7.645 & 13.69636 & 143.606 & 157.3023 \\
\hline R1336mzz(Z) & 400 & 6.422 & 13.79768 & 107.2355 & 121.0332 \\
\hline R114 & 376.9 & 6.897 & 14.09519 & 138.1366 & 152.2317 \\
\hline R218 & 310.5 & 6.771 & 13.00282 & 131.3963 & 144.3991 \\
\hline CF3I & 356.8 & 8.724 & 18.48168 & 150.0351 & 168.5168 \\
\hline RC318 & 349.5 & 6.379 & 13.79829 & 174.7525 & 188.5507 \\
\hline RE347mcc & 393.9 & 5.48 & 14.62967 & 135.4397 & 150.0694 \\
\hline perfluorobutane & 347.7 & 5.405 & 13.41915 & 124.3853 & 137.8044 \\
\hline perfluoropentane & 378.9 & 4.483 & 13.7027 & 146.0768 & 159.7795 \\
\hline perfluorohexane & 403.2 & 3.873 & 13.92935 & 156.9051 & 170.8344 \\
\hline methyl palmitate & 679.5 & 2.017 & 10.87873 & 135.0067 & 145.8854 \\
\hline methyl linolenate & 694.8 & 1.949 & 11.01249 & 114.1476 & 125.1601 \\
\hline methyl linoleate & 719.1 & 1.861 & 10.89515 & 137.4288 & 148.324 \\
\hline methyl oleate & 703.8 & 1.819 & 10.85542 & 176.9723 & \begin{tabular}{|l|}
187.8277 \\
\end{tabular} \\
\hline methyl stearate & 697.5 & 1.79 & 10.67771 & 140.4735 & 151.1512 \\
\hline neon & 40 & 45.956 & 6.006075 & 40.73116 & 46.73723 \\
\hline krypton & 188.5 & 21.274 & 16.32999 & 99.32927 & 115.6593 \\
\hline xenon & 260.8 & 16.517 & 20.11099 & 116.7744 & 136.8854 \\
\hline hydrogen sulfide* & 335.8 & 20.154 & 13.71425 & 83.45487 & 97.16912 \\
\hline carbonyl sulfide & 340.9 & 14.426 & 14.18906 & 90.60618 & 104.7952 \\
\hline carbon monoxide & 119.6 & 21.287 & 7.976226 & 48.72873 & 56.70495 \\
\hline nitrous oxide & 278.6 & 20.524 & 13.86694 & 77.10251 & 90.96945 \\
\hline ethylene oxide & 422 & 14.798 & 13.80012 & 85.49761 & 99.29773 \\
\hline propylene oxide & 439.3 & 10.502 & 12.48455 & 76.71464 & 89.19919 \\
\hline sulfur dioxide & 387.6 & 16.843 & 16.84906 & 110.5614 & 127.4105 \\
\hline
\end{tabular}




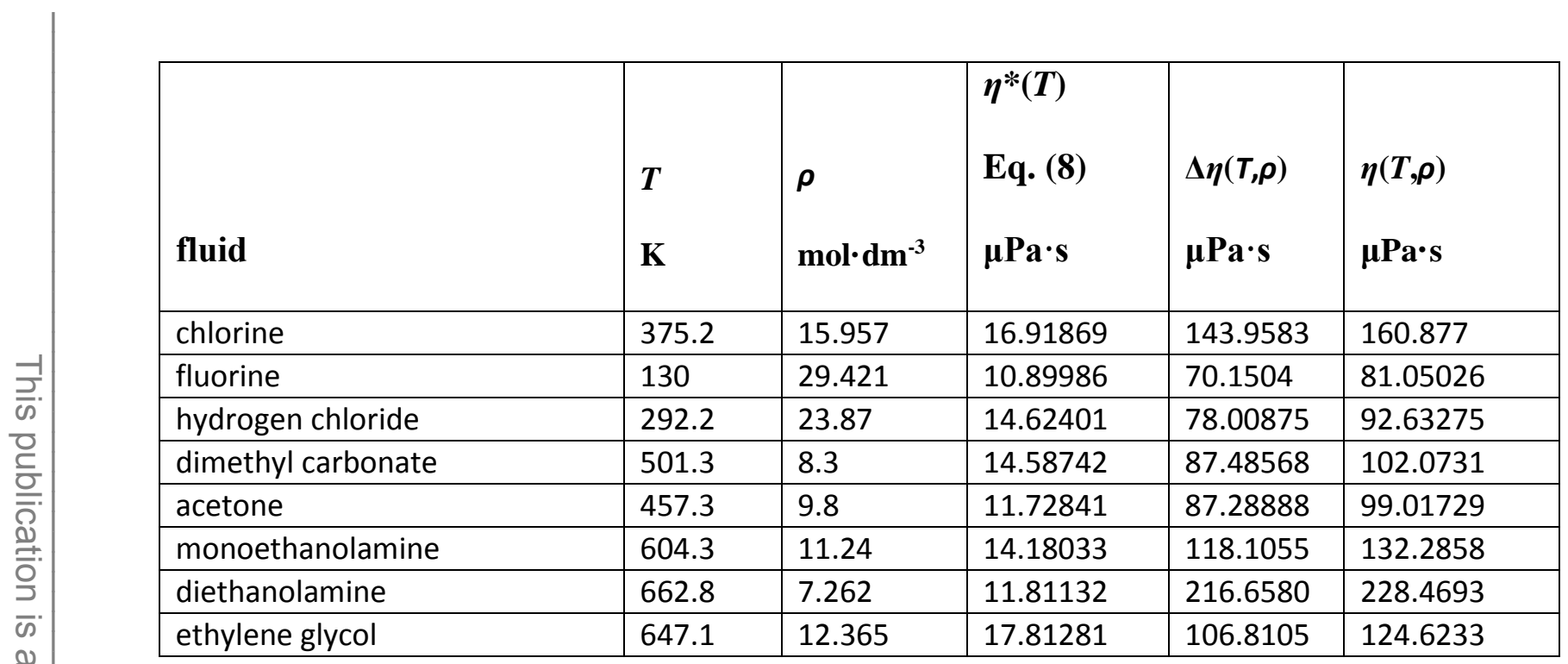

* Default model in REFPROP v10 is not ECS, values provided for information only, see text for specific fluid for recommended model. 
Table 8. Sample points for checking computer calculations for the thermal conductivity, Eq. (14).

\begin{tabular}{|c|c|c|c|c|c|c|}
\hline fluid & $\begin{array}{l}T \\
\mathbf{K}\end{array}$ & $\begin{array}{l}\rho \\
\mathbf{m o l} \cdot \mathbf{d m}^{-3}\end{array}$ & $\begin{array}{l}\lambda^{0}(T) \\
\text { Eq.(17)+Eq.(15) } \\
\mathrm{mW} \cdot \mathbf{m}^{-1} \cdot \mathrm{K}^{-1}\end{array}$ & $\begin{array}{l}\lambda^{\mathrm{r}}(T, \rho) \\
\text { Eq. (18) } \\
\mathrm{mW} \cdot \mathrm{m}^{-1} \cdot \mathrm{K}^{-1}\end{array}$ & $\begin{array}{l}\lambda^{\mathrm{crit}}(T, \rho) \\
\mathbf{m W} \cdot \mathbf{m}^{-1} \cdot \mathbf{K}^{-1}\end{array}$ & $\begin{array}{l}\lambda(T, \rho) \\
\mathbf{m W} \cdot \mathbf{m}^{-1} \cdot \mathbf{K}^{-1}\end{array}$ \\
\hline neopentane & 390.4 & 6.434 & 25.2462 & 41.7500 & 0.863 & 67.8593 \\
\hline isohexane & 447.9 & 5.581 & 28.9127 & 39.9509 & 1.033 & 69.8968 \\
\hline 3-methylpentane & 455.4 & 5.639 & 31.9953 & 37.4824 & 1.150 & 70.6275 \\
\hline 2,2-dimethylbutane & 441 & 5.585 & 29.1423 & 34.3672 & 1.067 & 64.5770 \\
\hline 2,3-dimethylbutane & 450.5 & 5.652 & 29.7736 & 36.5830 & 1.020 & 67.3762 \\
\hline isooctane & 489.6 & 4.325 & 29.5690 & 28.5203 & 1.009 & 59.0987 \\
\hline$n$-docosane & 713 & 1.568 & 34.4755 & 39.7879 & 0.608 & 74.8713 \\
\hline isobutene & 376.3 & 8.515 & 24.9523 & 47.5106 & 1.159 & 73.6222 \\
\hline trans-2-butene & 385.7 & 8.529 & 24.4650 & 48.7084 & 1.134 & 74.3078 \\
\hline cis-2-butene & 392.2 & 8.669 & 23.8568 & 49.2842 & 1.149 & 74.2905 \\
\hline 1-butene & 377.4 & 8.494 & 24.4889 & 47.3142 & 1.118 & 72.9207 \\
\hline 1-pentene & 419.2 & 6.898 & 26.6363 & 47.5056 & 1.166 & 75.3082 \\
\hline propadiene & 358.2 & 11.972 & 23.0754 & 65.3449 & 1.225 & 89.6455 \\
\hline 1,3-butadiene & 382.6 & 9.139 & 24.6868 & 49.3915 & 1.463 & 75.5409 \\
\hline acetylene & 277.5 & 17.772 & 19.4782 & 85.1698 & 1.316 & 105.9641 \\
\hline propyne & 362.1 & 12.571 & 23.3559 & 66.5147 & 1.336 & 91.2068 \\
\hline 1-butyne & 388.8 & 9.61 & 26.2465 & 53.8663 & 1.107 & 81.2199 \\
\hline cyclopropane & 358.5 & 12.343 & 22.4468 & 71.5362 & 1.212 & 95.1952 \\
\hline cyclobutene & 403.2 & 10.301 & 24.3786 & 54.0289 & 1.072 & 79.4797 \\
\hline dimethyl ether & 360.3 & 12.001 & 24.9937 & 72.8927 & 1.230 & 99.1164 \\
\hline diethyl ether & 420 & 7.267 & 27.3380 & 52.4383 & 1.069 & 80.8456 \\
\hline hexamethyldisiloxane & 466.8 & 3.375 & 26.2460 & 35.6256 & 0.751 & 62.6223 \\
\hline octamethyltrisiloxane & 508.8 & 2.366 & 26.6542 & 41.6701 & 0.449 & 68.7734 \\
\hline decamethyltetrasiloxane & 539.5 & 1.836 & 25.9943 & 42.1019 & 0.381 & 68.4771 \\
\hline dodecamethylpentasiloxane & 566.1 & 1.47 & 26.6158 & 36.0840 & 0.347 & 63.0474 \\
\hline tetradecamethylhexasiloxane & 587.9 & 1.229 & 26.1078 & 39.9592 & 0.326 & 66.3927 \\
\hline octamethylcyclotetrasiloxane & 527.8 & 2.176 & 27.3601 & 35.5189 & 0.307 & 63.1859 \\
\hline decamethylcyclopentasiloxane & 556.5 & 1.718 & 26.5248 & 32.6724 & 0.292 & 59.4893 \\
\hline dodecamethylcyclohexasiloxane & 581.2 & 1.371 & 25.4958 & 29.4701 & 0.395 & 55.3611 \\
\hline R40 & 374.7 & 14.671 & 15.6982 & 70.7431 & 1.213 & 87.6545 \\
\hline R1140 & 382.5 & 11.537 & 17.2473 & 66.3178 & 0.992 & 84.5575 \\
\hline R1123 & 298.6 & 12.527 & 15.7297 & 56.9454 & 0.572 & 73.2468 \\
\hline R1243zf & 339.2 & 8.901 & 17.5435 & 41.6143 & 0.768 & 59.9260 \\
\hline R150 & 505.4 & 8.821 & 21.4772 & 61.2642 & 0.931 & 83.6726 \\
\hline RE143a & 340.1 & 9.488 & 16.7241 & 45.2297 & 0.694 & 62.6479 \\
\hline chlorobenzene & 569.1 & 6.658 & 40.4643 & 43.4357 & 0.736 & 84.6360 \\
\hline R1234ze(Z) & 380.9 & 8.511 & 18.9540 & 45.7238 & 0.866 & 65.5442 \\
\hline
\end{tabular}




\begin{tabular}{|c|c|c|c|c|c|c|}
\hline fluid & $\begin{array}{c}T \\
\mathbf{K}\end{array}$ & $\begin{array}{l}\rho \\
\mathbf{m o l} \cdot \mathbf{d m}^{-3}\end{array}$ & $\begin{array}{l}\lambda^{0}(T) \\
\text { Eq.(17)+Eq.(15) } \\
\mathbf{m W} \cdot \mathbf{m}^{-1} \cdot \mathrm{K}^{-1}\end{array}$ & $\begin{array}{l}\lambda^{\mathrm{r}}(T, \rho) \\
\text { Eq. }(18) \\
\mathrm{mW} \cdot \mathrm{m}^{-1} \cdot \mathrm{K}^{-1}\end{array}$ & $\begin{array}{l}\lambda^{\mathrm{crit}}(T, \rho) \\
\mathbf{m W} \cdot \mathbf{m}^{-1} \cdot \mathbf{K}^{-1}\end{array}$ & $\begin{array}{l}\lambda(T, \rho) \\
\mathbf{m W} \cdot \mathbf{m}^{-1} \cdot \mathbf{K}^{-1}\end{array}$ \\
\hline R245ca & 402.8 & 8.056 & 24.1305 & 40.9368 & 0.644 & 65.7107 \\
\hline R365mfc & 414 & 6.494 & 24.7713 & 26.3136 & 0.665 & 51.7498 \\
\hline R1224yd(Z) & 385.8 & 7.275 & 15.7256 & 37.4867 & 0.720 & 53.9324 \\
\hline RE245cb2 & 366.1 & 6.908 & 17.1025 & 34.4065 & 0.611 & 52.1205 \\
\hline RE245fa2 & 400.4 & 7.154 & 19.2628 & 25.9076 & 0.638 & 45.8088 \\
\hline R236fa & 358.3 & 7.465 & 17.7008 & 38.6261 & 0.558 & 56.8852 \\
\hline R236ea & 371.2 & 7.645 & 20.0075 & 36.9195 & 0.546 & 57.4730 \\
\hline R1336mzz(Z) & 400 & 6.422 & 19.2298 & 33.6092 & 0.687 & 53.5264 \\
\hline R114 & 376.9 & 6.897 & 14.6517 & 30.6638 & 0.494 & 45.8090 \\
\hline $\mathrm{R} 218$ & 310.5 & 6.771 & 13.1834 & 28.1459 & 0.438 & 41.7675 \\
\hline CF3I & 356.8 & 8.724 & 10.0166 & 30.4463 & 0.389 & 40.8519 \\
\hline RC318 & 349.5 & 6.379 & 15.4231 & 34.4701 & 0.384 & 50.2769 \\
\hline perfluorobutane & 347.7 & 5.405 & 15.5928 & 40.4417 & 0.478 & 56.5125 \\
\hline perfluoropentane & 378.9 & 4.483 & 16.0248 & 38.1796 & 0.407 & 54.6119 \\
\hline perfluorohexane & 403.2 & 3.873 & 16.8869 & 36.9030 & 0.389 & 54.1785 \\
\hline neon & 40 & 45.956 & 9.2802 & 49.3757 & 0.662 & 59.3183 \\
\hline krypton & 188.5 & 21.274 & 6.0760 & 41.7658 & 0.465 & 48.3068 \\
\hline xenon & 260.8 & 16.517 & 4.7759 & 31.7721 & 0.385 & 36.9326 \\
\hline hydrogen sulfide & 335.8 & 20.154 & 16.3245 & 103.6125 & 1.321 & 121.2579 \\
\hline carbonyl sulfide & 340.9 & 14.426 & 14.0775 & 57.6791 & 0.865 & 72.6218 \\
\hline carbon monoxide & 119.6 & 21.287 & 10.4198 & 64.5627 & 0.747 & 75.7297 \\
\hline nitrous oxide & 278.6 & 20.524 & 15.7830 & 83.6194 & 1.226 & 100.6284 \\
\hline ethylene oxide & 422 & 14.798 & 25.8034 & 72.4105 & 2.061 & 100.2750 \\
\hline propylene oxide & 439.3 & 10.502 & 29.2792 & 69.1666 & 1.385 & 99.8309 \\
\hline sulfur dioxide & 387.6 & 16.843 & 13.7649 & 100.5614 & 1.095 & 115.4211 \\
\hline chlorine & 375.2 & 15.957 & 17.2956 & 76.9111 & 0.630 & 94.8363 \\
\hline fluorine & 130 & 29.421 & 11.4647 & 61.2024 & 0.934 & 73.6011 \\
\hline hydrogen chloride & 292.2 & 23.87 & 14.5130 & 183.6039 & 1.361 & 199.4777 \\
\hline dimethyl carbonate & 501.3 & 8.3 & 32.8743 & 58.1110 & 1.224 & 92.2091 \\
\hline acetone & 457.3 & 9.8 & 25.7637 & 69.4560 & 1.286 & 96.5053 \\
\hline monoethanolamine & 604.3 & 11.24 & 42.6644 & 122.9511 & 1.527 & 167.1429 \\
\hline diethanolamine & 662.8 & 7.262 & 46.7435 & 94.6571 & 0.757 & 142.1576 \\
\hline ethylene glycol & 647.1 & 12.365 & 62.0303 & 140.0711 & 1.976 & 204.0773 \\
\hline
\end{tabular}


Table 9. Sample points for checking computer calculations for the thermal conductivity, Eq. (22).

\begin{tabular}{|l|l|l|l|l|l|l|}
\hline Fluid & $\boldsymbol{T}$ & $\boldsymbol{\rho}$ & $\lambda^{\mathbf{0}}(\boldsymbol{T})$ & $\begin{array}{l}\Delta \lambda^{\text {res }}(\boldsymbol{T}, \boldsymbol{\rho}) \\
\mathbf{m W} \cdot \mathbf{m}^{-\mathbf{1}} \cdot \mathbf{K}^{-1}\end{array}$ & $\begin{array}{l}\lambda^{\text {crit }}(\boldsymbol{T}, \boldsymbol{\rho}) \\
\mathbf{m W} \cdot \mathbf{m}^{-\mathbf{1}} \cdot \mathbf{K}^{-1}\end{array}$ & $\begin{array}{l}\lambda(\boldsymbol{T}, \boldsymbol{\rho}) \\
\mathbf{m W} \cdot \mathbf{m}^{-\mathbf{1}} \cdot \mathbf{K}^{-1}\end{array}$ \\
\hline R143a & 311.27 & 10.6289 & 15.9637 & 46.0375 & 3.5104 & 65.5116 \\
\hline RE347mcc & 393.93 & 5.4797 & 20.2295 & 28.3688 & 0.7942 & 49.3925 \\
\hline
\end{tabular}

Table 10. Sample points for checking computer calculations for surface tension, Eq. (25).

\begin{tabular}{|l|l|l|}
\hline Fluid & $\boldsymbol{T}(\mathbf{K})$ & $\boldsymbol{\sigma}\left(\mathbf{N} \cdot \mathbf{m}^{-1}\right)$ \\
\hline 3-methylpentane & 455.4 & 0.0030857 \\
\hline 2,2-dimethylbutane & 441.0 & 0.0028459 \\
\hline 2,3-dimethylbutane & 450.5 & 0.0029538 \\
\hline$n$-docosane & 713.0 & 0.0027968 \\
\hline 1-pentene & 419.2 & 0.0034827 \\
\hline propadiene & 358.2 & 0.0034929 \\
\hline 1,3-butadiene & 382.6 & 0.0050290 \\
\hline acetylene & 277.5 & 0.0038951 \\
\hline 1-butyne & 388.8 & 0.0048117 \\
\hline cyclobutene & 403.2 & 0.0037848 \\
\hline R1140 & 382.5 & 0.0044838 \\
\hline R1123 & 298.6 & 0.0033577 \\
\hline R150 & 505.4 & 0.0050403 \\
\hline RE143a & 340.1 & 0.0038511 \\
\hline chlorobenzene & 569.1 & 0.0044341 \\
\hline R1224yd(Z) & 385.8 & 0.0032758 \\
\hline R1336mzz(Z) & 400.0 & 0.0036203 \\
\hline perfluorohexane & 403.2 & 0.0025401 \\
\hline propylene oxide & 439.3 & 0.0043986 \\
\hline chlorine & 375.2 & 0.0041019 \\
\hline monoethanolamine & 604.3 & 0.0122595 \\
\hline diethanolamine & 662.8 & 0.0059581 \\
\hline ethylene glycol & 647.1 & 0.0122213 \\
\hline & & \\
\hline
\end{tabular}

